\title{
Used Fuel Management System Architecture Evaluation, Fiscal Year 2012
}

\section{Fuel Cycle Research \& Development}

\author{
Prepared for \\ U.S. Department of Energy \\ Used Fuel Disposition Campaign and \\ Nuclear Fuel Storage and \\ Transportation Planning Project \\ Mark Nutt, Edgar Morris, Francesc Puig (ANL) \\ Joe Carter, Phil Rodwell, Alexcia Delley \\ (SRNL) \\ Rob Howard, Dominic Giuliano (ORNL)
}

October 31, 2012 FCRD-NFST-2013-000020 Rev. 0

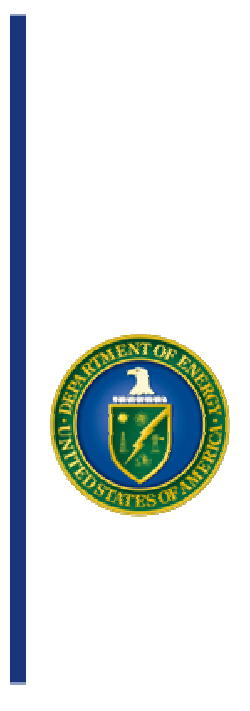




\section{DISCLAIMER}

This information was prepared as an account of work sponsored by an agency of the U.S. Government. Neither the U.S. Government nor any agency thereof, nor any of their employees, makes any warranty, expressed or implied, or assumes any legal liability or responsibility for the accuracy, completeness, or usefulness, of any information, apparatus, product, or process disclosed, or represents that its use would not infringe privately owned rights. References herein to any specific commercial product, process, or service by trade name, trade mark, manufacturer, or otherwise, does not necessarily constitute or imply its endorsement, recommendation, or favoring by the U.S. Government or any agency thereof. The views and opinions of authors expressed herein do not necessarily state or reflect those of the U.S. Government or any agency thereof. 


\section{FCT Quality Assurance Program Document}

\section{Appendix E}

\section{FCT Document Cover Sheet}

Name/Title of Deliverable/Milestone

Work Package Title and Number

Work Package WBS Number

Responsible Work Package Manager
Used Fuel Management System Architecture Evaluation, Fiscal Year 2012 / M2FT-12AN0814012

CX - Perspectives on NW Management - ANL/FT-12AN081401

$\underline{1.02 .08 .14}$

W. Mark Nutt (electronic signature)

(Name/Signature)

Date Submitted

Quality Rigor Level for

Deliverable/Milestone

This deliverable was prepared in accordance with

QA program which meets the requirements of
区 DOE Order 414.1
NQA-1-2000

This Deliverable was subjected to:

$\square$ Technical Review

Technical Review (TR)

Review Documentation Provided

$\square$ Signed TR Report or,

Signed TR Concurrence Sheet or,

Signature of TR Reviewer(s) below

Name and Signature of Reviewers

Thomas Cotton
Peer Review

Peer Review (PR)

Review Documentation Provided

Signed PR Report or,

Signed PR Concurrence Sheet or,

Signature of PR Reviewer(s) below

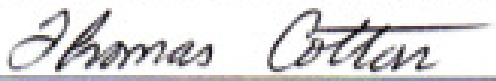

*Note: In some cases there may be a milestone where an item is being fabricated, maintenance is being performed on a facility, or a document is being issued through a formal document control process where it specifically calls out a formal review of the document. In these cases, documentation (e.g., inspection report, maintenance request, work planning package documentation or the documented review of the issued document through the document control process) of the completion of the activity along with the Document Cover Sheet is sufficient to demonstrate achieving the milestone. QRL for such milestones may be also be marked N/A in the work package provided the work package clearly specifies the requirement to use the Document Cover Sheet and provide supporting documentation. 


\section{SUMMARY}

\section{Introduction and Objectives}

In the 1990s the U.S. Department of Energy (DOE) completed a number of system analyses investigating consolidated interim storage as a part of the waste management solution. These analyses are "dated" and do not reflect the present situation regarding at-reactor used nuclear fuel (UNF) management, alternatives for away from reactor management of used nuclear fuel, and alternatives for the ultimate disposal of UNF. The Blue Ribbon Commission for America's Nuclear Future and the Nuclear Waste Technical Review Board have also pointed out the need for such analyses.

The Used Fuel Management System Architecture Evaluation effort provides the DOE and others with information regarding the various alternatives for managing UNF generated by the current fleet of light water reactors operating in the U.S. The objectives are to:

- Provide quantitative information with respect to a broad range of UNF management alternatives and considerations

- Develop an integrated approach to evaluating storage, transportation, and disposal options, with emphasis on flexibility

- Evaluate impacts of storage choices on UNF storage, handling, and disposal options

- Identify alternative strategies and evaluate with respect to cost and flexibility

- Consider a broad range of factors including repository emplacement capability, thermal constraints, repackaging needs, storage and transportation alternatives, and impacts on utility operations.

In Fiscal Year 2012 system-level analyses of the overall interface between at-reactor, consolidated storage, and ultimate disposition along with the development of supporting logistic simulation tools were initiated. The objective of the Fiscal Year 2012 effort was two-fold: 1) develop methodologies, approaches, and tools (capability development), and 2) evaluate select UNF disposition scenarios (capability demonstration). The scenarios chosen for evaluation and the assumptions, inputs, and boundary conditions selected allowed for an initial set of analyses to gain insight regarding integrated system dynamics and an understanding of trends. This initial set of analyses also points to where additional system architecture analyses should focus.

\section{R\&D Overview}

An important waste management system interface consideration is the need for ultimate disposal of UNF fuel assemblies contained in waste packages sized to be compatible with the geologic medium of the final repository. Thermal analyses completed by the Used Fuel Disposition Campaign indicate that waste package sizes for the geologic media under consideration by the Used Fuel Disposition Campaign are significantly smaller than the canisters being used for on-site dry storage by the nuclear utilities. Therefore, at some point along the UNF disposition pathway there may be a need to re-package fuel assemblies already loaded into the types of dry storage canisters currently in use unless the feasibility of direct disposal of these large canisters can be demonstrated. Note that evaluating the feasibility of the direct disposal of dual purpose canisters is underway.

A high-level diagram of the alternative UNF disposition pathways is shown in Figure S-1 and involves UNF storage at a consolidated storage facility (CSF) and UNF packaging/re-packaging prior to ultimate disposal.

\section{Fiscal Year 2012 Activities}

The analysis began with the development of a disposition pathway framework, which is a detailed and comprehensive expansion of Figure S-1.

While the reactors will continue to transfer UNF to dry storage, there will always be UNF in the used fuel pools, at least until a reactor is shut down and decommissioned. Another important aspect is how the UNF residing in the used fuel pools is managed when fuel acceptance from the reactor sites begins. UNF residing in 
the pools can be transported off-site in re-useable transportation casks, placed in dual-purpose canisters suitable for both storage and transportation, or placed in a standard canister once one is designed and licensed. This choice impacts the design of both a CSF (canistered fuel storage only or canistered and bare fuel storage) and the quantity of UNF that would ultimately have to be re-packaged.

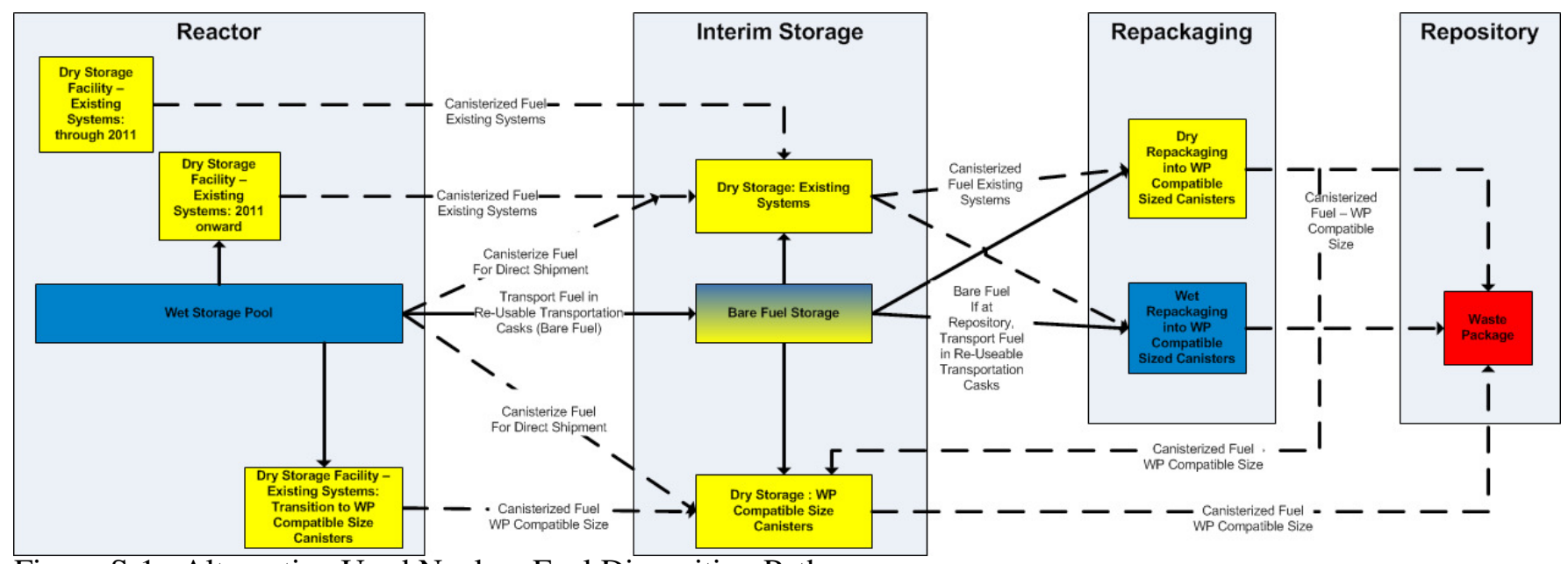

Figure S-1. Alternative Used Nuclear Fuel Disposition Pathways

These considerations resulted in the identification of nine potential disposition pathways that consider how UNF would be transported from the reactors, where UNF packaging/re-packaging would be performed (repository or CSF), and when UNF packaging/re-packaging would be performed (at CSF receipt or prior to shipment from the CSF to a repository). These nine disposition pathways were evaluated considering complexity and flexibility, resulting in a down-select of the disposition pathways that would be considered in FY12 to four, representing the possible combinations of two features: what would be accepted from reactors by the waste management system (fuel packaged into existing size canisters only, or bare fuel as well as canisterized fuel), and where/when the canisterized fuel would be packaged/re-packaged for disposal (at a CSF when the fuel is about to be sent to the repository, or at the repository when fuel is received there). The packaging/re-packaging of bare fuel/canisters into disposal size canisters at reactors or into either existing size or disposal size canisters at CSF receipt were not evaluated in this phase of the analysis.

The cases considered are summarized in Table ES-1 (see Section 3.1 for details regarding each case).

Table ES-1. TSL Case Matrix

\begin{tabular}{|c|c|c|c|c|}
\hline & Case 1 & Case 2 & Case 3 & Case 4 \\
\hline $\begin{array}{l}\text { Transport From } \\
\text { Reactors }\end{array}$ & $\begin{array}{l}\text { Existing Size } \\
\text { Canisters }\end{array}$ & $\begin{array}{c}\text { Existing Size } \\
\text { Canisters / } \\
\text { Bare Fuel }\end{array}$ & $\begin{array}{c}\text { Existing Size } \\
\text { Canisters }\end{array}$ & $\begin{array}{c}\text { Existing Size } \\
\text { Canisters / } \\
\text { Bare Fuel }\end{array}$ \\
\hline CSF & $\begin{array}{l}\text { Existing-Size } \\
\text { Canisters }\end{array}$ & $\begin{array}{c}\text { Existing Size } \\
\text { Canisters / } \\
\text { Bare Fuel }\end{array}$ & $\begin{array}{c}\text { Existing-Size } \\
\text { Canisters }\end{array}$ & $\begin{array}{c}\text { Existing Size } \\
\text { Canisters / } \\
\text { Bare Fuel }\end{array}$ \\
\hline $\begin{array}{l}\text { Package/ } \\
\text { Re-Package at ==> }\end{array}$ & Repository & Repository & CSF & CSF \\
\hline $\begin{array}{l}\text { Transport from CSF to } \\
\text { MGR }\end{array}$ & $\begin{array}{l}\text { Existing-Size } \\
\text { Canisters }\end{array}$ & $\begin{array}{c}\text { Existing Size } \\
\text { Canisters / } \\
\text { Bare Fuel }\end{array}$ & $\begin{array}{c}\text { Waste } \\
\text { Package Size } \\
\text { Canisters }\end{array}$ & $\begin{array}{c}\text { Waste } \\
\text { Package Size } \\
\text { Canisters }\end{array}$ \\
\hline
\end{tabular}


A range of input parameters was then determined for evaluating each disposition pathway. Parameters selected include start of CSF operations (2020, 2035), start of repository operations (2040, 2055), UNF acceptance rates (1500, 3000, and $6000 \mathrm{MTHM} / \mathrm{yr}$ ), and waste package size (4/9, 12/24, 21/44 PWR/BWR assemblies). The combination of disposition pathways and input parameters results in 36 individual scenarios that were evaluated.

In a parallel, the Transportation-Storage Logistics (TSL) simulator was developed. This effort involved the modification and enhancement of legacy UNF logistics simulators previously developed by the DOE to result in a logistic simulator capable of modeling the range of disposition pathways and input parameters discussed above. The TSL was used to simulate each of the 36 individual scenarios to provide information on a range of logistic parameters including quantities of UNF in at-reactor dry storage, shipping rates for the different types of dry storage canisters and bare fuel assemblies from the reactors, receipt rates at the CSF and the repository, quantities of UNF and canister types in dry storage, and number of canisters and UNF assemblies that are packaged and re-packaged.

This quantitative logistic information was then used as input to pre-conceptual design development for consolidated storage facilities, packaging/re-packaging facilities and repository surface facilities. The information was also used for making rough order of magnitude cost estimates.

The CSF design concepts and facility sizes differ depending on the scenario and UNF receipt rates (vertical, horizontal, and bare fuel storage). In addition, the following influence the CSF and packaging/re-packaging facility design concepts: 1) the way existing legacy and continued use of dual purpose canisters (and single purpose storage casks) are managed, 2) the inventory and mix of canisters (vertical/horizontal) entering the waste management system, and 3) the strategy for managing UNF in fuel pools once CSF begins operation.

\section{High-Level Insights and Observations}

The objectives of the Fiscal Year 2012 Used Fuel Management System Architecture Evaluation were achieved. Methodologies, approaches, and tools were developed (Capability Development) and used to evaluate select UNF disposition scenarios (Capability Demonstration). This effort re-established an important, foundational capability to assess potential UNF management options. The purpose of the evaluation and analysis at this stage is to use insights gained to refine and focus the next phase of analysis, rather than to provide a basis for any decisions about the design of operation of the waste management system. These high-level insights include:

The rate that UNF is processed has a significant effect on the used fuel management system: The results of this evaluation confirmed that the rate that UNF is transported between facilities, received at a CSF or a repository, and processed through a packaging/re-packaging facility effects the size of the facilities and associated infrastructure and the associated costs. Larger throughput rates result in larger facilities and higher costs.

There is also a trade-off with higher acceptance rates resulting in reduced at-reactor storage requirements, but larger facilities down-stream in the waste management system. A large UNF acceptance rate, $6000 \mathrm{MTHM} / \mathrm{yr}$, showed only incremental benefit in reducing on-site storage, but resulted in the largest facility requirements down-stream.

Thermal considerations have a major impact on the operation of the system: The entire UNF management system will have thermal constraints. There are thermal limits on storage canisters, transportation overpacks/casks, and on geologic media. Thermal constraints on transportation, which are more stringent than the constraints on storage canisters, mean that loading fuel into very large storage canisters at reactor sites may require storage of those canisters for decades before they could be moved due to thermal limits on the transportation overpacks/casks. These thermal constraints become more of an issue for higher UNF acceptance rates from the reactors because the older, cooler fuel is transported from the reactor sites relatively soon after acceptance begins, leaving the hotter, younger fuel to be managed. Lower acceptance rates allow this fuel to cool sufficiently and it can be transported. 
Thermal limits for geologic disposal design concepts, not considered in this evaluation, could also require extended decay storage and/or the blending of UNF during packaging/re-packaging, potentially impacting when UNF could be shipped to a repository and how it would be managed at the CSF or repository.

The implications of these thermal constraints and potential UNF management alternatives should be evaluated further.

A large acceptance rate, on the order of $6000 \mathrm{MTHM/yr}$ may result in under-utilized facilities and may not be cost-effective: The analysis of a $6000 \mathrm{MTHM} / \mathrm{yr}$ acceptance rate, which is nearly three times the used fuel generation rate ( $2000 \mathrm{MTHM} / \mathrm{yr})$, shows that such a high rate may not be cost-effective in the long run. Even though it removes fuel from reactor sites more quickly, and thereby reduces the costs of at reactor storage, it requires large initial infrastructure expenditure in both transportation equipment and receipt facilities at the CSF that can only be used at the full capacity for a relatively short period of time. This is due to fuel shipment restrictions based on thermal constraints. A $6000 \mathrm{MTHM} / \mathrm{yr}$ acceptance rate only occurs at the beginning of operation of the system until these thermal constraints restrict the achievable acceptance rate to a rate matching UNF discharge from the reactors (around $2000 \mathrm{MTHM} / \mathrm{yr}$ ). It is worth exploring rates in the area of 4000-4500 $\mathrm{MTHM} / \mathrm{yr}$ to determine if some of the benefits of an increased acceptance rate can be achieved without the spikes in UNF shipments that occur and under-utilization issues observed in the $6000 \mathrm{MTHM} / \mathrm{yr}$ acceptance scenarios.

The start of UNF acceptance from the reactors and the UNF acceptance rate will impact on-site dry storage requirements: The results presented in this report show that there is a significant decrease in the maximum amount of at-reactor dry storage required when the acceptance rate increases from $1500 \mathrm{MHMT} / \mathrm{yr}$ to 3000 $\mathrm{MTHM} / \mathrm{yr}$. However, there is a much smaller reduction in the maximum at-reactor dry storage capacity required when the acceptance rate is increased from $3000 \mathrm{MTHM} / \mathrm{yr}$ to $6000 \mathrm{MTHM} / \mathrm{yr}$.

Higher acceptance rates (3000 MTHM/yr, 6000 MTHM/yr) may not eliminate need for additional on-site dry storage when reactor fleet begins to shut down unless acceptance is "managed." A youngest-fuel-first acceptance preference will still require additional dry storage when reactors shut down. An oldest-fuel-first acceptance preference would require additional dry storage both during reactor operation and when the reactors shut down as preference would be given to shipping UNF already in dry storage from the reactor sites.

An oldest-fuel-first (OFF) acceptance preference would also require continued at-reactor dry storage. Generally, older fuel is the first UNF loaded into dry storage and would be the first shipped under such an acceptance preference. Since little fuel would be shipped directly from the used fuel pools, the reactors would have to transfer fuel from the pools to dry storage to maintain pool capacity.

Acceptance start date and acceptance rates can reduce flexibility: Lower UNF acceptance rates or delay in start of acceptance results in more UNF being placed into existing large canisters for at-reactor dry storage. This "hardens" this "boundary condition," resulting in reduced flexibility later.

The transportation capital cost is driven by the number of casks and transportation assets that need to be purchased during the campaign. The rate that UNF is transported between the reactors, CSF, and repository and the cycle time needed to transport the UNF affect the size of the transportation fleet. While the location of the storage facilities and repository drive the transportation time, transportation is actually only a small contributor to the overall cycle time. Loading, unloading, and maintenance activities make up the majority of the cycle time. Therefore, the acquisitions required are for the most part relatively independent of the location of the storage facility and repository.

Acceptance priority assumptions have a significant impact on the UNF management system: The management of UNF at the reactor sites define the "boundary condition" to which the system will respond. The acceptance priority assumptions, rates, and start dates assumed for this evaluation have a very significant impact on the transportation system and on the sizing of facilities. The preliminary analyses presented here suggest that there would be value to examining modifications to the acceptance priorities that would smooth out some of the sharp peaks and fluctuations that result from strict adherence to simple but rigid priority rules.

Alternate strategies for acceptance from reactors and subsequent shipment to a repository may allow for optimization of down-stream facilities: This initial evaluation assumed first-in-first-out shipping of UNF from the CSF to the repository. It is unlikely that the waste management system would be operated in this manner. 
A CSF could be treated as an integrated UNF management facility to act as a buffer between at-reactor UNF management needs and future repository requirements. This would allow for optimizing shipments from reactors to minimize additional on-site dry storage requirements and optimizing shipments from the CSF to the repository to meet repository requirements while minimizing processing facility requirements.

In addition, a managed UNF acceptance rate, perhaps giving preference to removing fuel from sites that are either shutdown or approaching shutdown, could potentially reduce long-term at-reactor dry storage requirements.

System benefits may also be gained by de-coupling acceptance rates from the reactors to a CSF with shipping rates between the CSF and a repository. For example, a shorter emplacement period with a higher emplacement rate at the repository than the acceptance rate from the reactors to the CSF combined with a later repository start could result in a large inventory of spent fuel at the CSF that could be more efficiently processed when the repository begins operating.

Such approaches may require additional CSF storage capacity. Additional evaluation of these approaches is needed.

A large-scale UNF handling effort will be needed regardless of the UNF management strategy, acceptance rates, and acceptance start dates: There will always be a need to re-package large canisters unless the direct disposability of such canisters is shown to be feasible. Approximately 11,200 canisters will have to be repackaged if all UNF is placed in such canisters. Maintaining some fraction of the UNF as bare fuel at central storage facilities can reduce the number of canisters that would have to be re-packaged. However, placing the entire UNF inventory in large canisters does not appear to require an increase in the packaging/re-packaging facility capabilities versus maintaining some fraction of the UNF as bare fuel. In addition, the capability to store bare fuel in addition to storing UNF in canisters would be required. There could also be broader systemlevel impacts associated with maintaining a fraction of the UNF as bare fuel that have yet to be evaluated (e.g., worker dose). Any potential benefit of not having to re-open canisters reduces for lower acceptance rates and/or delay in the start of acceptance.

Smaller waste package sizes have a significant impact on packaging/re-packaging facility and transportation system requirements. Processing a large number of smaller disposal canisters could result in the need for larger packaging/re-packaging facilities and a larger transportation infrastructure to meet the desired system throughput. Future work investigating alternative disposal canister/overpack and transportation equipment design concepts may identify more efficient concepts.

Bare fuel storage in wet pools at a CSF will likely lead to high CSF and overall system life-cycle costs. Future analyses of scenarios involving CSF storage of bare UNF should investigate alternative bare fuel storage concepts (such as dry storage vaults or single-purpose bolted lid bare UNF storage casks).

At-Reactor operational and logistic constraints could affect the actual rate that UNF could be loaded into dry storage canisters or transported off-site. Logistic analyses typically assume that there are no constraints on the ability of the reactor sites to load dry storage systems or transport UNF off-site. These assumptions could potentially be challenged while a reactor is in operation due to multiple requirements and demands on the used fuel pool during and operating fuel cycle. Such demands include receipt of fresh fuel, core re-load, fuel inspections/repair, and maintenance of the spent fuel pool. The actual window where fuel handling could occur may constrain the amount of used fuel that could be transferred to dry storage when either multiple fuel handling activities occur within a given operating fuel cycle (transfer to dry storage and loading for shipping off-site) or potentially when smaller capacity canisters are loaded (waste package compatible size canisters). These constraints should be further explored and their impacts on at-reactor logistics evaluated.

Rough order of magnitude life cycle cost (ROM LCC) estimates of the entire nuclear waste management system varied depending on the scenario. The estimated ROM LCC was found to be approximately $10 \%$ to $20 \%$ larger when UNF packaging/re-packaging is performed at the CSF versus at the repository. The estimated ROM LCC was also found to be approximately $20 \%$ to $85 \%$ larger than when all the UNF is loaded into existing size canisters at the reactor sites.

Higher throughput requires larger facilities and infrastructure. When considering the total away-from-reactor estimated ROM LCC, the lowest cost occurs for a UNF processing rate of $1500 \mathrm{MTHM} / \mathrm{yr}$ when re-packaging 
is done at the CSF and for a UNF processing rate of $3000 \mathrm{MTHM} / \mathrm{yr}$ when re-packaging is done at the repository.

However, the overall estimated ROM LCC of UNF management (all aspects of the UNF management system) is lowest when the UNF processing rate (acceptance from reactors, shipment from CSF to repository) is 3000 MTHM/yr, CSF operations begin in 2020, and repository operations begin in 2040 for each of the major cases considered..

Delaying the start of repository operations from 2040 to 2055 increases the estimated total UNF management ROM LCC by $5 \%-10 \%$ for a $1500 \mathrm{MTHM} / \mathrm{yr}$ throughput rate, $15 \%-20 \%$ for a throughput rate of 3000 $\mathrm{MTHM} / \mathrm{yr}$, and $10 \%-15 \%$ for a throughput rate of $6000 \mathrm{MTHM} / \mathrm{yr}$. 



\section{CONTENTS}

SUMMARY iv

ACRONYMS vii

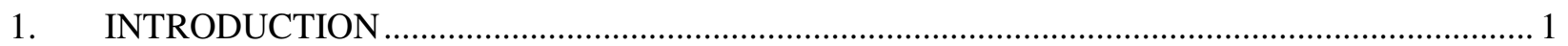

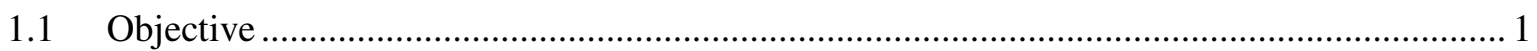

1.1.1 Fiscal Year 2012 Objectives .......................................................................... 2

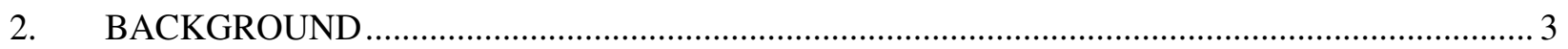

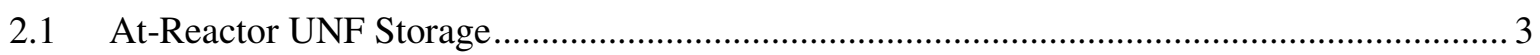

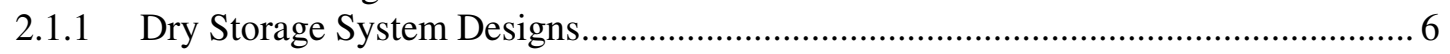

2.2 Disposal Media and Repository Design Concepts ........................................................ 7

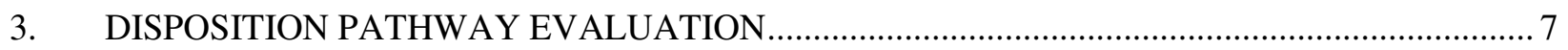

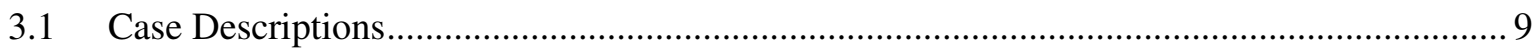

3.1.1 Case 1: Canisterize at Reactor / Re-Package into Waste Package

Compatible Size Canisters at a Re-Packaging Facility Co-Located with the Repository ..

3.1.2 Case 2: Bare Fuel Handling / Re-Packaging into Waste Package

Compatible Size Canisters at a Re-Packaging Facility Co-Located with the

Repository .....

3.1.3 Case 3: Canisterize at Reactor / Re-Package into Waste Package

Compatible Size Canisters at a Re-Packaging Facility Co-Located with the

CSF Prior to Repository Transport

3.1.4 Case 4: Bare Fuel Handling / Re-Packaging into Waste Package

Compatible Size Canisters at a Re-Packaging Facility Co-Located with the

CSF Prior to Repository Transport

3.1.5 Case 5: Canisterize at Reactor / Re-Package into Waste Package

Compatible Size Canisters at CFS Receipt at a Re-Packaging Facility Co-

Located with the CSF.

3.1.6 Case 6: Bare Fuel Processing / Re-Packaging into Waste Package

Compatible Size Canisters at CSF Receipt at a Re-Packaging Facility Co-

Located with the CSF.

3.1.7 Case 7: Bare Fuel Processing / Packaging into Existing Size Canisters at CSF Receipt -Re-Package into Waste Package Compatible Size Canisters at a Re-Packaging Facility Co-Located with the Repository .

3.1.8 Case 8: Bare Fuel Processing / Packaging at CSF Receipt - Existing Size Canisters, Re-Package at a Re-Packaging Facility Co-Located with the CSF

Prior to Repository Transport

3.1.9 Case 9: Transition to Loading Waste Package Compatible Size Canisters at Reactor at Future Time

3.2 Initial Qualitative Evaluation of Cases ................................................................... 25

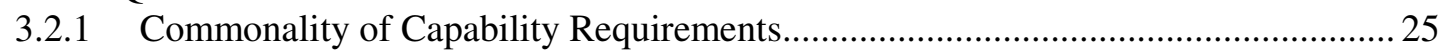

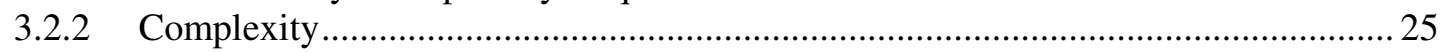

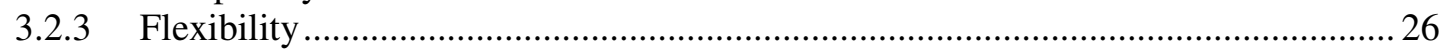

3.2.4 Cases for Detailed Qualitative and Quantitative Evaluation.................................. 27

3.3 Disposition Pathway Evaluation Assumptions and Input/Boundary Conditions .................. 28 


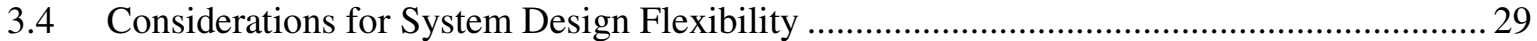

3.4.1 Sequential and Modular Facility Development........................................................ 30

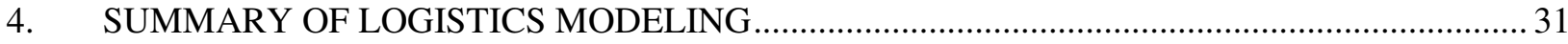

4.1 Summary of Logistic Simulation Results: At-Reactor UNF Management ........................... 32

4.2 Summary of Logistic Results: Consolidated Storage Facility UNF Management................ 36

4.3 Summary of Logistic Results: UNF Packaging/Re-Packaging ........................................ 41

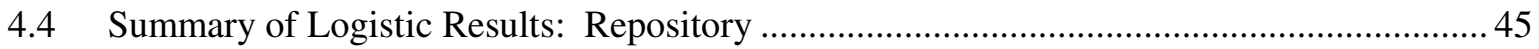

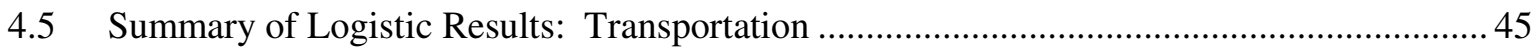

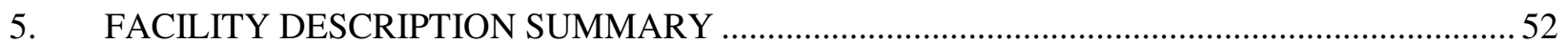

5.1 Consolidated Storage Facility (CSF) Scope and Functionality ......................................... 52

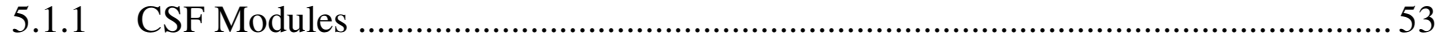

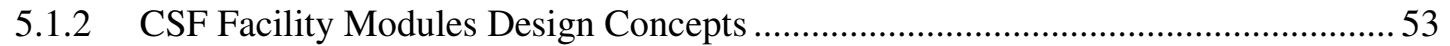

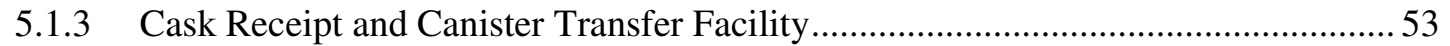

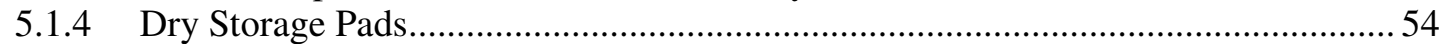

5.1.5 DSC Storage Vault and Canister Transfer Facility ..................................................... 54

5.1.6 Bare Fuel Receipt and Storage Facility ................................................................ 55

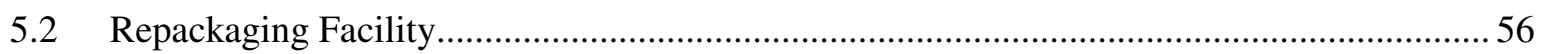

6. ROUGH ORDER OF MAGNITUDE COST ESTIMATES ….................................................... 58

6.1 At Reactor Rough Order of Magnitude Cost Estimates .................................................... 58

6.2 Consolidated Storage Rough Order of Magnitude Cost Estimates ...................................... 66

6.3 Packaging/Re-Packaging Facility Rough Order of Magnitude Cost Estimates .................... 73

6.4 Transportation Rough Order of Magnitude Cost Estimates ................................................... 76

6.5 Back-End Total Life Cycle Rough Order of Magnitude Cost Estimates ............................... 78

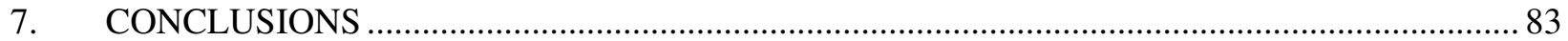

\section{FIGURES}

Figure S-1. Alternative Used Nuclear Fuel Disposition Pathways ........................................................

Figure 2-1. Historical and Projected UNF Discharges and Transfer to Dry Storage ................................ 3

Figure 2-2. Locations of independent spent fuel storage installations ................................................... 5

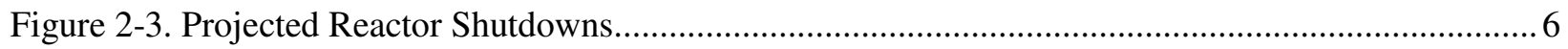

Figure 3-1. UNF Management - Overall Options and Alternatives ..................................................... 10

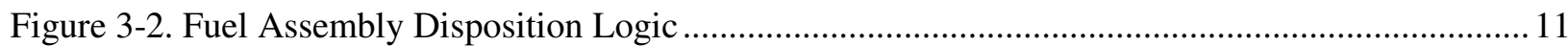

Figure 4-1. Cumulative Number of Canisters Placed in Dry Storage at Reactors .................................... 34

Figure 4-2. Cumulative Bare Fuel Assembly Shipments (Cases 2 and 4, Canisters and Bare Fuel)......... 37

Figure 4-3. Peak Inventory in Dry Storage at the CSF (Cases 1 and 3, Canisters Only)......................... 38

Figure 4-4. Peak Inventory in Bare Fuel Storage at the CSF (Cases 2 and 4, Canisters and Bare

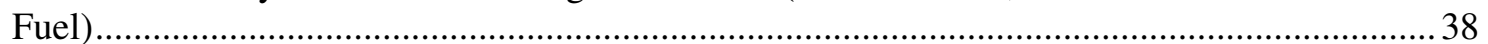

Figure 4-5. Peak Inventory in Dry Storage at the CSF (Cases 2 and 4, Canisters and Bare Fuel) ............. 39 
Figure 4-6. Peak Annual Canister Arrivals at the CSF (Cases 1 and 3, Canisters Only)...... 40

Figure 4-7. Peak Annual Bare Fuel Cask Arrivals at the CSF (Cases 2 and 4, Canisters and Bare Fuel)

Figure 4-8. Peak Annual Canister Arrivals at the CSF (Cases 2 and 4, Canisters and Bare Fuel) 41

Figure 4-9. Peak Annual Canister Re-Packaging Rates........................................................................ 43

Figure 4-10. Cumulative Canisters Re-Packaged ................................................................................ 44

Figure 5-1. Isometric view of the overall Repackaging Facility Module Concept, including Carrier Receipt Bay, Airlocks, Waste Handling Building and Carrier Release Bay..... .57

\section{TABLES}

Table ES-1. TSL Case Matrix. .. $\mathrm{V}$

Table 2-1. Waste Package Capacity for Generic Media ........................................................................ 7

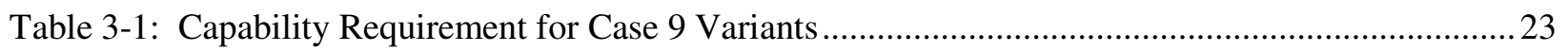

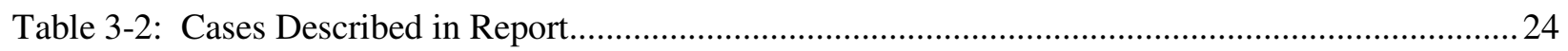

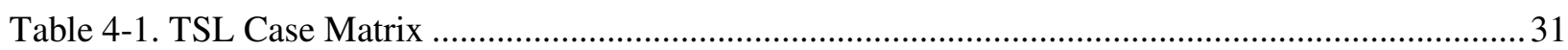

Table 4-2. Summary Level TSL Simulation Results for At-Reactor Dry Storage ................................... 32

Table 4-3. Peak Annual Bare Cask/Canister Shipping Rate from Reactors (Cask/Canister per year). . .46

Table 4-4. Cumulative Number of Canisters/Casks Shipped from Reactors ............................................46

Table 4-5. Average Capacity of Cask Shipments from Reactors............................................................ 47

Table 4-6. Consolidated Storage Facility Logistics Summary Results.................................................... 48

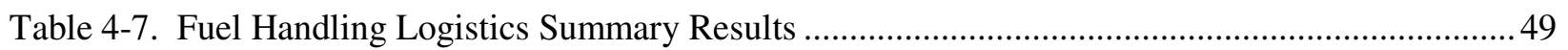

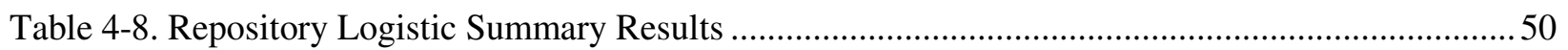

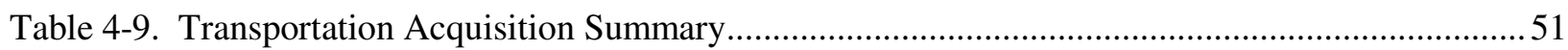

Table 6-1. Estimated Costs for At-Reactor Used Fuel Management .....................................................61

Table 6-2. Projected Total At-Reactor Used Fuel Management Costs: Cases 1 \& 3, Cases 2 \& 4 ........... 62

Table 6-3. Total At-Reactor Used Fuel Management Cost Drivers: Cases 1 \& 3, Cases 2 \& 4, and Case 9-1

Table 6-4 Consolidated Storage Annual Operations and Maintenance Costs ...........................................6 68

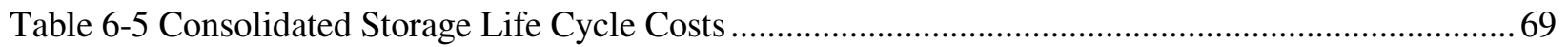

Table 6-6 Test and Validation Facility Annual Operations and Maintenance Costs .................................70

Table 6-7 Test and Validation Facility Life Cycle Cost ........................................................................ 71

Table 6-8 Comparison of Horizontal Modules and Vertical Cask Storage to a Large Vault for Dry

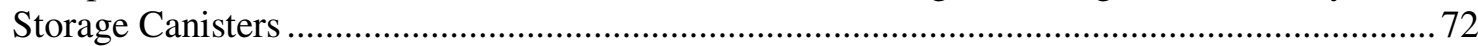

Table 6-9 Repackaging Facility Annual Operations and Maintenance Costs........................................... 74

Table 6-10 Repackaging Facility Life Cycle Costs ................................................................................. 75 


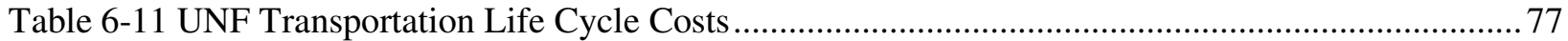

Table 6-12. Summary Roll-Up UNF Management Rough Order of Magnitude Cost Estimate ................. 80

Table 6-13. Summary Roll-Up UNF Management Rough Order of Magnitude Cost Estimate (2020-2040 CSF-Repository Start, 4/9 Assembly Capacity Disposal Canister...... 81

Note: Percentages for each category calculated as LCC for the category divided by the Total UNF Management LCC. .81

Table 6-14. Normalized UNF Management Cost 82 


\section{ACRONYMS}

$\begin{array}{ll}\text { BOP } & \text { Balance of Plant } \\ \text { BRC } & \text { Blue Ribbon Commission for America's Nuclear Future } \\ \text { BWR } & \text { Boiling Water Reactor } \\ \text { CRCT } & \text { Cask Receipt and Canister Transfer } \\ \text { CSF } & \text { Consolidated Storage Facility } \\ \text { D\&D } & \text { Decommissioning and Demolition } \\ \text { DOE } & \text { Department of Energy } \\ \text { DOE-NE } & \text { Department of Energy - Nuclear Energy } \\ \text { DPC } & \text { Dual Purpose Canister } \\ \text { DSC } & \text { Dry Storage Canister } \\ \text { EAS } & \text { Engineering Alternative Study } \\ \text { EPRI } & \text { Electric Power Research Institute } \\ \text { ESC } & \text { Existing Size Canister } \\ \text { FIFO } & \text { First-In-First-Out } \\ \text { FRS } & \text { Fuel Receipt and Storage } \\ \text { FTE } & \text { Full-time Employee } \\ \text { FY12 } & \text { Fiscal Year 2012 } \\ \text { GNEP } & \text { Global Nuclear Energy Plan } \\ \text { HEPA } & \text { High-Efficiency Particulate Air } \\ \text { ISFSI } & \text { Independent Spent Fuel Storage Installation } \\ \text { LCC } & \text { Life Cycle Cost } \\ \text { MAP } & \text { Mobile Access Platform } \\ \text { MRS } & \text { Monitored Retrievable Storage } \\ \text { MT } & \text { Metric Ton } \\ \text { MTHM } & \text { Metric Ton of Heavy Metal } \\ \text { MTU } & \text { Metric Ton of Uranium } \\ \text { NAC STC } & \text { NAC (Vendor) Storage and Transportation Cask System } \\ \text { NEI } & \text { Nuclear Energy Institute } \\ \text { NRC } & \text { Nuclear Regulatory Commission } \\ \text { NWPA } & \text { Nuclear Waste Policy Act } \\ \text { NWTRB } & \text { Nuclear Waste Technical Review Board } \\ \text { OFF } & \text { Oldest-Fuel-First } \\ \text { O\&M } & \text { Operation and Maintenance } \\ \text { PWR } & \text { Pressurized Water Reactor } \\ \text { RF } & \text { Repackaging Facility } \\ \text { SPMT } & \text { Self-propelled Modular Transport } \\ \text { SRNL } & \text { Savannah River National Laboratory } \\ \text { SRS } & \text { Savannah River Site } \\ \text { SVCT } & \text { Storage Vault Canister Transfer } \\ \text { TAD } & \text { Transportation Aging and Disposal Canister } \\ \text { TBD } & \text { To Be Determined } \\ \text { TPC } & \text { Total Project Cost } \\ \text { TSL } & \text { Transportation Storage Logistics Simulator } \\ \text { TVF } & \text { Test and Validation Facility } \\ \text { U.S. } & \text { United States } \\ \text { UFD } & \text { Used Fuel Disposition } \\ \text { WBS } & \text { Work Breakdown Structure } \\ \text { UNF } & \text { Used Nuclear Fuel } \\ \text { UOX } & \text { Uranium Dioxide nuclear fuel } \\ & \end{array}$


WBS Work Break-Down Structure

WHB Waste Handling Building

YFF Youngest-Fuel-First 


\section{USED FUEL MANAGEMENT SYSTEM ARCHITECTURE EVALUATION, FISCAL YEAR 2012}

\section{INTRODUCTION}

The nuclear power industry's used/spent fuel storage practices have evolved with changes in the nuclear industry. The fuel cycle originally envisioned in which low burn-up fuel is reprocessed quickly to provide fresh fuel has evolved to a once-through fuel cycle in which the fuel is burned to reasonably high values with the ultimate fuel disposal not yet accomplished. Delays (since 1989) in establishing a permanent repository have also forced evolution of the used/spent fuel storage concept. What was once envisioned as short term wet (pool) storage has been augmented by expanded pool storage (re-racking) and by the addition of dry fuel storage.

A variety of dry fuel storage systems have been and continue to be developed and deployed. Of the approximately 70,000 MTU of UNF estimated to have been generated through 2012, approximately $27 \%$ is stored in 1,650 dry storage casks. The amount of fuel that will be transferred from wet to dry storage is expected to increase steadily, at least until some off-site option is available. For economic reasons, the nuclear industry is currently using large dry storage systems with canister capacities up to 37 PWR and 80 BWR fuel assemblies, with larger capacity canisters being considered in the future. These systems are either single purpose (storage only) or dual-purpose (storage and transportation); none of them were designed or are currently licensed for disposal. In addition, such large capacity canisters may not be able to be emplaced in a geologic repository due to either physical emplacement constraints or the need for long periods of extended storage to allow for the thermal output of the fuel to decay so that repository thermal limits are met. While efforts are underway to evaluate the feasibility of directly disposing large capacity canisters, repackaging of the fuel assemblies in these large canisters may be required for disposal.

The Blue Ribbon Commission for America's Nuclear Future (BRC 2012), in its report to the Secretary of Energy, recommended that: "(Recommendation \#5) prompt efforts to develop one or more consolidated storage facilities be undertaken." The Commission further recommended that stranded fuel should be first in line for transfer to a consolidated facility so that these plant sites can be completely decommissioned and put to other beneficial uses. Finally, the Commission recommended that "Using existing authority in the NWPA, DOE should begin laying the groundwork for implementing consolidated storage and for improving the overall integration of storage as a planned part of the waste management system without further delay." The Nuclear Waste Technical Review Board (in June 30, 2011 correspondence to the BRC) also emphasized the importance of considering consolidated storage in the context of an integrated waste management system and plan, stating that "The Board believes that the system-wide implications of developing consolidated interim storage should be considered as part of a detailed evaluation that includes the advantages and disadvantages of such an approach," and "Information from the detailed analysis, suggested above, also will inform decisions about what technical capabilities may be required at SNF storage-site locations. The Board agrees that taking full account of the complex nature and integrated dependencies of the entire waste disposal system is vitally important in making any decisions about options for managing SNF and HLW. Thus, siting an interim storage facility without an integrated waste management plan is not recommended."

\subsection{Objective}

This study, termed the Used Fuel Disposition Campaign (UFD) System Architecture Evaluation starts to address the BRC and NWTRB recommendations and investigate the back-end of the current once-through nuclear fuel cycle in a systematic manner. It is a multi-year effort. 
The objective of the System Architecture Evaluation is to identify and evaluate integrated approaches to transportation, storage, and disposal in the waste management system with an emphasis on providing flexibility to respond to unexpected situations and developments. As identified by the BRC, much of the waste management system flexibility can be provided by consolidated storage. Consequently, the integration of consolidated storage into a waste management system is a key aspect of the System Architecture Evaluation.

This effort evaluates the implications of the current strategy for on-site storage of used nuclear fuel in large dry storage systems on the subsequent direct disposal of the stored used nuclear fuel in salt, clay/shale, and crystalline mined geologic repositories and in deep boreholes. Alternative strategies and approaches for managing the used nuclear fuel were identified and evaluated to identify potential benefits in cost and flexibility. Factors including emplacement capability, thermal constraints, the need for repackaging techniques, storage alternatives, transportation, and impacts on utility operations will ultimately be considered. Measures for flexibility and rough order of magnitude cost factors associated with each alternative were considered in the Fiscal Year 2012 (FY12) evaluation and, with additional factors, will be considered in future evaluations. Factors associated with potential repository system implementation, such as surface and subsurface design, emplacement capability, thermal management, the need for waste package overpacks, etc., are developed as part of a companion evaluation related repository design and thermal load management (Hardin, et. al. 2012).

Functional requirements for various cases of consolidated storage, re-packaging, and transportation concepts based on various initial conditions and disposition pathways will be determined. The functional requirements allow the development of facility design concepts that are flexible with regard to initial conditions, changes in the disposition pathway or changes in the implementation method. Once the consolidated storage functions are identified, alternatives for implementation can be developed and evaluated to identify potential benefits and disadvantages for several attributes.

This study evaluates potential future strategies for managing used nuclear fuel generated by commercial power reactors. DOE-managed spent nuclear fuel and high-level waste are excluded. This study is limited to the current once-through (open) fuel cycle and does not evaluate other fuel cycle technologies being investigated by the DOE Office of Fuel Cycle Technologies. This evaluation is based on the management of low enriched uranium dioxide (UOX) used nuclear fuel (UNF) generated by the existing fleet of light water reactors (LWR), termed as commercial used nuclear fuel (UNF). The evaluation is also based on current NRC licensing requirements and current transportation requirements (including packaging).

\subsubsection{Fiscal Year 2012 Objectives}

The objectives of the System Architecture Evaluation in Fiscal Year 2012 (FY12) are to:

1. Develop the overall framework of potential future commercial UNF disposition pathways.

2. Begin the analyses to support determining system operation conditions (i.e., method for transporting fuel from reactors, approach for fuel storage at a centralized storage facility) and system operating parameters (e.g., facility operation dates, acceptance rates).

3. Identify metrics that will be used to evaluate the disposition pathways and facility design concepts.

4. Select a limited set of potential disposition pathways and system operating conditions/parameters for evaluation and identify those where additional analysis is needed.

5. Evaluate commercial UNF management logistics for selected disposition pathways and system operating conditions/parameters. 
6. Develop facility functional requirements and design concepts for selected disposition pathways and system operating conditions/parameters.

7. Evaluate selected disposition pathways, system operating conditions/parameters, and facility design concepts using identified metrics.

The FY12 UFD System Architecture evaluation begins to address the BRC and NWTRB recommendations summarized above. It is not complete evaluation of all potential future commercial UNF disposition pathways. The FY12 efforts established the overall framework, methodologies, and approaches that will be applied and demonstrates the types of evaluations that can be conducted and the type of information that can be generated. The information generated in this report will be supplemented as additional disposition pathways are identified, alternative facility design concepts are developed, and different system operational assumptions are explored.

\section{BACKGROUND}

This section presents background material to provide the context for the UFD System Architecture Study. It summarizes current practices associated with long-term UNF storage and the types of systems in use. Potential disposal media and design concepts for those media are also summarized.

\subsection{At-Reactor UNF Storage}

Each operating nuclear reactor has a spent fuel pool to store UNF and provide the necessary cooling and shielding from the radiation the UNF emits. Typically, each reactor will remove between one-fourth and one-third of the total fuel in the core every 12, 18, or 24 months. As the spent fuel pools at reactors reach capacity, it is necessary to remove assemblies that have been sufficiently cooled to assure that the pool retains sufficient space to support refueling operations. Figure 1-1 shows projected UNF discharges, the projected amount of fuel that will be loaded into dry storage, and the projected amount of fuel that will be loaded into dry storage through 2060 assuming that no UNF is shipped from the reactor sites.

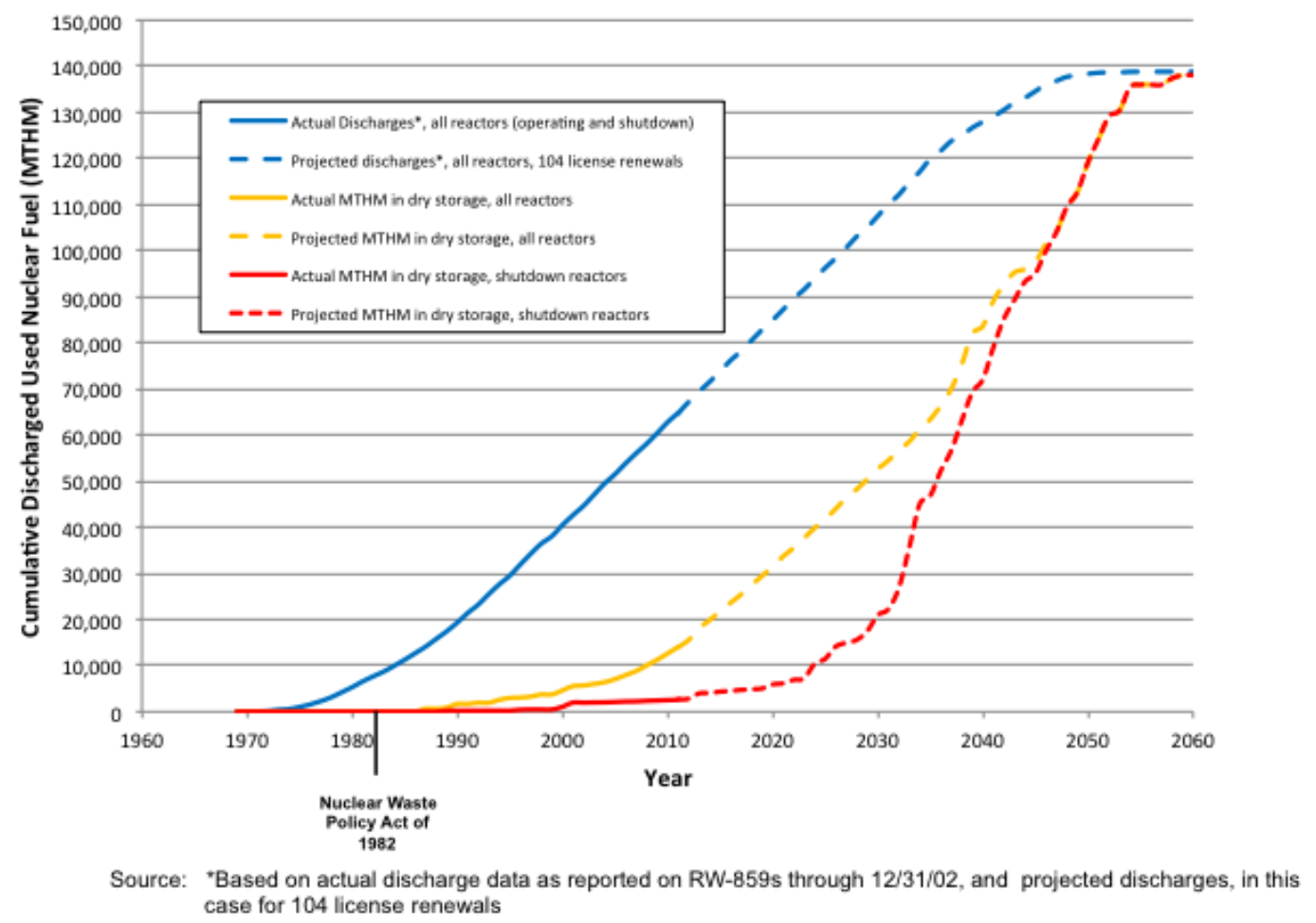

Figure 2-1. Historical and Projected UNF Discharges and Transfer to Dry Storage 
Without an operating repository, consolidated storage facility, or reprocessing facility, the only option is to build additional onsite storage, either wet or dry. Because at-reactor dry storage systems can be added in small increments as needed and are designed to allow passive cooling, their overall cost and maintenance are expected to be less than the cost and maintenance for an additional at-reactor pool. The commercial nuclear industry uses dry storage to meet its fuel storage needs; no utility has constructed an additional pool to meet a portion of its storage needs. Figure 2-2 is a map showing the location of currently licensed independent spent fuel storage installations (ISFSIs) as well as the additional 10 sites currently pursuing licenses.

Through 2012, it is estimated that the commercial nuclear industry will have generated approximately 70,000 MTU of UNF contained in about 245,000 assemblies (140,000 from BWRs and 105,000 from PWRs) (Carter et al. 2012). According to data collected by Ux Consulting, as of September 2012 a total of 65,261 assemblies were being stored in 1,650 casks, with approximately $73 \%$ of the total fuel inventory discharged remaining in wet storage (UxC 2011). It is projected that by 2020, total UNF discharges will be approximately 88,000 MTU (Carter et al. 2012). Roughly 35,000 MTHM of that is expected to be in dry storage by that time with the remaining 53,000 MTHM in the reactor pools. The fuel in dry storage by the time waste acceptance starts represent a legacy that must be dealt with regardless of what approach is taken to managing newly discharged fuel going forward. By 2060, when all currently licensed reactors will have reached the end of their operational lives, assuming a 60-year maximum, there will be approximately 140,000 MTU of UNF discharged from the reactor fleet (Carter et al. 2012).

Since the first dry storage facility was licensed in 1986, as of November 2010 the NRC has granted 63 licenses for ISFSIs for commercial power plants, as well as three additional licenses at DOE facilities (two at Idaho National Laboratory [INL] and one at Fort St. Vrain) (NRC 2012). A total of 48 of the ISFSIs received a general license granted under U.S. Code of Federal Regulations (CFR) Title 10, Part 72 (10 CFR 72). An example of an ISFSI is shown in Figure 1-2. One of the commercial licenses is for the Private Fuel Storage centralized ISFSI in Utah that has not begun construction because of ongoing litigation. It is estimated that by 2020, an additional 34 reactors will require dry storage capability and that by 2026, all but three of the currently operating commercial nuclear power plants will require dry storage for their UNF.

When a utility shuts down or decommissions a commercial nuclear power plant, the UNF remains onsite, even after all the infrastructure associated with the reactor and its operation is removed. The Blue Ribbon Commission for America's Nuclear Future has recommended that the UNF from these sites with no operating reactor be "first in line" for removal to a central storage facility when one is available. An additional issue can arise at a shutdown reactor when the spent fuel pool is decommissioned, thus limiting the ability to mitigate potential problems with the dry cask storage system (DCSS). The NRC refers to sites as "ISFSI Only" when the 10 CFR 50 license for the reactor has been terminated, which can occur only when all facilities including the spent fuel pool have been decommissioned, but fuel still remains in dry storage onsite. 


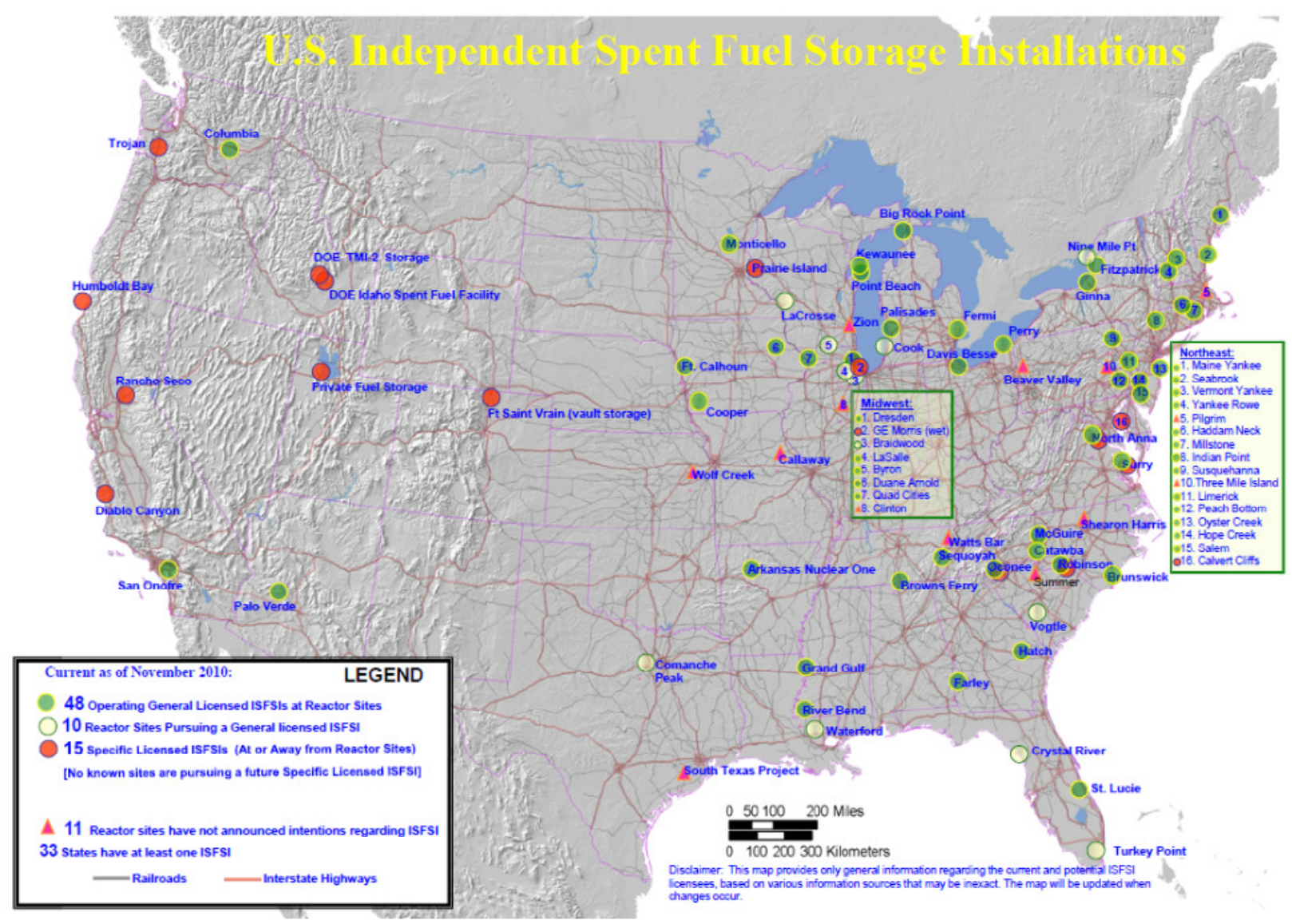

Figure 2-2. Locations of independent spent fuel storage installations (Source: http://www.nrc.gov/waste/spent-fuel-storage/locations.pdf).

There are 15 commercial light water power reactors that have been permanently shut down (NEI 2011b), not including the Shoreham reactor (Shoreham completed only low-power testing and has been fully decommissioned, with the fuel shipped to another utility). Three other non-light-water commercial reactors also have been permanently shut down. Another 10 reactors, either commercially owned but not considered power reactors or DOE-owned power reactors, also have been permanently shut down. Of the commercial LWR sites that have been shut down, six (Big Rock Point, Connecticut Yankee [Haddam Neck], Maine Yankee, Rancho Seco, Trojan, and Yankee Rowe) are currently classified as "ISFSI Only" (NRC 2011). Humboldt Bay is effectively in that category as well because the pool has been decommissioned, and the license for the reactor is expected to be terminated in 2015. LaCrosse is expected to transfer all its fuel to dry storage in the near future, and then to decommission its spent fuel pool. The two units at Zion are expected to do the same within the next 3 years.

Figure 2-3 shows the cumulative number of reactors projected to be shutdown over time, assuming all 104 operating reactors receive a 20 -year extension to their operating license. With the recent decision to shutdown the Kewaunee nuclear power plant, an additional eight reactors will move toward "ISFSI Only" by 2030 . Shortly after 2040, half the current fleet will become "ISFSI Only" sites as the reactors and supporting infrastructure are decommissioned.

Additional detail regarding the at-reactor management of commercial UNF is provided in Appendix A. 


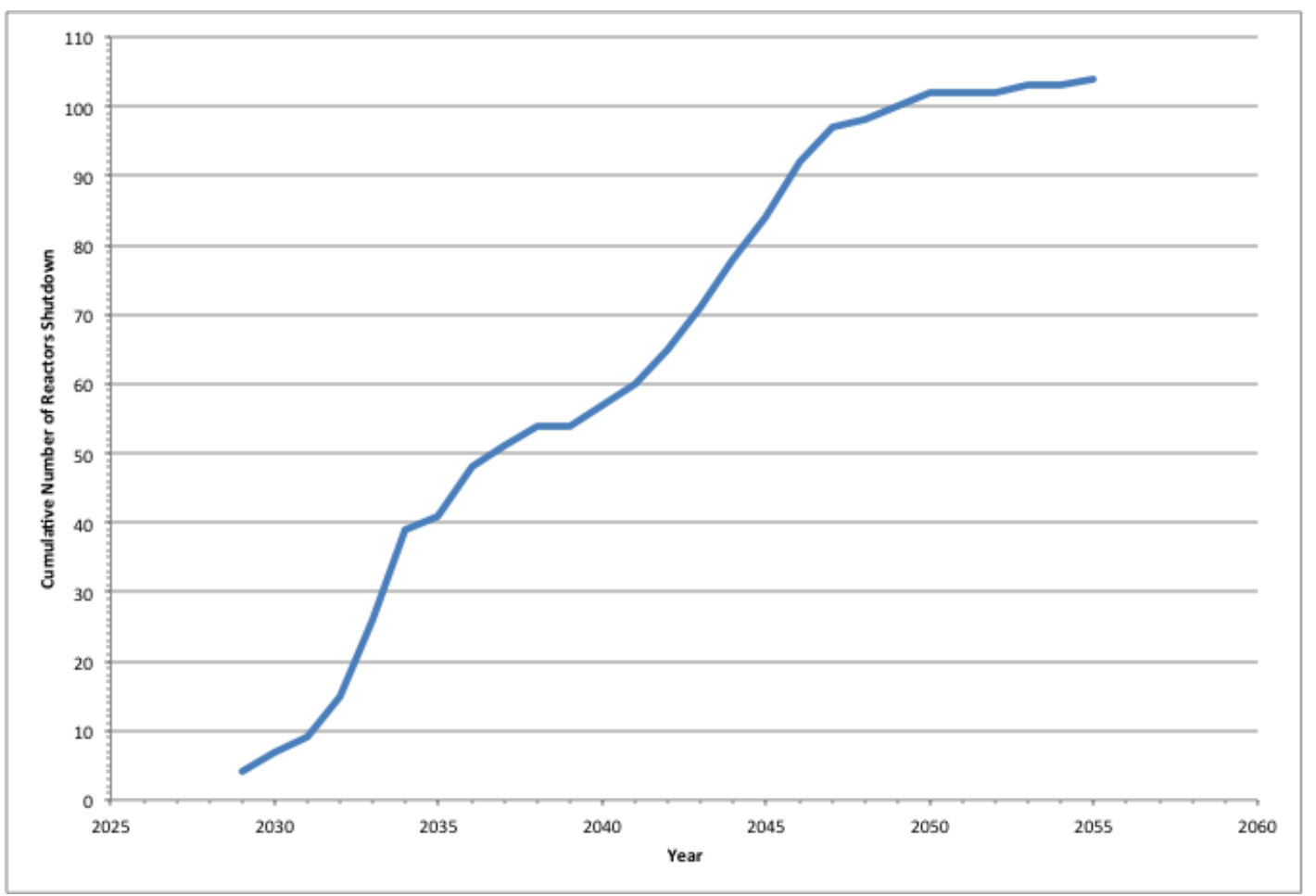

Note: Current license expirations from NRC 2012 and projected an additional 20 years for those reactors that have not received license extensions

Figure 2-3. Projected Reactor Shutdowns

\subsubsection{Dry Storage System Designs}

Since the mid 1980's 8 cask vendors have provided 11 cask systems comprised of 30 different canister types (Leduc 2012). There are four categorical descriptions of dry cask storage (Leduc and Carter 2012):

1. Metal canisters in vertical concrete overpacks or horizontal concrete modules,

2. Metal canisters in metal overpack/storage/shipping casks,

3. Metal canisters in concrete vaults, and

4. Bare fuel casks that provide both primary containment and shielding for storage and transportation. (Some of these casks have never been certified for transport)

Most fuel assemblies in dry storage in the U.S. are in metal canisters that are welded shut and stored inside vented concrete vertical overpacks or horizontal storage modules. For this configuration, the canister with its internal basket, fuel and fuel component contents is the only portion of the storage cask system which is transported. These systems all require a separate transportation cask with a type B containment vessel to overpack the fuel canister. The transfer usually requires the use of a transfer cask except for the NUHOMS transportation casks which can interface directly with the horizontal storage module (see Section 3.2). Some welded metal canisters cannot currently be transported for various design reasons.

Additional details regarding dry storage systems can be found in Appendix X and elsewhere (Leduc 2012). Future UNF management facilities concepts must be capable of receiving and handling any of these dry storage canister and transportation over-pack configurations. 


\subsection{Disposal Media and Repository Design Concepts}

The UFD campaign is currently evaluating the viability of mined geologic disposal concepts in three geologic media (clay/shale, salt, and crystalline rock) and deep borehole disposal in crystalline basement rock (Rechard et al. 2011). Mined geologic repository design concepts have been developed for each of these media (Hardin et al. 2012, Brady et al. 2009).

Mined geologic repository design concepts for the direct disposal of used/spent fuel fall into one of two classes: open or enclosed (Hardin et al. 2012). The enclosed repository concept calls for waste packages to be in direct contact with any surrounding medium such as buffer, backfill, or host geology, while the open concept allows an open gap around the packages at the time of waste emplacement. The choice of concepts impacts thermal management because it determines whether there is air space around the waste packages in which heat can be dispersed principally (and more efficiently) by thermal radiation and natural or forced convection. Open emplacement concepts are amenable to rock types where excavated openings persist for long time periods, because of either the inherent stability of the opening or reliance on long-lived ground support.

Thermal limits for the different repository media constrain the size of the waste packages that could be emplaced in them. Table 2-1 shows the waste package sizes for the different media and facility design concepts under consideration by the UFD campaign, based on thermal analyses considering generic properties for each medium (Hardin et al. 2012, Arnold et al. 2010). These waste package sizes are similar in size to those being considered in other countries developing geologic repositories.

It is recognized that evaluations are being conducted to evaluate the feasibility of directly disposing larger packages. However, in this UFD System Architecture evaluation, it is assumed that commercial UNF is ultimately packaged into what are termed "waste package compatible" size canisters (WPSCs) of the sizes shown in Table 2-1 that would be inserted into final disposal packages for emplacement in a geologic repository.

Table 2-1. Waste Package Capacity for Generic Media

\begin{tabular}{|c|c|c|}
\hline Media / Design Concept & Waste Package Size & Discussion \\
\hline Deep Borehole & 1 PWR / 2 BWR & $\begin{array}{c}\text { Limited by diameter of deep borehole (could } \\
\text { be 2 PWR / 4 BWR if fuel is consolidated }\end{array}$ \\
\hline Clay/Shale: Enclosed & 4 PWR / 9 BWR & $100^{\circ} \mathrm{C}$ Limit, 50 year cooled fuel \\
\hline Crystalline: Enclosed & 4 PWR / 9 BWR & $100^{\circ}$ C Limit, 100 year cooled fuel \\
\hline Salt: Enclosed & 12 PWR / 24 BWR & $200^{\circ}$ C Limit, 50 year cooled fuel \\
\hline Clay/Shale: Open & 21 PWR / 44 BWR & $100^{\circ}$ C Limit, 50 year cooled fuel \\
\hline
\end{tabular}

\section{DISPOSITION PATHWAY EVALUATION}

Where and when the packaging or re-packaging of commercial UNF will occur is a key question addressed in this evaluation. The evolution of the design for a repository in unsaturated tuff considered two alternatives:

- Shipment of individual fuel assemblies (referred to as "bare fuel") to the repository in re-useable transportation casks for packaging into waste packages and shipment of already loaded dry storage canisters/casks to the repository where they would be opened and the fuel assemblies repackaged into waste packages. 
- Canisterization of the majority of the fuel at reactor sites into large (21 PWR/ 44 BWR) assembly) Transportation, Aging, and Disposal (TAD) canisters that could be stored at the reactor site, transported to the repository, and ultimately disposed inside a waste package overpack. Shipment of already loaded dry storage casks to the repository where they would be opened and the fuel assemblies re-packaged into waste packages. Shipment of a limited number ( 10\%) of individual fuel assemblies to the repository in both dual-purpose canisters/casks and re-useable transportation casks for packaging into waste packages.

The surface facility designs for the two alternatives were significantly different. The first alternative, considered initially in the design process, required large throughput capacity bare fuel handling facilities. The second alternative required the majority of the canisterization to take place at the reactor sites, with lower throughput capacity bare fuel handling facilities at the repository site. The second alternative, based on the canisterization of most UNF into TADs at reactor sites, was adopted for the design included in the repository license application submitted to the U.S. NRC in 2008.

The disposition evaluation pathway evaluation discussed in this section considers a broader set of alternatives to investigate how they might contribute to the overall flexibility of the UNF management system. These alternatives include the storage of commercial UNF at an off-site Consolidated Storage Facility (CSF), canisterization of bare fuel at reactors, packaging/re-packaging of UNF at either the CSF or a repository, wet or dry packaging/re-packaging, multiple CSFs with different capacities, different repository environments having different waste package size limitations, and different throughputs at the CSF and at the repository.

The overall logistic framework describing the different options/alternatives and disposition pathways is shown in Figure 3-1. The logistic framework shows all the potential pathways for fuel assemblies stored at-reactor, either wet or dry, through a CSF (or multiple CSFs), to ultimate disposition in a geologic repository.

This framework begins with an initial state of conditions as they currently exist and are expected to continue:

- Fuel assemblies are stored as bare fuel in the wet pools at each reactor site.

- Fuel assemblies are transferred from the wet pools to dry storage systems at each reactor site to maintain full-core off-load capacity within the wet pools. The dry storage systems utilized are those currently being loaded (termed "existing size" casks/canisters, or ESCs).

- All remaining fuel assemblies are transferred from the wet pools to existing size dry storage systems at each reactor site after the reactor is shut down.

Three different alternatives for future at-reactor management of UNF were also considered in developing the broad UNF disposition framwork:

- Transfer of bare fuel in re-useable transportation casks directly to the CSF when the reactor is shut down and a CSF is available. This alternative requires bare fuel handling and storage capability at the CSF and maintaining the bare fuel in the pools at the reactor sites until it is all shipped.

- Transition from ESCs systems to loading WPSCs at the reactor sites. Such canisters would not have to be re-opened and the individual fuel assemblies re-packaged prior to emplacement in a repository. The maximum fuel assembly capacity of the waste package compatible size canisters depends on the media and design concept of the repository assumed (see Table 2-1).

- Accelerate the transfer of fuel assemblies from the wet pool to at-reactor dry storage. In this option, all fuel assemblies residing in the wet pools for a period of time greater than 5 years post reactor discharge would be transferred to dry storage as fast as possible. 
A range of different options are considered for the CSF facilities:

- Storage of existing size dry storage canisters

- Storage of waste package compatible size dry storage canisters

- Storage of bare fuel

- Re-packaging capability for existing size canisters (wet or dry) either upon receipt at the CSF or prior to transport to a repository

- Packaging capability for bare fuel (wet or dry) upon receipt at the CSF

- Bare fuel packaging and/or canister re-packaging either upon fuel assembly receipt at CSF or prior to transport to a repository

Three options are considered for the repository

- Direct disposal of waste package compatible size canisters

- Repackaging of existing size canisters (wet or dry)

- Packaging of bare fuel (wet or dry)

The overall logistics framework shown in Figure 3-1 was expanded to show the potential disposition pathways for an individual fuel assembly given all of the options described above. This expanded framework is shown in Figure 3-2. The color-coding in Figure 3-2 shows the stage in the disposition logic where existing size dry storage canisters (red) are re-packaged into waste package compatible size canisters (green) or when bare fuel assemblies (blue) are either packaged into existing size canisters (red) or into waste package compatible size canisters (green). The complexity of each disposition pathway can also be seen from the number of steps required between at-reactor storage and ultimate disposal.

\subsection{Case Descriptions}

The commercial used nuclear fuel assembly disposition logic shown in Figure 3-2 was used to develop the overall cases that will be evaluated, which are described below. Each of these cases has the common starting point described above - the current status of commercial UNF storage at-reactor and its expected evolution over a period of time until decisions are made:

- Fuel assemblies are stored as bare fuel in the wet pools at each reactor site.

- Fuel assemblies are transferred from the wet pools to dry storage systems at each reactor site to maintain full-core off-load capacity within the wet pools. The dry storage systems utilized are those currently loaded (termed "existing size" casks/canisters, or ESCs).

- All remaining fuel assemblies are transferred from the wet pools to existing size dry storage systems at each reactor site after the reactor is shut down.

The UNF processing steps for each case are described and the overall requirements to implement that case are provided. Each case is mapped to the individual fuel assembly pathways in Figure 3-2. Each case description below ends with case identification numbers that map to individual repository disposal boxes on the right of Figure 3-2. All cases assume that a CSF will be provided earlier than the repository.

Note that the case descriptions that follow do not make any assumptions regarding how the system architecture would be operated or the facility design concepts that could be utilized. The description below provides a pathway through which each fuel assembly could pass between at-reactor storage to ultimate disposal. The disposition cases that were selected for evaluation in FY12 are described in Section 3.2 and the assumptions, boundary conditions, and input parameters used in the evaluation are presented in Section 3.3. 

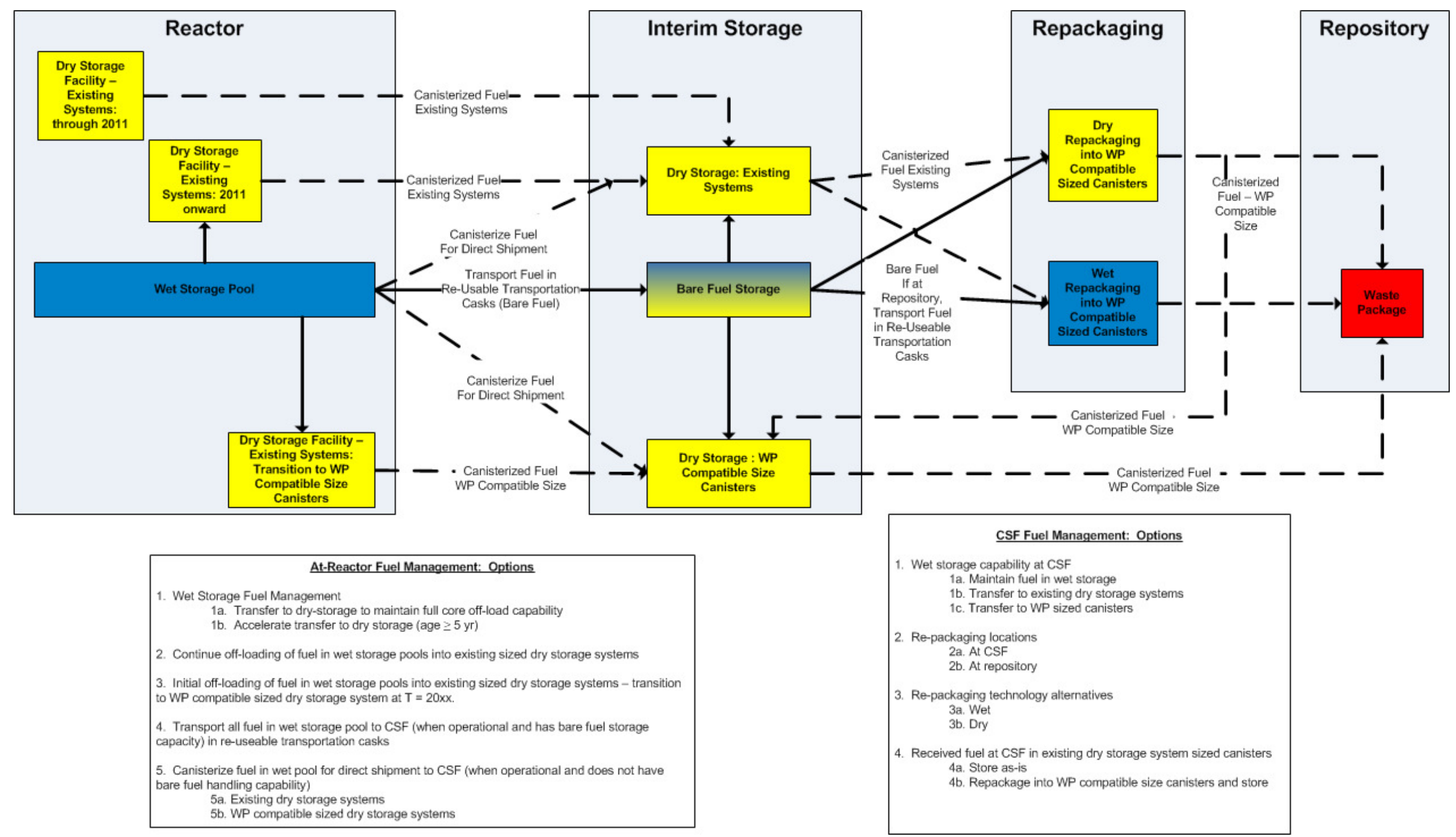

Figure 3-1. UNF Management - Overall Options and Alternatives 


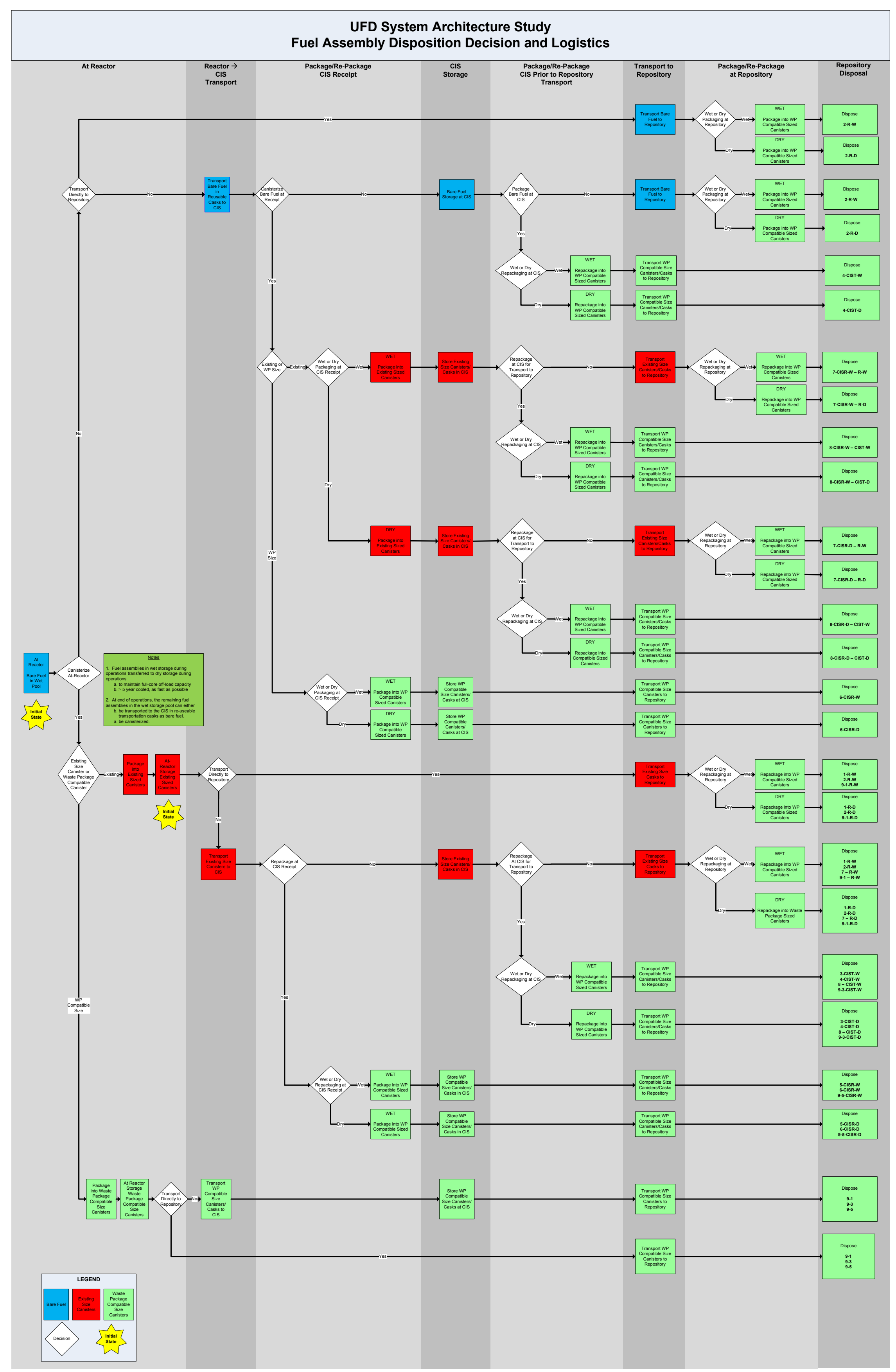

Figure 3-2. Fuel Assembly Disposition Logic 


\subsubsection{Case 1: Canisterize at Reactor / Re-Package into Waste Package Compatible Size Canisters at a Re-Packaging Facility Co-Located with the Repository}

This case is considered as the "base case" in that it is "closest" to the current once-through fuel cycle approach and involves the loading of existing size canisters at the reactor site, transporting them to the CSF for storage, and transporting them to the repository for re-packaging and ultimate disposal. Once the repository is available, the canisters are shipped from the reactors directly to the repository. The CSF serves only as a temporary buffer to allow canisters to be accepted from reactors before the repository is in operation.

Logistic Steps

- Bare fuel transferred to existing size canisters during reactor operation to maintain full core offload capacity in the at-reactor wet pool

- On-Site Storage prior to CSF or repository availability

- Transported, provided the fuel is sufficiently cool to meet transportation overpack/cask thermal limits, from Reactor Site to:

- CSF prior to repository availability; or

- Repository when available

- Bare fuel in the wet pool transferred to existing size canisters during reactor operation for direct transport, provided the fuel is sufficiently cool to meet transportation overpack/cask thermal limits, to

- CSF when available and prior to repository availability; or

○ Repository when available

- Bare fuel transferred to existing size canisters at reactor shutdown

- On-Site Storage prior to CSF or repository availability

- Transported provided the fuel is sufficiently cool to meet transportation overpack/cask thermal limits, from Reactor Site to:

- CSF prior to repository availability; or

- Repository when available

- Existing size canisters stored at CSF

- Existing size canisters transported from CSF to repository when repository is available

- Packaging/Re-Packaging at Repository

○ Wet (Case 1 - R-W, see Figure 3-2)

○ Dry (Case 1 - R-D, see Figure 3-2)

Capability Requirements:

Common to all cases:

- At-reactor bare fuel handling

- At-reactor existing size canister loading

- At-reactor storage of existing size canisters

- Existing size canister/overpack transportation from reactor to CSF

Specific to this case

- Existing size canister storage at the CSF

- Existing size canister/overpack transportation from CSF to the repository

- Canister re-packaging at the repository 


\subsubsection{Case 2: Bare Fuel Handling / Re-Packaging into Waste Package Compatible Size Canisters at a Re-Packaging Facility Co-Located with the Repository}

This case includes all aspects of Case 1, but transports bare fuel in re-useable transportation casks to the CSF when it becomes operational for storage only and to the repository when it becomes available. All packaging / re-packaging into waste package compatible size canisters is performed at a re-packaging facility co-located with the repository. This case allows for use of re-useable transportation casks to transport a significant fraction of fuel from reactor sites to the CSF and to the repository and from the CSF to the repository. Once the repository is available, the bare fuel and canisters are shipped from the reactors directly to the repository. The CSF serves only as a temporary buffer to allow bare fuel and canisters to be accepted before the repository is in operation.

\section{Logistic Steps}

- Bare fuel transferred to existing size canisters during reactor operation to maintain full core offload capacity in the at-reactor wet pool

- On-Site Storage prior to CSF or repository availability

- Transported, provided the fuel is sufficiently cool to meet transportation overpack/cask thermal limits, from Reactor Site to:

- CSF prior to repository availability; or

- Repository when available

- Bare fuel in the wet pool transported in re-useable transportation casks, provided the fuel is sufficiently cool to meet transportation cask thermal limits, during reactor operation directly to

$\circ$ CSF when available and prior to repository availability; or

○ Repository when available

- Bare fuel transferred to existing size canisters at reactor shutdown prior to CSF availability

- On-Site Storage prior to CSF or repository availability

- Transported, provided the fuel is sufficiently cool to meet transportation overpack/cask thermal limits, from Reactor Site to:

- CSF prior to repository availability; or

- Repository when available

- Existing size canisters stored at CSF

- Bare fuel stored at CSF (wet or dry)

- Bare fuel transferred to repository in re-useable casks when available

- Existing size canisters transported from CSF to repository when available

- Packaging/Re-Packaging at Repository

○ Wet (Case 2-R-W, see Figure 3-2)

○ Dry (Case 2 - R-D, see Figure 3-2)

A variant to this case is to maintain bare fuel in wet pools at the end of reactor operations in a safe-storage configuration until a CSF or a repository becomes available, when it would then be transported off-site to either the CSF or the repository in re-useable transportation casks, provided the fuel is sufficiently cool to meet transportation cask thermal limits. This variant requires that reactor decommissioning and demolition be delayed until the bare fuel is moved off-site. 
Capability Requirements

Common to all cases:

- At-reactor bare fuel handling

- At-reactor existing size canister loading

- At-reactor storage of existing size canisters

- Existing size canister/overpack transportation from reactor to CSF

Specific to this case

- Bare fuel transportation from reactor to CSF

- Existing size canister storage at the CSF

- Bare fuel storage at the CSF

- Existing size canister/overpack transportation from CSF to the repository

- Bare fuel transportation from CSF to the repository

- Canister re-packaging at the repository

- Bare fuel packaging at repository

\subsubsection{Case 3: Canisterize at Reactor / Re-Package into Waste Package Compatible Size Canisters at a Re-Packaging Facility Co-Located with the CSF Prior to Repository Transport}

This case similar to Case 1 except all packaging/re-packaging into waste package compatible size canisters is done at a re-packaging facility co-located with the CSF prior to transport to repository. Note that there is no direct transport between reactor and repository as all packaging is done at the CSF, which eventually handles all commercial UNF destined for the repository.

\section{$\underline{\text { Logistic Steps }}$}

- Bare fuel transferred to existing size canisters during reactor operation to maintain full core offload capacity in the at-reactor wet pool

- On-Site Storage prior to CSF availability; or

- Transported, provided the fuel is sufficiently cool to meet transportation overpack/cask thermal limits, from Reactor Site to CSF when available

- Bare fuel in the wet pool transferred to existing size canisters during reactor operation for transport to CSF when available, provided the fuel is sufficiently cool to meet transportation overpack/cask thermal limits

- Bare fuel transferred to existing size canisters at reactor shutdown

- On-Site Storage prior to CSF availability; or

- Transported, provided the fuel is sufficiently cool to meet transportation overpack/cask thermal limits, from Reactor Site to CSF when available

- Existing size canisters stored at CSF

- Existing size canisters re-packaged at CSF prior to Transport to Repository ( $>5 \mathrm{yr}$ before available)

- Wet (Case 3 - CIST-W, see Figure 3-2)

- Dry (Case 3 - CIST-D, see Figure 3-2)

- Waste Package compatible size canisters transported from CSF to repository when available 
Capability Requirements

Common to all cases:

- At-reactor bare fuel handling

- At-reactor existing size canister loading

- At-reactor storage of existing size canisters

Existing size canister/overpack transportation from reactor to CSF

Specific to this case

- Existing canister storage at the CSF

- Canister re-packaging at the CSF prior to transport to the repository

- There could be multiple CSFs to increase re-packaging rate

- There could be phased deployment: storage first, re-packaging capability later (when the specific repository requirements are known)

- Waste package compatible size canister/overpack transportation from CSF to the repository

\subsubsection{Case 4: Bare Fuel Handling / Re-Packaging into Waste Package Compatible Size Canisters at a Re-Packaging Facility Co-Located with the CSF Prior to Repository Transport}

This is similar to Case 2 except that all packaging/repackaging into waste package compatible size canisters is done at a re-packaging facility co-located with the CSF facility prior to transport to the repository. Bare fuel is transported to the CSF facility for storage with bare fuel packaging done at CSF prior to transport to the repository. Note that there is no direct transport between the reactor sites and the repository as all packaging and re-packaging is done at the CSF. This case allows for use of re-useable transportation casks to transport a significant fraction of fuel from reactor sites to the CSF.

\section{$\underline{\text { Logistic Steps }}$}

- Bare fuel transferred to existing size canisters during reactor operation to maintain full core offload capability in wet pool

- On-Site Storage prior to CSF availability

- Transported from Reactor Site to CSF when available, provided the fuel is sufficiently cool to meet transportation overpack/cask thermal limits

- Bare fuel in the wet pool transported in re-useable transportation casks, provided the fuel is sufficiently cool to meet transportation cask thermal limits, during reactor operation to CSF when available

- Bare fuel transferred to existing size canisters at reactor shutdown, prior to CSF availability

- On-Site Storage prior to CSF availability

- Transported from Reactor Site to CSF when available, provided the fuel is sufficiently cool to meet transportation overpack/cask thermal limits

- Bare fuel transferred to CSF in re-useable transportation casks for storage, when CSF becomes available, provided the fuel is sufficiently cool to meet transportation cask thermal limits,

- Existing size canisters stored at CSF

- Bare fuel stored at CSF (wet or dry)

- Bare fuel is packaged at CSF prior to transport to repository (>5 yr before available) 
- Existing size canisters re-packaged at CSF prior to Transport to Repository ( $>5 \mathrm{yr}$ before available)

- Wet (Case 4 - CIST-W, see Figure 3-2)

- Dry (Case 4 - CIST-D, see Figure 3-2)

- Waste Package compatible size canisters transported from CSF to repository when available

Capability Requirements

Common to all cases:

- At-reactor bare fuel handling

- At-reactor existing size canister loading

- At-reactor storage of existing size canisters

- Existing size canister/overpack transportation from reactor to CSF

Specific to this case

- Bare fuel transportation from reactor to CSF

- Existing canister storage at the CSF

- Bare fuel storage at the CSF

- Canister re-packaging at the CSF facility prior to transport to the repository

- There could be multiple CSFs/re-packaging facilities to increase re-packaging rate

- There could be phased deployment: storage first, re-packaging capability later(when the specific repository requirements are known)

- Bare fuel packaging at the CSF prior to transport to the repository

- There could be multiple CSFs/re-packaging facilities to increase bare fuel packaging rate

- There could be phased deployment: storage first, bare fuel packaging capability later(when the specific repository requirements are known)

- Waste package compatible size canister/overpack transportation from CSF to the repository

\subsubsection{Case 5: Canisterize at Reactor / Re-Package into Waste Package Compatible Size Canisters at CFS Receipt at a Re-Packaging Facility Co- Located with the CSF}

This case is similar to Case 1 except all re-packaging into waste package compatible size canisters is done at a re-packaging facility co-located with the CSF upon receipt. Note that there is no direct transport between the reactor sites and the repository as all packaging is done at the CSF.

This case presumes canister size for repository disposal is known, possibly well in advance of when the repository would be available (or sited), or that small canisters are used to maximize compatibility with any repository design. It also requires two canisterization steps in very close temporal proximity for the existing size canisters loaded at the reactor sites, then re-packaged upon receipt at the CSF facility.

\section{Logistic Steps}

- Bare fuel transferred to existing size canisters during reactor operation to maintain full core offload capacity in the at-reactor wet pool

- On-Site Storage prior to CSF availability 
- Transported from Reactor Site to CSF when available, provided the fuel is sufficiently cool to meet transportation overpack/cask thermal limits

- Bare fuel transferred to existing size canisters at reactor shutdown

- On-Site Storage prior to CSF availability

- Transported from Reactor Site to CSF when available, provided the fuel is sufficiently cool to meet transportation overpack/cask thermal limits,

- Existing size canisters re-packaged at CSF Receipt

- Wet (Case 5 - CISR-W, see Figure 3-2)

- Dry (Case 5 - CISR-D, see Figure 3-2)

- Waste Package compatible size canisters stored at CSF

- Waste Package compatible size canisters transported from CSF to repository when available

Capability Requirements

Common to all cases:

- At-reactor bare fuel handling

- At-reactor existing size canister loading

- At-reactor storage of existing size canisters

- Existing size canister/overpack transportation from reactor to CSF

Specific to this case

- Canister re-packaging into waste package compatible size canisters at the CSF upon receipt

- There could be multiple CSFs/re-packaging facilities to increase re-packaging rate

- There could NOT be phased deployment: re-packaging capabilities would be required at the start of facility operations to support storage

- Waste package compatible size canister storage at the CSF

- Waste package compatible size canister/overpack transportation from CSF to the repository

\subsubsection{Case 6: Bare Fuel Processing / Re-Packaging into Waste Package Compatible Size Canisters at CSF Receipt at a Re-Packaging Facility Co- Located with the CSF}

This case is identical to Case 2 except all bare fuel packaging and canister re-packaging into waste package compatible size canisters is done a re-packaging facility co-located at the CSF upon receipt. Note that there is no direct transport between reactor sites and the repository as all packaging and re-packaging is done at the CSF. This case allows for the use of re-useable transportation casks to transport a significant fraction of fuel from reactor sites to the CSF facility.

This case presumes that the disposal canister size for repository disposal is known, possibly well in advance of when a repository would be available (or sited); or that a canister small enough to be compatible with a wide range of repository designs is utilized. It also requires two canisterization steps in very close temporal proximity for the existing size canisters loaded at the reactor sites, then re-packaged upon receipt at the CSF facility.

\section{$\underline{\text { Logistic Steps }}$}

- Bare fuel transferred to existing size canisters during reactor operation to maintain full core offload capacity in the at-reactor wet pool 
- On-Site Storage prior to CSF availability

- Transported from Reactor Site to CSF when available, provided the fuel is sufficiently cool to meet transportation overpack/cask thermal limits

- Bare fuel transferred to existing size canisters at reactor shutdown prior to CSF availability

- On-Site Storage prior to CSF availability

- Transported from Reactor Site to CSF when available, provided the fuel is sufficiently cool to meet transportation overpack/cask thermal limits,

- Bare fuel transferred to CSF in re-useable casks, when available, provided the fuel is sufficiently cool to meet transportation cask thermal limits,

- Bare fuel is packaged and existing size canisters re-packaged into waste package compatible size canisters at CSF Receipt

$\circ$ Wet (Case 6 - CISR-W, see Figure 3-2)

○ Dry (Case 6 - CISR-D, see Figure 3-2)

- Waste Package compatible size canisters stored at CSF

- Waste Package compatible size canisters transported from CSF to repository when available

\section{Capability Requirements}

Common to all cases:

- At-reactor bare fuel handling

- At-reactor existing size canister loading

- At-reactor storage of existing size canisters

- Existing size canister/overpack transportation from reactor to CSF

Specific to this case

- Bare fuel transportation from reactor to CSF

- Existing-size canister re-packaging at the CSF upon receipt

- There could be multiple CSFs/re-packaging facilities to increase re-packaging rate

- There could NOT be phased deployment: re-packaging capabilities would be required to support storage

- Bare fuel packaging at the CSF upon receipt

- There could be multiple CSFS/re-packaging facilities to increase re-packaging rate

- There could NOT be phased deployment: re-packaging capabilities would be required at the start of facility operations to support storage

- Waste package compatible size canister storage at the CSF

- Waste package compatible size canister/overpack transportation from CSF to the repository

\subsubsection{Case 7: Bare Fuel Processing / Packaging into Existing Size Canisters at CSF Receipt -Re-Package into Waste Package Compatible Size Canisters at a Re-Packaging Facility Co-Located with the Repository}

This case is similar to Case 2 except bare fuel is transported to the CSF facility in re-useable transportation casks and packaged into existing size canisters (i.e., single canister design for PWR and BWR UNF) and stored, when the CSF becomes available. All existing size canisters previously loaded at the reactors are stored at the CSF facility, then transported to repository and re-packaged into waste 
package compatible size canisters for disposal. There is no shipment of fuel from reactors directly to the repository. This case allows for use of re-useable transportation casks to transport a significant fraction of fuel from reactor sites to the CSF. It includes two packaging/re-packaging capabilities; one at the CSF at receipt and one at the repository.

\section{$\underline{\text { Logistic Steps }}$}

- Bare fuel transferred to existing size canisters during reactor operation to maintain full core offload capacity in the at-reactor wet pool

- On-Site Storage prior to CSF availability

- Transported from Reactor Site to CSF when available, provided the fuel is sufficiently cool to meet transportation overpack/cask thermal limits,

- Bare fuel transferred to CSF at reactor shutdown - prior to CSF availability

- On-Site Storage prior to CSF availability

- Transported from Reactor Site to CSF when available, provided the fuel is sufficiently cool to meet transportation cask thermal limits

- Bare fuel transferred to CSF in re-useable casks, when available

- Bare fuel is packaged into existing size canisters at CSF Receipt

- Wet (Case 7 - CISR-W - \#)

○ Dry (Case 7 - CISR-D - \#)

- Existing Size canisters stored at CSF

- Existing size canisters transported from CSF to repository when available

- Packaging/re-packaging at repository

- Wet (Case 7 - CISR-W - R-W; Case 7 - CISR-D - R-W, see Figure 3-2)

○ Dry (Case 7 - CISR-W - R-D; Case 7 - CISR-D - R-D, see Figure 3-2)

\section{Capability Requirements}

Common to all cases:

- At-reactor bare fuel handling

- At-reactor existing size canister loading

- At-reactor storage of existing size canisters

- Existing size canister/overpack transportation from reactor to CSF

Specific to this case

- Bare fuel transportation from reactor to CSF

- Bare fuel packaging at the CSF upon receipt

- There could be multiple CSFs/packaging facilities to increase packaging rate

- There could NOT be phased deployment: Packaging capabilities would be required at the start of facility operations to support storage

- Existing size canister storage at the CSF

- Existing size canister/overpack transportation from CSF to the repository

- Canister re-packaging at the repository 


\subsubsection{Case 8: Bare Fuel Processing / Packaging at CSF Receipt - Existing Size Canisters, Re-Package at a Re-Packaging Facility Co-Located with the CSF Prior to Repository Transport}

This is identical to Case 7 except that all repackaging of fuel from existing size canisters into waste package compatible size canisters for disposal is done at the CSF before transportation to the repository for disposal. Like Case 7, this case allows for use of re-useable transportation casks to transport a significant fraction of bare fuel from reactor sites to the CSF. It includes two packaging/re-packaging capabilities; one at the CSF upon receipt to load bare fuel into existing size canisters and one at the CSF prior to transport to re-package UNF from existing size canisters into waste package compatible size canisters.

Logistic Steps

- Bare fuel transferred to existing size canisters during reactor operation to maintain full core offload capacity in the at-reactor wet pool

- On-Site Storage prior to CSF availability

- Transported from Reactor Site to CSF when available, provided the fuel is sufficiently cool to meet transportation overpack/cask thermal limits,

- Bare fuel transferred to CSF at reactor shutdown if CSF is available

○ On-Site Storage prior to CSF availability

- Transported from Reactor Site to CSF when available, provided the fuel is sufficiently cool to meet transportation cask thermal limits

- Bare fuel transferred to CSF in re-useable casks, when available, provided the fuel is sufficiently cool to meet transportation cask thermal limits,

- Bare fuel is packaged into existing size canisters at CSF Receipt

- Wet (Case 7 - CISR-W - \#) or Dry (Case 7 - CISR-D - \#)

- Existing Size canisters stored at CSF

- Existing size canisters re-packaged at CSF prior to repository transport ( $>5 \mathrm{yr}$ ahead of availability)

- Wet (Case 8 - CISR-W - CIST-W; Case 8 - CISR-D - CIST -W, see Figure 3-2)

- Dry (Case 8 - CISR-W - CIST -D; Case 8 - CISR-D - CIST -D, see Figure 3-2)

- Waste Package compatible size canisters transported from CSF to repository when available

Capability Requirements

Common to all cases:

- At-reactor bare fuel handling

- At-reactor existing size canister loading

- At-reactor storage of existing size canisters

- Existing size canister/overpack transportation from reactor to CSF

Specific to this case

- Bare fuel transportation from reactor to CSF

- Bare fuel packaging capabilities required at the CSF upon receipt

- There could be multiple CSFs/packaging facilities to increase packaging rate

- There could NOT be phased deployment of initial capabilities: Packaging capabilities would be required at the start of facility operations to support storage. There could be 
phased deployment of any additional re-packaging necessary for waste package compatible size canisters.

- Existing size canister storage at the CSF

- Re-packaging at the CSF; prior to transport to the repository

- Waste package compatible size canister/overpack transportation from CSF facility to the repository

\subsubsection{Case 9: Transition to Loading Waste Package Compatible Size Canisters at Reactor at Future Time}

This case is a variant to Cases 1,3, and 5. It assumes all bare fuel is canisterized at-reactor (no bare fuel handling at the CSF facility or at the repository). The capability and steps are identical to Cases 1,3 , and 5 above, except further growth of the inventory of existing size canisters is stopped by creation of waste package compatible size canisters at reactor sites instead.

- Bare fuel transferred to existing size canisters during operation, prior to transition to waste package compatible size canisters loaded at-reactor; Time $<$ X (i.e., 10 years in advance of repository becoming operational)

- On-Site Storage prior to CSF availability

- Transported from Reactor Site, provided the fuel is sufficiently cool to meet transportation overpack/cask thermal limits, to:

- CSF prior to repository availability

- Repository when available

- Bare fuel transferred to existing size canisters at shutdown, prior to transition to waste package compatible size canisters loaded at-reactor; Time $<\mathrm{X}$

- On-Site Storage prior to CSF availability

- Transported from Reactor Site, provided the fuel is sufficiently cool to meet transportation overpack/cask thermal limits, to:

- CSF prior to repository availability

- Repository when available

- Bare fuel transferred to waste package compatible size canisters during operation, after transition to waste package compatible size canisters loaded at-reactor; Time $\geq X$

- On-Site Storage prior to CSF availability

- Transported from Reactor Site, provided the fuel is sufficiently cool to meet transportation overpack/cask thermal limits, to:

- CSF prior to repository availability

- Repository when available

- Bare fuel transferred to waste package compatible size canisters at shutdown, after transition to waste package compatible size canisters loaded at-reactor; Time $\geq X$

- On-Site Storage prior to CSF availability

- Transported from Reactor Site, provided the fuel is sufficiently cool to meet transportation overpack/cask thermal limits, to:

- CSF prior to repository availability

- Repository when available

- Waste Package compatible size canisters stored at CSF

- Waste Package compatible size canisters transported from CSF to repository when available 
- Existing size canisters

- Stored at CSF; Transported to repository for repackaging and disposal

- Wet (Case 1-R-W, see Figure 3-2) (Case 9-R-W, see Figure 3-2)

- Dry (Case 1-R-D, see Figure 3-2) (Case 9-R-D, see Figure 3-2)

- Stored at CSF; Re-packaged at CSF prior to repository transport, transported and disposed

- Wet (Case 3-CIST-W, see Figure 3-2) (Case 9-CIST-W, see Figure 3-2)

- Dry (Case 3-CIST-D, see Figure 3-2) (Case 9-CIST-D, see Figure 3-2)

- Re-Packaged at CSF Receipt, waste package compatible size canisters stored, transported to repository and disposed

- Wet (Case 5-CISR-W, see Figure 3-2) (Case 9-CISR-W, see Figure 3-2)

- Dry (Case 5-CISR-D, see Figure 3-2) (Case 9-CISR-D, see Figure 3-2)

Capability Requirements

- Same capability requirements as Cases 1,3, and 5; plus

- Storage capability for waste package compatible size canisters required at the reactor and CSF

The capability requirements for each of the Case 9 variants are shown in Table 3-1.

Key elements of all cases are summarized briefly in Table 3-2 
Table 3-1: Capability Requirement for Case 9 Variants

\begin{tabular}{|c|c|c|}
\hline Case 9-1 & Case 9-3 & Case 9-5 \\
\hline $\begin{array}{l}\text { - At-reactor bare fuel handling } \\
\text { - } \text { At-reactor existing size } \\
\text { canister loading } \\
\text { At-reactor waste package } \\
\text { compatible size canister } \\
\text { loading } \\
\text { - At-reactor storage of existing } \\
\text { size canisters } \\
\text { At-reactor storage of waste } \\
\text { package compatible size } \\
\text { canisters } \\
\text { Existing size } \\
\text { canister/overpack } \\
\text { transportation from reactor to } \\
\text { CSF } \\
\text { Waste Package Compatible } \\
\text { size canister/overpack } \\
\text { transportation from reactor to } \\
\text { CSF } \\
\text { Waste package compatible } \\
\text { size canister storage at the } \\
\text { CSF } \\
\text { Existing size canister storage } \\
\text { at the CSF } \\
\text { Existing size } \\
\text { canister/overpack } \\
\text { transportation from CSF to } \\
\text { the repository } \\
\end{array}$ & 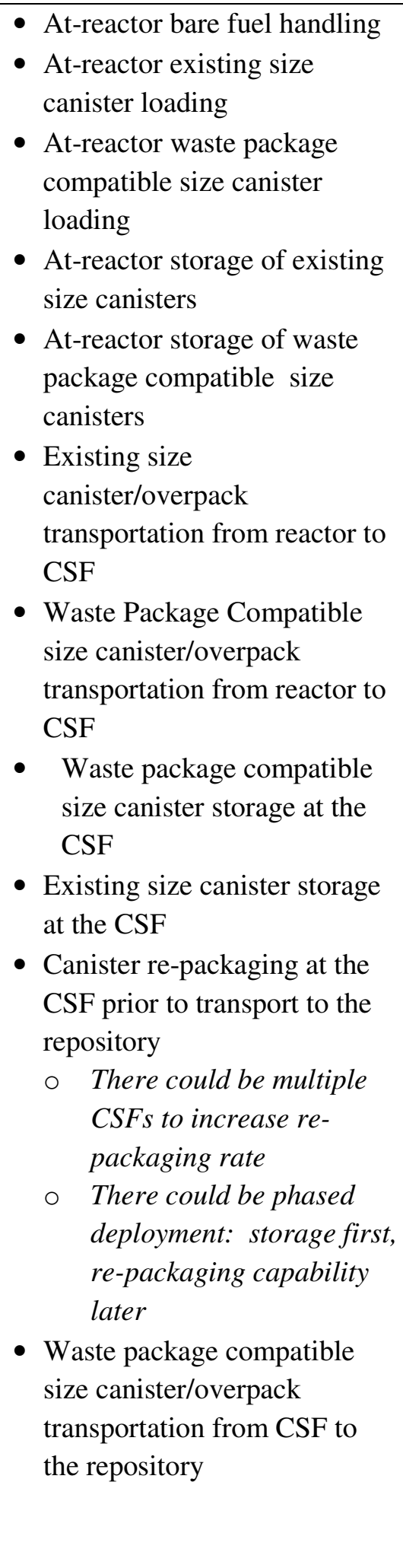 & $\begin{array}{l}\text { - } \text { At-reactor bare fuel handling } \\
\text { - At-reactor existing size } \\
\text { canister loading } \\
\text { At-reactor waste package } \\
\text { compatible size canister } \\
\text { loading } \\
\text { - At-reactor storage of existing } \\
\text { size canisters } \\
\text { At-reactor storage of waste } \\
\text { package compatible size } \\
\text { canisters } \\
\text { Existing size } \\
\text { canister/overpack } \\
\text { transportation from reactor to } \\
\text { CSF } \\
\text { Waste Package Compatible } \\
\text { size canister/overpack } \\
\text { transportation from reactor to } \\
\text { CSF facility } \\
\text { Waste package compatible } \\
\text { size canister storage at the } \\
\text { CSF } \\
\text { Canister re-packaging at the } \\
\text { CSF upon receipt } \\
\text { O There could be multiple } \\
\text { CSFs to increase re- } \\
\text { packaging rate } \\
\text { - There could NOT be } \\
\text { phased deployment: re- } \\
\text { packaging capabilities } \\
\text { would be required to } \\
\text { support storage }\end{array}$ \\
\hline
\end{tabular}


Table 3-2: Cases Described in Report

\begin{tabular}{|c|c|c|c|c|c|c|c|c|}
\hline & 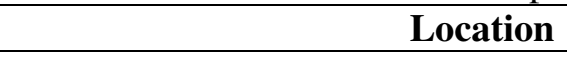 & Rea & tor & & CS & & & Repository \\
\hline & Activity & $\begin{array}{l}\text { Bare fuel } \\
\text { Packaging }\end{array}$ & $\begin{array}{l}\text { Ship to } \\
\text { CSF or } \\
\text { Repository }\end{array}$ & System function & $\begin{array}{l}\text { Packaging } \\
\text { into ESCs or } \\
\text { WPSCs - } \\
\text { on receipt }\end{array}$ & Storage & $\begin{array}{l}\text { Packaging into } \\
\text { WPSCs - before } \\
\text { transport to } \\
\text { Repository }\end{array}$ & $\begin{array}{l}\text { Packaging into } \\
\text { WPSCs - before } \\
\text { disposal }\end{array}$ \\
\hline & $\begin{array}{l}\text { ESCs at Reactor / Re-package } \\
\text { into WPSCs at Repository }\end{array}$ & ESCs & ESCs & $\begin{array}{l}\text { Early receipt until } \\
\text { Repository online }\end{array}$ & None & ESCs & None & ESCs \\
\hline & $\begin{array}{l}\text { Bare fuel Handling / Re-Package } \\
\text { into WPSCs at Repository }\end{array}$ & $\begin{array}{l}\text { ESCs until } \\
\text { CSF online }\end{array}$ & $\begin{array}{c}\text { ESCs } \\
\text { Bare fuel }\end{array}$ & $\begin{array}{l}\text { Early receipt until } \\
\text { Repository online }\end{array}$ & None & $\begin{array}{c}\text { ESCs } \\
\text { Bare fuel } \\
\end{array}$ & None & $\begin{array}{c}\text { ESCs } \\
\text { Bare fuel }\end{array}$ \\
\hline 3 & $\begin{array}{l}\text { ESCs at Reactor / Re-Package into } \\
\text { WPSCs at CSF before transport to } \\
\text { Repository }\end{array}$ & ESCs & ESCs & $\begin{array}{l}\text { Integral storage/ } \\
\text { processing all UNF }\end{array}$ & None & ESCs & ESCs & None \\
\hline & $\begin{array}{l}\text { Bare fuel Handling / Re-Packaging } \\
\text { into WPSCs at CSF before } \\
\text { transport to Repository }\end{array}$ & $\begin{array}{l}\text { ESCs until } \\
\text { CSF online }\end{array}$ & $\begin{array}{l}\text { ESCs } \\
\text { Bare fuel }\end{array}$ & $\begin{array}{l}\text { Integral storage/ } \\
\text { processing all UNF }\end{array}$ & None & $\begin{array}{c}\text { ESCs } \\
\text { Bare fuel }\end{array}$ & $\begin{array}{l}\text { ESCs } \\
\text { Bare fuel }\end{array}$ & None \\
\hline & $\begin{array}{l}\text { ESCs at Reactor / Re-Package into } \\
\text { WPSCs at CSF Receipt }\end{array}$ & ESCs & ESCs & $\begin{array}{l}\text { Integral storage/ } \\
\text { processing all UNF }\end{array}$ & $\begin{array}{l}\text { ESCs to } \\
\text { WPSCs }\end{array}$ & WPSCs & None & None \\
\hline 6 & $\begin{array}{l}\text { Bare fuel Handling / Re-Package } \\
\text { into WPSCs at CSF Receipt }\end{array}$ & $\begin{array}{l}\text { ESCs until } \\
\text { CSF online }\end{array}$ & $\begin{array}{l}\text { ESCs } \\
\text { Bare fuel }\end{array}$ & $\begin{array}{l}\text { Integral storage/ } \\
\text { processing all UNF }\end{array}$ & $\begin{array}{l}\text { ESCs and } \\
\text { Bare fuel to } \\
\text { WPSCs }\end{array}$ & WPSCs & None & None \\
\hline 7 & $\begin{array}{l}\text { Bare fuel Handling / Package into } \\
\text { ESCs at CSF Receipt, Re-package } \\
\text { into WPSCs at Repository }\end{array}$ & $\begin{array}{l}\text { ESCs until } \\
\text { CSF online }\end{array}$ & $\begin{array}{l}\text { ESCs } \\
\text { Bare fuel }\end{array}$ & $\begin{array}{l}\text { Integral storage/ } \\
\text { processing all UNF }\end{array}$ & $\begin{array}{l}\text { Bare fuel to } \\
\text { ESCs }\end{array}$ & ESCs & None & ESCs \\
\hline 8 & $\begin{array}{l}\text { Bare fuel Handling / Package into } \\
\text { ESCs at CSF Receipt, Re-package } \\
\text { into WPSCs at CSF before } \\
\text { transport to Repository }\end{array}$ & $\begin{array}{l}\text { ESCs until } \\
\text { CSF online }\end{array}$ & $\begin{array}{c}\text { ESCs } \\
\text { Bare fuel }\end{array}$ & $\begin{array}{l}\text { Integral storage/ } \\
\text { processing all UNF }\end{array}$ & $\begin{array}{l}\text { Bare fuel to } \\
\text { ESCs }\end{array}$ & ESCs & ESCs & None \\
\hline & $\begin{array}{l}\text { Transition to Loading WPSCs } \\
\text { at Reactor at Future Time }\end{array}$ & $\begin{array}{l}\text { ESCs until } \\
\text { transition to } \\
\text { WPSCs }\end{array}$ & $\begin{array}{l}\text { ESCs } \\
\text { WPSCs }\end{array}$ & $\begin{array}{l}\text { Integral storage/ } \\
\text { processing all UNF }\end{array}$ & None & $\begin{array}{l}\text { ESCs } \\
\text { WPSCs }\end{array}$ & None & ESCs \\
\hline $9-3$ & Same & Same & Same & Same & None & $\begin{array}{c}\text { ESCs } \\
\text { WPSCs }\end{array}$ & ESCs & None \\
\hline $9-5$ & Same & Same & Same & Same & $\begin{array}{l}\text { ESCs to } \\
\text { WPSCs }\end{array}$ & WPSCs & None & None \\
\hline
\end{tabular}

ESC - Existing Size Canister

WPSC - Waste Package-compatible Size Canister Highlighted cases - Evaluated in FY 2012 


\subsection{Initial Qualitative Evaluation of Cases}

This section provides an initial evaluation of the cases presented above. The commonality of capability requirements, the level of complexity, and the flexibility of each of the cases were evaluated to determine those cases considered in the Fiscal Year 2012 effort. This does not necessarily imply that any of the cases would not be pursued in the future, but that the focus of efforts will be prioritized.

\subsubsection{Commonality of Capability Requirements}

This section summarizes the commonality of capability requirements for each of the cases identified above. All cases would need to:

1. Be able to handle bare fuel at-reactor

It is noted that this is a "given" for all operating reactors and any shutdown reactors that have not been decommissioned and does not exist at those reactor sites where the reactor(s) have been decommissioned.

2. Canisterize into existing size dry storage systems

The quantity of canisters that would have to be stored either at-reactor or at the CSF would differ across the different cases (e.g., depending on whether fuel is stored bare, in waste package compatible canisters, or existing size canisters at the CSF) and due to the timing of CSF and/or repository availability. Throughput rates would also differ - due to the different rates considered in the evaluation.

3. Store existing size canisters on-site pending CSF and/or repository availability

The quantity of existing size canisters that would have to be stored on-site would depend on both the individual case and the timing of CSF and/or repository availability.

4. Package/re-package into waste package compatible size canisters

This would have to be done somewhere; either at the reactor sites, at fuel receipt at the CSF, at the CSF prior to repository transport, or at the repository.

5. Transport existing size canisters

Existing size canisters would have to be transported to the CSF, at a minimum. Existing size canisters have been and will continue to be loaded at-reactor, and will have to be dispositioned. The quantity of existing size casks that would have to be transported would differ across the cases and would be affected by the timing of the CSF and/or repository availability.

6. Storage at the CSF

The types (existing size canisters or waste package compatible size canisters) and quantities of each type would differ for the different cases and would be affected by the timing of the CSF and/or repository availability.

\subsubsection{Complexity}

This section summarizes the complexity associated with each of the cases identified above.

- Cases 1, 3, and 5 involve the least number of "steps" between the reactors and disposal.

- Existing size canisters have to be transported to the CSF (recognizing that there are a number of existing canister systems).

- Single "type" of storage at the CSF: existing size (number of different systems) or waste package compatible size. 
- A single repackaging step, somewhere: Case 1 at repository, Case 3 at CSF prior to repository transport, Case 5 upon receipt at the CSF.

- Cases 2, 4, and 6 are variants to Cases 1,3, and 5 to include bare fuel handling at the CSF.

- These cases could reduce the amount of canisterization at reactors. The trade-off is the need to canisterize either at the CSF or the repository as bare fuel ultimately has to be canisterized.

- These cases could require the capability to store fuel that has been shipped bare from the reactors at the CSF (Cases 2 and 4), or possibly at the repository. In Cases 2 and 4 it is stored bare (i.e. in wet pools or in single-purpose storage casks); in Case 6 it is stored in waste package size canisters upon receipt at the CSF and stored.

- Bare fuel canisterization would be needed, in addition to re-canistering of existing size canisters. Note that the same facility could perform both operations in that re-canistering is more complex and involves the same functions as bare fuel packaging.

- Cases 7 and 8 also include bare fuel handling at the CSF, but add more complexity by packaging bare fuel into existing size canisters at CSF receipt, which subsequently has to be re-packaged later

$\circ$ These cases could reduce the amount of canisterization at reactor.

- These cases eliminate the need for bare fuel storage at the CSF, as does Case 6 .

- However, a re-packaging step later is required, unlike Case 6.

- The Case 9 variants add a bit more complexity by introducing canisterization into waste package compatible size canisters at-reactor.

○ The same steps as Cases 1, 3, and 5 would still be required, but would reduce the number of canisters that would have to be re-packaged later.

- These cases would increase the amount of canisterization at reactors by having to load more, smaller-capacity canisters (compared to existing storage canisters).

\subsubsection{Flexibility}

This section summarizes the features of each case related to flexibility, focusing on two key aspects. (See the discussion of flexibility in Appendix A.)

- Timing of packaging/repackaging fuel into waste package compatible canisters. Decisions about the repository (i.e., the disposal medium and facility design) will not be known until well into the future and flexibility to respond to future decisions is important.

- The definition of a waste package compatible size canister would be unknown in situations where a CSF would be deployed with no repository defined. This results in two alternatives regarding timing of packaging/re-packaging that can be considered (and associated cases):

- Package as late as possible (after the repository design is known); either at the CSF or at the repository (Cases 1, 2, 3, 4, 7 8)

- Package/re-package into "small" canisters as early as possible, either at reactors or at the CSF upon receipt of fuel, and put in larger capacity waste package overpacks later if the final repository allows (Cases 5, 6, 9-3, and 9-5). This would minimize repackaging quantities later, but the Case 9 variants would increase canisterization efforts at the reactor 
- Location of re-packaging at the CSF instead of the repository

- Could deploy re-packaging in advance of repository availability so as to have canisters ready for disposal, showing early progress towards disposal and facilitating startup of the repository

- Could increase throughput of disposal once a repository is operational

- Would require individual licensing at each CSF as opposed to single licensing at a repository

- Allows a single transportation "mode" from CSF to repository

- No need to maintain a diverse shipping fleet once fuel is removed from reactors

- Still need diverse fleet to transport from reactors to the CSF, but would not have to maintain at the ready to go from the CSF to repository. However, a new fleet of specialized transportation equipment would be needed. As an example, such a fleet could include multi-canister overpacks for transporting several smaller capacity canisters.

\subsubsection{Cases for Detailed Qualitative and Quantitative Evaluation}

This section presents the cases that were qualitatively and quantitatively evaluated in Fiscal Year 2012. As noted earlier, this does not imply that other cases may not be pursued in the future. The commonality between cases and the complexity/flexibility of the cases, presented above, was used to determine those cases considered in Fiscal Year 2012.

The following cases (highlighted in table 3-2 above) and identified variants were evaluated:

- Cases 1 and 2: Re-packaging at repository, bare fuel variant (Case 2), with shipments directly from the reactors to the repository once it is available

- Case 1 variant for future consideration: Transition to bolted (rather than welded) casks at some point in time (to avoid having to cut open canisters that were welded shut for storage), store bolted casks at the CSF, re-package into waste package size compatible canisters at repository.

- Case 2 variant for future consideration: Maintain pool capability after reactor shutdown to store bare fuel and transfer to the CSF when available.

- Cases 3 and 4: Re-packaging at CSF, bare fuel variant (Case 4), with all fuel channeled through the $\mathrm{CSF}$

- Case 3 variant for future consideration: Transition to bolted casks at some point in time, store bolted casks at the CSF, re-package into waste package compatible size canisters at the CSF.

- Case 4 variant: Maintain pool capability after reactor shutdown to store bare fuel and transfer to the CSF when available.

Note that it is assumed for the purposes of this initial evaluation that a CSF and a repository are not colocated in any of the cases under consideration. This assumption results in there always being a transportation step between the CSF and the repository. In addition, it requires that the CSF and the repository be treated as being independent with no common or shared functions.

The following cases were not pursued in Fiscal Year 2012:

- Cases 7 and 8 (bare fuel packaging into existing size canisters at CSF receipt): These cases represent a situation where canisterization would be done close in time to shipment of the bare fuel from the reactor sites, which is covered by Cases 9-1 and 9-3. For bare fuel handling (Case 8), it is likely that if bare fuel were going to be handled at the CSF, it would likely not be packaged into very large 
storage canisters that might have a substantial likelihood of having to be reopened due to incompatibility with repository capabilities. It is more likely to be stored bare (Cases 2 and 4 ) or in small canisters designed to be compatible with any repository (Case 6). However, the use of large canisters could be evaluated in the future if other options for storing fuel received at the CSF bare prove challenging from a system perspective.

- Cases 5, 6, and 9-5 (canisterize bare fuel/re-package existing size fuel into waste package size canisters at CSF receipt): These are "close" to case 9-1 in terms of the time when waste is canistered into waste package compatible size packages. They also presume that the size of a waste package compatible canister would be known early on, or that a small canister that is likely to be compatible with any repository concept would be used.

- Cases 9-1, 9-3, and 9-5: Cases involving a transition to loading waste package compatible size canisters at-reactor will be evaluated under the UFD Standard Canister development effort (Howard et al. 2012).

Future cases for consideration:

- Variants to cases 1 and 3 that consider a transition to bolted casks (rather than welded canisters) at some point in time, store bolted casks at the CSF, re-package into waste package size compatible canisters at the repository (Case 1 variant) or at the CSF (Case 3 variant).

- Variants to cases 2 and 4 that maintain pool capability after reactor shutdown to store bare fuel and transfer to the CSF when available.

- Case 5, in which bare fuel received at CSF is packaged into small disposal-compatible canisters that are stored either in large multi-canister overpacks or in a dry vault system designed for canisters rather than bare fuel (similar to the Spanish central interim storage facility). Existing size canisters received at the CSF are stored until a repository is available, at which time they are reopened and the fuel is re-packaged into disposal compatible canisters for shipment to the repository. This option allows fuel to be shipped bare from reactors but stored dry at the CSF, avoiding large quantities of pool storage at the CSF. Use of small canisters minimizes the likelihood that they would have to be reopened for repackaging before disposal, while deferral of repackaging of fuel in existing size canisters until the repository is available allows repackaging of that fuel into canisters that are optimized for the repository.

- Variants of cases 1 and 2 in which the CSF continues to function as the receiving facility for all fuel shipped from reactors even after the repository comes online, stores the fuel until ready for disposal in the repository, and then selects the appropriate fuel to meet repository thermal constraints and ships the fuel to the repository using an efficient transportation campaign mode. This avoids the need for buffer storage at the repository to insulate fuel receipt rates from fuel emplacement rates and/or provide thermal management, and increases the efficiency of transportation of the fuel over part of the distance from the reactors to the repository.

\subsection{Disposition Pathway Evaluation Assumptions and Input/Boundary Conditions}

The following assumptions and input/boundary conditions were used to evaluate the disposition pathways discussed in Section 3.2 and to give insights about which assumptions and conditions have implications that might warrant consideration of alternative assumptions and conditions.

\section{Assumptions}

- Disposition of Used LWR Fuel in a Once-Through Fuel Cycle

- Reactor fleet is limited to the current 104 operating reactors 
- Reactors will receive life extensions to operate for 60 years

- Projected fuel inventory at reactor; wet and dry

- Oldest Fuel First allocation priority (determines which reactor sites ship and how much is shipped from each site in a given year)

- Youngest Fuel First shipment from reactors (determines the fuel within the annual allocation is shipped from each site)

- First-In-First Out shipment from storage facility

- Reactors off-load pools to dry storage 5 years after shutdown

- Single CSF and geologic repository

- CSF and repository are not co-located

- Wet storage for bare fuel at the CSF

- Wet fuel assembly packaging and re-packaging

- Thermal constraints on geologic media are not considered, beyond the size of the waste packages discussed below

\section{Input/Boundary Conditions}

- Single CSF and geologic repository

- CSF available: 2020, 2035

- Geologic repository available: 2040, 2055

- Acceptance rates: 1500, 3000, 6000 MTHM per year

- Waste package sizes: 4, 12, 21 PWR; 9, 24, 44 BWR

The assumptions and input/boundary conditions were selected to "constrain" the problem, but do provide a broad enough range to show trends and gain insight (i.e., the range in acceptance rages and start dates). The choice of these assumptions and input/boundary conditions are not meant to imply that the system would actually be operated as assumed. As an example, the allocation priority and shipment schemes are those that have been looked at in prior UNF system analyses and provide a logical starting point. Rather the initial set of assumptions, input/boundary conditions, and subsequent analyses allow for gaining insight regarding integrated system dynamics and understanding of trends, pointing to where the next set of system architecture analyses should focus.

\subsection{Considerations for System Design Flexibility}

The design concepts described here are intended to be part of an overall waste management system that is flexible and can adapt to various logistics, construction and operational conditions. This is consistent with the BRC's recommendation that DOE perform "the systems analyses and design studies needed to develop a conceptual design for a highly flexible, initial federal spent fuel storage facility." In this report, the phrase "flexibility in design" refers to capabilities built into a facility's design to accommodate a range of possible future conditions. Flexibility in design affords the capability to adapt to different waste management policy decisions and directions, waste receipt and operational scenarios and to unanticipated conditions encountered during construction.

Key contingencies that require flexible planning, design, and decision-making that have been identified include: 
1. Uncertainty in the availability and timing of new waste management facilities and any operational constraints (e.g. capacity limits or operational restrictions such as limitation to canistered fuel) that may be imposed on them.

2. Uncertainty about repository design

- Maximum waste package size

- Thermal constraints potentially requiring extended aging prior to emplacement

3. Potential need for design or operating mode changes after a repository has received an initial license.

4. Potential interruptions in emplacement at the repository.

5. Constrained and/or uncertain funding. (While the BRC recommended that the waste management funding system be fixed so that program funding will not be constrained or subject to the vagaries of the appropriations process, it is not certain when that will occur.)

6. Uncertainties about future fuel cycles and the associated amounts, types, and timing of waste forms requiring storage and disposal.

7. Uncertainties about whether currently-unanticipated measures will be required to retrieve, transport, and handle UNF after extended storage periods.

It should be noted that the preliminary analyses presented in this report were not designed to evaluate the robustness and flexibility of the various system configurations and operating scenarios with respect to these uncertainties. The capability to make such evaluations can be added later when potential modular or staged development approaches (discussed below) have been better defined, as appropriate.

\subsubsection{Sequential and Modular Facility Development}

The design concepts described in this document are intended to be compatible with a modular approach to facility construction. The phrase "modular or sequential implementation" describes a process in which decisions concerning facility design, development, operation, and closure are made in a stepwise manner: at each step in the process, a decision whether to proceed would be made based on policy direction, the licensing and regulatory requirements, funding profile, and operating experience. The next stage of construction would proceed informed by the experience gained from the previous stage. The impacts and full benefits of sequential and modular facility development have not yet been assessed.

The possible benefits of a modular or stepwise approach range from incorporation of lessons learned after each stage of construction to leveling annual construction costs. [Note: The National Research Council issued a report advising the DOE on possible repository development strategies, and the BRC endorsed the staged development concept presented in that report.]

\subsubsection{Potential Benefits}

\section{Ability to Learn and Improve the System}

Sequential development provides opportunities to apply lessons learned from the construction of one module to subsequent modules. The ability to refine designs based on pilot-scale testing of first-of-a-kind packaging, handling, and emplacement equipment could help ensure that the system will perform reliably and economically. 


\section{Increased Confidence in Meeting Schedule Expectations for Waste Receipt and Disposition -}

By reducing not only the investment but also the time required to construct the initial waste management and disposition capability, sequential and modular development can enhance confidence that the schedule for the start of facility operations can be met despite funding uncertainties. This development approach also offers the flexibility to adapt to unanticipated policy and budget changes and to unexpected developments during operation with fewer impacts on schedule and cost.

\subsubsection{Potential Impacts}

Sequential and modular facility development could increase the estimated total system life cycle costs of the waste management and disposition system by extending operating periods and foregoing some economies of scale in design and construction. However, the impact on the discounted value of disposal costs may be smaller because the investments in facilities are spread out over time rather than concentrated in the earlier years of the program, and because a sequential, modular approach allows unanticipated problems to be detected early and corrected before investment in full-scale facilities that could be difficult and expensive to retrofit.

\section{SUMMARY OF LOGISTICS MODELING}

The cases and input parameters presented in Section 3 were simulated using the UFD Transportation Storage Logistics (TSL) simulation model to provide quantitative information on (Nutt et al., 2012, Busch et.al., 2012):

- At-Reactor UNF management;

- UNF management at a CSF

- UNF handling operations at the CSF and the repository

- UNF transportation between the reactor sites and the CSF and between the CSF and the repository

Detailed results are provided in Appendix A and summary results and insights gained from the logistics analyses are provided in this section. The cases considered are summarized in Table 4-1 (see Section 3.1 for details regarding each case).

Table 4-1. TSL Case Matrix

\begin{tabular}{|l|c|c|c|c|}
\cline { 2 - 5 } \multicolumn{1}{c|}{} & Case 1 & Case 2 & Case 3 & Case 4 \\
\hline $\begin{array}{l}\text { Transport From } \\
\text { Reactors }\end{array}$ & $\begin{array}{c}\text { Existing Size } \\
\text { Canisters }\end{array}$ & $\begin{array}{c}\text { Existing Size } \\
\text { Canisters / } \\
\text { Bare Fuel }\end{array}$ & $\begin{array}{c}\text { Existing Size } \\
\text { Canisters }\end{array}$ & $\begin{array}{c}\text { Existing Size } \\
\text { Canisters / } \\
\text { Bare Fuel }\end{array}$ \\
\hline CSF & $\begin{array}{c}\text { Existing-Size } \\
\text { Canisters }\end{array}$ & $\begin{array}{c}\text { Existing Size } \\
\text { Canisters / } \\
\text { Bare Fuel }\end{array}$ & $\begin{array}{c}\text { Existing-Size } \\
\text { Canisters }\end{array}$ & $\begin{array}{c}\text { Existing Size } \\
\text { Canisters / } \\
\text { Bare Fuel }\end{array}$ \\
\hline $\begin{array}{l}\text { Package/ } \\
\text { Re-Package at ==> }\end{array}$ & Repository & Repository & CSF & CSF \\
\hline MGR & Existing-Size & $\begin{array}{c}\text { Existing Size } \\
\text { Canisters / } \\
\text { Banisters Fuel }\end{array}$ & $\begin{array}{c}\text { Wackage Size } \\
\text { Canisters }\end{array}$ & $\begin{array}{c}\text { Wackage Size } \\
\text { Canisters }\end{array}$ \\
\hline
\end{tabular}




\subsection{Summary of Logistic Simulation Results: At-Reactor UNF Management}

Current and projected trends in at-reactor UNF management, described in Section A-1 of Appendix A, projected UNF fuel discharges (Kalinina 2012), the rate that UNF is accepted from the reactor fleet (1500, 3000, $6000 \mathrm{MTHM} /$ year), and when acceptance begins (2020, 2035), and the preference for UNF acceptance from the reactor fleet (OFF, YFF) all affect on-site storage inventories and shipping characteristics. Detailed at-reactor UNF logistic results for the cases identified in Section 3.2.4 with the assumptions defined in Section 3.3 are presented in Sections A-2 of Appendix A and are summarized herein.

Summary results for at-reactor dry storage are shown in Table 4-2 and Figure 4-1. The cumulative number of canisters loaded into dry storage and the peak dry storage inventories are shown in Table 4-2 for the different UNF management cases, acceptance rates, and year that acceptance begins.

Table 4-2. Summary Level TSL Simulation Results for At-Reactor Dry Storage

\begin{tabular}{|c|c|c|c|c|c|c|}
\hline & $\begin{array}{l}\text { Acceptance } \\
\text { Rate } \\
\text { (MTHM/yr) }\end{array}$ & $\begin{array}{c}\text { Acceptance } \\
\text { Start }\end{array}$ & $\begin{array}{l}\text { Cumulative } \\
\text { Canisters Loaded } \\
\text { into Dry Storage }\end{array}$ & $\begin{array}{c}\text { Peak } \\
\text { Canister } \\
\text { Inventory }\end{array}$ & $\begin{array}{c}\text { Peak } \\
\text { MTHM } \\
\text { Inventory }\end{array}$ & $\begin{array}{c}\text { Peak } \\
\text { Inventory } \\
\text { Year }\end{array}$ \\
\hline \multirow{6}{*}{$\begin{array}{c}\text { Canistered } \\
\text { Fuel Transport } \\
\text { (Cases } 1 \text { \& 3) }\end{array}$} & 1500 & 2020 & 9226 & 6762 & 83384 & 2054 \\
\hline & 3000 & 2020 & 7910 & 2652 & 32433 & 2054 \\
\hline & 6000 & 2020 & 7420 & 2459 & 29552 & 2019 \\
\hline & 1500 & 2035 & 10308 & 8592 & 105872 & 2054 \\
\hline & 3000 & 2035 & 9638 & 6200 & 76674 & 2054 \\
\hline & 6000 & 2035 & 9141 & 5023 & 61339 & 2034 \\
\hline \multirow{6}{*}{$\begin{array}{c}\text { Bare and } \\
\text { Canistered } \\
\text { Fuel Transport } \\
\text { (Cases } 2 \text { \& 4) }\end{array}$} & 1500 & 2020 & 8196 & 6787 & 83532 & 2054 \\
\hline & 3000 & 2020 & 5106 & 3158 & 38538 & 2054 \\
\hline & 6000 & 2020 & 2768 & 2483 & 30179 & 2019 \\
\hline & 1500 & 2035 & 10054 & 8610 & 105953 & 2054 \\
\hline & 3000 & 2035 & 8547 & 6306 & 78061 & 2054 \\
\hline & 6000 & 2035 & 6629 & 5331 & 65592 & 2034 \\
\hline
\end{tabular}

The results show that the start of acceptance and the acceptance rate will impact on-site dry storage requirements. Peak dry storage inventories decrease significantly when the acceptance rate increases from $1500 \mathrm{MTHM} / \mathrm{yr}$ to $3000 \mathrm{MHMT} / \mathrm{yr}$ acceptance rate. However, the additional decrease is smaller when the acceptance rate is increased from $3000 \mathrm{MTHM} / \mathrm{yr}$ to $6000 \mathrm{MT} / \mathrm{yr}$. Extending the date that acceptance begins from 2020 to 2035 results in a significant increase in the amount of UNF placed in atreactor dry storage. There is little difference in the peak dry storage inventories for the different cases considered in this evaluation (all canisters, canisters and bare fuel).

A $1500 \mathrm{MTHM} / \mathrm{yr}$ acceptance rate does not overcome the need for additional on-site dry storage after UNF acceptance begins because the annual projected discharge rate of UNF from the current fleet is approximately $2000 \mathrm{MTHM} / \mathrm{yr}$ through approximately 2035 (Kalinina 2012). Acceptance rates of 3000 $\mathrm{MTHM} / \mathrm{yr}$ and $6000 \mathrm{MTHM} / \mathrm{yr}$ are larger than the projected discharge rate and reduce the dry storage inventory while the reactors are in operation. However, the logistic results indicate that a higher 
acceptance rates does not eliminate the need for additional on-site dry storage when the reactor fleet begins to shut down (starting in 2029) under a YFF-5 acceptance preference and the assumption that reactors will transfer all fuel from the pools to dry storage five years after reactor shutdown. Although the ultimate at-reactor dry storage inventory will be reduced in this case, some UNF will continue to be transferred to dry storage.

Figure 4-1 shows the cumulative number of canisters placed into storage at the reactor sites as a function of time for each of the acceptance scenarios. This figure reveals a very important difference between the scenarios in which all UNF is placed in existing size canisters at reactor sites before it is moved to a CSF or the repository (Cases 1 and 3) and those in which the bare fuel is removed from reactor pools and shipped to the CSF or the repository (Cases 2 and 4). For each acceptance rate and start date, more fuel is placed into dry storage at the reactor sites in the canister only scenarios (Cases 1 and 3) than in the bare fuel plus canister scenarios (Cases 2 and 4). While the expectation for the canister only cases was that each reactor's annual allocation would be loaded from the pool into an existing size DPC and shipped directly to the CSF or the repository, this proved not to be possible in a significant fraction of cases because of thermal loading constraints that prevented immediate shipment of the casks. Specifically, the maximum thermal load allowed for transportation is lower than the maximum load for storage. For Cases 2 and 4, at a $3000 \mathrm{MTHM} / \mathrm{yr}$ acceptance rate a significant amount of fuel residing in the pools does not meet the thermal limits on the DPC transportation overpacks and cannot be shipped off-site. In such instances, the model selects cooler canisterized fuel that is already in dry storage to be shipped off-site and, in order to maintain pool capacity, loads an equal amount of fuel from the pools into canisters that are stored onsite until they in turn are cool enough to be transported. In these cases, two operations are required at the reactor - loading a new DPC and placing it in storage on site, and removing an old DPC from storage and preparing it for shipment offsite.

In contrast, in the bare fuel scenarios (Cases 2 and 4), if the characteristics of the spent nuclear fuel projected for shipment exceeded the capabilities of one of the bare fuel transportation casks, it was assumed that the cask's capacity is reduced (de-rated) for the affected shipments. This increases the number of individual cask shipments that are required to move the amount of fuel that could be placed in an existing size canister within acceptable thermal limits, but avoids the additional dry storage at reactor sites that would be required to age that canister until it would meet transportation thermal limits.

The impact of these thermal considerations on the number of canisters that must be stored at reactor sites before they can be moved in the all-canisters cases is not small. For example, Figure 4-1 shows if acceptance starts in 2020 at a $3000 \mathrm{MTHM} / \mathrm{yr}$ acceptance rate, around twice as many additional canisters are stored at reactor sites in the canister only case than in the bare fuel case. In fact, the $3000 \mathrm{MTHM} / \mathrm{yr}$ acceptance rate, canister-only case performs only marginally better than the $1500 \mathrm{MTHM} / \mathrm{yr}$ acceptance rate bare fuel case in terms of ability to limit the increase in onsite canister storage. 


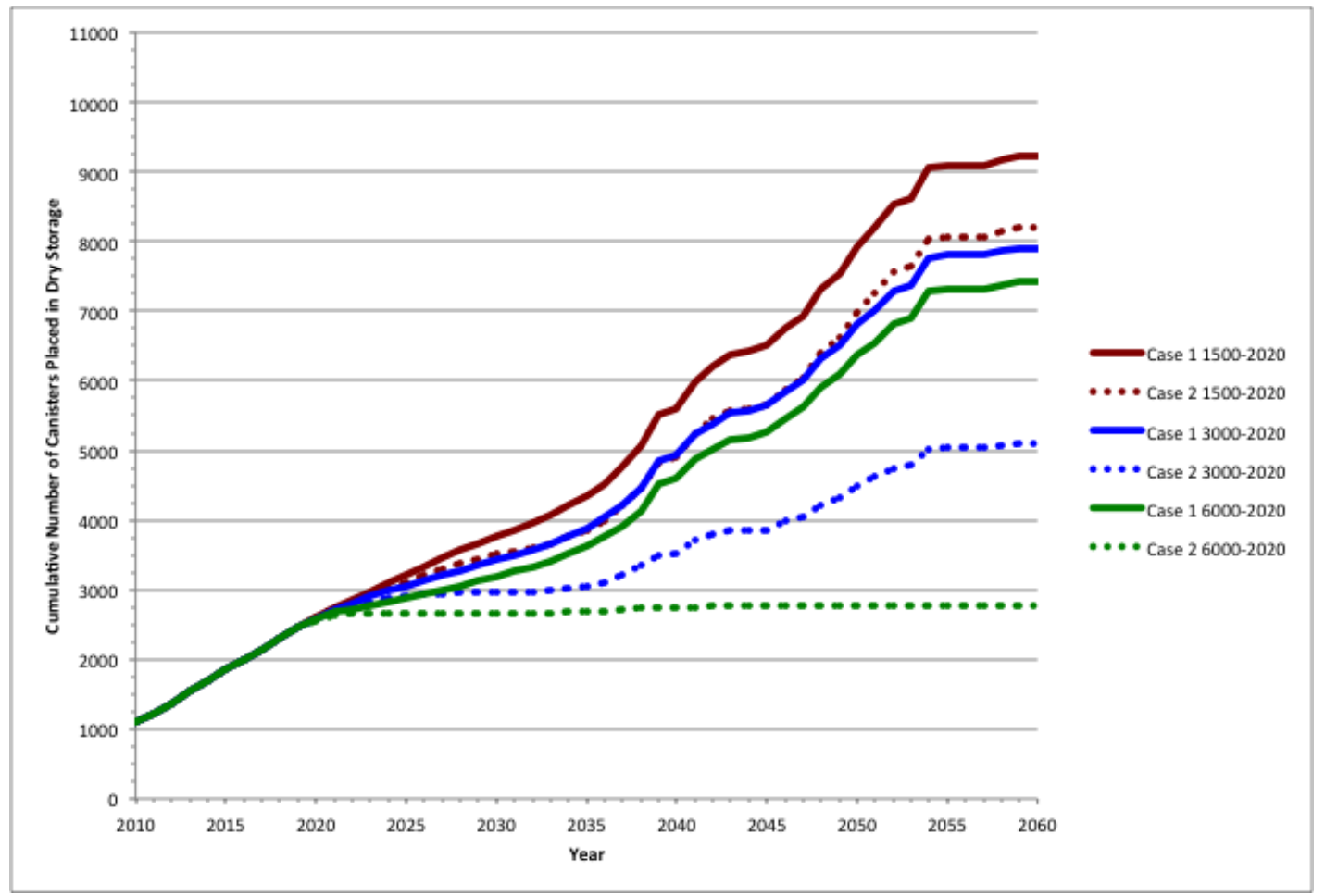

a) 2020 Start of Acceptance

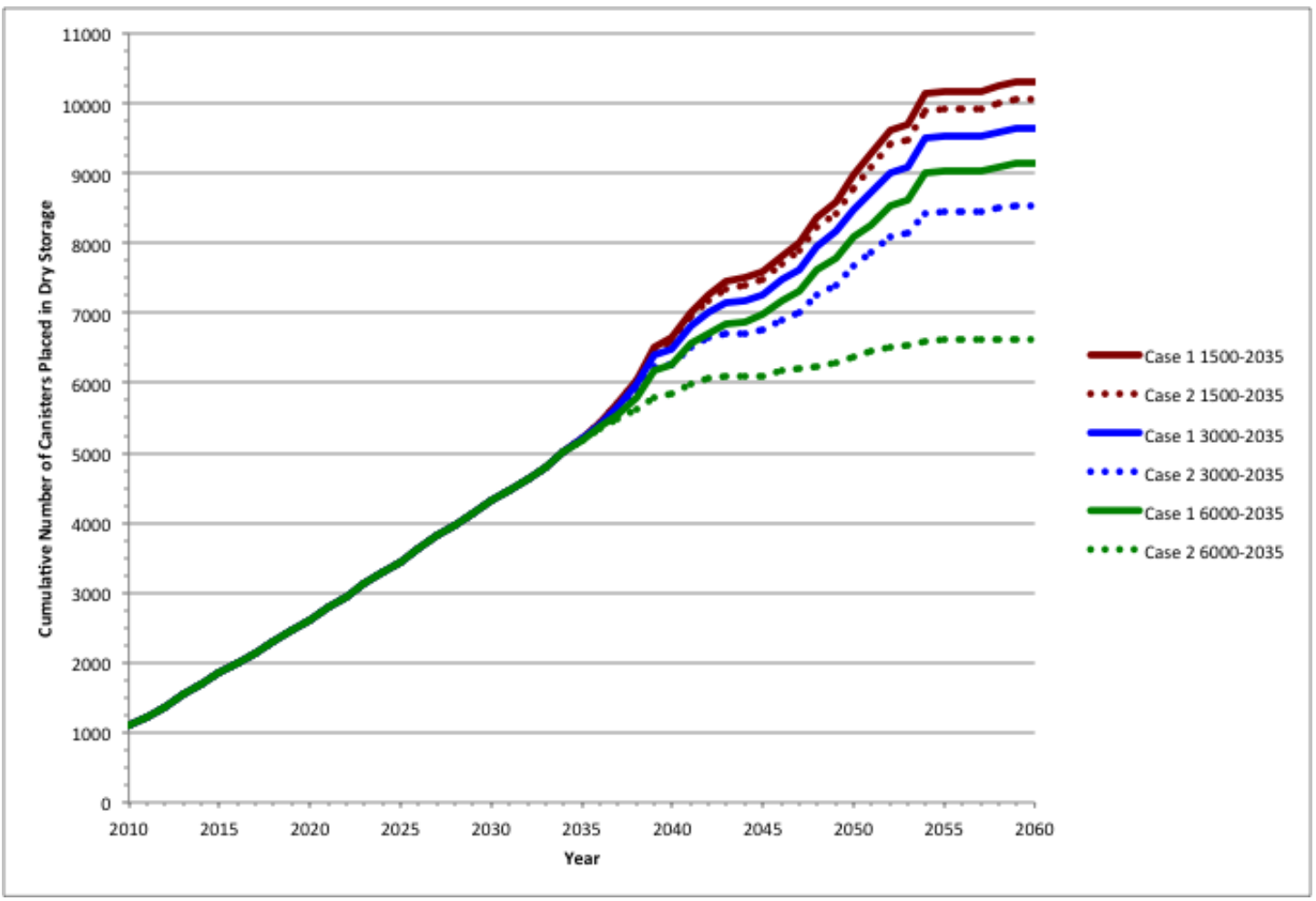

b) 2035 Start of Acceptance

Figure 4-1. Cumulative Number of Canisters Placed in Dry Storage at Reactors 
At-reactor logistic summary results pertaining to off-site shipping of used nuclear fuel, both from the used fuel pools and from dry storage, are shown in Tables 4-3 and 4-4. Unlike on-site dry storage inventories, there are significant differences in the resulting shipping characteristics for the different cases, acceptance rates, and when acceptance begins. More detailed discussions of the results and trends for each case and input variable set are provided in Section A-2.3 of Appendix A.

The results in Table 4-3 show that increasing the acceptance rate of fuel from the reactors increases the peak annual rate that canisters and bare fuel casks are shipped from the reactor sites, as expected. Except for the $6000 \mathrm{MTHM} / \mathrm{yr}$ all-canister case, delaying the start of UNF acceptance from the reactors results in a reduction in the peak annual shipping rate of canisters. This is due to having an increased amount of cooler fuel in storage that is within the thermal limits of transportation casks and is available for transport. This results in fewer "spikes" as can be seen in the results shown in Section A-2.3 of Appendix A and reduced peak annual arrivals ${ }^{\mathrm{a}}$. This same effect has the opposite impact for a $6000 \mathrm{MTHM} / \mathrm{yr}$ acceptance rate where the delay allows for a much larger fraction of the fuel to cool, which can then be transported at a higher rate as shown by comparing Figures A-23 and A-25 in Appendix A. Overall, these results show that cooler UNF (achieved in these scenarios through delay in the start of acceptance) allows for a much smoother shipping profile, either reducing "spikes" that occur in the 1500 and $3000 \mathrm{MTHM} / \mathrm{yr}$ acceptance rate scenarios or increasing the actual amount of fuel that can be shipped in $6000 \mathrm{MTHM} / \mathrm{yr}$ acceptance rate scenarios.

Comparing the cases that involve transporting all UNF in canisters with cases that include transporting bare UNF from the used fuel pools shows that the peak annual rate of canister shipments decreases with the fraction of the bare fuel being transported in re-useable transportation casks. On the average, the reuseable transportation casks carry smaller loads than dual-purpose canisters ${ }^{\mathrm{b}}$ because of the thermal constraints discussed above and require aging many fully-loaded DPCs on-site before they are cool enough to be shipped. This results in a large peak annual rate of bare fuel cask shipments that exceeds the reduction in the peak shipment rate of canisters. The effect of direct shipment of bare fuel on reduced average shipment capacity is shown in table 4-5 below, which presents the shipping data for the three acceptance rates with acceptance starting in 2020. While the average capacities for shipments of canistered fuel are about the same in both the all canistered and the canister plus bare cases, the average capacity of the bare fuel shipments is lower, reducing further as the shipment rate is increased. Thus, total peak annual canister arrivals are larger when transporting both canisters and bare fuel in re-useable transportation casks.

The cumulative canister/cask shipment results in Table 4-4 show that when all UNF is transported from the reactors in canisters (Cases 1 and 3) a total of approximately 11,200 canisters are shipped that must be subsequently stored and processed. These results also demonstrate that the amount of UNF shipped as bare fuel in re-useable transportation casks (Cases 2 and 4) decreases as the acceptance rate decreases and the time that fuel acceptance begins is delayed from 2020 to 2035. The amount of fuel shipped in canisters increases correspondingly. These results, also represented graphically in Figure 4-2 for individual UNF assemblies, show that lower the acceptance rates or delays in the start of acceptance of fuel from the reactors "hardens" the "boundary condition" of UNF entering the down-stream UNF

\footnotetext{
${ }^{a}$ Note that the TSL model assigns acceptance rights to the reactors every year. If a reactor site has insufficient fuel available to meet the allocated acceptance, it maintains the difference between fuel allocated and fuel shipped until the next year. This results in "spiky" behavior shown in the charts in Section A-2.3 of Appendix A.

${ }^{\mathrm{b}}$ It is assumed that bare fuel can be transported in either re-useable transportation casks (rail or legal weight truck) that can be derated or in specially designed high-heat re-useable transportation casks. De-rated casks and high-heat casks are of lower capacity than fully loaded bare fuel rail casks and dual purpose canisters. See Section A-1.3 of Appendix A.
} 
management system. This results in potential reduced flexibility later and is due to more UNF being placed in at-reactor dry canister storage systems for lower acceptance rates and/or delay in the start of UNF acceptance.

The at-reactor shipping logistic results are much smoother over time for acceptance rates of 1500 MTHM and $3000 \mathrm{MTHM} / \mathrm{yr}$ than for an acceptance rate of $6000 \mathrm{MTHM} / \mathrm{yr}$ when acceptance begins in 2020.

There is sufficient fuel in storage when shipping begins in either 2020 or 2035 that is cool enough and can be shipped while younger fuel cools. However, for a $6000 \mathrm{MTHM} / \mathrm{yr}$ acceptance rate the inventory of UNF that can be transported is rapidly depleted and actual acceptance decreases until the fuel cools sufficiently and can be transported. When acceptance begins in 2035 there is sufficient cool fuel available to support shipment at or near the desired acceptance rate.

\subsection{Summary of Logistic Results: Consolidated Storage Facility UNF Management}

The shipment of UNF from the reactors to the CSF, summarized above and discussed in more detail in Appendix A, has a very strong influence on the characteristics of the CSF. The rate that fuel arrives at and is shipped from the CSF dictates the number of processing facilities (or bays) needed. In addition, the duration between the start of CSF operations and the start of repository operations has a significant effect on the peak size of the CSF.

Detailed CSF UNF management logistic results for the cases identified in Section 3.2.4 with the assumptions defined in Section 3.3 are presented in Sections A-3 of Appendix 3 and are summarized herein. The results presented in this section establish requirements for the facilities described in Section 5 (and in detail in Appendix C) for each of the cases evaluated. Table 4-6 provides the summary logistics results for CSF UNF management. As expected, higher acceptance rates result in larger facility requirements, as indicated by peak canister arrival rates, bare fuel casks arrival rates, and peak inventories in storage.

Increasing the duration between the start of CSF and repository operations leads to a significant increase in the inventory of UNF in storage (either dry or as bare fuel). Higher acceptance rates combined with a longer duration between the start of CSF and repository operations results in the largest inventories. These trends are shown in Figures 4-3 through 4-5.

When all UNF is transported from the reactors to the CSF in canisters (Cases 1 and 3), peak annual arrival rates increase with larger UNF acceptance rates, as shown in Table 4-6 and in Figures 4-6 through 4-8. There is no difference in peak annual canister arrival rates at the CSF as the duration between start of CSF and repository operations increases for either re-packaging at the repository or at the CSF. 


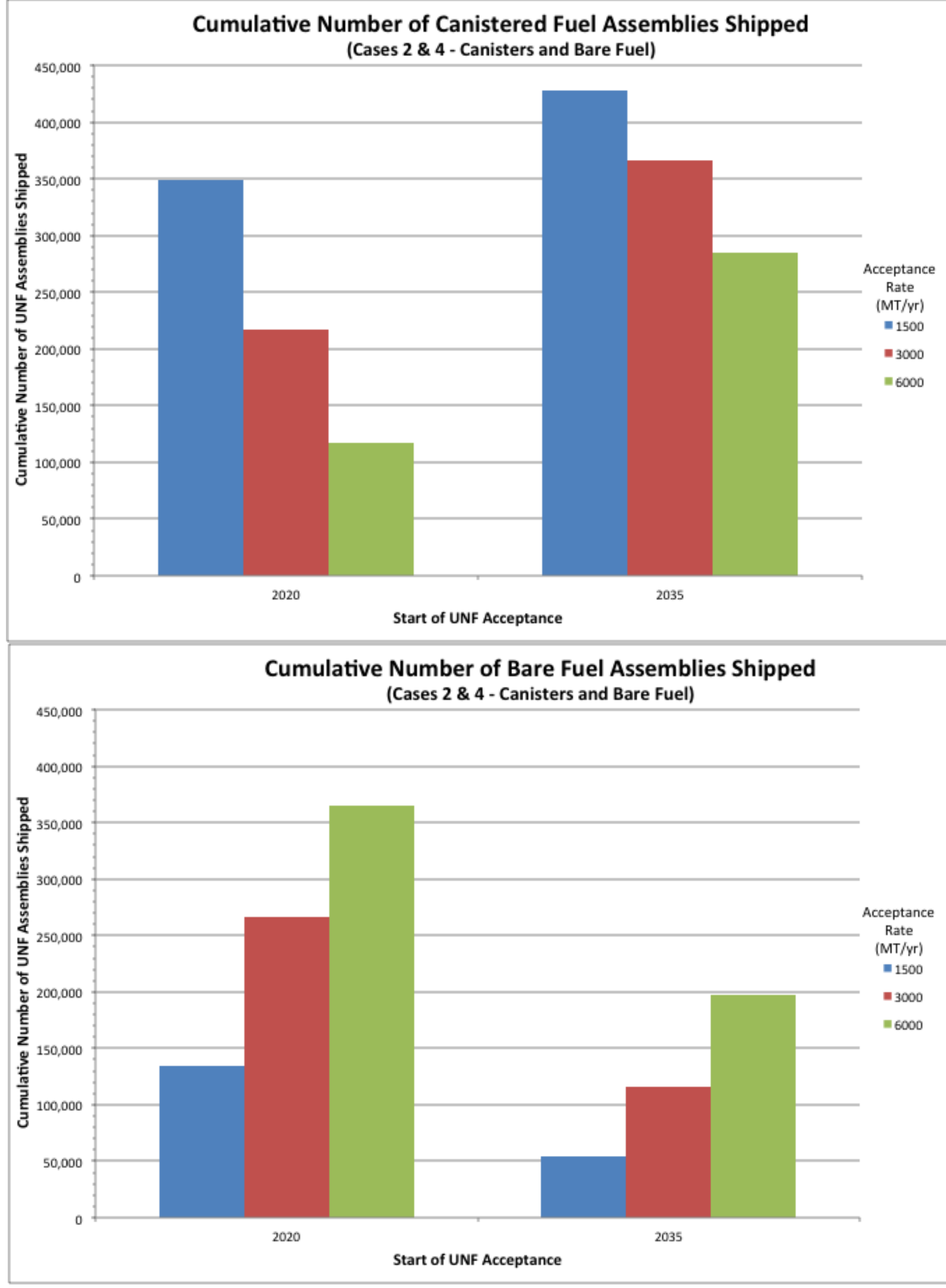

Figure 4-2. Cumulative Bare Fuel Assembly Shipments (Cases 2 and 4, Canisters and Bare Fuel) 


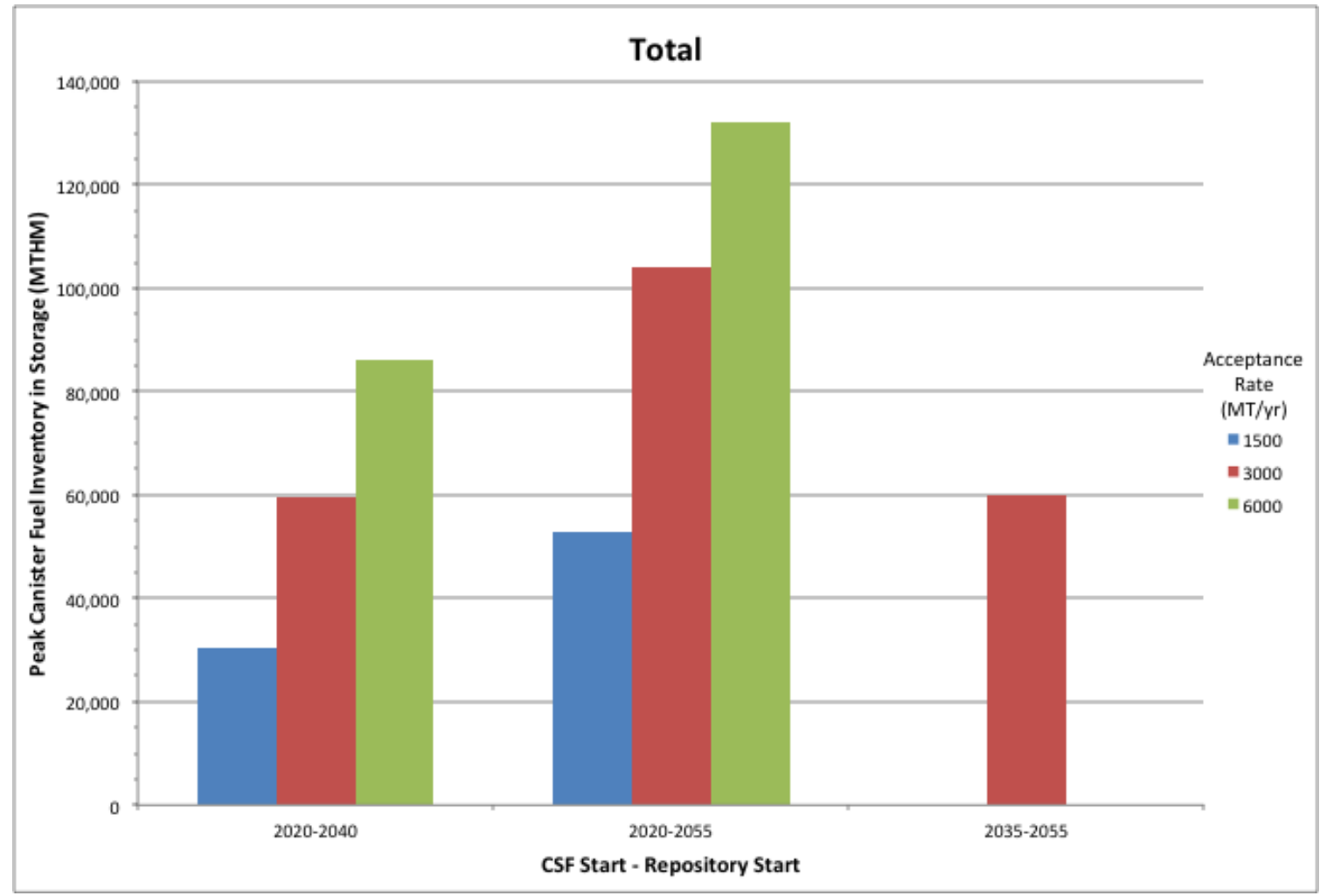

Figure 4-3. Peak Inventory in Dry Storage at the CSF (Cases 1 and 3, Canisters Only)

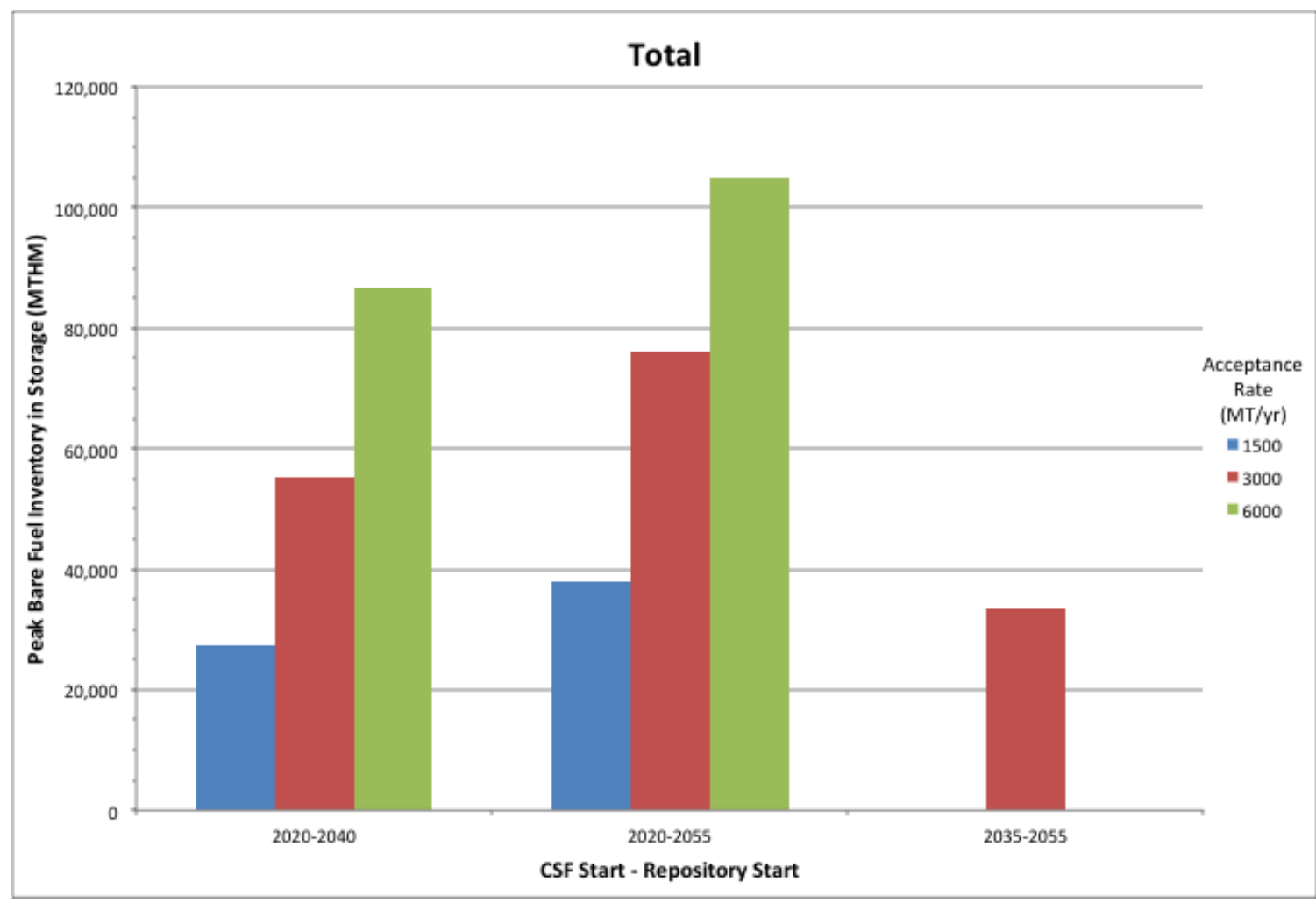

Figure 4-4. Peak Inventory in Bare Fuel Storage at the CSF (Cases 2 and 4, Canisters and Bare Fuel) 


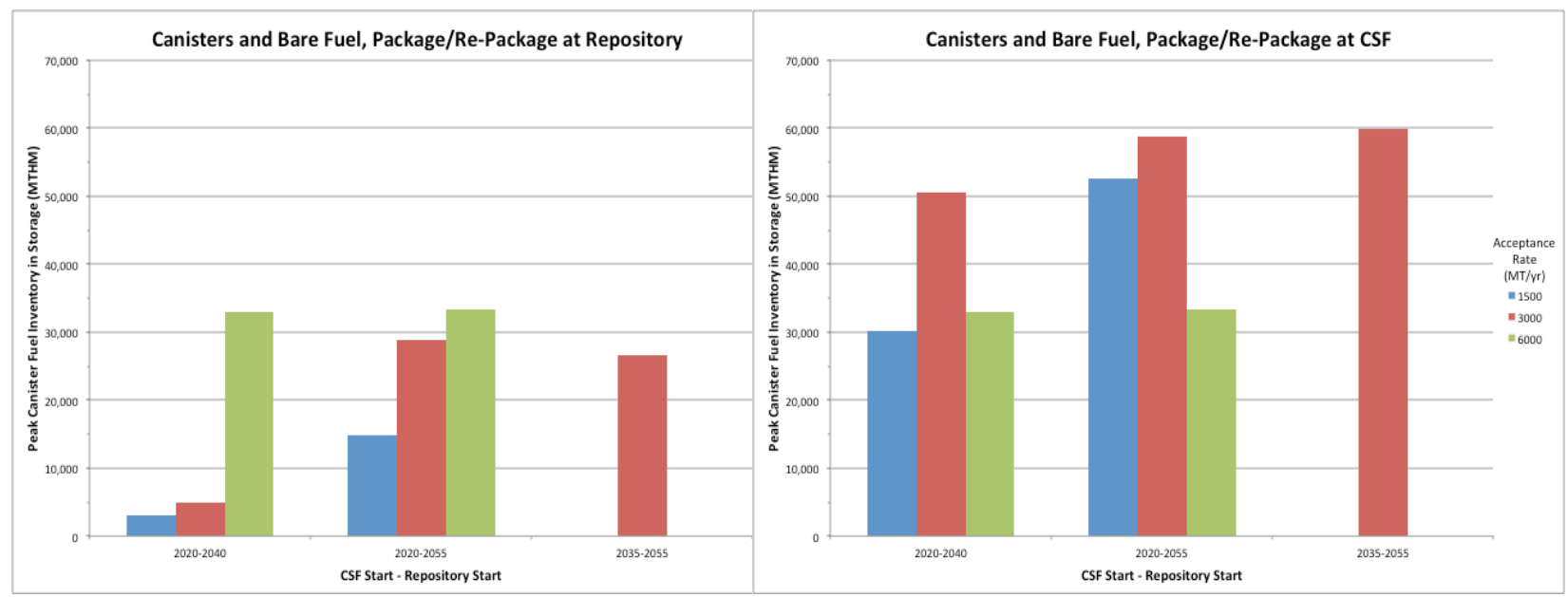

Figure 4-5. Peak Inventory in Dry Storage at the CSF (Cases 2 and 4, Canisters and Bare Fuel)

Where re-packaging occurs, either at the repository or at the CSF, does not affect the peak annual canister arrival rates at the CSF for acceptance rates of 1500 and $6000 \mathrm{MTHM} / \mathrm{yr}$. However, for an acceptance rate of $3000 \mathrm{MTHM} / \mathrm{yr}$, the peak canister acceptance rate is higher when re-packaging is performed at the CSF. This is solely due to a spike of vertical canisters arriving at the CSF in 2055 from the South Texas Project. Figures A-45 and A-63 in Appendix A show the comparison of arrivals at the CSF in more detail (the 3000 MTHM/yr curves overlap with the 6000 MTHM/yr curves in Figure A-63 and the South Texas spike is difficult to see). Figure A-19 in Appendix A clearly shows this spike occurring in shipments from the reactors. This spike occurs because of the thermal limits on the transportation overpacks/casks used for the South Texas canisters resulting in the canisters having to remain in at-reactor storage until they have sufficiently cooled ${ }^{\mathrm{c}}$ (not that this occurs for other reactors, but is most evident for the South Texas Project because their canisters/overpacks are tracked).

When bare fuel from the UNF pools at the reactor sites is sent to the CSF there is a reduction in the peak annual arrival rate of canisters to the CSF, corresponding with arrivals of re-useable transportation casks carrying bare fuel assemblies. The peak annual arrival rate of bare fuel casks increases with larger UNF acceptance rates as shown in Table 4-6, but is not affected by the duration between start of CSF and repository operations. Delaying the start of CSF operations decreases the peak annual arrival rate of bare fuel because more fuel is transferred to canisters for at-reactor dry storage, decreasing the bare fuel shipping rates from the reactors (see Table 4-3).

There is no difference in the peak annual arrival rate of bare fuel casks at the CSF when packaging/repackaging occurs at either the CSF or the repository. This is because the two cases have identical YFF-5 acceptance preferences, which result in identical bare UNF shipments from the reactors to the CSF.

While the peak annual arrival rates for bare fuel transportation casks are not affected by where packaging/re-packaging occurs or the duration between the start of CSF and repository operations, the peak annual canister arrival rates do differ. Peak canister arrival rates are higher when packaging/re-

\footnotetext{
${ }^{\mathrm{c}}$ Note that this also occurs for other reactors, but is most evident for the South Texas Project canisters those canisters are would be designed differently to accommodate the longer South Texas Project reactor fuel assemblies and as such the canisters/overpacks are tracked separately in TSL.
} 
packaging is performed at the CSF because all canisters are ultimately processed through the CSF and peak canister shipping rates from the reactors occur later, after the bare fuel has been shipped.

When packaging/re-packaging occurs at the CSF, there is no difference in peak annual canister arrival rate as the duration between the start of CSF and repository operations increases. Again, all canisters must be processed through the CSF. However, when packaging/re-packaging occurs at the repository, the peak annual canister arrival rates are larger when the period between the start of CSF and repository operations is longer. This is due to the later arrival of canisters to the CSF that occur under the YFF-5 acceptance preference.

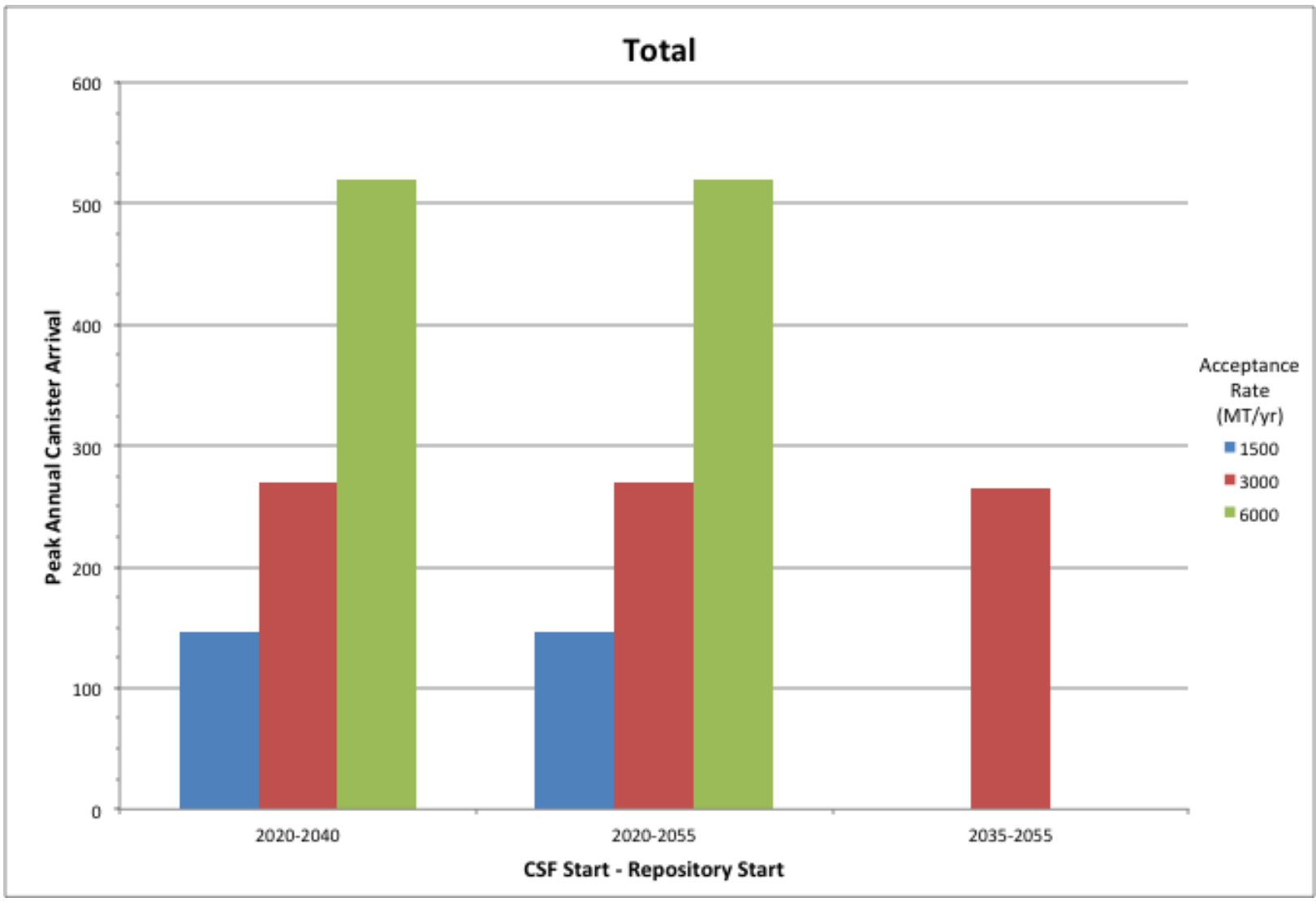

Figure 4-6. Peak Annual Canister Arrivals at the CSF (Cases 1 and 3, Canisters Only) 


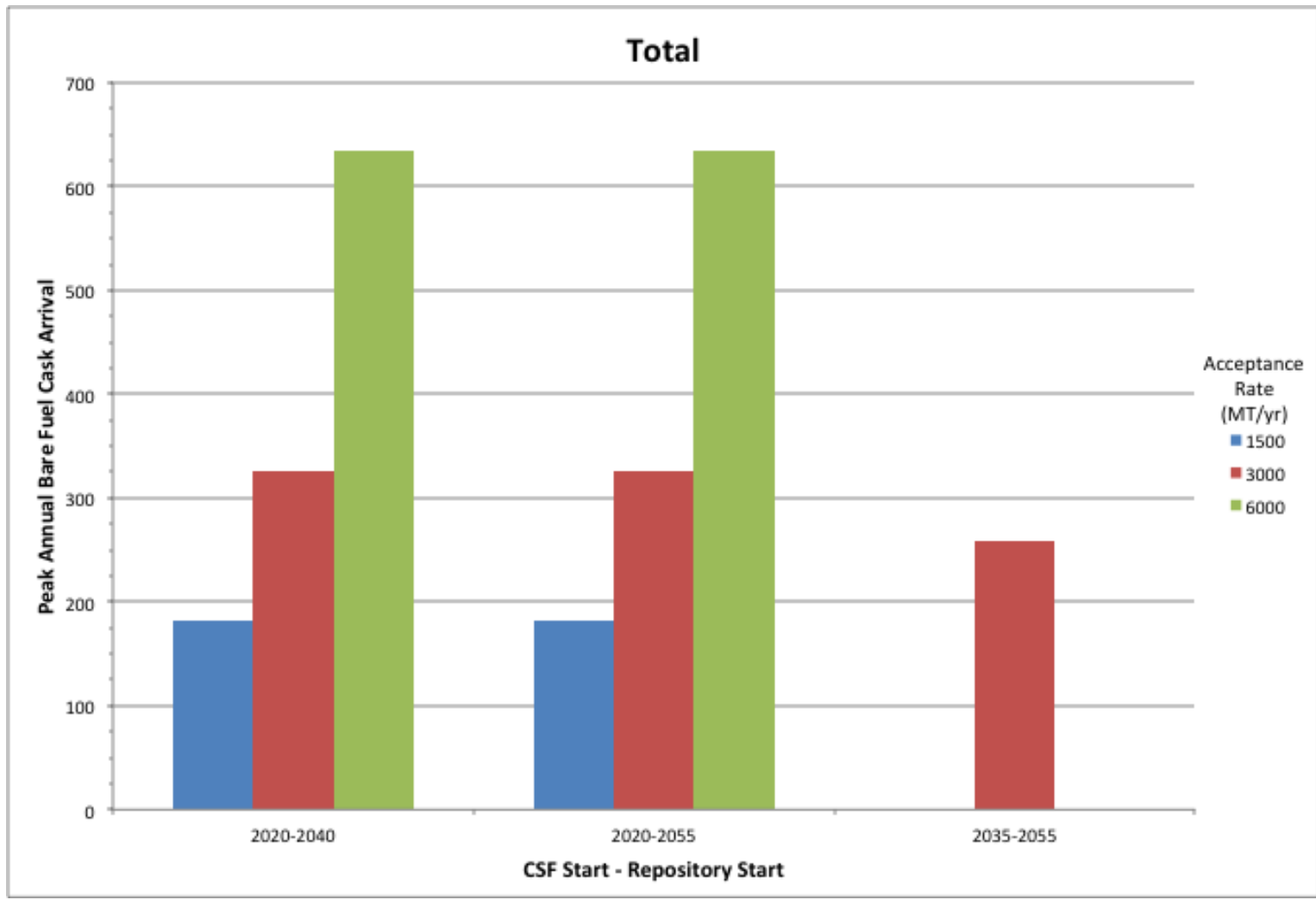

Figure 4-7. Peak Annual Bare Fuel Cask Arrivals at the CSF (Cases 2 and 4, Canisters and Bare Fuel)
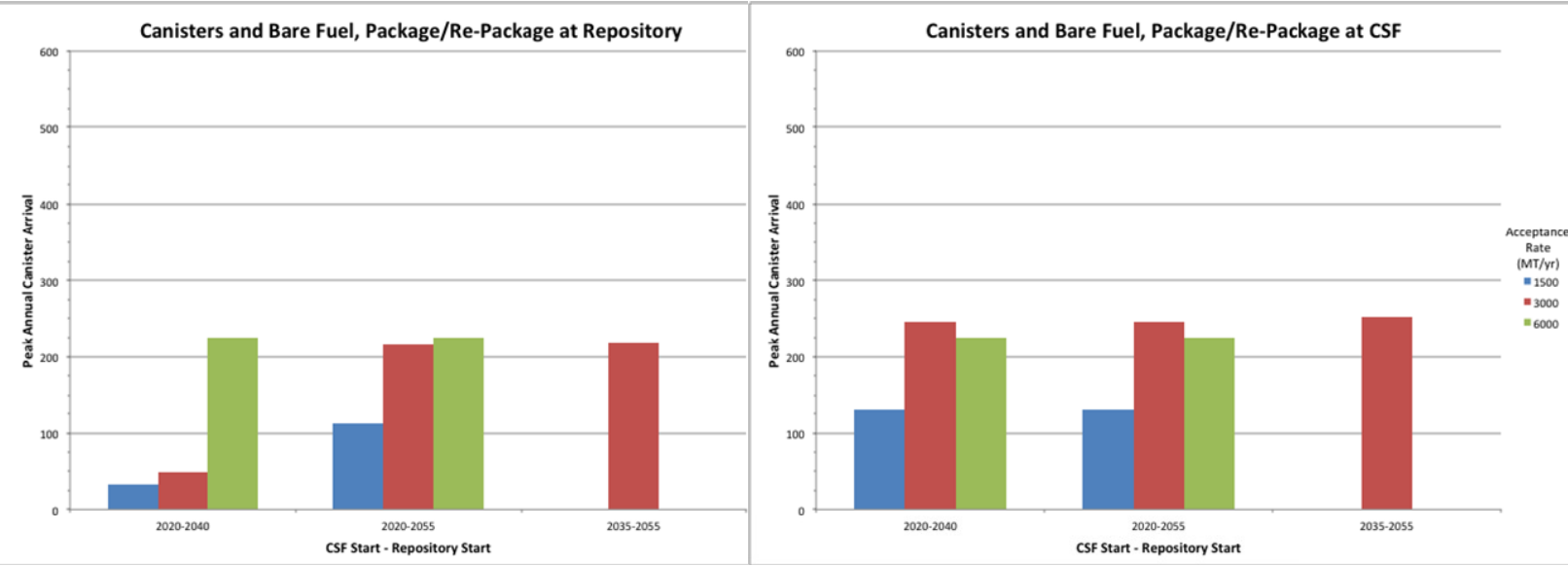

Figure 4-8. Peak Annual Canister Arrivals at the CSF (Cases 2 and 4, Canisters and Bare Fuel)

\subsection{Summary of Logistic Results: UNF Packaging/Re-Packaging}

This section presents summary logistics results for fuel handling operations to package bare fuel and to re-package canistered fuel (in existing size canisters) into waste package compatible size canisters. Detailed CSF UNF management logistic results for the cases identified in Section 3.2.4 with the assumptions defined in Section 3.3 are presented in Sections A-3 of Appendix 3 and are summarized. Table 4-7 provides summary level logistics results for fuel handling operations (packaging/re-packaging). 
The rate that UNF is shipped from the reactors and/or the CSF to the repository, and how the UNF is managed (all canisters or canisters and bare fuel) has a very strong influence on the characteristics of the packaging/re-packaging operation:

- The rate that UNF is transferred to waste package compatible size canisters affects the number of packaging stations required.

- The rate that canisters are opened affects the number of cutting stations required.

- The rate that waste package compatible size canisters are produced affects the number of welding stations and release bays required.

The results show that a large-scale UNF handling effort will be needed regardless of the UNF management strategy, acceptance rates, and acceptance start dates. There is always a need to re-package large, existing-size canisters for each scenario considered in this evaluation.

In total, approximately 205,000 PWR UNF assemblies and approximately 275,000 BWR assemblies have to be packaged into waste package compatible size canisters. If all UNF is transferred into existing size canisters prior to shipment from the reactors, approximately 11,200 canisters would have to be opened and the contents re-packaged.

Higher throughput leads to higher peak annual processing rates throughout the fuel handling operation. Loading smaller waste package compatible size canisters requires more handling operations (i.e., moving into the facility, applying closure welds, and preparing for off-site transport if at the CSF). It can be seen that where the packaging/re-packaging operation is performed results in very little difference in the peak annual re-packaging rates.

The results shown in Table 4-7 show that regardless of the whether the entire UNF inventory is canisterized or if some is maintained uncanisterized, peak annual UNF handling rates are similar for a given scenario. The peak UNF handling rates increase for larger throughput rates, as expected, because a finite number of fuel assemblies have to be processed to meet the throughput rate regardless of whether they are canistered or bare. The main difference between the two broad sets of cases (all canisters or canisters and bare fuel) is associated with the need to open the canisters, which is greater in the all canister cases.

Figure 4-9 shows the peak rates that existing size canisters are re-packaged for each of the different cases considered. It can be seen that maintaining UNF uncanistered reduces the peak annual rate that canisters must be re-packaged, most significantly for a throughput rate of $6000 \mathrm{MTHM} / \mathrm{yr}$ and less significantly for a throughput rate of $3000 \mathrm{MTHM} / \mathrm{yr}$. There is no significant difference in the peak canister re-packaging rate for a throughput rate of $1500 \mathrm{MTHM} / \mathrm{yr}$ because a larger fraction of the UNF assemblies end up being transferred to existing size canisters for dry storage at the reactors to maintain pool capacity. This indicates that the any potential benefit in terms of reducing the need to re-package existing size canisters would be realized at higher acceptance rates with little benefit associated with maintaining bare fuel at low acceptance rates (1500 MTHM/yr). This same trend can be seen with respect to the total number of canisters that are re-packaged shown in Figure 4-10.

The peak annual generation rate of waste package compatible size canisters increases with increasing throughput and decreasing fuel assembly capacity. These rates not only affect the number of welding stations and release bays required, but also the size of the transportation fleet to ship fuel from the CSF to the repository when the packaging/re-packaging is performed at the CSF (see Section 4.5 for additional discussion). Since a large number of waste package compatible size canisters would be generated, especially if smaller capacity canisters are used, evaluations and design of alternative canister concepts (i.e., can-in-canister, multi-overpack transportation casks) and processing techniques is future work worth considering. 


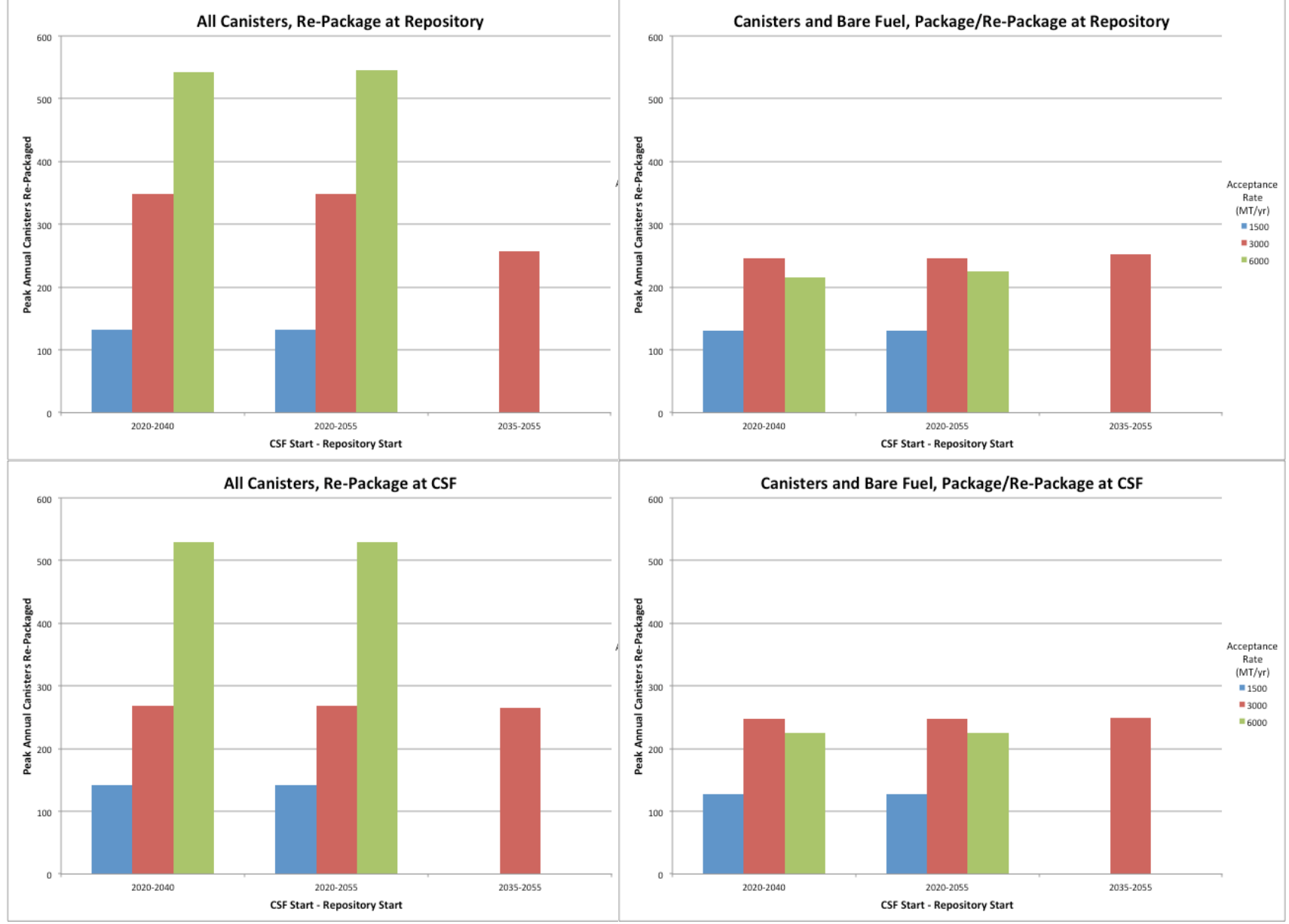

Figure 4-9. Peak Annual Canister Re-Packaging Rates 

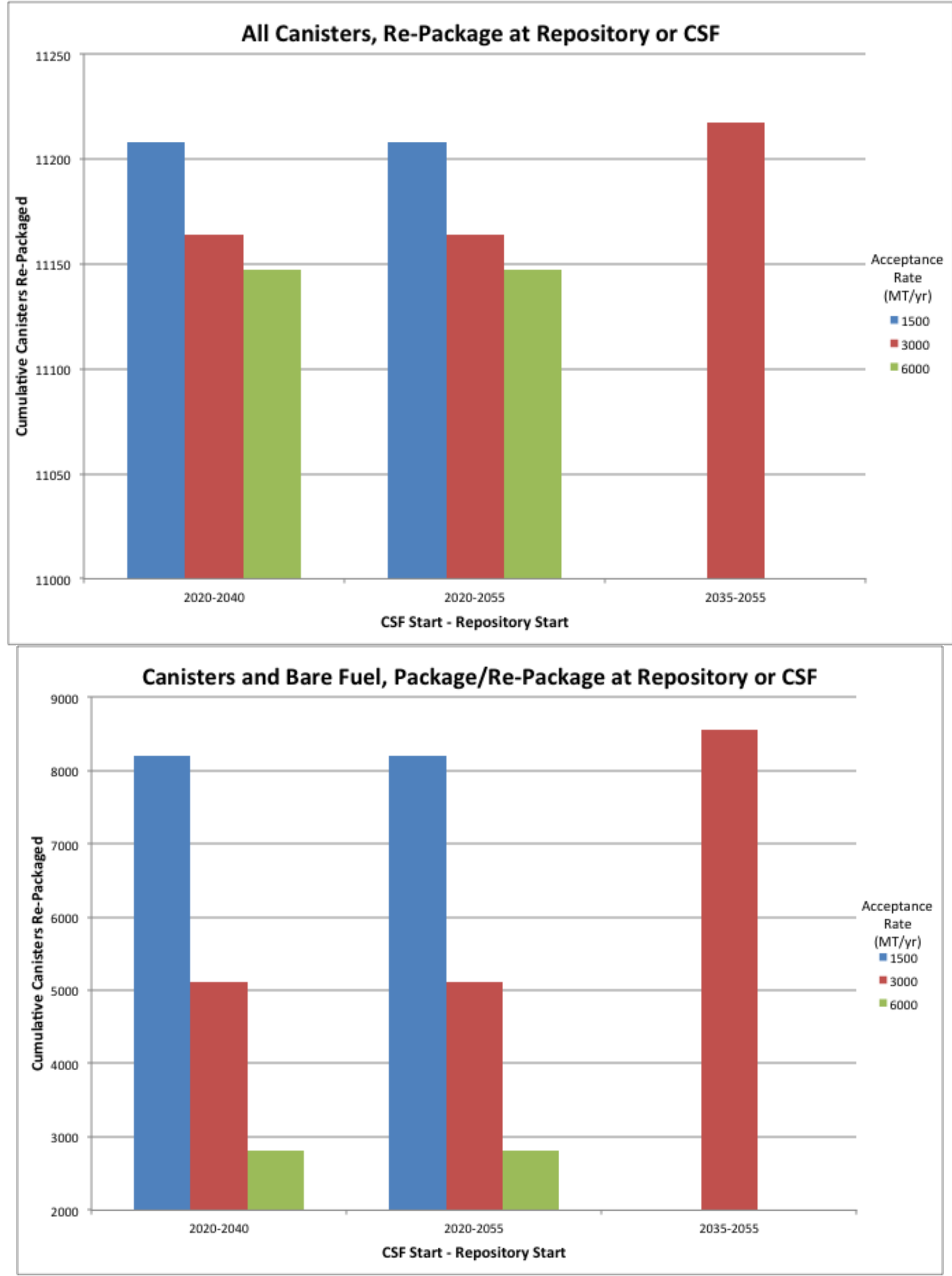

Figure 4-10. Cumulative Canisters Re-Packaged 


\subsection{Summary of Logistic Results: Repository}

Summary level results for repository operations are shown in Table 4-8. These results only show the quantities of canisters and re-useable transportation casks. When re-packaging occurs at the CSF, only waste package compatible size canisters arrive at the repository for disposal. When re-packaging occurs at the repository, canisters (Case 1) or canisters and bare fuel (Case 2) arrive for subsequent re-packaging prior to disposal. Operations related to disposing disposal canisters are discussed elsewhere (Hardin et al. 2012).

\subsection{Summary of Logistic Results: Transportation}

Summary results of the transportation logistics modeling for each case are shown in Table 4-9 and details are provided in Appendix A (Section A-4). Overall, the results show that higher UNF acceptance rates (either from the reactor sites or from the CSF) lead to larger infrastructure requirements, as expected.

When considering scenarios where all UNF is loaded into canisters at the reactor sites prior to transport either to the CSF or the repository (Cases 1 and 3), it can be seen that when the packaging/re-packaging facility is located at the CSF the required transportation fleet is larger. In a sense, essentially two transportation fleets are required to ship UNF both to the CSF and from the CSF to the repository. Recall, when the repository starts operations, the rate of UNF transfer to the repository is the same as the rate of UNF transfer from the reactors to the CSF.

In addition, a larger number of lower capacity canisters are shipped between the CSF and the repository to maintain the overall UNF transfer rate, resulting in larger transportation fleet requirements. This trend can be seen in Table 4-9 where there is a difference in fleet requirements for 12 and 21 PWR assembly capacity canisters at a throughput of $3000 \mathrm{MTHM} / \mathrm{yr}$ when all UNF is transported canisters and repackaging occurs at the CSF. No difference is seen when re-packaging occurs at the repository since the casks being transported from the CSF to the repository contain large, existing size canisters. In cases where re-packaging occurs at the CSF there is a need to acquire a large number of casks, rail cars, etc. when the repository begins operation in either 2040 or 2055 (see Table A-33 of Appendix A).

The results in Table 4-9 show that the transportation fleet requirements are larger for the cases that transport UNF in the used fuel pools to the CSF when it begins operations (Cases 2 and 4). This is because the re-useable transportation casks may be loaded to a lower capacity than dual-purpose canisters due to heat content of the UNF in the pools as opposed to the existing size dual-purpose canisters, which although fully loaded may have to be stored on-site for a period before they are cool enough to transport (see Table 4-5 above) ${ }^{\mathrm{d}}$. In addition, those reactor sites that currently cannot directly load rail casks are assumed to utilize legal-weight truck shipping in the scenarios (Cases 2 and 4) that involve bare fuel shipments (although it is assumed that they can ship dual purpose canisters by heavy-haul to the nearest rail head for the scenarios that involve all canisters, Cases 1 and 3). This assumption leads to the need for a legal weight truck transportation fleet in addition to the rail fleet in bare fuel scenarios (this assumption will be revisited in future analyses).

As before, the transportation fleet requirements are larger when bare fuel packaging and DPC repackaging occurs at the CSF for the same reasons as discussed above.

${ }^{d}$ It is assumed that bare fuel can be transported in either re-useable transportation casks (rail or legal weight truck) that can be derated or in specially designed high-heat re-useable transportation casks. De-rated casks and high-heat casks are of lower capacity than fully loaded bare fuel rail casks and dual purpose canisters. See Section A-1.3 of Appendix A. 
Table 4-3. Peak Annual Bare Cask/Canister Shipping Rate from Reactors (Cask/Canister per year)

\begin{tabular}{|c|c|c|c|c|c|c|c|c|}
\hline & $\begin{array}{c}\text { Acceptance Rate } \\
\text { (MT/yr) }\end{array}$ & $\begin{array}{l}\text { Acceptance } \\
\text { Start }\end{array}$ & PWR Bare & BWR Bare & Total Bare & $\begin{array}{c}\text { PWR } \\
\text { Canisterized }\end{array}$ & $\begin{array}{c}\text { BWR } \\
\text { Canisterized }\end{array}$ & $\begin{array}{c}\text { Total } \\
\text { Canisterized }\end{array}$ \\
\hline \multirow{6}{*}{$\begin{array}{l}\text { Canistered } \\
\text { Fuel Transport } \\
\text { (Cases } 1 \text { \& 3) }\end{array}$} & 1500 & 2020 & & & & 100 & 74 & 147 \\
\hline & 3000 & 2020 & & & & 236 & 132 & 348 \\
\hline & 6000 & 2020 & & & & 311 & 234 & 520 \\
\hline & 1500 & 2035 & & & & 97 & 74 & 145 \\
\hline & 3000 & 2035 & & & & 181 & 131 & 265 \\
\hline & 6000 & 2035 & & & & 452 & 232 & 619 \\
\hline \multirow{6}{*}{$\begin{array}{c}\text { Bare and } \\
\text { Canistered } \\
\text { Fuel Transport } \\
\text { (Cases } 2 \text { \& 4) }\end{array}$} & 1500 & 2020 & 119 & 63 & 182 & 98 & 62 & 130 \\
\hline & 3000 & 2020 & 202 & 133 & 325 & 179 & 124 & 246 \\
\hline & 6000 & 2020 & 404 & 303 & 636 & 153 & 121 & 225 \\
\hline & 1500 & 2035 & 83 & 57 & 137 & 94 & 62 & 131 \\
\hline & 3000 & 2035 & 146 & 112 & 258 & 179 & 126 & 252 \\
\hline & 6000 & 2035 & 316 & 245 & 544 & 323 & 196 & 482 \\
\hline
\end{tabular}

Note: The sum of PWR and BWR peak rates do not sum to the total as the peak rates occur in different years.

Table 4-4. Cumulative Number of Canisters/Casks Shipped from Reactors

\begin{tabular}{|c|c|c|c|c|c|c|c|c|}
\hline & $\begin{array}{c}\text { Acceptance Rate } \\
\text { (MT/yr) }\end{array}$ & $\begin{array}{c}\text { Acceptance } \\
\text { Start } \\
\end{array}$ & PWR Bare & BWR Bare & Total Bare & $\begin{array}{c}\text { PWR } \\
\text { Canisterized }\end{array}$ & $\begin{array}{c}\text { BWR } \\
\text { Canisterized }\end{array}$ & $\begin{array}{c}\text { Total } \\
\text { Canisterized } \\
\end{array}$ \\
\hline \multirow{6}{*}{$\begin{array}{c}\text { Canistered Fuel } \\
\text { Transport (Cases } \\
1 \text { \& 3) }\end{array}$} & 1500 & 2020 & & & & 6998 & 4210 & 11208 \\
\hline & 3000 & 2020 & & & & 6974 & 4190 & 11164 \\
\hline & 6000 & 2020 & & & & 6964 & 4183 & 11147 \\
\hline & 1500 & 2035 & & & & 7017 & 4223 & 11240 \\
\hline & 3000 & 2035 & & & & 7001 & 4216 & 11217 \\
\hline & 6000 & 2035 & & & & 6990 & 4208 & 11198 \\
\hline \multirow{6}{*}{$\begin{array}{c}\text { Bare and } \\
\text { Canistered Fuel } \\
\text { Transport (Cases } \\
\text { 2 \& 4) }\end{array}$} & 1500 & 2020 & 2338 & 1342 & 3680 & 5145 & 3051 & 8196 \\
\hline & 3000 & 2020 & 4848 & 2897 & 7745 & 3190 & 1916 & 5106 \\
\hline & 6000 & 2020 & 8376 & 4800 & 13176 & 1712 & 1056 & 2768 \\
\hline & 1500 & 2035 & 837 & 536 & 1373 & 6326 & 3728 & 10054 \\
\hline & 3000 & 2035 & 2020 & 1141 & 3161 & 5315 & 3232 & 8547 \\
\hline & 6000 & 2035 & 3783 & 2219 & 6002 & 4094 & 2535 & 6629 \\
\hline
\end{tabular}

Note: The sum of PWR and BWR peak rates do not sum to the total as the peak rates occur in different years. 
Used Fuel Management System Architecture Evaluation, Fiscal Year 2012

October 2012

Table 4-5. Average Capacity of Cask Shipments from Reactors

\begin{tabular}{|c|c|c|c|c|c|c|c|c|c|}
\hline \multirow{2}{*}{$\begin{array}{c}\text { Acceptance } \\
\text { Rate } \\
\text { (MTHM/yr) }\end{array}$} & \multicolumn{4}{|c|}{ Canisters and Bare Fuel (Cases 2 and 4) } & \multicolumn{3}{c|}{ All Canisters (Cases 1 and 3) } \\
\cline { 2 - 10 } & $\begin{array}{c}\text { Cumulative } \\
\text { Shipments } \\
\text { (MTHM) }\end{array}$ & $\begin{array}{c}\text { Cumulative } \\
\text { Shipments } \\
\text { (Casks) }\end{array}$ & $\begin{array}{c}\text { Average } \\
\text { Shipment } \\
\text { (MTHM / Cask) }\end{array}$ & $\begin{array}{c}\text { Cumulative } \\
\text { Shipments } \\
\text { (MTHM) }\end{array}$ & $\begin{array}{c}\text { Cumulative } \\
\text { Shipments } \\
\text { (Casks) }\end{array}$ & $\begin{array}{c}\text { Average } \\
\text { Shipment } \\
\text { (MTHM / Cask) }\end{array}$ & $\begin{array}{c}\text { Cumulative } \\
\text { Shipments } \\
\text { (MTHM) }\end{array}$ & $\begin{array}{c}\text { Cumulative } \\
\text { Shipments } \\
\text { (Casks) }\end{array}$ & $\begin{array}{c}\text { Average } \\
\text { Shipment } \\
\text { (MTHM / Cask) }\end{array}$ \\
\hline $1500^{\mathrm{a}}$ & 38179 & 3680 & 10.4 & 100556 & 8196 & 12.3 & 138735 & 11208 & 12.4 \\
\hline $3000^{\mathrm{b}}$ & 76544 & 7745 & 9.9 & 62191 & 5106 & 12.2 & 138735 & 11164 & 12.4 \\
\hline $6000^{\mathrm{c}}$ & 105541 & 13176 & 8.0 & 33194 & 2768 & 12.0 & 138735 & 11147 & 12.4 \\
\hline
\end{tabular}

a Tables A-21, A-15

b Tables A-23, A-17

c Tables A-25, A-19 
Table 4-6. Consolidated Storage Facility Logistics Summary Results

\begin{tabular}{|c|c|c|c|c|c|c|c|c|c|c|c|c|c|c|c|c|c|}
\hline \multirow[b]{3}{*}{ Scenario } & \multirow[b]{3}{*}{$\begin{array}{c}\text { Acceptance } \\
\text { Rate }\end{array}$} & \multirow[b]{3}{*}{$\begin{array}{l}\text { CSF } \\
\text { Start }\end{array}$} & \multirow[b]{3}{*}{$\begin{array}{l}\text { Repository } \\
\text { Start }\end{array}$} & \multicolumn{6}{|c|}{ Peak Annual Arrivals } & \multicolumn{8}{|c|}{ Peak Inventory in Storage } \\
\hline & & & & \multicolumn{3}{|c|}{ Bare Fuel Casks } & \multicolumn{3}{|c|}{ Canisters } & \multicolumn{2}{|c|}{$\begin{array}{c}\text { Bare Fuel } \\
\text { Assemblies }\end{array}$} & \multicolumn{2}{|c|}{ Vertical Canisters } & \multicolumn{2}{|c|}{ Horizontal Canisters } & \multicolumn{2}{|c|}{ Max MTHM } \\
\hline & & & & PWR & BWR & Total & $\begin{array}{c}\text { Horizontal } \\
\text { Total }\end{array}$ & $\begin{array}{l}\text { Vertical } \\
\text { Total }\end{array}$ & Total & PWR & BWR & 2-Year & Max & 2-Year & Max & Canisters & Bare \\
\hline \multirow{7}{*}{$\begin{array}{l}\text { Canisters Only - } \\
\text { Re-Package at } \\
\text { Repository } \\
\text { (Case 1) }\end{array}$} & 1500 & 2020 & 2040 & & & & 27 & 142 & 147 & & & 259 & 2155 & 13 & 325 & 30377 & \\
\hline & 3000 & 2020 & 2040 & & & & 61 & 257 & 269 & & & 480 & 3981 & 45 & 825 & 59731 & \\
\hline & 6000 & 2020 & 2040 & & & & 156 & 476 & 520 & & & 900 & 5349 & 108 & 1581 & 86031 & \\
\hline & 1500 & 2020 & 2055 & & & & 50 & 142 & 147 & & & 259 & 3471 & 13 & 791 & 52824 & \\
\hline & 3000 & 2020 & 2055 & & & & 85 & 257 & 269 & & & 480 & 6506 & 45 & 1839 & 104054 & \\
\hline & 6000 & 2020 & 2055 & & & & 162 & 476 & 520 & & & 900 & 8241 & 108 & 2347 & 132300 & \\
\hline & 3000 & 2035 & 2055 & & & & 81 & 236 & 265 & & & 448 & 3696 & 71 & 1150 & 60081 & \\
\hline \multirow{7}{*}{$\begin{array}{l}\text { Canisters and } \\
\text { Bare Fuel - } \\
\text { Re-Package at } \\
\text { Repository } \\
\text { (Case 2) }\end{array}$} & 1500 & 2020 & 2040 & 119 & 63 & 182 & 9 & 31 & 32 & 38746 & 60144 & 447 & 238 & 2 & 443 & 3010 & 27324 \\
\hline & 3000 & 2020 & 2040 & 202 & 133 & 325 & 11 & 47 & 49 & 79722 & 116490 & 84 & 346 & 7 & 91 & 4865 & 55216 \\
\hline & 6000 & 2020 & 2040 & 404 & 300 & 634 & 91 & 146 & 225 & 127651 & 174714 & 139 & 1790 & 14 & 953 & 32940 & 86605 \\
\hline & 1500 & 2020 & 2055 & 119 & 63 & 182 & 42 & 75 & 112 & 55862 & 77529 & 47 & 859 & 2 & 390 & 14834 & 37959 \\
\hline & 3000 & 2020 & 2055 & 202 & 133 & 325 & 80 & 143 & 215 & 112974 & 151895 & 84 & 1555 & 7 & 844 & 28925 & 76096 \\
\hline & 6000 & 2020 & 2055 & 404 & 300 & 634 & 91 & 146 & 225 & 156276 & 207289 & 139 & 1828 & 14 & 980 & 33280 & 104982 \\
\hline & 3000 & 2035 & 2055 & 146 & 112 & 258 & 79 & 139 & 218 & 49954 & 65464 & 84 & 1411 & 33 & 798 & 26671 & 33410 \\
\hline \multirow{7}{*}{$\begin{array}{c}\text { Canisters Only - } \\
\text { Re-Package at } \\
\text { CSF } \\
\text { (Case 3) }\end{array}$} & 1500 & 2020 & 2040 & & & & 55 & 142 & 147 & & & 259 & 2155 & 13 & 835 & 30377 & \\
\hline & 3000 & 2020 & 2040 & & & & 87 & 262 & 348 & & & 480 & 3981 & 45 & 1430 & 59979 & \\
\hline & 6000 & 2020 & 2040 & & & & 162 & 476 & 520 & & & 900 & 5349 & 108 & 1713 & 86031 & \\
\hline & 1500 & 2020 & 2055 & & & & 55 & 142 & 147 & & & 259 & 3471 & 13 & 1459 & 52839 & \\
\hline & 3000 & 2020 & 2055 & & & & 87 & 262 & 348 & & & 480 & 6512 & 45 & 2213 & 105073 & \\
\hline & 6000 & 2020 & 2055 & & & & 162 & 476 & 520 & & & 900 & 8241 & 108 & 2407 & 132300 & \\
\hline & 3000 & 2035 & 2055 & & & & 98 & 236 & 265 & & & 448 & 3696 & 71 & 1652 & 60109 & \\
\hline \multirow{7}{*}{$\begin{array}{c}\text { Canisters and } \\
\text { Bare Fuel - Re- } \\
\text { Package at CSF } \\
\text { (Case 4) }\end{array}$} & 1500 & 2020 & 2040 & 119 & 63 & 182 & 58 & 104 & 130 & 39280 & 60144 & 447 & 1721 & 2 & 839 & 30131 & 27324 \\
\hline & 3000 & 2020 & 2040 & 202 & 133 & 325 & 88 & 202 & 246 & 80044 & 116490 & 84 & 2800 & 7 & 1313 & 50451 & 55216 \\
\hline & 6000 & 2020 & 2040 & 404 & 300 & 634 & 91 & 146 & 225 & 127651 & 174714 & 139 & 1790 & 14 & 953 & 32940 & 86605 \\
\hline & 1500 & 2020 & 2055 & 119 & 63 & 182 & 58 & 104 & 130 & 55862 & 77529 & 47 & 2934 & 2 & 1459 & 52576 & 37959 \\
\hline & 3000 & 2020 & 2055 & 202 & 133 & 325 & 88 & 202 & 246 & 112974 & 151895 & 84 & 3203 & 7 & 1594 & 58845 & 76096 \\
\hline & 6000 & 2020 & 2055 & 404 & 300 & 634 & 91 & 146 & 225 & 156276 & 207289 & 139 & 1828 & 14 & 980 & 33280 & 104982 \\
\hline & 3000 & 2035 & 2055 & 146 & 112 & 258 & 94 & 219 & 252 & 49954 & 65464 & 84 & 3350 & 33 & 1655 & 59926 & 33410 \\
\hline
\end{tabular}

1. Peak BWR and PRW bare fuel cask arrivals do not necessarily sum to the total bare cask arrivals. The peaks do not necessarily occur on the same year.

2. Peak vertical and horizontal canister arrivals do not necessarily sum to the total canister arrivals. The peaks do not necessarily occur on the same year.

3. 2-Year UNF inventories are provided as these establish the storage capacity required for initial construction of the CSF (assumed part of the initial procurement and construction). 
Table 4-7. Fuel Handling Logistics Summary Results

\begin{tabular}{|c|c|c|c|c|c|c|c|c|c|c|c|c|c|c|c|c|c|c|c|c|c|c|c|c|c|c|c|c|c|c|c|c|c|c|c|}
\hline \multirow{3}{*}{ Scenario } & \multirow[b]{3}{*}{$\begin{array}{c}\text { Acceptance } \\
\text { Rate }\end{array}$} & \multirow[b]{3}{*}{$\begin{array}{c}\text { CSF } \\
\text { Start }\end{array}$} & \multirow[b]{3}{*}{$\begin{array}{c}\text { Repository } \\
\text { Start }\end{array}$} & \multirow[b]{3}{*}{$\begin{array}{c}\text { Disposal } \\
\text { casiser } \\
\text { siter }\end{array}$} & \multicolumn{6}{|c|}{ Peak Bare Fuel P } & \multirow{2}{*}{\multicolumn{4}{|c|}{ Cumulative Bare Fuel Packaging }} & \multicolumn{9}{|c|}{ Peak Canistered Fuel Re-Packaging } & \multicolumn{7}{|c|}{ Cumulative Canistered fuel Re-Packaging } & \multicolumn{5}{|c|}{ Disposal Canister Generation } \\
\hline & & & & & \multicolumn{3}{|c|}{ Assemblies } & \multicolumn{3}{|c|}{ мтнм } & & & & & & sters to 0 & & Asser & blies & & & мтнм & & & isters to 0 & & Assem & blies & MT1 & ннм & Peak A & Innual & & umulative & \\
\hline & & & & & PWR & BWR & Total & PWR & BWR & Total & PWR & BWR & PWR & BWR & PWR & BWR & Total & PWR & BWR & Total & PWR & BWR & Total & PWR & BWR & Total & PWR & BWR & PWR & BWR & PWR & BWR & PWR & BWR & Total \\
\hline & 1500 & 2020 & 2040 & 4 & & & & & & & & & & & 100 & 64 & 132 & 2879 & 4071 & 6145 & 1262 & 720 & 1647 & 6998 & 4210 & 11208 & 205983 & 276420 & 89838 & 48897 & 719 & 453 & 51496 & 30714 & 82210 \\
\hline & 3000 & 2020 & 2040 & 4 & & & & & & & & & & & 236 & 123 & 348 & 5899 & 8060 & 13170 & 2737 & 1420 & 4022 & 6974 & 4190 & 11164 & 205983 & 276420 & 89838 & 48897 & 1474 & 896 & 51496 & 30714 & 82210 \\
\hline & 3000 & 2020 & 2040 & 12 & & & & & & & & & & & 236 & 123 & 348 & 5899 & 8060 & 13170 & 2737 & 1420 & 4022 & 6974 & 4190 & 11164 & 205983 & 276420 & 89838 & 48897 & 491 & 336 & 17166 & 11518 & 28684 \\
\hline & 3000 & 2020 & 2040 & 21 & & & & & & & & & & & 236 & 123 & 348 & 5899 & 8060 & 13170 & 2737 & 1420 & 4022 & 6974 & 4190 & 11164 & 205983 & 276420 & 89838 & 48897 & 281 & 183 & 9809 & 6283 & 16092 \\
\hline & 6000 & 2020 & 2040 & 4 & & & & & & & & & & & 381 & 270 & 543 & 11300 & 17998 & 24904 & 4990 & 3141 & 6065 & 6964 & 4183 & 11147 & 205983 & 276420 & 89838 & 48897 & 2825 & 2000 & 51496 & 30714 & 82210 \\
\hline & 1500 & 2020 & 2055 & 4 & & & & & & & & & & & 100 & 64 & 132 & 2879 & 4071 & 6145 & 1262 & 720 & 1647 & 6998 & 4210 & 11208 & 205983 & 276420 & 89838 & 48897 & 720 & 452 & 51496 & 30714 & 82210 \\
\hline & 3000 & 2020 & 2055 & 4 & & & & & & & & & & & 236 & 123 & 348 & 5899 & 8060 & 13170 & 2737 & 1420 & 4022 & 6974 & 4190 & 11164 & 205983 & 276420 & 89838 & 48897 & 1474 & 896 & 51496 & 30714 & 82210 \\
\hline & 6000 & 2020 & 2055 & 4 & & & & & & & & & & & 410 & 274 & 546 & 12329 & 18022 & 24503 & 5394 & 3189 & 6063 & 6964 & 4183 & 11147 & 205983 & 276420 & 89838 & 48897 & 3082 & 2003 & 51496 & 30714 & 82210 \\
\hline & 3000 & 2035 & 2055 & 4 & & & & & & & & & & & 181 & 123 & 257 & 5261 & 8056 & 11756 & 2340 & 1421 & 3120 & 7001 & 4216 & 11217 & 205983 & 276420 & 89838 & 48897 & 1315 & 896 & 51496 & 30714 & 82210 \\
\hline & 1500 & 2020 & 2040 & 4 & 2306 & 3604 & 5236 & 1009 & 630 & 1471 & 56366 & 77529 & 24493 & 13686 & 98 & 62 & \begin{tabular}{ll|l}
130 \\
\end{tabular} & 2882 & 4029 & \begin{tabular}{l|l|}
6044 \\
\end{tabular} & 1264 & 715 & 1614 & 5145 & 3051 & 8196 & 149617 & 198891 & 65344 & 35212 & $\begin{array}{lll}720 \\
\end{array}$ & 455 & 51496 & 30714 & 82210 \\
\hline & 3000 & 2020 & 2040 & 4 & 4498 & 7208 & 10930 & 1970 & 1278 & 2935 & 114014 & 151895 & 49762 & 26782 & 179 & 124 & 246 & 5194 & 8084 & 11658 & 2322 & 1425 & 3069 & 3190 & 1916 & 5106 & 91969 & 124525 & 40075 & 22116 & \begin{tabular}{|l}
1298 \\
\end{tabular} & 898 & 51496 & 30714 & 82210 \\
\hline & 3000 & 2020 & 2040 & 12 & 4498 & 7208 & 10930 & 1970 & 1278 & 2935 & 114014 & 151895 & 49762 & 26782 & 179 & 124 & 246 & 5194 & 8084 & 11658 & 2322 & 1425 & 3069 & 3190 & 1916 & 5106 & 91969 & 124525 & 40075 & 22116 & 432 & 337 & 17166 & 11518 & 28684 \\
\hline Canisters and Bare Fuel & 3000 & 2020 & 2040 & 21 & 4498 & 7208 & 10930 & 1970 & 1278 & 2935 & 114014 & 151895 & 49762 & 26782 & 179 & 124 & 246 & 5194 & 8084 & 11658 & 2322 & 1425 & 3069 & 3190 & 1916 & 5106 & 91969 & 124525 & 40075 & 22116 & 248 & 183 & 9809 & 6283 & 16092 \\
\hline $\begin{array}{l}\text { Re-Packag } \\
\text { Rancat }\end{array}$ & 6000 & 2020 & 2040 & 4 & 9530 & 15592 & 21958 & 4162 & 2750 & 6011 & 157322 & 207289 & 689915 & 36522 & 139 & 118 & 216 & 4057 & 7642 & 9725 & 1752 & 1356 & 2670 & 1739 & 1073 & 2812 & 48661 & 69131 & 20923 & 12375 & 2768 & 1772 & 51496 & 30714 & 82210 \\
\hline (Case 2) & 1500 & 2020 & 2055 & 4 & 2306 & 3604 & 5236 & 1009 & 630 & 1471 & 56366 & 77529 & 24993 & 13686 & 98 & 62 & 130 & 2882 & 4029 & 6044 & 1264 & 715 & 1614 & 5145 & 3051 & 8196 & 149617 & 198891 & 65344 & 35212 & 721 & 456 & 51496 & 30714 & 82210 \\
\hline & 3000 & 2020 & 2055 & 4 & 4498 & 7208 & 10930 & 1970 & 1278 & 2935 & 114014 & 151895 & 49762 & 26782 & 179 & 124 & 246 & 5194 & 8084 & 11658 & 2322 & 1425 & 3069 & 3190 & 1916 & 5106 & 91969 & 124525 & 40075 & 22116 & 1298 & 898 & 51496 & 30714 & 82210 \\
\hline & 6000 & 2020 & 2055 & 4 & 9690 & 14144 & 21080 & 4270 & 2484 & 6023 & 157322 & 207289 & 68915 & 36522 & 153 & 121 & 225 & 4528 & 7801 & 10112 & 1953 & 1387 & 2814 & 1739 & 1073 & 2812 & 48661 & 69131 & 20923 & 12375 & 2439 & 1644 & 51496 & 30714 & 82210 \\
\hline & 3000 & 2035 & 2055 & 4 & 3520 & 6324 & 9076 & 1537 & 1120 & 2472 & 50962 & 65464 & 22248 & 11597 & 179 & 126 & 252 & 5205 & 8223 & 11940 & 2315 & 1451 & 3085 & 5315 & 3232 & 8547 & 155021 & 210956 & 67589 & 37301 & 1302 & 914 & 51496 & 30714 & 82210 \\
\hline & 1500 & 2020 & 2040 & 4 & & & & & & & & & & & 95 & 73 & 142 & 2852 & 4743 & 6667 & 1232 & 785 & 1514 & \begin{tabular}{|l}
6998 \\
\end{tabular} & 4210 & 11208 & 205983 & 276420 & 89838 & 48897 & $\begin{array}{lll}713 \\
\end{array}$ & 527 & 51496 & \begin{tabular}{ll|}
30714 \\
\end{tabular} & 82210 \\
\hline & 3000 & 2020 & 2040 & 4 & & & & & & & & & & & 182 & 132 & 268 & 5081 & 8768 & 12670 & 2263 & 1458 & 3015 & 6974 & 4190 & 11164 & 205983 & 276420 & 89838 & 48897 & \begin{tabular}{|l|l|}
1271 \\
\end{tabular} & 974 & 51496 & 30714 & \begin{tabular}{|l}
88210 \\
\end{tabular} \\
\hline & 3000 & 2020 & 2040 & 12 & & & & & & & & & & & 182 & 132 & 268 & 5081 & 8768 & 12660 & 2263 & 1458 & 3017 & 6974 & 4190 & 11164 & 205983 & 276420 & 89838 & 48897 & 424 & 365 & 17166 & 11518 & |28684 \\
\hline & 3000 & 2020 & 2040 & 21 & & & & & & & & & & & 182 & 132 & 268 & 5081 & 8768 & 12662 & 2263 & 1458 & 3019 & 6974 & 4190 & 11164 & 205983 & 276420 & 89838 & 48897 & 242 & 199 & 9809 & 6283 & 16092 \\
\hline & 6000 & 2020 & 2040 & 4 & & & & & & & & & & & 426 & 274 & 530 & 12234 & 18022 & 24338 & 5297 & 3189 & 6015 & 6964 & 4183 & 11147 & 205983 & 276420 & 89838 & 48897 & 3059 & 2002 & 51496 & 30714 & 82210 \\
\hline & 1500 & 2020 & 2055 & 4 & & & & & & & & & & & 95 & 73 & 142 & 2852 & 4743 & 6667 & 1232 & 785 & 1514 & 6998 & 4210 & 11208 & 205983 & 276420 & 89838 & 48897 & 713 & 527 & 51496 & 30714 & 82210 \\
\hline & 3000 & 2020 & 2055 & 4 & & & & & & & & & & & 182 & 132 & 268 & 5081 & 8768 & 12670 & 2263 & 1458 & 3015 & 6974 & 4190 & 11164 & 205983 & 276420 & 89838 & 48897 & 1271 & 974 & 51496 & 30714 & 82210 \\
\hline & 6000 & 2020 & 2055 & 4 & & & & & & & & & & & 426 & 274 & 530 & 12234 & 18022 & 24338 & 5297 & 3189 & 6015 & 6964 & 4183 & 11147 & 205983 & 276420 & 89838 & 48897 & 3059 & 2002 & 51496 & 30714 & 82210 \\
\hline & 3000 & 2035 & 2055 & 4 & & & & & & & & & & & 175 & 131 & 265 & 5077 & 8623 & 12486 & 2257 & 1454 & 3014 & 7001 & 4216 & 11217 & 205983 & 276420 & 89838 & 48897 & 1270 & 958 & 51496 & 30714 & 82210 \\
\hline & $\begin{array}{lll}1500 \\
\end{array}$ & 2020 & 2040 & 4 & 2296 & 4105 & 5587 & 1011 & 726 & 1463 & 56366 & $\begin{array}{l}77529 \\
\end{array}$ & 24502 & 13697 & 94 & 68 & 127 & 2792 & 4351 & 6476 & 1212 & 765 & 1514 & 5145 & 3051 & 8196 & 149617 & 198891 & 65344 & 35212 & 698 & 500 & 51496 & 30714 & 82210 \\
\hline & 3000 & 2020 & 2040 & 4 & 4498 & 7879 & 10881 & 1970 & 1383 & 2929 & 114014 & 151895 & 49773 & 26794 & 174 & 124 & 248 & 5042 & 8084 & 12651 & 2253 & 1425 & \begin{tabular}{|l|l|}
3012 \\
\end{tabular} & \begin{tabular}{|l|l|}
3190 \\
\end{tabular} & 1916 & 5106 & 91969 & 124525 & 40075 & \begin{tabular}{|l|l|}
22116 \\
\end{tabular} & 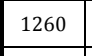 & 975 & 51496 & 30714 & 82210 \\
\hline & 3000 & 20. & 204 & 12 & 4498 & 7879 & 10881 & 1970 & 1383 & 2929 & \begin{tabular}{|l|l|l|l|}
14014 \\
\end{tabular} & 151895 & 49798 & 26812 & 174 & 124 & 248 & 5042 & 8084 & 12636 & 2253 & \begin{tabular}{l|l|l}
1425 \\
\end{tabular} & 3013 & 3190 & 1916 & 5106 & 91969 & 124525 & 75 & 22116 & 420 & 365 & 166 & 518 & 28684 \\
\hline & 3000 & 2020 & 2040 & 21 & 4498 & 7879 & 10881 & 1970 & 1383 & 2929 & 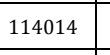 & 151895 & 49850 & 26856 & 174 & $\begin{array}{ll}124 \\
\end{array}$ & 248 & 5050 & 8084 & 12620 & $\begin{array}{l}2258 \\
\end{array}$ & 1425 & 3019 & 3190 & 1916 & 5106 & 91969 & 124525 & 40075 & 22116 & 241 & 199 & 9809 & 6283 & 16092 \\
\hline & 6000 & 2020 & 2040 & 4 & 9859 & 14152 & 20601 & 4328 & 2486 & 5934 & 157322 & 207289 & 68929 & 36538 & 153 & 121 & 225 & 4528 & 7801 & 23828 & \begin{tabular}{|l|l|}
1953 \\
\end{tabular} & 1387 & \begin{tabular}{|l|l|}
6011 \\
\end{tabular} & 1739 & 1073 & 2812 & 48661 & 69131 & 20923 & 12375 & 2508 & 1736 & 51496 & 30714 & 82210 \\
\hline & 1500 & 2020 & 2055 & 4 & 2296 & 4105 & 5587 & 1011 & 726 & $\begin{array}{l}1463 \\
\end{array}$ & 56366 & 77529 & 24495 & 13687 & 94 & 67 & 128 & 2810 & 4297 & 6476 & 1215 & 755 & 1515 & 5145 & 3051 & 8196 & 149617 & 198891 & 655344 & 35212 & 703 & 500 & 51496 & 30714 & 82210 \\
\hline & 3000 & 2020 & 2055 & 4 & 4498 & 7879 & 10881 & 1970 & $\begin{array}{ll}1383 \\
\end{array}$ & 2929 & 114014 & 151895 & 49763 & 26783 & 174 & $\begin{array}{l}124 \\
\end{array}$ & 248 & 5042 & 8084 & 12651 & 2253 & $\begin{array}{l}1425 \\
\end{array}$ & 3011 & \begin{tabular}{ll|}
3190 \\
\end{tabular} & 1916 & 5106 & 91969 & 124525 & 40075 & 22116 & 1260 & 975 & 51496 & 30714 & 82210 \\
\hline & 600 & & 205 & 4 & 985 & 14152 & 20601 & 4325 & 2486 & 5934 & 157322 & 207289 & 68920 & & 153 & 121 & 225 & 4528 & 7801 & 23828 & 1953 & 1387 & 6013 & 1739 & 1073 & 2812 & 48661 & 69131 & 20923 & 12375 & \begin{tabular}{|l|l|}
2506 \\
\end{tabular} & 1736 & 496 & 0714 & 2210 \\
\hline & 3000 & 2035 & 2055 & 4 & 3512 & 7102 & 9872 & 1533 & \begin{tabular}{ll|}
1254 \\
\end{tabular} & 2500 & 50962 & 65464 & 22249 & 11599 & 173 & 119 & 249 & 5021 & 7810 & \begin{tabular}{l|l|}
12440 \\
\end{tabular} & 2234 & 1375 & 3013 & \begin{tabular}{|l|}
5315 \\
\end{tabular} & 3232 & 8547 & 155021 & 210956 & 67589 & \begin{tabular}{|l|l|}
37301 \\
\end{tabular} & \begin{tabular}{|l|l|l}
1255 \\
\end{tabular} & 956 & 51496 & 30714 & \begin{tabular}{|l|l|}
82210 \\
\end{tabular} \\
\hline
\end{tabular}


Table 4-8. Repository Logistic Summary Results

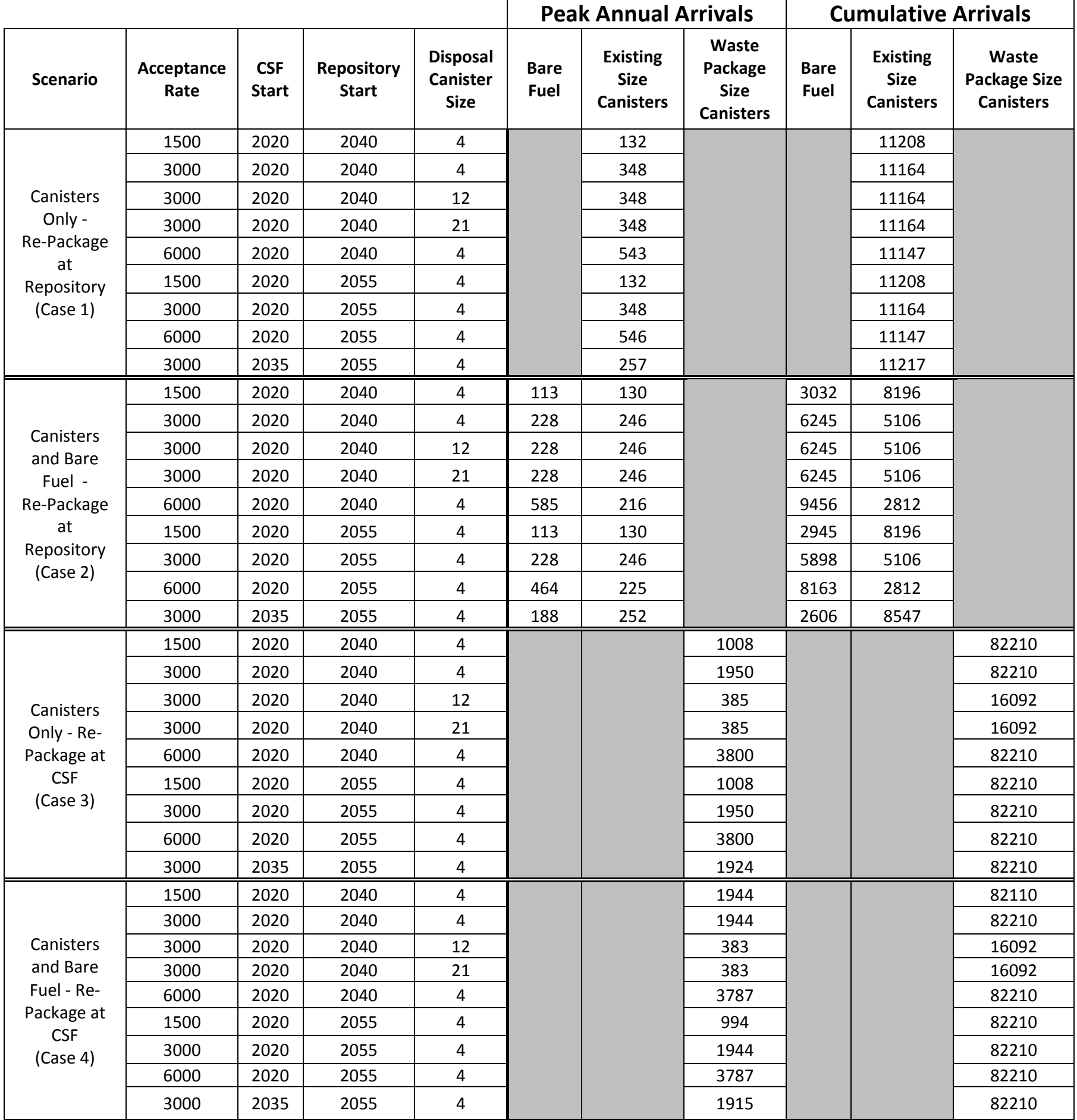


Table 4-9. Transportation Acquisition Summary

\begin{tabular}{|c|c|c|c|c|c|c|c|c|c|c|}
\hline Scenario & $\begin{array}{c}\text { Acceptance } \\
\text { Rate }\end{array}$ & $\begin{array}{l}\text { CSF } \\
\text { Start }\end{array}$ & $\begin{array}{l}\text { Repository } \\
\text { Start }\end{array}$ & $\begin{array}{l}\text { Disposal } \\
\text { Canister } \\
\text { Size }\end{array}$ & $\begin{array}{c}\text { Casks/ } \\
\text { Overpacks }\end{array}$ & $\begin{array}{l}\text { Buffer } \\
\text { Railcar }\end{array}$ & $\begin{array}{c}\text { Cask } \\
\text { Railcar }\end{array}$ & $\begin{array}{l}\text { Escort } \\
\text { Railcar }\end{array}$ & $\begin{array}{l}\text { Cask } \\
\text { Trailer }\end{array}$ & $\begin{array}{l}\text { Escort } \\
\text { Truck }\end{array}$ \\
\hline \multirow{9}{*}{$\begin{array}{c}\text { Canisters } \\
\text { Only - } \\
\text { Re-Package } \\
\text { at Repository } \\
\text { (Case 1) }\end{array}$} & 1,500 & 2020 & 2040 & 4 & 174 & 18 & 18 & 9 & & \\
\hline & 3,000 & 2020 & 2040 & 4 & 176 & 38 & 43 & 19 & & \\
\hline & 3,000 & 2020 & 2040 & 12 & 176 & 38 & 43 & 19 & & \\
\hline & 3,000 & 2020 & 2040 & 21 & 176 & 38 & 43 & 19 & & \\
\hline & 6,000 & 2020 & 2040 & 4 & 257 & 48 & 61 & 24 & & \\
\hline & 1,500 & 2020 & 2055 & 4 & 185 & 18 & 18 & 9 & & \\
\hline & 3,000 & 2020 & 2055 & 4 & 197 & 34 & 40 & 17 & & \\
\hline & 6,000 & 2020 & 2055 & 4 & 316 & 42 & 61 & 21 & & \\
\hline & 3,000 & 2035 & 2055 & 4 & 166 & 28 & 33 & 14 & & \\
\hline \multirow{9}{*}{$\begin{array}{l}\text { Canisters and } \\
\text { Bare Fuel - } \\
\text { Re-Package } \\
\text { at Repository } \\
\text { (Case 2) }\end{array}$} & 1,500 & 2020 & 2040 & 4 & 276 & 24 & 24 & 12 & 9 & 10 \\
\hline & 3,000 & 2020 & 2040 & 4 & 322 & 36 & 47 & 18 & 10 & 8 \\
\hline & 3,000 & 2020 & 2040 & 12 & 322 & 36 & 47 & 18 & 10 & 8 \\
\hline & 3,000 & 2020 & 2040 & 21 & 322 & 36 & 47 & 18 & 10 & 8 \\
\hline & 6,000 & 2020 & 2040 & 4 & 471 & 76 & 95 & 38 & 13 & 10 \\
\hline & 1,500 & 2020 & 2055 & 4 & 299 & 24 & 24 & 12 & 9 & 6 \\
\hline & 3,000 & 2020 & 2055 & 4 & 355 & 38 & 47 & 19 & 10 & 8 \\
\hline & 6,000 & 2020 & 2055 & 4 & 502 & 86 & 95 & 43 & 13 & 10 \\
\hline & 3,000 & 2035 & 2055 & 4 & 286 & 42 & 47 & 21 & 6 & 6 \\
\hline \multirow{9}{*}{$\begin{array}{c}\text { Canisters } \\
\text { Only - Re- } \\
\text { Package at } \\
\text { CSF } \\
\text { (Case 3) }\end{array}$} & 1,500 & 2020 & 2040 & 4 & 535 & 74 & 101 & 37 & & \\
\hline & 3,000 & 2020 & 2040 & 4 & 516 & 138 & 200 & 69 & & \\
\hline & 3,000 & 2020 & 2040 & 12 & 260 & 66 & 90 & 33 & & \\
\hline & 3,000 & 2020 & 2040 & 21 & 245 & 56 & 75 & 28 & & \\
\hline & 6,000 & 2020 & 2040 & 4 & 664 & 266 & 395 & 133 & & \\
\hline & 1,500 & 2020 & 2055 & 4 & 537 & 76 & 102 & 38 & & \\
\hline & 3,000 & 2020 & 2055 & 4 & 515 & 146 & 213 & 73 & & \\
\hline & 6,000 & 2020 & 2055 & 4 & 664 & 252 & 377 & 126 & & \\
\hline & 3,000 & 2035 & 2055 & 4 & 506 & 138 & 198 & 69 & & \\
\hline \multirow{9}{*}{$\begin{array}{c}\text { Canisters and } \\
\text { Bare Fuel - } \\
\text { Re-Package } \\
\text { at CSF } \\
\text { (Case 4) }\end{array}$} & 1,500 & 2020 & 2040 & 4 & 626 & 80 & 108 & 40 & 9 & 8 \\
\hline & 3,000 & 2020 & 2040 & 4 & 647 & 148 & 212 & 74 & 12 & 8 \\
\hline & 3,000 & 2020 & 2040 & 12 & 378 & 76 & 97 & 38 & 10 & 8 \\
\hline & 3,000 & 2020 & 2040 & 21 & 368 & 60 & 86 & 30 & 12 & 8 \\
\hline & 6,000 & 2020 & 2040 & 4 & 753 & 260 & 379 & 130 & 13 & 10 \\
\hline & 1,500 & 2020 & 2055 & 4 & 619 & 70 & 101 & 35 & 9 & 8 \\
\hline & 3,000 & 2020 & 2055 & 4 & 641 & 140 & 203 & 70 & 12 & 8 \\
\hline & 6,000 & 2020 & 2055 & 4 & 757 & 236 & 353 & 118 & 13 & 10 \\
\hline & 3,000 & 2035 & 2055 & 4 & 600 & 136 & 199 & 68 & 6 & 6 \\
\hline
\end{tabular}




\section{FACILITY DESCRIPTION SUMMARY}

As noted in Section 3.4 above, the design concepts described here are intended to be part of an overall waste management system that is flexible and can adapt to various logistics, construction and operational conditions. Flexibility in design affords the capability to adapt to different waste management policy decisions and directions, waste receipt and operational scenarios, and unanticipated conditions encountered during construction.

The type, total quantity (capacity) and annual quantity (throughput) of material that must be handled under a given UNF system architecture scenario will determine the size, configuration, and functional capability of the facilities that are needed to support that scenario. Each facility required by a scenario must be equipped with sufficient infrastructure to process the quantity of material, or throughput, assigned to it by the scenario. The throughput capacity is a function of the time needed to accomplish each material handling or processing sequence through each element of the facility. Because a given throughput must be processed through all facility elements and different facility elements may require different time periods to process the same throughput quantities, each facility processing element must be sized accordingly. In order to address this variability in size, configuration, and functional capability of facilities required by a wide spectrum of waste management scenarios, modular design concepts have been developed and adopted to support the system analyses. The modules are then combined in appropriate configurations, combinations, and quantities to address the system needs dictated by a given scenario's functional capability, capacity, and throughput requirements. Separate unit costs for each module or facility element are developed (described in Appendix B and summarized here) are combined to develop the various Scenario Costs in Section 6.

Using a modular approach is not only useful for developing system costs using a consistent basis across all scenarios, it is also a reasonable approach for developing and implementing a flexible and integrated commercial used nuclear fuel system over a multi-decade period of time. As noted in Section 3.4.1, modularity allows an incremental and stepwise approach to system implementation rather than committing to and locking in on exceptionally large, single function facilities up front.

The disposition pathway evaluation described in Section 3 suggests an integrated used fuel management system will account for the following major system components:

- used fuel management at utilities

- used fuel acceptance and transportation

- used fuel management at one or more consolidated storage facilities

- used fuel repackaging (if necessary) either at a consolidated storage facility, a repository, or as a standalone facility.

- used fuel management at a repository

Utility facilities and equipment are generally assumed to already exist and are not described further. Transportation facilities (cask and fleet maintenance facilities) have not been developed and their associated construction costs are not included in this study although they are assumed to be co-located with a consolidated storage facility as noted in Section 6.4. Repository waste handling facilities are developed separately in Disposal Concepts/Thermal Load Management (FY11/12 Summary Report) (DOE 2012c). The remainder of Section 5 focuses on the facility elements needed to address the consolidated storage and UNF re-packaging functions.

\subsection{Consolidated Storage Facility (CSF) Scope and Functionality}

The scope of the CSF includes the following major functions:

- Receiving fuel from reactors, currently in various wet and dry storage configurations, that will need to be considered by consolidated storage (all Cases) 
- Receiving canisterized fuel from a co-located re-packaging facility (Case 5, Case 6, Case 7, Case 8, and Case 9-5)

- Storing dry fuel on pads (all Cases)

- Storing dry canisterized fuel in vaults (as an alternative to pads)

- Storing bare fuel in pools (Case 2 and Case 4)

- Transferring fuel to a Repackaging Facility where it would be processed into waste package sized containers, with the size dependent upon specific repository geologic conditions (Case 3, Case 4, Case 8, Case 9-3)

- Transferring fuel to a Repository for final disposition (all Cases)

\subsubsection{CSF Modules}

The CSF physical features were subdivided into the following potential stand-alone facility modules. These include:

- Cask Receipt and Canister Transfer (CRCT) Facility

- Dry Storage Pads (Vertical and Horizontal)

- Dry Storage Canister (DSC) Storage Vault Canister Transfer (SVCT) Facility

- Bare Fuel Receipt and Storage (FRS) Facility

Basic facility modules are then combined into configurations necessary to the facility size and throughput requirements dictated by each given scenario. Sections 5.1.2 through 5.1.6 below summarize these facilities and associated operations. Appendix B summarizes the unit operations activities times associated with the facilities and tabulates the key facility element quantities (e.g. number of carrier receipt bays, number of carrier release bays, number of fuel pools and basins etc.) associated with the scenarios developed in this study.

\subsubsection{CSF Facility Modules Design Concepts}

The CSF will receive commercial UNF, currently in various wet and dry storage configurations, from reactor sites. The CSF module dry storage design concepts are based on receiving and storing cask/canister systems whose designs are similar to the commercially available vertical and horizontal systems designs.

In general, the cask and canister handling, and storage design concepts are similar to concepts developed for DOE's past repository surface facility designs and the Private Fuel Storage L.L.C. (PFS) facility proposed for Toole County, Utah. The receipt and the vertical storage design concepts are based closely on the PFS facility that the US NRC licensed (US NRC-PFS).

\subsubsection{Cask Receipt and Canister Transfer Facility}

The scope for the CRCT facility includes the following processes. The pre-conceptual layout of the CRCT facility is illustrated in Figures B-17, B-18, and B-19 of Appendix B.

- Receiving containerized fuel from the reactors in legacy existing storage systems

- Removing canisters from the transport overpacks (or casks)

- Transferring canisters to facility overpacks (or casks)

- Transferring loaded overpacks to CSF dry storage

The CRCT facility will be located at the CSF and is envisioned as a structural steel high-bay structure, consisting of one or more receipt bays, each on the order of $168.5 \mathrm{ft}$. wide, $162 \mathrm{ft}$. long, and between 70 and $90 \mathrm{ft}$. high. This structure will have rail carrier access into each cask bay for cask handling and 
canister unloading/transfer operations. Each CRCT building transfer bay would contain a 250 ton single trolley overhead bridge crane. This crane would also be equipped with a 25 ton auxiliary hoist

Each bay would receive the transportation cask on its rail carrier, remove the transportation cask impact limiters and remove the transportation cask from the carrier, open the transportation casks, unload the UNF canisters from the cask, and place the canisters into a facility storage cask for transfer to the dry storage pads.

The loaded storage casks and empty transportation casks would be removed from the building by the same rail carrier "pusher engine" used to deliver the loaded rail cars from the Site security rail inspection siding, located at the site perimeter, as reflected in, Figures B-4 and B-5 The CRCT facility will have the ability to provide localized (at the work site) HEPA filtered ventilation for cask venting and sampling operations.

Following removal of the loaded storage cask from the building, the transportation cask would be radiologically surveyed, decontaminated if necessary, and reassembled for shipment offsite. Each loading bay would provide for rail carrier pass through for fuel transportation cask receipt/returns, and storage cask transfer to dry storage.

\subsubsection{Dry Storage Pads}

The loaded CSF storage cask would be mounted on a self-propelled modular transporter (SPMT) at the CRCT facility and transferred to a dry storage pad or vault. The storage casks are equipped with vents and channels that provide cooling by passive, natural convection processes. It is assumed that the storage casks and pads would be constructed to the respective licensed storage unit design on an as needed basis. A concrete batch plant will be located within the boundary of the CSF to support on-site final fabrication of the storage units.

\subsubsection{Dry Storage - Pads (Horizontal)}

Horizontal storage systems, such as shown in Appendix B, Figure B-6, can be aligned with multiple single-canister horizontal storage units in a module. Horizontal storage module arrays containing 12 canisters are assumed for layout purposes. Each module is approximately $52 \mathrm{ft}$. wide by $89 \mathrm{ft}$. long, containing 12 horizontal storage units. Rows of modules would be separated by approximately $50 \mathrm{ft}$. to allow access for the SPMT shielded transporter. Areas between the modules would have transporter access and be surfaced sufficiently to allow travel of heavy lift equipment.

\subsubsection{Dry Storage - Pads (Vertical)}

There are multiple licensed vertical dry storage systems and the vertical dry storage design concept would have to accommodate all vertical dry storage systems in use. Multiple vertical cask storage pads are needed. Each pad is assumed to hold 8 storage casks. Each storage pad would be constructed flush with grade level and accept up to eight storage casks in a $2 \times 4$ array. The typical vertical storage pad dimensions would be on the order of approximately 67 feet long by 30 feet wide by 3 feet thick. Each cask would contain approximately 10 to 14 MTHM of UNF, assuming a basis of 68 BWR or 32 PWR assemblies in each cask, although canister loading densities will be a function of licensed canister design and actual loading densities achieved at reactor source sites. Although other pad configurations can be designed, each pad is assumed to be surrounded by a compacted gravel skirt, on the order of 30 feet wide. Areas between the pads would have transporter access and be surfaced sufficiently to allow travel of heavy lift and transfer equipment such as a SPMT.

\subsubsection{DSC Storage Vault and Canister Transfer Facility}

The scope for the Storage Vault Canister Transfer (SVCT) facility includes the following functions. The pre-conceptual layout of the SVCT has been illustrated in Figure B-21 of Appendix B. 
- Receiving canisterized fuel from the reactors in either existing storage systems or newly designed disposal canisters that are compatible with future repository disposal criteria.

- Removing canisters from the transport over packs (or casks)

- Transferring canisterized fuel to CSF DSC vaults

A dry storage vault concept for the receipt, unloading, and storage of 3,000 MTHM of fuel in existing size canisters was previously developed in the Engineering Alternative Study (EAS) for Separations Summary Report (McConnel et al. 2007). The basic receipt and storage concept has been adopted for the SVCT with an expansion of the module storage capacity to 7,500 MT.

The SVCT facility is envisioned to receive commercial UNF packaged in welded or bolted canisters in transportation overpacks. The initial inspection and receipt operations would be similar to the receipt bay operations described in Section 5.1.3 above. When received, the licensed transportation cask will be opened and unloaded by removal of its sealed fuel canister into a transfer cask. The canister will be moved to an underground transfer shuttle cart, which moves the canister into the vault operations area. Overhead crane mounted shielded transfer casks are then used to locate the canister in the desired vault storage location. The underground vault ventilation is assumed to provide cooling by passive, natural convection; thermal analyses will be necessary to evaluate this assumption for canisters with higher burnup, newly discharged (5-10 years old) used fuel.

Following transfer of the fuel canisters to the storage vault, the transportation cask would be radiologically surveyed, decontaminated if necessary, and reassembled for shipment offsite. Each loading bay would provide for railroad pass through of fuel transportation cask carriers.

\subsubsection{Bare Fuel Receipt and Storage Facility}

The scope of the bare Fuel Receipt and Storage (FRS) Facility for the pool storage includes the following functions. The pre-conceptual layout is illustrated in, Figures B-22, B-23, and B-24.

- Receiving bare fuel transportation cask from the reactors loaded on a transportation cask carrier.

- Removing bare fuel transportation cask from the transportation cask carrier

- Transferring bare fuel from the transportation cask to CSF pool storage

A pool storage concept for the receipt, unloading, and storage of 3,000 MTHM was previously developed in the Engineering Alternative Study (EAS) for Separations-Summary Report (McConnell et al. 2007). The basic receipt concept was adopted for this study with an expansion of the storage capacity to 7,500 MT.

The wet storage pool is separated from the cask receipt bays by an airlock. The transportation cask is moved from the cask receipt bays, through the airlock, lowered into the pool and flooded with water prior to removal of the inner container lid. Once the lid is removed, the individual fuel assemblies are transferred to the desired fuel assembly storage rack location.

The pool consists of 8 interconnected basins. Each basin is approximately $158 \mathrm{ft}$. long by $60 \mathrm{ft}$. wide and $55 \mathrm{ft}$. deep. Each basin contains 100 storage racks providing 35 assembly storage positions using a 15 by 15 inch array. Thus each fuel pool basin corresponds to 3500 bare fuel assembly storage positions.

If an 8 basin, 28,000 assembly pool (3500 assemblies per basin, separate PWR and BWR UNF assembly basins) were fully loaded with high-burn-up UNF, on a ratio of $43 \%$ PWR fuel assemblies and 57\% BWR assemblies, the total assemblies of each type would be about 12,040 PWR and 15,960 BWR. On that basis, at approximately 0.436 MTU per PWR assembly and 0.179 MTU per BWR assembly, the total decay heat (60GWd/MT PWR fuel is $\sim 3,530$ watts/MT and $50 \mathrm{GWd} / \mathrm{MT}$ BWR fuel is $\sim 2,920$ watts/MT) would be about $26.9 \mathrm{MW}$. The decay heat is discharged to the atmosphere by cooling towers. Water treatment, ventilation, and support areas are adjacent to the pools. 
Used nuclear fuel assemblies will arrive onsite via commercially licensed transport vehicle. The transport vehicle will consist of a special railcar or special truck with casks specifically designed for the safe and secure transport of UNF. All shipping casks will be NRC licensed, and contents will be within license constraints. For baseline planning purposes, it can be assumed that rail casks contain approximately 26 PWR fuel assemblies (or $61 \mathrm{BWR}$ assemblies) and truck casks contain approximately 4 PWR fuel assemblies (or 9 BWR assemblies).

The fuel transportation casks will be received and initially staged in a receipt area where contamination surveys, other integrity checks and transportation \& shipment documentation verification can be performed, to assure that receipt documentation and package condition are in order and that decontamination or repairs are not required before unloading.

Upon completion of the radiological survey, the transport vehicle and transportation cask will proceed to the Fuel Receipt and Storage (FRS) facility for removal, inspection, survey and storage of the bare spent fuel assemblies. Cameras, scanners, manipulators and similar equipment are likely to be required to perform this function.

\subsection{Repackaging Facility}

The repackaging of the canisters could be accomplished either at a stand- alone facility or a facility colocated with a CSF or Mined Geologic Repository. The scenarios defined in this study only contemplate waste management system configurations where the repackaging facility is co-located with another system facility (CSF or repository) thus eliminating another major transportation step if the repackaging facility were to be totally stand alone. The pre-conceptual design of the RF is illustrated in Figure 5-1 and in more detail in Appendix B The scope for the Repackaging Facility (RF) includes the following processes.

- Receiving bare fuel or canisterized fuel from either a utility site, a consolidated storage facility or both

- Unloading the transportation cask from the transportation carrier or unloading a transfer cask from a CSF SPMT

- Opening bare fuel transportation casks

- Cutting open welded dual purpose canisters

- Unloading used fuel assemblies from casks or canisters and packaging the assemblies into disposal canisters

- Transferring re-packaged fuel to a CSF or a Repository

The RF module is sized for 1500 MTU/yr throughput. The main sub-structures within the module include a Carrier Receipt Bay, a Waste Handling Building (WHB), and a Carrier Release Bay. Two air locks are included - one between the Receipt Bay and the WHB and one between the WHB and the Release Bay (Figure 3-1). The configurations of the Receipt Bay and the Release Bay may vary considerably depending on whether the RF is co-located with a CSF or a Mined Geologic Repository, or is a standalone facility. For example, if the repackaging facility is co-located with a Mined Geologic Repository, then the Release Bay would not necessarily be needed and could potentially be replaced with a transfer corridor to a facility for placing waste package over packs on the canisters as described in Disposal Concepts/Thermal Load Management (FY11/12 Summary Report) (Hardin et al. 2012). 


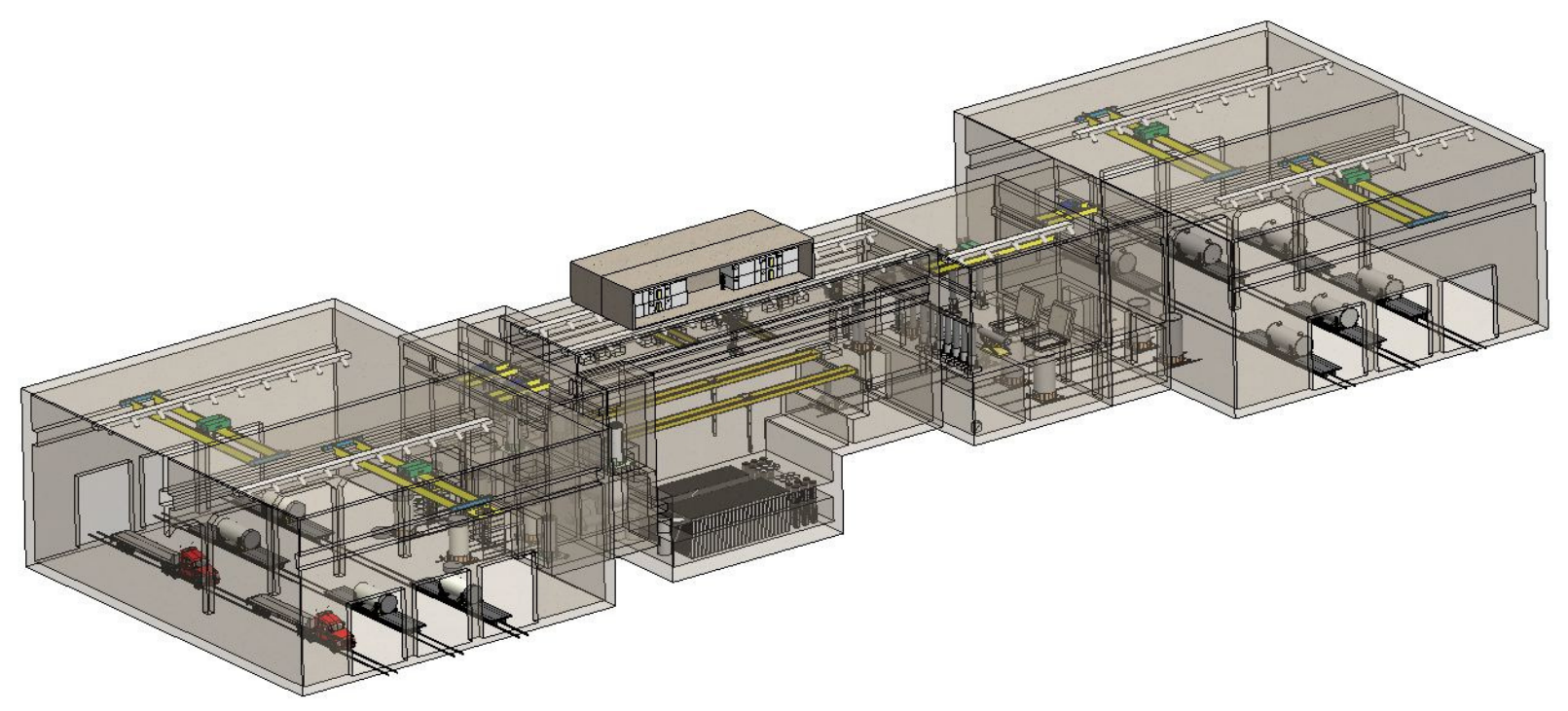

Figure 5-1. Isometric view of the overall Repackaging Facility Module Concept, including Carrier Receipt Bay, Airlocks, Waste Handling Building and Carrier Release Bay

The Carrier Receipt Bay and Carrier Release Bay designs are similar to the receipt facility design concepts for the CSF concepts. The bulk of the operations in the Re-Packaging Facility would be conducted in the Waste Handling Building (WHB) module, where the packaging of UNF into waste package compatible size canisters occurs. The WHB is a multi-level reinforced concrete structure made of noncombustible materials with interior and exterior shear walls, concrete floor, concrete roof slab diaphragms, concrete mat foundations, and a transfer pool. The nominal footprint of the WHB, including air locks is about $282 \mathrm{ft}$ by $92 \mathrm{ft}$. The maximum height of the building is about $100 \mathrm{ft}$ above grade, with the majority of the building under a roof approximately $80 \mathrm{ft}$ above grade. The WHB pool substructure includes the rooms surrounding the pool that provide internal buttresses for the actual pool and space for make-up tanks, pumps and filters, ion exchangers, etc. The concrete base mat for the basement structure (pool and surrounding rooms) is $55 \mathrm{ft}$ below the top of the at-grade concrete mat. The spent fuel pool is sized hold approximately $750 \mathrm{MTU}$ of used nuclear fuel (6 month worth of spent fuel assembly inventory for a 1500 MTU module.) This will allow some flexibility for fuel blending as a thermal management strategy as well as decouple waste receipt and unloading critical path operations from waste package canister loading and closure operations. The pool is split into separate basins and storage racks for BWR assemblies and PWR assemblies and includes separate spent fuel transfer machines (handling cranes) for BWR and PWR assemblies to avoid change out operations of lifting grapples. 


\section{ROUGH ORDER OF MAGNITUDE COST ESTIMATES}

Facilities that are needed to support a given UNF management scenario vary in size and configuration, relative to other scenarios, principally as a function of the type and quantity of material that must be handled under that scenario. To support its mission, a facility must be equipped with sufficient infrastructure to process the quantity of material, or throughput, assigned to each operational scenario as a function of the time needed to accomplish each material handling or processing sequence through each element of the facility.

The UNF logistic analyses summarized in Section 4 above and described in more detail in Appendix A are used along with unit cost factors for the facilities summarized in Section 5 and described in more detail in Appendix B to estimate the annual and life cycle costs for each scenario described above. The unit cost factors used are described in Appendix C.

This section presents rough order of magnitude cost estimates for:

- At reactor costs

- Consolidated storage and an associated test and validation facility to support extended dry storage,

- Fuel repackaging, and

- Transportation

All cost used are presented in calendar year 2012 dollars.

Again, as discussed in Sections 3.2 and 3.3, disposition pathways to evaluate along with the assumptions and input/boundary conditions were selected to "constrain" the problem, but do provide a broad enough range to show trends and gain insight. The choice of these assumptions and input/boundary conditions are not meant to imply that the system would actually be operated as assumed. Rather the initial set of assumptions, input/boundary conditions, and subsequent analyses allows for gaining insight regarding integrated system dynamics and an understanding of trends to give overall insights about which assumptions and conditions have implications that might warrant consideration of alternative assumptions and conditions.

The rough order of magnitude cost estimates presented in this section are affected by the selected assumptions and input/boundary conditions through the logistic modeling results. As such, the costs should not be considered as "absolute" costs of a UNF management system. Rather, as with all the results presented in this report, they should be used to determine overall trends and insights associated with potential UNF management system architectures.

\subsection{At Reactor Rough Order of Magnitude Cost Estimates}

The costs for at-reactor used fuel management have been estimated in a number of different sources as shown in Table 6-1. The estimated unit costs assumed in this evaluation for at-reactor used fuel management, based on these sources, is also shown in Table 6-1.

These estimated unit costs assumed in this evaluation for at-reactor used fuel management were used to project annual and total costs of at-reactor used fuel management based on the at-reactor logistic results obtained using the TSL. The estimated total cost is provided in Table 6-2 for the cases described in Section 3.2.4 with the assumptions presented in Section 3.3; from 2012 until the last fuel assembly is transported off-site. The estimated total costs of each major item associated with at-reactor used fuel management are also shown. Table 6-3 shows the fraction of the total cost associated with each of these major items. 
As expected, the total at-reactor storage cost decreases as the acceptance rate increases and increases as the start of acceptance is delayed from 2020 to 2035. There is no significant difference in total cost between the cases where fuel is transported from the used fuel pools in transportable canisters (Case $1 \&$ 3 ) or in re-useable transportation casks (Case $2 \& 4$ ). This trend is shown in Figure 6-1.

The major cost drivers are the continued maintenance of the dry storage facility after reactor shutdown and the cost of procuring dry storage canisters and overpacks. An exception is for an acceptance rate of $6000 \mathrm{MTHM} / \mathrm{yr}$ starting in 2020 where the cost of maintaining the dry storage facility after reactor shutdown is a small fraction of the total cost since the entire dry storage inventory is removed by 2040 .

The cost of loading fuel into canisters (for dry storage or transportation), into re-useable transportation casks, and preparing canisters in dry storage for transportation are each a relatively small contributor to the total cost. However, when combined these fuel handling operations can become significant.

The cost of deploying new dry storage facilities at reactor sites is relatively insignificant (1-3\% of total projected cost) and does not change for the different cases. This is because most dry storage facilities have been deployed prior to 2012 and each case requires the deployment of 22 new at-reactor dry storage facilities which will need to be put in place before the earliest assumed date for the start of UNF acceptance. The cost of dry storage facility maintenance and operation while reactors are in operation is also a small contributor to the total cost (2-6\% of total projected cost) and does not change significantly for the different cases.

The cost of maintaining the used fuel pools after the reactors have shut down is relatively insensitive to the cases considered. This is because it is assumed that any fuel remaining in the used fuel pools is transferred to dry storage five years following reactor shutdown. Giving acceptance priority to removal of fuel from shutdown sites, combined with acceptance of bare fuel directly from the pools, might lead to significant differences, however.

Figures 6-2 and 6-3 show the annual cost of at-reactor management of used nuclear fuel. It can be seen that annual costs peak during the period when the reactors shut down and all fuel is being transferred to dry storage because of the large number of storage casks being purchased, loaded, and stored to unload the pools at the reactors. 


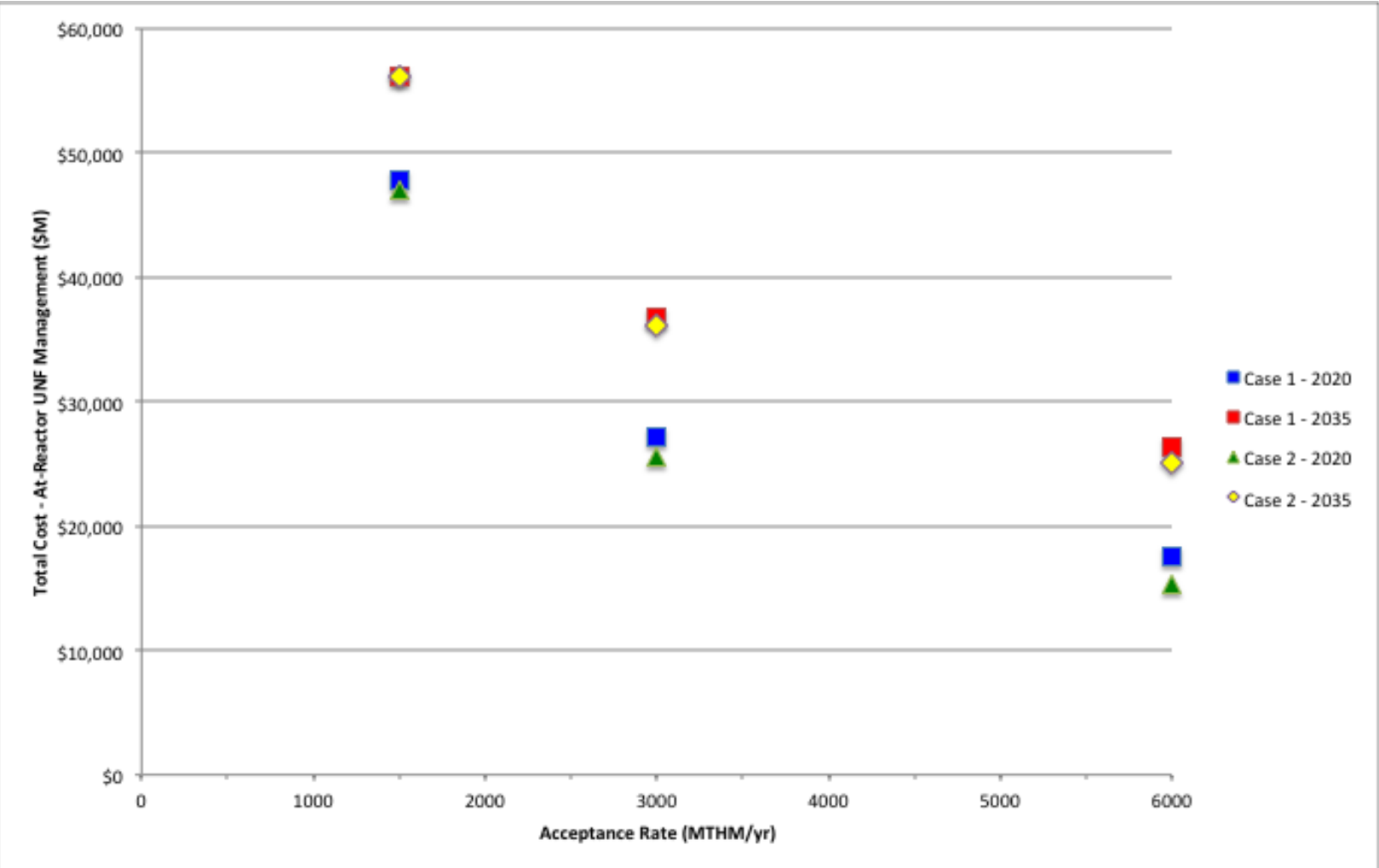

Figure 6-1. Projected Total At-Reactor Used Fuel Management Costs: Cases $1 \& 3$ and 2 \& 4 
UFD System Architecture

Storage, Transportation, and Disposal Interface - Life Cycle Cost Study

September, 2012

Table 6-1. Estimated Costs for At-Reactor Used Fuel Management

\begin{tabular}{|c|c|c|c|c|c|c|c|c|}
\hline & Cost Item & $\mathrm{GAO}^{1}$ & EPRI $I^{2}$ & $\mathrm{EPRI}^{3}$ & OCRWM $^{4}$ & OCRWM ${ }^{5}$ & Storefuel $^{6}$ & Assumed Cost for Analysis \\
\hline \multirow{4}{*}{$\begin{array}{l}\text { Dry Storage } \\
\text { Facility } \\
\text { Construction and } \\
\text { Operation }\end{array}$} & $\begin{array}{l}\text { Dry Storage Up-Front: } \\
\text { Design, Licensing, } \\
\text { Construction, Testing }\end{array}$ & $\$ 30 \mathrm{M} \pm 40 \%$ per site & $\begin{array}{l}\text { \$625 K per storage system. } \\
\text { Based on estimates of \$21.5M } \\
\text { total up-front cost for ISFSI at } \\
\text { Monticello and \$22M for ISFSI } \\
\text { at Pilgrim }\end{array}$ & & & & & $\$ 25 \mathrm{M}$ per site \\
\hline & $\begin{array}{l}\text { Annual Dry Storage } \\
\text { Operating - Reactor in } \\
\text { Operation }\end{array}$ & $\$ 200 \mathrm{~K} \pm 50 \%$ per site & $\begin{array}{l}\$ 600 \mathrm{~K} \text { per site. Cited a range } \\
\text { of } \$ 200 \mathrm{~K} \text { to } 1,000 \mathrm{~K} \text { per site }\end{array}$ & & & & & $\$ 600 \mathrm{~K}$ per site \\
\hline & $\begin{array}{l}\text { Annual Dry Storage } \\
\text { Operating - Reactor } \\
\text { Shutdown/Decommissioned }\end{array}$ & $\$ 4.5 \mathrm{M} \pm 40 \%$ per site & $\$ 6 \mathrm{M}$ per site & & & & & $\$ 6 \mathrm{M}$ per site \\
\hline & $\begin{array}{l}\text { Annual Wet Storage } \\
\text { Operating - Reactor } \\
\text { Shutdown/Decommissioned }\end{array}$ & $\$ 10 \mathrm{M} \pm 20 \%$ per site & & & & & & $\$ 10 \mathrm{M}$ per site \\
\hline \multirow{2}{*}{$\begin{array}{l}\text { Storage Canisters } \\
\text { and Overpacks }\end{array}$} & DPC & \multirow{2}{*}{$\begin{array}{c}\$ 900 \mathrm{~K} \pm 25 \% \text { per canister } \\
\text { (assume includes canister } \\
\text { and storage overpack) }\end{array}$} & \multirow{2}{*}{$\begin{array}{l}\$ 950 \mathrm{~K} \text { per canister } \\
\text { (includes overpack) }\end{array}$} & $\begin{array}{l}21 \text { PWR }-\$ 700 \mathrm{~K} \text { per canister } \\
44 \text { BWR }-\$ 800 \mathrm{~K} \text { per canister }\end{array}$ & \multirow{2}{*}{$\begin{array}{c}21 \text { PWR TAD - \$700 K per } \\
\text { canister } \\
44 \text { BWR TAD - \$800 K per } \\
\text { canister } \\
\text { (includes overpack) }\end{array}$} & $\begin{array}{c}\$ 500-600 \mathrm{~K} \text { per } \\
\text { canister }\end{array}$ & & $\begin{array}{l}21 \text { PWR - } \$ 700 \mathrm{~K} \text { per canister } \\
44 \mathrm{BWR}-\$ 800 \mathrm{~K} \text { per canister }\end{array}$ \\
\hline & DPC Storage Overpack & & & $\$ 200 \mathrm{~K}$ & & $\$ 300 \mathrm{~K}$ per overpack & & $\$ 200 \mathrm{~K}$ \\
\hline \multirow{4}{*}{$\begin{array}{l}\text { Loading / Load- } \\
\text { Out Operations }\end{array}$} & $\begin{array}{l}\text { Loading into Dry Storage } \\
\text { Canister }\end{array}$ & $\$ 275 \mathrm{~K} \pm 45 \%$ per canister & $\$ 300 \mathrm{~K}$ per canister & & & & & $\$ 300 \mathrm{~K}$ per canister \\
\hline & $\begin{array}{l}\text { Dry Storage Loading } \\
\text { Campaign (set-up, clean-up, } \\
\text { training, labor) }\end{array}$ & $\$ 750 \mathrm{~K} \pm 5 \%$ per campaign & & & & & & $\$ 750 \mathrm{~K} \mathrm{per} \mathrm{campaign}$ \\
\hline & $\begin{array}{l}\text { DPC Load-Out for } \\
\text { Transportation }\end{array}$ & $\$ 150 \mathrm{~K} \pm 40 \%$ per canister & & & & & & $\$ 150 \mathrm{~K}$ per canister \\
\hline & Bare Fuel loading & & & & & & & $\begin{array}{l}\text { Assume } 100 \% \text { of the cost of } \\
\text { loading a DPC a large rail bare fuel } \\
\text { cask. Assume } 65 \% \text { for legal } \\
\text { weight cost. } \\
\$ 300 \mathrm{~K} \text { for Rail } \\
\$ 180 \mathrm{~K} \text { for LWT }\end{array}$ \\
\hline & \multicolumn{8}{|c|}{$\begin{array}{l}\text { Sources: } \\
{ }^{1} \text { Nuclear Waste Management - Key Attributes, Challenges, and Costs for the Yucca Mountain Repository and Two Potential Alternatives, U.S. Government Accountability Office, GAO-10-148, November 2009. } \\
2 \text { Impacts Associated with Transfer of Spent Nuclear Fuel from Spent Fuel Storage Pools to Dry Storage After Five Years of Cooling. EPRI, Palo Alto, CA: 2010. 1021049, November 2010. } \\
{ }^{3} \text { Cost Estimate for an Away-From-Reactor Generic Interim Storage Facility (GISF) for Spent Nuclear Fuel. EPRI, Palo Alto, CA: 2009, 1018722, May 2009. } \\
{ }^{4} \text { Analysis of the Total System Life Cycle Cost o the Civilian Radioactive Waste Management Program, Fiscal Year 2007, U.S. Department of Energy, DOE/RW-0591, July 2008. } \\
\text { Analysis of the Total System Life Cycle Cost o the Civilian Radioactive Waste Management Program, U.S. Department of Energy, DOE/RW-0533, May 2001. } \\
{ }^{6} \text { StoreFUEL, Ux Consulting Company, LLC, 13-163, March 6, 2012. }\end{array}$} \\
\hline
\end{tabular}


Table 6-2. Projected Total At-Reactor Used Fuel Management Costs: Cases 1 \& 3, Cases 2 \& 4

\begin{tabular}{|c|c|c|c|c|c|c|c|c|c|c|c|}
\hline & $\begin{array}{c}\text { Acceptance } \\
\text { Rate } \\
\text { (MTHM/year) }\end{array}$ & $\begin{array}{c}\text { Start of } \\
\text { Acceptance }\end{array}$ & $\begin{array}{l}\text { Dry Storage } \\
\text { Facility } \\
\text { Construction } \\
\text { (\$B) }\end{array}$ & $\begin{array}{l}\text { Dry-Storage } \\
\text { Facility } \\
\text { Maintenance } \\
\text { - Reactor in } \\
\text { Operation } \\
\text { (\$B) }\end{array}$ & $\begin{array}{l}\text { Used Fuel } \\
\text { Pool } \\
\text { Maintenance } \\
\text { - Reactor } \\
\text { Shutdown } \\
\text { (\$B) }\end{array}$ & $\begin{array}{l}\text { Dry Storage } \\
\text { Facility } \\
\text { Maintenance } \\
\text { - Reactor } \\
\text { Shutdown } \\
\text { (\$B) }\end{array}$ & $\begin{array}{l}\text { Loading } \\
\text { Fuel } \\
\text { into } \\
\text { Dry } \\
\text { Storage } \\
\text { (\$B) }\end{array}$ & $\begin{array}{l}\text { Loading Bare } \\
\text { Fuel into Re- } \\
\text { Useable } \\
\text { Transportation } \\
\text { Casks } \\
\text { (\$B) }\end{array}$ & $\begin{array}{l}\text { Loading } \\
\text { Dry } \\
\text { Storage } \\
\text { Canisters } \\
\text { for } \\
\text { Shipping } \\
\text { (\$B) }\end{array}$ & $\begin{array}{c}\text { Dry } \\
\text { Storage } \\
\text { Canister/ } \\
\text { Overpack } \\
\text { (\$B) }\end{array}$ & $\begin{array}{l}\text { Total } \\
\text { (\$B) }\end{array}$ \\
\hline \multirow{6}{*}{$\begin{array}{c}\text { Canistered } \\
\text { Fuel } \\
\text { Transport } \\
\text { (Cases } 1 \text { \& } \\
\text { 3) }\end{array}$} & 1500 & 2020 & $\$ 0.6$ & $\$ 1.0$ & $\$ 4.6$ & $\$ 26.7$ & $\$ 3.4$ & & $\$ 4.8$ & $\$ 6.7$ & $\$ 47.8$ \\
\hline & 1500 & 2035 & $\$ 0.6$ & $\$ 1.1$ & $\$ 4.8$ & $\$ 33.4$ & $\$ 3.9$ & & $\$ 4.9$ & $\$ 7.6$ & $\$ 56.2$ \\
\hline & 3000 & 2020 & $\$ 0.6$ & $\$ 1.0$ & $\$ 4.6$ & $\$ 8.9$ & $\$ 2.8$ & & $\$ 3.6$ & $\$ 5.6$ & $\$ 27.2$ \\
\hline & 3000 & 2035 & $\$ 0.6$ & $\$ 1.1$ & $\$ 4.6$ & $\$ 16.3$ & $\$ 3.7$ & & $\$ 3.7$ & $\$ 7.0$ & $\$ 36.8$ \\
\hline & 6000 & 2020 & $\$ 0.6$ & $\$ 1.0$ & $\$ 4.5$ & $\$ 0.8$ & $\$ 2.6$ & & $\$ 2.9$ & $\$ 5.1$ & $\$ 17.6$ \\
\hline & 6000 & 2035 & $\$ 0.6$ & $\$ 1.1$ & $\$ 4.6$ & $\$ 7.4$ & $\$ 3.5$ & & $\$ 2.7$ & $\$ 6.6$ & $\$ 26.4$ \\
\hline \multirow{6}{*}{$\begin{array}{c}\text { Bare and } \\
\text { Canistered } \\
\text { Fuel } \\
\text { Transport } \\
\text { (Cases } 2 \text { \& } \\
4)\end{array}$} & 1500 & 2020 & $\$ 0.6$ & $\$ 1.0$ & $\$ 4.6$ & $\$ 26.7$ & $\$ 2.9$ & $\$ 1.9$ & $\$ 3.5$ & $\$ 5.9$ & $\$ 47.0$ \\
\hline & 1500 & 2035 & $\$ 0.6$ & $\$ 1.1$ & $\$ 4.8$ & $\$ 33.4$ & $\$ 3.8$ & $\$ 0.7$ & $\$ 4.4$ & $\$ 7.4$ & $\$ 56.1$ \\
\hline & 3000 & 2020 & $\$ 0.6$ & $\$ 1.0$ & $\$ 4.6$ & $\$ 9.5$ & $\$ 1.7$ & $\$ 3.3$ & $\$ 1.7$ & $\$ 3.3$ & $\$ 25.6$ \\
\hline & 3000 & 2035 & $\$ 0.6$ & $\$ 1.1$ & $\$ 4.6$ & $\$ 16.3$ & $\$ 3.3$ & $\$ 1.3$ & $\$ 2.8$ & $\$ 6.1$ & $\$ 36.1$ \\
\hline & 6000 & 2020 & $\$ 0.5$ & $\$ 1.0$ & $\$ 4.5$ & $\$ 1.4$ & $\$ 0.9$ & $\$ 4.9$ & $\$ 0.9$ & $\$ 1.3$ & $\$ 15.4$ \\
\hline & 6000 & 2035 & $\$ 0.6$ & $\$ 1.1$ & $\$ 4.6$ & $\$ 7.7$ & $\$ 2.6$ & $\$ 2.2$ & $\$ 1.7$ & $\$ 4.5$ & $\$ 25.0$ \\
\hline
\end{tabular}


Table 6-3. Total At-Reactor Used Fuel Management Cost Drivers: Cases 1 \& 3, Cases 2 \& 4, and Case 9-1

\begin{tabular}{|c|c|c|c|c|c|c|c|c|c|c|}
\hline & $\begin{array}{l}\text { Acceptance } \\
\text { Rate } \\
\text { (MTHM/year) }\end{array}$ & $\begin{array}{c}\text { Start of } \\
\text { Acceptance }\end{array}$ & $\begin{array}{l}\text { Dry Storage } \\
\text { Facility } \\
\text { Construction } \\
\text { (\% of Total) }\end{array}$ & $\begin{array}{l}\text { Dry-Storage } \\
\text { Facility } \\
\text { Maintenance } \\
\text { - Reactor in } \\
\text { Operation } \\
\text { (\% of Total) }\end{array}$ & $\begin{array}{l}\text { Used Fuel } \\
\text { Pool } \\
\text { Maintenance } \\
\text { - Reactor } \\
\text { Shutdown } \\
\text { (\% of Total) }\end{array}$ & $\begin{array}{l}\text { Dry Storage } \\
\text { Facility } \\
\text { Maintenance } \\
\text { - Reactor } \\
\text { Shutdown } \\
\text { (\% of Total) }\end{array}$ & $\begin{array}{l}\text { Loading } \\
\text { Fuel into } \\
\text { Dry } \\
\text { Storage } \\
\text { (\% of } \\
\text { Total) }\end{array}$ & $\begin{array}{l}\text { Loading Bare } \\
\text { Fuel into Re- } \\
\text { Useable } \\
\text { Transportation } \\
\text { Casks } \\
\text { (\% of Total ) }\end{array}$ & $\begin{array}{c}\text { Loading } \\
\text { Dry } \\
\text { Storage } \\
\text { Canisters } \\
\text { for } \\
\text { Shipping } \\
\text { (\% of } \\
\text { Total) } \\
\end{array}$ & $\begin{array}{c}\text { Dry Storage } \\
\text { Canister/Overpack } \\
\text { (\% of Total ) }\end{array}$ \\
\hline \multirow{6}{*}{ Cases 1 \& 3} & 1500 & 2020 & $1.2 \%$ & $2.2 \%$ & $9.6 \%$ & $55.8 \%$ & $7.1 \%$ & & $10.2 \%$ & $14.0 \%$ \\
\hline & 1500 & 2035 & $1.0 \%$ & $1.9 \%$ & $8.5 \%$ & $59.5 \%$ & $7.0 \%$ & & $8.7 \%$ & $13.5 \%$ \\
\hline & 3000 & 2020 & $2.0 \%$ & $3.8 \%$ & $16.9 \%$ & $32.9 \%$ & $10.5 \%$ & & $13.4 \%$ & $20.5 \%$ \\
\hline & 3000 & 2035 & $1.5 \%$ & $2.9 \%$ & $12.5 \%$ & $44.2 \%$ & $10.0 \%$ & & $9.9 \%$ & $19.1 \%$ \\
\hline & 6000 & 2020 & $3.1 \%$ & $5.8 \%$ & $25.8 \%$ & $4.5 \%$ & $15.0 \%$ & & $16.5 \%$ & $29.2 \%$ \\
\hline & 6000 & 2035 & $2.1 \%$ & $4.0 \%$ & $17.5 \%$ & $27.9 \%$ & $13.2 \%$ & & $10.4 \%$ & $25.0 \%$ \\
\hline \multirow{6}{*}{ Cases $2 \& 4$} & 1500 & 2020 & $1.2 \%$ & $2.2 \%$ & $9.8 \%$ & $56.7 \%$ & $6.1 \%$ & $4.1 \%$ & $7.5 \%$ & $12.5 \%$ \\
\hline & 1500 & 2035 & $1.0 \%$ & $1.9 \%$ & $8.5 \%$ & $59.6 \%$ & $6.8 \%$ & $1.2 \%$ & $7.8 \%$ & $13.2 \%$ \\
\hline & 3000 & 2020 & $2.2 \%$ & $4.0 \%$ & $18.0 \%$ & $37.0 \%$ & $6.5 \%$ & $13.0 \%$ & $6.6 \%$ & $12.8 \%$ \\
\hline & 3000 & 2035 & $1.5 \%$ & $2.9 \%$ & $12.8 \%$ & $45.1 \%$ & $9.1 \%$ & $3.7 \%$ & $7.9 \%$ & $17.0 \%$ \\
\hline & 6000 & 2020 & $2.9 \%$ & $6.6 \%$ & $29.5 \%$ & $9.2 \%$ & $5.7 \%$ & $31.7 \%$ & $5.8 \%$ & $8.7 \%$ \\
\hline & 6000 & 2035 & $2.2 \%$ & $4.2 \%$ & $18.4 \%$ & $30.7 \%$ & $10.5 \%$ & $9.0 \%$ & $6.9 \%$ & $18.2 \%$ \\
\hline
\end{tabular}




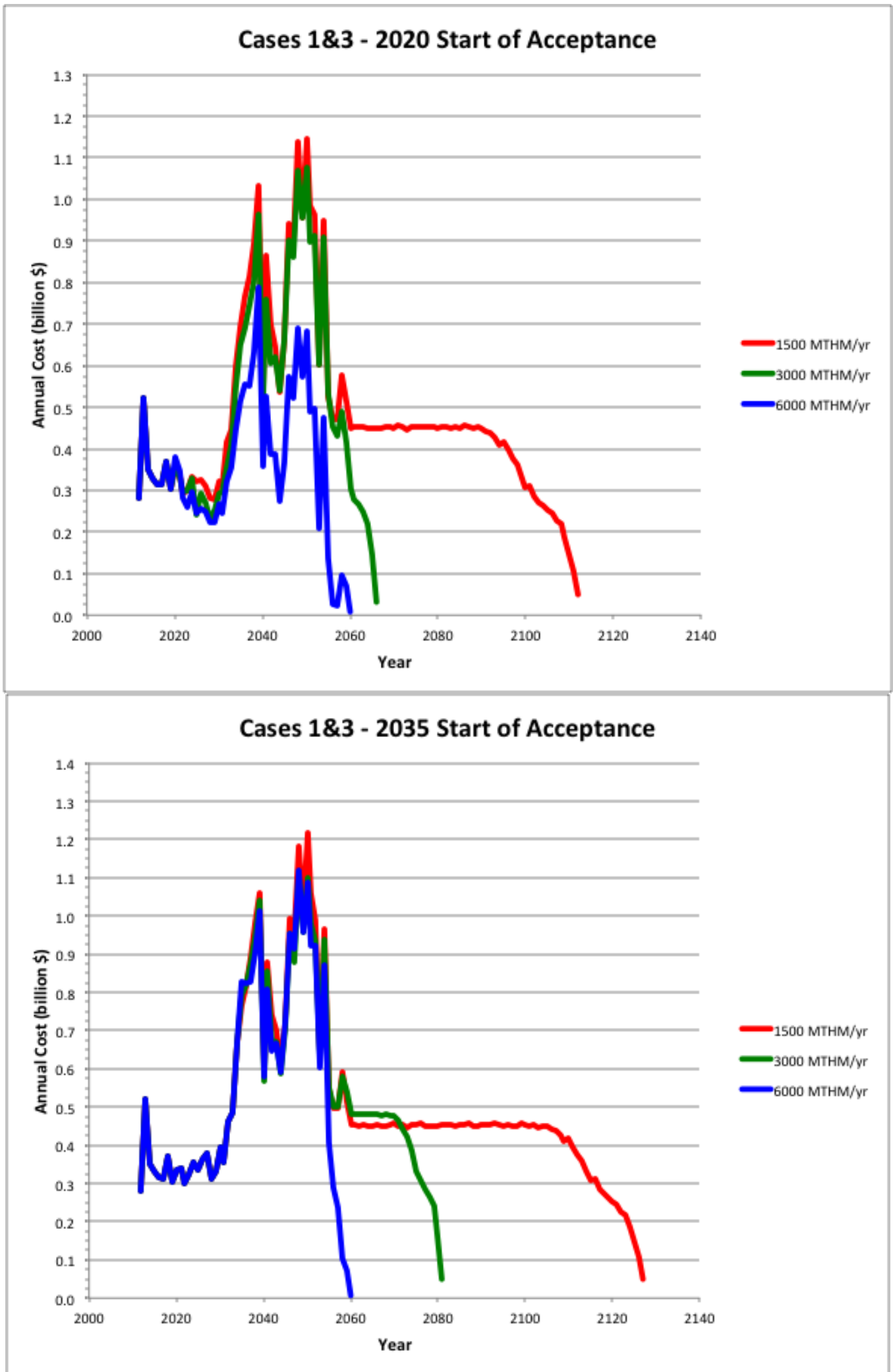

Figure 6-2. Annual At-Reactor Used Fuel Management Costs, Cases 1\&3, Canisters Only 


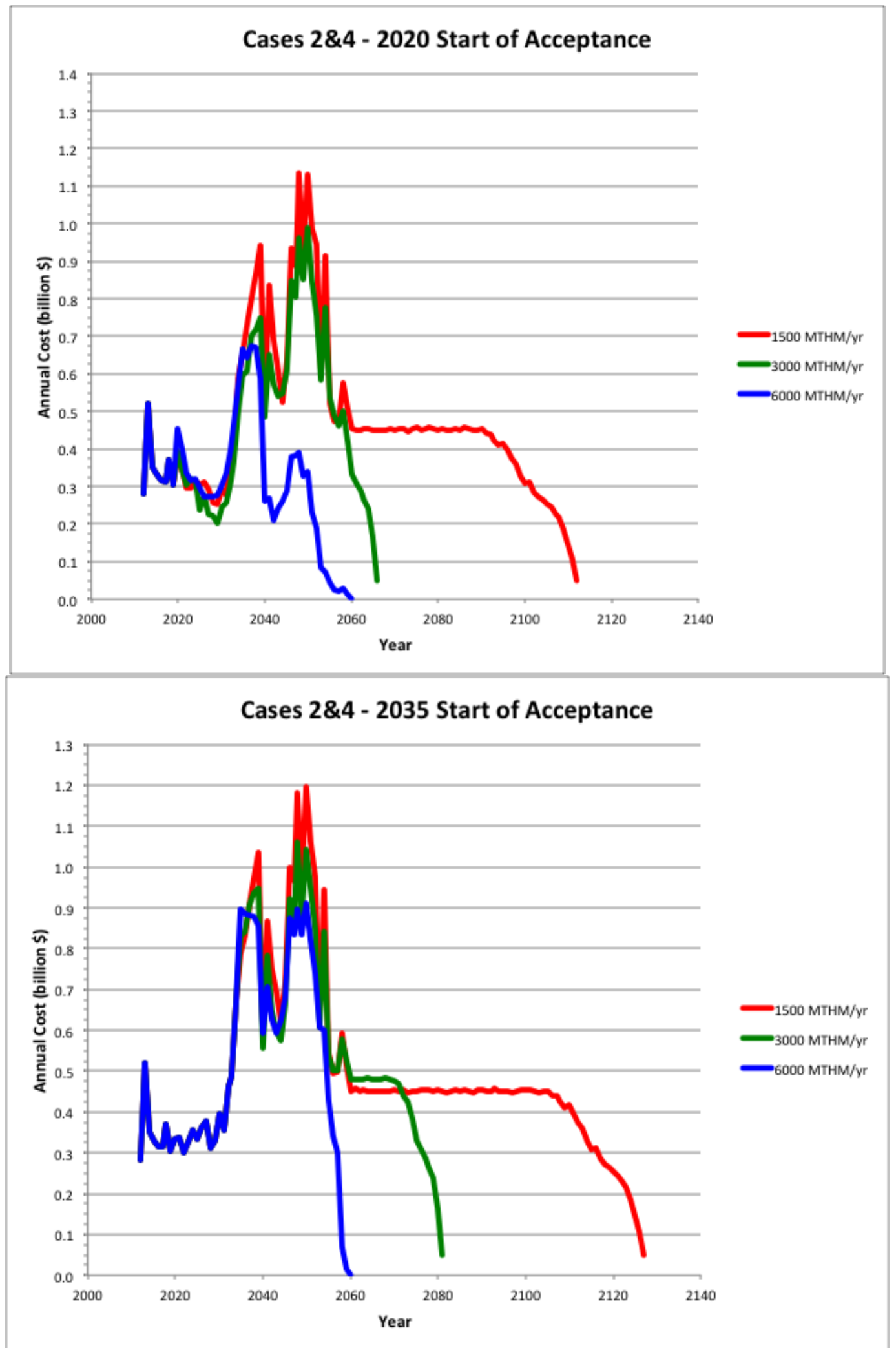

Figure 6-3. Annual At-Reactor Used Fuel Management Costs, Cases 2\&4, Canisters and Bare Fuel 


\subsection{Consolidated Storage Rough Order of Magnitude Cost Estimates}

Previously developed unit costs for UNF storage, presented in Appendix C, were used as the basis for estimating the rough order of magnitude costs for the facility configurations used in this evaluation (Rodwell et al. 2007, McConnell et al. 2007, DOE 2008, Carter et al 2012). These costs are then applied via the methodology described in Appendix C to the CSF requirements presented in Section 4.

The resulting CSF annual operations and maintenance costs are summarized in Table 6-4. As expected, the annual costs vary with fuel acceptance rate and the storage type (dry only or a combination of dry and pool). There are small variations driven by small differences in the characteristics of the UNF received at the CSF (canisters only or canisters and bare fuel).

Table 6-5 extends the annual cost to the total life cycle. The annual cost for labor, utilities, materials \& contracts and decommissioning and demolition (D\&D) are extended as point estimates based on the scenario schedule (see below). The D\&D and additional capital costs are derived by algorithm (Appendix C) based on Total Project Cost (TPC). The point values can be converted to a low and high range by an $80 \%, 120 \%$ algorithm.

The operations and maintenance (O\&M) life-cycle costs (LCC) vary due to differences in the operations schedules for the different cases. The CSF is expected to be in operation from the CSF start date (Table 6-5) through the repository start date (20 or 35 years depending on the scenario) and until the repository emplacement is finished (23, 47 or 97 years to dispose of 140,000 MTHM of UNF, depending upon the acceptance rate). The scenario schedule ranges from 43 to 120 years and is the largest driver in the variation of the O\&M LCC.

The TPC, shown in Table 6-5, is limited to the facilities required for storage for the first two years of operation. The variation reflects the storage type for each scenario. The additional capital, also shown in Table 6-5, provides the cost of the vertical and horizontal dry and the pool storage facilities required for the UNF inventory storage beyond the first two years.

For scenarios that include bare fuel storage, the capital requirements for pool storage of bare fuel are greater than those for dry storage for the canisters. Wet storage is a large driver in the total consolidated storage costs for these scenarios.

The Blue Ribbon Commission on America's Nuclear Future (BRC 2012, Section 5.2.5) recommended that a laboratory with hot cell facilities for research and development on long term performance of storage systems be included in the considerations on consolidated storage. Table 6-6 summarizes the annual cost of a Test and Validation Facility (TVF) that would perform such functions. In all scenarios the annual cost are the same as the facility is assumed to not vary in the analytical capacity with acceptance rate, overall storage capacity nor storage type.

The facility is assumed to operate over the same time period as the consolidated storage facility. Because the assumed annual TVF O\&M costs are comparable to those for the storage facility and represent a significant fraction of the TVF LCC costs, those cost (Table 6-7) vary by the length of time the storage facility operates in each scenario. The LCC cost is indicated to range from about $\$ 2.9 \mathrm{~B}$ to $\$ 4.7 \mathrm{~B}$ across all scenarios, or an additional $4 \%$ of the cost for a large CSF to $60 \%$ of the cost for a small CSF, depending upon the scenario selected.

An alternative to the vertical pad and horizontal module dry storage configurations substituting a large dry storage vault to hold the large existing size dry storage canisters received from utilities was considered. The total capital (TPC and additional capital) required for the horizontal plus vertical dry storage is compared to the large vault concept in Table 6-8. The comparison does not indicate a cost advantage for the large vault system for storing large canisters received from utilities, which was expected to be more capital intensive, compared to using modular storage systems for which such canisters were designed. The LCC for the two alternatives were not compared but the alternate large vault does not 
appear to be attractive based on the large difference in the TPC, which would have to be "overcome" by a lower operational cost.

Note that the option discussed in section 3.2.4 of packaging bare fuel received at the CSF into small disposal-compatible canisters that are stored in a dry vault system designed for such canisters and would not have to be re-opened, as would have to be done with the large canisters considered in this comparison, has not yet been evaluated. 
Table 6-4 Consolidated Storage Annual Operations and Maintenance Costs

\begin{tabular}{|c|c|c|c|c|c|c|c|c|c|c|c|}
\hline \multirow[b]{2}{*}{ Scenario } & \multirow[b]{2}{*}{$\begin{array}{c}\text { Acceptance } \\
\text { Rate }\end{array}$} & \multirow[b]{2}{*}{ CSF Start } & \multirow[b]{2}{*}{$\begin{array}{c}\text { Repository } \\
\text { Start }\end{array}$} & \multirow[b]{2}{*}{$\begin{array}{c}\text { Disposal } \\
\text { Canister } \\
\text { Size } \\
\end{array}$} & \multicolumn{3}{|c|}{ Total Staffing } & \multicolumn{4}{|c|}{ Annual Operations and Maintenance } \\
\hline & & & & & Management & Exempt & Hourly & $\begin{array}{c}\text { Labor } \\
\text { FY } 2012 \text { (\$M) }\end{array}$ & $\begin{array}{c}\text { Utilities } \\
\text { FY } 2012 \text { (\$M) }\end{array}$ & $\begin{array}{l}\text { Materials and } \\
\text { Contracts } \\
\text { FY } 2012 \text { (\$M) }\end{array}$ & $\begin{array}{c}\text { Base Total Annual } \\
\text { FY } 2012 \text { (\$M) }\end{array}$ \\
\hline \multirow{9}{*}{$\begin{array}{c}\text { Canisters } \\
\text { Only - } \\
\text { Re-Package } \\
\text { at Repository } \\
\text { (Case 1) }\end{array}$} & 1,500 & 2020 & 2040 & 4 & 12 & 63 & 56 & $\$ 20.4$ & $\$ 1.7$ & $\$ 1.6$ & $\$ 23.7$ \\
\hline & 3,000 & 2020 & 2040 & 4 & 12 & 63 & 56 & $\$ 20.4$ & $\$ 1.7$ & $\$ 1.6$ & $\$ 23.7$ \\
\hline & 3,000 & 2020 & 2040 & 12 & 12 & 63 & 56 & $\$ 20.4$ & $\$ 1.7$ & $\$ 1.6$ & $\$ 23.7$ \\
\hline & 3,000 & 2020 & 2040 & 21 & 12 & 63 & 56 & $\$ 20.4$ & $\$ 1.7$ & $\$ 1.6$ & $\$ 23.7$ \\
\hline & 6,000 & 2020 & 2040 & 4 & 17 & 83 & 86 & $\$ 28.4$ & $\$ 3.4$ & $\$ 2.9$ & $\$ 34.8$ \\
\hline & 1,500 & 2020 & 2055 & 4 & 12 & 63 & 56 & $\$ 20.4$ & $\$ 1.7$ & $\$ 1.6$ & $\$ 23.7$ \\
\hline & 3,000 & 2020 & 2055 & 4 & 12 & 63 & 56 & $\$ 20.4$ & $\$ 1.7$ & $\$ 1.6$ & $\$ 23.7$ \\
\hline & 6,000 & 2020 & 2055 & 4 & 17 & 83 & 86 & $\$ 28.4$ & $\$ 3.4$ & $\$ 2.9$ & $\$ 34.8$ \\
\hline & 3,000 & 2035 & 2055 & 4 & 12 & 63 & 56 & $\$ 20.4$ & $\$ 1.7$ & $\$ 1.6$ & $\$ 23.7$ \\
\hline \multirow{9}{*}{$\begin{array}{c}\text { Canisters and } \\
\text { Bare Fuel - } \\
\text { Re-Package } \\
\text { at Repository } \\
\text { (Case 2) }\end{array}$} & 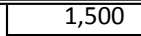 & 2020 & 2040 & 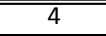 & 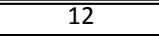 & "63 & 256 & $\$ 20.1$ & $\begin{array}{l}\$ 3.4 \\
\end{array}$ & $\begin{array}{l}\$ 1.2 \\
\end{array}$ & \$24.6 \\
\hline & 3,000 & 2020 & 2040 & 4 & 13 & 71 & 108 & $\$ 27.7$ & $\$ 3.4$ & $\$ 1.2$ & $\$ 32.3$ \\
\hline & 3,000 & 2020 & 2040 & 12 & 13 & 71 & 108 & $\$ 27.7$ & $\$ 3.4$ & $\$ 1.2$ & $\$ 32.3$ \\
\hline & 3,000 & 2020 & 2040 & 21 & 13 & 71 & 108 & $\$ 27.7$ & $\$ 3.4$ & $\$ 1.2$ & $\$ 32.3$ \\
\hline & 6,000 & 2020 & 2040 & 4 & 19 & 99 & 190 & $\$ 43.0$ & $\$ 6.8$ & $\$ 1.8$ & $\$ 51.6$ \\
\hline & 1,500 & 2020 & 2055 & 4 & 10 & 57 & 67 & $\$ 20.1$ & $\$ 3.4$ & $\$ 1.2$ & $\$ 24.6$ \\
\hline & 3,000 & 2020 & 2055 & 4 & 13 & 71 & 108 & $\$ 27.7$ & $\$ 3.4$ & $\$ 1.8$ & $\$ 32.9$ \\
\hline & 6,000 & 2020 & 2055 & 4 & 19 & 99 & 190 & $\$ 43.0$ & $\$ 6.8$ & $\$ 1.8$ & $\$ 51.6$ \\
\hline & 3,000 & 2035 & 2055 & 4 & 13 & 71 & 108 & $\$ 27.7$ & $\$ 3.4$ & $\$ 1.8$ & $\$ 32.9$ \\
\hline \multirow{9}{*}{$\begin{array}{c}\text { Canisters } \\
\text { Only - Re- } \\
\text { Package at } \\
\text { CSF (Case 3) }\end{array}$} & 1,500 & 2020 & 2040 & 4 & 12 & 263 & 256 & $\$ 20.4$ & $\$ 1.7$ & $\$ 1.6$ & $\$ 23.7$ \\
\hline & 3,000 & 2020 & 2040 & 4 & 15 & 73 & 71 & $\$ 24.4$ & $\$ 1.7$ & $\$ 2.2$ & $\$ 28.4$ \\
\hline & 3,000 & 2020 & 2040 & 12 & 15 & 73 & 71 & $\$ 24.4$ & $\$ 1.7$ & $\$ 2.2$ & $\$ 28.4$ \\
\hline & 3,000 & 2020 & 2040 & 21 & 15 & 73 & 71 & $\$ 24.4$ & $\$ 1.7$ & $\$ 2.2$ & $\$ 28.4$ \\
\hline & 6,000 & 2020 & 2040 & 4 & 17 & 83 & 86 & $\$ 28.4$ & $\$ 3.4$ & $\$ 2.9$ & $\$ 34.8$ \\
\hline & 1,500 & 2020 & 2055 & 4 & 12 & 63 & 56 & $\$ 20.4$ & $\$ 1.7$ & $\$ 1.6$ & $\$ 23.7$ \\
\hline & 3,000 & 2020 & 2055 & 4 & 15 & 73 & 71 & $\$ 24.4$ & $\$ 1.7$ & $\$ 2.2$ & $\$ 28.4$ \\
\hline & 6,000 & 2020 & 2055 & 4 & 17 & 83 & 86 & $\$ 28.4$ & $\$ 3.4$ & $\$ 2.9$ & $\$ 34.8$ \\
\hline & 3,000 & 2035 & 2055 & 4 & 12 & 63 & 56 & $\$ 20.4$ & $\$ 1.7$ & $\$ 1.6$ & $\$ 23.7$ \\
\hline \multirow{9}{*}{$\begin{array}{c}\text { Canisters and } \\
\text { Bare Fuel - } \\
\text { Re-Package } \\
\text { at CSF (Case } \\
\text { 4) }\end{array}$} & 1,500 & 2020 & 2040 & $\bar{~} 4$ & 10 & "57 & 67 & \$20.1 & $\$ 3.4$ & \$1.2 & $\$ 24.6$ \\
\hline & 3,000 & 2020 & 2040 & 4 & 13 & 71 & 108 & $\$ 27.7$ & $\$ 3.4$ & $\$ 1.8$ & $\$ 32.9$ \\
\hline & 3,000 & 2020 & 2040 & 12 & 13 & 71 & 108 & $\$ 27.7$ & $\$ 3.4$ & $\$ 1.8$ & $\$ 32.9$ \\
\hline & 3,000 & 2020 & 2040 & 21 & 13 & 71 & 108 & $\$ 27.7$ & $\$ 3.4$ & $\$ 1.8$ & $\$ 32.9$ \\
\hline & 6,000 & 2020 & 2040 & 4 & 19 & 99 & 190 & $\$ 43.0$ & $\$ 6.8$ & $\$ 1.8$ & $\$ 51.6$ \\
\hline & 1,500 & 2020 & 2055 & 4 & 10 & 57 & 67 & $\$ 20.1$ & $\$ 3.4$ & $\$ 1.2$ & $\$ 24.6$ \\
\hline & 3,000 & 2020 & 2055 & 4 & 13 & 71 & 108 & $\$ 27.7$ & $\$ 3.4$ & $\$ 1.8$ & $\$ 32.9$ \\
\hline & 6,000 & 2020 & 2055 & 4 & 19 & 99 & 190 & $\$ 43.0$ & $\$ 6.8$ & $\$ 1.8$ & $\$ 51.6$ \\
\hline & 3,000 & 2035 & 2055 & 4 & 13 & 71 & 108 & $\$ 27.7$ & $\$ 3.4$ & $\$ 1.8$ & $\$ 32.9$ \\
\hline
\end{tabular}


Used Fuel Management System Architecture Evaluation, Fiscal Year 2012

Table 6-5 Consolidated Storage Life Cycle Costs

\begin{tabular}{|c|c|c|c|c|c|c|c|c|c|c|c|c|c|c|c|}
\hline \multirow[b]{2}{*}{ Scenario } & \multirow[b]{2}{*}{$\begin{array}{c}\text { Acceptance } \\
\text { Rate }\end{array}$} & \multirow[b]{2}{*}{ CSF Start } & \multirow[b]{2}{*}{$\begin{array}{c}\text { Repository } \\
\text { Start }\end{array}$} & \multirow[b]{2}{*}{$\begin{array}{c}\text { Disposal } \\
\text { Canister } \\
\text { Size }\end{array}$} & \multicolumn{5}{|c|}{ Life Cycle O\&M } & \multirow{2}{*}{$\begin{array}{c}\text { TPC } \\
\text { FY } 2012 \text { (\$B) }\end{array}$} & \multicolumn{4}{|c|}{ Additional Capital } & \multirow{2}{*}{$\begin{array}{l}\text { Total Life Cycl } \\
\text { FY } 2012 \text { (\$B) }\end{array}$} \\
\hline & & & & & $\begin{array}{c}\text { Labor } \\
\text { FY } 2012 \text { (\$B) }\end{array}$ & $\begin{array}{l}\text { Utilities } \\
\text { FY } 2012 \text { (\$B) }\end{array}$ & $\begin{array}{l}\text { Materials and } \\
\text { Contracts } \\
\text { FY } 2012 \text { (\$B) }\end{array}$ & $\begin{array}{c}\text { D\&D } \\
\text { FY } 2012 \text { (SB) }\end{array}$ & $\begin{array}{c}\text { Total } \\
\text { FY } 2012 \text { (\$B) }\end{array}$ & & $\begin{array}{l}\text { Vertical Storage } \\
\text { Modules } \\
\text { FY } 2012 \text { (\$B) }\end{array}$ & $\begin{array}{c}\text { Horizontal } \\
\text { Modules } \\
\text { FY } 2012 \text { (\$B) }\end{array}$ & $\begin{array}{c}\text { Storage Pools and } \\
\text { Infrastructure } \\
\text { FY } 2012 \text { (SB) }\end{array}$ & $\begin{array}{c}\text { Total } \\
\text { FY } 2012 \text { (\$B) }\end{array}$ & \\
\hline \multirow{9}{*}{$\begin{array}{c}\text { Canisters } \\
\text { Only - } \\
\text { Re-Package } \\
\text { at Repository } \\
\text { (Case 1) }\end{array}$} & 1,500 & 2020 & 2040 & 4 & $\$ 2.4$ & $\$ 0.2$ & $\$ 0.2$ & $\$ 0.1$ & $\$ 2.9$ & $\$ 0.8$ & $\$ 1.7$ & $\$ 1.6$ & & $\$ 3.8$ & $\$ 7.5$ \\
\hline & 3,000 & 2020 & 2040 & 4 & $\$ 1.4$ & $\$ 0.1$ & $\$ 0.1$ & $\$ 0.3$ & $\$ 1.9$ & $\$ 1.2$ & $\$ 3.2$ & $\$ 4.1$ & & $\$ 8.2$ & $\$ 11.3$ \\
\hline & 3,000 & 2020 & 2040 & 12 & $\$ 1.4$ & $\$ 0.1$ & $\$ 0.1$ & $\$ 0.3$ & $\$ 1.9$ & $\$ 1.2$ & $\$ 3.2$ & $\$ 4.1$ & & $\$ 8.2$ & $\$ 11.3$ \\
\hline & 3,000 & 2020 & 2040 & 21 & $\$ 1.4$ & $\$ 0.1$ & $\$ 0.1$ & $\$ 0.3$ & $\$ 1.9$ & $\$ 1.2$ & $\$ 3.2$ & $\$ 4.1$ & & $\$ 8.2$ & $\$ 11.3$ \\
\hline & 6,000 & 2020 & 2040 & 4 & $\$ 1.3$ & $\$ 0.2$ & $\$ 0.1$ & $\$ 0.4$ & $\$ 2.0$ & $\$ 2.3$ & $\$ 4.1$ & $\$ 7.7$ & & $\$ 13.2$ & $\$ 17.5$ \\
\hline & 1,500 & 2020 & 2055 & 4 & $\$ 2.7$ & $\$ 0.2$ & $\$ 0.2$ & $\$ 0.2$ & $\$ 3.3$ & $\$ 0.8$ & $\$ 2.9$ & $\$ 4.0$ & & $\$ 7.8$ & $\$ 11.9$ \\
\hline & 3,000 & 2020 & 2055 & 4 & $\$ 1.7$ & $\$ 0.1$ & $\$ 0.1$ & $\$ 0.4$ & $\$ 2.4$ & $\$ 1.2$ & $\$ 5.5$ & $\$ 9.4$ & & $\$ 16.8$ & $\$ 20.4$ \\
\hline & 6,000 & 2020 & 2055 & 4 & $\$ 1.7$ & $\$ 0.2$ & $\$ 0.2$ & $\$ 0.6$ & $\$ 2.7$ & $\$ 2.3$ & $\$ 6.7$ & $\$ 11.8$ & & $\$ 20.7$ & $\$ 25.7$ \\
\hline & 3,000 & 2035 & 2055 & 4 & $\$ 1.4$ & $\$ 0.1$ & $\$ 0.1$ & $\$ 0.3$ & $\$ 1.9$ & $\$ 1.3$ & $\$ 3.0$ & $\$ 5.7$ & & $\$ 9.7$ & $\$ 12.9$ \\
\hline \multirow{9}{*}{$\begin{array}{c}\text { Canisters and } \\
\text { Bare Fuel - } \\
\text { Re-Package } \\
\text { at Repository } \\
\text { (Case 2) }\end{array}$} & 1,500 & 2020 & 2040 & 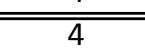 & $\$ 2.4$ & $\$ 0.2$ & $\$ 0.1$ & $\$ 1.5$ & $\$ 4.3$ & $\$ 5.7$ & $\$ 0.2$ & $\$ 0.2$ & $\$ 9.9$ & $\$ 12.4$ & $\$ 22.5$ \\
\hline & 3,000 & 2020 & 2040 & 4 & $\$ 1.9$ & $\$ 0.2$ & $\$ 0.1$ & $\$ 2.9$ & $\$ 5.1$ & $\$ 6.2$ & $\$ 0.2$ & $\$ 0.4$ & $\$ 23.2$ & $\$ 28.8$ & $\$ 40.2$ \\
\hline & 3,000 & 2020 & 2040 & 12 & $\$ 1.9$ & $\$ 0.2$ & $\$ 0.1$ & $\$ 2.9$ & $\$ 5.1$ & $\$ 6.2$ & $\$ 0.2$ & $\$ 0.4$ & $\$ 23.2$ & $\$ 28.8$ & $\$ 40.2$ \\
\hline & 3,000 & 2020 & 2040 & 21 & $\$ 1.9$ & $\$ 0.2$ & $\$ 0.1$ & $\$ 2.9$ & $\$ 5.1$ & $\$ 6.2$ & $\$ 0.2$ & $\$ 0.4$ & $\$ 23.2$ & $\$ 28.8$ & $\$ 40.2$ \\
\hline & 6,000 & 2020 & 2040 & 4 & $\$ 2.0$ & $\$ 0.3$ & $\$ 0.1$ & $\$ 4.5$ & $\$ 6.9$ & $\$ 7.9$ & $\$ 1.5$ & $\$ 4.9$ & $\$ 37.4$ & $\$ 52.4$ & $\$ 67.2$ \\
\hline & 1,500 & 2020 & 2055 & 4 & $\$ 2.6$ & $\$ 0.4$ & $\$ 0.1$ & $\$ 2.0$ & $\$ 5.2$ & $\$ 5.7$ & $\$ 0.7$ & $\$ 2.0$ & $\$ 14.7$ & $\$ 20.8$ & $\$ 31.8$ \\
\hline & 3,000 & 2020 & 2055 & 4 & $\$ 2.3$ & $\$ 0.3$ & $\$ 0.1$ & $\$ 3.9$ & $\$ 6.6$ & $\$ 6.4$ & $\$ 1.4$ & $\$ 4.4$ & $\$ 32.2$ & $\$ 45.4$ & $\$ 58.4$ \\
\hline & 6,000 & 2020 & 2055 & 4 & $\$ 2.6$ & $\$ 0.4$ & $\$ 0.1$ & $\$ 5.3$ & $\$ 8.5$ & $\$ 7.9$ & $\$ 1.6$ & $\$ 5.0$ & $\$ 45.4$ & $\$ 62.4$ & $\$ 78.7$ \\
\hline & 3,000 & 2035 & 2055 & 4 & $\$ 1.9$ & $\$ 0.2$ & $\$ 0.1$ & $\$ 1.8$ & $\$ 4.1$ & $\$ 6.2$ & $\$ 1.2$ & $\$ 4.0$ & $\$ 11.8$ & $\$ 20.2$ & $\$ 30.5$ \\
\hline \multirow{9}{*}{$\begin{array}{c}\text { Canisters } \\
\text { Only - Re- } \\
\text { Package at } \\
\text { CSF } \\
\text { (Case 3) }\end{array}$} & 1,500 & 2020 & 2040 & 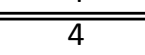 & $\$ 2.4$ & $\$ 0.2$ & $\$ 0.2$ & $\$ 0.2$ & $\$ 2.9$ & $\$ 0.7$ & $\$ 1.7$ & $\$ 4.3$ & & $\$ 6.7$ & $\$ 10.3$ \\
\hline & 3,000 & 2020 & 2040 & 4 & $\$ 1.7$ & $\$ 0.1$ & $\$ 0.2$ & $\$ 0.3$ & $\$ 2.3$ & $\$ 1.1$ & $\$ 3.2$ & $\$ 7.3$ & & $\$ 11.8$ & $\$ 15.2$ \\
\hline & 3,000 & 2020 & 2040 & 12 & $\$ 1.7$ & $\$ 0.1$ & $\$ 0.2$ & $\$ 0.3$ & $\$ 2.3$ & $\$ 1.1$ & $\$ 3.2$ & $\$ 7.3$ & & $\$ 11.8$ & $\$ 15.2$ \\
\hline & 3,000 & 2020 & 2040 & 21 & $\$ 1.7$ & $\$ 0.1$ & $\$ 0.2$ & $\$ 0.3$ & $\$ 2.3$ & $\$ 1.1$ & $\$ 3.2$ & $\$ 7.3$ & & $\$ 11.8$ & $\$ 15.2$ \\
\hline & 6,000 & 2020 & 2040 & 4 & $\$ 1.3$ & $\$ 0.2$ & $\$ 0.1$ & $\$ 0.4$ & $\$ 2.0$ & $\$ 2.0$ & $\$ 4.1$ & $\$ 8.4$ & & $\$ 14.0$ & $\$ 18.0$ \\
\hline & 1,500 & 2020 & 2055 & 4 & $\$ 2.7$ & $\$ 0.2$ & $\$ 0.2$ & $\$ 0.3$ & $\$ 3.4$ & $\$ 0.7$ & $\$ 2.9$ & $\$ 7.5$ & & $\$ 11.7$ & $\$ 15.8$ \\
\hline & 3,000 & 2020 & 2055 & 4 & $\$ 2.1$ & $\$ 0.1$ & $\$ 0.2$ & $\$ 0.4$ & $\$ 2.8$ & $\$ 1.1$ & $\$ 5.5$ & $\$ 11.4$ & & $\$ 18.9$ & $\$ 22.9$ \\
\hline & 6,000 & 2020 & 2055 & 4 & $\$ 1.7$ & $\$ 0.2$ & $\$ 0.2$ & $\$ 0.6$ & $\$ 2.7$ & $\$ 2.0$ & $\$ 6.7$ & $\$ 12.1$ & & $\$ 21.1$ & $\$ 25.7$ \\
\hline & 3,000 & 2035 & 2055 & 4 & $\$ 1.4$ & $\$ 0.1$ & $\$ 0.1$ & $\$ 0.3$ & $\$ 2.0$ & $\$ 1.1$ & $\$ 3.0$ & $\$ 8.3$ & & $\$ 12.6$ & $\$ 15.8$ \\
\hline \multirow{9}{*}{$\begin{array}{c}\text { Canisters and } \\
\text { Bare Fuel - } \\
\text { Re-Package } \\
\text { at CSF } \\
\text { (Case 4) }\end{array}$} & 1,500 & 2020 & 2040 & $\overline{4} 4$ & $\$ 2.3$ & $\$ 0.4$ & $\$ 0.1$ & $\$ 1.6$ & $\$ 4.4$ & $\$ 5.7$ & $\$ 1.5$ & $\$ 4.3$ & $\$ 9.9$ & $\$ 18.6$ & $\$ 28.7$ \\
\hline & 3,000 & 2020 & 2040 & 4 & $\$ 1.9$ & $\$ 0.2$ & $\$ 0.1$ & $\$ 3.0$ & $\$ 5.3$ & $\$ 6.2$ & $\$ 2.5$ & $\$ 6.9$ & $\$ 23.2$ & $\$ 38.5$ & $\$ 50.1$ \\
\hline & 3,000 & 2020 & 2040 & 12 & $\$ 1.9$ & $\$ 0.2$ & $\$ 0.1$ & $\$ 3.0$ & $\$ 5.3$ & $\$ 6.2$ & $\$ 2.5$ & $\$ 6.9$ & $\$ 23.2$ & $\$ 38.5$ & $\$ 50.1$ \\
\hline & 3,000 & 2020 & 2040 & 21 & $\$ 1.9$ & $\$ 0.2$ & $\$ 0.1$ & $\$ 3.0$ & $\$ 5.3$ & $\$ 6.2$ & $\$ 2.5$ & $\$ 6.9$ & $\$ 23.2$ & $\$ 38.5$ & $\$ 50.1$ \\
\hline & 6,000 & 2020 & 2040 & 4 & $\$ 2.0$ & $\$ 0.3$ & $\$ 0.1$ & $\$ 4.5$ & $\$ 6.9$ & $\$ 7.8$ & $\$ 1.5$ & $\$ 4.9$ & $\$ 37.4$ & $\$ 52.4$ & $\$ 67.1$ \\
\hline & 1,500 & 2020 & 2055 & 4 & $\$ 2.6$ & $\$ 0.4$ & $\$ 0.2$ & $\$ 2.1$ & $\$ 5.4$ & $\$ 5.7$ & $\$ 2.7$ & $\$ 7.6$ & $\$ 14.7$ & $\$ 29.2$ & $\$ 40.3$ \\
\hline & 3,000 & 2020 & 2055 & 4 & $\$ 2.3$ & $\$ 0.3$ & $\$ 0.2$ & $\$ 4.0$ & $\$ 6.7$ & $\$ 6.2$ & $\$ 2.9$ & $\$ 8.3$ & $\$ 32.2$ & $\$ 51.4$ & $\$ 64.4$ \\
\hline & 6,000 & 2020 & 2055 & 4 & $\$ 2.6$ & $\$ 0.4$ & $\$ 0.1$ & $\$ 5.3$ & $\$ 8.5$ & $\$ 7.8$ & $\$ 1.6$ & $\$ 5.0$ & $\$ 45.4$ & $\$ 62.4$ & $\$ 78.6$ \\
\hline & 3,000 & 2035 & 2055 & 4 & $\$ 1.9$ & $\$ 0.2$ & $\$ 0.1$ & $\$ 1.9$ & $\$ 4.2$ & $\$ 6.1$ & $\$ 3.0$ & $\$ 8.5$ & $\$ 11.8$ & $\$ 27.2$ & $\$ 37.5$ \\
\hline
\end{tabular}


Table 6-6 Test and Validation Facility Annual Operations and Maintenance Costs

\begin{tabular}{|c|c|c|c|c|c|c|c|c|c|c|c|}
\hline \multirow[b]{2}{*}{ Scenario } & \multirow[b]{2}{*}{$\begin{array}{c}\text { Acceptance } \\
\text { Rate }\end{array}$} & \multirow[b]{2}{*}{ CSF Start } & \multirow[b]{2}{*}{$\begin{array}{l}\text { Repository } \\
\text { Start }\end{array}$} & \multirow[b]{2}{*}{$\begin{array}{c}\text { Disposal } \\
\text { Canister } \\
\text { Size }\end{array}$} & \multicolumn{3}{|c|}{ Total Staffing } & \multicolumn{4}{|c|}{ Annual Operations and Maintenance } \\
\hline & & & & & Management & Exempt & Hourly & $\begin{array}{c}\text { Labor } \\
\text { FY } 2012 \text { (\$M) }\end{array}$ & $\begin{array}{c}\text { Utilities } \\
\text { FY } 2012 \text { (\$M) }\end{array}$ & $\begin{array}{l}\text { Materials and } \\
\text { Contracts } \\
\text { FY } 2012 \text { (\$M) }\end{array}$ & $\begin{array}{l}\text { Base Total Annual } \\
\text { FY } 2012 \text { (\$M) }\end{array}$ \\
\hline \multirow{9}{*}{$\begin{array}{c}\text { Canisters } \\
\text { Only - } \\
\text { Re-Package } \\
\text { at Repository } \\
\text { (Case 1) }\end{array}$} & 1,500 & 2020 & 2040 & 4 & 7 & 59 & 58 & $\$ 18.9$ & $\$ 0.2$ & $\$ 2.0$ & $\$ 21.1$ \\
\hline & 3,000 & 2020 & 2040 & 4 & 7 & 59 & 58 & $\$ 18.9$ & $\$ 0.2$ & $\$ 2.0$ & $\$ 21.1$ \\
\hline & 3,000 & 2020 & 2040 & 12 & 7 & 59 & 58 & $\$ 18.9$ & $\$ 0.2$ & $\$ 2.0$ & $\$ 21.1$ \\
\hline & 3,000 & 2020 & 2040 & 21 & 7 & 59 & 58 & $\$ 18.9$ & $\$ 0.2$ & $\$ 2.0$ & $\$ 21.1$ \\
\hline & 6,000 & 2020 & 2040 & 4 & 7 & 59 & 58 & $\$ 18.9$ & $\$ 0.2$ & $\$ 2.0$ & $\$ 21.1$ \\
\hline & 1,500 & 2020 & 2055 & 4 & 7 & 59 & 58 & $\$ 18.9$ & $\$ 0.2$ & $\$ 2.0$ & $\$ 21.1$ \\
\hline & 3,000 & 2020 & 2055 & 4 & 7 & 59 & 58 & $\$ 18.9$ & $\$ 0.2$ & $\$ 2.0$ & $\$ 21.1$ \\
\hline & 6,000 & 2020 & 2055 & 4 & 7 & 59 & 58 & $\$ 18.9$ & $\$ 0.2$ & $\$ 2.0$ & $\$ 21.1$ \\
\hline & 3,000 & 2035 & 2055 & 4 & 7 & 59 & 58 & $\$ 18.9$ & $\$ 0.2$ & $\$ 2.0$ & $\$ 21.1$ \\
\hline \multirow{9}{*}{$\begin{array}{c}\text { Canisters and } \\
\text { Bare Fuel - } \\
\text { Re-Package } \\
\text { at Repository } \\
\text { (Case 2) }\end{array}$} & 1,500 & 2020 & 2040 & 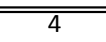 & 7 & $\overline{59}$ & 258 & $\$ 18.9$ & $\$ 0.2$ & $\$ 2.0$ & $\$ 21.1$ \\
\hline & 3,000 & 2020 & 2040 & 4 & 7 & 59 & 58 & $\$ 18.9$ & $\$ 0.2$ & $\$ 2.0$ & $\$ 21.1$ \\
\hline & 3,000 & 2020 & 2040 & 12 & 7 & 59 & 58 & $\$ 18.9$ & $\$ 0.2$ & $\$ 2.0$ & $\$ 21.1$ \\
\hline & 3,000 & 2020 & 2040 & 21 & 7 & 59 & 58 & $\$ 18.9$ & $\$ 0.2$ & $\$ 2.0$ & $\$ 21.1$ \\
\hline & 6,000 & 2020 & 2040 & 4 & 7 & 59 & 58 & $\$ 18.9$ & $\$ 0.2$ & $\$ 2.0$ & $\$ 21.1$ \\
\hline & 1,500 & 2020 & 2055 & 4 & 7 & 59 & 58 & $\$ 18.9$ & $\$ 0.2$ & $\$ 2.0$ & $\$ 21.1$ \\
\hline & 3,000 & 2020 & 2055 & 4 & 7 & 59 & 58 & $\$ 18.9$ & $\$ 0.2$ & $\$ 2.0$ & $\$ 21.1$ \\
\hline & 6,000 & 2020 & 2055 & 4 & 7 & 59 & 58 & $\$ 18.9$ & $\$ 0.2$ & $\$ 2.0$ & $\$ 21.1$ \\
\hline & 3,000 & 2035 & 2055 & 4 & 7 & 59 & 58 & $\$ 18.9$ & $\$ 0.2$ & $\$ 2.0$ & $\$ 21.1$ \\
\hline \multirow{9}{*}{$\begin{array}{c}\text { Canisters } \\
\text { Only - Re- } \\
\text { Package at } \\
\text { CSF } \\
\text { (Case 3) }\end{array}$} & $\begin{array}{l}1,500 \\
\end{array}$ & 2020 & 2040 & 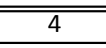 & 7 & 59 & 58 & $\begin{array}{l}18.9 \\
\end{array}$ & $\$ 0.2$ & $\$ 2.0$ & $\$ 21.1$ \\
\hline & 3,000 & 2020 & 2040 & 4 & 7 & 59 & 58 & $\$ 18.9$ & $\$ 0.2$ & $\$ 2.0$ & $\$ 21.1$ \\
\hline & 3,000 & 2020 & 2040 & 12 & 7 & 59 & 58 & $\$ 18.9$ & $\$ 0.2$ & $\$ 2.0$ & $\$ 21.1$ \\
\hline & 3,000 & 2020 & 2040 & 21 & 7 & 59 & 58 & $\$ 18.9$ & $\$ 0.2$ & $\$ 2.0$ & $\$ 21.1$ \\
\hline & 6,000 & 2020 & 2040 & 4 & 7 & 59 & 58 & $\$ 18.9$ & $\$ 0.2$ & $\$ 2.0$ & $\$ 21.1$ \\
\hline & 1,500 & 2020 & 2055 & 4 & 7 & 59 & 58 & $\$ 18.9$ & $\$ 0.2$ & $\$ 2.0$ & $\$ 21.1$ \\
\hline & 3,000 & 2020 & 2055 & 4 & 7 & 59 & 58 & $\$ 18.9$ & $\$ 0.2$ & $\$ 2.0$ & $\$ 21.1$ \\
\hline & 6,000 & 2020 & 2055 & 4 & 7 & 59 & 58 & $\$ 18.9$ & $\$ 0.2$ & $\$ 2.0$ & $\$ 21.1$ \\
\hline & 3,000 & 2035 & 2055 & 4 & 7 & 59 & 58 & $\$ 18.9$ & $\$ 0.2$ & $\$ 2.0$ & $\$ 21.1$ \\
\hline \multirow{9}{*}{$\begin{array}{c}\text { Canisters and } \\
\text { Bare Fuel - } \\
\text { Re-Package } \\
\text { at CSF } \\
\text { (Case 4) }\end{array}$} & 1,500 & 2020 & 2040 & 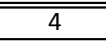 & 7 & 59 & 58 & "\$18.9 & $\$ 0.2$ & $\$ 2.0$ & $\$ 21.1$ \\
\hline & 3,000 & 2020 & 2040 & 4 & 7 & 59 & 58 & $\$ 18.9$ & $\$ 0.2$ & $\$ 2.0$ & $\$ 21.1$ \\
\hline & 3,000 & 2020 & 2040 & 12 & 7 & 59 & 58 & $\$ 18.9$ & $\$ 0.2$ & $\$ 2.0$ & $\$ 21.1$ \\
\hline & 3,000 & 2020 & 2040 & 21 & 7 & 59 & 58 & $\$ 18.9$ & $\$ 0.2$ & $\$ 2.0$ & $\$ 21.1$ \\
\hline & 6,000 & 2020 & 2040 & 4 & 7 & 59 & 58 & $\$ 18.9$ & $\$ 0.2$ & $\$ 2.0$ & $\$ 21.1$ \\
\hline & 1,500 & 2020 & 2055 & 4 & 7 & 59 & 58 & $\$ 18.9$ & $\$ 0.2$ & $\$ 2.0$ & $\$ 21.1$ \\
\hline & 3,000 & 2020 & 2055 & 4 & 7 & 59 & 58 & $\$ 18.9$ & $\$ 0.2$ & $\$ 2.0$ & $\$ 21.1$ \\
\hline & 6,000 & 2020 & 2055 & 4 & 7 & 59 & 58 & $\$ 18.9$ & $\$ 0.2$ & $\$ 2.0$ & $\$ 21.1$ \\
\hline & 3,000 & 2035 & 2055 & 4 & 7 & 59 & 58 & $\$ 18.9$ & $\$ 0.2$ & $\$ 2.0$ & $\$ 21.1$ \\
\hline
\end{tabular}


Used Fuel Management System Architecture Evaluation, Fiscal Year 2012

October, 2012

Table 6-7 Test and Validation Facility Life Cycle Cost

\begin{tabular}{|c|c|c|c|c|c|c|c|c|c|c|c|c|}
\hline & & & & & & & Life Cycle O\&M & & & & & \\
\hline Scenario & $\begin{array}{c}\text { Acceptance } \\
\text { Rate }\end{array}$ & CSF Start & $\begin{array}{c}\text { Repository } \\
\text { Start }\end{array}$ & $\begin{array}{c}\text { Disposal } \\
\text { Canister } \\
\text { Size }\end{array}$ & $\begin{array}{l}\text { Labor } \\
\text { FY } 2012 \text { (\$B) }\end{array}$ & $\begin{array}{l}\text { Utilities } \\
\text { FY } 2012 \text { (\$B) }\end{array}$ & $\begin{array}{l}\text { Materials and } \\
\text { Contracts } \\
\text { FY } 2012(\$ B)\end{array}$ & $\begin{array}{c}\text { D\&D } \\
\text { FY } 2012 \text { (\$B) }\end{array}$ & $\begin{array}{c}\text { Total } \\
\text { FY } 2012 \text { (\$B) }\end{array}$ & $\begin{array}{c}\text { TPC } \\
\text { FY } 2012 \text { (\$B) }\end{array}$ & $\begin{array}{l}\text { Total Life Cycle } \\
\text { FY } 2012 \text { (\$B) }\end{array}$ & $\begin{array}{l}\text { Relative to CSF } \\
\text { LCC Cost }\end{array}$ \\
\hline & 1,500 & 2020 & 2040 & 4 & $\$ 2.2$ & $\$ 0.0$ & $\$ 0.2$ & $\$ 0.1$ & $\$ 2.6$ & $\$ 1.8$ & $\$ 4.4$ & $59 \%$ \\
\hline & 3,000 & 2020 & 2040 & 4 & $\$ 1.3$ & $\$ 0.0$ & $\$ 0.1$ & $\$ 0.1$ & $\$ 1.6$ & $\$ 1.8$ & $\$ 3.4$ & $30 \%$ \\
\hline Canisters & 3,000 & 2020 & 2040 & 12 & $\$ 1.3$ & $\$ 0.0$ & $\$ 0.1$ & $\$ 0.1$ & $\$ 1.6$ & $\$ 1.8$ & $\$ 3.4$ & $30 \%$ \\
\hline Only - & 3,000 & 2020 & 2040 & 21 & $\$ 1.3$ & $\$ 0.0$ & $\$ 0.1$ & $\$ 0.1$ & $\$ 1.6$ & $\$ 1.8$ & $\$ 3.4$ & $30 \%$ \\
\hline Re-Package & 6,000 & 2020 & 2040 & 4 & $\$ 0.9$ & $\$ 0.0$ & $\$ 0.1$ & $\$ 0.1$ & $\$ 1.1$ & $\$ 1.8$ & $\$ 2.9$ & $17 \%$ \\
\hline at Repository & 1,500 & 2020 & 2055 & 4 & $\$ 2.5$ & $\$ 0.0$ & $\$ 0.3$ & $\$ 0.1$ & $\$ 2.9$ & $\$ 1.8$ & $\$ 4.7$ & $40 \%$ \\
\hline (Case 1) & 3,000 & 2020 & 2055 & 4 & $\$ 1.6$ & $\$ 0.0$ & $\$ 0.2$ & $\$ 0.1$ & $\$ 1.9$ & $\$ 1.8$ & $\$ 3.7$ & $18 \%$ \\
\hline & 6,000 & 2020 & 2055 & 4 & $\$ 1.2$ & $\$ 0.0$ & $\$ 0.1$ & $\$ 0.1$ & $\$ 1.4$ & $\$ 1.8$ & $\$ 3.3$ & $13 \%$ \\
\hline & 3,000 & 2035 & 2055 & 4 & $\$ 1.3$ & $\$ 0.0$ & $\$ 0.1$ & $\$ 0.1$ & $\$ 1.6$ & $\$ 1.8$ & $\$ 3.4$ & $27 \%$ \\
\hline & $\begin{array}{ll}1,500 \\
\end{array}$ & 2020 & 2040 & 4 & $\$ 2.2$ & $\$ 0.0$ & $\$ \$ 0.2$ & $\begin{array}{l}0.1 \\
\end{array}$ & $\$ 2.6$ & $\$ 1.8$ & $\$ 44.4$ & $20 \%$ \\
\hline & 3,000 & 2020 & 2040 & 4 & $\$ 1.3$ & $\$ 0.0$ & $\$ 0.1$ & $\$ 0.1$ & $\$ 1.6$ & $\$ 1.8$ & $\$ 3.4$ & $9 \%$ \\
\hline Canisters and & 3,000 & 2020 & 2040 & 12 & $\$ 1.3$ & $\$ 0.0$ & $\$ 0.1$ & $\$ 0.1$ & $\$ 1.6$ & $\$ 1.8$ & $\$ 3.4$ & $9 \%$ \\
\hline Bare Fuel - & 3,000 & 2020 & 2040 & 21 & $\$ 1.3$ & $\$ 0.0$ & $\$ 0.1$ & $\$ 0.1$ & $\$ 1.6$ & $\$ 1.8$ & $\$ 3.4$ & $9 \%$ \\
\hline Re-Package & 6,000 & 2020 & 2040 & 4 & $\$ 0.9$ & $\$ 0.0$ & $\$ 0.1$ & $\$ 0.1$ & $\$ 1.1$ & $\$ 1.8$ & $\$ 2.9$ & $4 \%$ \\
\hline at Repository & 1,500 & 2020 & 2055 & 4 & $\$ 2.5$ & $\$ 0.0$ & $\$ 0.3$ & $\$ 0.1$ & $\$ 2.9$ & $\$ 1.8$ & $\$ 4.7$ & $15 \%$ \\
\hline (Case 2) & 3,000 & 2020 & 2055 & 4 & $\$ 1.6$ & $\$ 0.0$ & $\$ 0.2$ & $\$ 0.1$ & $\$ 1.9$ & $\$ 1.8$ & $\$ 3.7$ & $6 \%$ \\
\hline & 6,000 & 2020 & 2055 & 4 & $\$ 1.2$ & $\$ 0.0$ & $\$ 0.1$ & $\$ 0.1$ & $\$ 1.4$ & $\$ 1.8$ & $\$ 3.2$ & $4 \%$ \\
\hline & 3,000 & 2035 & 2055 & 4 & $\$ 1.3$ & $\$ 0.0$ & $\$ 0.1$ & $\$ 0.1$ & $\$ 1.6$ & $\$ 1.8$ & $\$ 3.4$ & $11 \%$ \\
\hline & 1,500 & 2020 & 2040 & 4 & $\$ 2.2$ & $\$ 0.0$ & $\$ \$ \$ 0.2$ & $\$ 0.1$ & $\$ 2.6$ & $\begin{array}{l}1.8 \\
\end{array}$ & $\begin{array}{l}4.4 \\
\end{array}$ & $43 \%$ \\
\hline & 3,000 & 2020 & 2040 & 4 & $\$ 1.3$ & $\$ 0.0$ & $\$ 0.1$ & $\$ 0.1$ & $\$ 1.6$ & $\$ 1.8$ & $\$ 3.4$ & $23 \%$ \\
\hline Canisters & 3,000 & 2020 & 2040 & 12 & $\$ 1.3$ & $\$ 0.0$ & $\$ 0.1$ & $\$ 0.1$ & $\$ 1.6$ & $\$ 1.8$ & $\$ 3.4$ & $23 \%$ \\
\hline Only - Re- & 3,000 & 2020 & 2040 & 21 & $\$ 1.3$ & $\$ 0.0$ & $\$ 0.1$ & $\$ 0.1$ & $\$ 1.6$ & $\$ 1.8$ & $\$ 3.4$ & $23 \%$ \\
\hline Package at & 6,000 & 2020 & 2040 & 4 & $\$ 0.9$ & $\$ 0.0$ & $\$ 0.1$ & $\$ 0.1$ & $\$ 1.1$ & $\$ 1.8$ & $\$ 2.9$ & $16 \%$ \\
\hline CSF & 1,500 & 2020 & 2055 & 4 & $\$ 2.5$ & $\$ 0.0$ & $\$ 0.3$ & $\$ 0.1$ & $\$ 2.9$ & $\$ 1.8$ & $\$ 4.7$ & $30 \%$ \\
\hline (Case 3) & 3,000 & 2020 & 2055 & 4 & $\$ 1.6$ & $\$ 0.0$ & $\$ 0.2$ & $\$ 0.1$ & $\$ 1.9$ & $\$ 1.8$ & $\$ 3.7$ & $16 \%$ \\
\hline & 6,000 & 2020 & 2055 & 4 & $\$ 1.2$ & $\$ 0.0$ & $\$ 0.1$ & $\$ 0.1$ & $\$ 1.4$ & $\$ 1.8$ & $\$ 3.3$ & $13 \%$ \\
\hline & 3,000 & 2035 & 2055 & 4 & $\$ 1.3$ & $\$ 0.0$ & $\$ 0.1$ & $\$ 0.1$ & $\$ 1.6$ & $\$ 1.8$ & $\$ 3.4$ & $22 \%$ \\
\hline & 1,500 & 2020 & 2040 & 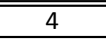 & $\$ 2.2$ & $\$ \$ 0.0$ & $\$ 0.2$ & \$\$0.1 & $\$ 2.6$ & \$1.8 & $\$ \$ \$ 4.4$ & $15 \%$ \\
\hline & 3,000 & 2020 & 2040 & 4 & $\$ 1.3$ & $\$ 0.0$ & $\$ 0.1$ & $\$ 0.1$ & $\$ 1.6$ & $\$ 1.8$ & $\$ 3.4$ & $7 \%$ \\
\hline Canisters and & 3,000 & 2020 & 2040 & 12 & $\$ 1.3$ & $\$ 0.0$ & $\$ 0.1$ & $\$ 0.1$ & $\$ 1.6$ & $\$ 1.8$ & $\$ 3.4$ & $7 \%$ \\
\hline Bare Fuel - & 3,000 & 2020 & 2040 & 21 & $\$ 1.3$ & $\$ 0.0$ & $\$ 0.1$ & $\$ 0.1$ & $\$ 1.6$ & $\$ 1.8$ & $\$ 3.4$ & $7 \%$ \\
\hline Re-Package & 6,000 & 2020 & 2040 & 4 & $\$ 0.9$ & $\$ 0.0$ & $\$ 0.1$ & $\$ 0.1$ & $\$ 1.1$ & $\$ 1.8$ & $\$ 2.9$ & $4 \%$ \\
\hline at CSF & 1,500 & 2020 & 2055 & 4 & $\$ 2.5$ & $\$ 0.0$ & $\$ 0.2$ & $\$ 0.1$ & $\$ 2.8$ & $\$ 1.8$ & $\$ 4.7$ & $12 \%$ \\
\hline & 3,000 & 2020 & 2055 & 4 & $\$ 1.6$ & $\$ 0.0$ & $\$ 0.1$ & $\$ 0.1$ & $\$ 1.9$ & $\$ 1.8$ & $\$ 3.7$ & $6 \%$ \\
\hline & 6,000 & 2020 & 2055 & 4 & $\$ 1.2$ & $\$ 0.0$ & $\$ 0.1$ & $\$ 0.1$ & $\$ 1.4$ & $\$ 1.8$ & $\$ 3.2$ & $4 \%$ \\
\hline & 3,000 & 2035 & 2055 & 4 & $\$ 1.3$ & $\$ 0.0$ & $\$ 0.1$ & $\$ 0.1$ & $\$ 1.6$ & $\$ 1.8$ & $\$ 3.4$ & $9 \%$ \\
\hline
\end{tabular}


Table 6-8 Comparison of Horizontal Modules and Vertical Cask Storage to a Large Vault for Dry Storage Canisters

\begin{tabular}{|c|c|c|c|c|c|c|}
\hline & & & & & $\begin{array}{l}\text { Large Dry } \\
\text { Storage Vault }\end{array}$ & $\begin{array}{l}\text { Horizontal Dry } \\
\text { Storage }\end{array}$ \\
\hline Scenario & $\begin{array}{c}\text { Acceptance } \\
\text { Rate }\end{array}$ & CSF Start & $\begin{array}{c}\text { Repository } \\
\text { Start }\end{array}$ & $\begin{array}{c}\text { Disposal } \\
\text { Canister } \\
\text { Size }\end{array}$ & FY 2012 (\$B) & FY 2012 (\$B) \\
\hline \multirow{9}{*}{$\begin{array}{c}\text { Canisters } \\
\text { Only - } \\
\text { Re-Package } \\
\text { at Repository }\end{array}$} & 1,500 & 2020 & 2040 & 4 & $\$ 10.8$ & $\$ 4.2$ \\
\hline & 3,000 & 2020 & 2040 & 4 & $\$ 20.3$ & $\$ 9.0$ \\
\hline & 3,000 & 2020 & 2040 & 12 & $\$ 20.3$ & $\$ 9.0$ \\
\hline & 3,000 & 2020 & 2040 & 21 & $\$ 20.3$ & $\$ 9.0$ \\
\hline & 6,000 & 2020 & 2040 & 4 & $\$ 28.7$ & $\$ 14.8$ \\
\hline & 1,500 & 2020 & 2055 & 4 & $\$ 17.9$ & $\$ 8.2$ \\
\hline & 3,000 & 2020 & 2055 & 4 & $\$ 34.7$ & $\$ 17.5$ \\
\hline & 6,000 & 2020 & 2055 & 4 & $\$ 43.1$ & $\$ 22.3$ \\
\hline & 3,000 & 2035 & 2055 & 4 & $\$ 20.3$ & $\$ 10.6$ \\
\hline \multirow{9}{*}{$\begin{array}{l}\text { Canisters and } \\
\text { Bare Fuel - } \\
\text { Re-Package } \\
\text { at Repository }\end{array}$} & 1,500 & 2020 & 2040 & 4 & $\$ 1.2$ & $\$ 0.5$ \\
\hline & 3,000 & 2020 & 2040 & 4 & $\$ 2.4$ & $\$ 0.9$ \\
\hline & 3,000 & 2020 & 2040 & 12 & $\$ 2.4$ & $\$ 0.9$ \\
\hline & 3,000 & 2020 & 2040 & 21 & $\$ 2.4$ & $\$ 0.9$ \\
\hline & 6,000 & 2020 & 2040 & 4 & $\$ 12.0$ & $\$ 7.5$ \\
\hline & 1,500 & 2020 & 2055 & 4 & $\$ 6.0$ & $\$ 3.2$ \\
\hline & 3,000 & 2020 & 2055 & 4 & $\$ 10.8$ & $\$ 6.6$ \\
\hline & 6,000 & 2020 & 2055 & 4 & $\$ 12.0$ & $\$ 7.7$ \\
\hline & 3,000 & 2035 & 2055 & 4 & $\$ 9.6$ & $\$ 6.2$ \\
\hline \multirow{9}{*}{$\begin{array}{c}\text { Canisters } \\
\text { Only - Re- } \\
\text { Package at } \\
\text { CSF }\end{array}$} & 1,500 & 2020 & 2040 & 4 & $\$ 13.2$ & $\$ 7.1$ \\
\hline & 3,000 & 2020 & 2040 & 4 & $\$ 22.7$ & $\$ 12.5$ \\
\hline & 3,000 & 2020 & 2040 & 12 & $\$ 22.7$ & $\$ 12.5$ \\
\hline & 3,000 & 2020 & 2040 & 21 & $\$ 22.7$ & $\$ 12.5$ \\
\hline & 6,000 & 2020 & 2040 & 4 & $\$ 28.7$ & $\$ 15.6$ \\
\hline & 1,500 & 2020 & 2055 & 4 & $\$ 20.3$ & $\$ 12.2$ \\
\hline & 3,000 & 2020 & 2055 & 4 & $\$ 35.9$ & \$19.7 \\
\hline & 6,000 & 2020 & 2055 & 4 & $\$ 43.1$ & $\$ 22.6$ \\
\hline & 3,000 & 2035 & 2055 & 4 & $\$ 22.7$ & $\$ 13.5$ \\
\hline \multirow{9}{*}{$\begin{array}{c}\text { Canisters and } \\
\text { Bare Fuel - } \\
\text { Re-Package } \\
\text { at CSF }\end{array}$} & 1,500 & 2020 & 2040 & 4 & $\begin{array}{l}\$ 10.8 \\
\end{array}$ & $\$ 6.7$ \\
\hline & 3,000 & 2020 & 2040 & 4 & $\$ 16.7$ & $\$ 10.6$ \\
\hline & 3,000 & 2020 & 2040 & 12 & $\$ 16.7$ & $\$ 10.6$ \\
\hline & 3,000 & 2020 & 2040 & 21 & $\$ 16.7$ & $\$ 10.6$ \\
\hline & 6,000 & 2020 & 2040 & 4 & $\$ 12.0$ & $\$ 7.5$ \\
\hline & 1,500 & 2020 & 2055 & 4 & $\$ 17.9$ & $\$ 11.6$ \\
\hline & 3,000 & 2020 & 2055 & 4 & $\$ 20.3$ & $\$ 12.7$ \\
\hline & 6,000 & 2020 & 2055 & 4 & $\$ 12.0$ & $\$ 7.7$ \\
\hline & 3,000 & 2035 & 2055 & 4 & $\$ 20.3$ & $\$ 13.2$ \\
\hline
\end{tabular}




\subsection{Packaging/Re-Packaging Facility Rough Order of Magnitude Cost Estimates}

Previously developed unit costs for fuel handling, presented in Appendix C, were used as the basis for estimating the rough order of magnitude costs for the packaging/re-packaging facility configurations (Rodwell et al. 2007, McConnell et al. 2007, DOE 2008, Carter et al 2012). These costs are then applied via the methodology described in Appendix $\mathrm{C}$ to the packaging/re-packaging facility requirements presented in Section 4.

The resulting packaging/re-packaging facility annual operations and maintenance costs are summarized in Table 6-9. As expected, the annual costs vary with fuel acceptance rate and the waste package disposal canister size, which is directly related to the processing repackaging throughput. There are small variations driven by small differences in the characteristics of the UNF received at the CSF (canisters only or canisters and bare fuel).

Table 6-10 extends the annual cost to the total life cycle. The annual cost for labor, utilities, materials \& contracts and D\&D are extended as point estimates, based on the scenario schedule (see below). The D\&D cost is derived by algorithm (Appendix C) based TPC and additional capital (see below).

The O\&M life-cycle costs (LCC) vary due to differences in the operations schedules for the different cases. The packaging/re-packaging facility is expected to be in operation from the start date of repository operations (Table 6-10) until the repository emplacement is finished (23, 47 or 97 years to dispose of 140,000 MTHM of UNF, depending upon the acceptance rate). The scenario schedule ranges from 43 to 120 years and is the largest driver in the variation of the O\&M LCC.

The variation in TPC reflects the differences in functional requirements for each scenario, presented in Section 5. The packaging/re-packaging facility complex is large and will likely be split into multiple buildings to obtain the desired acceptance rate, especially if the $6,000 \mathrm{MT} / \mathrm{yr}$ processing rate were selected. For this LCC study, all packaging/re-packaging facility costs are included in TPC and no additional capital is assumed to be required.

Locating the packaging/re-packaging facility at the repository has a lower LCC compared to the same scenario when the facility is located at the CSF. This difference is mostly due to the lower capital cost associated with reduced shipping bay facility requirements since a Department of Transportation shipping overpack would not be required when the waste package is transferred directly to the repository from the packaging/re-packaging facility for emplacement. 
Table 6-9 Repackaging Facility Annual Operations and Maintenance Costs

\begin{tabular}{|c|c|c|c|c|c|c|c|c|c|c|c|}
\hline \multirow[b]{2}{*}{ Scenario } & \multirow[b]{2}{*}{$\begin{array}{c}\text { Acceptance } \\
\text { Rate }\end{array}$} & \multirow[b]{2}{*}{ CSF Start } & \multirow[b]{2}{*}{$\begin{array}{l}\text { Repository } \\
\text { Start }\end{array}$} & \multirow[b]{2}{*}{$\begin{array}{l}\text { Disposal } \\
\text { Canister } \\
\text { Size }\end{array}$} & \multicolumn{3}{|c|}{ Total Staffing } & \multicolumn{4}{|c|}{ Annual Operations and Maintenance } \\
\hline & & & & & Management & Exempt & Hourly & $\begin{array}{c}\text { Labor } \\
\text { FY } 2012 \text { (\$M) }\end{array}$ & $\begin{array}{c}\text { Utilities } \\
\text { FY } 2012 \text { (\$M) }\end{array}$ & $\begin{array}{l}\text { Materials and } \\
\text { Contracts } \\
\text { FY } 2012 \text { (\$M) }\end{array}$ & $\begin{array}{l}\text { Base Total Annual } \\
\text { FY } 2012 \text { (\$M) }\end{array}$ \\
\hline \multirow{9}{*}{$\begin{array}{c}\text { Canisters } \\
\text { Only - } \\
\text { Re-Package } \\
\text { at Repository } \\
\text { (Case 1) }\end{array}$} & 1,500 & 2020 & 2040 & 4 & 15 & 70 & 118 & $\$ 28.9$ & $\$ 1.5$ & $\$ 61.8$ & $\$ 92.2$ \\
\hline & 3,000 & 2020 & 2040 & 4 & 23 & 114 & 194 & $\$ 46.9$ & $\$ 3.0$ & $\$ 125.9$ & $\$ 175.8$ \\
\hline & 3,000 & 2020 & 2040 & 12 & 13 & 57 & 97 & $\$ 23.8$ & $\$ 1.1$ & $\$ 71.5$ & $\$ 96.3$ \\
\hline & 3,000 & 2020 & 2040 & 21 & 10 & 45 & 75 & $\$ 18.7$ & $\$ 0.6$ & $\$ 55.6$ & $\$ 74.9$ \\
\hline & 6,000 & 2020 & 2040 & 4 & 39 & 201 & 344 & $\$ 82.9$ & $\$ 6.0$ & $\$ 250.1$ & $\$ 338.9$ \\
\hline & 1,500 & 2020 & 2055 & 4 & 15 & 70 & 118 & $\$ 28.9$ & $\$ 1.5$ & $\$ 61.8$ & $\$ 92.2$ \\
\hline & 3,000 & 2020 & 2055 & 4 & 23 & 114 & 194 & $\$ 46.9$ & $\$ 3.0$ & $\$ 125.9$ & $\$ 175.8$ \\
\hline & 6,000 & 2020 & 2055 & 4 & 41 & 214 & 366 & $\$ 88.0$ & $\$ 6.4$ & $\$ 251.2$ & $\$ 345.6$ \\
\hline & 3,000 & 2035 & 2055 & 4 & 22 & 107 & 183 & $\$ 44.3$ & $\$ 2.8$ & $\$ 121.7$ & $\$ 168.8$ \\
\hline \multirow{9}{*}{$\begin{array}{c}\text { Canisters and } \\
\text { Bare Fuel - } \\
\text { Re-Package } \\
\text { at Repository } \\
\text { (Case 2) }\end{array}$} & 1,500 & 2020 & 2040 & 4 & 16 & 76 & 129 & $\$ 28.9$ & $\$ 1.5$ & $\$ 61.7$ & $\$ 92.1$ \\
\hline & 3,000 & 2020 & 2040 & 4 & 22 & 107 & 183 & $\$ 44.3$ & $\$ 2.8$ & $\$ 121.3$ & $\$ 168.4$ \\
\hline & 3,000 & 2020 & 2040 & 12 & 13 & 57 & 97 & $\$ 23.8$ & $\$ 1.1$ & $\$ 67.3$ & $\$ 92.2$ \\
\hline & 3,000 & 2020 & 2040 & 21 & 10 & 45 & 75 & $\$ 18.7$ & $\$ 0.6$ & $\$ 51.5$ & $\$ 70.8$ \\
\hline & 6,000 & 2020 & 2040 & 4 & 36 & 189 & 323 & $\$ 77.7$ & $\$ 5.5$ & $\$ 235.9$ & $\$ 319.2$ \\
\hline & 1,500 & 2020 & 2055 & 4 & 15 & 70 & 118 & $\$ 28.9$ & $\$ 1.5$ & $\$ 61.7$ & $\$ 92.1$ \\
\hline & 3,000 & 2020 & 2055 & 4 & 22 & 107 & 183 & $\$ 44.3$ & $\$ 2.8$ & $\$ 121.3$ & $\$ 168.4$ \\
\hline & 6,000 & 2020 & 2055 & 4 & 34 & 176 & 301 & $\$ 72.6$ & $\$ 5.1$ & $\$ 235.3$ & $\$ 313.0$ \\
\hline & 3,000 & 2035 & 2055 & 4 & 22 & 107 & 183 & $\$ 44.3$ & $\$ 2.8$ & $\$ 121.5$ & $\$ 168.6$ \\
\hline \multirow{9}{*}{$\begin{array}{c}\text { Canisters } \\
\text { Only - Re- } \\
\text { Package at } \\
\text { CSF } \\
\text { (Case 3) }\end{array}$} & 1,500 & 2020 & 2040 & 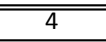 & 16 & 76 & 129 & $\$ 31.5$ & $\$ 1.7$ & $\$ 62.7$ & $\$ 95.9$ \\
\hline & 3,000 & 2020 & 2040 & 4 & 22 & 107 & 183 & $\$ 44.3$ & $\$ 2.8$ & $\$ 122.2$ & $\$ 169.3$ \\
\hline & 3,000 & 2020 & 2040 & 12 & 13 & 57 & 97 & $\$ 23.8$ & $\$ 1.1$ & $\$ 68.2$ & $\$ 93.1$ \\
\hline & 3,000 & 2020 & 2040 & 21 & 10 & 45 & 75 & $\$ 18.7$ & $\$ 0.6$ & $\$ 52.4$ & $\$ 71.7$ \\
\hline & 6,000 & 2020 & 2040 & 4 & 40 & 207 & 355 & $\$ 85.4$ & $\$ 6.2$ & $\$ 250.1$ & $\$ 341.7$ \\
\hline & 1,500 & 2020 & 2055 & 4 & 16 & 76 & 129 & $\$ 31.5$ & $\$ 1.7$ & $\$ 62.7$ & $\$ 95.9$ \\
\hline & 3,000 & 2020 & 2055 & 4 & 22 & 107 & 183 & $\$ 44.3$ & $\$ 2.8$ & $\$ 122.2$ & $\$ 169.3$ \\
\hline & 6,000 & 2020 & 2055 & 4 & 40 & 207 & 355 & $\$ 85.4$ & $\$ 6.2$ & $\$ 250.1$ & $\$ 341.7$ \\
\hline & 3,000 & 2035 & 2055 & 4 & 22 & 107 & 183 & $\$ 44.3$ & $\$ 2.8$ & $\$ 122.0$ & \$169.1 \\
\hline \multirow{9}{*}{$\begin{array}{c}\text { Canisters and } \\
\text { Bare Fuel - } \\
\text { Re-Package } \\
\text { at CSF } \\
\text { (Case 4) }\end{array}$} & 1,500 & 2020 & 2040 & 4 & 15 & 70 & 118 & $\$ 28.9$ & \$1.5 & "\$61.6 & $\$ \$ 92.0$ \\
\hline & 3,000 & 2020 & 2040 & 4 & 22 & 107 & 183 & $\$ 44.3$ & $\$ 2.8$ & $\$ 121.3$ & $\$ 168.5$ \\
\hline & 3,000 & 2020 & 2040 & 12 & 13 & 57 & 97 & $\$ 23.8$ & $\$ 1.1$ & $\$ 67.4$ & $\$ 92.3$ \\
\hline & 3,000 & 2020 & 2040 & 21 & 10 & 45 & 75 & $\$ 18.7$ & $\$ 0.6$ & $\$ 51.6$ & $\$ 70.9$ \\
\hline & 6,000 & 2020 & 2040 & 4 & 35 & 182 & 312 & $\$ 75.1$ & $\$ 5.3$ & $\$ 235.8$ & $\$ 316.3$ \\
\hline & 1,500 & 2020 & 2055 & 4 & 15 & 70 & 118 & $\$ 28.9$ & $\$ 1.5$ & $\$ 61.6$ & $\$ 92.1$ \\
\hline & 3,000 & 2020 & 2055 & 4 & 22 & 107 & 183 & $\$ 44.3$ & $\$ 2.8$ & $\$ 121.3$ & $\$ 168.5$ \\
\hline & 6,000 & 2020 & 2055 & 4 & 35 & 182 & 312 & $\$ 75.1$ & $\$ 5.3$ & $\$ 235.8$ & $\$ 316.3$ \\
\hline & 3,000 & 2035 & 2055 & 4 & 22 & 107 & 183 & $\$ 44.3$ & $\$ 2.8$ & $\$ 121.4$ & $\$ 168.5$ \\
\hline
\end{tabular}


Table 6-10 Repackaging Facility Life Cycle Costs

\begin{tabular}{|c|c|c|c|c|c|c|c|c|c|c|c|}
\hline \multirow[b]{2}{*}{ Scenario } & \multirow[b]{2}{*}{\begin{tabular}{|c}
$\begin{array}{c}\text { Acceptance } \\
\text { Rate }\end{array}$ \\
\end{tabular}} & \multirow[b]{2}{*}{ CSF Start } & \multirow[b]{2}{*}{$\begin{array}{l}\text { Repository } \\
\text { Start }\end{array}$} & \multirow[b]{2}{*}{$\begin{array}{c}\text { Disposal } \\
\text { Canister } \\
\text { Size }\end{array}$} & \multicolumn{5}{|c|}{ Life Cycle O\&M } & \multirow[b]{2}{*}{$\begin{array}{c}\text { TPC } \\
\text { FY } 2012 \text { (\$ B) }\end{array}$} & \multirow[b]{2}{*}{$\begin{array}{l}\text { Total Life Cycle } \\
\text { FY } 2012 \text { (\$ B) }\end{array}$} \\
\hline & & & & & $\begin{array}{c}\text { Labor } \\
\text { FY } 2012 \text { (\$ B) }\end{array}$ & $\begin{array}{c}\text { Utilities } \\
\text { FY } 2012 \text { (\$ B) }\end{array}$ & $\begin{array}{l}\text { Materials and } \\
\text { Contracts } \\
\text { FY } 2012 \text { (\$ B) }\end{array}$ & $\begin{array}{c}\text { D\&D } \\
\text { FY } 2012 \text { (\$ B) }\end{array}$ & $\begin{array}{c}\text { Total } \\
\text { FY } 2012 \text { (\$ B) }\end{array}$ & & \\
\hline \multirow{7}{*}{\begin{tabular}{|c|} 
Canisters \\
Only - \\
Re-Package \\
at Repository \\
(Case 1)
\end{tabular}} & 1,500 & 2020 & 2040 & 4 & $\$ 2.8$ & $\$ 0.1$ & $\$ 6.0$ & $\$ 2.3$ & $\$ 9.1$ & $\$ 2.9$ & $\$ 12.0$ \\
\hline & 3,000 & 2020 & 2040 & 12 & $\$ 1.2$ & $\$ 0.1$ & $\$ 3.5$ & $\$ 2.7$ & $\$ 5.1$ & $\$ 3.3$ & $\$ 8.4$ \\
\hline & 3,000 & 2020 & 2040 & 21 & $\$ 0.9$ & $\$ 0.0$ & $\$ 2.8$ & $\$ 2.2$ & $\$ 3.9$ & $\$ 2.6$ & $\$ 6.6$ \\
\hline & 6,000 & 2020 & 2040 & 4 & $\$ 2.2$ & $\$ 0.2$ & $\$ 6.6$ & $\$ 8.1$ & $\$ 9.7$ & $\$ 9.9$ & $\$ 19.6$ \\
\hline & 1,500 & 2020 & 2055 & 4 & $\$ 2.8$ & $\$ 0.1$ & $\$ 6.0$ & $\$ 2.3$ & $\$ 9.1$ & $\$ 2.9$ & $\$ 12.0$ \\
\hline & 6,000 & 2020 & 2055 & 4 & $\$ 2.3$ & $\$ 0.2$ & $\$ 6.6$ & $\$ 8.4$ & $\$ 9.9$ & $\$ 10.3$ & $\$ 20.3$ \\
\hline & 3,000 & 2035 & 2055 & 4 & $\$ 2.2$ & $\$ 0.1$ & $\$ 6.0$ & $\$ 3.9$ & $\$ 8.8$ & $\$ 4.8$ & $\$ 13.6$ \\
\hline \multirow{8}{*}{$\begin{array}{c}\text { Canisters and } \\
\text { Bare Fuel - } \\
\text { Re-Package } \\
\text { at Repository } \\
\text { (Case 2) }\end{array}$} & 1,500 & 2020 & 2040 & 4 & $\$ 3.0$ & $\$ 0.2$ & $\$ 5.9$ & $\$ 2.7$ & $\$ 9.1$ & $\$ 3.3$ & $\$ 12.5$ \\
\hline & 3,000 & 2020 & 2040 & 4 & $\$ 2.2$ & $\$ 0.1$ & $\$ 6.0$ & $\$ 4.5$ & $\$ 8.8$ & $\$ 5.6$ & $\$ 14.4$ \\
\hline & 3,000 & 2020 & 2040 & 12 & $\$ 1.2$ & $\$ 0.1$ & $\$ 3.3$ & $\$ 3.0$ & $\$ 4.9$ & $\$ 3.7$ & $\$ 8.5$ \\
\hline & 3,000 & 2020 & 2040 & 21 & $\$ 0.9$ & $\$ 0.0$ & $\$ 2.6$ & $\$ 2.8$ & $\$ 3.8$ & $\$ 3.4$ & $\$ 7.2$ \\
\hline & 6,000 & 2020 & 2040 & 4 & $\$ 2.0$ & $\$ 0.1$ & $\$ 6.2$ & $\$ 8.9$ & $\$ 9.3$ & $\$ 11.0$ & $\$ 20.3$ \\
\hline & 1,500 & 2020 & 2055 & 4 & $\$ 2.8$ & $\$ 0.1$ & $\$ 5.9$ & $\$ 2.7$ & $\$ 9.1$ & $\$ 3.3$ & $\$ 12.5$ \\
\hline & 6,000 & 2020 & 2055 & 4 & $\$ 1.9$ & $\$ 0.1$ & $\$ 6.2$ & $\$ 8.4$ & $\$ 9.1$ & $\$ 10.3$ & $\$ 19.3$ \\
\hline & 3,000 & 2035 & 2055 & 4 & $\$ 2.2$ & $\$ 0.1$ & $\$ 6.0$ & $\$ 4.3$ & $\$ 8.8$ & $\$ 5.3$ & $\$ 14.1$ \\
\hline \multirow{9}{*}{$\begin{array}{c}\text { Canisters } \\
\text { Only - Re- } \\
\text { Package at } \\
\text { CSF (Case 3) }\end{array}$} & 1,500 & 2020 & 2040 & 4 & $\$ 3.0$ & $\$ 0.2$ & $\$ 6.0$ & $\$ 3.3$ & $\$ 9.6$ & $\$ 4.0$ & $\$ 13.6$ \\
\hline & 3,000 & 2020 & 2040 & 4 & $\$ 2.2$ & $\$ 0.1$ & $\$ 6.1$ & $\$ 5.6$ & $\$ 9.0$ & $\$ 6.8$ & $\$ 15.8$ \\
\hline & 3,000 & 2020 & 2040 & 12 & $\$ 1.2$ & $\$ 0.1$ & $\$ 3.4$ & $\$ 3.1$ & $\$ 4.9$ & $\$ 3.8$ & $\$ 8.7$ \\
\hline & 3,000 & 2020 & 2040 & 21 & $\$ 0.9$ & $\$ 0.0$ & $\$ 2.6$ & $\$ 2.4$ & $\$ 3.8$ & $\$ 2.9$ & $\$ 6.7$ \\
\hline & 6,000 & 2020 & 2040 & 4 & $\$ 2.2$ & $\$ 0.2$ & $\$ 6.6$ & $\$ 11.8$ & $\$ 10.2$ & $\$ 14.4$ & $\$ 24.6$ \\
\hline & 1,500 & 2020 & 2055 & 4 & $\$ 3.0$ & $\$ 0.2$ & $\$ 6.0$ & $\$ 3.3$ & $\$ 9.6$ & $\$ 4.0$ & $\$ 13.6$ \\
\hline & 3,000 & 2020 & 2055 & 4 & $\$ 2.2$ & $\$ 0.1$ & $\$ 6.1$ & $\$ 5.6$ & $\$ 9.0$ & $\$ 6.8$ & $\$ 15.8$ \\
\hline & 6,000 & 2020 & 2055 & 4 & $\$ 2.2$ & $\$ 0.2$ & $\$ 6.6$ & $\$ 11.8$ & $\$ 10.2$ & $\$ 14.4$ & $\$ 24.6$ \\
\hline & 3,000 & 2035 & 2055 & 4 & $\$ 2.2$ & $\$ 0.1$ & $\$ 6.1$ & $\$ 5.6$ & $\$ 9.0$ & $\$ 6.8$ & $\$ 15.8$ \\
\hline \multirow{7}{*}{$\begin{array}{l}\text { Canisters and } \\
\text { Bare Fuel - } \\
\text { Re-Package } \\
\text { at CSF (Case } \\
4 \text { ) }\end{array}$} & 1,500 & 2020 & 2040 & 4 & $\$ 2.8$ & $\$ 0.1$ & $\$ 5.9$ & $\$ 3.1$ & $\$ 9.2$ & $\$ 3.8$ & $\$ 13.0$ \\
\hline & 3,000 & 2020 & 2040 & 4 & $\$ 2.2$ & $\$ 0.1$ & $\$ 6.0$ & $\$ 5.6$ & $\$ 8.9$ & $\$ 6.8$ & $\$ 15.8$ \\
\hline & 3,000 & 2020 & 2040 & 12 & $\$ 1.2$ & $\$ 0.1$ & $\$ 3.3$ & $\$ 3.1$ & $\$ 4.9$ & $\$ 3.8$ & $\$ 8.7$ \\
\hline & 3,000 & 2020 & 2040 & 21 & $\$ 0.9$ & $\$ 0.0$ & $\$ 2.6$ & $\$ 2.4$ & $\$ 3.8$ & $\$ 2.9$ & $\$ 6.7$ \\
\hline & 6,000 & 2020 & 2040 & 4 & $\$ 2.0$ & $\$ 0.1$ & $\$ 6.2$ & $\$ 9.7$ & $\$ 9.3$ & $\$ 11.9$ & $\$ 21.2$ \\
\hline & 1,500 & 2020 & 2055 & 4 & $\$ 2.8$ & $\$ 0.1$ & $\$ 5.9$ & $\$ 3.1$ & $\$ 9.2$ & $\$ 3.8$ & $\$ 13.0$ \\
\hline & 3,000 & 2020 & 2055 & 4 & $\$ 2.2$ & $\$ 0.1$ & $\$ 6.0$ & $\$ 5.6$ & $\$ 8.9$ & $\$ 6.8$ & $\$ 15.8$ \\
\hline
\end{tabular}




\subsection{Transportation Rough Order of Magnitude Cost Estimates}

Table 6-11 presents the estimated costs for each of transporting UNF for each of the cases considered in this evaluation. These estimated costs were calculated using the Transportation Operations Model (TOM) portion of the TSL framework (Busch et al., 2012).

The capital cost is driven by the number of casks and transportation assets that need to be purchased during the campaign. This is in turn driven by the cycle time needed to transport the spent fuel. While the location of the storage facilities and repository drive the transportation time, transportation is actually only a small contributor to the overall cycle time. Loading, unloading, and maintenance activities make up the majority of the cycle time. Therefore, the acquisitions required are for the most part relatively independent of the location of the storage facility and repository.

Maintenance costs are directly related to the number of casks and transportation assets that are being used, so these costs will vary as the capital cost value does.

The operations cost is a function of the distance that the spent fuel is transported. This distance is highly dependent on where the storage facilities and repository are located. Since these locations have not yet been determined, the analysis arbitrarily assumed that the CSF was located in the Eastern United States and the repository was located in the Western United States. The cask maintenance facility and fleet maintenance facility were assumed to be co-located with the CSF. Operations costs are expected to have the most variability as the storage locations change.

Overall, the largest transportation costs occur when packaging/re-packaging occurs at the CSF (Cases 3 and 4). This results from having to acquire essentially a "second" transportation fleet to ship the smaller waste package compatible size canisters from the CSF to the repository. The capital and maintenance costs are larger simply because the fleet is larger. However, operations costs are also larger because the smaller capacity canisters require significantly more shipments to achieve a given UNF transportation rate (i.e. $3000 \mathrm{MTHM} / \mathrm{yr}$ ) between the CSF and the repository. Future work investigating alternative waste package compatible canister/overpack and transportation equipment design concepts may identify more efficient concepts for transportation.

Transportation costs are also larger when bare fuel is accepted from the reactor sites (Cases 2 and 4). As discussed above, re-useable transportation casks are generally of smaller capacity than dual-purpose canisters, requiring a larger number of shipments to achieve a desired UNF transportation rate (i.e., 3000 $\mathrm{MTHM} / \mathrm{yr}$ ) and therefore a larger transportation fleet.

Overall, the largest transportation costs are when bare fuel is accepted from the reactor sites and repackaging occurs at the CSF. The smallest transportation costs are when all fuel is placed in dual-purpose canisters and they are re-packaged at the repository. 
Table 6-11 UNF Transportation Life Cycle Costs

\begin{tabular}{|c|c|c|c|c|c|c|c|c|}
\hline Scenario & $\begin{array}{c}\text { Acceptance } \\
\text { Rate }\end{array}$ & $\begin{array}{l}\text { CSF } \\
\text { Start }\end{array}$ & $\begin{array}{c}\text { Repository } \\
\text { Start }\end{array}$ & $\begin{array}{c}\text { Disposal } \\
\text { Canister } \\
\text { Size } \\
\end{array}$ & $\begin{array}{c}\text { Capital Cost } \\
\text { (\$B) }\end{array}$ & $\begin{array}{c}\text { Maintenance } \\
\text { Cost (\$B) }\end{array}$ & $\begin{array}{c}\text { Operations } \\
\text { Cost (\$B) }\end{array}$ & $\begin{array}{c}\text { Total Cost } \\
\text { (\$B) }\end{array}$ \\
\hline \multirow{9}{*}{$\begin{array}{l}\text { Canisters Only - } \\
\text { Re-Package at } \\
\text { Repository }\end{array}$} & 1500 & 2020 & 2040 & 4 & $\$ 0.5$ & $\$ 0.4$ & $\$ 2.3$ & $\$ 3.2$ \\
\hline & 3000 & 2020 & 2040 & 4 & $\$ 0.6$ & $\$ 0.4$ & $\$ 2.2$ & $\$ 3.2$ \\
\hline & 3000 & 2020 & 2040 & 12 & $\$ 0.6$ & $\$ 0.4$ & $\$ 2.2$ & $\$ 3.2$ \\
\hline & 3000 & 2020 & 2040 & 21 & $\$ 0.6$ & $\$ 0.4$ & $\$ 2.2$ & $\$ 3.2$ \\
\hline & 6000 & 2020 & 2040 & 4 & $\$ 0.8$ & $\$ 0.5$ & $\$ 2.1$ & $\$ 3.4$ \\
\hline & 1500 & 2020 & 2055 & 4 & $\$ 0.5$ & $\$ 0.5$ & $\$ 2.3$ & $\$ 3.3$ \\
\hline & 3000 & 2020 & 2055 & 4 & $\$ 0.6$ & $\$ 0.5$ & $\$ 2.3$ & $\$ 3.4$ \\
\hline & 6000 & 2020 & 2055 & 4 & $\$ 0.9$ & $\$ 0.6$ & $\$ 2.3$ & $\$ 3.8$ \\
\hline & 3000 & 2035 & 2055 & 4 & $\$ 0.5$ & $\$ 0.4$ & $\$ 2.1$ & $\$ 3.1$ \\
\hline \multirow{9}{*}{$\begin{array}{l}\text { Canisters and } \\
\text { Bare Fuel - } \\
\text { Re-Package at } \\
\text { Repository }\end{array}$} & 1500 & 2020 & 2040 & 4 & $\$ 0.8$ & $\$ 0.5$ & $\$ 2.5$ & $\$ 3.8$ \\
\hline & 3000 & 2020 & 2040 & 4 & $\$ 1.0$ & $\$ 0.5$ & $\$ 2.6$ & $\$ 4.1$ \\
\hline & 3000 & 2020 & 2040 & 12 & $\$ 1.0$ & $\$ 0.5$ & $\$ 2.6$ & $\$ 4.1$ \\
\hline & 3000 & 2020 & 2040 & 21 & $\$ 1.0$ & $\$ 0.5$ & $\$ 2.6$ & $\$ 4.1$ \\
\hline & 6000 & 2020 & 2040 & 4 & $\$ 1.5$ & $\$ 0.8$ & $\$ 3.2$ & $\$ 5.5$ \\
\hline & 1500 & 2020 & 2055 & 4 & $\$ 0.9$ & $\$ 0.5$ & $\$ 2.6$ & $\$ 4.0$ \\
\hline & 3000 & 2020 & 2055 & 4 & $\$ 1.1$ & $\$ 0.6$ & $\$ 2.7$ & $\$ 4.5$ \\
\hline & 6000 & 2020 & 2055 & 4 & $\$ 1.6$ & $\$ 0.8$ & $\$ 3.1$ & $\$ 5.6$ \\
\hline & 3000 & 2035 & 2055 & 4 & $\$ 0.9$ & $\$ 0.5$ & $\$ 2.4$ & $\$ 3.8$ \\
\hline \multirow{9}{*}{$\begin{array}{c}\text { Canisters Only - } \\
\text { Re-Package at } \\
\text { CSF }\end{array}$} & 1500 & 2020 & 2040 & 4 & $\$ 1.6$ & $\$ 2.2$ & $\$ 9.6$ & $\$ 13.4$ \\
\hline & 3000 & 2020 & 2040 & 4 & $\$ 1.7$ & $\$ 2.2$ & $\$ 9.5$ & $\$ 13.4$ \\
\hline & 3000 & 2020 & 2040 & 12 & $\$ 0.8$ & $\$ 1.0$ & $\$ 4.1$ & $\$ 5.9$ \\
\hline & 3000 & 2020 & 2040 & 21 & $\$ 0.8$ & $\$ 0.7$ & $\$ 2.7$ & $\$ 4.2$ \\
\hline & 6000 & 2020 & 2040 & 4 & $\$ 2.4$ & $\$ 2.2$ & $\$ 9.4$ & $\$ 14.0$ \\
\hline & 1500 & 2020 & 2055 & 4 & $\$ 1.6$ & $\$ 2.2$ & $\$ 9.6$ & $\$ 13.4$ \\
\hline & 3000 & 2020 & 2055 & 4 & $\$ 1.7$ & $\$ 2.2$ & $\$ 9.5$ & $\$ 13.4$ \\
\hline & 6000 & 2020 & 2055 & 4 & $\$ 2.3$ & $\$ 2.2$ & $\$ 9.4$ & $\$ 14.0$ \\
\hline & 3000 & 2035 & 2055 & 4 & $\$ 1.6$ & $\$ 2.2$ & $\$ 9.5$ & $\$ 13.3$ \\
\hline \multirow{9}{*}{$\begin{array}{l}\text { Canisters and } \\
\text { Bare Fuel - Re- } \\
\text { Package at CSF }\end{array}$} & 1500 & 2020 & 2040 & 4 & $\$ 1.8$ & $\$ 2.3$ & $\$ 10.9$ & $\$ 15.0$ \\
\hline & 3000 & 2020 & 2040 & 4 & $\$ 2.1$ & $\$ 2.3$ & $\$ 10.9$ & $\$ 15.3$ \\
\hline & 3000 & 2020 & 2040 & 12 & $\$ 1.2$ & $\$ 1.1$ & $\$ 4.8$ & $\$ 7.1$ \\
\hline & 3000 & 2020 & 2040 & 21 & $\$ 1.2$ & $\$ 0.8$ & $\$ 3.3$ & $\$ 5.3$ \\
\hline & 6000 & 2020 & 2040 & 4 & $\$ 2.7$ & $\$ 2.4$ & $\$ 11.2$ & $\$ 16.3$ \\
\hline & 1500 & 2020 & 2055 & 4 & $\$ 1.8$ & $\$ 2.3$ & $\$ 10.9$ & $\$ 15.0$ \\
\hline & 3000 & 2020 & 2055 & 4 & $\$ 2.0$ & $\$ 2.3$ & $\$ 10.9$ & $\$ 15.3$ \\
\hline & 6000 & 2020 & 2055 & 4 & $\$ 2.6$ & $\$ 2.4$ & $\$ 11.2$ & $\$ 16.2$ \\
\hline & 3000 & 2035 & 2055 & 4 & $\$ 1.9$ & $\$ 2.2$ & $\$ 10.8$ & $\$ 14.9$ \\
\hline
\end{tabular}




\subsection{Back-End Total Life Cycle Rough Order of Magnitude Cost Estimates}

Table 6-12 summarizes all of the LCC information presented above and provides a roll-up of the total cost of UNF management for each of the cases considered in this evaluation. Two sets of roll-up costs are provided. One considers all aspects of the UNF management system, less the at-reactor estimated costs. This allows for comparison of the costs of future waste management options and how they compare with on-site UNF management requirements. The second considers all aspects of the UNF management system and allows for an overall estimate of the potential costs of managing UNF.

The rows in Table 6-12 representing the three acceptance rates considered in this study are shaded with different colors to facilitate comparisons. Since Table 6-12 presents a number of 3,000 MTHM/year cases that are not comparable to the cases for the other acceptance rates because of assumptions about the CSF start, repository start, or the disposal canister size that are considered only for the 3,000 MTHM/year cases, Table 6-13 presents a subset of the data in Table 6-12 related to the comparable 4/9 PWR/BWR assembly capacity waste package compatible size canister cases with the CSF starting in 2020 and the repository starting in 2040. Table 6-13 also shows both the magnitude of the costs in each category and the percentage of the total. This simpler table allows the main effects (except for the effect of a delayed repository or CSF) to be seen more clearly.

Table 6-12 shows that the total estimated LCC of UNF management is largest when 1) re-packaging occurs at the CSF, and 2) when bare fuel is accepted from the reactors and stored in wet pools at the CSF. The combination of the two (canisters and bare fuel, re-packaging at the CSF) yields the largest costs. These larger costs are driven by:

- The cost of wet storage pools at the CSF

- An increased number of release bays at the packaging/re-packaging facility to process the smaller capacity waste package compatible size canisters

- The need for a "second" transportation fleet to ship the larger number of smaller capacity canisters from the CSF to the repository.

The total estimated UNF management LCC when packaging/re-packaging is approximately $10 \%$ to $20 \%$ larger when packaging/re-packaging is performed at the CSF, depending on the assumed processing rate and CSF/repository start dates, regardless of whether the UNF is all loaded into canisters at the reactor sites or whether some fraction is maintained as bare fuel.

The total estimated UNF LCC when some fraction of UNF is maintained as bare fuel ranges from $20 \%$ to $85 \%$ larger than when all the UNF is loaded into existing size canisters at the reactor sites, depending on the assume processing rate and CSF/repository start dates, regardless of where the UNF is packaged/repackaged.

Table 6-12 shows that the total estimated UNF management LCC of away from reactor UNF management increases with increasing UNF throughput. As discussed above and shown in the summary LCCs in Table 6-12, higher throughput requires a larger CSF, packaging/re-packaging facility, and transportation fleet. When considering the total away-from-reactor costs, the lowest cost occurs for a UNF processing rate of $1500 \mathrm{MTHM} / \mathrm{yr}$ when re-packaging is done at the CSF and for a UNF processing rate of 3000 $\mathrm{MTHM} / \mathrm{yr}$ when re-packaging is done at the repository.

However, the overall cost of UNF management (all aspects of the UNF management system) is lowest when the UNF processing rate (acceptance from reactors, shipment from CSF to repository) is 3000 MTHM/yr, CSF operations begin in 2020, and repository operations begin in 2040 for each of the major cases considered (all canisters/bare fuel, re-package at the CSF/repository). 
Delaying the start of repository operations from 2040 to 2055 increases the total UNF management LCC by $5 \%-10 \%$ for a $1500 \mathrm{MTHM} / \mathrm{yr}$ throughput rate, $15 \%-20 \%$ for a throughput rate of $3000 \mathrm{MTHM} / \mathrm{yr}$, and $10 \%-15 \%$ for a throughput rate of $6000 \mathrm{MTHM} / \mathrm{yr}$. This is driven by increased storage capacity required at the CSF.

For a UNF throughput rate of $3000 \mathrm{MTH} / \mathrm{yr}$, delaying the start of CSF operations from 2020 to 2035 increases the total estimated UNF management LCC by $15 \%$ - $18 \%$ when all UNF is loaded into dualpurpose canisters at the reactor sites regardless of where re-packaging is performed. However, when UNF is transported from the reactors in re-useable transportation casks as bare fuel there is essentially no difference. When bare fuel is considered, the delay in beginning acceptance from the reactors results in more fuel being transferred to canisters for at-reactor dry storage and a reduction in the amount of bare fuel that has to be stored at the CSF. Thus, the delay results in having to construct a reduced number of wet storage basins at the CSF and a cost savings. However, this savings is essentially equal to the increase in at-reactor LCC costs due to the delay.

Utilizing larger waste packages, should that prove possible at the repository, also results in lower estimated total UNF management LCC. This is because of a reduced number of closure and receipt bays within the packaging/re-packaging facility and lower transportation fleet requirements. Of course, this is not a design choice, but rather will be an option dictated by the capabilities of the repository site and medium ultimately selected.

Throughput rates of $6000 \mathrm{MTHM} / \mathrm{yr}$ result in the largest estimated away from reactor UNF management LCC. This is partially compensated by the reduced at-reactor UNF management LCC. However, as discussed above, the lowest total UNF management LCC occurs at a throughput rate of $3000 \mathrm{MTHM} / \mathrm{yr}$.

Table 6-14 shows the total UNF management cost and the away from reactor UNF management cost, normalized to 140,000 MTHM of UNF ultimately processed into disposal canisters. 
Table 6-12. Summary Roll-Up UNF Management Rough Order of Magnitude Cost Estimate

\begin{tabular}{|c|c|c|c|c|c|c|c|c|c|c|c|}
\hline Scenario & $\begin{array}{c}\text { Acceptance } \\
\text { Rate }\end{array}$ & CSF Start & $\begin{array}{c}\text { Repository } \\
\text { Start }\end{array}$ & $\begin{array}{c}\text { Disposal } \\
\text { Canister } \\
\text { Size }\end{array}$ & $\begin{array}{c}\text { At-Reactor Life } \\
\text { Cycle } \\
\text { FY } 2012 \text { (\$B) }\end{array}$ & $\begin{array}{l}\text { Transportation } \\
\text { Life Cycle } \\
\text { FY } 2012 \text { (\$B) }\end{array}$ & $\begin{array}{l}\text { CSF Total Life } \\
\text { Cycle } \\
\text { FY } 2012 \text { (\$B) }\end{array}$ & $\begin{array}{l}\text { TVF Total Life } \\
\text { Cycle } \\
\text { FY } 2012 \text { (\$B) }\end{array}$ & $\begin{array}{c}\text { RF Total Life Cycle } \\
\text { FY } 2012 \text { (\$B) }\end{array}$ & \begin{tabular}{|c|} 
Away From \\
Reactor UNF \\
Management Life \\
Cycle \\
(Trans. + CSF + TVF \\
+ RF) \\
FY 2012 (\$B)
\end{tabular} & $\begin{array}{c}\text { Total UNF } \\
\text { Management Life } \\
\text { Cycle } \\
\text { (React. + Trans. + } \\
\text { CSF + TVF + RF) } \\
\text { FY } 2012 \text { (\$B) }\end{array}$ \\
\hline \multirow{9}{*}{$\begin{array}{l}\text { Canisters } \\
\text { Only - } \\
\text { Re-Package } \\
\text { at Repository } \\
\text { (Case 1) }\end{array}$} & 1,500 & 2020 & 2040 & 4 & $\$ 47.8$ & $\$ 3.2$ & $\$ 7.5$ & $\$ 4.4$ & $\$ 12.0$ & $\$ 27.1$ & $\$ 74.9$ \\
\hline & 3,000 & 2020 & 2040 & 4 & $\$ 27.2$ & $\$ 3.2$ & $\$ 11.3$ & $\$ 3.4$ & $\$ 14.4$ & $\$ 32.2$ & $\$ 59.3$ \\
\hline & 3,000 & 2020 & 2040 & 12 & $\$ 27.2$ & $\$ 3.2$ & $\$ 11.3$ & $\$ 3.4$ & $\$ 8.4$ & $\$ 26.2$ & $\$ 53.4$ \\
\hline & 3,000 & 2020 & 2040 & 21 & $\$ 27.2$ & $\$ 3.2$ & $\$ 11.3$ & $\$ 3.4$ & $\$ 6.6$ & $\$ 24.4$ & $\$ 51.6$ \\
\hline & 6,000 & 2020 & 2040 & 4 & $\$ 17.6$ & $\$ 3.4$ & $\$ 17.5$ & $\$ 2.9$ & $\$ 19.6$ & $\$ 43.5$ & $\$ 61.1$ \\
\hline & 1,500 & 2020 & 2055 & 4 & $\$ 47.8$ & $\$ 3.3$ & $\$ 11.9$ & $\$ 4.7$ & $\$ 12.0$ & $\$ 32.0$ & $\$ 79.8$ \\
\hline & 3,000 & 2020 & 2055 & 4 & $\$ 27.2$ & $\$ 3.4$ & $\$ 20.4$ & $\$ 3.7$ & $\$ 14.4$ & $\$ 41.8$ & $\$ 69.0$ \\
\hline & 6,000 & 2020 & 2055 & 4 & $\$ 17.6$ & $\$ 3.8$ & $\$ 25.7$ & $\$ 3.3$ & $\$ 20.3$ & $\$ 53.0$ & $\$ 70.6$ \\
\hline & 3,000 & 2035 & 2055 & 4 & $\$ 36.8$ & $\$ 3.1$ & $\$ 12.9$ & $\$ 3.4$ & $\$ 13.6$ & $\$ 33.0$ & $\$ 69.8$ \\
\hline \multirow{9}{*}{$\begin{array}{c}\text { Canisters and } \\
\text { Bare Fuel - } \\
\text { Re-Package } \\
\text { at Repository } \\
\text { (Case 2) }\end{array}$} & 1,500 & 2020 & 2040 & 4 & $\begin{array}{l}47.8 \\
\end{array}$ & $\$ 3.8$ & $\$ 22.5$ & $\begin{array}{l}4.4 \\
\end{array}$ & $\$ 12.5$ & $\$ 43.2$ & $\begin{array}{l}\$ 91.0 \\
\end{array}$ \\
\hline & 3,000 & 2020 & 2040 & 4 & $\$ 27.2$ & $\$ 4.1$ & $\$ 40.2$ & $\$ 3.4$ & $\$ 14.4$ & $\$ 62.1$ & $\$ 89.3$ \\
\hline & 3,000 & 2020 & 2040 & 12 & $\$ 27.2$ & $\$ 4.1$ & $\$ 40.2$ & $\$ 3.4$ & $\$ 8.5$ & $\$ 56.3$ & $\$ 83.4$ \\
\hline & 3,000 & 2020 & 2040 & 21 & $\$ 27.2$ & $\$ 4.1$ & $\$ 40.2$ & $\$ 3.4$ & $\$ 7.2$ & $\$ 54.9$ & $\$ 82.0$ \\
\hline & 6,000 & 2020 & 2040 & 4 & $\$ 17.6$ & $\$ 5.5$ & $\$ 67.2$ & $\$ 2.9$ & $\$ 20.3$ & $\$ 95.9$ & $\$ 113.5$ \\
\hline & 1,500 & 2020 & 2055 & 4 & $\$ 47.8$ & $\$ 4.0$ & $\$ 31.8$ & $\$ 4.7$ & $\$ 12.5$ & $\$ 53.0$ & $\$ 100.8$ \\
\hline & 3,000 & 2020 & 2055 & 4 & $\$ 27.2$ & $\$ 4.5$ & $\$ 58.4$ & $\$ 3.7$ & $\$ 14.4$ & $\$ 81.0$ & $\$ 108.2$ \\
\hline & 6,000 & 2020 & 2055 & 4 & $\$ 17.6$ & $\$ 5.6$ & $\$ 78.7$ & $\$ 3.2$ & $\$ 19.3$ & $\$ 106.9$ & $\$ 124.5$ \\
\hline & 3,000 & 2035 & 2055 & 4 & $\$ 36.8$ & $\$ 3.8$ & $\$ 30.5$ & $\$ 3.4$ & $\$ 14.1$ & $\$ 51.8$ & $\$ 88.6$ \\
\hline \multirow{9}{*}{$\begin{array}{c}\text { Canisters } \\
\text { Only - Re- } \\
\text { Package at } \\
\text { CSF } \\
\text { (Case 3) }\end{array}$} & $\overline{11,500}$ & 2020 & 2040 & $\overline{4}$ & $\begin{array}{l}\$ 47.0 \\
\end{array}$ & $\$ 13.4$ & $\$ 10.3$ & $\begin{array}{l}\$ 4.4 \\
\end{array}$ & $\begin{array}{l}\$ 13.6 \\
\end{array}$ & $\begin{array}{l}\$ 1.8 \\
\end{array}$ & $\begin{array}{l}\$ 88.8 \\
\end{array}$ \\
\hline & 3,000 & 2020 & 2040 & 4 & $\$ 25.6$ & $\$ 13.4$ & $\$ 15.2$ & $\$ 3.4$ & $\$ 15.8$ & $\$ 47.8$ & $\$ 73.3$ \\
\hline & 3,000 & 2020 & 2040 & 12 & $\$ 25.6$ & $\$ 5.9$ & $\$ 15.2$ & $\$ 3.4$ & $\$ 8.7$ & $\$ 33.2$ & $\$ 58.7$ \\
\hline & 3,000 & 2020 & 2040 & 21 & $\$ 25.6$ & $\$ 4.2$ & $\$ 15.2$ & $\$ 3.4$ & $\$ 6.7$ & $\$ 29.6$ & $\$ 55.1$ \\
\hline & 6,000 & 2020 & 2040 & 4 & $\$ 15.4$ & $\$ 14.0$ & $\$ 18.0$ & $\$ 2.9$ & $\$ 24.6$ & $\$ 59.6$ & $\$ 75.0$ \\
\hline & 1,500 & 2020 & 2055 & 4 & $\$ 47.0$ & $\$ 13.4$ & $\$ 15.8$ & $\$ 4.7$ & $\$ 13.6$ & $\$ 47.6$ & $\$ 94.6$ \\
\hline & 3,000 & 2020 & 2055 & 4 & $\$ 25.6$ & $\$ 13.4$ & $\$ 22.9$ & $\$ 3.7$ & $\$ 15.8$ & $\$ 55.8$ & $\$ 81.4$ \\
\hline & 6,000 & 2020 & 2055 & 4 & $\$ 15.4$ & $\$ 14.0$ & $\$ 25.7$ & $\$ 3.3$ & $\$ 24.6$ & $\$ 67.6$ & $\$ 83.0$ \\
\hline & 3,000 & 2035 & 2055 & 4 & $\$ 36.1$ & $\$ 13.3$ & $\$ 15.8$ & $\$ 3.4$ & $\$ 15.8$ & $\$ 48.3$ & $\$ 84.4$ \\
\hline \multirow{9}{*}{$\begin{array}{c}\text { Canisters and } \\
\text { Bare Fuel - } \\
\text { Re-Package } \\
\text { at CSF } \\
\text { (Case 4) }\end{array}$} & 1, & 2020 & 2040 & 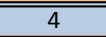 & (\$47.0 & \$15.0 & \$28.7 & $\begin{array}{l}\$ 4.4 \\
\end{array}$ & \$13.0 & "\$61.1 & $\$ 108.2$ \\
\hline & 3,000 & 2020 & 2040 & 4 & $\$ 25.6$ & $\$ 15.3$ & $\$ 50.1$ & $\$ 3.4$ & $\$ 15.8$ & $\$ 84.5$ & $\$ 110.1$ \\
\hline & 3,000 & 2020 & 2040 & 12 & $\$ 25.6$ & $\$ 7.1$ & $\$ 50.1$ & $\$ 3.4$ & $\$ 8.7$ & $\$ 69.2$ & $\$ 94.8$ \\
\hline & 3,000 & 2020 & 2040 & 21 & $\$ 25.6$ & $\$ 5.3$ & $\$ 50.1$ & $\$ 3.4$ & $\$ 6.7$ & $\$ 65.5$ & $\$ 91.0$ \\
\hline & 6,000 & 2020 & 2040 & 4 & $\$ 15.4$ & $\$ 16.3$ & $\$ 67.1$ & $\$ 2.9$ & $\$ 21.2$ & $\$ 107.5$ & $\$ 122.8$ \\
\hline & 1,500 & 2020 & 2055 & 4 & $\$ 47.0$ & $\$ 15.0$ & $\$ 40.3$ & $\$ 4.7$ & $\$ 13.0$ & $\$ 72.9$ & $\$ 120.0$ \\
\hline & 3,000 & 2020 & 2055 & 4 & $\$ 25.6$ & $\$ 15.3$ & $\$ 64.4$ & $\$ 3.7$ & $\$ 15.8$ & $\$ 99.1$ & $\$ 124.7$ \\
\hline & 6,000 & 2020 & 2055 & 4 & $\$ 15.4$ & $\$ 16.2$ & $\$ 78.6$ & $\$ 3.2$ & $\$ 21.2$ & $\$ 119.2$ & $\$ 134.6$ \\
\hline & 3,000 & 2035 & 2055 & 4 & $\$ 36.1$ & $\$ 14.9$ & $\$ 37.5$ & $\$ 3.4$ & $\$ 15.6$ & $\$ 71.3$ & $\$ 107.4$ \\
\hline
\end{tabular}


Table 6-13. Summary Roll-Up UNF Management Rough Order of Magnitude Cost Estimate (2020-2040 CSF-Repository Start, 4/9 Assembly Capacity Disposal Canister

\begin{tabular}{|c|c|c|c|c|c|c|c|c|c|c|c|}
\hline Scenario & $\begin{array}{c}\text { Acceptance } \\
\text { Rate }\end{array}$ & CSF Start & $\begin{array}{c}\text { Repository } \\
\text { Start }\end{array}$ & $\begin{array}{c}\text { Disposal } \\
\text { Canister } \\
\text { Size }\end{array}$ & $\begin{array}{l}\text { At-Reactor Life } \\
\text { Cycle } \\
\text { FY } 2012 \text { (\$B) }\end{array}$ & $\begin{array}{l}\text { Transportation } \\
\text { Life Cycle } \\
\text { FY } 2012 \text { (\$B) }\end{array}$ & $\begin{array}{l}\text { CSF Total Life } \\
\text { Cycle } \\
\text { FY } 2012 \text { (\$B) }\end{array}$ & $\begin{array}{l}\text { TVF Total Life } \\
\text { Cycle } \\
\text { FY } 2012 \text { (\$B) }\end{array}$ & $\begin{array}{c}\text { RF Total Life Cycle } \\
\text { FY } 2012 \text { (\$B) }\end{array}$ & \begin{tabular}{|c|} 
Away From \\
Reactor UNF \\
Management Life \\
Cycle \\
(Trans. + CSF + TVF \\
+ RF) \\
FY 2012 (\$B) \\
\end{tabular} & $\begin{array}{c}\text { Total UNF } \\
\text { Management Life } \\
\text { Cycle } \\
\text { (React. + Trans. + } \\
\text { CSF + TVF + RF) } \\
\text { FY } 2012 \text { (\$B) }\end{array}$ \\
\hline \multirow{3}{*}{$\begin{array}{l}\text { Canisters } \\
\text { Only - } \\
\text { Re-Package } \\
\text { at Repository } \\
\text { (Case 1) }\end{array}$} & 1,500 & 2020 & 2040 & 4 & $\begin{array}{c}\$ 47.8 \\
64 \%\end{array}$ & $\begin{array}{c}\$ 3.2 \\
4 \%\end{array}$ & $\begin{array}{l}\$ 7.5 \\
10 \%\end{array}$ & $\begin{array}{c}\$ 4.4 \\
6 \%\end{array}$ & $\begin{array}{c}\$ 12.0 \\
16 \%\end{array}$ & $\begin{array}{c}\$ 27.1 \\
36 \%\end{array}$ & $\$ 74.9$ \\
\hline & 3,000 & 2020 & 2040 & 4 & $\begin{array}{c}\$ 27.2 \\
46 \%\end{array}$ & $\begin{array}{c}\$ 3.2 \\
5 \%\end{array}$ & $\begin{array}{c}\$ 11.3 \\
19 \%\end{array}$ & $\begin{array}{c}\$ 3.4 \\
6 \%\end{array}$ & $\begin{array}{c}\$ 14.4 \\
24 \%\end{array}$ & $\begin{array}{c}\$ 32.2 \\
54 \%\end{array}$ & $\$ 59.3$ \\
\hline & 6,000 & 2020 & 2040 & 4 & $\begin{array}{c}\$ 17.6 \\
29 \% \\
\end{array}$ & $\begin{array}{c}\$ 3.4 \\
6 \% \\
\end{array}$ & $\begin{array}{c}\$ 17.5 \\
29 \% \\
\end{array}$ & $\begin{array}{c}\$ 2.9 \\
5 \% \\
\end{array}$ & $\begin{array}{c}\$ 19.6 \\
32 \%\end{array}$ & $\begin{array}{c}\$ 43.5 \\
71 \% \\
\end{array}$ & $\$ 61.1$ \\
\hline \multirow{3}{*}{$\begin{array}{c}\text { Canisters and } \\
\text { Bare Fuel - } \\
\text { Re-Package } \\
\text { at Repository } \\
\text { (Case 2) }\end{array}$} & 1,500 & 2020 & 2040 & 4 & $\begin{array}{c}\$ 47.8 \\
52 \%\end{array}$ & $\begin{array}{c}3.8 \\
4 \%\end{array}$ & $\begin{array}{c}22.5 \\
25 \%\end{array}$ & $\begin{array}{c}4.4 \\
5 \%\end{array}$ & $\begin{array}{c}12.5 \\
14 \%\end{array}$ & $\begin{array}{c}43.2 \\
48 \%\end{array}$ & \$\$91.0 \\
\hline & 3,000 & 2020 & 2040 & 4 & $\begin{array}{c}\$ 27.2 \\
30 \%\end{array}$ & $\begin{array}{c}\$ 4.1 \\
5 \%\end{array}$ & $\begin{array}{c}\$ 40.2 \\
45 \%\end{array}$ & $\begin{array}{c}\$ 3.4 \\
4 \%\end{array}$ & $\begin{array}{c}\$ 14.4 \\
16 \%\end{array}$ & $\begin{array}{c}\$ 62.1 \\
70 \%\end{array}$ & $\$ 89.3$ \\
\hline & 6,000 & 2020 & 2040 & 4 & $\begin{array}{c}\$ 17.6 \\
16 \% \\
\end{array}$ & $\begin{array}{c}\$ 5.5 \\
5 \% \\
\end{array}$ & $\begin{array}{c}\$ 67.2 \\
59 \% \\
\end{array}$ & $\begin{array}{c}\$ 2.9 \\
3 \% \\
\end{array}$ & $\begin{array}{c}\$ 20.3 \\
18 \% \\
\end{array}$ & $\begin{array}{r}\$ 95.9 \\
84 \% \\
\end{array}$ & $\$ 113.5$ \\
\hline \multirow{3}{*}{$\begin{array}{l}\text { Canisters } \\
\text { Only - Re- } \\
\text { Package at } \\
\text { CSF } \\
\text { (Case 3) }\end{array}$} & 1,500 & 2020 & 2040 & 4 & $\begin{array}{c}\$ 47.0 \\
53 \% \\
\end{array}$ & $\begin{array}{c}13.4 \\
15 \% \\
\end{array}$ & $\begin{array}{c}10.3 \\
12 \%\end{array}$ & $\begin{array}{c}\$ 4.4 \\
5 \%\end{array}$ & $\begin{array}{c}13.6 \\
15 \%\end{array}$ & $\begin{array}{c}41.8 \\
47 \%\end{array}$ & "\$88.8 \\
\hline & 3,000 & 2020 & 2040 & 4 & $\begin{array}{c}\$ 25.6 \\
35 \%\end{array}$ & $\begin{array}{c}\$ 13.4 \\
18 \%\end{array}$ & $\begin{array}{c}\$ 15.2 \\
21 \%\end{array}$ & $\begin{array}{c}\$ 3.4 \\
5 \%\end{array}$ & $\begin{array}{c}\$ 15.8 \\
22 \%\end{array}$ & $\begin{array}{c}\$ 47.8 \\
65 \%\end{array}$ & $\$ 73.3$ \\
\hline & 6,000 & 2020 & 2040 & 4 & $\begin{array}{c}\$ 15.4 \\
21 \%\end{array}$ & $\begin{array}{c}\$ 14.0 \\
19 \%\end{array}$ & $\begin{array}{c}\$ 18.0 \\
24 \%\end{array}$ & $\begin{array}{c}\$ 2.9 \\
4 \%\end{array}$ & $\begin{array}{c}\$ 24.6 \\
33 \%\end{array}$ & $\begin{array}{c}\$ 59.6 \\
79 \%\end{array}$ & $\$ 75.0$ \\
\hline \multirow{3}{*}{\begin{tabular}{|c|} 
Canisters and \\
Bare Fuel - \\
Re-Package \\
at CSF \\
(Case 4)
\end{tabular}} & 1,500 & 2020 & 2040 & 4 & $\begin{array}{c}47.0 \\
43 \%\end{array}$ & $\begin{array}{c}15.0 \\
14 \%\end{array}$ & $\begin{array}{c}28.7 \\
27 \%\end{array}$ & $\begin{array}{l}\$ 4.4 \\
4 \%\end{array}$ & $\begin{array}{c}13.0 \\
12 \%\end{array}$ & $\begin{array}{c}561.1 \\
57 \%\end{array}$ & \$108.2 \\
\hline & 3,000 & 2020 & 2040 & 4 & $\begin{array}{c}\$ 25.6 \\
23 \%\end{array}$ & $\begin{array}{c}\$ 15.3 \\
14 \%\end{array}$ & $\begin{array}{c}\$ 50.1 \\
45 \%\end{array}$ & $\begin{array}{c}\$ 3.4 \\
3 \%\end{array}$ & $\begin{array}{c}\$ 15.8 \\
14 \%\end{array}$ & $\begin{array}{c}\$ 84.5 \\
77 \%\end{array}$ & $\$ 110.1$ \\
\hline & 6,000 & 2020 & 2040 & 4 & $\begin{array}{c}\$ 15.4 \\
13 \%\end{array}$ & $\begin{array}{c}\$ 16.3 \\
13 \%\end{array}$ & $\begin{array}{c}\$ 67.1 \\
55 \%\end{array}$ & $\begin{array}{c}\$ 2.9 \\
2 \%\end{array}$ & $\begin{array}{c}\$ 21.2 \\
17 \%\end{array}$ & $\begin{array}{c}\$ 107.5 \\
87 \%\end{array}$ & $\$ 122.8$ \\
\hline
\end{tabular}

Note: Percentages for each category calculated as LCC for the category divided by the Total UNF Management LCC 
Table 6-14. Normalized UNF Management Cost

\begin{tabular}{|c|c|c|c|c|c|c|c|c|c|c|c|}
\hline Scenario & $\begin{array}{l}\text { Acceptance } \\
\text { Rate }\end{array}$ & CSF Start & $\begin{array}{c}\text { Repository } \\
\text { Start }\end{array}$ & $\begin{array}{l}\text { Disposal } \\
\text { Canister } \\
\text { Size }\end{array}$ & $\begin{array}{l}\text { At-Reactor Total } \\
\text { Life Cycle } \\
\text { FY } 2012(\$ / \mathrm{kg})\end{array}$ & $\begin{array}{l}\text { Transportation } \\
\text { Total Life Cycle } \\
\text { FY } 2012(\$ / \mathrm{kg})\end{array}$ & $\begin{array}{l}\text { CSF Total Life } \\
\text { Cycle } \\
\text { FY } 2012(\$ / \mathrm{kg})\end{array}$ & $\begin{array}{l}\text { TVF Total Life } \\
\text { Cycle } \\
\text { FY } 2012(\$ / \mathrm{kg})\end{array}$ & \begin{tabular}{|c|} 
RF Total Life Cycle \\
FY $2012(\$ / \mathrm{kg})$ \\
\end{tabular} & \begin{tabular}{|c|} 
Away from \\
Reactor UNF \\
Management Life \\
Cycle \\
FY $2012(\$ / \mathrm{kg})$ \\
\end{tabular} & $\begin{array}{c}\text { Total UNF } \\
\text { Management Life } \\
\text { Cycle } \\
\text { FY } 2012(\$ / \mathrm{kg})\end{array}$ \\
\hline \multirow{9}{*}{$\begin{array}{l}\text { Canisters } \\
\text { Only - } \\
\text { Re-Package } \\
\text { at Repository } \\
\text { (Case 1) }\end{array}$} & 1,500 & 2020 & 2040 & 4 & $\$ 341.1$ & $\$ 22.9$ & $\$ 53.6$ & $\$ 31.5$ & $\$ 85.6$ & $\$ 193.7$ & $\$ 534.8$ \\
\hline & 3,000 & 2020 & 2040 & 4 & $\$ 193.9$ & $\$ 22.5$ & $\$ 80.5$ & $\$ 24.5$ & $\$ 102.5$ & $\$ 230.0$ & $\$ 423.9$ \\
\hline & 3,000 & 2020 & 2040 & 12 & $\$ 193.9$ & $\$ 22.5$ & $\$ 80.5$ & $\$ 24.5$ & $\$ 59.8$ & $\$ 187.3$ & $\$ 381.2$ \\
\hline & 3,000 & 2020 & 2040 & 21 & $\$ 193.9$ & $\$ 22.5$ & $\$ 80.5$ & $\$ 24.5$ & $\$ 47.0$ & $\$ 174.4$ & $\$ 368.4$ \\
\hline & 6,000 & 2020 & 2040 & 4 & $\$ 125.7$ & $\$ 24.5$ & $\$ 125.3$ & $\$ 21.0$ & $\$ 140.2$ & $\$ 311.1$ & $\$ 436.7$ \\
\hline & 1,500 & 2020 & 2055 & 4 & $\$ 341.1$ & $\$ 23.8$ & $\$ 85.4$ & $\$ 33.8$ & $\$ 85.6$ & $\$ 228.6$ & $\$ 569.7$ \\
\hline & 3,000 & 2020 & 2055 & 4 & $\$ 193.9$ & $\$ 24.2$ & $\$ 145.4$ & $\$ 26.7$ & $\$ 102.5$ & $\$ 298.8$ & $\$ 492.8$ \\
\hline & 6,000 & 2020 & 2055 & 4 & $\$ 125.7$ & $\$ 27.1$ & $\$ 183.4$ & $\$ 23.2$ & $\$ 144.9$ & $\$ 378.6$ & $\$ 504.3$ \\
\hline & 3,000 & 2035 & 2055 & 4 & $\$ 263.2$ & $\$ 21.9$ & $\$ 92.2$ & $\$ 24.5$ & $\$ 96.9$ & $\$ 235.5$ & $\$ 498.7$ \\
\hline \multirow{9}{*}{$\begin{array}{l}\text { Canisters and } \\
\text { Bare Fuel - } \\
\text { Re-Package } \\
\text { at Repository } \\
\text { (Case 2) }\end{array}$} & 1,500 & 2020 & 2040 & 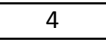 & $\$ 341.1$ & $\begin{array}{l}\$ 27.4 \\
\end{array}$ & \$160.7 & $\begin{array}{l}\$ 31.5 \\
\end{array}$ & $\$ 89.2$ & \$308.8 & $\$ 650.0$ \\
\hline & 3,000 & 2020 & 2040 & 4 & $\$ 193.9$ & $\$ 29.6$ & $\$ 286.9$ & $\$ 24.5$ & $\$ 102.7$ & $\$ 443.7$ & $\$ 637.6$ \\
\hline & 3,000 & 2020 & 2040 & 12 & $\$ 193.9$ & $\$ 29.6$ & $\$ 286.9$ & $\$ 24.5$ & $\$ 61.0$ & $\$ 402.0$ & $\$ 595.9$ \\
\hline & 3,000 & 2020 & 2040 & 21 & $\$ 193.9$ & $\$ 29.6$ & $\$ 286.9$ & $\$ 24.5$ & $\$ 51.1$ & $\$ 392.1$ & $\$ 586.0$ \\
\hline & 6,000 & 2020 & 2040 & 4 & $\$ 125.7$ & $\$ 39.1$ & $\$ 480.3$ & $\$ 21.0$ & $\$ 144.7$ & $\$ 685.1$ & $\$ 810.8$ \\
\hline & 1,500 & 2020 & 2055 & 4 & $\$ 341.1$ & $\$ 28.7$ & $\$ 227.2$ & $\$ 33.7$ & $\$ 89.2$ & $\$ 378.8$ & $\$ 719.9$ \\
\hline & 3,000 & 2020 & 2055 & 4 & $\$ 193.9$ & $\$ 32.0$ & $\$ 417.3$ & $\$ 26.7$ & $\$ 102.7$ & $\$ 578.6$ & $\$ 772.5$ \\
\hline & 6,000 & 2020 & 2055 & 4 & $\$ 125.7$ & $\$ 40.0$ & $\$ 562.5$ & $\$ 23.2$ & $\$ 138.1$ & $\$ 763.7$ & $\$ 889.4$ \\
\hline & 3,000 & 2035 & 2055 & 4 & $\$ 263.2$ & $\$ 27.2$ & $\$ 217.9$ & $\$ 24.5$ & $\$ 100.5$ & $\$ 370.0$ & $\$ 633.2$ \\
\hline \multirow{9}{*}{$\begin{array}{c}\text { Canisters } \\
\text { Only - Re- } \\
\text { Package at } \\
\text { CSF } \\
\text { (Case 3) }\end{array}$} & 1,500 & 2020 & 2040 & 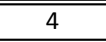 & "\$336.0 & $\begin{array}{l}\$ 95.9 \\
\end{array}$ & $\begin{array}{l}\$ 73.9 \\
\end{array}$ & $\begin{array}{l}\$ 31.6 \\
\end{array}$ & "\$97.2 & "\$298.6 & $\begin{array}{l}\$ 634.6 \\
\end{array}$ \\
\hline & 3,000 & 2020 & 2040 & 4 & $\$ 182.5$ & $\$ 95.4$ & $\$ 108.3$ & $\$ 24.5$ & $\$ 112.9$ & $\$ 341.1$ & $\$ 523.7$ \\
\hline & 3,000 & 2020 & 2040 & 12 & $\$ 182.5$ & $\$ 42.1$ & $\$ 108.3$ & $\$ 24.5$ & $\$ 62.1$ & $\$ 237.1$ & $\$ 419.6$ \\
\hline & 3,000 & 2020 & 2040 & 21 & $\$ 182.5$ & $\$ 30.3$ & $\$ 108.3$ & $\$ 24.5$ & $\$ 48.2$ & $\$ 211.3$ & $\$ 393.8$ \\
\hline & 6,000 & 2020 & 2040 & 4 & $\$ 109.8$ & $\$ 100.2$ & $\$ 128.7$ & $\$ 21.0$ & $\$ 175.9$ & $\$ 425.8$ & $\$ 535.6$ \\
\hline & 1,500 & 2020 & 2055 & 4 & $\$ 336.0$ & $\$ 96.0$ & $\$ 112.9$ & $\$ 33.8$ & $\$ 97.2$ & $\$ 339.9$ & $\$ 675.9$ \\
\hline & 3,000 & 2020 & 2055 & 4 & $\$ 182.5$ & $\$ 95.6$ & $\$ 163.5$ & $\$ 26.8$ & $\$ 112.9$ & $\$ 398.7$ & $\$ 581.2$ \\
\hline & 6,000 & 2020 & 2055 & 4 & $\$ 109.8$ & $\$ 100.0$ & $\$ 183.7$ & $\$ 23.3$ & $\$ 175.9$ & $\$ 482.8$ & $\$ 592.6$ \\
\hline & 3,000 & 2035 & 2055 & 4 & $\$ 257.9$ & $\$ 95.0$ & $\$ 112.5$ & $\$ 24.5$ & $\$ 112.7$ & $\$ 344.8$ & $\$ 602.7$ \\
\hline \multirow{9}{*}{$\begin{array}{c}\text { Canisters and } \\
\text { Bare Fuel - } \\
\text { Re-Package } \\
\text { at CSF } \\
\text { (Case 4) }\end{array}$} & 1,500 & 2020 & 2040 & 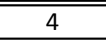 & "\$336.0 & "\$107.4 & "\$205.1 & $\begin{array}{l}31.3 \\
\end{array}$ & "\$92.8 & "\$436.6 & 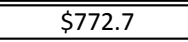 \\
\hline & 3,000 & 2020 & 2040 & 4 & $\$ 182.5$ & $\$ 109.3$ & $\$ 357.7$ & $\$ 24.2$ & $\$ 112.6$ & $\$ 603.9$ & $\$ 786.4$ \\
\hline & 3,000 & 2020 & 2040 & 12 & $\$ 182.5$ & $\$ 50.7$ & $\$ 357.7$ & $\$ 24.2$ & $\$ 61.8$ & $\$ 494.5$ & $\$ 677.0$ \\
\hline & 3,000 & 2020 & 2040 & 21 & $\$ 182.5$ & $\$ 37.8$ & $\$ 357.7$ & $\$ 24.2$ & $\$ 47.9$ & $\$ 467.6$ & $\$ 650.1$ \\
\hline & 6,000 & 2020 & 2040 & 4 & $\$ 109.8$ & $\$ 116.4$ & $\$ 479.2$ & $\$ 20.7$ & $\$ 151.3$ & $\$ 767.6$ & $\$ 877.5$ \\
\hline & 1,500 & 2020 & 2055 & 4 & $\$ 336.0$ & $\$ 107.1$ & $\$ 287.7$ & $\$ 33.3$ & $\$ 92.9$ & $\$ 520.9$ & $\$ 857.0$ \\
\hline & 3,000 & 2020 & 2055 & 4 & $\$ 182.5$ & $\$ 109.0$ & $\$ 460.2$ & $\$ 26.3$ & $\$ 112.6$ & $\$ 708.1$ & $\$ 890.6$ \\
\hline & 6,000 & 2020 & 2055 & 4 & $\$ 109.8$ & $\$ 116.1$ & $\$ 561.4$ & $\$ 22.8$ & $\$ 151.3$ & $\$ 851.5$ & $\$ 961.3$ \\
\hline & 3,000 & 2035 & 2055 & 4 & $\$ 257.9$ & $\$ 106.5$ & $\$ 267.6$ & $\$ 24.2$ & $\$ 111.3$ & $\$ 509.6$ & $\$ 767.4$ \\
\hline
\end{tabular}




\section{CONCLUSIONS}

The objectives of the Fiscal Year 2012 Used Fuel Management System Architecture Evaluation were achieved. Methodologies, approaches, and tools were developed (Capability Development) and used to evaluate select UNF disposition scenarios (Capability Demonstration). This effort re-established an important, foundational capability to assess potential UNF management options. The purpose of the evaluation and analysis at this stage is to use insights gained to refine and focus the next phase of analysis, rather than to provide a basis for any decisions about the design of operation of the waste management system.

The rate that $U N F$ is processed has a significant effect on the used fuel management system: The results of this evaluation confirmed that the rate that UNF is transported between facilities, received at a CSF or a repository, and processed through a packaging/re-packaging facility effects the size of the facilities and associated infrastructure and the associated costs. Larger throughput rates result in larger facilities and higher costs.

There is also a trade-off with higher acceptance rates resulting in reduced at-reactor storage requirements, but larger facilities down-stream in the waste management system. A large UNF acceptance rate, 6000 MTHM/yr, showed only incremental benefit in reducing on-site storage, but resulted in the largest facility requirements down-stream.

Thermal considerations have a major impact on the operation of the system: The entire UNF management system will have thermal constraints. There are thermal limits on storage canisters, transportation overpacks/casks, and on geologic media. Thermal constraints on transportation, which are more stringent than the constraints on storage canisters, mean that loading fuel into very large storage canisters at reactor sites may require storage of those canisters for decades before they could be moved due to thermal limits on the transportation overpacks/casks. These thermal constraints become more of an issue for higher UNF acceptance rates from the reactors because the older, cooler fuel is transported from the reactor sites relatively soon after acceptance begins, leaving the hotter, younger fuel to be managed. Lower acceptance rates allow this fuel to cool sufficiently and it can be transported.

Thermal limits for geologic disposal design concepts, not considered in this evaluation, could also require extended decay storage and/or the blending of UNF during packaging/re-packaging, potentially impacting when UNF could be shipped to a repository and how it would be managed at the CSF or repository.

The implications of these thermal constraints and potential UNF management alternatives should be evaluated further.

A large acceptance rate, on the order of $6000 \mathrm{MTHM} / \mathrm{yr}$ may result in under-utilized facilities and may not be cost-effective: The analysis of a $6000 \mathrm{MTHM} / \mathrm{yr}$ acceptance rate, which is nearly three times the used fuel generation rate ( 2000 MTHM/yr), shows that such a high rate may not be cost-effective in the long run. Even though it removes fuel from reactor sites more quickly, and thereby reduces the costs of at reactor storage, it requires large initial infrastructure expenditure in both transportation equipment and receipt facilities at the CSF that can only be used at the full capacity for a relatively short period of time. This is due to fuel shipment restrictions based on thermal constraints. A $6000 \mathrm{MTHM} / \mathrm{yr}$ acceptance rate only occurs at the beginning of operation of the system until these thermal constraints restrict the achievable acceptance rate to a rate matching UNF discharge from the reactors (around $2000 \mathrm{MTHM} / \mathrm{yr}$ ). It is worth exploring rates in the area of 4000-4500 MTHM/yr to determine if some of the benefits of an increased acceptance rate can be achieved without the spikes in UNF shipments that occur and underutilization issues observed in the $6000 \mathrm{MTHM} / \mathrm{yr}$ acceptance scenarios. 
The start of $U N F$ acceptance from the reactors and the UNF acceptance rate will impact on-site dry storage requirements: The results presented in this report show that there is a significant decrease in the maximum amount of at-reactor dry storage required when the acceptance rate increases from 1500 MHMT/yr to $3000 \mathrm{MTHM} / \mathrm{yr}$. However, there is a much smaller reduction in the maximum at-reactor dry storage capacity required when the acceptance rate is increased from $3000 \mathrm{MTHM} / \mathrm{yr}$ to 6000 MTHM/yr.

Higher acceptance rates (3000 MTHM/yr, $6000 \mathrm{MTHM} / \mathrm{yr}$ ) may not eliminate need for additional on-site dry storage when reactor fleet begins to shut down unless acceptance is "managed." A youngest-fuel-first acceptance preference will still require additional dry storage when reactors shut down. An oldest-fuelfirst acceptance preference would require additional dry storage both during reactor operation and when the reactors shut down as preference would be given to shipping UNF already in dry storage from the reactor sites.

An oldest-fuel-first (OFF) acceptance preference would also require continued at-reactor dry storage. Generally, older fuel is the first UNF loaded into dry storage and would be the first shipped under such an acceptance preference. Since little fuel would be shipped directly from the used fuel pools, the reactors would have to transfer fuel from the pools to dry storage to maintain pool capacity.

Acceptance start date and acceptance rates can reduce flexibility: Lower UNF acceptance rates or delay in start of acceptance results in more UNF being placed into existing large canisters for at-reactor dry storage. This "hardens" this "boundary condition," resulting in reduced flexibility later.

The transportation capital cost is driven by the number of casks and transportation assets that need to be purchased during the campaign. The rate that UNF is transported between the reactors, CSF, and repository and the cycle time needed to transport the UNF affect the size of the transportation fleet. While the location of the storage facilities and repository drive the transportation time, transportation is actually only a small contributor to the overall cycle time. Loading, unloading, and maintenance activities make up the majority of the cycle time. Therefore, the acquisitions required are for the most part relatively independent of the location of the storage facility and repository.

Acceptance priority assumptions have a significant impact on the UNF management system: The management of UNF at the reactor sites define the "boundary condition" to which the system will respond. The acceptance priority assumptions, rates, and start dates assumed for this evaluation have a very significant impact on the transportation system and on the sizing of facilities. The preliminary analyses presented here suggest that there would be value to examining modifications to the acceptance priorities that would smooth out some of the sharp peaks and fluctuations that result from strict adherence to simple but rigid priority rules.

Alternate strategies for acceptance from reactors and subsequent shipment to a repository may allow for optimization of down-stream facilities: This initial evaluation assumed first-in-first-out shipping of UNF from the CSF to the repository. It is unlikely that the waste management system would be operated in this manner. A CSF could be treated as an integrated UNF management facility to act as a buffer between at-reactor UNF management needs and future repository requirements. This would allow for optimizing shipments from reactors to minimize additional on-site dry storage requirements and optimizing shipments from the CSF to the repository to meet repository requirements while minimizing processing facility requirements.

In addition, a managed UNF acceptance rate, perhaps giving preference to removing fuel from sites that are either shutdown or approaching shutdown, could potentially reduce long-term at-reactor dry storage requirements.

System benefits may also be gained by de-coupling acceptance rates from the reactors to a CSF with shipping rates between the CSF and a repository. For example, a shorter emplacement period with a 
higher emplacement rate at the repository than the acceptance rate from the reactors to the CSF combined with a later repository start could result in a large inventory of spent fuel at the CSF that could be more efficiently processed when the repository begins operating.

Such approaches may require additional CSF storage capacity. Additional evaluation of these approaches is needed.

A large-scale UNF handling effort will be needed regardless of the UNF management strategy, acceptance rates, and acceptance start dates: There will always be a need to re-package large canisters unless the direct disposability of such canisters is shown to be feasible. Approximately 11,200 canisters will have to be re-packaged if all UNF is placed in such canisters. Maintaining some fraction of the UNF as bare fuel at central storage facilities can reduce the number of canisters that would have to be repackaged. However, placing the entire UNF inventory in large canisters does not appear to require an increase in the packaging/re-packaging facility capabilities versus maintaining some fraction of the UNF as bare fuel. In addition, the capability to store bare fuel in addition to storing UNF in canisters would be required. There could also be broader system-level impacts associated with maintaining a fraction of the UNF as bare fuel that have yet to be evaluated (e.g., worker dose). Any potential benefit of not having to re-open canisters reduces for lower acceptance rates and/or delay in the start of acceptance.

Smaller waste package sizes have a significant impact on packaging/re-packaging facility and transportation system requirements. Processing a large number of smaller disposal canisters could result in the need for larger packaging/re-packaging facilities and a larger transportation infrastructure to meet the desired system throughput. Future work investigating alternative disposal canister/overpack and transportation equipment design concepts may identify more efficient concepts.

Bare fuel storage in wet pools at a CSF will likely lead to high CSF and overall system life-cycle costs. Future analyses of scenarios involving CSF storage of bare UNF should investigate alternative bare fuel storage concepts (such as dry storage vaults or single-purpose bolted lid bare UNF storage casks).

At-Reactor operational and logistic constraints could affect the actual rate that UNF could be loaded into dry storage canisters or transported off-site. Logistic analyses typically assume that there are no constraints on the ability of the reactor sites to load dry storage systems or transport UNF off-site. These assumptions could potentially be challenged while a reactor is in operation due to multiple requirements and demands on the used fuel pool during and operating fuel cycle. Such demands include receipt of fresh fuel, core re-load, fuel inspections/repair, and maintenance of the spent fuel pool. The actual window where fuel handling could occur may constrain the amount of used fuel that could be transferred to dry storage when either multiple fuel handling activities occur within a given operating fuel cycle (transfer to dry storage and loading for shipping off-site) or potentially when smaller capacity canisters are loaded (waste package compatible size canisters). These constraints should be further explored and their impacts on at-reactor logistics evaluated.

Rough order of magnitude life cycle cost (ROM LCC) estimates of the entire nuclear waste management system varied depending on the scenario. The estimated ROM LCC was found to be approximately $10 \%$ to $20 \%$ larger when UNF packaging/re-packaging is performed at the CSF versus at the repository. The estimated ROM LCC was also found to be approximately $20 \%$ to $85 \%$ larger than when all the UNF is loaded into existing size canisters at the reactor sites.

Higher throughput requires larger facilities and infrastructure. When considering the total away-fromreactor estimated ROM LCC, the lowest cost occurs for a UNF processing rate of $1500 \mathrm{MTHM} / \mathrm{yr}$ when re-packaging is done at the CSF and for a UNF processing rate of $3000 \mathrm{MTHM} / \mathrm{yr}$ when re-packaging is done at the repository.

However, the overall estimated ROM LCC of UNF management (all aspects of the UNF management system) is lowest when the UNF processing rate (acceptance from reactors, shipment from CSF to 
repository) is $3000 \mathrm{MTHM} / \mathrm{yr}$, CSF operations begin in 2020, and repository operations begin in 2040 for each of the major cases considered..

Delaying the start of repository operations from 2040 to 2055 increases the estimated total UNF management ROM LCC by $5 \%-10 \%$ for a $1500 \mathrm{MTHM} / \mathrm{yr}$ throughput rate, $15 \%-20 \%$ for a throughput rate of $3000 \mathrm{MTHM} / \mathrm{yr}$, and $10 \%$ - 15\% for a throughput rate of $6000 \mathrm{MTHM} / \mathrm{yr}$. 


\section{REFERENCES}

Blue Ribbon Commission for America's Nuclear Future, Report to the Secretary of Energy, January 2012. Available at

http://cybercemetery.unt.edu/archive/brc/20120620220235/http://brc.gov/sites/default/files/documents/brc finalreport_jan2012.pdf

Brady, P.V., Arnold, B.W., Freeze, G.A., Swift, P.N., Bauer, S.J., Kanney, J.L., Rechard, R.P., Stein, J.S., Deep Borehole Disposal of High-Level Radioactive Waste, SAND2009-4401, August 2009.

Busch, I., Howard, R., Transportation Operations Model (TOM) Technical Manual, FCRD-NFST-2012000425 , October 2012.

Carter, J.T. Luptak, A.J., Gastelum, J., Stockman, C., Miller, A., Fuel Cycle Potential Waste Inventory for Disposition, FCR\&D-USED-2010-000031 Rev 5, July 2012.

Carter, J., Delley, A., et al., Used Fuel Research and Development Test and Validation Facility Cost Study, FCRD-UFD-2012-000206, Rev. 0, August 2012.

Hardin E., Blink, J., Greenberg, H., Sutton, M., Fratoni, M., Carter, J., Dupont, M. Howard, R., Generic Design Concepts and Thermal Analysis (FY11), FCRD-USED-2011-000143 REV. 0, August 2011.

Hardin, E., Hadgu, T., Clayton, D., Howard, R. Greenberg, H., Blink, J., Sharma, M., Sutton, M. Carter., J. Dupont, M. Rodwell, P., Disposal Concepts/Thermal Load Management (FY11/12 Summary Report), FCRD-USED-2012-000219 REV. 1, September 2012.

Howard, R., Scaglione, J., Wagner, J., Hardin, E., Nutt, M., Implementation Plan for the Development and Licensing of Standardized Transportation, Aging, and Disposal Canisters and the Feasibility of Direct Disposal of Dual Purpose Canisters, FCRD-UFD-2012-000106, May 14, 2012.

Kalinina, E., Calvin Database Update in Support of UFD System Architecture Study, FCRD-UFD-2012000135, May 2012.

Leduc D.R., Carter J.T., Dry Storage of Used Fuel Transition to Transport, FCRD-UFD-2012-000253, August 2012.

McConnell S., Ostby, D.W. et al., Engineering Alternative Studies (EAS) for Separations-Summary Report, EAS-G-ESR-G-00049, June 2007.

Nutt, M., Morris, E., Puig, F., Kalinina, E., Gillespie, S., Transportation Storage Logistics Model CALVIN (TSL-CALVIN) , FCRD-NFST-2012-000424, October 2012.

Rechard R.P., Goldstein, B., Brush, L.H., Blink, J.A., Sutton, M., Perry, F.V., Basis for Identification of Disposal Options for Research and Development for Spent Nuclear Fuel and High-Level Waste, FCRDUSED-2011-000071, March 2011.

Rodwell, P., Geddes, R., McConnell, S., Follow-on Engineering Alternative Studies for Separations (FOEAS), Early Fuel Receipt \& Storage (EFR\&S) Considerations, FRS-G-ESR-G-00053, Rev. 0, September 2007. 
Ux Consulting, StoreFuel and Decommissioning Report, Vol 13 No 169, September 4, 2011

U.S. Department of Energy, Follow-on Engineering Alternative Studies (FOEAS) Summary Report, Global Nuclear Energy Partnership (GNEP), Consolidated Fuel Treatment Center, GNEP-CFTC-PMOMI-DV-2008-00087, March 2008.

U.S. Nuclear Regulatory Commission, U.S. Independent Spent Fuel Storage Installations - Current as of November 2010, Available at http://www.nrc.gov/waste/spent-fuel-storage/locations.pdf

U.S. Nuclear Regulatory Commission, Information Digest 2012-2013, NUREG-1350, Volume 24, August 2012. 


\section{APPENDIX A AT-REACTOR LOGISTICS RESULTS}

\section{A-1. At-Reactor Used Fuel Management}

This section discusses the management of used nuclear fuel at reactor sites in the U.S. The first section describes the overall strategy for managing UNF both during normal reactors and when the reactors are shut down. This section also discusses the types of dry storage systems currently in use and the general processes for loading on-site dry storage facilities. A discussion of the processes used for transporting UNF from the reactor sites and potential re-useable transportation casks systems is then presented. Last, assumptions used in the conduct of logistic evaluations of at-reactor management and cost estimating information are then presented.

\section{A-1.1 Used Fuel Management Strategy}

This section discusses the current strategy in use at the existing reactor fleet for managing UNF while the reactors are in operation and when they are shut down.

\section{A-1.1.1 Normal Operations}

UNF is initially stored at the nuclear plants in water filled pools. Typically, a power plant desires to maintain sufficient capacity within the used fuel pool to off-load the entire content of the reactor core to the pool. The used fuel pools were not originally designed for long-term storage and the nuclear utilities have implemented two strategies for managing used nuclear fuel on-site. First, the fuel assembly capacity of most used fuel pools has been increased, typically termed as "re-racking" the pool. This increased the capacity of the used fuel pools beyond the original design.

However, even with increased capacity many facilities have run out of capacity to store all of the UNF in their pools. At these facilities, above ground dry storage systems are utilized to store the UNF. As more facilities run out of pool storage the amount of used fuel placed in dry storage will increase. Through 2012, it is estimated that the commercial nuclear industry will have generated an estimated approximately 70,000 MTU of UNF contained in approximately 242,000 assemblies (175,000 from BWRs and 105,000 from PWRs) (Carter et al. 2012). It is projected that by 2020, total UNF discharges will be approximately 88,000 MTU (Carter et al. 2012). By 2060, when all currently licensed reactors will have reached the end of their operational lives, assuming a 60-year maximum, there will be approximately 140,000 MTU of UNF in storage (Carter et al. 2012). According to data collected by Ux Consulting, a total of 65,261 assemblies were being stored in 1,650 casks (UxC 2012), with approximately $73 \%$ of the total fuel inventory discharged remaining in wet storage.

In order to decrease the risk of terrorism and to decrease the potentially vulnerability of the used fuel pools during severe accidents, it has been suggested that used nuclear fuel be moved to dry storage as early as possible, for example five years after discharge from the reactors. The Electric Power Research Institute evaluated both the economic and worker exposure impacts of such early movement of spent fuel and concluded that the impacts would be significant while providing no safety benefit to the public (EPRI 2010a). However, this is being evaluated by the U.S. NRC as reported at the Nuclear Waste Technical Review Board meeting on October $17^{\text {th }}, 2012$. 


\section{A-1.1.2 Reactor Shutdown}

When a reactor is shut down all fuel must be removed from the used fuel pool before the plant can be decommissioned and demolished. At present, there are nine sites where plants that have been shutdown. These sites are shown in Table A-1. The used nuclear fuel at seven of the sites has been transferred from the pool to dry storage and the plants have been decommissioned and demolished. The fuel at the LaCrosse and Zion sites has just begun to move or is planned to be moved to dry storage in the near-term and those plants will then be decommissioned and demolished.

Table A-1. Shutdown Reactor Sites

\begin{tabular}{|l|c|c|c|c|c|c|}
\hline Reactor Site & Type & $\begin{array}{c}\text { Shutdown } \\
\text { Date }\end{array}$ & $\begin{array}{c}\text { ISFSI Load } \\
\text { Date }\end{array}$ & $\begin{array}{c}\text { Total } \\
\text { Casks } \\
\text { Fuel/GTCC }\end{array}$ & $\begin{array}{c}\text { Total } \\
\text { Assemblies }\end{array}$ & $\begin{array}{c}\text { Total } \\
\text { MTHM }\end{array}$ \\
\hline Big Rock Point & BWR & $8 / 97$ & $12 / 02-3 / 03$ & $8 / 1$ & 441 & 58 \\
\hline $\begin{array}{l}\text { Connecticut } \\
\text { Yankee }\end{array}$ & PWR & $12 / 96$ & $5 / 04-3 / 05$ & $40 / 3$ & 1019 & 412 \\
\hline Maine Yankee & PWR & $8 / 97$ & $8 / 02-3 / 04$ & $60 / 4$ & 1434 & 29 \\
\hline Yankee Rowe & PWR & $9 / 91$ & $6 / 02-6 / 03$ & $15 / 1$ & 5333 & 127 \\
\hline Rancho Seco & PWR & $6 / 89$ & $4 / 01-8 / 02$ & $21 / 1$ & 493 & 228 \\
\hline Trojan & PWR & $11 / 92$ & $12 / 02-9 / 03$ & $34 /$ & 780 & 359 \\
\hline Humbolt Bay & BWR & $7 / 76$ & $8 / 08-12 / 08$ & $5 / 1$ & 390 & 29 \\
\hline LaCrosse & BWR & $4 / 87$ & Planned 3/12 & 5 (estimated) & 333 & 38 \\
\hline Zion 1 and 2 & PWR & $7 / 98$ & Planned 2013 & $\begin{array}{c}61 \\
\text { (estimated) }\end{array}$ & 2226 & 1018 \\
\hline
\end{tabular}

Table A-2 shows the status of commercial reactor operating licenses, including when the operating license was renewed and the current operating license expires for each reactor (NRC 2011). Some reactors have yet to have their operating license renewed. Table A-2 also shows the anticipated operating license expiration date for those plants assuming they would receive a twenty-year license extension. The current and anticipated license expiration dates shown in Table A-2 were assumed for this evaluation.

There is considerable uncertainty regarding when the used nuclear fuel in the pool at a site that shuts down in the future would be transferred to dry storage and the plant decommissioned and demolished. Some utilities may desire to proceed to decommission and demolish the shutdown plant as soon as possible while others may desire to maintain the plant in a safe-storage mode for a period of time. Table A-1 shows a significant range in time between when a reactor was shutdown and when the used fuel was transitioned to dry storage. However, it must be recognized that only a few reactors have been shutdown and their decommissioning was somewhat of a unique, first-of-a-kind effort. Future reactor decommissioning may be more efficient, resulting in shorter durations between reactor shutdown and fuel off-load.

For this evaluation, it was assumed that the transfer the fuel from the pools of future shutdown reactors to dry storage this transfer occurrs five years after shutdown. As discussed in Section 3.2.4, a variant to Case 2 that assumes the used fuel remains in the pools for a more extended period after reactor shutdown will be evaluated in the future. 
Table A-2. Operating Commercial Reactors

\begin{tabular}{|c|c|c|c|c|}
\hline Plant Name, Unit Number & Licensee & $\begin{array}{l}\text { Renewed } \\
\text { Operating } \\
\text { License } \\
\text { Issued }\end{array}$ & $\begin{array}{l}\text { Current } \\
\text { Operating } \\
\text { License } \\
\text { Expires }\end{array}$ & $\begin{array}{c}\text { Anticipated } \\
\text { Operating } \\
\text { License } \\
\text { Expiration w/ } \\
\text { License Renewal }\end{array}$ \\
\hline Oyster Creek Nuclear Generating Station, Unit 1 & Exelon Generation Co., LLC & $4 / 8 / 09$ & $4 / 9 / 29$ & \\
\hline Nine Mile Point Nuclear Station, Unit 1 & Nine Mile Point Nuclear Station, LLC & $10 / 31 / 06$ & $8 / 22 / 29$ & \\
\hline R.E. Ginna Nuclear Power Plant & R.E. Ginna Nuclear Power Plant, LLC & $5 / 19 / 04$ & $9 / 18 / 29$ & \\
\hline Dresden Nuclear Power Station, Unit 2 & Exelon Generation Co., LLC & $10 / 28 / 04$ & $12 / 22 / 29$ & \\
\hline H. B. Robinson Steam Electric Plant, Unit 2 & Carolina Power \& Light Co., & 4/19/04 & $7 / 31 / 30$ & \\
\hline Monticello Nuclear Generating Plant, Unit 1 & Northern States Power Company & $11 / 8 / 06$ & $9 / 8 / 30$ & \\
\hline Point Beach Nuclear Plant, Unit 1 & FPL Energy Duane Arnold, LLC & $12 / 22 / 05$ & $10 / 5 / 30$ & \\
\hline Dresden Nuclear Power Station, Unit 3 & Exelon Generation Co., LLC & $10 / 28 / 04$ & $1 / 12 / 31$ & \\
\hline Palisades Nuclear Plant & Entergy Nuclear Operations, Inc. & $1 / 17 / 07$ & $3 / 24 / 31$ & \\
\hline Vermont Yankee Nuclear Power Plant, Unit 1 & Entergy Nuclear Operations, Inc. & & $3 / 21 / 12$ & $3 / 16 / 32$ \\
\hline Surry Nuclear Power Station, Unit 1 & Virginia Electric \& Power Co. & $3 / 20 / 03$ & $5 / 25 / 32$ & \\
\hline Pilgrim Nuclear Power Station & Entergy Nuclear Operations, Inc. & & $6 / 8 / 12$ & $6 / 3 / 32$ \\
\hline Turkey Point Nuclear Generating, Unit 3 & Florida Power \& Light Co. & 6/6/02 & 7/19/32 & \\
\hline Quad Cities Nuclear Power Station, Unit 1 & Exelon Generation Co., LLC & $10 / 28 / 04$ & $12 / 14 / 32$ & \\
\hline Quad Cities Nuclear Power Station, Unit 2 & Exelon Generation Co., LLC & $10 / 28 / 04$ & $12 / 14 / 32$ & \\
\hline Surry Nuclear Power Station, Unit 2 & Virginia Electric \& Power Co. & $3 / 20 / 03$ & $1 / 29 / 33$ & \\
\hline Oconee Nuclear Station, Unit 1 & Duke Energy Carolinas, LLC & $5 / 23 / 00$ & $2 / 6 / 33$ & \\
\hline Point Beach Nuclear Plant, Unit 2 & FPL Energy Duane Arnold, LLC & $12 / 22 / 05$ & $3 / 8 / 33$ & \\
\hline Turkey Point Nuclear Generating, Unit 4 & Florida Power \& Light Co. & 6/6/02 & 4/10/33 & \\
\hline Peach Bottom Atomic Power Station, Unit 2 & Exelon Generation Co., LLC & $5 / 7 / 03$ & $8 / 8 / 33$ & \\
\hline Fort Calhoun Station, Unit 1 & Omaha Public Power District & $11 / 4 / 03$ & $8 / 9 / 33$ & \\
\hline Prairie Island Nuclear Generating Plant, Unit 1 & Northern States Power Co. Minnesota & $6 / 27 / 11$ & $8 / 9 / 33$ & \\
\hline Indian Point Nuclear Generating, Unit 2 & Entergy Nuclear Operations, Inc. & & $9 / 28 / 13$ & $9 / 23 / 33$ \\
\hline Oconee Nuclear Station, Unit 2 & Duke Energy Carolinas, LLC & $5 / 23 / 00$ & $10 / 6 / 33$ & \\
\hline Browns Ferry Nuclear Plant, Unit 1 & Tenessee Valley Authority & $5 / 4 / 06$ & $12 / 20 / 33$ & \\
\hline Kewaunee Power Station & Dominion Energy Kewaunee, Inc. & $2 / 24 / 11$ & $12 / 21 / 33$ & \\
\hline Cooper Nuclear Station & Nebraska Public Power District & $11 / 29 / 10$ & $1 / 18 / 34$ & \\
\hline Duane Arnold Energy Center & FPL Energy Duane Arnold, LLC & $12 / 16 / 10$ & $2 / 21 / 34$ & \\
\hline Three Mile Island Nuclear Station, Unit 1 & Exelon Generation Co., LLC & $10 / 22 / 09$ & $4 / 19 / 34$ & \\
\hline Arkansas Nuclear One, Unit 1 & Entergy Nuclear Operations, Inc. & 6/20/01 & $5 / 20 / 34$ & \\
\hline
\end{tabular}


Table A-2. Operating Commercial Reactors (continued)

\begin{tabular}{|c|c|c|c|c|}
\hline Plant Name, Unit Number & Licensee & $\begin{array}{l}\text { Renewed } \\
\text { Operating } \\
\text { License } \\
\text { Issued }\end{array}$ & $\begin{array}{l}\text { Current } \\
\text { Operating } \\
\text { License } \\
\text { Expires }\end{array}$ & $\begin{array}{c}\text { Anticipated } \\
\text { Operating } \\
\text { License } \\
\text { Expiration w/ } \\
\text { License Renewal }\end{array}$ \\
\hline Browns Ferry Nuclear Plant, Unit 2 & Tenessee Valley Authority & $5 / 4 / 06$ & $6 / 28 / 34$ & \\
\hline Peach Bottom Atomic Power Station, Unit 3 & Exelon Generation Co., LLC & $5 / 7 / 03$ & $7 / 2 / 34$ & \\
\hline Oconee Nuclear Station, Unit 3 & Duke Energy Carolinas, LLC & $5 / 23 / 00$ & $7 / 19 / 34$ & \\
\hline Calvert Cliffs Nuclear Power Plant, Unit 1 & Calvert Cliffs Nuclear Power Plant Inc. & $3 / 23 / 00$ & $7 / 31 / 34$ & \\
\hline Edwin I. Hatch Nuclear Plant, Unit 1 & Southern Nuclear Operating Co. & $1 / 15 / 02$ & $8 / 6 / 34$ & \\
\hline James A. FitzPatrick Nuclear Power Plant & Entergy Nuclear Operations, Inc. & 9/8/08 & $10 / 17 / 34$ & \\
\hline Donald C. Cook Nuclear Power Plant, Unit 1 & Indiana Michigan Power Co. & $8 / 30 / 05$ & $10 / 25 / 34$ & \\
\hline Prairie Island Nuclear Generating Plant, Unit 2 & Northern States Power Co. Minnesota & $6 / 27 / 11$ & $10 / 29 / 34$ & \\
\hline Brunswick Steam Electric Plant, Unit 2 & Carolina Power \& Light Co. & $6 / 26 / 06$ & $12 / 27 / 34$ & \\
\hline Millstone Power Station, Unit 2 & Dominion Nuclear Conneticut, Inc. & $11 / 28 / 05$ & $7 / 31 / 35$ & \\
\hline Indian Point Nuclear Generating, Unit 3 & Entergy Nuclear Operations, Inc. & & $12 / 12 / 15$ & $12 / 7 / 35$ \\
\hline Beaver Valley Power Station, Unit 1 & First Energy Nuclear Operating Co. & $11 / 5 / 09$ & $1 / 29 / 36$ & \\
\hline St. Lucie Plant, Unit 1 & Florida Power \& Light Co. & $10 / 2 / 03$ & $3 / 1 / 36$ & \\
\hline Browns Ferry Nuclear Plant, Unit 3 & Tennessee Valley Authority & $5 / 4 / 06$ & $7 / 2 / 36$ & \\
\hline Calvert Cliffs Nuclear Power Plant, Unit 2 & Calvert Cliffs Nuclear Power Plant Inc. & $3 / 23 / 00$ & $8 / 13 / 36$ & \\
\hline Salem Nuclear Generating Station, Unit 1 & PSEG Nuclear, LLC & $6 / 30 / 11$ & $8 / 13 / 36$ & \\
\hline Brunswick Steam Electric Plant, Unit 1 & Carolina Power \& Light Co. & $6 / 26 / 06$ & $9 / 8 / 36$ & \\
\hline Crystal River Nuclear Generating Plant, Unit 3 & Florida Power Corp. & & $12 / 3 / 16$ & $11 / 28 / 36$ \\
\hline Davis-Besse Nuclear Power Station, Unit 1 & First Energy Nuclear Operating Co. & & $4 / 22 / 17$ & $4 / 17 / 37$ \\
\hline Joseph M. Farley Nuclear Plant, Unit 1 & Southern Nuclear Operating Co. & $5 / 12 / 05$ & $6 / 25 / 37$ & \\
\hline Donald C. Cook Nuclear Power Plant, Unit 2 & Indiana Michigan Power Co. & $8 / 30 / 05$ & $12 / 23 / 37$ & \\
\hline North Anna Power Station, Unit 1 & Virginia Electric \& Power Co. & $3 / 20 / 03$ & $4 / 1 / 38$ & \\
\hline Edwin I. Hatch Nuclear Plant, Unit 2 & Southern Nuclear Operating Co. & $1 / 15 / 02$ & $6 / 13 / 38$ & \\
\hline Arkansas Nuclear One, Unit 2 & Entergy Nuclear Operations, Inc. & $6 / 30 / 05$ & $7 / 17 / 38$ & \\
\hline Salem Nuclear Generating Station, Unit 2 & PSEG Nuclear, LLC & $6 / 30 / 11$ & $4 / 18 / 40$ & \\
\hline North Anna Power Station, Unit 2 & Virginia Electric \& Power Co. & $3 / 20 / 03$ & $8 / 21 / 40$ & \\
\hline Sequoyah Nuclear Plant, Unit 1 & Tennessee Valley Authority & & 9/17/20 & $9 / 12 / 40$ \\
\hline Joseph M. Farley Nuclear Plant, Unit 2 & Southern Nuclear Operating Co. & $5 / 12 / 05$ & $3 / 31 / 41$ & \\
\hline McGuire Nuclear Station, Unit 1 & Duke Energy Carolinas, LLC & $12 / 5 / 03$ & $6 / 12 / 41$ & \\
\hline Sequoyah Nuclear Plant, Unit 2 & Tennessee Valley Authority & & $9 / 15 / 21$ & $9 / 10 / 41$ \\
\hline San Onofre Nuclear Generating Station, Unit 2 & Southern California Edison Co. & & $2 / 16 / 22$ & $2 / 11 / 42$ \\
\hline
\end{tabular}


Table A-2. Operating Commercial Reactors (continued)

\begin{tabular}{|c|c|c|c|c|}
\hline Plant Name, Unit Number & Licensee & $\begin{array}{l}\text { Renewed } \\
\text { Operating } \\
\text { License } \\
\text { Issued }\end{array}$ & $\begin{array}{l}\text { Current } \\
\text { Operating } \\
\text { License } \\
\text { Expires }\end{array}$ & $\begin{array}{c}\text { Anticipated } \\
\text { Operating } \\
\text { License } \\
\text { Expiration w/ } \\
\text { License Renewal }\end{array}$ \\
\hline LaSalle County Station, Unit 1 & Exelon Generation Co., LLC & & $4 / 17 / 22$ & $4 / 12 / 42$ \\
\hline Susquehanna Steam Electric Station, Unit 1 & PPL Susquehanna, LLC & $11 / 24 / 09$ & $7 / 17 / 42$ & \\
\hline Virgil C. Summer Nuclear Station, Unit 1 & South Carolina Electric \& Gas Co. & $4 / 23 / 04$ & $8 / 6 / 42$ & \\
\hline San Onofre Nuclear Generating Station, Unit 3 & Southern California Edison Co. & & $11 / 15 / 22$ & $11 / 10 / 42$ \\
\hline McGuire Nuclear Station, Unit 2 & Duke Energy Carolinas, LLC & $12 / 5 / 03$ & $3 / 3 / 43$ & \\
\hline St. Lucie Plant, Unit 2 & Florida Power \& Light Co. & $10 / 2 / 03$ & $4 / 6 / 43$ & \\
\hline Catawba Nuclear Station, Unit 1 & Duke Energy Carolinas, LLC & $12 / 5 / 03$ & $12 / 5 / 43$ & \\
\hline Catawba Nuclear Station, Unit 2 & Duke Energy Carolinas, LLC & $12 / 5 / 03$ & $12 / 5 / 43$ & \\
\hline LaSalle County Station, Unit 2 & Exelon Generation Co., LLC & & $12 / 16 / 23$ & $12 / 11 / 43$ \\
\hline Columbia Generating Station, Unit 2 & Energy Northwest & & $12 / 20 / 23$ & $12 / 15 / 43$ \\
\hline Susquehanna Steam Electric Station, Unit 2 & PPL Susquehanna, LLC & $11 / 24 / 09$ & $3 / 23 / 44$ & \\
\hline Callaway Plant & Union Electric Co. & & $10 / 18 / 24$ & $10 / 13 / 44$ \\
\hline Limerick Generating Station, Unit 1 & Exelon Generation Co., LLC & & $10 / 26 / 24$ & $10 / 21 / 44$ \\
\hline Byron Station, Unit 1 & Exelon Generation Co., LLC & & $10 / 31 / 24$ & $10 / 26 / 44$ \\
\hline Grand Gulf Nuclear Station, Unit 1 & Entergy Nuclear Operations, Inc. & & $11 / 1 / 24$ & $10 / 27 / 44$ \\
\hline Diablo Canyon Nuclear Power Plant, Unit 1 & Pacific Gas \& Electric Co. & & $11 / 2 / 24$ & $10 / 28 / 44$ \\
\hline Waterford Steam Electric Station, Unit 3 & Entergy Nuclear Operations, Inc. & & $12 / 18 / 24$ & $12 / 13 / 44$ \\
\hline Palo Verde Nuclear Generating Station, Unit 1 & Arizona Public Service Company & $4 / 21 / 11$ & $12 / 31 / 44$ & \\
\hline Wolf Creek Generating Station, Unit 1 & Wolf Creek Nuclear Operating Corp. & $11 / 20 / 08$ & $3 / 11 / 45$ & \\
\hline Fermi, Unit 2 & The Detroit Edison Co. & & $3 / 20 / 25$ & $3 / 15 / 45$ \\
\hline Diablo Canyon Nuclear Power Plant, Unit 2 & Pacific Gas \& Electric Co. & & $8 / 26 / 25$ & $8 / 21 / 45$ \\
\hline River Bend Station, Unit 1 & Entergy Nuclear Operations, Inc. & & $8 / 29 / 25$ & $8 / 24 / 45$ \\
\hline Millstone Power Station, Unit 3 & Dominion Nuclear Connecticut, Inc. & $11 / 28 / 05$ & $11 / 25 / 45$ & \\
\hline Perry Nuclear Power Plant, Unit 1 & First Energy Nuclear Operating Co. & & $3 / 18 / 26$ & $3 / 13 / 46$ \\
\hline Hope Creek Generating Station, Unit 1 & PSEG Nuclear, LLC & $7 / 20 / 11$ & $4 / 11 / 46$ & \\
\hline Palo Verde Nuclear Generating Station, Unit 2 & Arizona Public Service Company & $4 / 21 / 11$ & $4 / 24 / 46$ & \\
\hline Clinton Power Station, Unit 1 & Exelon Generation Co., LLC & & $9 / 29 / 26$ & 9/24/46 \\
\hline Braidwood Station, Unit 1 & Exelon Generation Co., LLC & & $10 / 17 / 26$ & $10 / 12 / 46$ \\
\hline Shearon Harris Nuclear Power Plant, Unit 1 & Carolina Power \& Light Co. & $12 / 17 / 08$ & $10 / 24 / 46$ & \\
\hline Nine Mile Point Nuclear Station, Unit 2 & Nine Mile Point Nuclear Station, LLC & $10 / 31 / 06$ & $10 / 31 / 46$ & \\
\hline Byron Station, Unit 2 & Exelon Generation Co., LLC & & $11 / 6 / 26$ & $11 / 1 / 46$ \\
\hline
\end{tabular}


Table A-2. Operating Commercial Reactors (continued)

\begin{tabular}{|c|c|c|c|c|}
\hline Plant Name, Unit Number & Licensee & $\begin{array}{l}\text { Renewed } \\
\text { Operating } \\
\text { License } \\
\text { Issued }\end{array}$ & $\begin{array}{l}\text { Current } \\
\text { Operating } \\
\text { License } \\
\text { Expires }\end{array}$ & $\begin{array}{c}\text { Anticipated } \\
\text { Operating } \\
\text { License } \\
\text { Expiration w/ } \\
\text { License Renewal }\end{array}$ \\
\hline Vogtle Electric Generating Plant, Unit 1 & Southern Nuclear Operating Co. & $6 / 3 / 09$ & $1 / 16 / 47$ & \\
\hline Beaver Valley Power Station, Unit 2 & First Energy Nuclear Operating Co. & $11 / 5 / 09$ & $5 / 27 / 47$ & \\
\hline South Texas Project, Unit 1 & STP Nuclear Operating Co. & & $8 / 20 / 27$ & 8/15/47 \\
\hline Palo Verde Nuclear Generating Station, Unit 3 & Arizona Public Service Company & $4 / 21 / 11$ & $11 / 25 / 47$ & \\
\hline Braidwood Station, Unit 2 & Exelon Generation Co., LLC & & $12 / 18 / 27$ & $12 / 13 / 47$ \\
\hline South Texas Project, Unit 2 & STP Nuclear Operating Co. & & $12 / 15 / 28$ & $12 / 10 / 48$ \\
\hline Vogtle Electric Generating Plant, Unit 2 & Southern Nuclear Operating Co. & $6 / 3 / 09$ & $2 / 9 / 49$ & \\
\hline Limerick Generating Station, Unit 2 & Exelon Generation Co., LLC & & $6 / 22 / 29$ & $6 / 17 / 49$ \\
\hline Comanche Peak Steam Electric Station, Unit 1 & Luminant Generation Co., LLC & & $2 / 8 / 30$ & $2 / 3 / 50$ \\
\hline Seabrook Station, Unit 1 & FPL Energy Seabrook, LLC & & $3 / 15 / 30$ & $3 / 10 / 50$ \\
\hline Comanche Peak Steam Electric Station, Unit 2 & Luminant Generation Co., LLC & & $2 / 2 / 33$ & $1 / 28 / 53$ \\
\hline Watts Bar Nuclear Plant, Unit 1 & Tennessee Valley Authority & & $11 / 9 / 35$ & $11 / 4 / 55$ \\
\hline
\end{tabular}

Source: U.S. Nuclear Regulatory Commission, 2011-2012 Information Digest, NUREG-1350, Volume 23, August 2011, Appendix A 


\section{A-1.2 Dry Storage Systems}

Dry storage in the U.S can be divided into two broad categories, those in which the fuel is stored bare in a fuel basket inside a metal cask and those in which the fuel is stored in a welded canister inside a vented concrete overpack or a metal dual purpose cask.

Most fuel assemblies in dry storage in the U.S. are in welded metal canisters inside vented concrete vertical overpacks or a horizontal storage module. For this configuration, the canister with its internal basket, fuel and fuel component contents is the only portion of the storage cask system that is transported. These systems all require a separate transportation cask with a type B containment vessel to overpack the fuel canister. The transfer usually requires the use of a transfer cask except for the NUHOMS transportation casks, which can interface directly with the horizontal storage module. Some welded metal canisters cannot currently be transported for various design reasons.

There are four categorical descriptions of dry cask storage:

1. Metal canisters in vertical concrete overpacks or horizontal concrete modules,

2. Metal canisters in metal overpack/storage/shipping casks,

3. Metal canisters in concrete vaults and

4. Bare fuel casks that provide both primary containment and shielding for storage and transportation.

Details on these categories are provided elsewhere (Leduc 2012).

The type of dry storage system cask utilized at each reactor site, number of casks loaded, and the number of fuel assemblies loaded are shown in Table A-3 (UxC 2012). A summary of near- and longer-term plans for dry storage at plants with existing ISFSIs is shown in Table A-4 (UxC 2012) and a summary of plants that plan to deploy ISFSIs in the next few years is shown in Table A-5 (UxC 2012).

The information shown in Table A-3 shows that while a number of different casks types have been utilized over the years, recent trends and future plans indicate that the nuclear industry is primarily loading fuel into the following systems at operating plants:

- Holtec HISTORM MPC-32 (32 assembly capacity) PWR casks;

- Holtec HISTORM MPC-68 (68 assembly capacity) BWR casks;

- $\quad$ NAC UMS-24 (24 assembly capacity) PWR casks ;

- NUHOMS 24 and 32 assembly capacity PWR casks; and

- NUHOMS 61 assembly capacity BWR casks.

The information shown in Tables A-4 and A-5 also indicate that there is significant inertia within the nuclear industry regarding the dry storage systems. Several utilities have made significant investments into ISFSI licensing, design, pad/module construction, and have established contracts with vendors to procure canisters/casks to supply storage capacity for a number of years. Many utilities envision using the same dry storage system already in use or planned to be deployed in the next few years through the operational life of their plants. Thus, it is expected that a variety of dry storage systems will continue to be loaded for the foreseeable future. 
Table A-3 Commercial UNF Dry Storage by Cask System

\begin{tabular}{|c|c|c|c|c|c|c|c|c|}
\hline Vendor & Cask System & $\begin{array}{c}\text { Canister } \\
\text { Type }\end{array}$ & Reactor & $\begin{array}{l}\text { Reactor } \\
\text { Type }\end{array}$ & Reactor State & Utility & Casks & Assemblies \\
\hline GNB & Castor & $\begin{array}{l}\mathrm{V} / 21 \text { and } \\
\mathrm{X} 33\end{array}$ & Surry & PWR & Virginia & Dominion & 26 & 558 \\
\hline DOE & Foster Wheeler & MVDS & Ft. St. Vrain & HTGR & Colorado & PS Colorado & & 1464 \\
\hline \multirow[t]{4}{*}{ BFS/ES } & Fuel Solutions & VSC-24 & $\begin{array}{l}\text { ANO } \\
\text { Big Rock }\end{array}$ & PWR & Arkansas & Entergy & 24 & 576 \\
\hline & Fuel Solutions & W150 & Point $1,{ }^{3}$ & BWR & Michigan & Consumers & 8 & 441 \\
\hline & Fuel Solutions & VSC-24 & Palisades & PWR & Michigan & Entergy & 18 & 432 \\
\hline & Fuel Solutions & VSC-24 & Point Beach & PWR & Wisconsin & FPL & 16 & 384 \\
\hline \multirow[t]{27}{*}{ Holtec } & HI-STAR & MPC-80 & Humboldt Bay ${ }^{1,3}$ & BWR & California & PG\&E & 5 & 390 \\
\hline & HI-STAR & MPC-68 & Hatch & BWR & Georgia & Southern Nuclear & 3 & 204 \\
\hline & Hi-STAR & $\begin{array}{l}\text { MPC-68 } \\
\text { MPC- }\end{array}$ & Dresden & BWR & Illinois & Exelon & 4 & 272 \\
\hline & TranStor & 24E/EF & Trojan & PWR & Oregon & Portland GE & 34 & 780 \\
\hline & HI-STORM & MPC-68 & Browns Ferry & BWR & Alabama & TVA & 39 & 2652 \\
\hline & HI-STORM & MPC-32 & Farley & PWR & Alabama & Southern Nuclear & 16 & 512 \\
\hline & HI-STORM & MPC-24 & ANO & PWR & Arkansas & Entergy & 22 & 528 \\
\hline & HI-STORM & MPC-32 & ANO & PWR & Arkansas & Entergy & 16 & 512 \\
\hline & HI-STORM & MPC-32 & Diablo Canyon & PWR & California & PG\&E & 23 & 736 \\
\hline & HI-STORM & MPC-32 & Comanche Peak & PWR & Texas & Luminant & 9 & 288 \\
\hline & HI-STORM & MPC-68 & Hatch & BWR & Georgia & Southern Nuclear & 47 & 3196 \\
\hline & HI-STORM & MPC-32 & Byron & PWR & Illinois & Exelon & 14 & 448 \\
\hline & HI-STORM & MPC-32 & Braidwood & PWR & Illinois & Exelon & 3 & 96 \\
\hline & HI-STORM & MPC-68 & Dresden & BWR & Illinois & Exelon & 49 & 3332 \\
\hline & HI-STORM & MPC-68 & LaSalle & BWR & Illinois & Exelon & 6 & 408 \\
\hline & HI-STORM & MPC-68 & Quad Cities & BWR & Illinois & Exelon & 35 & 2380 \\
\hline & HI-STORM & MPC-68 & River Bend & BWR & Louisiana & Entergy & 15 & 1020 \\
\hline & HI-STORM & MPC-68 & Grand Gulf & BWR & Mississippi & Entergy & 17 & 1156 \\
\hline & HI-STORM & MPC-68 & Hope Creek & BWR & New Jersey & PSE\&G & 16 & 1088 \\
\hline & HI-STORM & MPC-32 & Salem & PWR & New Jersey & PSE\&G & 16 & 512 \\
\hline & HI-STORM & MPC-68 & Fitzpatrick & BWR & New York & Entergy & 15 & 1020 \\
\hline & HI-STORM & MPC-32 & Indian Point $1^{3}$ & PWR & New York & Entergy & 5 & 160 \\
\hline & HI-STORM & MPC-32 & Indian Point 2 & PWR & New York & Entergy & 14 & 448 \\
\hline & HI-STORM & MPC-32 & Sequoyah & PWR & Tennessee & TVA & 32 & 1024 \\
\hline & HI-STORM & MPC-68 & Vermont Yanke & BWR & Vermont & Entergy & 14 & 952 \\
\hline & HI-STORM & MPC-32 & Waterford & PWR & Louisiana & Entergy & 9 & 288 \\
\hline & HI-STORM & MPC-68 & Columbia & BWR & Washington & Energy Northwest & 27 & 1836 \\
\hline Westinghouse & MC-10 & MC-10 & Surry & PWR & Virginia & Dominion & 1 & 24 \\
\hline \multirow[t]{8}{*}{ NAC } & NAC-I28 & NAC-128 & Surry & PWR & Virginia & Dominion & 2 & 56 \\
\hline & NAC-MPC & MPC-26 & Conn Yankee ${ }^{2,3}$ & PWR & Connecticut & Ct. Yankee & 43 & 1019 \\
\hline & NAC-MPC & MPC-36 & Yankee Rowe $e^{2,3}$ & PWR & Massachusetts & YAEC & 16 & 533 \\
\hline & NAC-MPC & LACBWR & LaCrosse & BWR & Wisconsin & Dairyland & 4 & 272 \\
\hline & NAC-UMS & UMS-24 & Palo Verde & PWR & Arizona & APS & 91 & 2184 \\
\hline & NAC-UMS & UMS-24 & Maine Yankee $e^{2,3}$ & PWR & Maine & Maine Yankee & 64 & 1434 \\
\hline & NAC-UMS & UMS-24 & McGuire & PWR & North Carolina & Duke & 28 & 672 \\
\hline & NAC-UMS & UMS-24 & Catawba & PWR & South Carolina & Duke & 24 & 576 \\
\hline
\end{tabular}


Table A-3 (Con't)

\begin{tabular}{|c|c|c|c|c|c|c|c|c|}
\hline Vendor & Cask System & $\begin{array}{c}\text { Canister } \\
\text { Type }\end{array}$ & Reactor & $\begin{array}{c}\text { Reactor } \\
\text { Type }\end{array}$ & Reactor State & Utility & Casks & Assemblies \\
\hline \multirow[t]{36}{*}{ Trans Nuclear } & NUHOMS & 24PT & Rancho Seco ${ }^{1}$ & PWR & California & SMUD & 22 & 493 \\
\hline & NUHOMS & 24PT1 & SONGS $1^{1,3}$ & PWR & California & Edison & 18 & 395 \\
\hline & NUHOMS & 24PT4 & SONGS 2, 3 & PWR & California & $\begin{array}{l}\text { Southern Cal } \\
\text { Edison }\end{array}$ & 29 & 696 \\
\hline & NUHOMS & 32PT & Millstone & PWR & Connecticut & Dominion & 18 & 576 \\
\hline & NUHOMS & 32PTH & St. Lucie & PWR & Florida & FPL & 14 & 448 \\
\hline & NUHOMS & 32PTH & Turkey Point & PWR & Florida & FPL & 18 & 576 \\
\hline & NUHOMS & $12 \mathrm{~T}$ & INEEL & PWR & Idaho & DOE & 20 & 1220 \\
\hline & NUHOMS & 61BT & Duane Arnold & BWR & lowa & FPL & 48 & 1152 \\
\hline & NUHOMS & $24 \mathrm{P}$ & Calvert Cliffs & PWR & Maryland & Constellation & 22 & 704 \\
\hline & NUHOMS & $32 \mathrm{P}$ & Calvert Cliffs & PWR & Maryland & Constellation & 13 & 312 \\
\hline & NUHOMS & 24PHT & Palisades & PWR & Michigan & Entergy & 11 & 352 \\
\hline & NUHOMS & 32PT & Palisades & PWR & Michigan & Entergy & 10 & 610 \\
\hline & NUHOMS & 61BT & Monticello & BWR & Minnesota & Xcel Energy & 8 & 488 \\
\hline & NUHOMS & 61BT & Cooper & BWR & Nebraska & NPPD & 10 & 320 \\
\hline & NUHOMS & 32PT & Fort Calhoun & PWR & $\begin{array}{l}\text { Nebraska } \\
\text { New }\end{array}$ & OPPD & 6 & 192 \\
\hline & NUHOMS & 32PHT & Seabrook & PWR & Hampshire & FPL & 23 & 1403 \\
\hline & NUHOMS & $61 \mathrm{BT}$ & Oyster Creek & BWR & New Jersey & Exelon & 6 & 192 \\
\hline & NUHOMS & 32PT & Ginna & PWR & New York & Constellation & 8 & 488 \\
\hline & NUHOMS & 61BTH & Brunswick & BWR & North Carolina & Progress & 3 & 72 \\
\hline & NUHOMS & $24 \mathrm{P}$ & Davis-Besse & PWR & Ohio & FirstEnergy & 19 & 1159 \\
\hline & NUHOMS & 61BT & Limerick & BWR & Pennsylvania & Exelon & 27 & 1404 \\
\hline & NUHOMS & $52 \mathrm{~B}$ & Susquehanna & BWR & Pennsylvania & PPL & 41 & 2501 \\
\hline & NUHOMS & 61BT & Susquehanna & BWR & Pennsylvania & PPL & 38 & 912 \\
\hline & NUHOMS & 24PHB & Oconee & PWR & South Carolina & Duke & 84 & 2016 \\
\hline & NUHOMS & $24 \mathrm{P}$ & Oconee & PWR & South Carolina & Duke & 14 & 336 \\
\hline & NUHOMS & 24PTH & Robinson & PWR & South Carolina & Progress & 8 & 56 \\
\hline & NUHOMS & $7 \mathrm{P}$ & Robinson & PWR & South Carolina & Progress & 13 & 416 \\
\hline & NUHOMS & 32PTH & North Anna & PWR & Virginia & Dominion & 18 & 576 \\
\hline & NUHOMS & 32РTH & Surry & PWR & Virginia & Dominion & 8 & 256 \\
\hline & NUHOMS & 32PT & Kewaunee & PWR & Wisconsin & Dominion & 17 & 544 \\
\hline & NUHOMS & 32PT & Point Beach & PWR & Wisconsin & FPL & 29 & 1160 \\
\hline & TN Metal Casks & $\mathrm{TN}-40$ & Prairie Island & PWR & Minnesota & Ecel Energy & 10 & 320 \\
\hline & TN Metal Casks & TN-32 & McGuire & PWR & North Carolina & Duke & 59 & 4012 \\
\hline & TN Metal Casks & TN-68 & Peach Bottom & BWR & Pennsylvania & Exelon & 27 & 864 \\
\hline & TN Metal Casks & TN-32 & North Anna & PWR & Virginia & Dominion & 26 & 832 \\
\hline & TN Metal Casks & TN-32 & Surry & PWR & Virginia & Dominion & 22 & 493 \\
\hline
\end{tabular}

Source: The Ux Consulting Company, LLC, StoreFUEL and Decomissioning Report, Volume 13, No. 169, September 4, 2012

${ }^{1}$ One cask is storing GTCC waste is in use

${ }^{2} \mathrm{CY}$ has 3 casks storing GTCC waste; Yankee Rowe has one and Maine Yankee has four casks

${ }^{3}$ All spent fuel from the shutdown plant

Yellow highlight shows sites where used fuel loading into dry storage occurred between December 2010 and September 2012 , showing recent activity 
Table A-4 Summary of Near- and Long-Term Plans for Existing ISFIs

\begin{tabular}{|c|c|}
\hline Reactor Site & Near-Term and Long-Term Plans \\
\hline D.C. Cook & Plan to load 14 casks (Holtec MPC 32/HISTORM) by November 2012. \\
\hline Palo Verde & $\begin{array}{l}\text { ISFSI has sufficient capacity for all spent fuel that will be generated. Has ordered a total of } 104 \text { NAC UMS systems. } \\
\text { Will eventually transition to MAGNASTOR system }\end{array}$ \\
\hline Calvert Cliffs & $\begin{array}{l}\text { Has two open horizontal storage modules of the } 72 \text { currently (NUHOMS 32P) installed with plans to load in } \\
\text { September 2012. Another } 24 \text { storage modules to be added in } 2013 \text {. Pad being expanded to accommodate a total of } \\
60 \text { more horizontal storage modules. }\end{array}$ \\
\hline Ginna & $\begin{array}{l}\text { ISFSI designed for a } 30 \text { horizontal storage module capacity. } 12 \text { modules are in place and } 6 \text { NUHOMS } 32 \text { PT } \\
\text { canisters have been loaded. Next loadings planned in } 2016\end{array}$ \\
\hline LaCrosse & $\begin{array}{l}\text { Initial dry storage campaign began in June 2012. } 68 \text { assemblies per canister. } 333 \text { assemblies total, } 155 \text { are } \\
\text { damaged and will be put in damaged fuel canisters }\end{array}$ \\
\hline Surry & $\begin{array}{l}55 \text { metal storage casks are on two pads, using } 5 \text { different casks designs storing } 1470 \text { assemblies under site specific } \\
\text { license. This is complete. A third pad, operating under general license, using NUHOMS 32PTH canisters, is being } \\
\text { used. This pad is designed for } 40 \text { modules and } 18 \text { have been loaded (through 2020). }\end{array}$ \\
\hline North Anna & $\begin{array}{l}\text { The loading of one pad, under a site specific license, that has } 27 \text { TN-32 casks is complete. A second pad, operating } \\
\text { under general license, using NUHOMS } 32 \mathrm{PTH} \text { canisters is being used. The pad is designed for } 40 \text { modules, } 26 \text { are } \\
\text { in place, and } 13 \text { have been loaded. }\end{array}$ \\
\hline Millstone & $\begin{array}{l}18 \text { NUHOMS 32PT canisters are in place, and } 19 \text { horizatonal storage modules have been installed. Has permission } \\
\text { to place } 135 \text { storage modules at site (for Units } 1,2 \text {, and } 3 \text { ). }\end{array}$ \\
\hline Catawba & $\begin{array}{l}24 \text { NAC UMS systems on storage pad. Has two storage pads capable of various dry storage sytems (horizontal and } \\
\text { vertical) }\end{array}$ \\
\hline McGuire & Plans to begin using NAC MAGNASTOR system in 2012, but has not yet deployed any systems \\
\hline Oconee & $\begin{array}{l}\text { Site specific ISFSI license has been renewed until January 31, 2050. The licensed capacity is } 88 \text { NUHOMS } 24 \mathrm{P} \\
\text { storage units. }\end{array}$ \\
\hline Columbia & $\begin{array}{l}27 \text { MPC- } 68 \text { canisters are in place. Two separate pads have been built, each can hold } 18 \text { casks. } 9 \text { additional cask } \\
\text { loads are planned in } 2014 \text {. Three more pads are scheduled to be constructed in } 2016 \text {, bringing total to } 90 \text { (enough to } \\
\text { store all fuel that would be generated through more than } 60 \text { years of operation). }\end{array}$ \\
\hline ANO & Plan to load 3 MPC-24 casks from unit 1 pool and 3 MPC-32s from unit pool beginning in fall of 2012 \\
\hline River Bend & ISFSI has a 44-cask capacity with capability to expand. Plan to load 4 MPC-68 casks in 2012. \\
\hline Waterford & ISFSI has 72 cask capacity (MPC-32); 9 have been loaded to-date \\
\hline Indian Point & $\begin{array}{l}\text { ISFSI is sized to accommodate Unit } 2 \text { and Unit } 3 \text { operations (full-core discharge capability) through } 20 \text { years beyond } \\
\text { current operating license and to store } 5 \text { casks with } 160 \text { Unit } 1 \text { assemblies. }\end{array}$ \\
\hline $\begin{array}{l}\text { Vermont } \\
\text { Yankee }\end{array}$ & ISFSI has capacity to store 36 systems (MPC-68 canisters). \\
\hline Palisades & Plans to continue using higher heat load NUHOMS 24PTH casks for all remaining loads. \\
\hline Braidwood & Braidwood contract is for 24 HI-STORM 100 systems (MPC 32). \\
\hline Byron & Byron contract is for $24 \mathrm{HI}-\mathrm{STORM} 100$ systems (MPC-32) \\
\hline LaSalle & Contract is for $24 \mathrm{HI}$-STORM 100 systems (MPC-68). \\
\hline Limerick & Contract is for 24 NUHOMS 61 BT systems \\
\hline Oyster Creek & Expanding ISFSI and installing additional horizontal storage modules (NUHOMS 61BT) \\
\hline St. Lucie & 14 NUHOMS 32PTH systems deployed. Will procure additional modules as needed \\
\hline Seabrook & Construction of eight horizontal storage modules (NUHOMS 32 PTH) on-going in 2012 and will be loaded in 2013 \\
\hline Cooper & ISFSI designed to support 52 horizontal storage modules. Next loading campaign is planned for 2013. \\
\hline $\begin{array}{l}\text { Diablo } \\
\text { Canyon }\end{array}$ & $\begin{array}{l}\text { Currently in a campaign to load seven casks. Holtec has delivered } 12 \text { additional systems in June } 2011 \text {. PG\&E is } \\
\text { ordering } 10 \text { more systems for delivery at the end of } 2012 \text {. } \\
\text { Initial ISFSI has capacity for } 40 \text { casks, which is sufficient capacity through end of operating license; could add } \\
\text { additional capacity for } 3,136 \text { assemblies. The ISFSI is planned for a total of } 7 \text { pads, } 138 \text { casks. } 2 \text { pads have been } \\
\text { constructed and expansion is needed in the } 2012-2013 \text { timeframe }\end{array}$ \\
\hline H.B. Robinson & ISFSI designed to provide life-of-plant storage \\
\hline
\end{tabular}


Table A-4 (cont) Summary of Near- and Long-Term Plans for Existing ISFIs

\begin{tabular}{|l|l|}
\hline \multicolumn{1}{|c|}{ Reactor Site } & \multicolumn{1}{c|}{ Near-Term and Long-Term Plans } \\
\hline Brunswick & A loading campaign is planned in 2013. Anticipate loading campaigns every 2 years for life of plant \\
\hline Salem/Hope Creek & Share single ISFSI, designed to store 200 casks; 89 from Salem, the rest from Hope Creek. \\
\hline Sequoyah & ISFSI has capacity for 90 casks. Plans to add a second pad by 2028 if needed. \\
\hline Browns Ferry & ISFSI has capacity for 96 casks. A second pad could be built in 2018 if needed. \\
\hline Prairie Island & $\begin{array}{l}\text { ISFSI has two pads; } 24 \text { cask capacity each. The Minnesota Public Utilities Commission approved plan to } \\
\text { accommodate up to 35 additional casks to support continued operation through licenses. }\end{array}$ \\
\hline Monticello & 30 systems are expected to be needed and the facility has room to add 35 more if needed. \\
\hline
\end{tabular}

Source: The Ux Consulting Company, LLC, StoreFUEL and Decomissioning Report, Volume 13, No. 169, September 4, 2012

Table A-5. Near-Term Planned New ISFSIs and Loadings

\begin{tabular}{|c|c|c|c|c|c|c|c|}
\hline Vendor & Cask System & $\begin{array}{l}\text { Canister } \\
\text { Type }\end{array}$ & Reactor & $\begin{array}{l}\text { Reactor } \\
\text { Type }\end{array}$ & Utility & $\begin{array}{l}\text { Estimated } \\
\text { First Load }\end{array}$ & Comments \\
\hline \multirow[t]{7}{*}{ Holtec } & HI-STORM 100 & MPC-68 & Fermi-2 & BWR & $\begin{array}{l}\text { Detroit } \\
\text { Edison }\end{array}$ & 2014 & $\begin{array}{l}\text { Contracted with HOLTEC for } 12 \text { systems for } \\
\text { capacity through } 2016 \text {. ISFSI will } \\
\text { accommodate } 64 \text { casks, but can be expanded }\end{array}$ \\
\hline & HI-STORM 100 & MPC-68 & Perry & BWR & FirstEnergy & 2012 & $\begin{array}{l}\text { Under contract for } 16 \text { canisters/overpacks. } \\
\text { ISFSI designed for } 80 \text { casks capacity. Six } \\
\text { casks planned to be loaded in } 2012 \text {. Up to } 74 \\
\text { additional systems will be added as needed. }\end{array}$ \\
\hline & HI-STORM 100 & MPC-32 & DC Cook & PWR & $\begin{array}{l}\text { American } \\
\text { Electric }\end{array}$ & 2012 & $\begin{array}{l}\text { Estimates cask loading every } 2-3 \text { years. } 90 \\
\text { casks needed to get to end of operating } \\
\text { license in } 2037 \text {. Additional } 112 \text { for } \\
\text { decommissioning. }\end{array}$ \\
\hline & HI-STORM 100 & MPC-32 & Vogtle & PWR & $\begin{array}{l}\text { Southern } \\
\text { Nuclear }\end{array}$ & 2013 & $\begin{array}{l}\text { Plan loading } 4 \text { systems in } 2013,6 \text { in } 2015,6 \\
\text { in } 2016,8 \text { in } 2018 \text { - then } 8 \text { in every year } \\
\text { except in years where both units are re- } \\
\text { fuelled. } 80 \text { casks expected to be loaded by } \\
2035\end{array}$ \\
\hline & HI-STORM 100 & MPC-32 & $\begin{array}{l}\text { Indian } \\
\text { Point } 3\end{array}$ & PWR & Entergy & 2013 & $\begin{array}{l}\text { Plan to move Unit } 3 \text { fuel to Unit } 2 \text { pool for } \\
\text { transfer into dry storage at existing ISFSI }\end{array}$ \\
\hline & HI-STORM 100 & MPC-68 & Pilgrim & BWR & Entergy & $\begin{array}{l}2013- \\
2014\end{array}$ & $\begin{array}{l}\text { Plans to load } 3 \text { MPC- } 68 \text { casks in } 2013 \text { or } \\
\text { 2014. Pad construction to begin in } 2012\end{array}$ \\
\hline & Hi-STORM FW & MPC-32 & Summer & PWR & $\begin{array}{l}\text { South } \\
\text { Caroling } \\
\text { Electric \& } \\
\text { Gas }\end{array}$ & 2015 & $\begin{array}{l}\text { Contract with Holtec for three loading } \\
\text { campaigns (initial loading of } 4 \text { systems in } \\
\text { 2015, then seven in } 2019 \text { and seven in } 2022\end{array}$ \\
\hline NAC & MAGNASTOR & MPC-37 & Zion & PWR & Exelon & 2013 & $\begin{array}{l}2226 \text { fuel assemblies to be loaded in up to } 61 \\
\text { storage systems, plus } 4 \text { for GTCC waste }\end{array}$ \\
\hline \multirow[t]{2}{*}{$\begin{array}{l}\text { Trans- } \\
\text { nuclear }\end{array}$} & $\begin{array}{l}\text { NUHOMS } \\
\text { 32PT }\end{array}$ & 32PT & $\begin{array}{l}\text { Crystal } \\
\text { River }\end{array}$ & PWR & Progress & 2014 & $\begin{array}{l}\text { Date being delayed due to extended } \\
\text { shutdown. ISFSI will have a } 80 \text { storage } \\
\text { module capacity }\end{array}$ \\
\hline & $\begin{array}{l}\text { NUHOMS } \\
61 \mathrm{BT}\end{array}$ & $61 \mathrm{BT}$ & $\begin{array}{l}\text { Nine Mile } \\
\text { Point }\end{array}$ & BWR & Constellation & 2012 & $\begin{array}{l}\text { First loading scheduled for September } 2012 \text {; } \\
\text { six canisters to be loaded. Thirty horiztonal } \\
\text { modules have been constructed and the } \\
\text { future capacity is planned up to } 200 \text { horizontal } \\
\text { storage modules. Plans for initial construction } \\
\text { of } 30 \text { modules to load between } 2012 \text { and } \\
2015.30 \text { canisters are being acquired. }\end{array}$ \\
\hline
\end{tabular}


Table A-5. Near-Term Planned New ISFSIs and Loadings (continued)

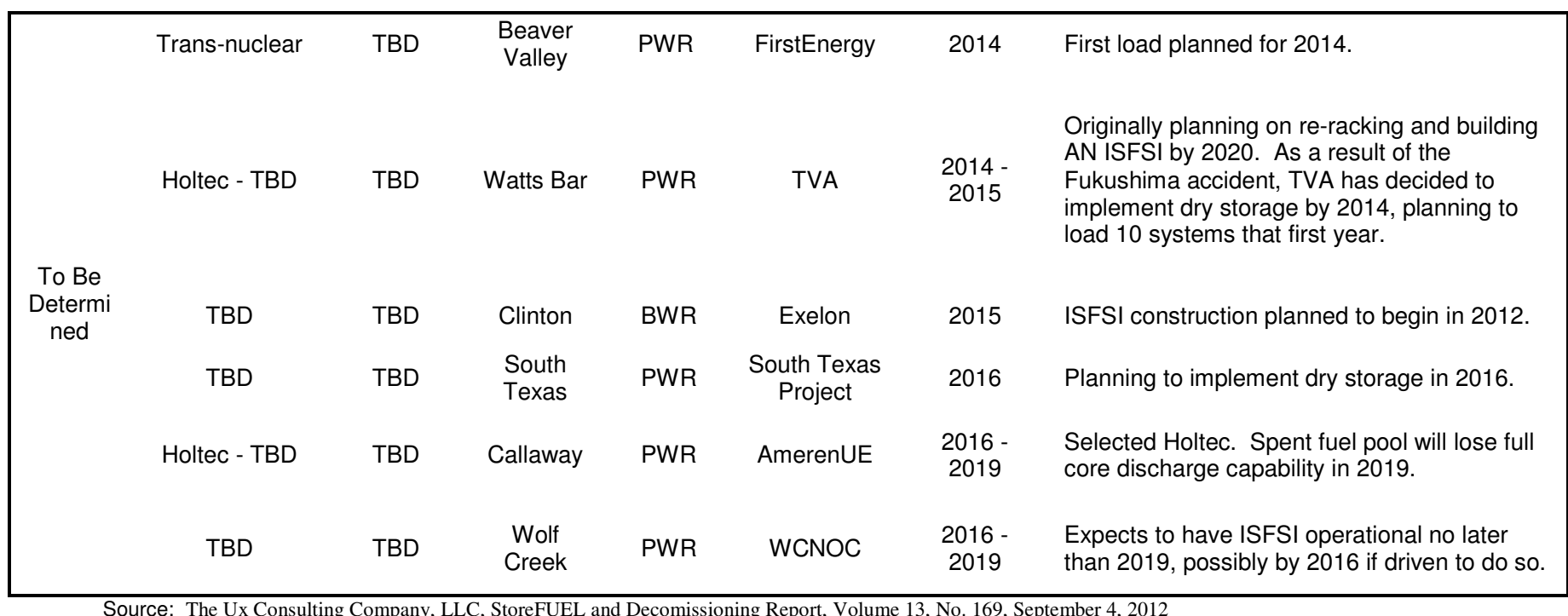

Thus, it was assumed in this evaluation that plants already loading fuel into dry storage will continue to utilize the systems they are already loading or plan to load. To ensure consistency for purposes of this analysis, plants that have not yet made decisions regarding dry storage systems were assumed to load Holtec MPC-32 and MPC-68 systems. This assumes that the utilities will make whatever upgrades necessary to handle these large size casks.

The Transportation Logistics Simulation - CALVIN (TSL-CALVIN) model (Nutt et al. 2012) was used to project dry storage inventories through 2035. Figure A-1 shows the number of casks systems that will be loaded over time through 2035 and Table A- 6 shows the projected number of casks, the number of fuel assemblies, and the amount (MTHM) of material loaded for each system in 2020 and 2035.

\section{A-1.2.1 At-Reactor Operations for On-Site Dry Storage}

Each canister-based or cask storage system has specific procedures associated with the fuel loading operation, however in general the steps are similar. The high level steps are (EPRI 2010b):

1. Receipt and inspection of canister/cask

2. Cleaning, decontamination, and inspection of transfer cask (if utilized)

3. Transfer of canister/cask (and transfer cask if utilized) into the fuel pool

4. Filling the canister/cask with water

5. Transfer fuel from the fuel pool storage racks into the canister/cask

6. Seat closure lid on top of the canister/cask

7. Remove the loaded canister/cask (and transfer cask if utilized) from the fuel pool

8. Seal the canister/cask (welded or bolted closure)

9. Drain water from the canister/cask cavity (vacuum or forced helium drying system)

10. Backfill cavity with helium to provide an inert atmosphere

11. For canister systems

a. Place canister in transfer cask

b. Move canister/transfer cask to ISFSI and place canister inside storage overpack (vertical) or inside storage module (horizontal)

12. For storage casks - transfer cask to ISFSI 


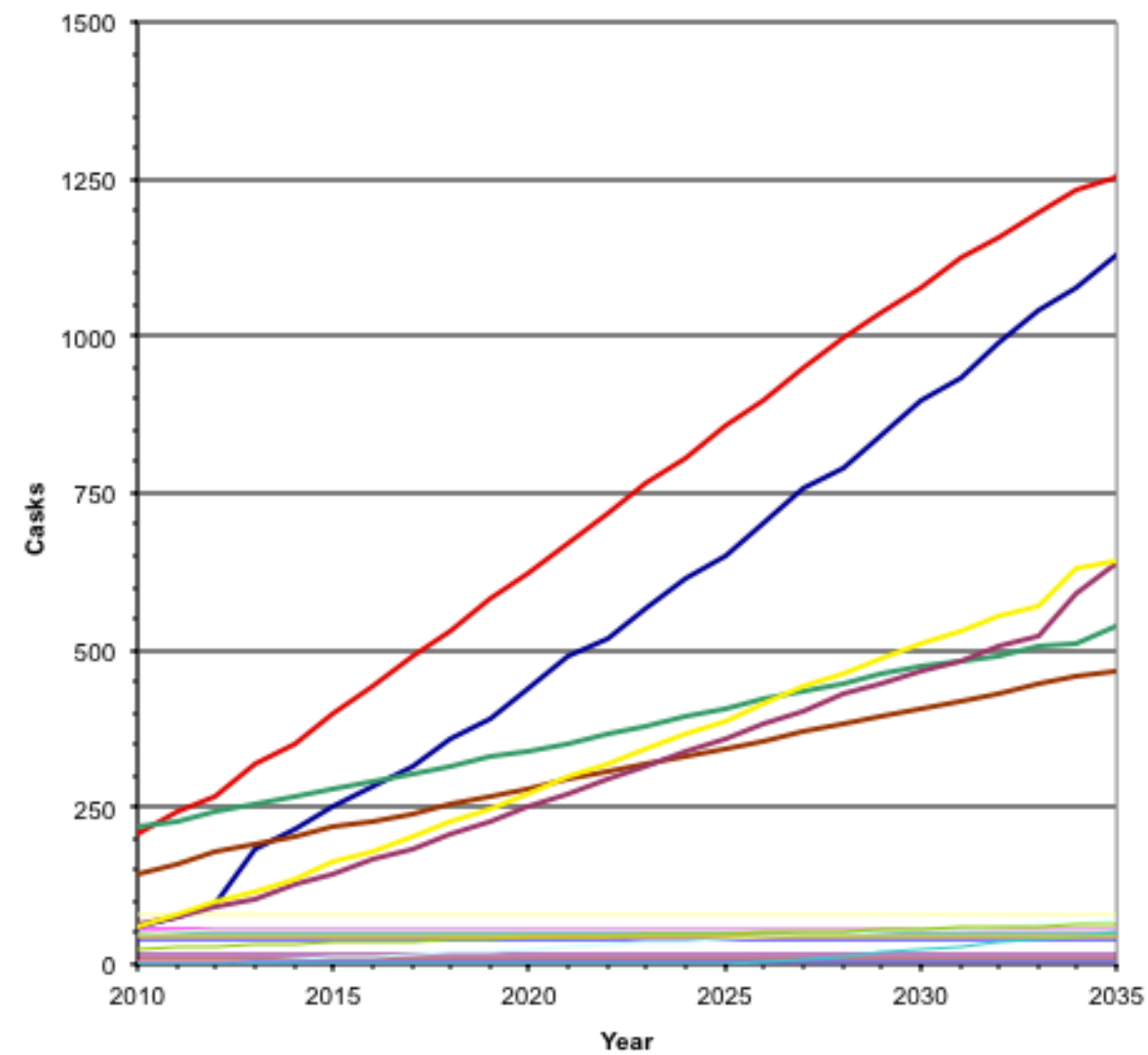

-Fuel Solutions VSC-24

Holtec MPC-68

- Holtec MPC-32

- NAC MPC-36 (Maine Yankee)

- Fuel Solutions W-150 (Big Rock Point)

Holtec MPC-24

NAC MPC-26

NUHOMS 24PT NAC MPC-37 TransNuclear TN-68 TransNuclear TN-32

- NAC UMS-24

Holtec MPC-80 (Humbolt Bay)

-NUHOMS 61BT

NUHOMS 32PT

NAC MPC-68 (LaCrosse BWR)

- South Texas (18)

- TransNuclear TN-40 - NUHOMS 52B (Susquehanna)

Note: Does not include Castor V21/X33 and NUHOMS 7P

Figure A-1. Projected Dry Storage Cask Inventory 
Table A-6. Projected Dry Storage Inventory at 2020 and 2035

\begin{tabular}{|l|c|c|c|}
\hline \multicolumn{4}{|c|}{$\mathbf{2 0 2 0}$} \\
\hline \multicolumn{1}{|c|}{ System } & Assemblies & Casks & MTHM \\
\hline Total & $\mathbf{1 1 1 1 4 9}$ & $\mathbf{2 6 1 8}$ & $\mathbf{3 1 5 6 8 . 0}$ \\
\hline Holtec MPC-68 & 42131 & 621 & 7599.2 \\
\hline Holtec MPC-32 & 13970 & 437 & 6275.5 \\
\hline NUHOMS 24P & 8100 & 340 & 3527.8 \\
\hline NAC UMS-24 & 6690 & 279 & 2807.3 \\
\hline NUHOMS 32P & 8672 & 271 & 3605.4 \\
\hline NUHOMS 61B & 15311 & 251 & 2749.2 \\
\hline TransNuclear TN-32 & 2494 & 78 & 1137.6 \\
\hline Fuel Solutions VSC-24 & 1368 & 57 & 567.5 \\
\hline TransNuclear TN-68 & 3740 & 55 & 694.8 \\
\hline Holtec MPC-24 & 1116 & 47 & 504.3 \\
\hline NUHOMS 52B (Susquehanna) & 2236 & 43 & 404.5 \\
\hline TransNuclear TN-40 & 1680 & 42 & 625.2 \\
\hline NAC MPC-26 & 1019 & 40 & 412.3 \\
\hline NAC MPC-37 & 925 & 25 & 405.5 \\
\hline NAC MPC-36 (Maine Yankee) & 533 & 15 & 127.1 \\
\hline Fuel Solutions W-150 (Big Rock Point) & 441 & 7 & 57.9 \\
\hline Holtec MPC-80 (Humbolt Bay) & 390 & 5 & 28.9 \\
\hline NAC MPC-68 (LaCrosse BWR) & 333 & 5 & 38.0 \\
\hline Castor V21 and X33 & 558 & 26 & \\
\hline NUHOMS 7P & 56 & 8 & \\
\hline
\end{tabular}

\begin{tabular}{|l|c|c|c|}
\hline \multicolumn{4}{|c|}{$\mathbf{2 0 3 5}$} \\
\hline \multicolumn{1}{|c|}{ System } & Assemblies & Casks & MTHM \\
\hline Total & $\mathbf{2 2 4 3 2 0}$ & $\mathbf{5 2 0 5}$ & $\mathbf{6 3 5 1 7 . 0}$ \\
\hline Holtec MPC-68 & 84971 & 1251 & 15121.5 \\
\hline Holtec MPC-32 & 36146 & 1130 & 15951.2 \\
\hline NUHOMS 32P & 20520 & 642 & 8539.6 \\
\hline NUHOMS 61B & 38847 & 638 & 6866.2 \\
\hline NUHOMS 24P & 12890 & 540 & 5662.3 \\
\hline NAC UMS-24 & 11226 & 468 & 4818.3 \\
\hline TransNuclear TN-32 & 2494 & 78 & 1137.6 \\
\hline NAC MPC-37 & 2590 & 70 & 1164.2 \\
\hline TransNuclear TN-40 & 2560 & 64 & 934.2 \\
\hline Fuel Solutions VSC-24 & 1368 & 57 & 567.5 \\
\hline TransNuclear TN-68 & 3740 & 55 & 694.8 \\
\hline South Texas (18) & 900 & 50 & 486.7 \\
\hline Holtec MPC-24 & 1116 & 47 & 504.3 \\
\hline NUHOMS 52B (Susquehanna) & 2236 & 43 & 404.5 \\
\hline NAC MPC-26 & 1019 & 40 & 412.3 \\
\hline NAC MPC-36 (Maine Yankee) & 533 & 15 & 127.1 \\
\hline Fuel Solutions W-150 (Big Rock Point) & 441 & 7 & 57.9 \\
\hline Holtec MPC-80 (Humbolt Bay) & 390 & 5 & 28.9 \\
\hline NAC MPC-68 (LaCrosse BWR) & 333 & 5 & 38.0 \\
\hline Castor V21 and X33 & 558 & 26 & \\
\hline NUHOMS 7P & 56 & 8 & \\
\hline
\end{tabular}




\section{A-1.3 At-Reactor Operations for Transportation}

\section{A-1.3.1 Bare Fuel}

The cases being evaluated consider two alternatives for transporting bare fuel from the used nuclear fuel pools; using re-useable transportation casks and using dual- or multi-purpose canisters/overpacks.

The concept for at-reactor-loading of bare fuel into re-useable transportation casks includes the following activities (DOE 2002, Section J.1.3.1.1):

1. Receiving the empty transportation cask at the site fence

2. Preparing and moving the cask into the facility loading area

3. Removing the cask from the site prime mover trailer

4. Preparing the cask for loading and placing it in the water-filled loading pit

5. Transferring spent nuclear fuel from its pool storage location to the cask

6. Removing the cask from the pool and preparing it for shipment

7. Placing the cask on the site prime mover trailer

8. Moving the loaded cask to the site fence where the trailer is connected to the transportation carrier's prime mover for offsite shipment

Consistent with the mostly rail scenario previously considered by the DOE (DOE 2012), 17 shipping cask configurations were assumed for the transportation of bare fuel assemblies from reactor sites: 8 for legalweight truck casks and 9 for rail casks. Table A-7 lists the legal-weight truck and rail cask configurations used in the analysis and their capacities. It was assumed that all shipments would use one of the 17 configurations. If the characteristics of the spent nuclear fuel projected for shipment exceeded the capabilities of one of the casks, it was assumed that the cask's capacity is reduced for the affected shipments. The reduction, which is sometimes referred to as cask de-rating, is needed in certain instances to satisfy nuclear criticality, shielding, and thermal constraints.

For the concept for at-reactor loading of bare fuel residing in the used fuel pools into dual-purpose canisters for off-site transportation involves all steps for loading a dry storage canister described in Section A-1.2.1, through step 10 followed by steps 7 and 8 immediately above. For such scenarios, it was assumed that Holtec MPC-32 and MPC-68 systems would be used.

\section{A-1.3.2 Casks/Canisters in Dry Storage}

Table A- 8 shows the dry storage systems that have been or are currently being loaded at reactor sites (Leduc 2012). Table A-8 also shows the storage configuration and the required operations to transition to transporting those canisters/casks from the reactor sites. These operations involve either direct transport of a transportable shielded cask or the transfer of a dry storage canister to a transportation overpack/cask. 
Table A-7. Bare Fuel Shipping Cask Configurations

\begin{tabular}{|c|c|c|}
\hline Shipping Cask & $\begin{array}{c}\text { Capacity } \\
\text { (number of UNF } \\
\text { Assemblies) }\end{array}$ & Description \\
\hline \multicolumn{3}{|l|}{ Rail } \\
\hline B-R-32-SP & 32 & BWR single-purpose shipping container \\
\hline B-R-32-SP-HH & 32 & BWR single-purpose high-heat-capacity shipping container \\
\hline B-R-44-SP & 44 & Medium BWR single-purpose shipping container \\
\hline B-R-68-SP & 68 & Large BWR single-purpose shipping container \\
\hline P-R-12-SP & 12 & Small PWR single-purpose shipping container \\
\hline P-R-12-SP-HH & 12 & Small PWR single-purpose high-heat capacity shipping container \\
\hline P-R-21-SP & 21 & Medium PWR single-purpose shipping container \\
\hline P-R-24-SP & 24 & Large PWR single-purpose shipping container \\
\hline P-R-7-SP-HH & 7 & PWR high-heat capacity shipping container \\
\hline \multicolumn{3}{|l|}{ Truck } \\
\hline B-T-9/9-SP & 9 & BWR single-purpose shipping container \\
\hline B-T-9/7-SP & 7 & Derated BWR single-purpose shipping container \\
\hline P-T-4/4-SP & 4 & PWR single-purpose shipping container \\
\hline P-T-4/3-SP & 3 & Derated PWR single-purpose shipping container \\
\hline $\mathrm{P}-\mathrm{T}-4 / 2-\mathrm{SP}$ & 2 & Derated PWR single-purpose shipping container \\
\hline P-T-4/4-SP-ST & 4 & PWR plant-unique single-purpose shipping container \\
\hline P-T-4/3-SP-ST & 3 & Derated PWR plant-unique single-purpose shipping container \\
\hline P-T-4/4-SP-BP & 1 & PWR plant-unique single-purpose shipping container \\
\hline
\end{tabular}

Source: DOE 2002, Table J-3. 
Table A-8 UNF Dry Storage Cask/Vault Systems

\begin{tabular}{|c|c|c|c|c|}
\hline Vendor & Cask System & $\begin{array}{l}\text { Canister } \\
\text { Type }\end{array}$ & $\begin{array}{l}\text { Storage } \\
\text { Configuration }\end{array}$ & $\begin{array}{l}\text { Transition to } \\
\text { Transport Required } \\
\text { Operation }\end{array}$ \\
\hline \multicolumn{5}{|c|}{ Welded Metal Canister in Vented Concrete Overpack $(84.1 \%)^{\mathrm{a}}$} \\
\hline \multirow[t]{2}{*}{ Fuel Solutions } & & W150 & $\begin{array}{l}\text { Vertical } \\
\text { Cylinder }\end{array}$ & $\begin{array}{l}\text { Canister Transfer to } \\
\text { Transport Cask }\end{array}$ \\
\hline & & VSC-24 & $\begin{array}{l}\text { Vertical } \\
\text { Cylinder }\end{array}$ & $\begin{array}{l}\text { Canister Transfer to } \\
\text { Transport Cask }\end{array}$ \\
\hline \multirow[t]{3}{*}{$\mathrm{NAC}$} & \multirow[t]{2}{*}{ NAC-MPC } & MPC-26 & $\begin{array}{l}\text { Vertical } \\
\text { Cylinder }\end{array}$ & $\begin{array}{l}\text { Canister Transfer to } \\
\text { Transport Cask }\end{array}$ \\
\hline & & MPC-36 & $\begin{array}{l}\text { Vertical } \\
\text { Cylinder }\end{array}$ & $\begin{array}{l}\text { Canister Transfer to } \\
\text { Transport Cask }\end{array}$ \\
\hline & NAC-UMS & UMS-24 & $\begin{array}{l}\text { Vertical } \\
\text { Cylinder }\end{array}$ & $\begin{array}{l}\text { Canister Transfer to } \\
\text { Transport Cask }\end{array}$ \\
\hline \multirow[t]{14}{*}{ TransNuclear } & \multirow[t]{14}{*}{ NUHOMS } & $7 \mathrm{P}$ & $\begin{array}{l}\text { Horizontal } \\
\text { Rectangular }\end{array}$ & $\begin{array}{l}\text { Canister Transfer to } \\
\text { Transport Cask }\end{array}$ \\
\hline & & $24 \mathrm{P}$ & $\begin{array}{l}\text { Horizontal } \\
\text { Rectangular }\end{array}$ & $\begin{array}{l}\text { Canister Transfer to } \\
\text { Transport Cask }\end{array}$ \\
\hline & & $32 \mathrm{P}$ & $\begin{array}{l}\text { Horizontal } \\
\text { Rectangular }\end{array}$ & $\begin{array}{l}\text { Canister Transfer to } \\
\text { Transport Cask }\end{array}$ \\
\hline & & 24PT & $\begin{array}{l}\text { Horizontal } \\
\text { Rectangular }\end{array}$ & $\begin{array}{l}\text { Canister Transfer to } \\
\text { Transport Cask }\end{array}$ \\
\hline & & 24PT1 & $\begin{array}{l}\text { Horizontal } \\
\text { Rectangular }\end{array}$ & $\begin{array}{l}\text { Canister Transfer to } \\
\text { Transport Cask }\end{array}$ \\
\hline & & 24PT4 & $\begin{array}{l}\text { Horizontal } \\
\text { Rectangular }\end{array}$ & $\begin{array}{l}\text { Canister Transfer to } \\
\text { Transport Cask }\end{array}$ \\
\hline & & 32PT & $\begin{array}{l}\text { Horizontal } \\
\text { Rectangular }\end{array}$ & $\begin{array}{l}\text { Canister Transfer to } \\
\text { Transport Cask }\end{array}$ \\
\hline & & $12 \mathrm{~T}$ & $\begin{array}{l}\text { Horizontal } \\
\text { Rectangular }\end{array}$ & $\begin{array}{l}\text { Canister Transfer to } \\
\text { Transport Cask }\end{array}$ \\
\hline & & 24PTH & $\begin{array}{l}\text { Horizontal } \\
\text { Rectangular }\end{array}$ & $\begin{array}{l}\text { Canister Transfer to } \\
\text { Transport Cask }\end{array}$ \\
\hline & & 32PTH & $\begin{array}{l}\text { Horizontal } \\
\text { Rectangular }\end{array}$ & $\begin{array}{l}\text { Canister Transfer to } \\
\text { Transport Cask }\end{array}$ \\
\hline & & 24BHP & $\begin{array}{l}\text { Horizontal } \\
\text { Rectangular }\end{array}$ & $\begin{array}{l}\text { Canister Transfer to } \\
\text { Transport Cask }\end{array}$ \\
\hline & & 61BT & $\begin{array}{l}\text { Horizontal } \\
\text { Rectangular }\end{array}$ & $\begin{array}{l}\text { Canister Transfer to } \\
\text { Transport Cask }\end{array}$ \\
\hline & & 61BTH & $\begin{array}{l}\text { Horizontal } \\
\text { Rectangular }\end{array}$ & $\begin{array}{l}\text { Canister Transfer to } \\
\text { Transport Cask }\end{array}$ \\
\hline & & 52B & $\begin{array}{l}\text { Horizontal } \\
\text { Rectangular }\end{array}$ & $\begin{array}{l}\text { Canister Transfer to } \\
\text { Transport Cask }\end{array}$ \\
\hline
\end{tabular}


Table A-8 (Continued)

\begin{tabular}{|c|c|c|c|c|}
\hline Vendor & Cask System & $\begin{array}{l}\text { Canister } \\
\text { Type }\end{array}$ & $\begin{array}{l}\text { Storage } \\
\text { Configuration }\end{array}$ & $\begin{array}{l}\text { Transition to } \\
\text { Transport Required } \\
\text { Operation }\end{array}$ \\
\hline \multirow[t]{3}{*}{ HOLTEC } & \multirow[t]{3}{*}{ HI-STORM } & MPC-24 & $\begin{array}{l}\text { Vertical } \\
\text { Cylinder }\end{array}$ & $\begin{array}{l}\text { Canister Transfer to } \\
\text { Transport Cask }\end{array}$ \\
\hline & & MPC-32 & $\begin{array}{l}\text { Vertical } \\
\text { Cylinder }\end{array}$ & $\begin{array}{l}\text { Canister Transfer to } \\
\text { Transport Cask }\end{array}$ \\
\hline & & MPC-68 & $\begin{array}{l}\text { Vertical } \\
\text { Cylinder }\end{array}$ & $\begin{array}{l}\text { Canister Transfer to } \\
\text { Transport Cask }\end{array}$ \\
\hline HOLTEC & TransStor & $\begin{array}{l}\text { MPC- } \\
\text { 24E/EF }\end{array}$ & $\begin{array}{l}\text { Vertical } \\
\text { Cylinder }\end{array}$ & $\begin{array}{l}\text { Canister Transfer to } \\
\text { Transport Cask }\end{array}$ \\
\hline \multicolumn{5}{|c|}{ Welded Metal Canister in Metal Sealed Overpack (1.4\%) } \\
\hline \multirow[t]{2}{*}{ HOLTEC } & \multirow[t]{2}{*}{ HISTAR 100} & MPC-68 & $\begin{array}{l}\text { Vertical } \\
\text { Cylinder }\end{array}$ & Direct Ship Possible \\
\hline & & MPC-80 & $\begin{array}{l}\text { Vertical } \\
\text { Cylinder }\end{array}$ & Direct Ship Possible \\
\hline \multicolumn{5}{|c|}{ Welded Metal Canister in Vault Storage $(2.4 \%)$} \\
\hline Foster Wheeler & MVDS & $\begin{array}{l}6 \\
\text { assembly } \\
\text { canisters }\end{array}$ & Vault & $\begin{array}{l}\text { Canister Transfer to } \\
\text { Transport Cask }\end{array}$ \\
\hline \multicolumn{5}{|c|}{ Bare Fuel Casks with Bolted Closure (12.8\%) } \\
\hline NAC & NAC I28 & $\mathrm{I} 28$ & $\begin{array}{l}\text { Vertical } \\
\text { Cylinder }\end{array}$ & $\begin{array}{l}\text { Fuel Transfer to } \\
\text { Transport. Cask }\end{array}$ \\
\hline \multirow[t]{3}{*}{ TransNuclear } & \multirow[t]{3}{*}{$\begin{array}{l}\text { TN Metal } \\
\text { Casks }\end{array}$} & TN-32 & $\begin{array}{l}\text { Vertical } \\
\text { Cylinder }\end{array}$ & $\begin{array}{l}\text { Fuel Transfer to } \\
\text { Transport. Cask }^{\mathrm{c}}\end{array}$ \\
\hline & & TN-40 & $\begin{array}{l}\text { Vertical } \\
\text { Cylinder }\end{array}$ & Direct Ship Possible \\
\hline & & TN-68 & $\begin{array}{l}\text { Vertical } \\
\text { Cylinder }\end{array}$ & Direct Ship Possible \\
\hline GNB & CASTOR & $\mathrm{V} / 21$ & $\begin{array}{l}\text { Vertical } \\
\text { Cylinder }\end{array}$ & $\begin{array}{l}\text { Fuel Transfer to } \\
\text { Transport Cask }^{\mathrm{d}}\end{array}$ \\
\hline Westinghouse & MC-10 & MC-10 & $\begin{array}{l}\text { Vertical } \\
\text { Cylinder }\end{array}$ & $\begin{array}{l}\text { Fuel Transfer to } \\
\text { Transport. Cask }\end{array}$ \\
\hline
\end{tabular}

Source: Leduc 2010, Table 2-1

${ }^{\mathrm{a}} \%$ of assemblies in dry storage

${ }^{\mathrm{b}}$ Direct shipment of the NAC I28 may be possible (see Leduc 2012, Section 3.1.3)

${ }^{\mathrm{c}}$ Direct shipment of the TN-32 may be possible (see Leduc 2012, Section 3.1.1)

${ }^{\mathrm{d}}$ Cannot currently be transported for various design reasons (see Leduc 2012, Sections 3.1 .2 and 3.1.4)

${ }^{\mathrm{e}}$ NUHOMS 7P, 12T, 24P, 24PHB, 32P, and 52B cannot currently be transported for various design reasons; however, NUHOMS 24PT, 24PT1, 24PT4, 24PTH, 32PT, 32PTH, 61BT, and $61 \mathrm{BTH}$ are transportable by canister transfer to transport cask. 
Table A-8 shows that several of the canisters/casks cannot presently be transported for different design reasons (Leduc 2010). If an engineering solution cannot be developed to allow for the transport of these casks or canisters, it would be necessary to re-package the used fuel assemblies into a transportable cask or canister. Such a re-packaging operation could be performed within the used fuel pool, provided that the pool is still available. This would not be the case for shut down reactors that have been decommissioned and demolished. In such a situation, a facility to re-package the fuel would have to be either constructed on-site or a portable re-packaging facility, which has yet to be developed, would have to be deployed. The U.S. Government Accountability Office has estimated the cost of constructing a generic wet or dry re-packaging facility at a reactor site at $\$ 300 \mathrm{M}( \pm 50 \%)$ (GAO 2009).

In general, the dry storage cask or canister would have to be moved from the on-site storage facility to a re-packaging facility. A new transportable canister or cask would have to be moved into the re-packaging facility. The lid would have to be removed to access the fuel; either by un-bolting the lid or cutting it off if it is a welded canister. The fuel assemblies would then be transferred to the new cask/canister following the steps described above. The old canister/cask would then have to be cleaned, decontaminated and disposed of, likely as low-level radioactive waste.

A canister/cask re-packaging operation would be costly and increase worker radiation exposures. As an example, the Virginia Electric Power Company (Dominion) has estimated that the total cost of repackaging some of their dry storage canisters would be $\$ 1.5$ million per storage canister $(\$ 150 \mathrm{~K}$ for unloading, $\$ 150 \mathrm{~K}$ for re-loading, $\$ 1 \mathrm{M}$ for a new canister, and $\$ 200 \mathrm{~K}$ for disposal of the old canister/cask) (Rice 2011). In addition, they estimate that re-packaging would increase personnel radiation exposure by an estimated $250 \mathrm{mRem}$ per canister.

Comparing Table A-3 and Table A-8 indicates that seven sites have dry storage systems that are presently not transportable for different design reasons. Table A-9 shows these sites, the canisters types, and the number of canisters that are not presently transportable. These seven reactor sites have pools in operation, so re-packaging could be done there until the reactors shut down and are decommissioned and demolished. The fuel assemblies either in dry storage or soon to be placed in dry storage at the nine shutdown reactor sites are in transportable dry storage systems and could be transported from the sites provided that the infrastructure is in place to support transportation off-site.

Table A-9 also shows the estimated costs and worker exposures that would be incurred to re-package the fuel assemblies in these casks (presumably into the same cask design), assuming the Virginia Electric Power Company estimates. Table A-9 also shows the estimated cost of constructing new re-packaging facilities at these sites in the future, should they be required. Re-packaging costs may be lower as this simple analysis assumes re-packaging into the same size canisters. In reality, re-packaging would likely be into larger capacity canisters and the costs would be lower.

Table A-9 shows the potential costs and worker exposures that could be avoided by identifying an engineering solution for the transportation of these canisters (based on the Virginia Electric Power Company estimates presented above). This evaluation does not address this issue further and assumes that these dry storage canisters are transportable when evaluating the various cases described in Section 3. 
Table A-9 Impacts of On-Site Used Nuclear Fuel Re-Packaging

\begin{tabular}{|c|c|c|c|c|}
\hline Site & $\begin{array}{l}\text { Canister Type/ } \\
\text { Number }\end{array}$ & $\begin{array}{c}\text { Estimated } \\
\text { Re-Packaging } \\
\text { Cost (\$M) }\end{array}$ & $\begin{array}{c}\text { Estimated } \\
\text { Re-Packaging } \\
\text { Exposure } \\
\text { (mRem) }\end{array}$ & $\begin{array}{c}\text { Estimated } \\
\text { Re-Packaging } \\
\text { Facility Cost } \\
\text { (\$M) }\end{array}$ \\
\hline Surry & $\begin{array}{c}\mathrm{V} / 21 \text { and X33: } 26 \\
\text { MC-10: } 1\end{array}$ & 40.5 & 6,750 & 300 \\
\hline ANO & VSC-24: 24 & 36 & 6,000 & 300 \\
\hline Calvert Cliffs & $\begin{array}{l}24 \mathrm{P}: 48 \\
32 \mathrm{P}: 32\end{array}$ & 103.5 & 17,250 & 300 \\
\hline Davis Besse & 24P: 3 & 4.5 & 750 & 300 \\
\hline Susquehanna & 52B: 27 & 40.5 & 6,750 & 300 \\
\hline Oconee & $\begin{array}{c}\text { 24PHB: } 38 \\
24 \mathrm{P}: 84\end{array}$ & 183 & 30,500 & 300 \\
\hline Robinson & $7 \mathrm{P}: 8$ & 12 & 2,000 & 300 \\
\hline Total & 280 & 420 & 70,000 & 2,100 \\
\hline
\end{tabular}

Note: Calvert Cliffs plans to continue loading 32P canisters and Oconee plans to continue loading 24PHP canisters

\section{A-1.3.3 Additional Evaluation Assumptions for At-Reactor Operations}

The following additional assumptions, beyond those described above, were made to evaluate at-reactor impacts for the different evaluation cases described in Section 3. Generally speaking, these are analytical assumptions for convenience, rather than hard and fast constraints. Future analyses will explore the implications of changing these assumptions.

- Reactor sites utilize dry storage to maintain full-core off-load reserve capacity in the used fuel pools. When the fuel pool assembly inventory exceeds the full-core off-load reserve capacity in a year, it is assumed that fuel is moved to dry storage. It is recognized that some utilities may allow the available pool capacity to be smaller than the full-core off-load reserve capacity and also strive to load fuel in campaigns that transfer large amounts of fuel to dry storage. As such, the actual rate that fuel would be moved from the pools to dry storage over time will likely differ from a rate projected using the full-core off-load reserve capacity assumption, however the overall trends and amounts of fuel being transferred to dry storage will be similar.

- The ability to load fuel assemblies into dry storage at reactor sites is unconstrained, except by canister/cask thermal limits. In other words, all fuel that needs to be transferred to dry storage in a given year to maintain pool capacity can be moved, within the thermal limits of the storage canister/cask.

- An oldest-fuel-first (OFF) allocation is used to determine the amount of fuel, in terms of MTHM, that will be accepted from each reactor site when fuel is transported away from reactor sites.

- A youngest-fuel-first, minimum 5-year out of reactor (YFF-5) fuel prioritization is used to determine the number of fuel assemblies that are transported within the allocated MTHM amount for each reactor site. It is assumed that reactor operators would prefer to transfer younger fuel from the used fuel pools first and leaving the generally older fuel in dry storage to both increase the available capacity in the used fuel pools and to reduce or eliminate the need to transfer additional fuel to dry storage. 
- No inter-utility or intra-utility trading of allocation rights.

- The ability to load fuel assemblies into either re-useable transportation casks or transportable canisters for subsequent shipping from reactor sites is not constrained, except by thermal limits. All fuel that is allocated for shipping from a reactor site in a year is loaded into a re-useable transportation casks or transportable canisters can be moved, unless constrained by thermal limits.

- Thermal limits on transportation overpacks/casks for canisters, and re-useable bare fuel transportation casks apply. Only full canisters and/or re-useable transportation casks that meet the thermal limits are shipped. Any remaining fuel allocation that cannot be shipped due to thermal constraints is carried into the next year and added to the reactor's allocation for that year.

- For cases where all fuel assemblies from the pool are transferred to dry storage at reactor shutdown, this transition occurs five years after shutdown.

- The minimum time that fuel can either be moved to dry storage or transported off site is five years.

The following assumptions were made with respect to transport away from the operating and shutdown reactor sites for a mostly rail transportation scenario involving the transport of bare fuel from the reactor sites, consistent with the Yucca Mountain FEIS:

- Six sites without sufficient crane capacity to lift a rail cask or without other factors such as sufficient floor loading capacity or ceiling height were assumed to ship by legal-weight truck.

- These six sites will be upgraded to handle rail casks once the reactors are shut down, and all remaining spent nuclear fuel would ship by rail.

- Of these six sites, two are direct rail and four are indirect rail sites. Of the four with indirect rail access, three have access to a navigable waterway.

- Twenty-four sites with sufficient crane capacity but without direct rail access were assumed to ship by heavy-haul truck to the nearest railhead.

It was assumed for scenarios involving the transport of all fuel from the reactor sites in large existing size canisters that

- Sufficient crane capacity would be added to the six sites currently without sufficient capacity to handle large canisters.

- Shipment of canisters away from these sites would be by direct rail or heavy-haul truck

- Twenty-four sites with sufficient crane capacity but without direct rail access were assumed to ship by heavy-haul truck to the nearest railhead.

\section{A-2. Projections of At-Reactor Used Nuclear Fuel Inventories and Acceptance}

The TSL model was used to project used nuclear fuel inventories at each of the reactor sites, the number of bare fuel casks and dry storage canisters that would be shipped for the different start dates for fuel acceptance $(2020,2035)$ and fuel acceptance rates (1500, 3000, 6000 MT/yr). Again, a YFF-5 fuel shipping prioritizaton was used.

Summary results are presented and discussed in this section. The TSL produces much more detailed results (i.e., individual cask/canister shipments and quantities) that can be used for additional analysis. 


\section{A-2.1 At Reactor Logistics}

The logistic steps for the cases under consideration are described in detail in Section 3.1. This section repeats those logistic steps; focusing on those that affect at-reactor used fuel management operations.

\section{Cases 1 and 3}

Cases 1 and 3, described in Sections 3.1.1 and 3.1.3, respectively, involve the loading of exising size canisters/casks at the reactor sites for storage and subsequent transportation. The logistic steps are:

- Bare fuel transferred to existing size canisters during reactor operation to maintain full core offload capacity in the at-reactor wet pool

- On-Site Storage prior to destination availability (CSF or repository)

- Transported from Reactor Site when destination is available, provided the canisters are within the thermal limits of transportation casks/overpacks (CSF or repository)

- Bare fuel in the wet pool transferred to existing size canisters during reactor operation for transport to destination when available (CSF or repository)

- Bare fuel transferred to existing size canisters at reactor shutdown

- On-Site Storage prior to destination availability (CSF or repository)

- Transported from Reactor Site when destination is available, provided the canisters are within the thermal limits of transportation casks/overpacks (CSF or repository)

For these cases, it was assumed that the Holtec MPC-32 and MPC-68 systems would be used for transporting bare fuel residing in the used nuclear fuel pools.

\section{Cases 2 and 4}

Cases 2 and 4, described in Sections 3.1.2 and 3.1.4, respectively, both involve the loading of exising size canisters/casks at the reactor sites for storage to maintain fuel pool capacity and when a reactor shuts down. However, any fuel transportation to the CSF from the spent fuel pools is done as bare fuel transport using re-usable transporation casks. The logistic steps are:

- Bare fuel transferred to existing size canisters during reactor operation to maintain full core offload capacity in the at-reactor wet pool

- On-Site Storage prior to destination availability (CSF or repository)

- Transported from Reactor Site when destination is available, provided the canisters are within the thermal limits of transportation casks/overpacks (CSF or repository)

- Bare fuel in the wet pool transported in re-useable transportation casks during reactor operation when destination is available (CSF or repository)

- Bare fuel transferred to existing size canisters at reactor shutdown

- On-Site Storage prior to destination availability (CSF or repository)

- Transported from Reactor Site when destination is available, provided the canisters are within the thermal limits of transportation casks/overpacks (CSF or repository)

\section{A-2.2 At Reactor Logistic Results: On-Site Dry Storage Inventories}

This section presents the at-reactor logistic results obtained from the TSL simulations pertaining to atreactor dry storage inventories and requirements. The results of the TSL simulations indicate that the inventory of used nuclear fuel in on-site dry storage depends primarily on the acceptance rate and when acceptance begins. There is little to no difference in the maximum used nuclear fuel inventory in dry storage for the different cases for a given acceptance rate and acceptance start date. Except for the 3000 
$\mathrm{MTHM} / \mathrm{yr}$ acceptance rate, the time dependent trend in the at-reactor dry storage inventory is also very similar for the different cases for a given acceptance rate and acceptance start date (see Section A-2.2.3 for more details). Summary-level results are shown in Table A-10.

The total amount of used fuel loaded into at-reactor dry storage also depends on the acceptance rate and when acceptance begins. However, unlike the maximum dry storage inventory, the total number of canisters loaded into at-reactor dry storage does change for the different cases for a given acceptance rate and acceptance start date. In particular, those cases where used fuel from the pools is transported from the reactors in DPCs (Cases 1 and 3) result in a larger total amount of fuel being loaded into at-reactor dry storage compared to cases where fuel from the pools is transported using re-useable transportation casks (Cases 2 and 4).

Table A-10. Summary Level TSL Simulation Results for At-Reactor Dry Storage

\begin{tabular}{|c|c|c|c|c|c|}
\hline & $\begin{array}{l}\text { Acceptance } \\
\text { Rate } \\
\text { (MT/yr) }\end{array}$ & $\begin{array}{l}\text { Acceptance } \\
\text { Start }\end{array}$ & $\begin{array}{l}\text { Cumulative } \\
\text { Canisters Loaded } \\
\text { into Dry Storage }\end{array}$ & $\begin{array}{c}\text { Peak } \\
\text { Canister } \\
\text { Inventory }\end{array}$ & $\begin{array}{c}\text { Peak } \\
\text { MTHM } \\
\text { Inventory }\end{array}$ \\
\hline \multirow{6}{*}{$\begin{array}{c}\text { Canistered } \\
\text { Fuel Transport } \\
\text { (Cases } 2 \text { \& 4) }\end{array}$} & 1500 & 2020 & 9226 & 6762 & 83384 \\
\hline & 3000 & 2020 & 7910 & 2652 & 32433 \\
\hline & 6000 & 2020 & 7420 & 2459 & 29552 \\
\hline & 1500 & 2035 & 10308 & 8592 & 105872 \\
\hline & 3000 & 2035 & 9638 & 6200 & 76674 \\
\hline & 6000 & 2035 & 9141 & 5023 & 61339 \\
\hline \multirow{6}{*}{$\begin{array}{c}\text { Bare and } \\
\text { Canistered } \\
\text { Fuel Transport } \\
\text { (Cases } 1 \text { \& 3) }\end{array}$} & 1500 & 2020 & 8196 & 6787 & 83532 \\
\hline & 3000 & 2020 & 5106 & 3158 & 38538 \\
\hline & 6000 & 2020 & 2768 & 2483 & 30179 \\
\hline & 1500 & 2035 & 10054 & 8610 & 105953 \\
\hline & 3000 & 2035 & 8547 & 6306 & 78061 \\
\hline & 6000 & 2035 & 6629 & 5331 & 65592 \\
\hline
\end{tabular}

Recall that in Cases 1 and 3 it is assumed that the Holtec MPC-32 and MPC-68 systems would be used for transporting bare fuel residing in the used nuclear fuel pools and no other bare fuel transportation casks would be used. It was assumed that the thermal output of a fuel assembly was limited at 201 watts for BWR fuel and 532 watts for PWR fuel to maintain the total thermal output of the transportation overpacks within their certified limits. Under a YFF-5 fuel acceptance scenario, in some years there was no fuel available in the used fuel pools at certain reactors that met these thermal output limits and fuel in dry storage was transported off-site instead to achieve that reactor's annual allocation of fuel to ship. This then necessitated those reactors to off-load fuel from the pools to dry storage to maintain pool capacity.

This behavior results from the assumptions presented in Sections A-1.3 and A-1.4: 1) reactor sites will utilize dry storage to maintain full-core off-load reserve capacity in the used fuel pools, and 2) the ability to load fuel assemblies into dry storage at reactor sites is unconstrained.

These assumptions could potentially be challenged while a reactor is in operation due to multiple requirements and demands on the used fuel pool during and operating fuel cycle. Such demands include 
receipt of fresh fuel, core re-load, fuel inspections/repair, and maintenance of the spent fuel pool. As an example, it has been estimated that a used fuel pool would potentially be available for approximately 30 weeks during an operating fuel cycle to support used fuel transfer activities (Leblang 2011). Such a window may constrain the amount of used fuel that could be transferred to dry storage when either multiple fuel handling activities occur within a given operating fuel cycle (transfer to dry storage and loading for shipping off-site) or potentially when smaller capacity canisters are loaded (waste package compatible size canisters). These constraints should be further explored and their impacts on at-reactor logistics evaluated.

\section{A-2.2.1 1500 MTHM/year YFF-5 Acceptance, Starting in 2020}

Figure A-2 shows the inventory of used nuclear fuel (assemblies, MTHM) and casks in at-reactor dry storage as a function of time for a $1500 \mathrm{MTHM} / \mathrm{yr}$ - YFF-5 acceptance rate, starting in 2020 assuming $\mathrm{UNF}$ in the pools is all placed in DPCs prior to transport (Cases 1 and 3). Figure A-3 shows the inventory of used nuclear fuel (assemblies, MTHM) and casks in at-reactor dry storage as a function of time for a $1500 \mathrm{MTHM} / \mathrm{yr}$ - YFF-5 acceptance rate, starting in 2020 assuming UNF in the pools is transported using re-useable transportation casks (Cases 2 and 4). Figure A-14 shows the cumulative number of canisters placed in dry storage.

A $1500 \mathrm{MTHM} / \mathrm{yr}$ used fuel acceptance rate beginning in 2020 does not overcome the need for reactor sites to continue transferring fuel from the used fuel pools into dry storage because it does not even keep up with the rate at which fuel is being discharged from the reactor fleet ( 2000 MTHM/yr). The sharp increase in the rate that used fuel is transferred to dry storage coincides with the shutdown of the reactor fleet that begins in 2029 and runs through 2055. The peak total inventory of approximately 83,000 MTHM (53,000 MTHM PWR, 30,000 MTHM BWR) occurs in 2054, coinciding with the date of the last reactor shutdown. It is only after all the reactors have shutdown does the inventory of used fuel in dry storage begin to decrease with the inventory of used nuclear fuel being removed from the reactor sites in 2113.

\section{A-2.2.2 1500 MTHM/year YFF-5 Acceptance, Starting in 2035}

Figure A-4 shows the inventory of used nuclear fuel (assemblies, MTHM) and casks in at-reactor dry storage as a function of time for a $1500 \mathrm{MTHM} / \mathrm{yr}$ - YFF-5 acceptance rate, starting in 2035 assuming UNF in the pools is all placed in DPCs prior to transport (Cases 1 and 3). Figure A-5 shows the inventory of used nuclear fuel (assemblies, MTHM) and casks in at-reactor dry storage as a function of time for a $1500 \mathrm{MTHM} / \mathrm{yr}$ - YFF-5 acceptance rate, starting in 2035 assuming UNF in the pools is transported using re-useable transportation casks (Cases 2 and 4). Figure A-14 shows the cumulative number of canisters placed in dry storage.

A $1500 \mathrm{MTHM} / \mathrm{yr}$ used fuel acceptance rate beginning in 2035 also does not overcome the need for reactor sites to continue transferring fuel from the used fuel pools into dry storage. The sharp increase in the rate that used fuel is transferred to dry storage again coincides with the shutdown of the reactor fleet that begins in 2029 and runs through 2054. The peak total inventory of approximately 105,000 MTHM (68,000 MTHM PWR, 37,000 MTHM BWR) occurs in 2055, coinciding with the date of the last reactor shutdown. It is only after all the reactors have shutdown does the inventory of used fuel in dry storage begin to decrease with the inventory of used nuclear fuel being removed from the reactor sites in 2128 (15 years later than starting in 2020). Initiating acceptance in 2035 results in an additional 20,000 MTHM of used nuclear fuel being transferred to dry storage. 

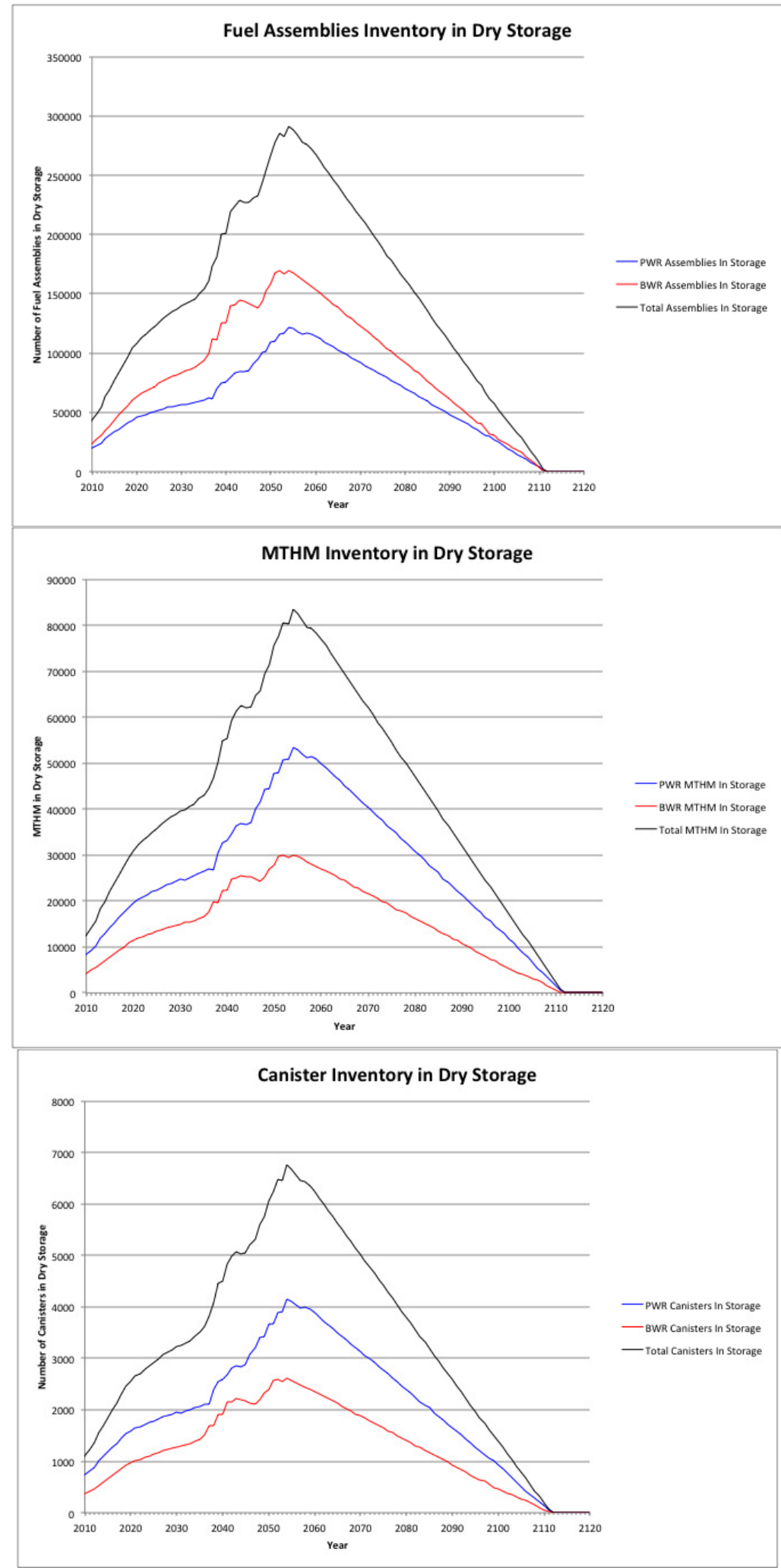

Figure A-2. Cases $1 \&$ 3: Total At-Reactor Dry Storage Inventory, 1500 MTHM YFF-5 Acceptance 2020 

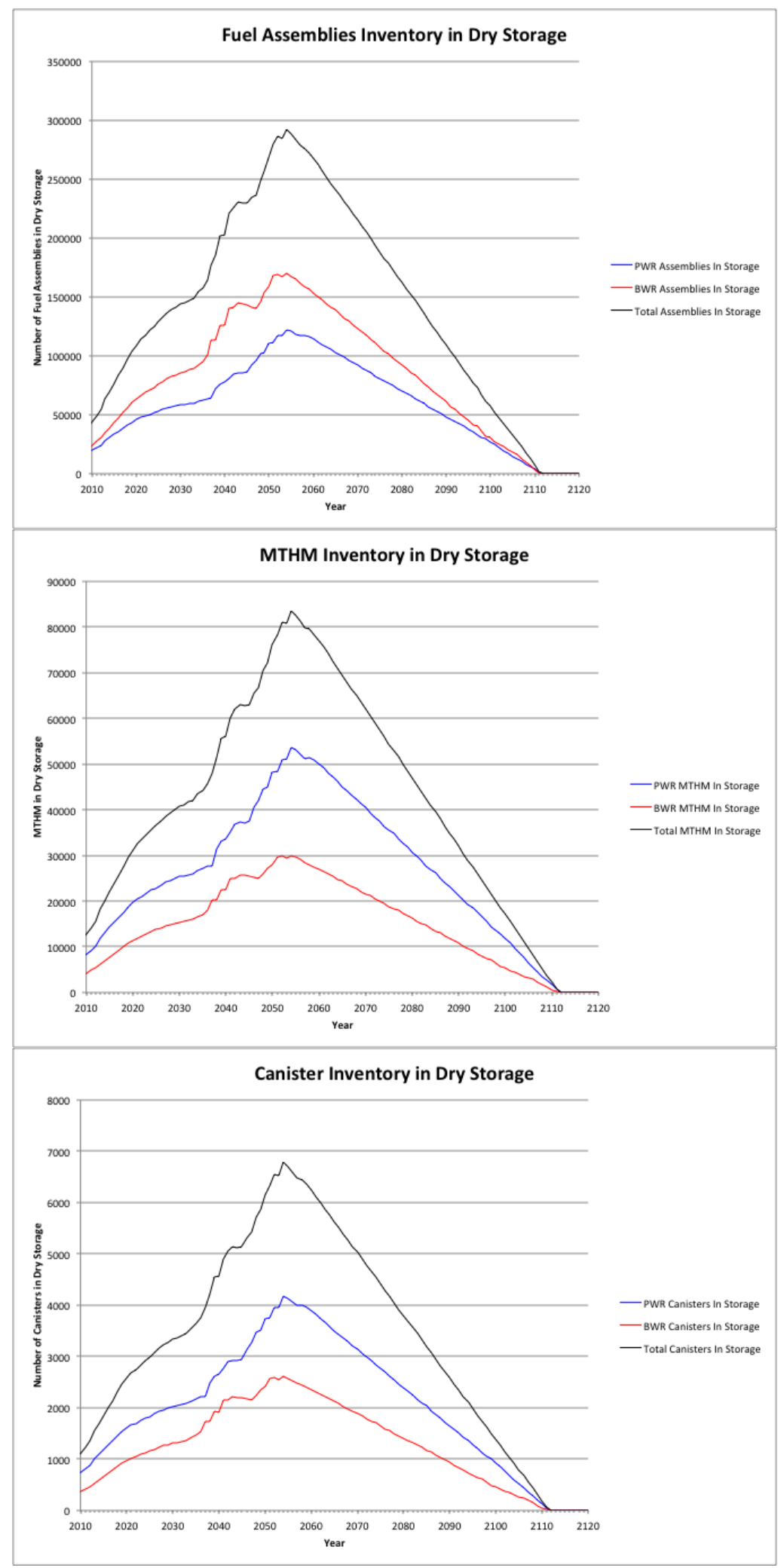

Figure A-3. Cases 2 \& 4: Total At-Reactor Dry Storage Inventory, 1500 MTHM YFF-5 Acceptance 2020 

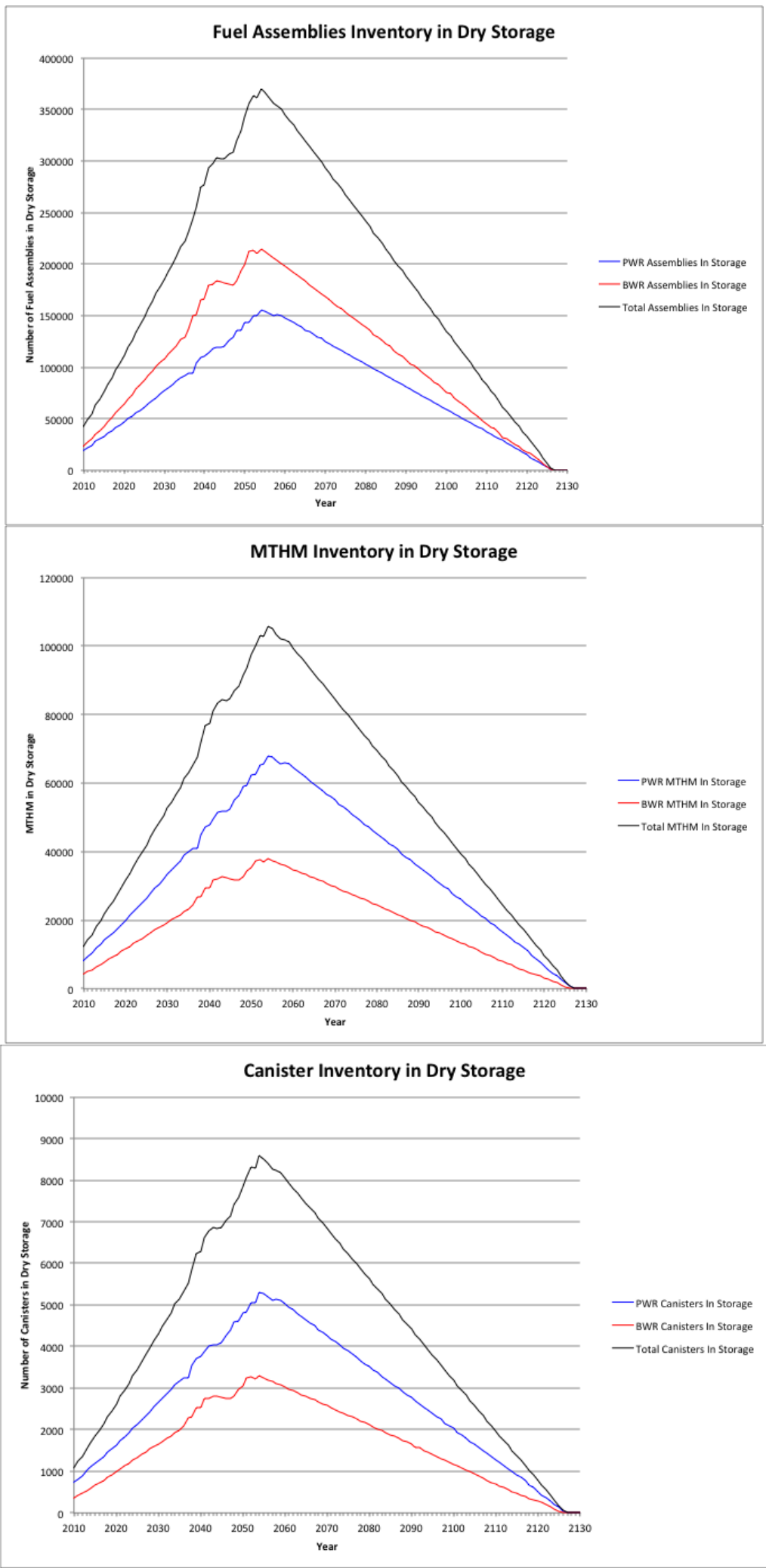

Figure A-4. Cases 1 \& 3: Total At-Reactor Dry Storage Inventory, 1500 MTHM YFF-5 Acceptance 2035 

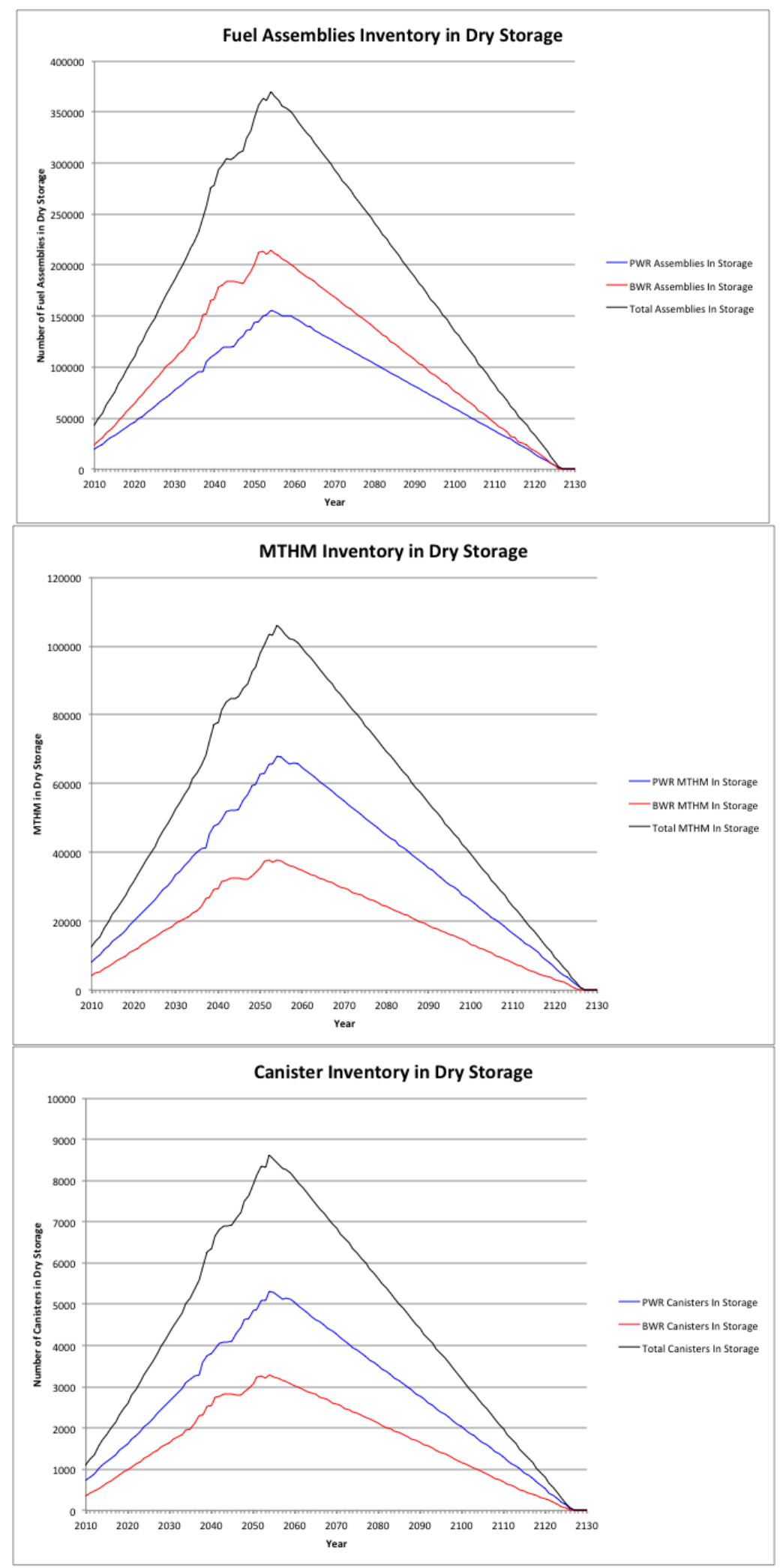

Figure A-5. Cases $2 \&$ 4: Total At-Reactor Dry Storage Inventory, 1500 MTHM YFF-5 Acceptance 2035 


\section{A-2.2.3 3000 MTHM/year YFF-5 Acceptance, Starting in 2020}

Figure A-6 shows the inventory of used nuclear fuel (MTHM) and casks in at-reactor dry storage as a function of time for a $3000 \mathrm{MTHM} / \mathrm{yr}$ - YFF-5 acceptance rate, starting in 2020 assuming UNF in the pools is all placed in DPCs prior to transport (Cases 1 and 3). Figure A-7 shows the inventory of used nuclear fuel (assemblies, MTHM) and casks in at-reactor dry storage as a function of time for a 3000 MTHM/yr - YFF-5 acceptance rate, starting in 2020 assuming UNF in the pools is transported using reuseable transportation casks (Cases 2 and 4). Figure A-14 shows the cumulative number of canisters placed in dry storage.

A $3000 \mathrm{MTHM} / \mathrm{yr}$ used fuel acceptance rate beginning in 2020 overcomes the need for reactor sites to continue transferring fuel from the used fuel pools into dry storage while in the reactors are in operation. This is shown in the decreasing dry storage inventory that occurs when acceptance begins in 2020 .

However, when the reactor fleet begins to shutdown, starting in 2029 and running through 2055, the 3000 $\mathrm{MTHM} / \mathrm{yr}$ acceptance rate is not sufficient to overcome the need to transfer the remaining used fuel from the wet pools to dry storage. This is shown in the increase in the dry storage inventory that begins in 2029.

This trend is seen in both Figure A-6 (Cases $1 \& 3$ ) and Figure A-7 (Cases $2 \& 4$ ) and is evident by the two peaks that occur. The first is at 2020, when acceptance begins, and the second at 2055, coinciding with the date of the last reactor shutdown. Only after all the reactors have shutdown does the inventory of used fuel in dry storage begin to decrease with the inventory of used nuclear fuel being removed from the reactor sites in 2067.

The time-dependent dry storage inventory shown in Figure A-6 (Cases $1 \&$ 3) and Figure A-7 (Cases 2 \& 4) are quite different as is the cumulative number of canisters placed in dry storage, shown in Figure A14. For Cases 2 and 4, at $3000 \mathrm{MTHM} /$ year fuel is predominately shipped from the pools, however there is still a need to transfer fuel into dry storage at some reactor sites. Shipments from dry storage do not occur until later, after the pools are depleted of fuel available for transport. There is a larger amount of fuel being transferred into dry storage than is being removed after acceptance begins. When the reactor fleet begins to shut down, the additional canisters loaded to dry storage add to a larger inventory already in dry storage. Thus, although the cumulative number of canisters loaded into dry storage is larger for Cases 1 and 3, the peak number of canisters loaded into dry storage is larger for Cases 2 and 4.

The peak total inventory is approximately 34,000 MTHM (20,000 MTHM PWR, 14,000 MTHM BWR), with two peaks occurring for Cases 1 and 3. The peak total inventory is approximately 39,000 MTHM (22,000 MTHM PWR, 17,000 MTHM BWR) for Cases 2 and 4.

The peak on-site dry storage requirements are significantly less for an acceptance rate of $3000 \mathrm{MTHM} / \mathrm{yr}$ as compared to $1500 \mathrm{MTHM} / \mathrm{yr}$; 34,000 - 39,000 MTHM versus 83,000 MTHM. Thus, a 3000 MTHM/yr acceptance rate beginning in 2020 essentially "maintains" the overall on-site dry storage needs at 2020 levels, with the inventory first decreasing, then increasing as reactors shut down.

\section{A-2.2.4 3000 MTHM/year YFF-5 Acceptance, Starting in 2035}

Figure A-8 shows the inventory of used nuclear fuel (MTHM) and casks in at-reactor dry storage as a function of time for a $3000 \mathrm{MTHM} / \mathrm{yr}$ - YFF-5 acceptance rate, starting in 2035 assuming UNF in the pools is all placed in DPCs prior to transport (Cases 1 and 3). Figure A-9 shows the inventory of used nuclear fuel (assemblies, MTHM) and casks in at-reactor dry storage as a function of time for a 3000 MTHM/yr - YFF-5 acceptance rate, starting in 2035 assuming UNF in the pools is transported using reuseable transportation casks (Cases 2 and 4). Figure A-14 shows the cumulative number of canisters placed in dry storage.

A $3000 \mathrm{MTHM} / \mathrm{yr}$ used fuel acceptance rate beginning in 2035 does not overcome the need for reactor sites to continue transferring fuel from the used fuel pools into dry storage while in they are in operation 
and after the reactors begin to shut down, starting in 2029 and running through 2055 . The acceptance of used nuclear fuel, beginning in 2035, reduces the rate that fuel is transferred to dry storage, but the 3000 $\mathrm{MTHM} / \mathrm{yr}$ acceptance rate is not sufficient to eliminate the need to transfer fuel from operating and shutdown reactors to dry storage.

The peak total inventory is approximately 77,000 MTHM (49,000 MTHM PWR, 28,000 MTHM BWR) and occurs in 2055. It is only after all the reactors have shutdown does the inventory of used fuel in dry storage begin to decrease with the inventory of used nuclear fuel being removed from the reactor sites in 2081. The peak inventories are essentially double that of a $3000 \mathrm{MTHM} / \mathrm{yr}$ acceptance rate starting in 2020. The peak inventory is significantly less than that for a $1500 \mathrm{MTHM} / \mathrm{yr}$ acceptance rate starting in 2035 (105,000 MTHM total, 68,000 MTHM PWR, 37,000 MTHM BWR). 

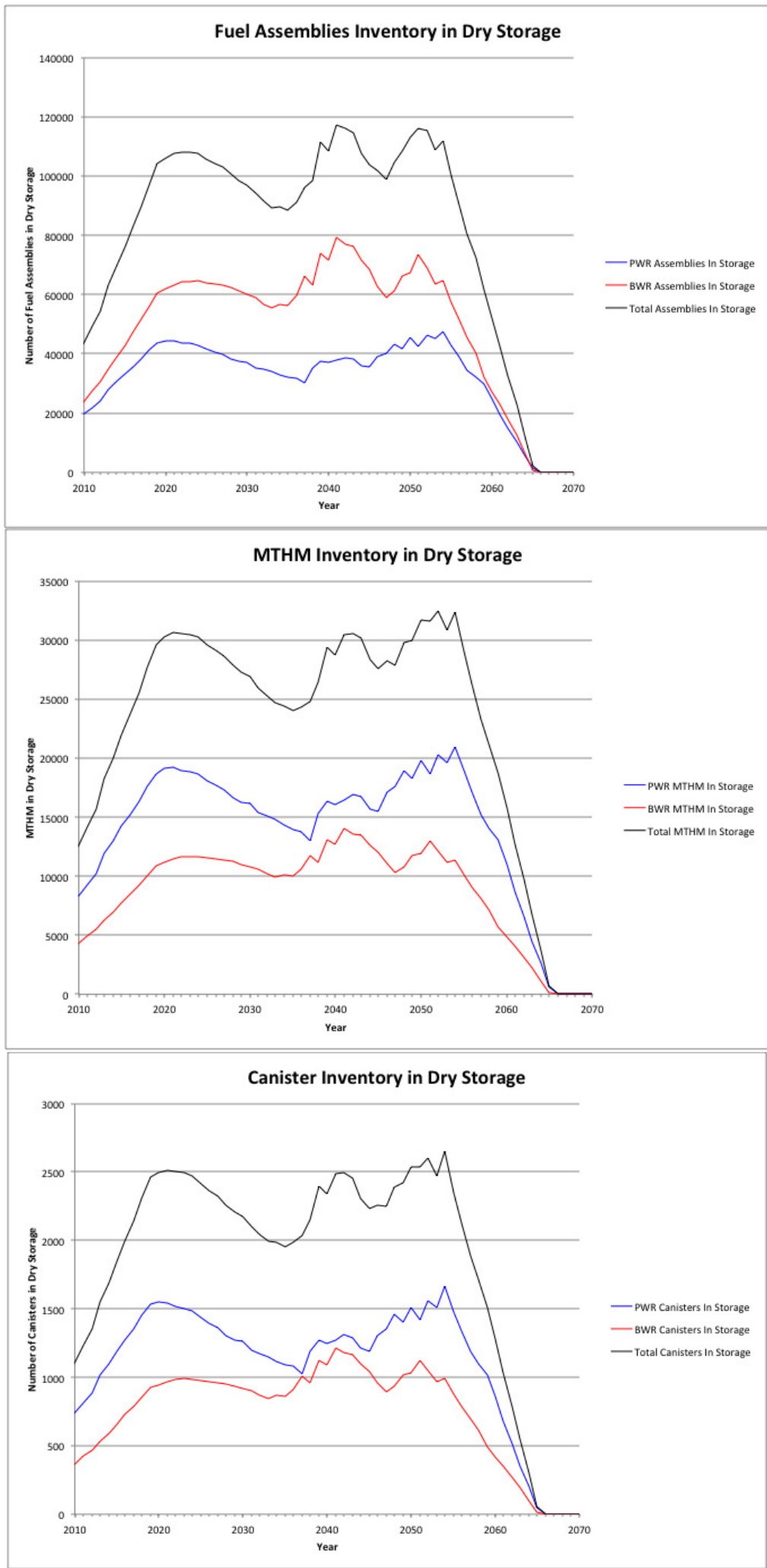

Figure A-6. Cases 1 \& 3: Total At-Reactor Dry Storage Inventory, 3000 MTHM YFF-5 Acceptance 2020 

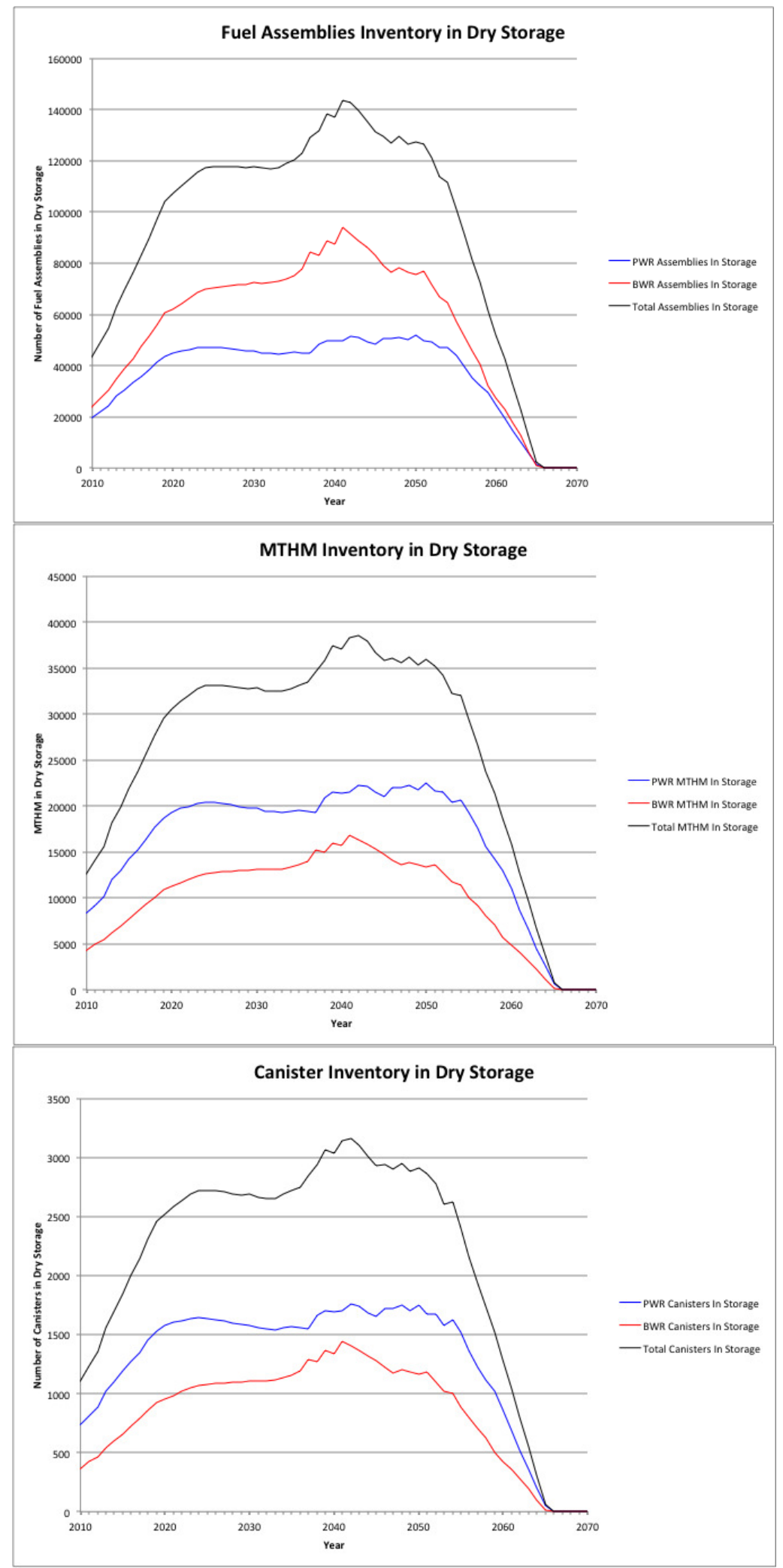

Figure A-7. Cases 2 \& 4: Total At-Reactor Dry Storage Inventory, 3000 MTHM YFF-5 Acceptance 2020 

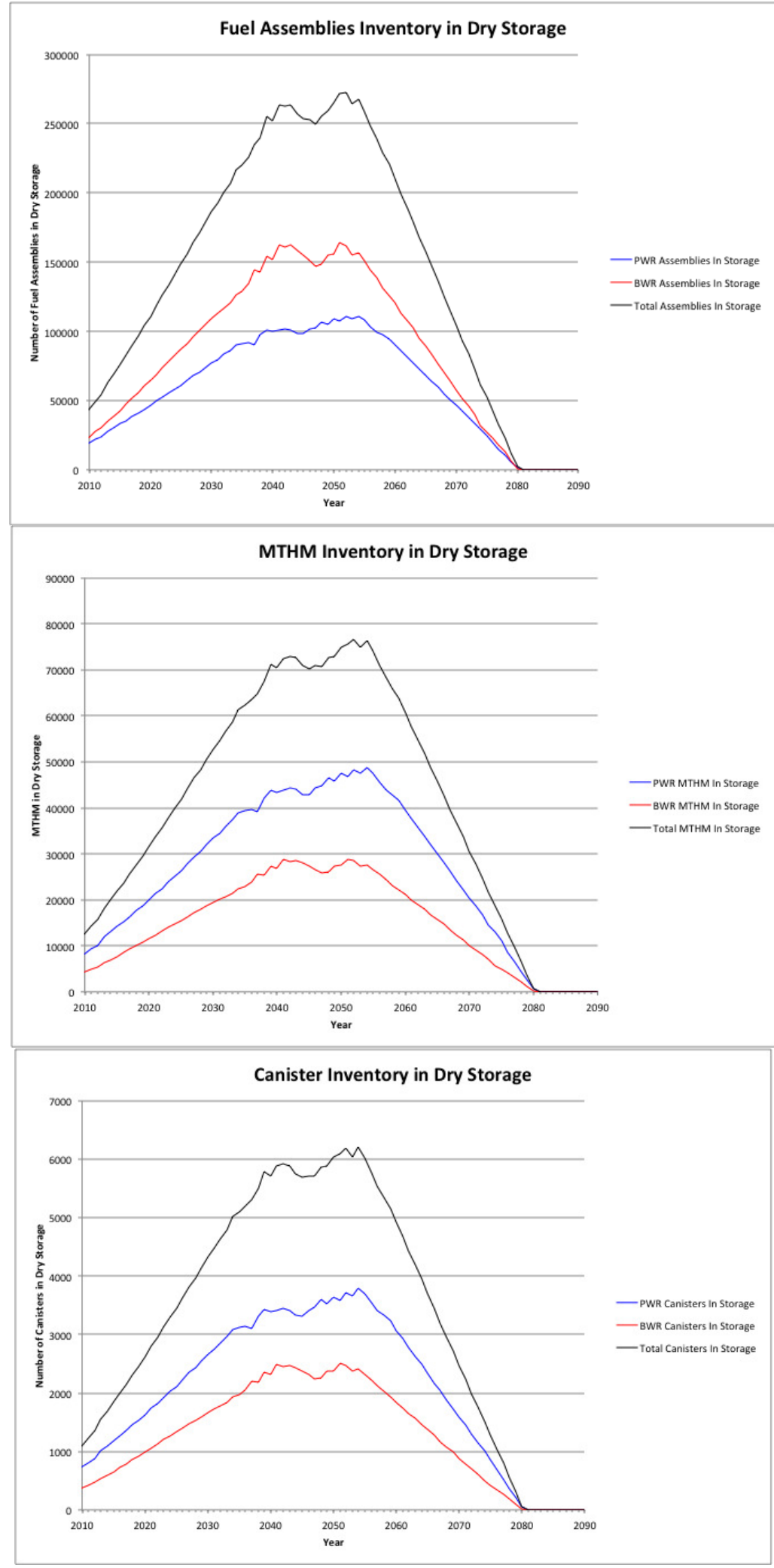

Figure A-8. Cases 1 \& 3: Total At-Reactor Dry Storage Inventory, 3000 MTHM YFF-5 Acceptance 2035 

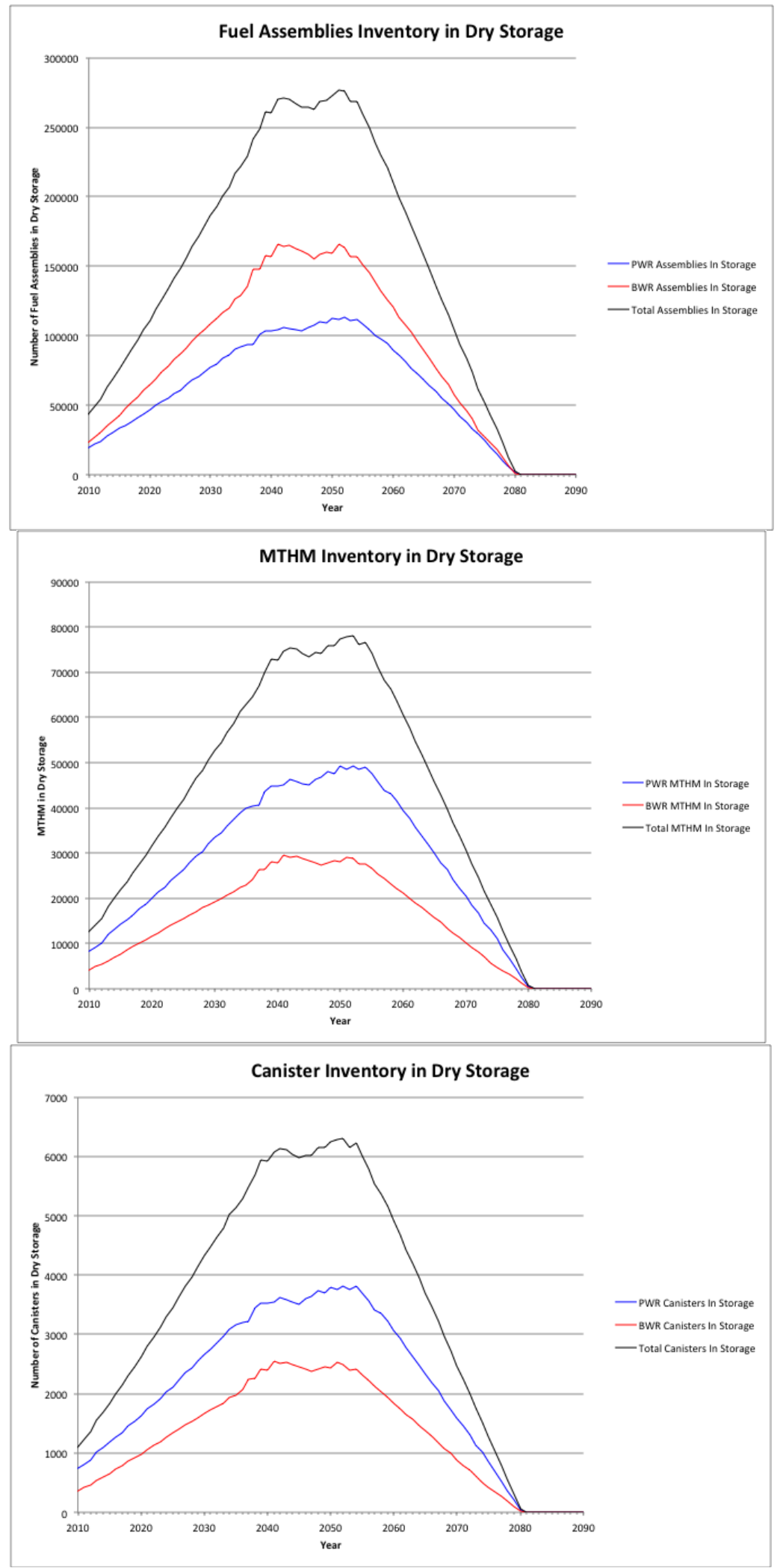

Figure A-9. Cases 2 \& 4: Total At-Reactor Dry Storage Inventory, 3000 MTHM YFF-5 Acceptance 2035 


\section{A-2.2.5 6000 MTHM/year YFF-5 Acceptance, Starting in 2020}

Figure A-10 shows the inventory of used nuclear fuel (assemblies, MTHM) and casks in at-reactor dry storage as a function of time for a $6000 \mathrm{MTHM} / \mathrm{yr}$ - YFF-5 acceptance rate, starting in 2020 assuming UNF in the pools is all placed in DPCs prior to transport (Cases 1 and 3). Figure A-11 shows the inventory of used nuclear fuel (assemblies, MTHM) and casks in at-reactor dry storage as a function of time for a $6000 \mathrm{MTHM} / \mathrm{yr}$ - YFF-5 acceptance rate, starting in 2020 assuming UNF in the pools is transported using re-useable transportation casks (Cases 2 and 4). Figure A-14 shows the cumulative number of canisters placed in dry storage.

A $6000 \mathrm{MTHM} / \mathrm{yr}$ used fuel acceptance rate beginning in 2020 overcomes the need for reactor sites to continue transferring fuel from the used fuel pools into dry storage while in they are in operation when fuel residing in the pools is transported off-site using re-useable transportation casks (Cases 2 and 4). This is shown in the decreasing dry storage inventory that occurs when acceptance begins in 2020 . The results show that all of the used fuel placed in dry storage by 2020 would be removed by 2040 . No additional dry storage is likely beyond that required through 2020.

However, as discussed above, when fuel residing in the pools is transported off-site using DPCs, the thermal constraints on the DPC transportation overpacks limits the amount of fuel that is shipped directly from the pools. This necessitates shipping cooler fuel from dry storage and the subsequent transfer of fuel from the pools to dry storage. Thus, although additional dry storage capacity would not be needed because old casks would be removed at the same rate as new ones are added, loading additional storage casks and continued use of the at reactor dry storage facilities would be needed. This is evident by the trends shown in Figure A-10 after about 2035.

The peak total inventory is approximately 30,000 MTHM (19,000 MTHM PWR, 11,000 MTHM BWR), with the peak occurring in 2020. The dry storage requirements are similar to that of the $3000 \mathrm{MTHM} / \mathrm{yr}$ starting in 2020 case (39,000 MTHM total, 22,000 MTHM PWR, 17,000 MTHM BWR). This indicates that the increased acceptance rate somewhat reduces the peak at-reactor dry storage requirements. In addition, the increased acceptance rate decreases the duration that dry storage needs to be in place at reactor sites by approximately 25 years (primarily for Cases 2 and 4 which utilize re-useable transportation casks).

\section{A-2.2.6 6000 MTHM/year YFF-5 Acceptance, Starting in 2035}

Figure A-12 shows the inventory of used nuclear fuel (assemblies, MTHM) and casks in at-reactor dry storage as a function of time for a $6000 \mathrm{MTHM} / \mathrm{yr}$ - YFF-5 acceptance rate, starting in 2035 assuming UNF in the pools is all placed in DPCs prior to transport (Cases 1 and 3). Figure A-13 shows the inventory of used nuclear fuel (assemblies, MTHM) and casks in at-reactor dry storage as a function of time for a $6000 \mathrm{MTHM} / \mathrm{yr}$ - YFF-5 acceptance rate, starting in 2035 assuming UNF in the pools is transported using re-useable transportation casks (Cases 2 and 4). Figure A-14 shows the cumulative number of canisters placed in dry storage.

A $6000 \mathrm{MTHM} / \mathrm{yr}$ used fuel acceptance rate beginning in 2035 overcomes the need for reactor sites to continue transferring fuel from the used fuel pools into dry storage while in they are in operation.

Beginning acceptance in 2035 coincides with the time that reactors begin to shutdown (starting in 2029 and running through 2055). The TSL results show a non-linear decrease in the dry storage inventory once acceptance begins. This again results from used fuel at shutdown reactors being transferred to dry storage five years following shutdown and due to thermal constraints on the DPC transportation overpacks when used to transport fuel from the pools (Cases $1 \& 3$ ).

When the reactor fleet begins to shutdown, starting in 2029 and running through 2055, the 6000 MTHM/yr TSL simulations indicate that some used nuclear fuel will be transferred to dry storage five years after reactor shutdown (note the continued inventory increase after 2035 shown in Figure A-13). In reality, it is likely that the fuel prioritization strategy under a $6000 \mathrm{MTHM} / \mathrm{yr}$ acceptance rate (or any 
acceptance rate) would not follow a strict OFF allocation/YFF acceptance approach and would give priority to removing fuel from those reactors sites that are ready to move to decommissioning and demolition. If that were done, no additional dry storage would be likely beyond that required through 2035.

The peak total inventory at 2035 is approximately 61,000 MTHM (39,000 MTHM PWR, 22,000 MTHM BWR) for Cases 1 and 3 and approximately 65,000 MTHM (40,000 MTHM PWR, 25,000 MTHM BWR) for Cases 2 and 4. These are essentially double that seen for the 6000 MTHM acceptance rate starting in 2020 (30,000 MTHM total, 19,000 MTHM PWR, 11,000 MTHM BWR). The dry storage requirements are less than that of the $3000 \mathrm{MTHM} / \mathrm{yr}$ starting in 2035 case (77,000 MTHM total, 49,000 MTHM PWR, 28,000 MTHM BWR). This is due to not having to transfer any fuel from shutdown reactors to dry storage for the $6000 \mathrm{MTHM} / \mathrm{yr}$ acceptance rate. 

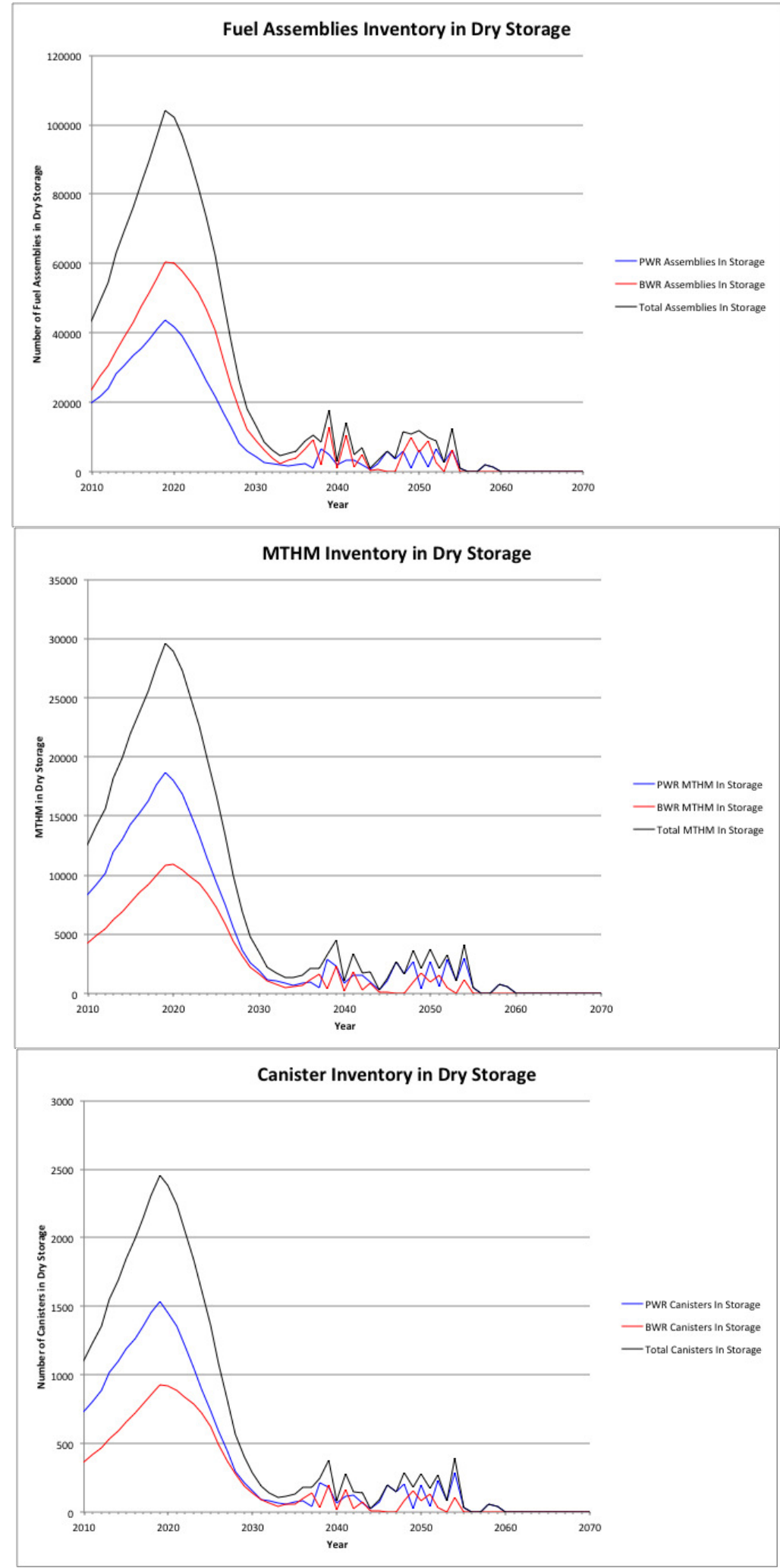

Figure A-10. Cases $1 \&$ 3: Total At-Reactor Dry Storage Inventory, 6000 MTHM YFF-5 Acceptance 2020 


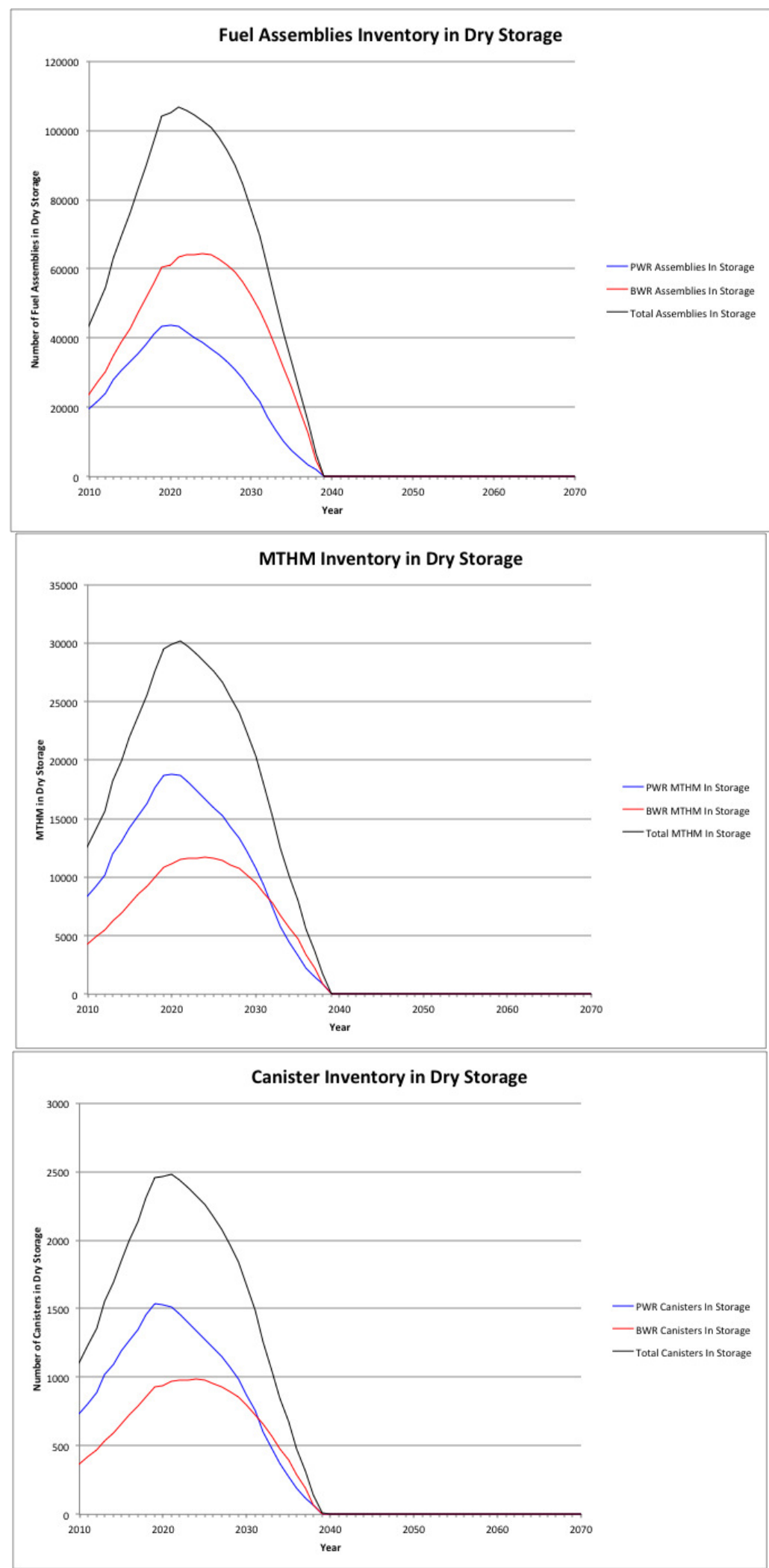

Figure A-11. Cases 2 \& 4: Total At-Reactor Dry Storage Inventory, 6000 MTHM YFF-5 Acceptance 2020 

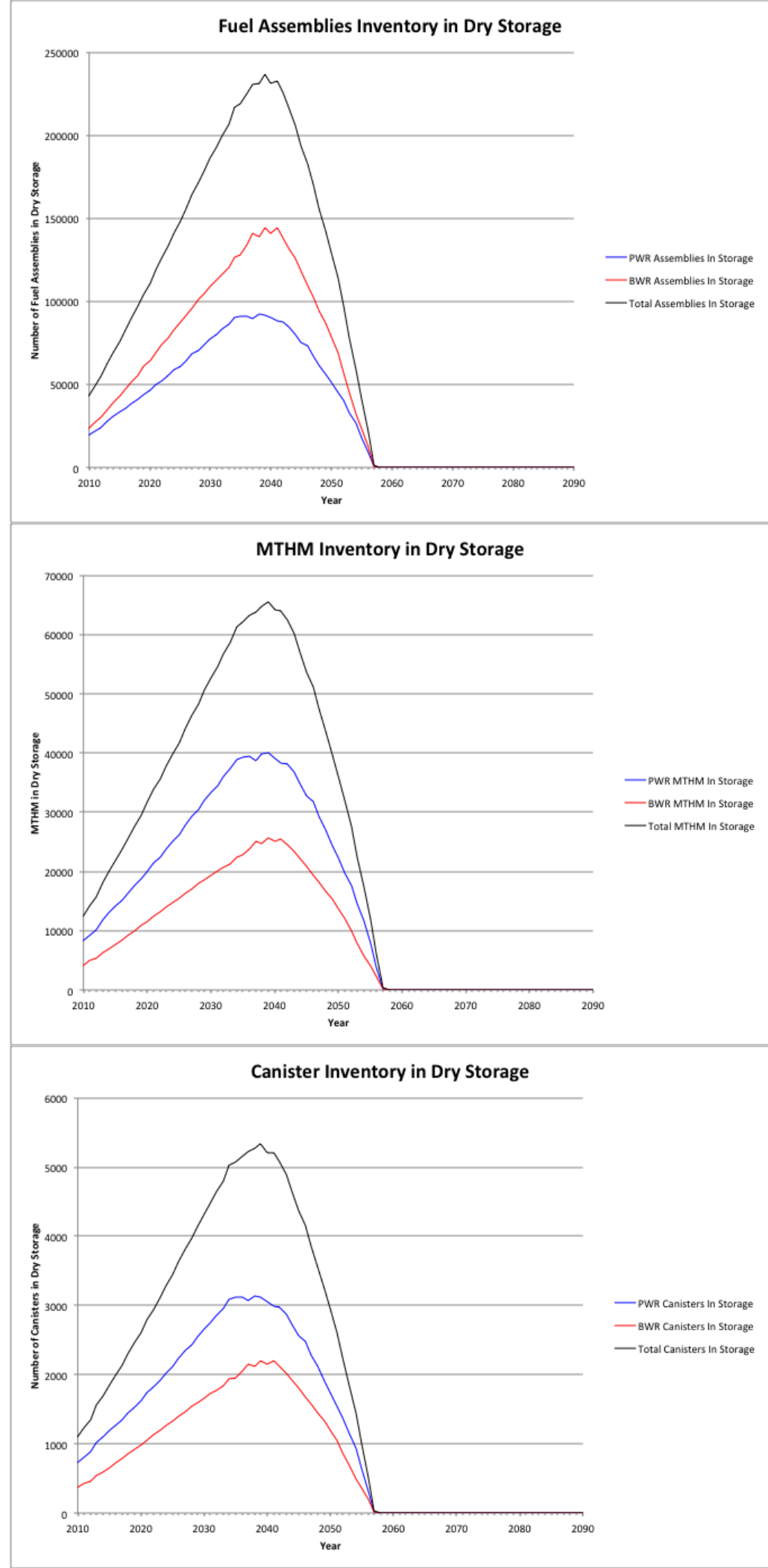

Figure A-12. Cases $1 \&$ 3: Total At-Reactor Dry Storage Inventory, 6000 MTHM YFF-5 Acceptance 2035 

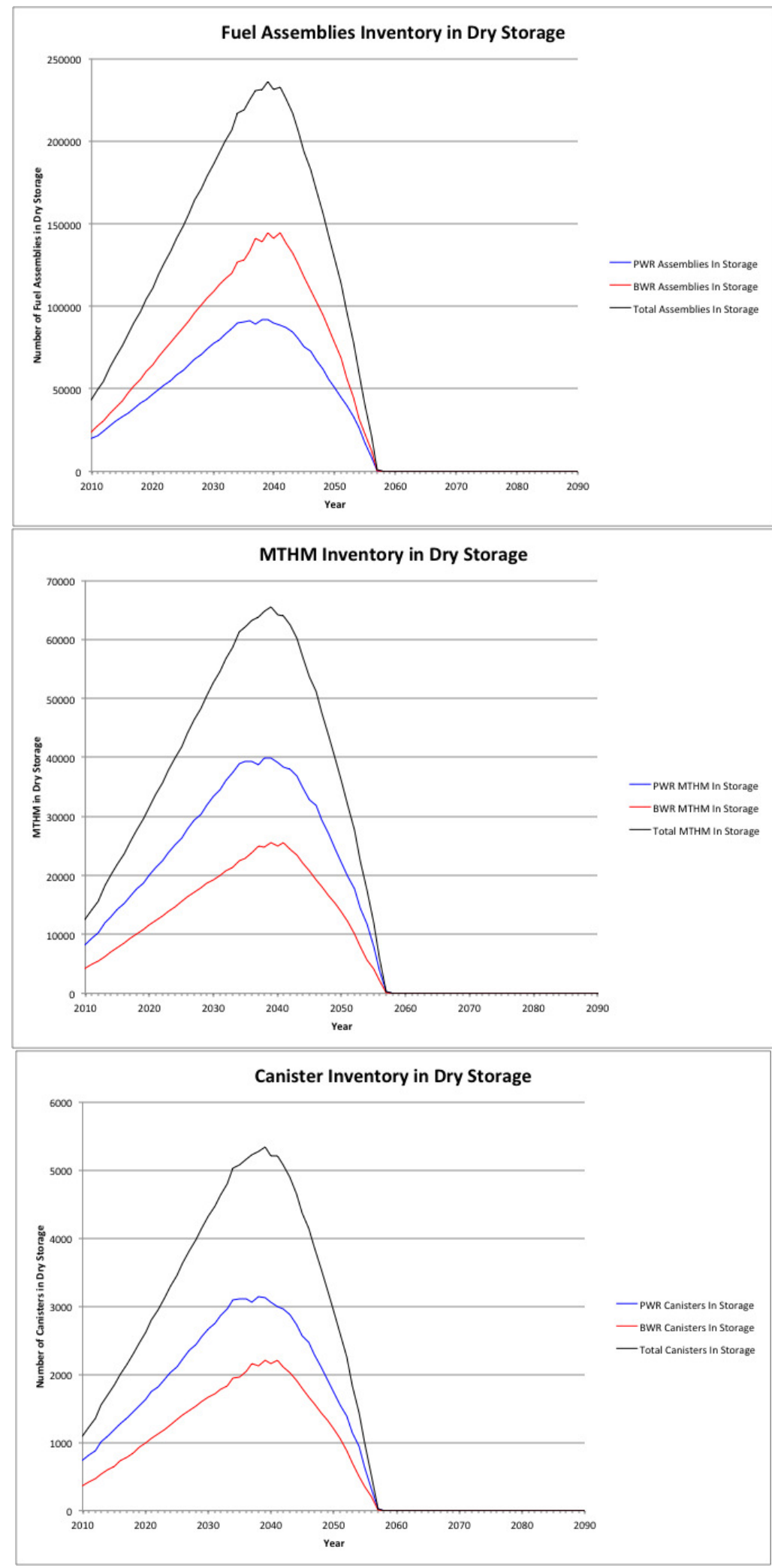

Figure A-13. Cases 2 \& 4: Total At-Reactor Dry Storage Inventory, 6000 MTHM YFF-5 Acceptance 2035 


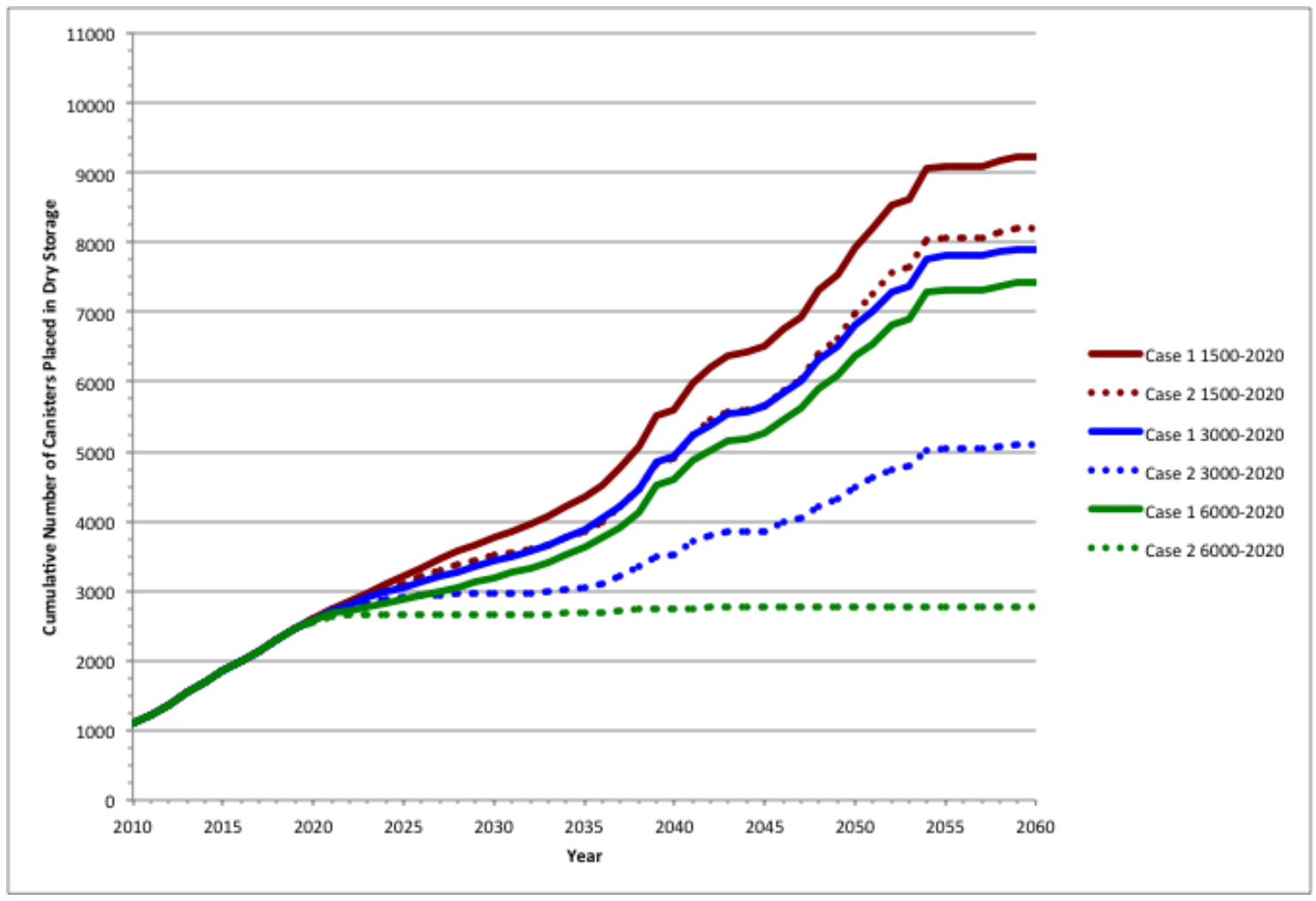

a) 2020 Start of Acceptance

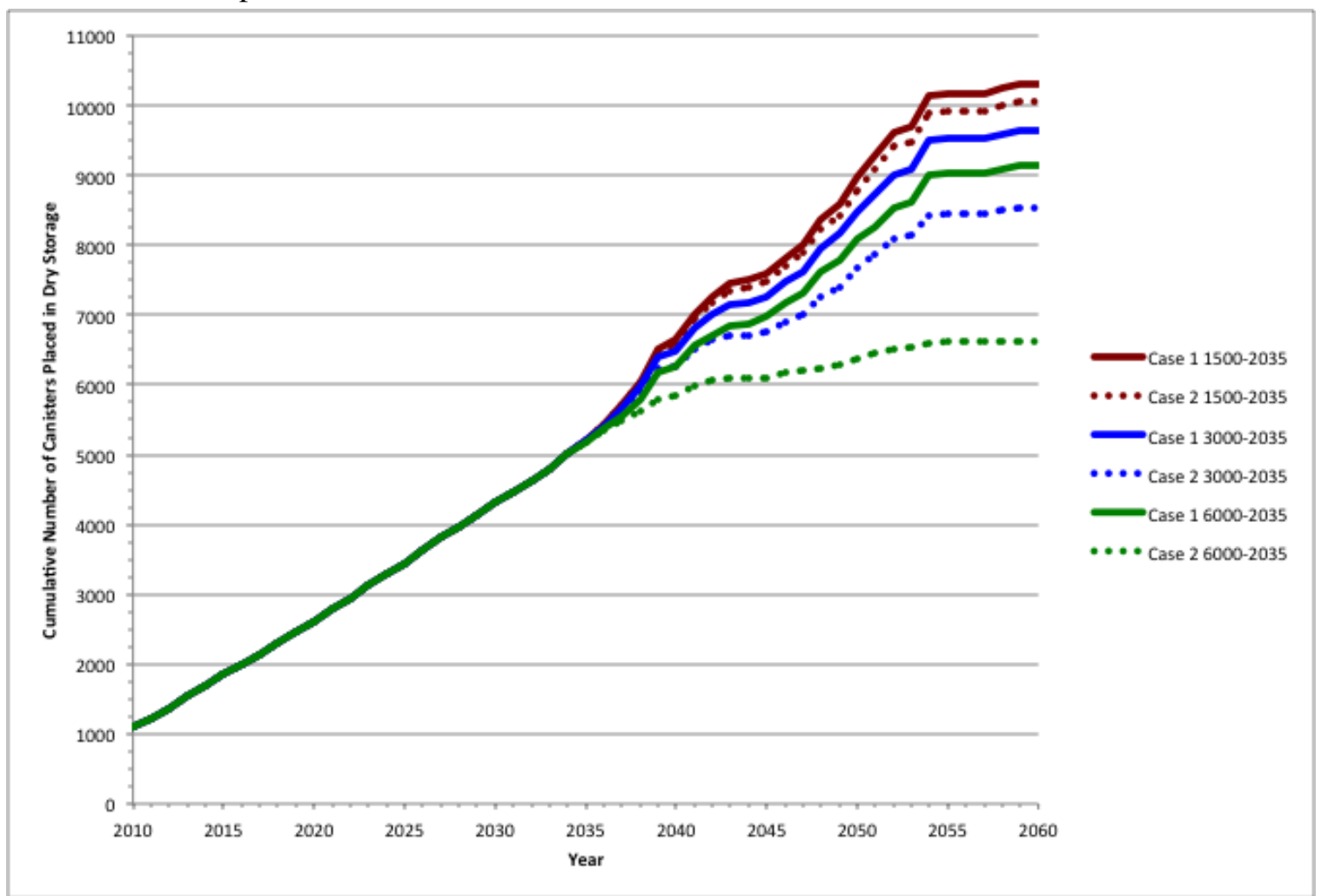

b) 2035 Start of Acceptance

Figure A-14. Cumulative Number of Canisters Placed in Dry Storage 


\section{A-2.3 At Reactor Logistic Results: Shipping Logistic Simulations - Cases $1 \& 3$ and Cases $2 \& 4$}

This section presents the at-reactor logistic results obtained from the TSL simulations pertaining to offsite shipping of used nuclear fuel, both from the used fuel pools and from dry storage. Unlike on-site dry storage inventories presented in Section A-2.2, there are significant differences in the resulting shipping characteristics for the different cases, acceptance rates, and when acceptance begins.

Summary-level results are shown in Tables A-11 through A-14. More detailed discussions of the results and trends for each case and input variable set are provided further in this section.

\section{A-2.3.1 At Reactor Logistic Results: Shipping Logistic Simulations - Cases 1 and 3}

This section presents the at-reactor logistics results for case 1 which considers that all used fuel is transported off-site, either from the used fuel pool or from dry storage, in existing size canisters. No used fuel is transported in re-useable transportation casks. The assumptions presented in Section A-1.3.3 regarding site capabilities were considered in these analyses and it was assumed that all fuel could be transported from every reactor using existing size canisters.

\section{MTHM/year YFF-5 Acceptance, Starting in 2020}

Figure A-15 shows the annual shipping of canisterized fuel from the reactor sites for this case. The shipping remains relatively constant over the entire 92-year period, ending in 2112, due to the relatively low acceptance rate. Figure A-16 shows the annual shipping of casks for each of the major cask vendors.

Table A-15 shows the summary results for shipping used nuclear fuel from the reactor sites. As shown in Table A-15, these cases do not involve the transportation of used fuel in re-useable transportation casks and all fuel is shipped in existing size canisters. The peak shipping rate of assemblies, MTHM, and canisters are shown along with the average shipping rate over the period where transportation occurs. Also shown are the cumulative amount of assemblies, canisters, and MTHM that are shipped. Table A-15 also shows the peak, average, and cumulative number of canisters that are shipped for each of the major dry storage system vendors.

\section{MTHM/year YFF-5 Acceptance, Starting in 2035}

Figure A-17 shows the annual shipping of canisterized fuel from the reactor sites for this case. The shipping remains relatively constant over the entire 92-year period due to the relatively low acceptance rate. Figure A-18 shows the annual shipping of casks for each of the major cask vendors.

Table A-16 shows the summary results for shipping used nuclear fuel from the reactor sites. As shown in Table A-16, these cases do not involve the transportation of used fuel in re-useable transportation casks and all fuel is shipped in existing size canisters. The peak shipping rate of assemblies, MTHM, and canisters are shown along with the average shipping rate over the period where transportation occurs. Also shown are the cumulative amount of assemblies, canisters, and MTHM that are shipped. Table A-12 also shows the peak, average, and cumulative number of canisters that are shipped for each of the major dry storage system vendors.

The summary results shown in Table A-16 are similar to those shown in Table A-15 for $1500 \mathrm{MTHM} / \mathrm{yr}$ acceptance starting in 2020. This is as expected since all used fuel is being shipped in canisters with the only difference being the date that acceptance begins (and ends). 
Table A-11. Peak Annual Bare Fuel Shipping Rate from Reactors (Assemblies per year)

\begin{tabular}{|c|c|c|c|c|c|c|c|c|}
\hline & $\begin{array}{l}\text { Acceptance Rate } \\
\text { (MT/yr) }\end{array}$ & $\begin{array}{l}\text { Acceptance } \\
\text { Start }\end{array}$ & PWR Bare & BWR Bare & Total Bare & $\begin{array}{c}\text { PWR } \\
\text { Canisterized }\end{array}$ & $\begin{array}{c}\text { BWR } \\
\text { Canisterized }\end{array}$ & $\begin{array}{c}\text { Total } \\
\text { Canisterized }\end{array}$ \\
\hline \multirow{6}{*}{$\begin{array}{l}\text { Canistered } \\
\text { Fuel Transport } \\
\text { (Cases } 1 \text { \& 3) }\end{array}$} & 1500 & 2020 & & & & 2879 & 4807 & 6861 \\
\hline & 3000 & 2020 & & & & 5899 & 8768 & 13170 \\
\hline & 6000 & 2020 & & & & 9245 & 15636 & 23888 \\
\hline & 1500 & 2035 & & & & 2812 & 4751 & 6781 \\
\hline & 3000 & 2035 & & & & 5261 & 8623 & 12489 \\
\hline & 6000 & 2035 & & & & 11896 & 15456 & 23564 \\
\hline \multirow{6}{*}{$\begin{array}{l}\text { Bare and } \\
\text { Canistered } \\
\text { Fuel Transport } \\
\text { (Cases } 2 \text { \& 4) }\end{array}$} & 1500 & 2020 & 2296 & 4105 & 5587 & 2882 & 4029 & 6044 \\
\hline & 3000 & 2020 & 4498 & 7879 & 10881 & 5194 & 8084 & 11658 \\
\hline & 6000 & 2020 & 7734 & 14152 & 20582 & 4528 & 7801 & 10112 \\
\hline & 1500 & 2035 & 1678 & 3598 & 5070 & 2748 & 4071 & 5921 \\
\hline & 3000 & 2035 & 3512 & 7102 & 9872 & 5205 & 8223 & 11940 \\
\hline & 6000 & 2035 & 7618 & 13004 & 19880 & 9460 & 12985 & 20792 \\
\hline
\end{tabular}

Note: The sum of PWR and BWR peak rates do not sum to the total as the peak rates occur in different years.

Table A-12. Peak Annual Bare Cask/Canister Shipping Rate from Reactors (Cask/Canister per year)

\begin{tabular}{|c|c|c|c|c|c|c|c|c|}
\hline & $\begin{array}{c}\text { Acceptance Rate } \\
\text { (MT/yr) }\end{array}$ & $\begin{array}{l}\text { Acceptance } \\
\text { Start }\end{array}$ & PWR Bare & BWR Bare & Total Bare & $\begin{array}{c}\text { PWR } \\
\text { Canisterized }\end{array}$ & $\begin{array}{c}\text { BWR } \\
\text { Canisterized }\end{array}$ & $\begin{array}{c}\text { Total } \\
\text { Canisterized }\end{array}$ \\
\hline \multirow{6}{*}{$\begin{array}{l}\text { Canistered } \\
\text { Fuel Transport } \\
\text { (Cases } 1 \text { \& 3) }\end{array}$} & 1500 & 2020 & & & & 100 & 74 & 147 \\
\hline & 3000 & 2020 & & & & 236 & 132 & 348 \\
\hline & 6000 & 2020 & & & & 311 & 234 & 520 \\
\hline & 1500 & 2035 & & & & 97 & 74 & 145 \\
\hline & 3000 & 2035 & & & & 181 & 131 & 265 \\
\hline & 6000 & 2035 & & & & 452 & 232 & 619 \\
\hline \multirow{6}{*}{$\begin{array}{c}\text { Bare and } \\
\text { Canistered } \\
\text { Fuel Transport } \\
\text { (Cases } 2 \text { \& 4) }\end{array}$} & 1500 & 2020 & 119 & 63 & 182 & 98 & 62 & 130 \\
\hline & 3000 & 2020 & 202 & 133 & 325 & 179 & 124 & 246 \\
\hline & 6000 & 2020 & 404 & 303 & 636 & 153 & 121 & 225 \\
\hline & 1500 & 2035 & 83 & 57 & 137 & 94 & 62 & 131 \\
\hline & 3000 & 2035 & 146 & 112 & 258 & 179 & 126 & 252 \\
\hline & 6000 & 2035 & 316 & 245 & 544 & 323 & 196 & 482 \\
\hline
\end{tabular}


Table A-13. Cumulative Number of Fuel Assemblies Shipped from Reactors

\begin{tabular}{|c|c|c|c|c|c|c|c|c|}
\hline & $\begin{array}{c}\text { Acceptance Rate } \\
\text { (MT/yr) }\end{array}$ & $\begin{array}{l}\text { Acceptance } \\
\text { Start }\end{array}$ & PWR Bare & BWR Bare & Total Bare & $\begin{array}{c}\text { PWR } \\
\text { Canisterized }\end{array}$ & $\begin{array}{c}\text { BWR } \\
\text { Canisterized }\end{array}$ & $\begin{array}{c}\text { Total } \\
\text { Canisterized }\end{array}$ \\
\hline \multirow{6}{*}{$\begin{array}{l}\text { Canistered } \\
\text { Fuel Transport } \\
\text { (Cases } 1 \text { \& 3) }\end{array}$} & 1500 & 2020 & & & & 205983 & 276420 & 482403 \\
\hline & 3000 & 2020 & & & & 205983 & 276420 & 482403 \\
\hline & 6000 & 2020 & & & & 205983 & 276420 & 482403 \\
\hline & 1500 & 2035 & & & & 205983 & 276420 & 482403 \\
\hline & 3000 & 2035 & & & & 205983 & 276420 & 482403 \\
\hline & 6000 & 2035 & & & & 205983 & 276420 & 482403 \\
\hline \multirow{6}{*}{$\begin{array}{c}\text { Bare and } \\
\text { Canistered } \\
\text { Fuel Transport } \\
\text { (Cases } 2 \text { \& 4) }\end{array}$} & 1500 & 2020 & 56366 & 77529 & 133895 & 149617 & 198891 & 348508 \\
\hline & 3000 & 2020 & 114014 & 151895 & 265909 & 91969 & 124525 & 216494 \\
\hline & 6000 & 2020 & 157443 & 207591 & 365034 & 48540 & 68829 & 117369 \\
\hline & 1500 & 2035 & 21552 & 33099 & 54651 & 184431 & 243321 & 427752 \\
\hline & 3000 & 2035 & 50962 & 65464 & 116426 & 155021 & 210956 & 365977 \\
\hline & 6000 & 2035 & 86290 & 111269 & 197559 & 119693 & 165151 & 284844 \\
\hline
\end{tabular}

Note: The sum of PWR and BWR peak rates do not sum to the total as the peak rates occur in different years.

Table A-14. Cumulative Number of Canisters/Casks Shipped from Reactors

\begin{tabular}{|c|c|c|c|c|c|c|c|c|}
\hline & \\
\hline & $\begin{array}{c}\text { Acceptance Rate } \\
\text { (MT/yr) }\end{array}$ & $\begin{array}{l}\text { Acceptance } \\
\text { Start }\end{array}$ & PWR Bare & BWR Bare & Total Bare & $\begin{array}{c}\text { PWR } \\
\text { Canisterized }\end{array}$ & $\begin{array}{c}\text { BWR } \\
\text { Canisterized }\end{array}$ & $\begin{array}{c}\text { Total } \\
\text { Canisterized }\end{array}$ \\
\hline \multirow{6}{*}{$\begin{array}{c}\text { Canistered Fuel } \\
\text { Transport (Cases } \\
1 \text { \& 3) }\end{array}$} & 1500 & 2020 & & & & 6998 & 4210 & 11208 \\
\hline & 3000 & 2020 & & & & 6974 & 4190 & 11164 \\
\hline & 6000 & 2020 & & & & 6964 & 4183 & 11147 \\
\hline & 1500 & 2035 & & & & 7017 & 42223 & 11240 \\
\hline & 3000 & 2035 & & & & 7001 & 4216 & 11217 \\
\hline & 6000 & 2035 & & & & 6990 & 4208 & 11198 \\
\hline \multirow{6}{*}{$\begin{array}{c}\text { Bare and } \\
\text { Canistered Fuel } \\
\text { Transport (Cases } \\
2 \text { \& 4) }\end{array}$} & 1500 & 2020 & 2338 & 1342 & 3680 & 5145 & 3051 & 8196 \\
\hline & 3000 & 2020 & 4848 & 2897 & 7745 & 3190 & 1916 & 5106 \\
\hline & 6000 & 2020 & 8376 & 4800 & 13176 & 1712 & 1056 & 2768 \\
\hline & 1500 & 2035 & 837 & 536 & 1373 & 6326 & 3728 & 10054 \\
\hline & 3000 & 2035 & 2020 & 1141 & 3161 & 5315 & 3232 & 8547 \\
\hline & 6000 & 2035 & 3783 & 2219 & 6002 & 4094 & 2535 & 6629 \\
\hline
\end{tabular}




\section{MTHM/year YFF-5 Acceptance, Starting in 2020}

Figure A-19 shows the annual shipping of canisterized fuel from the reactor sites for this case. The shipping remains relatively constant over the entire 47-year period, ending in 2067, due to the availability of used fuel for shipping. Figure A-20 shows the annual shipping of casks for each of the major cask vendors.

Table A-17 shows the summary results for shipping used nuclear fuel from the reactor sites. As shown in Table A-17, these cases do not involve the transportation of used fuel in re-useable transportation casks and all fuel is shipped in existing size canisters. The peak shipping rate of assemblies, MTHM, and canisters are shown along with the average shipping rate over the period where transportation occurs. Also shown are the cumulative amount of assemblies, canisters, and MTHM that are shipped. Table A-17 also shows the peak, average, and cumulative number of canisters that are shipped for each of the major dry storage system vendors.

While the cumulative shipments are essentially identical to the $1500 \mathrm{MTHM} / \mathrm{yr}$ - 2020 acceptance case, both the peak and average annual acceptance rates are roughly a factor of two larger due to the larger acceptance rate (also by a factor of two).

\section{MTHM/year YFF-5 Acceptance, Starting in 2035}

Figure A-21 shows the annual shipping of canisterized fuel from the reactor sites for this case. The shipping remains relatively constant over the entire 47 -year period, ending in 2082 , due to the availability of used fuel for shipping. Figure A-22 shows the annual shipping of casks for each of the major cask vendors.

Table A-18 shows the summary results for shipping used nuclear fuel from the reactor sites. As shown in Table A-18, these cases do not involve the transportation of used fuel in re-useable transportation casks and all fuel is shipped in existing size canisters. The peak shipping rate of assemblies, MTHM, and canisters are shown along with the average shipping rate over the period where transportation occurs. Also shown are the cumulative amount of assemblies, canisters, and MTHM that are shipped. Table A-18 also shows the peak, average, and cumulative number of canisters that are shipped for each of the major dry storage system vendors.

The summary results shown in Table A-18 are similar to those shown in Table A-17 for 3000 MTHM/yr acceptance starting in 2020. This is as expected since all used fuel is being shipped in canisters with the only difference being the date that acceptance begins (and ends).

While the cumulative shipments are essentially identical to the $1500 \mathrm{MTHM} / \mathrm{yr}$ - 2035 acceptance case, both the peak and average annual acceptance rates are roughly a factor of two larger due to the larger acceptance rate (also by a factor of two).

\section{MTHM/year YFF-5 Acceptance, Starting in 2020}

Figure A-23 shows the annual shipping of canisterized fuel from the reactor sites for this case. It can be seen that $6000 \mathrm{MTHM} / \mathrm{yr}$ is shipped only for the first six years after acceptance begins in 2020. At this point the acceptance rate decreases to about $3000 \mathrm{MTHM} / \mathrm{year}$ and then fluctuates around an average of approximately $3000 \mathrm{MTHM} / \mathrm{yr}$. This fluctuation results from needing to meet fuel assembly thermal limits within the dual-purpose canisters being used for both storage and transportation.

Figure A-24 shows the annual shipping of casks for each of the major cask vendors. A similar decrease and fluctuating trend is seen.

Table A-19 shows the summary results for shipping used nuclear fuel from the reactor sites. As shown in Table A-19, these cases do not involve the transportation of used fuel in re-useable transportation casks and all fuel is shipped in existing size canisters. The peak shipping rate of assemblies, MTHM, and canisters are shown along with the average shipping rate over the period where transportation occurs. 
Also shown are the cumulative amount of assemblies, canisters, and MTHM that are shipped. Table A-19 also shows the peak, average, and cumulative number of canisters that are shipped for each of the major dry storage system vendors.

While the cumulative shipments are essentially identical to the $3000 \mathrm{MTHM} / \mathrm{yr}$ - 2020 acceptance case, both the peak and average annual acceptance rates are roughly a factor of two larger due to the larger acceptance rate (also by a factor of two).

\section{MTHM/year YFF-5 Acceptance, Starting in 2035}

Figure A-25 shows the annual shipping of canisterized fuel from the reactor sites for this case. It can be seen that $6000 \mathrm{MTHM} / \mathrm{yr}$ is shipped only for the first six years after acceptance begins in 2020. At this point the acceptance rate decreases to about $3000 \mathrm{MTHM} / \mathrm{year}$ and then fluctuates around an average of approximately $3000 \mathrm{MTHM} / \mathrm{yr}$. This fluctuation results from needing to meet fuel assembly thermal limits within the dual-purpose canisters being used for both storage and transportation.

Figure A-26 shows the annual shipping of casks for each of the major cask vendors. A similar decrease and fluctuating trend is seen.

Table A-20 shows the summary results for shipping used nuclear fuel from the reactor sites. As shown in Table A-20, these cases do not involve the transportation of used fuel in re-useable transportation casks and all fuel is shipped in existing size canisters. The peak shipping rate of assemblies, MTHM, and canisters are shown along with the average shipping rate over the period where transportation occurs. Also shown are the cumulative amount of assemblies, canisters, and MTHM that are shipped. Table A-20 also shows the peak, average, and cumulative number of canisters that are shipped for each of the major dry storage system vendors.

While the cumulative shipments are essentially identical to the $3000 \mathrm{MTHM} / \mathrm{yr}$ - 2035 acceptance case, both the peak and average annual acceptance rates are roughly a factor of two larger due to the larger acceptance rate (also by a factor of two). 

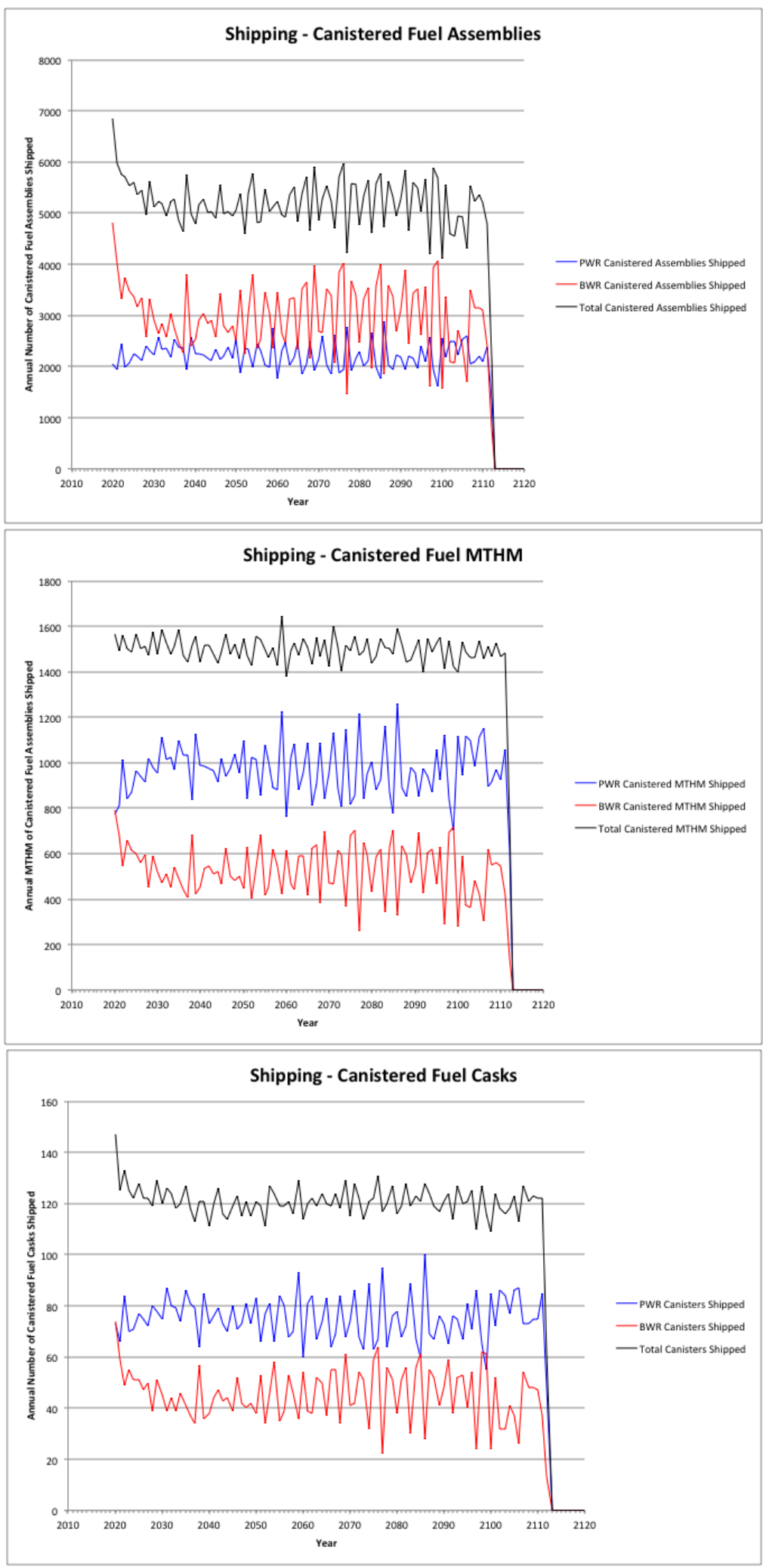

Figure A-15. Cases $1 \&$ 3: Annual Shipping of Canisterized Fuel, 1500 MTHM YFF-5 Acceptance 2020 


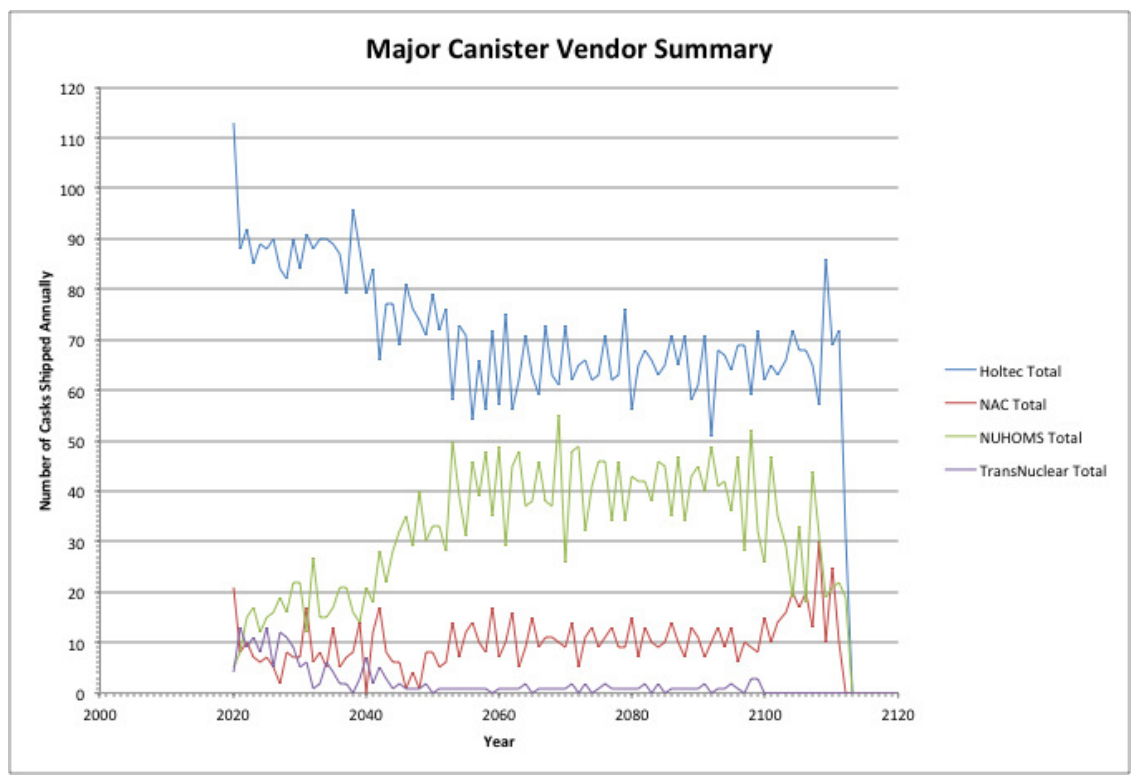

Figure A-16. Cases 1 \& 3: Annual Shipping - Major Canister Vendor Summary, 1500 MTHM YFF-5 Acceptance - 2020

Table A-15. Cases 1 \& 3: Summary of Shipping from Reactor Sites, 1500 MTHM YFF-5 Acceptance 2020

\begin{tabular}{|c|c|c|c|c|c|c|}
\hline \multicolumn{7}{|c|}{ Assemblies } \\
\hline & $\begin{array}{l}\text { PWR Bare } \\
\text { Assemblies } \\
\text { Shipped }\end{array}$ & $\begin{array}{l}\text { BWR Bare } \\
\text { Assemblies } \\
\text { Shipped }\end{array}$ & $\begin{array}{c}\text { Total Bare } \\
\text { Assemblies } \\
\text { Shipped }\end{array}$ & $\begin{array}{c}\text { PWR } \\
\text { Canistered } \\
\text { Assemblies } \\
\text { Shipped }\end{array}$ & $\begin{array}{c}\text { BWR } \\
\text { Canistered } \\
\text { Assemblies } \\
\text { Shipped }\end{array}$ & $\begin{array}{c}\text { Total } \\
\text { Canistered } \\
\text { Assemblies } \\
\text { Shipped }\end{array}$ \\
\hline Peak & 0 & 0 & 0 & 2879 & 4807 & 6861 \\
\hline Average & 0 & 0 & 0 & 2224 & 2996 & 5220 \\
\hline Cumulative & 0 & 0 & 0 & 205983 & 276420 & 482403 \\
\hline \multicolumn{7}{|c|}{ MTHM } \\
\hline & $\begin{array}{c}\text { PWR Bare } \\
\text { MTHM Shipped }\end{array}$ & $\begin{array}{l}\text { BWR Bare } \\
\text { MTHM } \\
\text { Shipped }\end{array}$ & $\begin{array}{l}\text { Total Bare } \\
\text { MTHM } \\
\text { Shipped }\end{array}$ & $\begin{array}{c}\text { PWR } \\
\text { Canistered } \\
\text { MTHM } \\
\text { Shipped }\end{array}$ & $\begin{array}{c}\text { BWR } \\
\text { Canistered } \\
\text { MTHM } \\
\text { Shipped }\end{array}$ & $\begin{array}{c}\text { Total } \\
\text { Canistered } \\
\text { MTHM } \\
\text { Shipped }\end{array}$ \\
\hline Peak & 0 & 0 & 0 & 1262 & 793 & 1647 \\
\hline Average & 0 & 0 & 0 & 970 & 530 & 1500 \\
\hline Cumulative & 0 & 0 & 0 & 89838 & 48897 & 138735 \\
\hline \multicolumn{7}{|c|}{ Canisters/Casks } \\
\hline & $\begin{array}{l}\text { PWR Bare Fuel } \\
\text { Casks Shipped }\end{array}$ & $\begin{array}{l}\text { BWR Bare Fuel } \\
\text { Casks Shipped }\end{array}$ & $\begin{array}{c}\text { Total Bare } \\
\text { Fuel Casks } \\
\text { Shipped }\end{array}$ & $\begin{array}{l}\text { PWR Canisters } \\
\text { Shipped }\end{array}$ & $\begin{array}{l}\text { BWR Canisters } \\
\text { Shipped }\end{array}$ & $\begin{array}{l}\text { Total Canisters } \\
\text { Shipped }\end{array}$ \\
\hline Peak & 0 & 0 & 0 & 100 & 74 & 147 \\
\hline Average & 0 & 0 & 0 & 76 & 46 & 121 \\
\hline Cumulative & 0 & 0 & 0 & 6998 & 4210 & 11208 \\
\hline
\end{tabular}

\begin{tabular}{|l|c|c|c|c|c|c|}
\hline \multicolumn{9}{|c|}{ Major Dry Storage System Vendors } \\
\hline & $\begin{array}{c}\text { Holtec } \\
\text { Canisters }\end{array}$ & $\begin{array}{c}\text { NAC } \\
\text { Canisters }\end{array}$ & $\begin{array}{c}\text { NUHOMS } \\
\text { Canisters }\end{array}$ & $\begin{array}{c}\text { TransNuclear } \\
\text { Canisters }\end{array}$ & $\begin{array}{c}\text { Fuel Solutions } \\
\text { Canisters }\end{array}$ & $\begin{array}{c}\text { South Texas } \\
\text { Project Canisters }\end{array}$ \\
\hline Peak & 113 & 30 & 55 & 13 & 8 & 18 \\
\hline Average & 68 & 11 & 36 & 1 & NA & 4 \\
\hline Cumulative & 6660 & 945 & 3004 & 204 & 64 & 331 \\
\hline
\end{tabular}




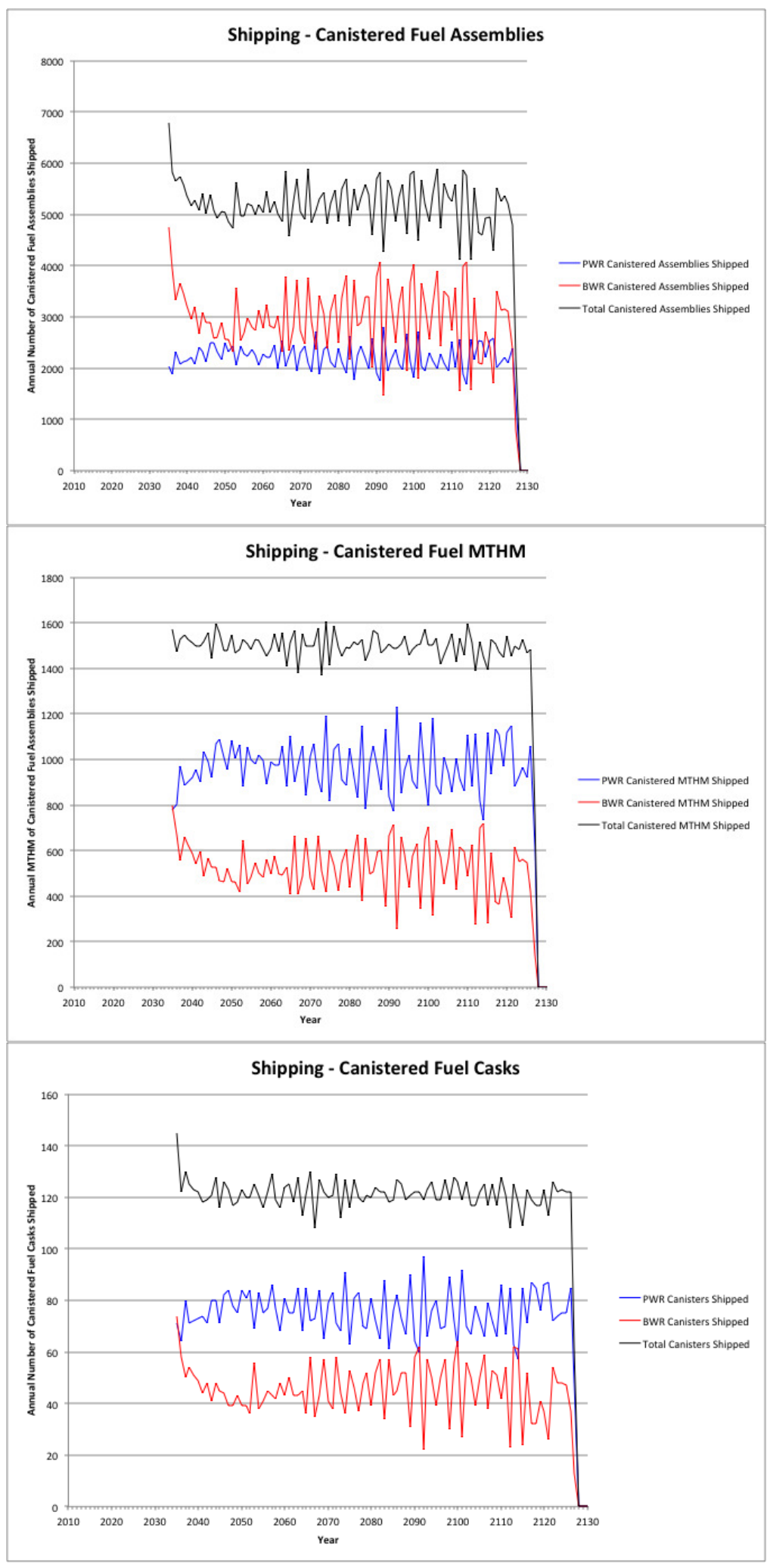

Figure A-17. Cases 1 \& 3: Annual Shipping of Canisterized Fuel, 1500 MTHM YFF-5 Acceptance2035 


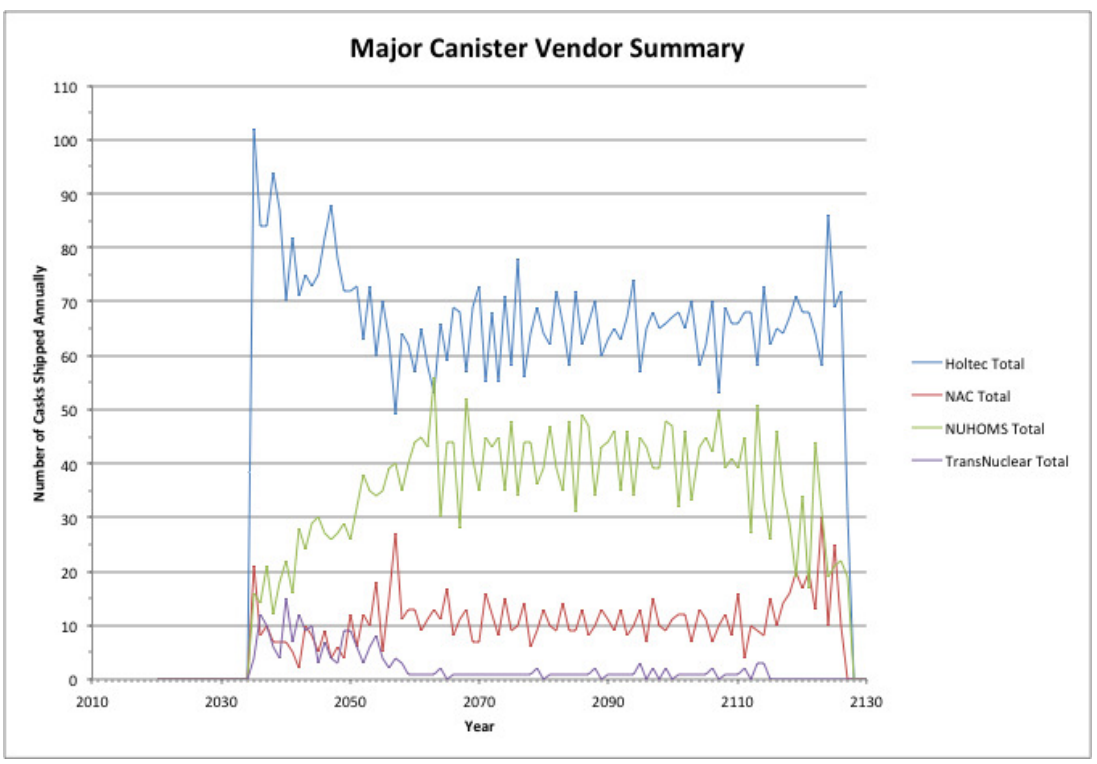

Figure A-18. Cases $1 \&$ 3: Annual Shipping - Major Canister Vendor Summary, 1500 MTHM YFF-5 Acceptance - 2035

Table A-16. Cases 1 \& 3: Summary of Shipping from Reactor Sites, 1500 MTHM YFF-5 Acceptance 2035

\begin{tabular}{|c|c|c|c|c|c|c|}
\hline \multicolumn{7}{|c|}{ Assemblies } \\
\hline & $\begin{array}{l}\text { PWR Bare } \\
\text { Assemblies } \\
\text { Shipped }\end{array}$ & $\begin{array}{l}\text { BWR Bare } \\
\text { Assemblies } \\
\text { Shipped }\end{array}$ & $\begin{array}{c}\text { Total Bare } \\
\text { Assemblies } \\
\text { Shipped }\end{array}$ & $\begin{array}{c}\text { PWR } \\
\text { Canistered } \\
\text { Assemblies } \\
\text { Shipped }\end{array}$ & $\begin{array}{c}\text { BWR } \\
\text { Canistered } \\
\text { Assemblies } \\
\text { Shipped }\end{array}$ & $\begin{array}{c}\text { Total } \\
\text { Canistered } \\
\text { Assemblies } \\
\text { Shipped }\end{array}$ \\
\hline Peak & 0 & 0 & 0 & 2812 & 4751 & 6781 \\
\hline Average & 0 & 0 & 0 & 2224 & 2996 & 5220 \\
\hline Cumulative & 0 & 0 & 0 & 205983 & 276420 & 482403 \\
\hline \multicolumn{7}{|c|}{ MTHM } \\
\hline & $\begin{array}{c}\text { PWR Bare } \\
\text { MTHM Shipped }\end{array}$ & $\begin{array}{l}\text { BWR Bare } \\
\text { MTHM } \\
\text { Shipped }\end{array}$ & $\begin{array}{l}\text { Total Bare } \\
\text { MTHM } \\
\text { Shipped }\end{array}$ & $\begin{array}{c}\text { PWR } \\
\text { Canistered } \\
\text { MTHM } \\
\text { Shipped }\end{array}$ & $\begin{array}{c}\text { BWR } \\
\text { Canistered } \\
\text { MTHM } \\
\text { Shipped }\end{array}$ & $\begin{array}{c}\text { Total } \\
\text { Canistered } \\
\text { MTHM } \\
\text { Shipped }\end{array}$ \\
\hline Peak & 0 & 0 & 0 & 1230 & 796 & 1607 \\
\hline Average & 0 & 0 & 0 & 970 & 530 & 1500 \\
\hline Cumulative & 0 & 0 & 0 & 89838 & 48897 & 138735 \\
\hline \multicolumn{7}{|c|}{ Canisters/Casks } \\
\hline & $\begin{array}{l}\text { PWR Bare Fuel } \\
\text { Casks Shipped }\end{array}$ & $\begin{array}{l}\text { BWR Bare Fuel } \\
\text { Casks Shipped }\end{array}$ & $\begin{array}{l}\text { Total Bare } \\
\text { Fuel Casks } \\
\text { Shipped }\end{array}$ & $\begin{array}{l}\text { PWR Canisters } \\
\text { Shipped }\end{array}$ & $\begin{array}{l}\text { BWR Canisters } \\
\text { Shipped }\end{array}$ & $\begin{array}{l}\text { Total Canisters } \\
\text { Shipped }\end{array}$ \\
\hline Peak & 0 & 0 & 0 & 97 & 74 & 145 \\
\hline Average & 0 & 0 & 0 & 76 & 46 & 122 \\
\hline Cumulative & 0 & 0 & 0 & 7017 & 4223 & 11240 \\
\hline
\end{tabular}

\begin{tabular}{|l|c|c|c|c|c|c|}
\hline \multicolumn{9}{|c|}{} & $\begin{array}{c}\text { Moltec } \\
\text { Canisters }\end{array}$ & $\begin{array}{c}\text { NAC } \\
\text { Canisters }\end{array}$ & $\begin{array}{c}\text { NUHOMS } \\
\text { Canisters }\end{array}$ & $\begin{array}{c}\text { TransNuclear } \\
\text { Canisters }\end{array}$ & $\begin{array}{c}\text { Fuel Solutions } \\
\text { Canisters }\end{array}$ & $\begin{array}{c}\text { South Texas } \\
\text { Project Canisters }\end{array}$ \\
\hline Peak & 102 & 30 & 56 & 15 & 8 & 18 \\
\hline Average & 68 & 11 & 36 & 2 & NA & 3 \\
\hline Cumulative & 6265 & 1024 & 3335 & 221 & 64 & 331 \\
\hline
\end{tabular}



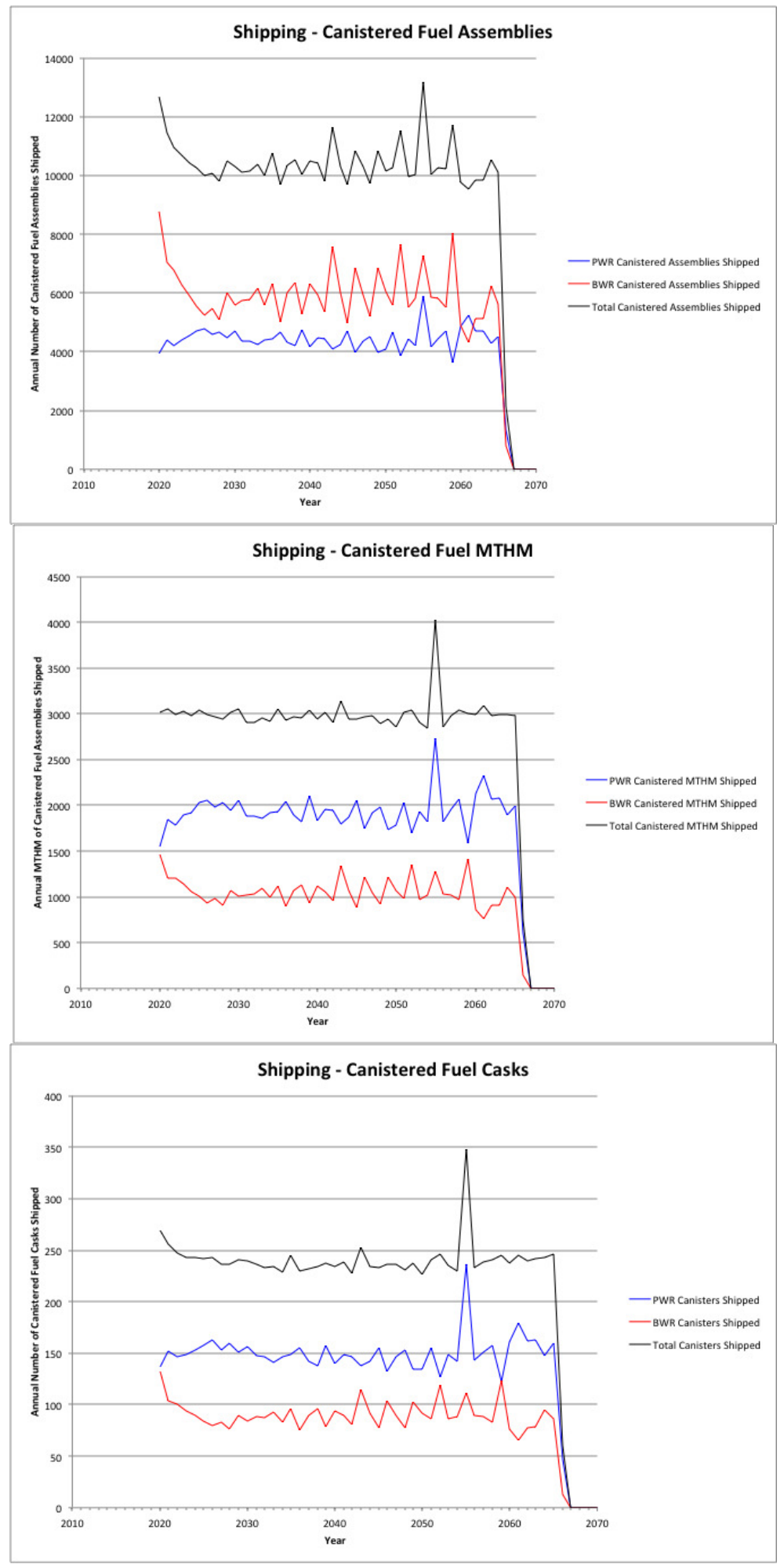

Figure A-19. Cases $1 \&$ 3: Annual Shipping of Canisterized Fuel, 3000 MTHM YFF-5 Acceptance 2020 


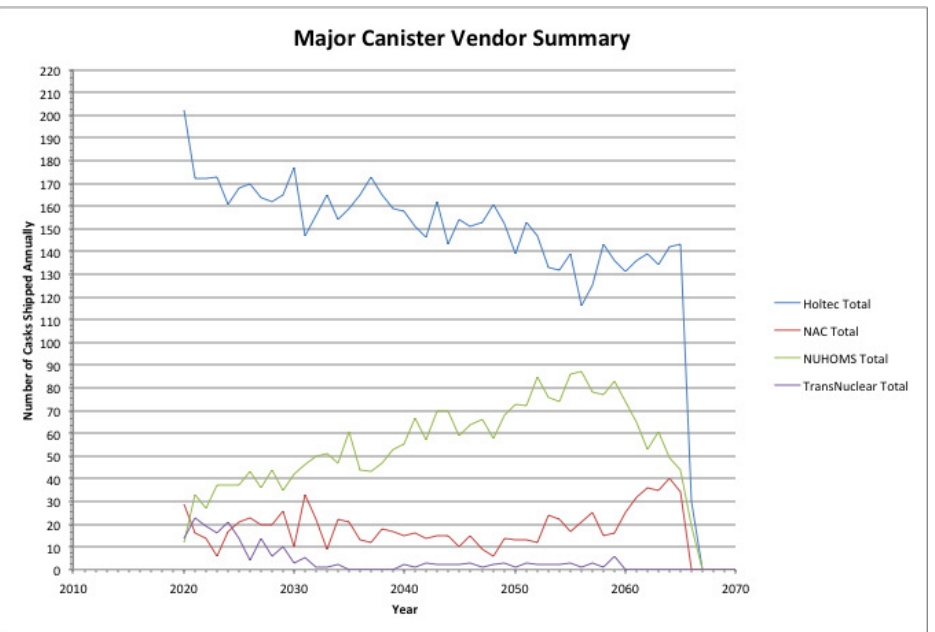

Figure A-20. Cases 1 \& 3: Annual Shipping - Major Canister Vendor Summary, 3000 MTHM YFF-5 Acceptance -2020

Table A-17. Cases 1 \& 3: Summary of Shipping from Reactor Sites, 3000 MTHM YFF-5 Acceptance 2020

\begin{tabular}{|c|c|c|c|c|c|c|}
\hline \multicolumn{7}{|c|}{ Assemblies } \\
\hline & $\begin{array}{l}\text { PWR Bare } \\
\text { Assemblies } \\
\text { Shipped }\end{array}$ & $\begin{array}{l}\text { BWR Bare } \\
\text { Assemblies } \\
\text { Shipped }\end{array}$ & $\begin{array}{l}\text { Total Bare } \\
\text { Assemblies } \\
\text { Shipped }\end{array}$ & $\begin{array}{c}\text { PWR } \\
\text { Canistered } \\
\text { Assemblies } \\
\text { Shipped }\end{array}$ & $\begin{array}{c}\text { BWR } \\
\text { Canistered } \\
\text { Assemblies } \\
\text { Shipped }\end{array}$ & $\begin{array}{c}\text { Total } \\
\text { Canistered } \\
\text { Assemblies } \\
\text { Shipped }\end{array}$ \\
\hline Peak & 0 & 0 & 0 & 5899 & 8768 & 13170 \\
\hline Average & 0 & 0 & 0 & 4449 & 5992 & 10440 \\
\hline Cumulative & 0 & 0 & 0 & 205983 & 276420 & 482403 \\
\hline \multicolumn{7}{|c|}{ MTHM } \\
\hline & $\begin{array}{c}\text { PWR Bare } \\
\text { MTHM Shipped }\end{array}$ & $\begin{array}{l}\text { BWR Bare } \\
\text { MTHM } \\
\text { Shipped }\end{array}$ & $\begin{array}{l}\text { Total Bare } \\
\text { MTHM } \\
\text { Shipped }\end{array}$ & $\begin{array}{c}\text { PWR } \\
\text { Canistered } \\
\text { MTHM } \\
\text { Shipped } \\
\end{array}$ & $\begin{array}{c}\text { BWR } \\
\text { Canistered } \\
\text { MTHM } \\
\text { Shipped } \\
\end{array}$ & $\begin{array}{c}\text { Total } \\
\text { Canistered } \\
\text { MTHM } \\
\text { Shipped } \\
\end{array}$ \\
\hline Peak & 0 & 0 & 0 & 2737 & 1458 & 4022 \\
\hline Average & 0 & 0 & 0 & 1940 & 1060 & 3000 \\
\hline Cumulative & 0 & 0 & 0 & 89838 & 48897 & 138735 \\
\hline \multicolumn{7}{|c|}{ Canisters/Casks } \\
\hline & $\begin{array}{l}\text { PWR Bare Fuel } \\
\text { Casks Shipped }\end{array}$ & $\begin{array}{l}\text { BWR Bare Fuel } \\
\text { Casks Shipped }\end{array}$ & $\begin{array}{c}\text { Total Bare } \\
\text { Fuel Casks } \\
\text { Shipped }\end{array}$ & $\begin{array}{l}\text { PWR Canisters } \\
\text { Shipped }\end{array}$ & $\begin{array}{l}\text { BWR Canisters } \\
\text { Shipped }\end{array}$ & $\begin{array}{l}\text { Total Canisters } \\
\text { Shipped }\end{array}$ \\
\hline Peak & 0 & 0 & 0 & 236 & 132 & 348 \\
\hline Average & 0 & 0 & 0 & 151 & 91 & 241 \\
\hline Cumulative & 0 & 0 & 0 & 6974 & 4190 & 11164 \\
\hline
\end{tabular}

\begin{tabular}{|l|c|c|c|c|c|c|}
\hline \multicolumn{9}{|c|}{ Major Dry Storage System Vendors } \\
\hline & $\begin{array}{c}\text { Holtec } \\
\text { Canisters }\end{array}$ & $\begin{array}{c}\text { NAC } \\
\text { Canisters }\end{array}$ & $\begin{array}{c}\text { NUHOMS } \\
\text { Canisters }\end{array}$ & $\begin{array}{c}\text { TransNuclear } \\
\text { Canisters }\end{array}$ & $\begin{array}{c}\text { Fuel Solutions } \\
\text { Canisters }\end{array}$ & $\begin{array}{c}\text { South Texas } \\
\text { Project Canisters }\end{array}$ \\
\hline Peak & 202 & 40 & 87 & 23 & 15 & 103 \\
\hline Average & 151 & 19 & 56 & 4 & NA & 5 \\
\hline Cumulative & 7078 & 878 & 2615 & 198 & 64 & 331 \\
\hline
\end{tabular}



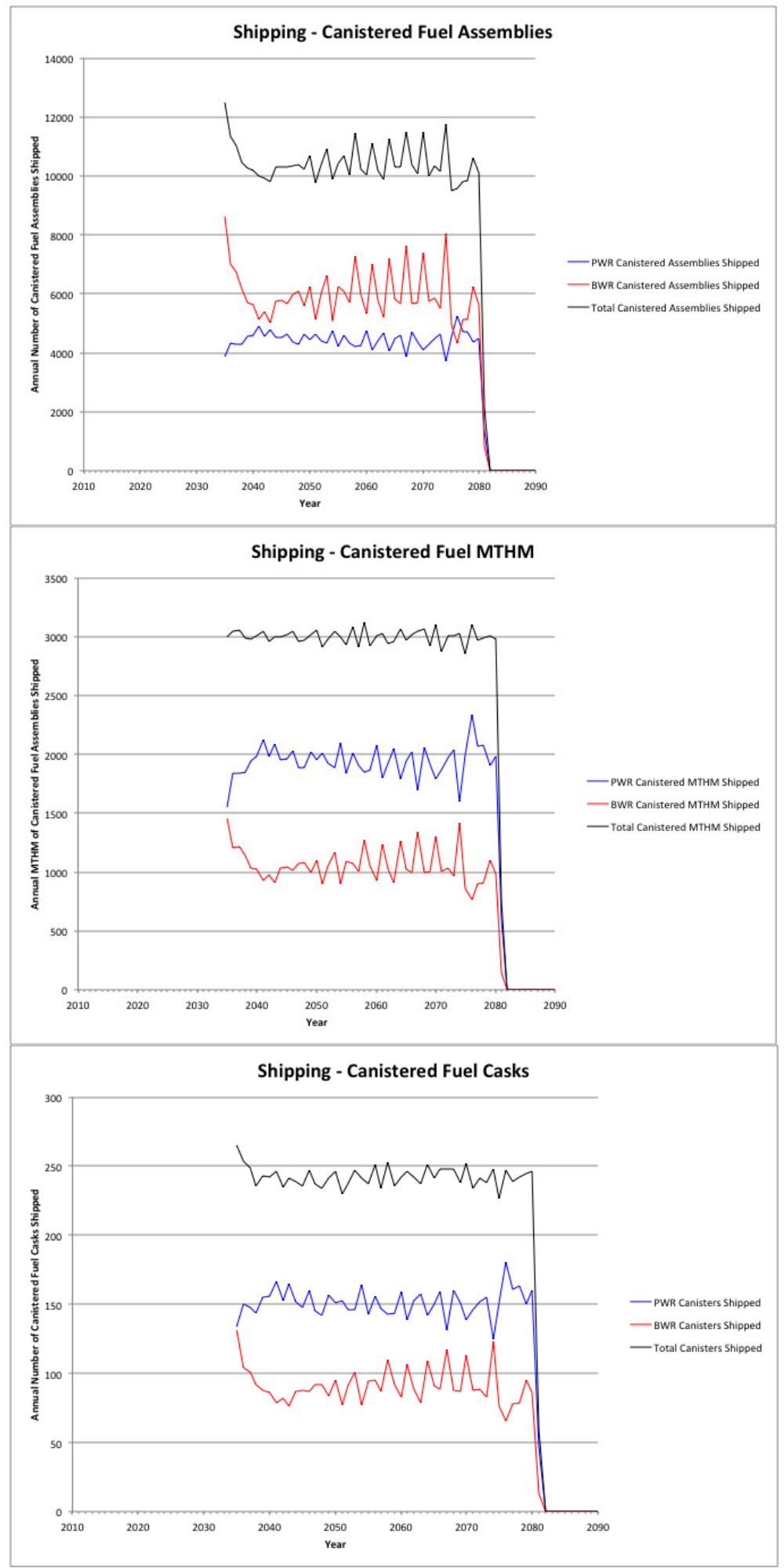

Figure A-21. Cases $1 \&$ 3: Annual Shipping of Canisterized Fuel, 3000 MTHM YFF-5 Acceptance 2035 


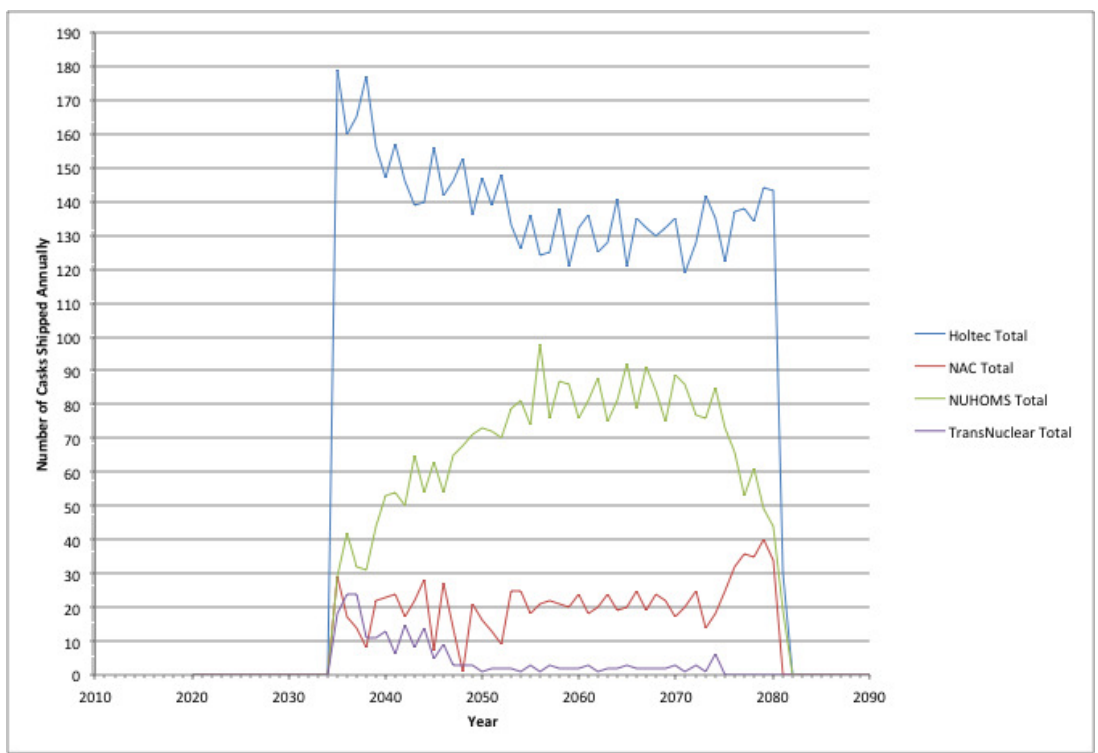

Figure A-22. Cases 1 \& 3: Annual Shipping - Major Canister Vendor Summary, 3000 MTHM YFF-5 Acceptance - 2035

Table A-18. Cases 1 \& 3: Summary of Shipping from Reactor Sites, 3000 MTHM YFF-5 Acceptance 2035

\begin{tabular}{|c|c|c|c|c|c|c|}
\hline \multicolumn{7}{|c|}{ Assemblies } \\
\hline & $\begin{array}{l}\text { PWR Bare } \\
\text { Assemblies } \\
\text { Shipped }\end{array}$ & $\begin{array}{l}\text { BWR Bare } \\
\text { Assemblies } \\
\text { Shipped }\end{array}$ & $\begin{array}{c}\text { Total Bare } \\
\text { Assemblies } \\
\text { Shipped }\end{array}$ & $\begin{array}{c}\text { PWR } \\
\text { Canistered } \\
\text { Assemblies } \\
\text { Shipped } \\
\end{array}$ & $\begin{array}{c}\text { BWR } \\
\text { Canistered } \\
\text { Assemblies } \\
\text { Shipped } \\
\end{array}$ & $\begin{array}{c}\text { Total } \\
\text { Canistered } \\
\text { Assemblies } \\
\text { Shipped } \\
\end{array}$ \\
\hline Peak & 0 & 0 & 0 & 5261 & 8623 & 12489 \\
\hline Average & 0 & 0 & 0 & 4449 & 5992 & 10440 \\
\hline Cumulative & 0 & 0 & 0 & 205983 & 276420 & 482403 \\
\hline \multicolumn{7}{|c|}{ MTHM } \\
\hline & $\begin{array}{c}\text { PWR Bare } \\
\text { MTHM Shipped }\end{array}$ & $\begin{array}{l}\text { BWR Bare } \\
\text { MTHM } \\
\text { Shipped }\end{array}$ & $\begin{array}{l}\text { Total Bare } \\
\text { MTHM } \\
\text { Shipped }\end{array}$ & $\begin{array}{c}\text { PWR } \\
\text { Canistered } \\
\text { MTHM } \\
\text { Shipped } \\
\end{array}$ & $\begin{array}{c}\text { BWR } \\
\text { Canistered } \\
\text { MTHM } \\
\text { Shipped } \\
\end{array}$ & $\begin{array}{c}\text { Total } \\
\text { Canistered } \\
\text { MTHM } \\
\text { Shipped } \\
\end{array}$ \\
\hline Peak & 0 & 0 & 0 & 2340 & 1454 & 3120 \\
\hline Average & 0 & 0 & 0 & 1940 & 1060 & 3000 \\
\hline Cumulative & 0 & 0 & 0 & 89838 & 48898 & 138735 \\
\hline \multicolumn{7}{|c|}{ Canisters/Casks } \\
\hline & $\begin{array}{l}\text { PWR Bare Fuel } \\
\text { Casks Shipped }\end{array}$ & $\begin{array}{l}\text { BWR Bare Fuel } \\
\text { Casks Shipped }\end{array}$ & $\begin{array}{c}\text { Total Bare } \\
\text { Fuel Casks } \\
\text { Shipped }\end{array}$ & $\begin{array}{l}\text { PWR Canisters } \\
\text { Shipped }\end{array}$ & $\begin{array}{l}\text { BWR Canisters } \\
\text { Shipped }\end{array}$ & $\begin{array}{l}\text { Total Canisters } \\
\text { Shipped }\end{array}$ \\
\hline Peak & 0 & 0 & 0 & 181 & 131 & 265 \\
\hline Average & 0 & 0 & 0 & 151 & 91 & 243 \\
\hline Cumulative & 0 & 0 & 0 & 7001 & 4216 & 11217 \\
\hline
\end{tabular}

\begin{tabular}{|l|c|c|c|c|c|c|}
\hline \multicolumn{9}{|c|}{ Major Dry Storage System Vendors } \\
\hline & $\begin{array}{c}\text { Holtec } \\
\text { Canisters }\end{array}$ & $\begin{array}{c}\text { NAC } \\
\text { Canisters }\end{array}$ & $\begin{array}{c}\text { NUHOMS } \\
\text { Canisters }\end{array}$ & $\begin{array}{c}\text { TransNuclear } \\
\text { Canisters }\end{array}$ & $\begin{array}{c}\text { Fuel Solutions } \\
\text { Canisters }\end{array}$ & $\begin{array}{c}\text { South Texas } \\
\text { Project Canisters }\end{array}$ \\
\hline Peak & 179 & 40 & 98 & 24 & 14 & 25 \\
\hline Average & 140 & 21 & 69 & 5 & NA & 8 \\
\hline Cumulative & 6455 & 975 & 3171 & 221 & 64 & 331 \\
\hline
\end{tabular}



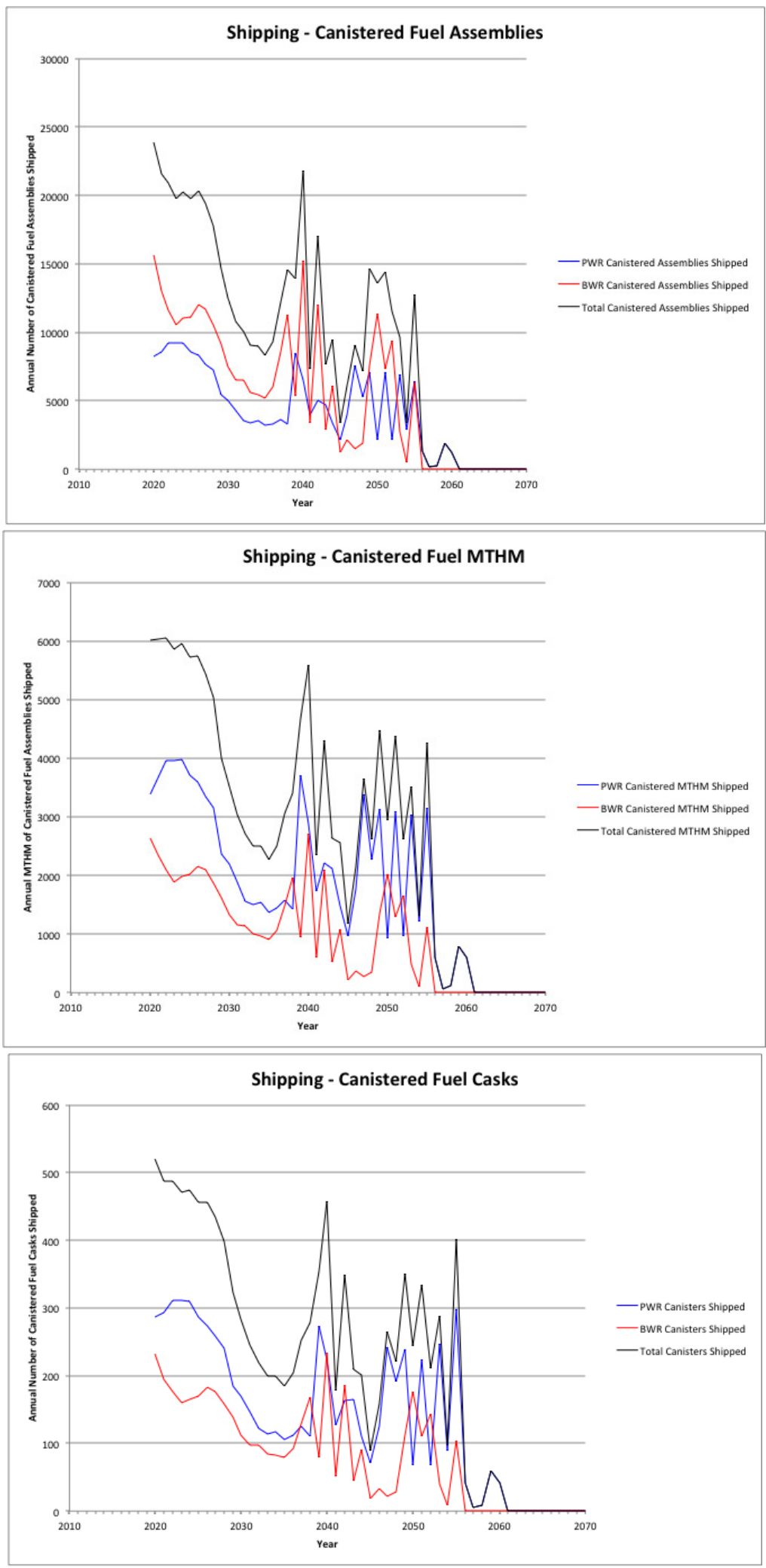

Figure A-23. Cases 1 \& 3: Annual Shipping of Canisterized Fuel, 6000 MTHM YFF-5 Acceptance 2020 


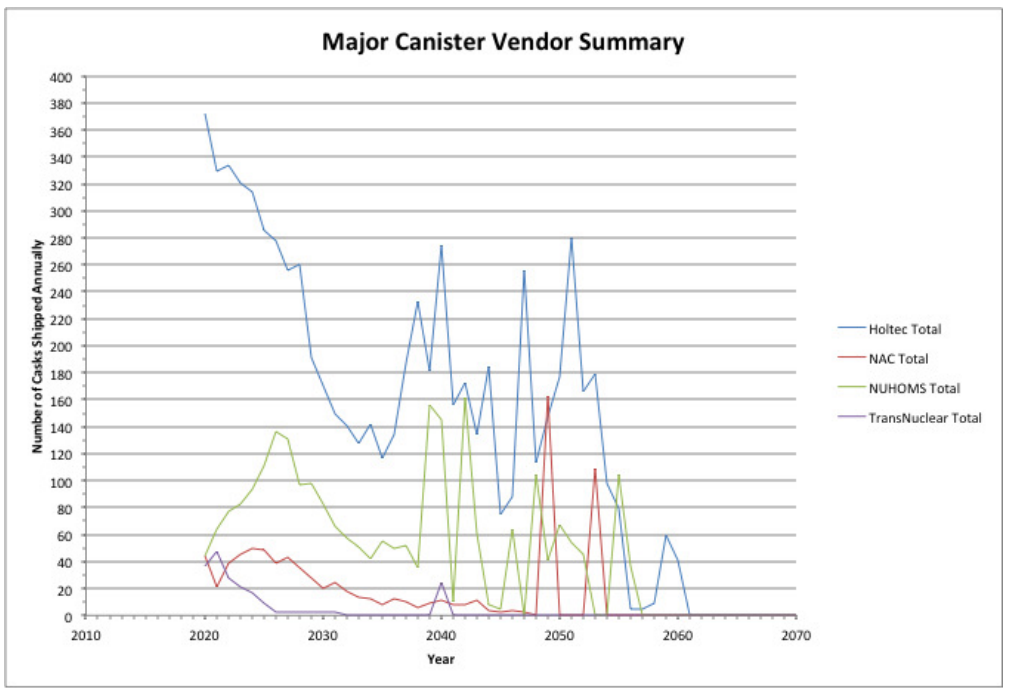

Figure A-24. Cases 1 \& 3: Annual Shipping - Major Canister Vendor Summary, 6000 MTHM YFF-5 Acceptance - 2020

Table A-19. Cases 1 \& 3: Summary of Shipping from Reactor Sites, 6000 MTHM YFF-5 Acceptance 2020

\begin{tabular}{|c|c|c|c|c|c|c|}
\hline \multicolumn{7}{|c|}{ Assemblies } \\
\hline & $\begin{array}{l}\text { PWR Bare } \\
\text { Assemblies } \\
\text { Shipped }\end{array}$ & $\begin{array}{l}\text { BWR Bare } \\
\text { Assemblies } \\
\text { Shipped }\end{array}$ & $\begin{array}{c}\text { Total Bare } \\
\text { Assemblies } \\
\text { Shipped }\end{array}$ & $\begin{array}{c}\text { PWR } \\
\text { Canistered } \\
\text { Assemblies } \\
\text { Shipped }\end{array}$ & $\begin{array}{c}\text { BWR } \\
\text { Canistered } \\
\text { Assemblies } \\
\text { Shipped }\end{array}$ & $\begin{array}{c}\text { Total } \\
\text { Canistered } \\
\text { Assemblies } \\
\text { Shipped }\end{array}$ \\
\hline Peak & 0 & 0 & 0 & 9245 & 15636 & 23888 \\
\hline Average & 0 & 0 & 0 & 6048 & 9359 & 15407 \\
\hline Cumulative & 0 & 0 & 0 & 205983 & 276420 & 482403 \\
\hline \multicolumn{7}{|c|}{ MTHM } \\
\hline & $\begin{array}{c}\text { PWR Bare } \\
\text { MTHM Shipped }\end{array}$ & $\begin{array}{l}\text { BWR Bare } \\
\text { MTHM } \\
\text { Shipped }\end{array}$ & $\begin{array}{l}\text { Total Bare } \\
\text { MTHM } \\
\text { Shipped }\end{array}$ & $\begin{array}{c}\text { PWR } \\
\text { Canistered } \\
\text { MTHM } \\
\text { Shipped }\end{array}$ & $\begin{array}{c}\text { BWR } \\
\text { Canistered } \\
\text { MTHM } \\
\text { Shipped }\end{array}$ & $\begin{array}{c}\text { Total } \\
\text { Canistered } \\
\text { MTHM } \\
\text { Shipped }\end{array}$ \\
\hline Peak & 0 & 0 & 0 & 3972 & 2711 & 6052 \\
\hline Average & 0 & 0 & 0 & 2617 & 1656 & 4273 \\
\hline Cumulative & 0 & 0 & 0 & 89838 & 48897 & 138735 \\
\hline \multicolumn{7}{|c|}{ Canisters/Casks } \\
\hline & $\begin{array}{l}\text { PWR Bare Fuel } \\
\text { Casks Shipped }\end{array}$ & $\begin{array}{l}\text { BWR Bare Fuel } \\
\text { Casks Shipped }\end{array}$ & $\begin{array}{l}\text { Total Bare } \\
\text { Fuel Casks } \\
\text { Shipped }\end{array}$ & $\begin{array}{l}\text { PWR Canisters } \\
\text { Shipped }\end{array}$ & $\begin{array}{l}\text { BWR Canisters } \\
\text { Shipped }\end{array}$ & $\begin{array}{l}\text { Total Canisters } \\
\text { Shipped }\end{array}$ \\
\hline Peak & 0 & 0 & 0 & 311 & 234 & 520 \\
\hline Average & 0 & 0 & 0 & 203 & 141 & 344 \\
\hline Cumulative & 0 & 0 & 0 & 6964 & 4183 & 11147 \\
\hline
\end{tabular}

\begin{tabular}{|c|c|c|c|c|c|c|}
\hline \multicolumn{7}{|c|}{ Major Dry Storage System Vendors } \\
\hline & $\begin{array}{c}\text { Holtec } \\
\text { Canisters }\end{array}$ & NAC Canisters & $\begin{array}{l}\text { NUHOMS } \\
\text { Canisters }\end{array}$ & $\begin{array}{l}\text { TransNuclear } \\
\text { Canisters }\end{array}$ & $\begin{array}{l}\text { Fuel Solutions } \\
\text { Canisters }\end{array}$ & $\begin{array}{c}\text { South Texas } \\
\text { Project Canisters }\end{array}$ \\
\hline Peak & 372 & 163 & 162 & 47 & 27 & 219 \\
\hline Average & 204 & 25 & 73 & 7 & NA & 5 \\
\hline Cumulative & 7221 & 847 & 2488 & 196 & 64 & 331 \\
\hline
\end{tabular}



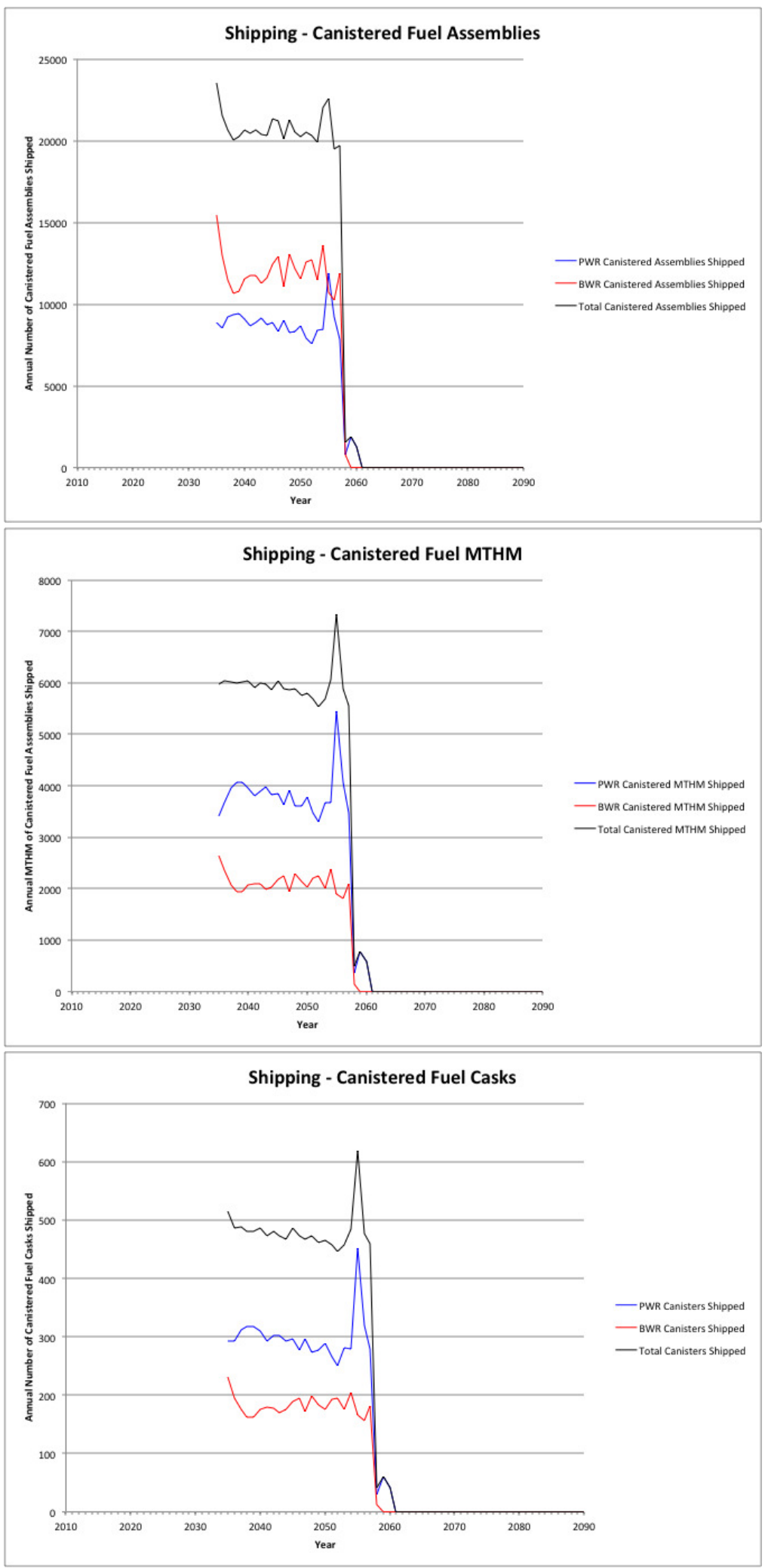

Figure A-25. Cases $1 \&$ 3: Annual Shipping of Canisterized Fuel, 6000 MTHM YFF-5 Acceptance 2035 


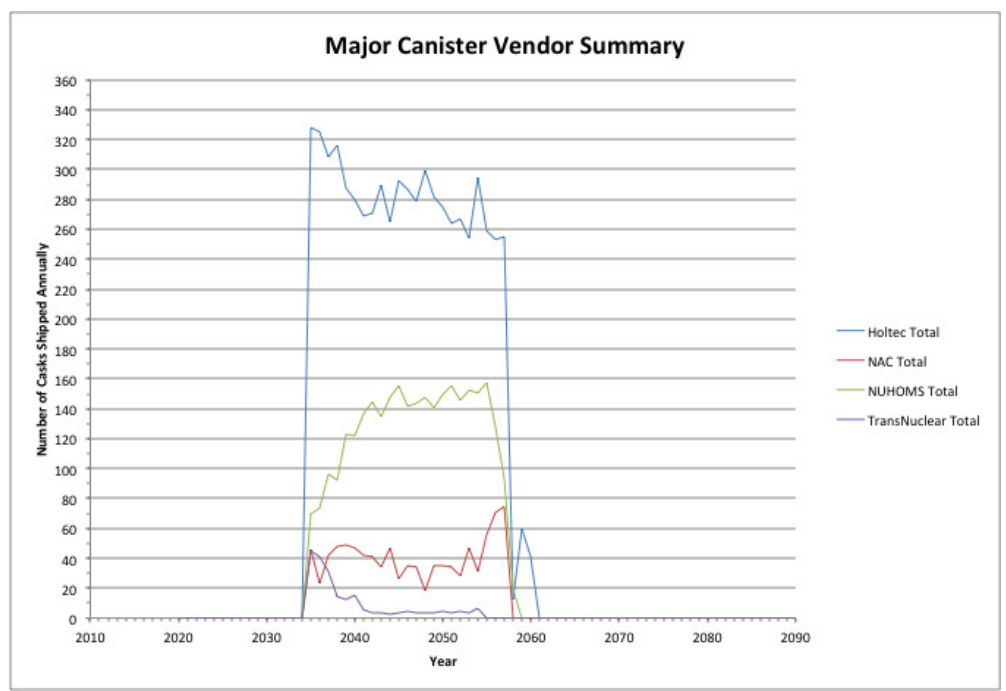

Figure A-26. Cases 1 \& 3: Annual Shipping - Major Canister Vendor Summary, 6000 MTHM YFF-5 Acceptance -2035

Table A-20. Cases 1 \& 3: Summary of Shipping from Reactor Sites, 6000 MTHM YFF-5 Acceptance 2035

\begin{tabular}{|c|c|c|c|c|c|c|}
\hline \multicolumn{7}{|c|}{ Assemblies } \\
\hline & $\begin{array}{l}\text { PWR Bare } \\
\text { Assemblies } \\
\text { Shipped }\end{array}$ & $\begin{array}{l}\text { BWR Bare } \\
\text { Assemblies } \\
\text { Shipped }\end{array}$ & $\begin{array}{l}\text { Total Bare } \\
\text { Assemblies } \\
\text { Shipped }\end{array}$ & $\begin{array}{c}\text { PWR } \\
\text { Canistered } \\
\text { Assemblies } \\
\text { Shipped } \\
\end{array}$ & $\begin{array}{c}\text { BWR } \\
\text { Canistered } \\
\text { Assemblies } \\
\text { Shipped } \\
\end{array}$ & $\begin{array}{c}\text { Total } \\
\text { Canistered } \\
\text { Assemblies } \\
\text { Shipped } \\
\end{array}$ \\
\hline Peak & 0 & 0 & 0 & 11896 & 15456 & 23564 \\
\hline Average & 0 & 0 & 0 & 8784 & 11984 & 20768 \\
\hline Cumulative & 0 & 0 & 0 & 205983 & 276420 & 482403 \\
\hline \multicolumn{7}{|c|}{ MTHM } \\
\hline & $\begin{array}{c}\text { PWR Bare } \\
\text { MTHM Shipped }\end{array}$ & $\begin{array}{l}\text { BWR Bare } \\
\text { MTHM } \\
\text { Shipped }\end{array}$ & $\begin{array}{l}\text { Total Bare } \\
\text { MTHM } \\
\text { Shipped }\end{array}$ & $\begin{array}{c}\text { PWR } \\
\text { Canistered } \\
\text { MTHM } \\
\text { Shipped }\end{array}$ & $\begin{array}{c}\text { BWR } \\
\text { Canistered } \\
\text { MTHM } \\
\text { Shipped }\end{array}$ & $\begin{array}{c}\text { Total } \\
\text { Canistered } \\
\text { MTHM } \\
\text { Shipped }\end{array}$ \\
\hline Peak & 0 & 0 & 0 & 5449 & 2639 & 7336 \\
\hline Average & 0 & 0 & 0 & 3831 & 2120 & 5950 \\
\hline Cumulative & 0 & 0 & 0 & 89838 & 48897 & 138735 \\
\hline \multicolumn{7}{|c|}{ Canisters/Casks } \\
\hline & $\begin{array}{l}\text { PWR Bare Fuel } \\
\text { Casks Shipped }\end{array}$ & $\begin{array}{l}\text { BWR Bare Fuel } \\
\text { Casks Shipped }\end{array}$ & $\begin{array}{c}\text { Total Bare } \\
\text { Fuel Casks } \\
\text { Shipped }\end{array}$ & $\begin{array}{l}\text { PWR Canisters } \\
\text { Shipped }\end{array}$ & $\begin{array}{l}\text { BWR Canisters } \\
\text { Shipped }\end{array}$ & $\begin{array}{l}\text { Total Canisters } \\
\text { Shipped }\end{array}$ \\
\hline Peak & 0 & 0 & 0 & 452 & 232 & 619 \\
\hline Average & 0 & 0 & 0 & 298 & 182 & 481 \\
\hline Cumulative & 0 & 0 & 0 & 6990 & 4208 & 11198 \\
\hline
\end{tabular}

\begin{tabular}{|c|c|c|c|c|c|c|}
\hline \multicolumn{7}{|c|}{ Major Dry Storage System Vendors } \\
\hline & $\begin{array}{l}\text { Holtec } \\
\text { Canisters }\end{array}$ & NAC Canisters & $\begin{array}{l}\text { NUHOMS } \\
\text { Canisters }\end{array}$ & $\begin{array}{l}\text { TransNuclear } \\
\text { Canisters }\end{array}$ & $\begin{array}{l}\text { Fuel Solutions } \\
\text { Canisters }\end{array}$ & $\begin{array}{c}\text { South Texas } \\
\text { Project Canisters }\end{array}$ \\
\hline Peak & 328 & 75 & 157 & 45 & 26 & 147 \\
\hline Average & 283 & 41 & 131 & 10 & NA & 11 \\
\hline Cumulative & 6615 & 944 & 3023 & 221 & 64 & 331 \\
\hline
\end{tabular}




\section{A-2.3.2 Results of At-Reactor Logistic Simulations: Cases 2 and 4}

This section presents the at-reactor logistics results for case 2 which considers that all used fuel in the used fuel pools is transported off-site in re-usable transportation casks. All used fuel in dry storage is transported off-site in the existing size dry storage canisters canisters. Fuel is transported both in reuseable transportation casks and in the existing size dry storage canisters. The assumptions presented in Section A-1.3.3 regarding site capabilities (i.e., crane capacities necessitating the use of legal weigh trucks) were considered in these analyses.

\section{MTHM/year YFF-5 Acceptance, Starting in 2020}

Figure A-27 and A-28 show the annual shipping of bare fuel and canisterized fuel, respectively from the reactor sites for this case. Used fuel from the pools is primarily shipped first (using re-usable transportation casks) until the inventory of fuel that has cooled at least five years is depleted. As the inventory of fuel in the pools available for shipment is depleted, shipments from dry storage increase. Reactors begin to shutdown, starting in 2029 and running through 2055 and after 2060 there is no longer any fuel to ship from the pools. All fuel shipments after 2060 through 2114 are in canisters, from on-site dry storage. Figure A-29 shows the annual shipments from each of the major dry storage system vendors.

Table A-21 shows the summary results for shipping used nuclear fuel from the reactor sites. The peak shipping rate of assemblies, MTHM, and canisters are shown along with the average shipping rate over the period where transportation occurs. Also shown are the cumulative amount of assemblies, canisters, and MTHM that are shipped. Table A-21 also shows the peak, average, and cumulative number of canisters that are shipped for each of the major dry storage system vendors.

\section{MTHM/year YFF-5 Acceptance, Starting in 2035}

Figure A-30 and A-31 show the annual shipping of bare fuel and canisterized fuel, respectively from the reactor sites for this case. Used fuel from the pools is primarily shipped first (using re-usable transportation casks) until the inventory of fuel that has cooled at least five years is depleted. As the inventory of fuel in the pools available for shipment is depleted, shipments from dry storage increase. Reactors begin to shutdown, starting in 2029 and running through 2055 and after 2060 there is no longer any fuel to ship from the pools. All fuel shipments after 2060 through 2114 are in canisters, from on-site dry storage. Figure A-32 shows the annual shipments from each of the major dry storage system vendors.

Table A-22 shows the summary results for shipping used nuclear fuel from the reactor sites. The peak shipping rate of assemblies, MTHM, and canisters are shown along with the average shipping rate over the period where transportation occurs. Also shown are the cumulative amount of assemblies, canisters, and MTHM that are shipped. Table A-22 also shows the peak, average, and cumulative number of canisters that are shipped for each of the major dry storage system vendors.

Comparing Tables A-22 and A-21 shows that the cumulative shipments of bare fuel decreases and the cumulative shipments of canistered fuel increases as the start of accepting $1500 \mathrm{MTHM} / \mathrm{yr}$ of used fuel changes from 2020 to 2035. The delay in starting acceptance results in more fuel being transferred from the used fuel pools to on-site dry storage and subsequent transport of the fuel in canisters. The peak and average shipping rates of bare fuel also are lower. The peak annual shipping rates of canistered fuel are similar, primarily constrained by the $1500 \mathrm{MTHM} / \mathrm{yr}$ acceptance rates. The average annual shipping rate of canistered fuel increases since more fuel is being shipped in canisters.

\section{MTHM/year YFF-5 Acceptance, Starting in 2020}

Figure A-33 and A-34 show the annual shipping of bare fuel and canisterized fuel, respectively from the reactor sites for this case. Used fuel from the pools is primarily shipped first (using re-usable transportation casks) until the inventory of fuel that has cooled at least five years is depleted. As the inventory of fuel in the pools available for shipment is depleted, shipments from dry storage increase. 
Reactors begin to shutdown, starting in 2029 and running through 2055 and after 2060 there is no longer any fuel to ship from the pools. All fuel shipments after 2060 through 2067 are in canisters, from on-site dry storage. Figure A-35 shows the annual shipments from each of the major dry storage system vendors.

Table A-23 shows the summary results for shipping used nuclear fuel from the reactor sites. The peak shipping rate of assemblies, MTHM, and canisters are shown along with the average shipping rate over the period where transportation occurs. Also shown are the cumulative amount of assemblies, canisters, and MTHM that are shipped. Table A-23 also shows the peak, average, and cumulative number of canisters that are shipped for each of the major dry storage system vendors.

Comparing Tables A-23 and A-21 shows that the cumulative shipments of bare fuel increases and the cumulative shipments of canistered fuel deceases as the acceptance rate increases from $1500 \mathrm{MTHM} / \mathrm{yr}$ to 3000 MTHM, starting in 2020. Peak and average annual shipping rates show a similar trend. The increased acceptance rate results in a reduction in the need to transfer fuel from the pools to dry storage, allowing for more used fuel to be shipped directly from the used fuel pools in re-useable transportation casks.

\section{MTHM/year YFF-5 Acceptance, Starting in 2035}

Figure A-36 and A-37 show the annual shipping of bare fuel and canisterized fuel, respectively from the reactor sites for this case. Used fuel from the pools is primarily shipped first (using re-usable transportation casks) until the inventory of fuel that has cooled at least five years is depleted. As the inventory of fuel in the pools available for shipment is depleted, shipments from dry storage increase. Reactors begin to shutdown, starting in 2029 and running through 2055 and after 2060 there is no longer any fuel to ship from the pools. All fuel shipments after 2060 through 2082 are in canisters, from on-site dry storage. Figure A-38 shows the annual shipments from each of the major dry storage system vendors.

Table A-24 shows the summary results for shipping used nuclear fuel from the reactor sites. The peak shipping rate of assemblies, MTHM, and canisters are shown along with the average shipping rate over the period where transportation occurs. Also shown are the cumulative amount of assemblies, canisters, and MTHM that are shipped. Table A-24 also shows the peak, average, and cumulative number of canisters that are shipped for each of the major dry storage system vendors.

Comparing Tables A-24 and A-22 shows that the cumulative shipments of bare fuel increases and the cumulative shipments of canistered fuel deceases as the acceptance rate increases from $1500 \mathrm{MTHM} / \mathrm{yr}$ to 3000 MTHM, starting in 2035. Peak and average annual shipping rates show a similar trend. The increased acceptance rate results in a reduction in the need to transfer fuel from the pools to dry storage, allowing for more used fuel to be shipped directly from the used fuel pools in re-useable transportation casks.

Comparing Tables A-24 and A-23 shows that delaying the beginning of acceptance from 2020 to 2035 reduces the cumulative amount of bare fuel and increases the amount of canistered fuel shipped from the reactor sites. This is again because the 15-year delay results in more fuel being placed into dry storage and subsequently being shipped in canisters. Peak and annual shipping rates show similar trends.

\section{MTHM/year YFF-5 Acceptance, Starting in 2020}

Figure A-39 and A-40 show the annual shipping of bare fuel and canisterized fuel, respectively from the reactor sites for this case. Used fuel from the pools is primarily shipped first (using re-usable transportation casks) until the inventory of fuel that has cooled at least five years is depleted. As the inventory of fuel in the pools available for shipment is depleted, shipments from dry storage increase. Ultimately, all the fuel placed in dry storage by 2020 is shipped (by approximately 2040) and no additional shipments of canistered fuel occur. At this point, the acceptance rate of $6000 \mathrm{MTHM} / \mathrm{yr}$ cannot be maintained because there is insufficient fuel available to transport. From 2040 through 2060 only bare fuel shipments occur, essentially at the annual rate that fuel is discharged from the reactors (5-years 
following discharge). Figure A-41 shows the annual shipments from each of the major dry storage system vendors.

Table A-25 shows the summary results for shipping used nuclear fuel from the reactor sites. The peak shipping rate of assemblies, MTHM, and canisters are shown along with the average shipping rate over the period where transportation occurs. Also shown are the cumulative amount of assemblies, canisters, and MTHM that are shipped. Table A-25 also shows the peak, average, and cumulative number of canisters that are shipped for each of the major dry storage system vendors.

Comparing Tables A-25 and A-23 shows that the cumulative shipments of bare fuel increases and the cumulative shipments of canistered fuel deceases as the acceptance rate increases from $3000 \mathrm{MTHM} / \mathrm{yr}$ to 6000 MTHM, starting in 2020. Peak and average annual shipping rates show a similar trend. The increased acceptance rate results in a reduction in the need to transfer fuel from the pools to dry storage, allowing for more used fuel to be shipped directly from the used fuel pools in re-useable transportation casks.

\section{MTHM/year YFF-5 Acceptance, Starting in 2035}

Figure A-42 and A-43 show the annual shipping of bare fuel and canisterized fuel, respectively from the reactor sites for this case. Used fuel from the pools is primarily shipped first (using re-usable transportation casks) until the inventory of fuel that has cooled at least five years is depleted. As the inventory of fuel in the pools available for shipment is depleted, shipments from dry storage increase. Reactors begin to shutdown, starting in 2029 and running through 2055 and after 2060 there is no longer any fuel to ship from the pools. All fuel shipments after 2060 through 2082 are in canisters, from on-site dry storage. Figure A-44 shows the annual shipments from each of the major dry storage system vendors.

Table A-26 shows the summary results for shipping used nuclear fuel from the reactor sites. The peak shipping rate of assemblies, MTHM, and canisters are shown along with the average shipping rate over the period where transportation occurs. Also shown are the cumulative amount of assemblies, canisters, and MTHM that are shipped. Table A-26 also shows the peak, average, and cumulative number of canisters that are shipped for each of the major dry storage system vendors.

Comparing Tables A-26 and A-24 shows that the cumulative shipments of bare fuel increases and the cumulative shipments of canistered fuel deceases as the acceptance rate increases from $3000 \mathrm{MTHM} / \mathrm{yr}$ to 6000 MTHM, starting in 2035. Peak and average annual shipping rates show a similar trend. The increased acceptance rate results in a reduction in the need to transfer fuel from the pools to dry storage, allowing for more used fuel to be shipped directly from the used fuel pools in re-useable transportation casks.

Comparing Tables A-26 and A-19 shows that delaying the beginning of acceptance from 2020 to 2035 reduces the cumulative amount of bare fuel and increases the amount of canistered fuel shipped from the reactor sites. This is again because the 15-year delay results in more fuel being placed into dry storage and subsequently being shipped in canisters. Peak and annual shipping rates show similar trends. 

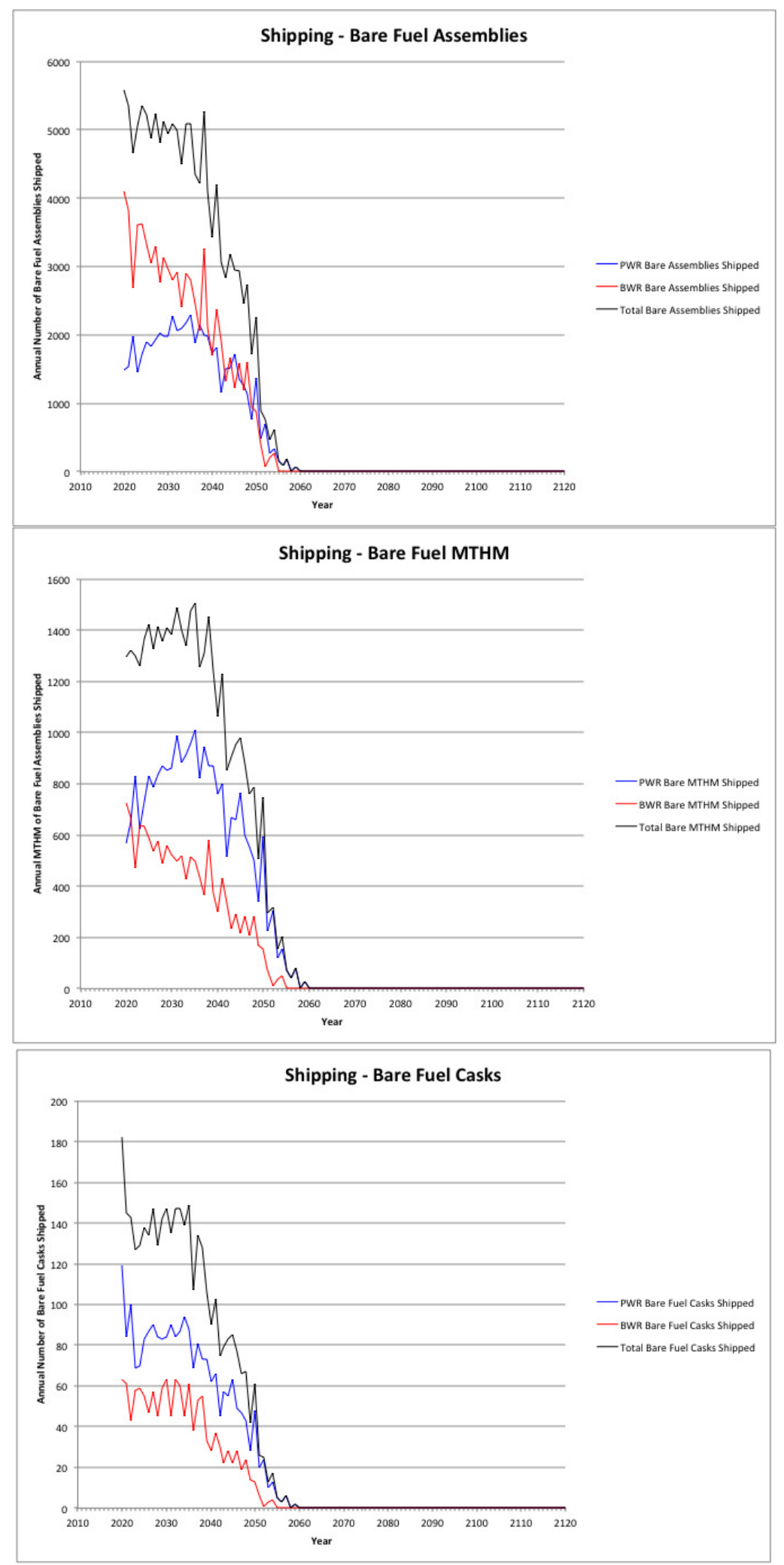

Figure A-27. Cases 2 \& 4: Annual Shipping of Bare Fuel, 1500 MTHM YFF-5 Acceptance - 2020 

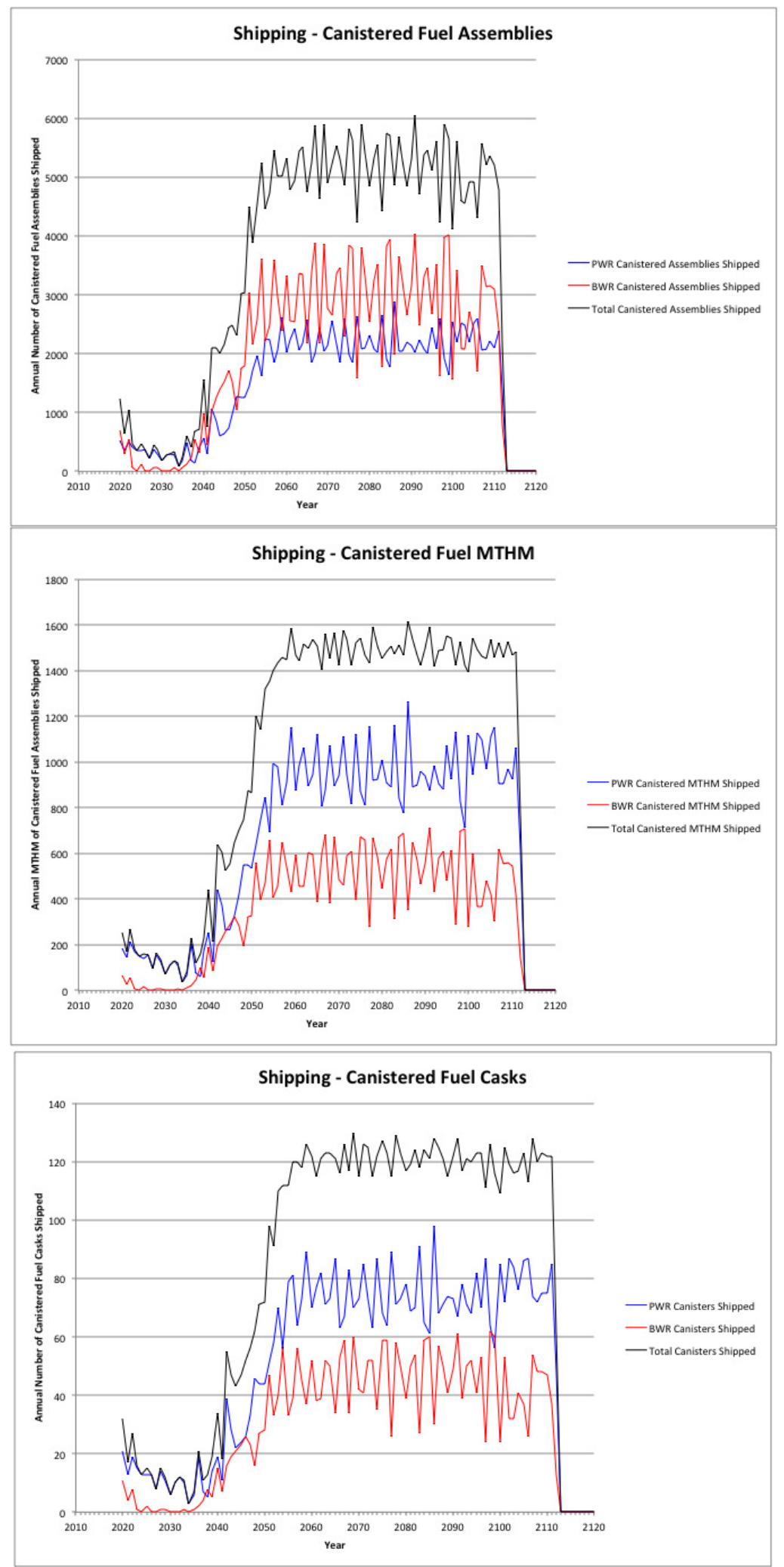

Figure A-28. Cases 2 \& 4: Annual Shipping of Canisterized Fuel, 1500 MTHM YFF-5 Acceptance 2020 


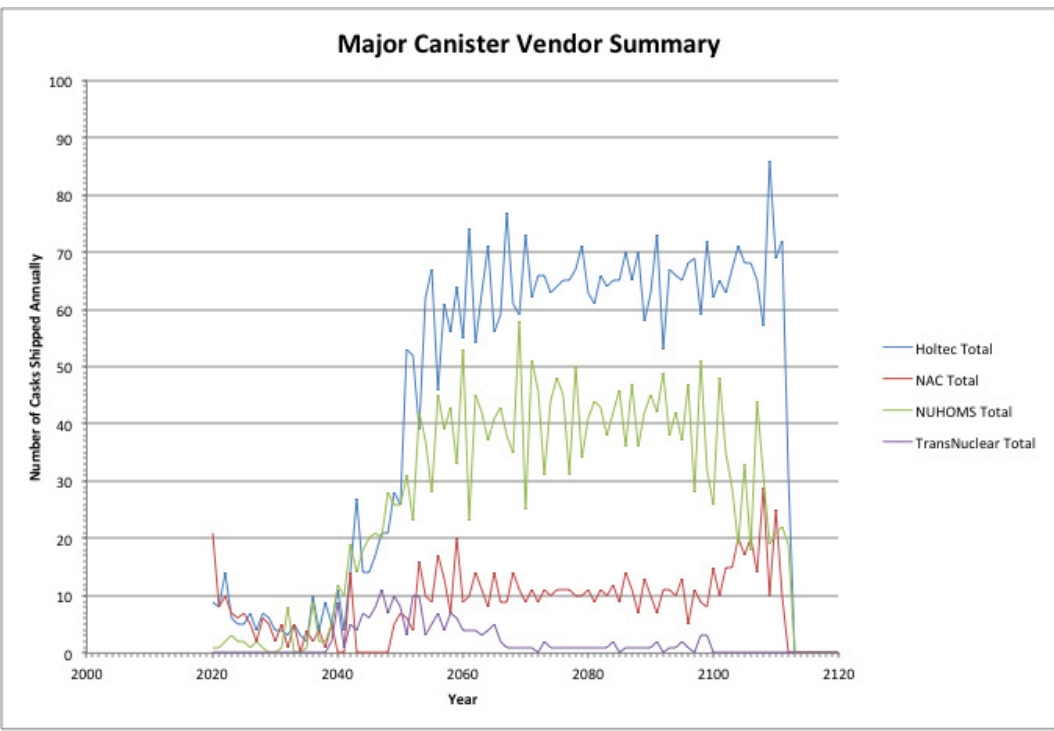

Figure A-29. Cases 2 \& 4: Annual Shipping - Major Canister Vendor Summary, 1500 MTHM YFF-5 Acceptance - 2020

Table A-21. Cases 2 \& 4: Summary of Shipping from Reactor Sites, 1500 MTHM YFF-5 Acceptance 2020

\begin{tabular}{|c|c|c|c|c|c|c|c|c|}
\hline \multicolumn{9}{|c|}{ Assemblies } \\
\hline & \multicolumn{2}{|c|}{$\begin{array}{l}\text { PWR Bare } \\
\text { Assemblies } \\
\text { Shipped }\end{array}$} & $\begin{array}{l}\text { BWR Bare } \\
\text { Assemblies } \\
\text { Shipped }\end{array}$ & $\begin{array}{c}\text { Total Bare } \\
\text { Assemblies } \\
\text { Shipped }\end{array}$ & \multicolumn{2}{|c|}{$\begin{array}{c}\text { PWR } \\
\text { Canistered } \\
\text { Assemblies } \\
\text { Shipped }\end{array}$} & $\begin{array}{c}\text { BWR } \\
\text { Canistered } \\
\text { Assemblies } \\
\text { Shipped }\end{array}$ & $\begin{array}{c}\text { Total } \\
\text { Canistered } \\
\text { Assemblies } \\
\text { Shipped }\end{array}$ \\
\hline Peak & \multicolumn{2}{|l|}{2296} & 4105 & 5587 & \multicolumn{2}{|c|}{2882} & 4029 & 6044 \\
\hline Average & \multicolumn{2}{|l|}{1873} & 2811 & 4684 & \multicolumn{2}{|l|}{1611} & 2153 & 3764 \\
\hline Cumulative & \multicolumn{2}{|l|}{56366} & 77529 & 133895 & \multicolumn{2}{|c|}{149617} & 198891 & 348508 \\
\hline \multicolumn{9}{|c|}{ MTHM } \\
\hline & \multicolumn{2}{|c|}{$\begin{array}{l}\text { PWR Bare } \\
\text { MTHM Shipped }\end{array}$} & $\begin{array}{l}\text { BWR Bare } \\
\text { MTHM } \\
\text { Shipped }\end{array}$ & $\begin{array}{l}\text { Total Bare } \\
\text { MTHM } \\
\text { Shipped }\end{array}$ & \multicolumn{2}{|c|}{$\begin{array}{c}\text { PWR } \\
\text { Canistered } \\
\text { MTHM } \\
\text { Shipped }\end{array}$} & $\begin{array}{c}\text { BWR } \\
\text { Canistered } \\
\text { MTHM } \\
\text { Shipped }\end{array}$ & $\begin{array}{c}\text { Total } \\
\text { Canistered } \\
\text { MTHM } \\
\text { Shipped } \\
\end{array}$ \\
\hline Peak & \multicolumn{2}{|l|}{1011} & 726 & 1507 & \multicolumn{2}{|c|}{1264} & 715 & 1614 \\
\hline Average & \multicolumn{2}{|l|}{811} & 496 & 1307 & \multicolumn{2}{|l|}{704} & 381 & 1085 \\
\hline Cumulative & \multicolumn{2}{|l|}{24493} & 13686 & 38179 & \multicolumn{2}{|c|}{65344} & 35212 & 100556 \\
\hline \multicolumn{9}{|c|}{ Canisters/Casks } \\
\hline & $\begin{array}{l}\text { PWR Bare } \\
\text { Casks Ship }\end{array}$ & & $\begin{array}{l}\text { BWR Bare } \\
\text { Casks Ship }\end{array}$ & $\begin{array}{c}\text { Total Bare } \\
\text { Fuel Casks } \\
\text { Shipped }\end{array}$ & $\begin{array}{r}\text { PWR Canis } \\
\text { Shippe }\end{array}$ & & $\begin{array}{l}\text { BWR Canisters } \\
\text { Shipped }\end{array}$ & $\begin{array}{l}\text { Total Canisters } \\
\text { Shipped }\end{array}$ \\
\hline Peak & 119 & & 63 & 182 & 98 & & 62 & 130 \\
\hline Average & 80 & & 49 & 129 & 55 & & 33 & 88 \\
\hline Cumulative & 2338 & & 1342 & 3680 & 5145 & & 3051 & 8196 \\
\hline & & & & Dry Storage Sys & m Vendors & & & \\
\hline & $\begin{array}{l}\text { Holtec } \\
\text { Canisters }\end{array}$ & & C Canisters & $\begin{array}{l}\text { NUHOMS } \\
\text { Canisters }\end{array}$ & $\begin{array}{l}\text { TransNuclear } \\
\text { Canisters }\end{array}$ & & $\begin{array}{l}\text { el Solutions } \\
\text { Canisters }\end{array}$ & $\begin{array}{c}\text { South Texas } \\
\text { Project Canisters }\end{array}$ \\
\hline Peak & 86 & & 29 & 58 & 11 & & 7 & 18 \\
\hline Average & 54 & & 10 & 34 & 3 & & NA & 4 \\
\hline Cumulative & 4254 & & 848 & 2588 & 196 & & 64 & 246 \\
\hline
\end{tabular}



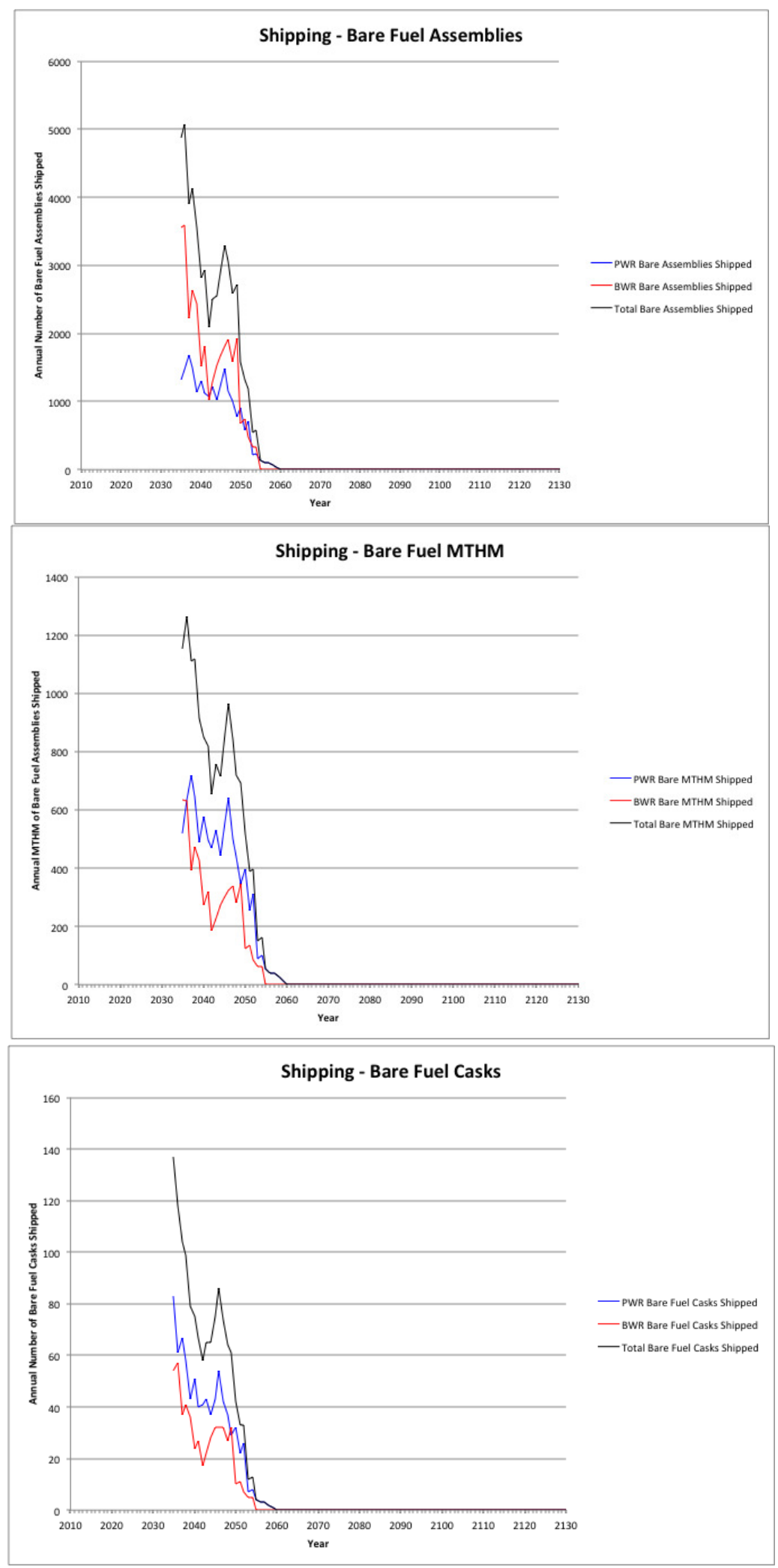

Figure A-30. Cases $1 \&$ 3: Annual Shipping of Bare Fuel, 1500 MTHM YFF-5 Acceptance - 2035 

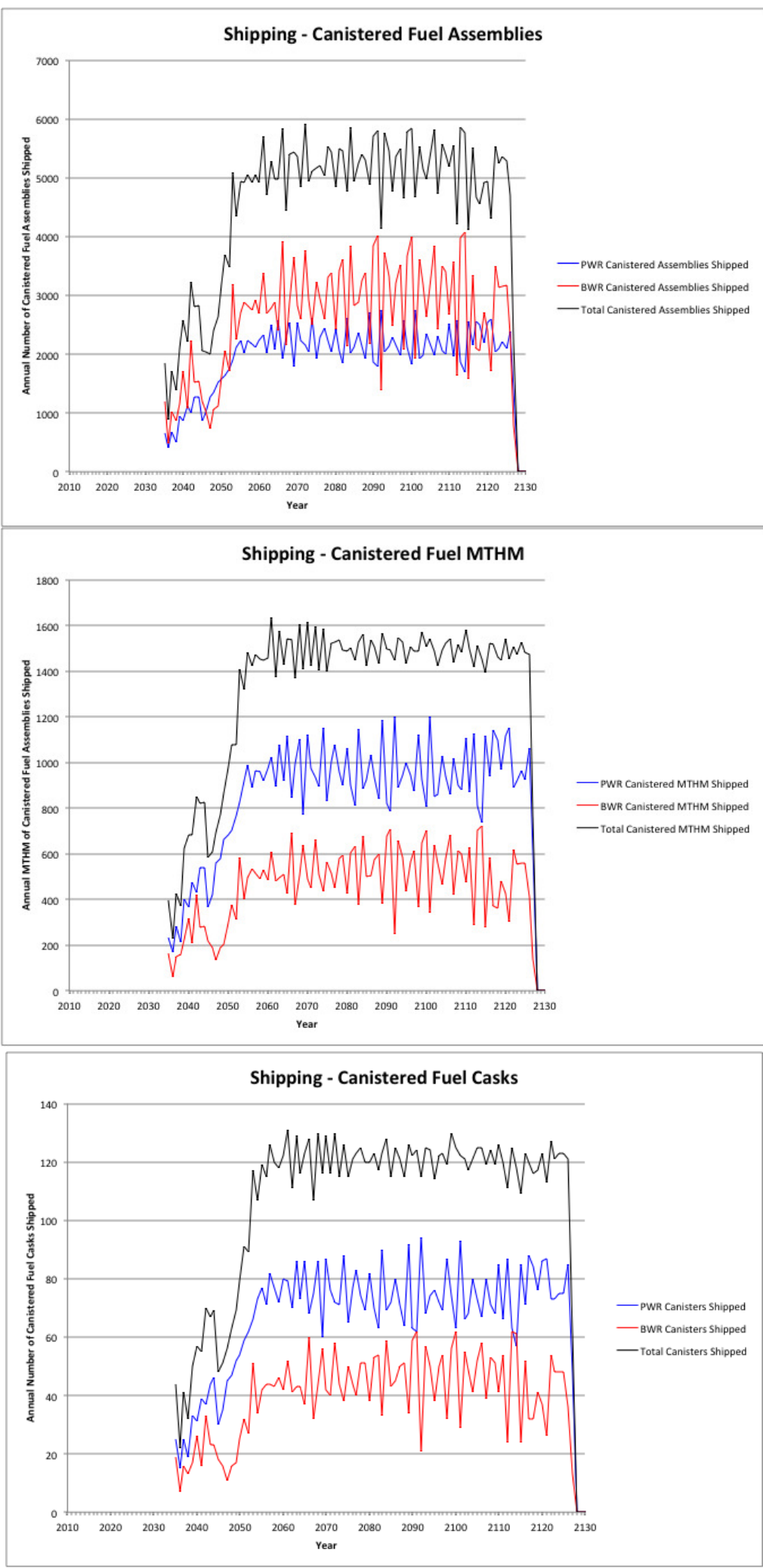

Figure A-31. Cases 2 \& 4: Annual Shipping of Canisterized Fuel, 1500 MTHM YFF-5 Acceptance 2035 


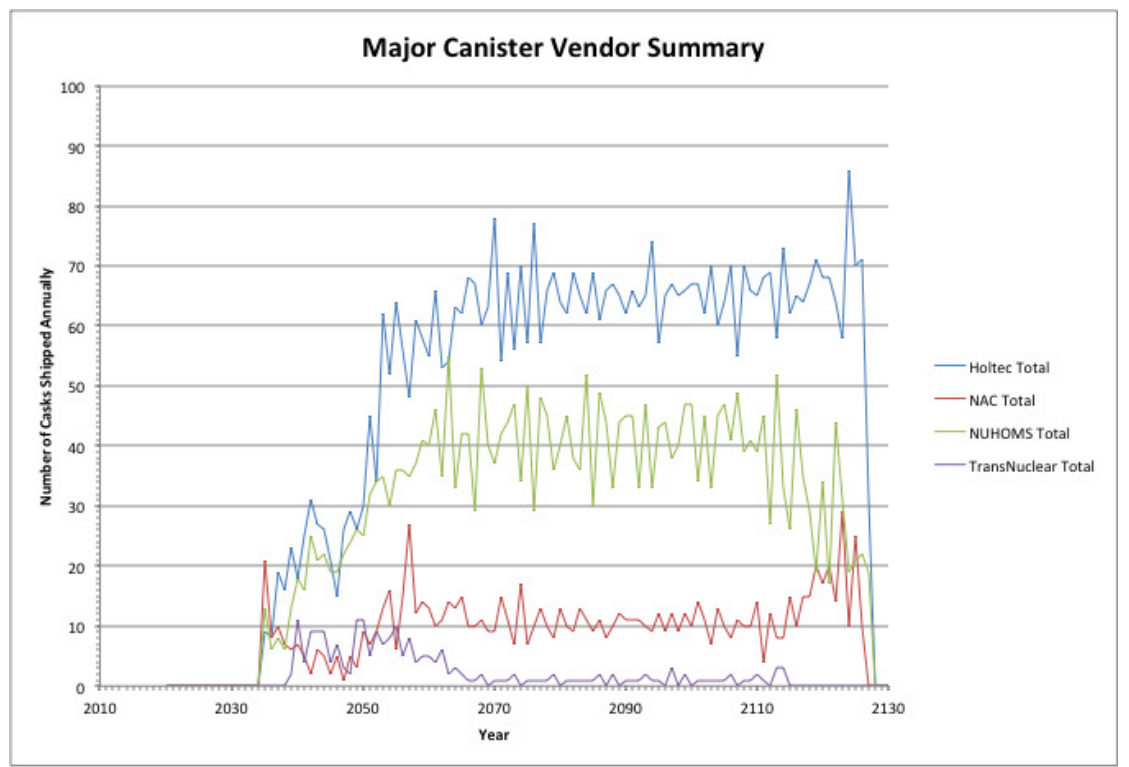

Figure A-32. Cases 2 \& 4: Annual Shipping - Major Canister Vendor Summary, 1500 MTHM YFF-5 Acceptance - 2035

Table A-22. Cases $2 \&$ 4: Summary of Shipping from Reactor Sites, 1500 MTHM YFF-5 Acceptance 2035

\begin{tabular}{|c|c|c|c|c|c|c|}
\hline \multicolumn{7}{|c|}{ Assemblies } \\
\hline & $\begin{array}{l}\text { PWR Bare } \\
\text { Assemblies } \\
\text { Shipped }\end{array}$ & $\begin{array}{c}\text { BWR Bare } \\
\text { Assemblies } \\
\text { Shipped }\end{array}$ & $\begin{array}{c}\text { Total Bare } \\
\text { Assemblies } \\
\text { Shipped }\end{array}$ & $\begin{array}{c}\text { PWR } \\
\text { Canistered } \\
\text { Assemblies } \\
\text { Shipped }\end{array}$ & $\begin{array}{c}\text { BWR } \\
\text { Canistered } \\
\text { Assemblies } \\
\text { Shipped }\end{array}$ & $\begin{array}{c}\text { Total } \\
\text { Canistered } \\
\text { Assemblies } \\
\text { Shipped }\end{array}$ \\
\hline Peak & 1678 & 3598 & 5070 & 2748 & 4071 & 5921 \\
\hline Average & 1265 & 2042 & 3307 & 2213 & 2969 & 5181 \\
\hline Cumulative & 21552 & 33099 & 54651 & 184431 & 243321 & 427752 \\
\hline \multicolumn{7}{|c|}{ MTHM } \\
\hline & $\begin{array}{c}\text { PWR Bare } \\
\text { MTHM Shipped }\end{array}$ & $\begin{array}{l}\text { BWR Bare } \\
\text { MTHM } \\
\text { Shipped }\end{array}$ & $\begin{array}{l}\text { Total Bare } \\
\text { MTHM } \\
\text { Shipped }\end{array}$ & $\begin{array}{c}\text { PWR } \\
\text { Canistered } \\
\text { MTHM } \\
\text { Shipped }\end{array}$ & $\begin{array}{c}\text { BWR } \\
\text { Canistered } \\
\text { MTHM } \\
\text { Shipped }\end{array}$ & $\begin{array}{c}\text { Total } \\
\text { Canistered } \\
\text { MTHM } \\
\text { Shipped }\end{array}$ \\
\hline Peak & 717 & 633 & 1265 & 1201 & 720 & 1634 \\
\hline Average & 545 & 362 & 908 & 969 & 524 & 1493 \\
\hline Cumulative & 9316 & 5879 & 15195 & 80522 & 43019 & 123541 \\
\hline \multicolumn{7}{|c|}{ Canisters/Casks } \\
\hline & $\begin{array}{l}\text { PWR Bare Fuel } \\
\text { Casks Shipped }\end{array}$ & $\begin{array}{l}\text { BWR Bare Fuel } \\
\text { Casks Shipped }\end{array}$ & $\begin{array}{c}\text { Total Bare } \\
\text { Fuel Casks } \\
\text { Shipped }\end{array}$ & $\begin{array}{l}\text { PWR Canisters } \\
\text { Shipped }\end{array}$ & $\begin{array}{l}\text { BWR Canisters } \\
\text { Shipped }\end{array}$ & $\begin{array}{l}\text { Total Canisters } \\
\text { Shipped }\end{array}$ \\
\hline Peak & 83 & 57 & 137 & 94 & 62 & 131 \\
\hline Average & 50 & 33 & 83 & 76 & 45 & 121 \\
\hline Cumulative & 837 & 536 & 1373 & 6326 & 3728 & 10054 \\
\hline
\end{tabular}

\begin{tabular}{|l|c|c|c|c|c|c|}
\hline \multicolumn{7}{|c|}{ Major Dry Storage System Vendors } \\
\hline & $\begin{array}{c}\text { Holtec } \\
\text { Canisters }\end{array}$ & $\begin{array}{c}\text { NAC } \\
\text { Canisters }\end{array}$ & $\begin{array}{c}\text { NUHOMS } \\
\text { Canisters }\end{array}$ & $\begin{array}{c}\text { TransNuclear } \\
\text { Canisters }\end{array}$ & $\begin{array}{c}\text { Fuel Solutions } \\
\text { Canisters }\end{array}$ & $\begin{array}{c}\text { South Texas } \\
\text { Project Canisters }\end{array}$ \\
\hline Peak & 86 & 29 & 55 & 11 & 7 & 18 \\
\hline Average & 52 & 10 & 32 & 2 & NA & 4 \\
\hline Cumulative & 5222 & 1006 & 3231 & 219 & 64 & 312 \\
\hline
\end{tabular}



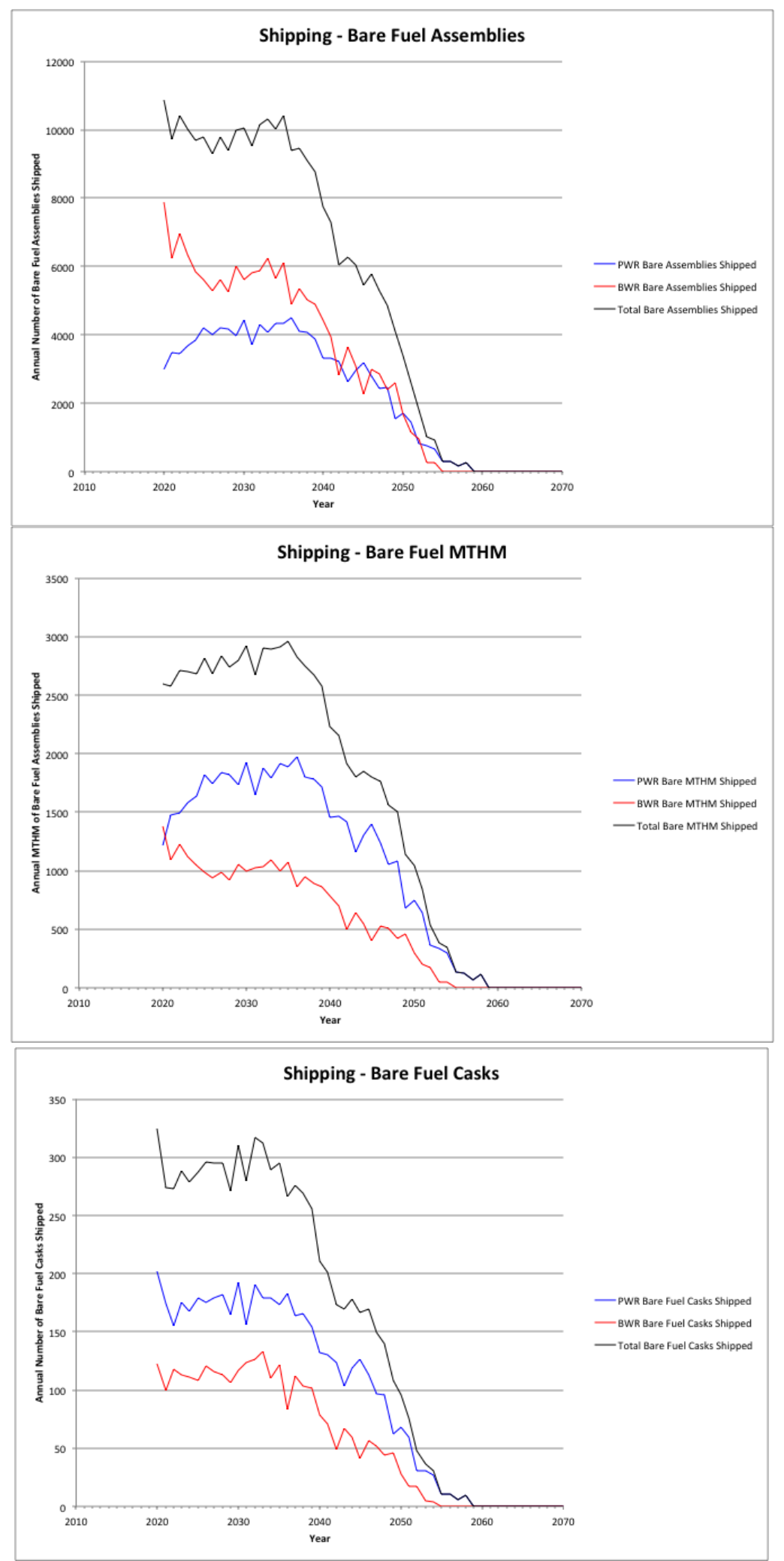

Figure A-33. Cases 2 \& 4: Annual Shipping of Bare Fuel, 3000 MTHM YFF-5 Acceptance - 2020 

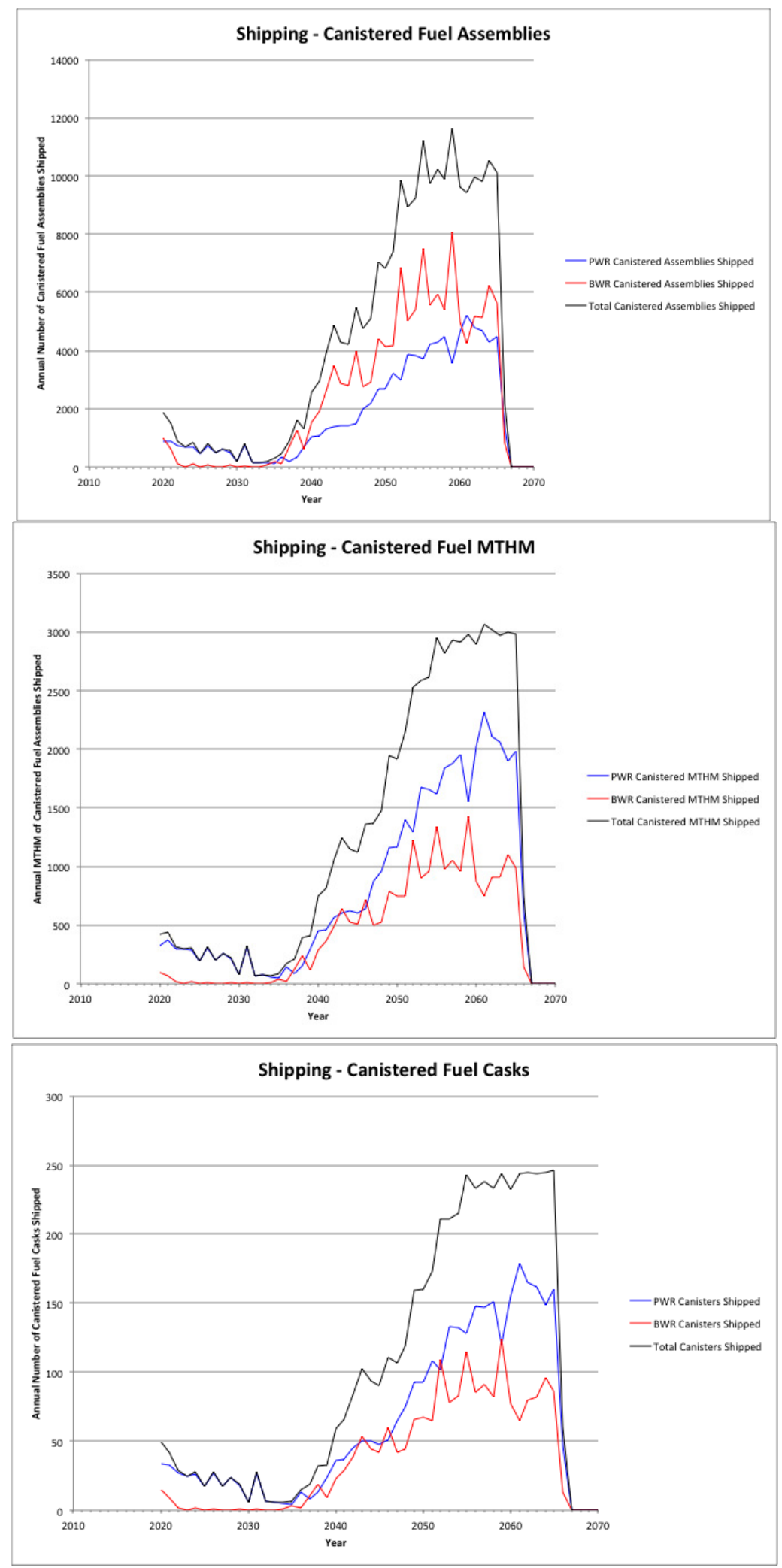

Figure A-34. Cases $2 \&$ 4: Annual Shipping of Canisterized Fuel, 3000 MTHM YFF-5 Acceptance 2020 


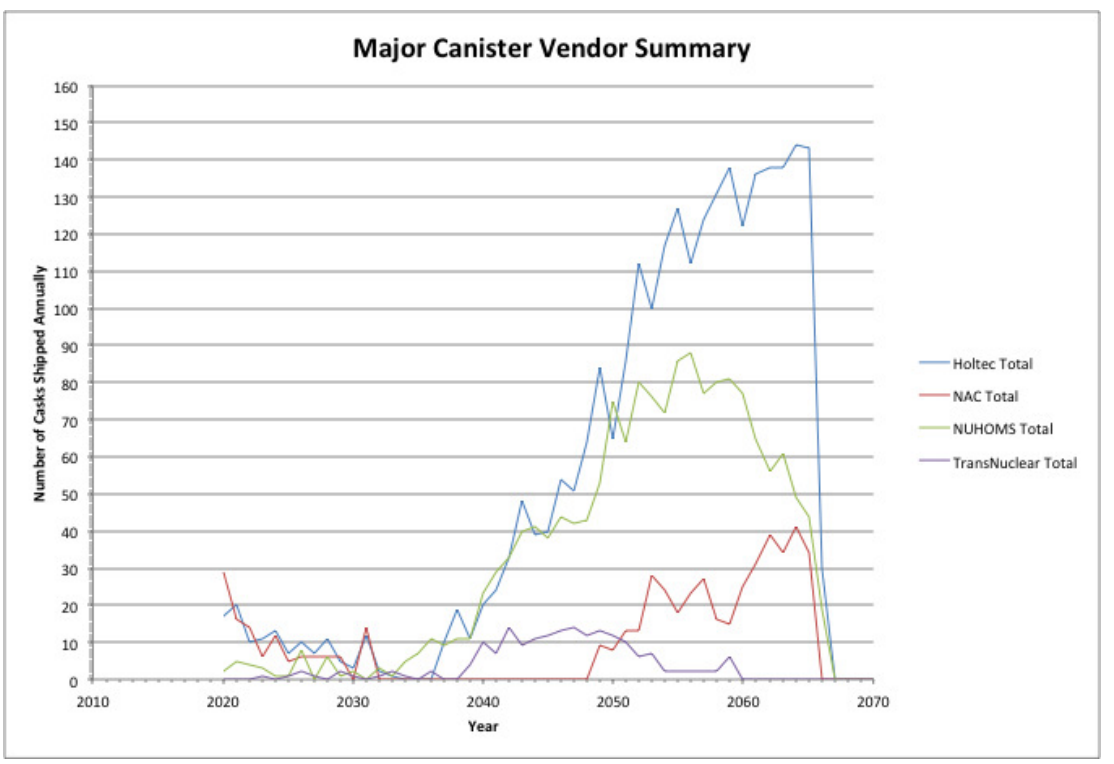

Figure A-35. Cases 2 \& 4: Annual Shipping - Major Canister Vendor Summary, 3000 MTHM YFF-5 Acceptance - 2020

Table A-23. Cases $2 \&$ 4: Summary of Shipping from Reactor Sites, 3000 MTHM YFF-5 Acceptance 2020

\begin{tabular}{|c|c|c|c|c|c|c|}
\hline \multicolumn{7}{|c|}{ Assemblies } \\
\hline & $\begin{array}{l}\text { PWR Bare } \\
\text { Assemblies } \\
\text { Shipped }\end{array}$ & $\begin{array}{l}\text { BWR Bare } \\
\text { Assemblies } \\
\text { Shipped }\end{array}$ & $\begin{array}{c}\text { Total Bare } \\
\text { Assemblies } \\
\text { Shipped }\end{array}$ & $\begin{array}{c}\text { PWR } \\
\text { Canistered } \\
\text { Assemblies } \\
\text { Shipped }\end{array}$ & $\begin{array}{c}\text { BWR } \\
\text { Canistered } \\
\text { Assemblies } \\
\text { Shipped }\end{array}$ & $\begin{array}{c}\text { Total } \\
\text { Canistered } \\
\text { Assemblies } \\
\text { Shipped }\end{array}$ \\
\hline Peak & 4498 & 7879 & 10881 & 5194 & 8084 & 11658 \\
\hline Average & 3460 & 4700 & 8160 & 1970 & 2690 & 4660 \\
\hline Cumulative & 114014 & 151895 & 265909 & 91969 & 124525 & 216494 \\
\hline \multicolumn{7}{|c|}{ MTHM } \\
\hline & $\begin{array}{c}\text { PWR Bare } \\
\text { MTHM Shipped }\end{array}$ & $\begin{array}{l}\text { BWR Bare } \\
\text { MTHM } \\
\text { Shipped }\end{array}$ & $\begin{array}{l}\text { Total Bare } \\
\text { MTHM } \\
\text { Shipped }\end{array}$ & $\begin{array}{c}\text { PWR } \\
\text { Canistered } \\
\text { MTHM } \\
\text { Shipped } \\
\end{array}$ & $\begin{array}{c}\text { BWR } \\
\text { Canistered } \\
\text { MTHM } \\
\text { Shipped } \\
\end{array}$ & $\begin{array}{c}\text { Total } \\
\text { Canistered } \\
\text { MTHM } \\
\text { Shipped } \\
\end{array}$ \\
\hline Peak & 1970 & 1383 & 2960 & 2322 & 1425 & 3069 \\
\hline Average & 1510 & 829 & 2338 & 858 & 478 & 1336 \\
\hline Cumulative & 49762 & 26782 & 76544 & 40075 & 22116 & 62191 \\
\hline \multicolumn{7}{|c|}{ Canisters/Casks } \\
\hline & $\begin{array}{l}\text { PWR Bare Fuel } \\
\text { Casks Shipped }\end{array}$ & $\begin{array}{l}\text { BWR Bare Fuel } \\
\text { Casks Shipped }\end{array}$ & $\begin{array}{c}\text { Total Bare } \\
\text { Fuel Casks } \\
\text { Shipped }\end{array}$ & $\begin{array}{l}\text { PWR Canisters } \\
\text { Shipped }\end{array}$ & $\begin{array}{l}\text { BWR Canisters } \\
\text { Shipped }\end{array}$ & $\begin{array}{l}\text { Total Canisters } \\
\text { Shipped }\end{array}$ \\
\hline Peak & 202 & 133 & 325 & 179 & 124 & 246 \\
\hline Average & 148 & 90 & 237 & 68 & 41 & 110 \\
\hline Cumulative & 4848 & 2897 & 7745 & 3190 & 1916 & 5106 \\
\hline
\end{tabular}

\begin{tabular}{|l|c|c|c|c|c|c|}
\hline & $\begin{array}{c}\text { Moltec } \\
\text { Canisters }\end{array}$ & $\begin{array}{c}\text { NAC } \\
\text { Canisters }\end{array}$ & $\begin{array}{c}\text { NUHOMS } \\
\text { Canisters }\end{array}$ & $\begin{array}{c}\text { TransNuclear } \\
\text { Canisters }\end{array}$ & $\begin{array}{c}\text { Fuel Solutions } \\
\text { Canisters }\end{array}$ & $\begin{array}{c}\text { South Texas } \\
\text { Project Canisters }\end{array}$ \\
\hline Peak & 144 & 41 & 88 & 14 & 7 & 25 \\
\hline Average & 55 & 11 & 35 & 4 & NA & 10 \\
\hline Cumulative & 2589 & 518 & 1627 & 184 & 64 & 124 \\
\hline
\end{tabular}



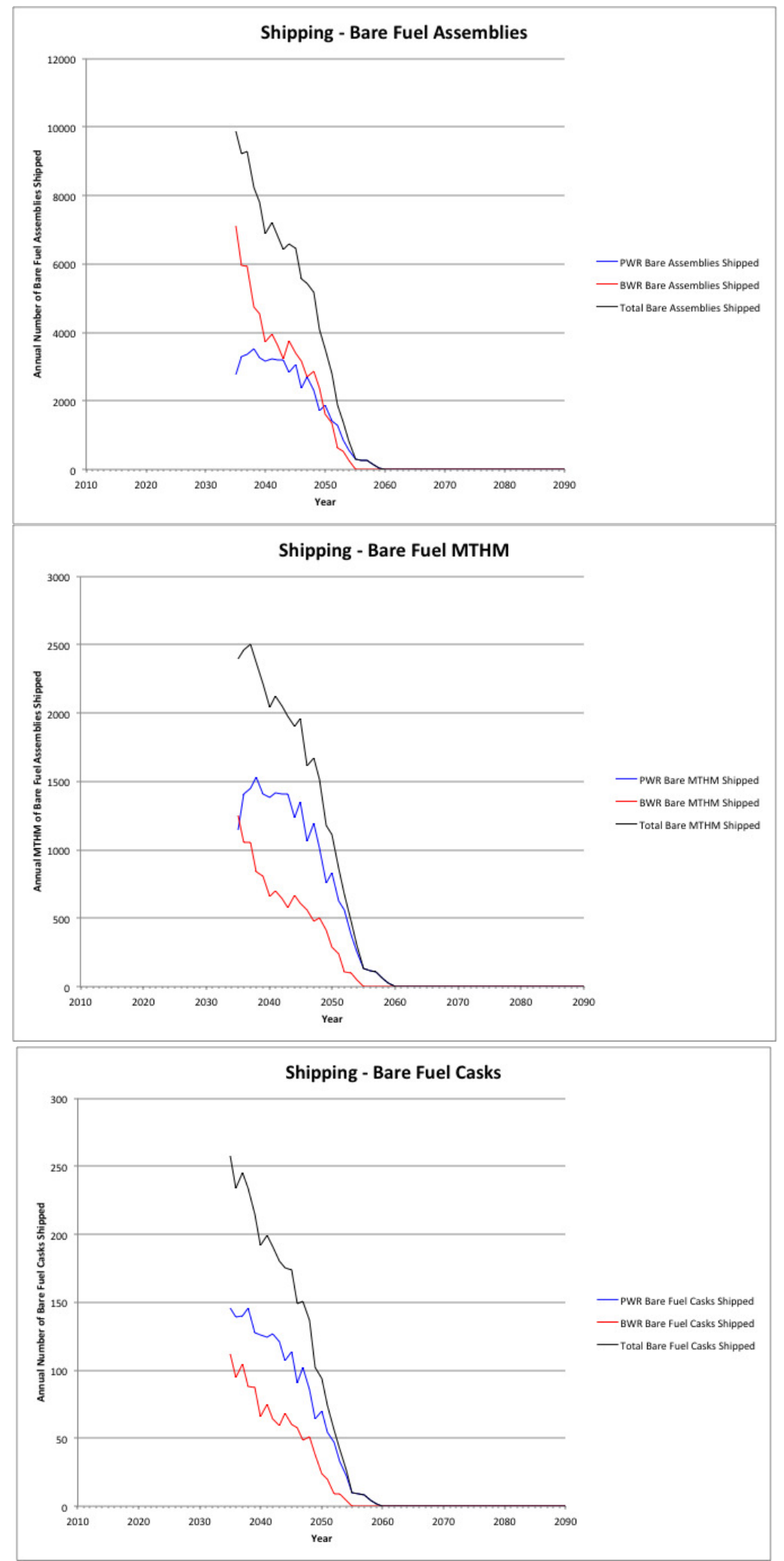

Figure A-36. Cases 2 \& 4: Annual Shipping of Bare Fuel, 3000 MTHM YFF-5 Acceptance - 2035 

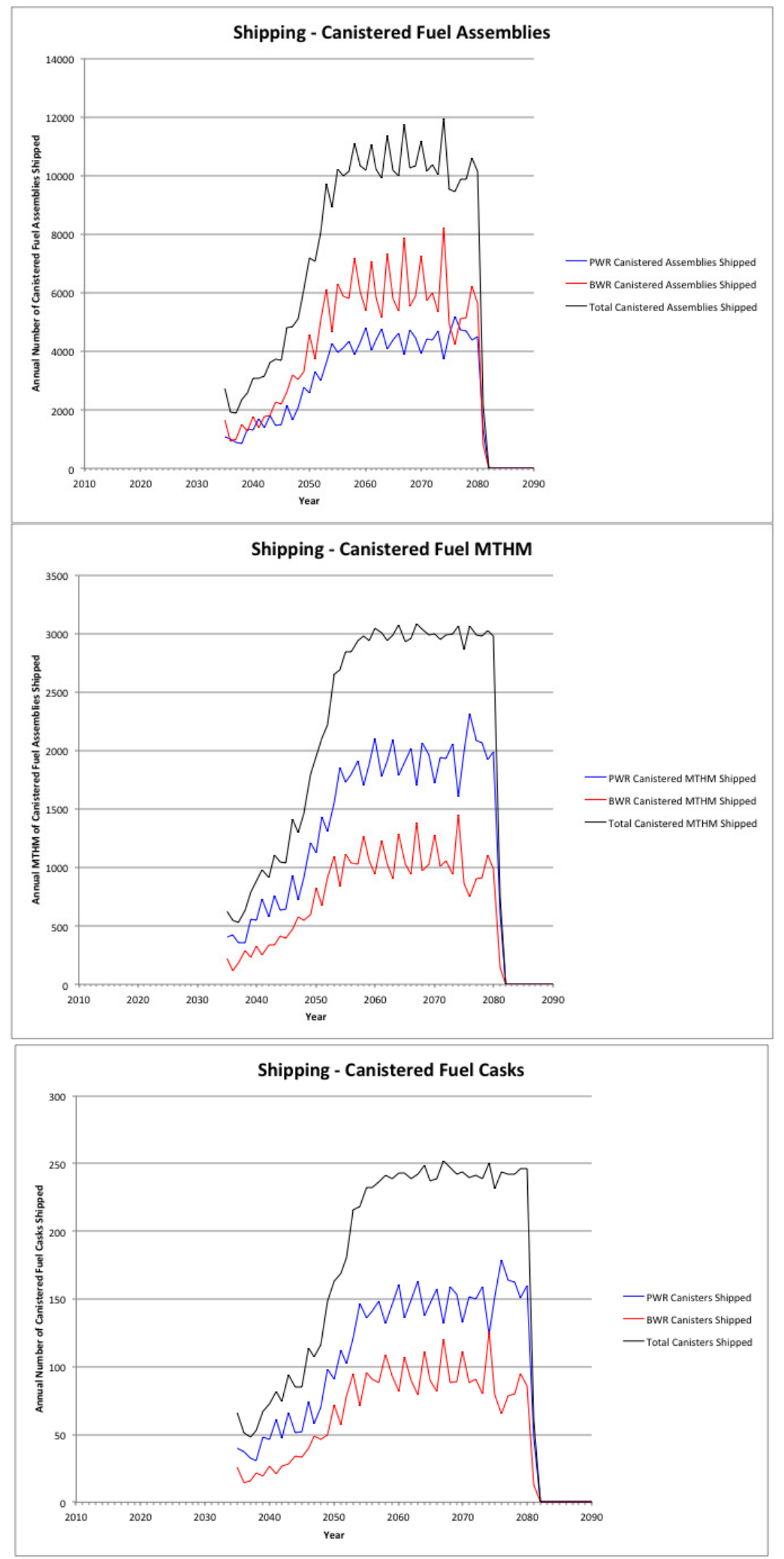

Figure A-37. Cases 2 \& 4: Annual Shipping of Canisterized Fuel, 3000 MTHM YFF-5 Acceptance 2035 


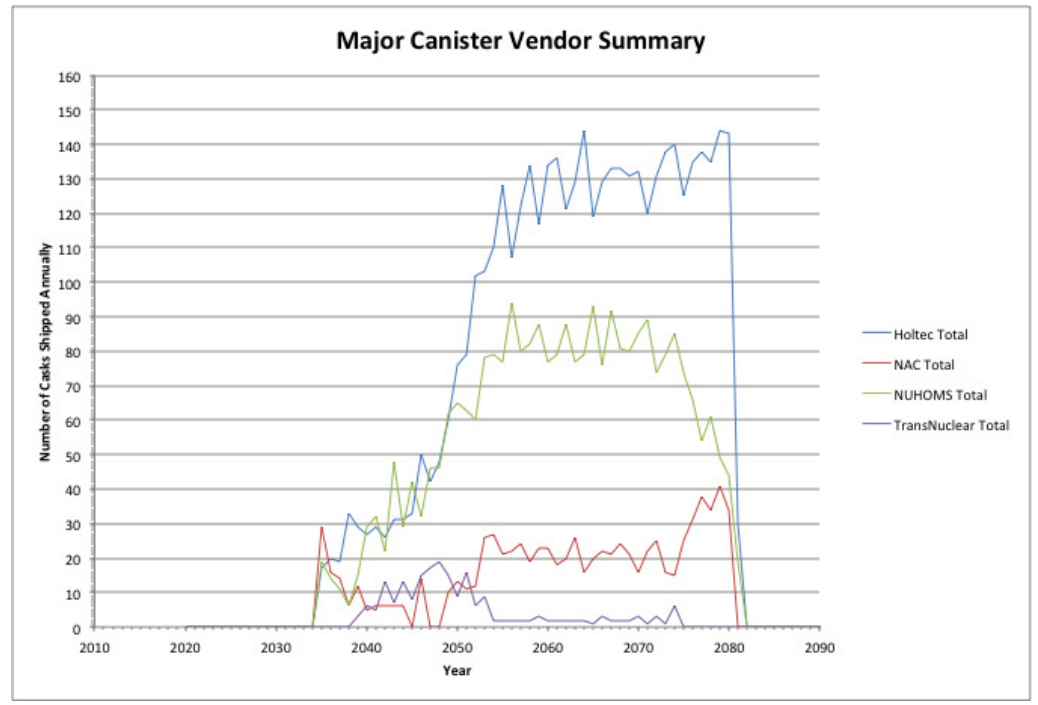

Figure A-38. Cases 2 \& 4: Annual Shipping - Major Canister Vendor Summary, 3000 MTHM YFF-5 Acceptance - 2035

Table A-24. Cases 2 \& 4: Summary of Shipping from Reactor Sites, 3000 MTHM YFF-5 Acceptance 2035

\begin{tabular}{|c|c|c|c|c|c|c|}
\hline \multicolumn{7}{|c|}{ Assemblies } \\
\hline & $\begin{array}{l}\text { PWR Bare } \\
\text { Assemblies } \\
\text { Shipped }\end{array}$ & $\begin{array}{l}\text { BWR Bare } \\
\text { Assemblies } \\
\text { Shipped }\end{array}$ & $\begin{array}{c}\text { Total Bare } \\
\text { Assemblies } \\
\text { Shipped }\end{array}$ & $\begin{array}{c}\text { PWR } \\
\text { Canistered } \\
\text { Assemblies } \\
\text { Shipped }\end{array}$ & $\begin{array}{c}\text { BWR } \\
\text { Canistered } \\
\text { Assemblies } \\
\text { Shipped }\end{array}$ & $\begin{array}{c}\text { Total } \\
\text { Canistered } \\
\text { Assemblies } \\
\text { Shipped }\end{array}$ \\
\hline Peak & 3512 & 7102 & 9872 & 5205 & 8223 & 11940 \\
\hline Average & 2697 & 3592 & 6289 & 4383 & 6012 & 10395 \\
\hline Cumulative & 50962 & 65464 & 116426 & 155021 & 210956 & 365977 \\
\hline \multicolumn{7}{|c|}{ MTHM } \\
\hline & $\begin{array}{c}\text { PWR Bare } \\
\text { MTHM Shipped }\end{array}$ & $\begin{array}{l}\text { BWR Bare } \\
\text { MTHM } \\
\text { Shipped }\end{array}$ & $\begin{array}{l}\text { Total Bare } \\
\text { MTHM } \\
\text { Shipped }\end{array}$ & $\begin{array}{c}\text { PWR } \\
\text { Canistered } \\
\text { MTHM } \\
\text { Shipped }\end{array}$ & $\begin{array}{c}\text { BWR } \\
\text { Canistered } \\
\text { MTHM } \\
\text { Shipped }\end{array}$ & $\begin{array}{c}\text { Total } \\
\text { Canistered } \\
\text { MTHM } \\
\text { Shipped } \\
\end{array}$ \\
\hline Peak & 1533 & 1254 & 2500 & 2315 & 1451 & 3085 \\
\hline Average & 1177 & 636 & 1813 & 1922 & 1058 & 2980 \\
\hline Cumulative & 22248 & 11597 & 33845 & 67589 & 37301 & 104890 \\
\hline \multicolumn{7}{|c|}{ Canisters/Casks } \\
\hline & $\begin{array}{l}\text { PWR Bare Fuel } \\
\text { Casks Shipped }\end{array}$ & $\begin{array}{l}\text { BWR Bare Fuel } \\
\text { Casks Shipped }\end{array}$ & $\begin{array}{c}\text { Total Bare } \\
\text { Fuel Casks } \\
\text { Shipped }\end{array}$ & $\begin{array}{l}\text { PWR Canisters } \\
\text { Shipped }\end{array}$ & $\begin{array}{l}\text { BWR Canisters } \\
\text { Shipped }\end{array}$ & $\begin{array}{l}\text { Total Canisters } \\
\text { Shipped }\end{array}$ \\
\hline Peak & 146 & 112 & 258 & 179 & 126 & 252 \\
\hline Average & 107 & 63 & 170 & 149 & 92 & 241 \\
\hline Cumulative & 2020 & 1141 & 3161 & 5315 & 3232 & 8547 \\
\hline
\end{tabular}

\begin{tabular}{|c|c|c|c|c|c|c|}
\hline \multicolumn{7}{|c|}{ Major Dry Storage System Vendors } \\
\hline & $\begin{array}{l}\text { Holtec } \\
\text { Canisters }\end{array}$ & $\begin{array}{c}\text { NAC } \\
\text { Canisters }\end{array}$ & $\begin{array}{l}\text { NUHOMS } \\
\text { Canisters }\end{array}$ & $\begin{array}{l}\text { TransNuclear } \\
\text { Canisters }\end{array}$ & $\begin{array}{l}\text { Fuel Solutions } \\
\text { Canisters }\end{array}$ & $\begin{array}{c}\text { South Texas } \\
\text { Project Canisters }\end{array}$ \\
\hline Peak & 144 & 41 & 94 & 19 & 10 & 25 \\
\hline Average & 128 & 24 & 77 & 2 & NA & 7 \\
\hline Cumulative & 4393 & 836 & 2820 & 208 & 64 & 226 \\
\hline
\end{tabular}



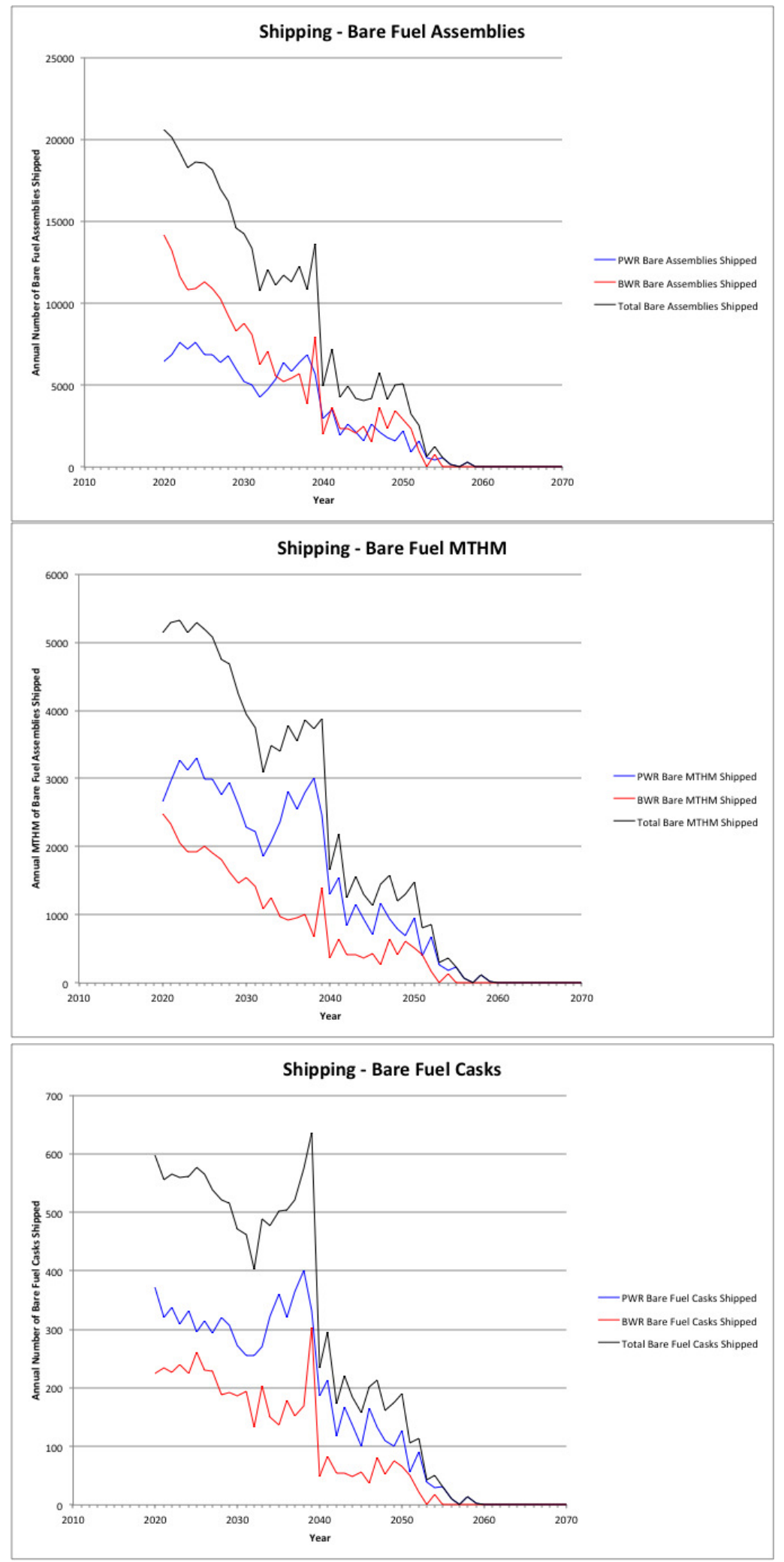

Figure A-39. Cases 2 \& 4: Annual Shipping of Bare Fuel, 6000 MTHM YFF-5 Acceptance - 2020 

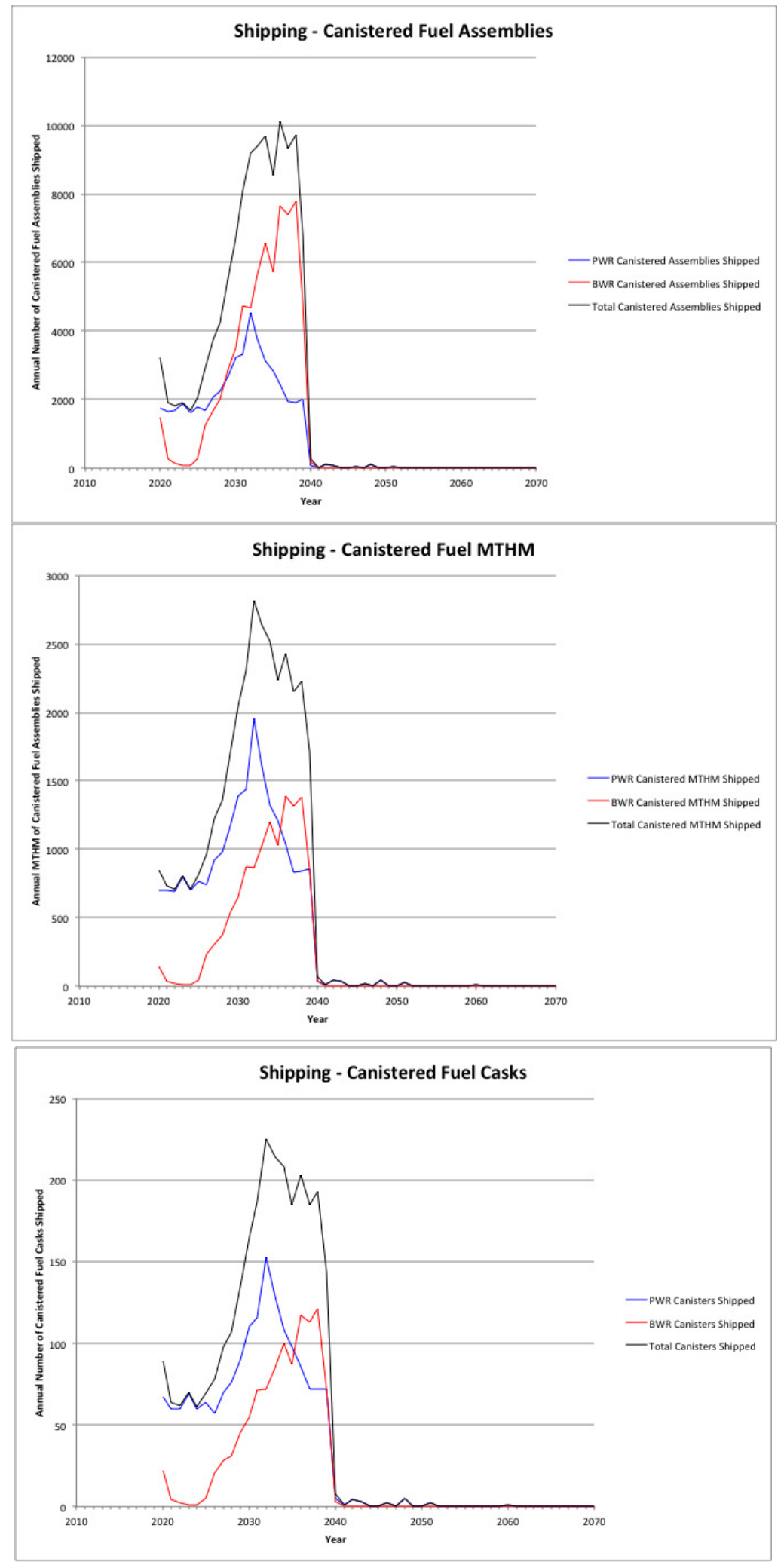

Figure A-40. Cases 2 \& 4: Annual Shipping of Canisterized Fuel, 6000 MTHM YFF-5 Acceptance 2020 


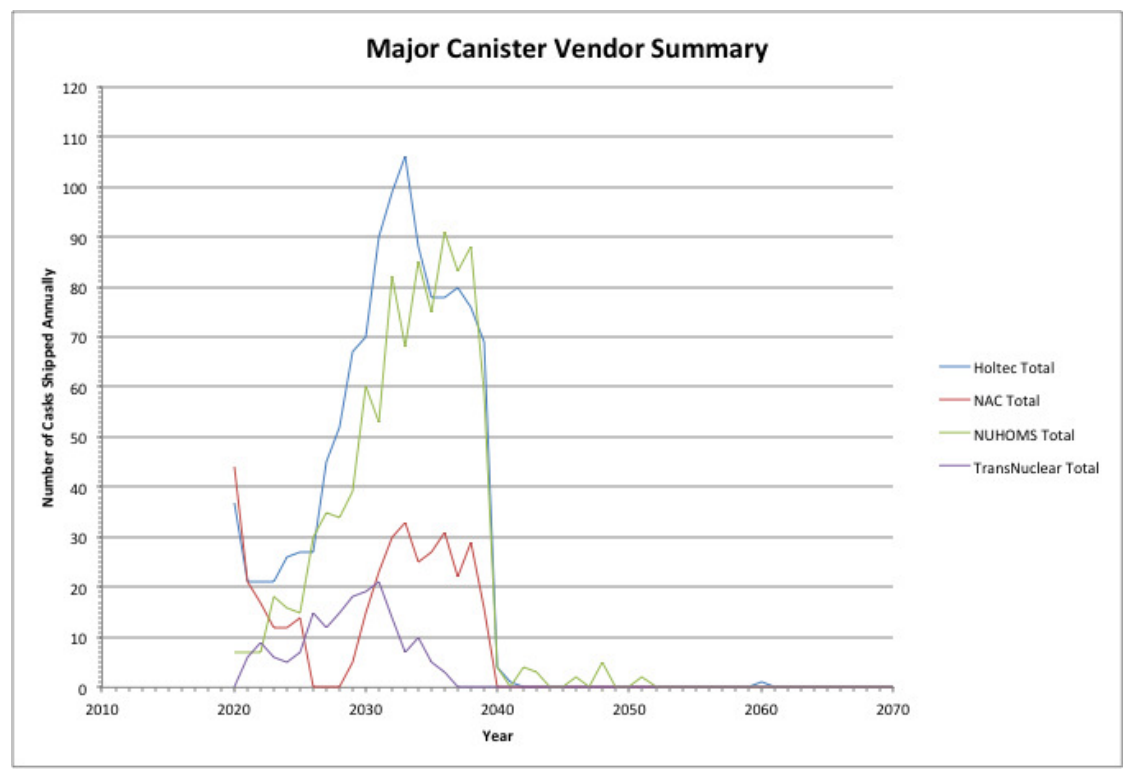

Figure A-41. Cases 2 \& 4: Annual Shipping - Major Canister Vendor Summary, 6000 MTHM YFF-5 Acceptance -2020

Table A-25. Cases 2 \& 4: Summary of Shipping from Reactor Sites, 6000 MTHM YFF-5 Acceptance 2020

\begin{tabular}{|c|c|c|c|c|c|c|}
\hline \multicolumn{7}{|c|}{ Assemblies } \\
\hline & $\begin{array}{l}\text { PWR Bare } \\
\text { Assemblies } \\
\text { Shipped }\end{array}$ & $\begin{array}{l}\text { BWR Bare } \\
\text { Assemblies } \\
\text { Shipped }\end{array}$ & $\begin{array}{c}\text { Total Bare } \\
\text { Assemblies } \\
\text { Shipped }\end{array}$ & $\begin{array}{c}\text { PWR } \\
\text { Canistered } \\
\text { Assemblies } \\
\text { Shipped }\end{array}$ & $\begin{array}{c}\text { BWR } \\
\text { Canistered } \\
\text { Assemblies } \\
\text { Shipped }\end{array}$ & $\begin{array}{c}\text { Total } \\
\text { Canistered } \\
\text { Assemblies } \\
\text { Shipped }\end{array}$ \\
\hline Peak & 7734 & 14152 & 20582 & 4528 & 7801 & 10112 \\
\hline Average & 6382 & 8738 & 15120 & 2403 & 3432 & 5835 \\
\hline Cumulative & 157443 & 207591 & 365034 & 48540 & 68829 & 117369 \\
\hline \multicolumn{7}{|c|}{ MTHM } \\
\hline & $\begin{array}{c}\text { PWR Bare } \\
\text { MTHM Shipped }\end{array}$ & $\begin{array}{l}\text { BWR Bare } \\
\text { MTHM } \\
\text { Shipped }\end{array}$ & $\begin{array}{l}\text { Total Bare } \\
\text { MTHM } \\
\text { Shipped }\end{array}$ & $\begin{array}{c}\text { PWR } \\
\text { Canistered } \\
\text { MTHM } \\
\text { Shipped }\end{array}$ & $\begin{array}{c}\text { BWR } \\
\text { Canistered } \\
\text { MTHM } \\
\text { Shipped }\end{array}$ & $\begin{array}{c}\text { Total } \\
\text { Canistered } \\
\text { MTHM } \\
\text { Shipped }\end{array}$ \\
\hline Peak & 3370 & 2485 & 5324 & 1953 & 1387 & 2814 \\
\hline Average & 2791 & 1539 & 4331 & 1032 & 614 & 1647 \\
\hline Cumulative & 68966 & 36575 & 105541 & 20872 & 12322 & 33194 \\
\hline \multicolumn{7}{|c|}{ Canisters/Casks } \\
\hline & $\begin{array}{l}\text { PWR Bare Fuel } \\
\text { Casks Shipped }\end{array}$ & $\begin{array}{l}\text { BWR Bare Fuel } \\
\text { Casks Shipped }\end{array}$ & $\begin{array}{c}\text { Total Bare } \\
\text { Fuel Casks } \\
\text { Shipped }\end{array}$ & $\begin{array}{l}\text { PWR Canisters } \\
\text { Shipped }\end{array}$ & $\begin{array}{l}\text { BWR Canisters } \\
\text { Shipped }\end{array}$ & $\begin{array}{l}\text { Total Canisters } \\
\text { Shipped }\end{array}$ \\
\hline Peak & 404 & 303 & 636 & 153 & 121 & 225 \\
\hline Average & 327 & 203 & 530 & 84 & 53 & 137 \\
\hline Cumulative & 8376 & 4800 & 13176 & 1712 & 1056 & 2768 \\
\hline
\end{tabular}

\begin{tabular}{|c|c|c|c|c|c|c|}
\hline \multicolumn{7}{|c|}{ Major Dry Storage System Vendors } \\
\hline & $\begin{array}{c}\text { Holtec } \\
\text { Canisters }\end{array}$ & $\begin{array}{c}\text { NAC } \\
\text { Canisters }\end{array}$ & $\begin{array}{l}\text { NUHOMS } \\
\text { Canisters }\end{array}$ & $\begin{array}{c}\text { TransNuclear } \\
\text { Canisters }\end{array}$ & $\begin{array}{c}\text { Fuel Solutions } \\
\text { Canisters }\end{array}$ & $\begin{array}{c}\text { South Texas } \\
\text { Project Canisters }\end{array}$ \\
\hline Peak & 106 & 44 & 91 & 21 & 13 & 0 \\
\hline Average & 60 & 17 & 50 & 9 & NA & NA \\
\hline Cumulative & 1184 & 376 & 972 & 172 & 64 & 0 \\
\hline
\end{tabular}



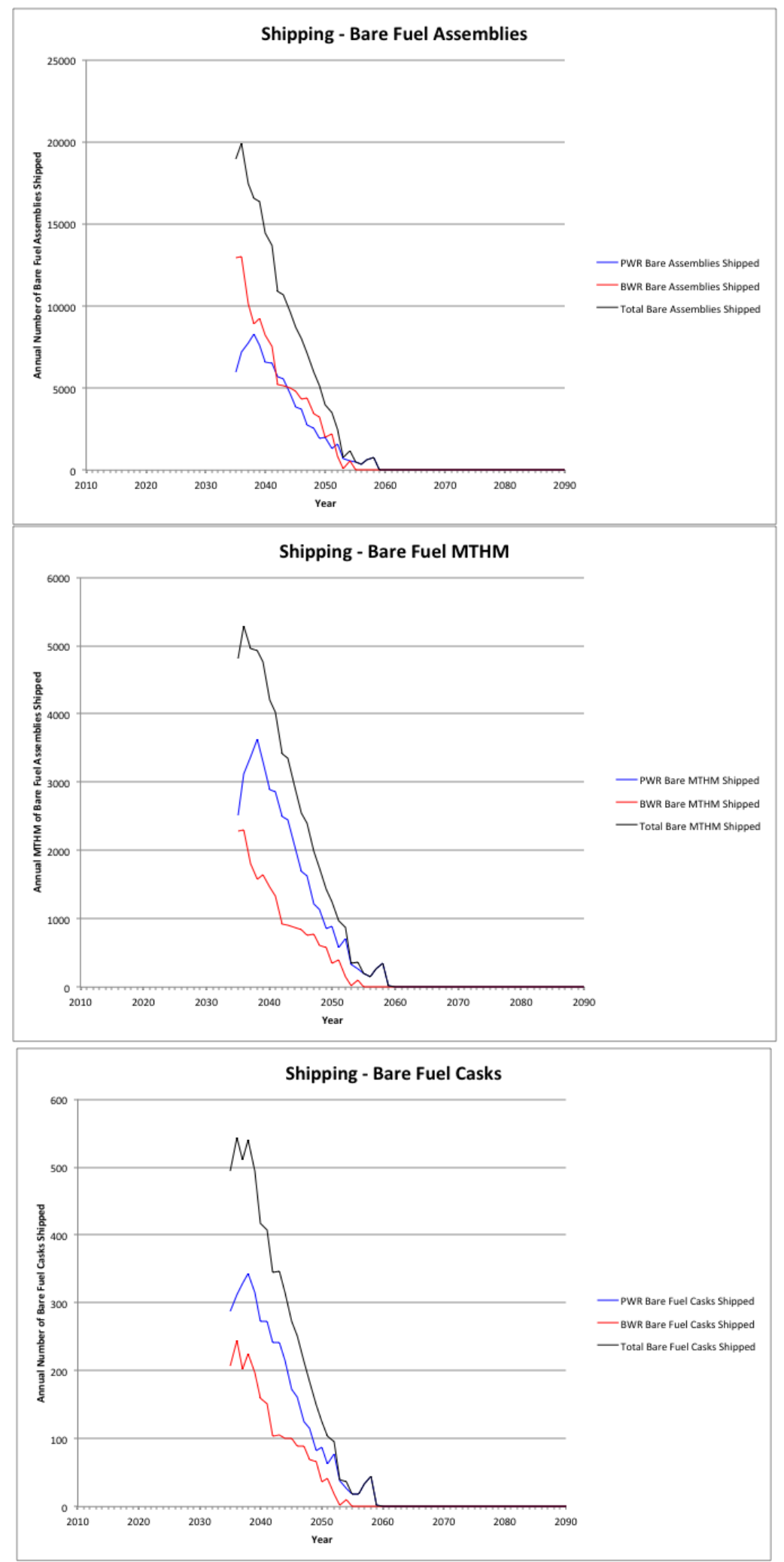

Figure A-42. Cases 2 \& 4: Annual Shipping of Bare Fuel, 6000 MTHM YFF-5 Acceptance - 2035 

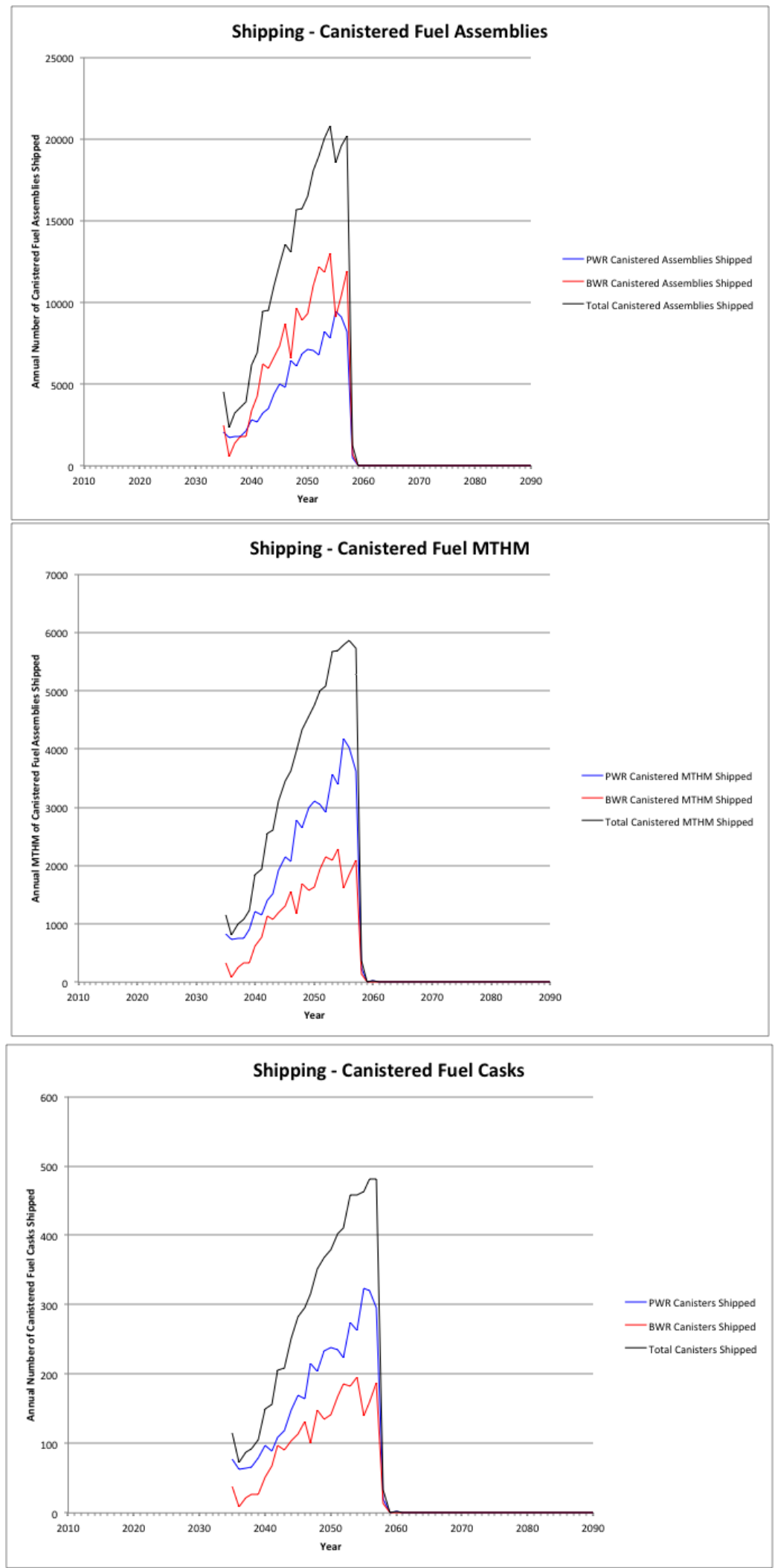

Figure A-43. Cases 2 \& 4: Annual Shipping of Canisterized Fuel, 6000 MTHM YFF-5 Acceptance 2035 


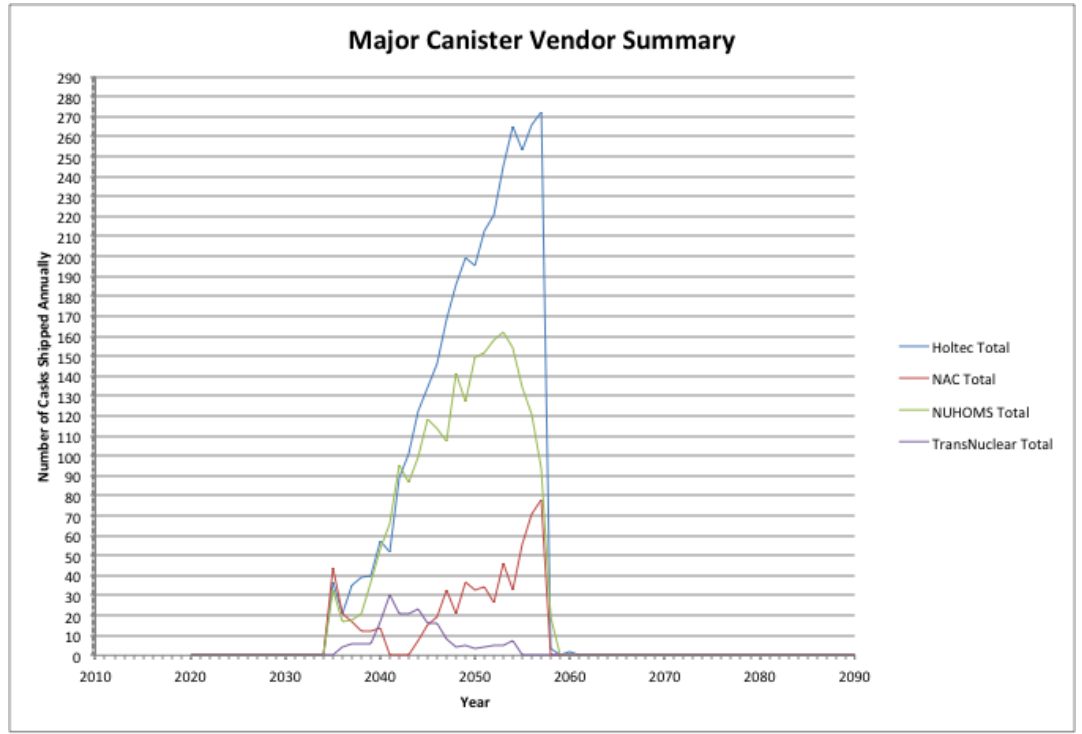

Figure A-44. Cases 2 \& 4: Annual Shipping - Major Canister Vendor Summary, 6000 MTHM YFF-5 Acceptance -2035

Table A-26. Cases 2 \& 4: Summary of Shipping from Reactor Sites, 6000 MTHM YFF-5 Acceptance 2035

\begin{tabular}{|c|c|c|c|c|c|c|}
\hline \multicolumn{7}{|c|}{ Assemblies } \\
\hline & $\begin{array}{l}\text { PWR Bare } \\
\text { Assemblies } \\
\text { Shipped }\end{array}$ & $\begin{array}{l}\text { BWR Bare } \\
\text { Assemblies } \\
\text { Shipped }\end{array}$ & $\begin{array}{l}\text { Total Bare } \\
\text { Assemblies } \\
\text { Shipped }\end{array}$ & $\begin{array}{c}\text { PWR } \\
\text { Canistered } \\
\text { Assemblies } \\
\text { Shipped } \\
\end{array}$ & $\begin{array}{c}\text { BWR } \\
\text { Canistered } \\
\text { Assemblies } \\
\text { Shipped } \\
\end{array}$ & $\begin{array}{c}\text { Total } \\
\text { Canistered } \\
\text { Assemblies } \\
\text { Shipped } \\
\end{array}$ \\
\hline Peak & 7618 & 13004 & 19880 & 9460 & 12985 & 20792 \\
\hline Average & 4596 & 6146 & 10742 & 5181 & 7149 & 12330 \\
\hline Cumulative & 8620 & 111269 & 197559 & 119693 & 165151 & 284844 \\
\hline \multicolumn{7}{|c|}{ MTHM } \\
\hline & $\begin{array}{c}\text { PWR Bare } \\
\text { MTHM Shipped }\end{array}$ & $\begin{array}{l}\text { BWR Bare } \\
\text { MTHM } \\
\text { Shipped }\end{array}$ & $\begin{array}{l}\text { Total Bare } \\
\text { MTHM } \\
\text { Shipped }\end{array}$ & $\begin{array}{c}\text { PWR } \\
\text { Canistered } \\
\text { MTHM } \\
\text { Shipped }\end{array}$ & $\begin{array}{c}\text { BWR } \\
\text { Canistered } \\
\text { MTHM } \\
\text { Shipped }\end{array}$ & $\begin{array}{c}\text { Total } \\
\text { Canistered } \\
\text { MTHM } \\
\text { Shipped }\end{array}$ \\
\hline Peak & 3341 & 2294 & 5281 & 4180 & 2288 & 5858 \\
\hline Average & 2017 & 1087 & 3104 & 2077 & 1169 & 3246 \\
\hline Cumulative & 37894 & 19676 & 57570 & 51943 & 29221 & 81165 \\
\hline \multicolumn{7}{|c|}{ Canisters/Casks } \\
\hline & $\begin{array}{l}\text { PWR Bare Fuel } \\
\text { Casks Shipped }\end{array}$ & $\begin{array}{l}\text { BWR Bare Fuel } \\
\text { Casks Shipped }\end{array}$ & $\begin{array}{c}\text { Total Bare } \\
\text { Fuel Casks } \\
\text { Shipped }\end{array}$ & $\begin{array}{l}\text { PWR Canisters } \\
\text { Shipped }\end{array}$ & $\begin{array}{l}\text { BWR Canisters } \\
\text { Shipped }\end{array}$ & $\begin{array}{l}\text { Total Canisters } \\
\text { Shipped }\end{array}$ \\
\hline Peak & 316 & 245 & 544 & 323 & 196 & 482 \\
\hline Average & 200 & 123 & 323 & 164 & 101 & 265 \\
\hline Cumulative & 3783 & 2219 & 6002 & 4094 & 2535 & 6629 \\
\hline
\end{tabular}

\begin{tabular}{|l|c|c|c|c|c|c|}
\hline \multicolumn{9}{|c|}{ Major Dry Storage System Vendors } \\
\hline & $\begin{array}{c}\text { Holtec } \\
\text { Canisters }\end{array}$ & NAC Canisters & $\begin{array}{c}\text { NUHOMS } \\
\text { Canisters }\end{array}$ & $\begin{array}{c}\text { TransNuclear } \\
\text { Canisters }\end{array}$ & $\begin{array}{c}\text { Fuel Solutions } \\
\text { Canisters }\end{array}$ & $\begin{array}{c}\text { South Texas } \\
\text { Project Canisters }\end{array}$ \\
\hline Peak & 272 & 78 & 162 & 30 & 15 & 38 \\
\hline Average & 146 & 27 & 98 & 9 & NA & NA \\
\hline
\end{tabular}




\section{A-3. Consolidated Storage Facility and Fuel Handling Logistics}

The cases and input parameters presented in Section 3 were simulated using the TSL simulation model (Nutt et al. 2012) to evaluate at reactor and fuel handling logistics. The cases considered are summarized in Table A-27 (see Section 3.1 for details regarding each case). The assumptions and input/boundary conditions are provided in Section 3.3. The shipment of UNF from the reactors, explicitly considered in the TSL simulations as discussed in Section A-2 above, has a very strong influence on the characteristics of the CSF and fuel handling facilities.

It should be noted that in Cases 2 and 4, packaging/re-packaging of the UNF at the CSF, occurs relatively soon before transportation of the UNF to the repository. As discussed in Section 3, UNF disposition scenarios in which packaging for disposal occurs at reactors or upon receipt at the CSF have been given preliminary consideration and may be evaluated in more detail later.

Table A-27. TSL Case Matrix

\begin{tabular}{|l|c|c|c|c|}
\cline { 2 - 5 } \multicolumn{1}{l|}{} & Case 1 & Case 2 & Case 3 & Case 4 \\
\hline $\begin{array}{l}\text { Transport From } \\
\text { Reactors }\end{array}$ & $\begin{array}{c}\text { Existing Size } \\
\text { Canisters }\end{array}$ & $\begin{array}{c}\text { Existing Size } \\
\text { Canisters / } \\
\text { Bare Fuel }\end{array}$ & $\begin{array}{c}\text { Existing Size } \\
\text { Canisters }\end{array}$ & $\begin{array}{c}\text { Existing Size } \\
\text { Canisters / } \\
\text { Bare Fuel }\end{array}$ \\
\hline CSF & $\begin{array}{c}\text { Existing-Size } \\
\text { Canisters }\end{array}$ & $\begin{array}{c}\text { Existing Size } \\
\text { Canisters / } \\
\text { Bare Fuel }\end{array}$ & $\begin{array}{c}\text { Existing-Size } \\
\text { Canisters }\end{array}$ & $\begin{array}{c}\text { Existing Size } \\
\text { Canisters / } \\
\text { Bare Fuel }\end{array}$ \\
\hline $\begin{array}{l}\text { Package/ } \\
\text { Re-Package at ==> }\end{array}$ & Repository & Repository & CSF & CSF \\
\hline MGR & Existing-Size & $\begin{array}{c}\text { Existing Size } \\
\text { Canisters / } \\
\text { Bare Fuel }\end{array}$ & $\begin{array}{c}\text { Package Size } \\
\text { Canisters }\end{array}$ & $\begin{array}{c}\text { Package Size } \\
\text { Canisters }\end{array}$ \\
\hline
\end{tabular}

\section{A-3.1 Summary-Level Results Of CSF and Fuel Handling}

Summary level results of CSF logistics are shown in Table A-28 and more detailed results and charts are provided in the following discussion. The capabilities of the CSF were assumed to be unconstrained. The peak annual bare fuel cask and dual-purpose cask arrivals are dictated by the rate that UNF (canisters and bare fuel) is shipped from the reactors to the CSF and establish the required processing capability of the CSF. Peak bare fuel assembly and dual-purpose canister inventories are dictated by the rates that UNF arrives at and is shipped from the CSF and the duration between the start of CSF and repository operations. These establish the required capacity for the CSF. Canisters and bare fuel assemblies counts and rates are shown primarily, rather than mass (MTHM) because it is these discrete units that dictate facility capability requirements. However, inventories are also shown in MTHM.

Summary level results of fuel handling logistics are shown in Table A-29 and more detailed results and charts are provided in the following discussion. The capabilities of the fuel handling facilities were assumed to be unconstrained. The peak annual bare fuel and dual-purpose cask packaging/re-packaging rates and quantities are dictated by the rate that UNF (canisters and bare fuel) is shipped to the repository and the fraction of UNF being handled as either bare fuel or in canisters. Peak annual bare fuel cask and dual purpose cask shipments from the reactors and/or the CSF to the repository establish the required 
throughput capability for the packaging/re-packaging operations. Canisters and bare/canisterized fuel assembly counts are shown, rather than mass (MTHM), because it is these discrete units that dictate capability requirements.

\section{A-3.2 Detailed Results of CSF Logistics}

Detailed results for each of the cases are provided in a number of figures as shown in Table A-30. These figures show time-dependent and peak arrivals and inventories at the CSF for both canistered and bare fuel.

\section{Case 1 - All Canisters, Re-Package at Repository}

The results for Case 1 are shown in Figures A-45 through A-50. Canisters arrive at the CSF until the repository starts operations (2040 or 2055), as shown in Figures A-45 and A-46. When the repository starts operations, the repository first receives UNF from the reactor fleet until such time that there is no longer UNF available. UNF is then shipped from the CSF to the repository. As shown in Figure A-44, the CSF canister inventory increases until the repository begins operation, then decreases once the repository begins to receive UNF from the CSF.

The peak annual arrival rate of all canisters, shown in Figure A-47 (based on the results shown in Figure A-45), increases for higher UNF acceptance rates, but does not depend on when the CSF starts operation and the period between CSF and repository operations. The total annual arrival rate of all canisters is dominated by the arrival of vertical canisters, which represents the majority of canisters that arrive at the CSF.

The peak annual arrival rate of horizontal canisters at the CSF is larger when the repository start time is delayed until 2050, as shown in Figure A-47. This is because the arrival rate of horizontal canisters to the CSF increases after 2040 (as can be seen in Figure A-45), rather than those canisters being sent to the repository. This is a direct effect of the assumed YFF shipment of fuel from the reactors as shown in Figure A-20 and discussed in Section A-2.3 which gives preference to vertical dry storage systems being shipped earlier. Since the fraction of vertical canisters increases over time, peaking around 2055, the youngest fuel stored in canisters at the reactor sites will tend to be in vertical canisters.

The peak inventory of canisters in dry storage, shown in Figures A-49 and A-50, increases for:

- Higher UNF acceptance rates,

- Longer period between the start of CSF and repository operations, and

- Later start of CSF operations.

\section{Case 2 - Canisters and Bare Fuel, Re-Package at Repository}

The results for Case 2 are shown in Figures A-51 through A-62. Canisters and bare fuel casks arrive at the CSF until the repository starts operations (2040 or 2055), as shown in Figures A-51 and A-52. Again, when the repository starts operations, the repository first receives UNF from the reactor fleet until such time that there is no longer UNF available. UNF is then received from the CSF. As shown in Figures A54 and A-60, the CSF canister and bare fuel inventory increases until the repository begins operation, then decreases once the repository begins to receive UNF from the CSF.

The peak annual arrival rate of bare fuel casks, shown in Figure A-59 (derived from the peaks shown in Figure B-51), increases for higher UNF acceptance rates, but does not depend on the period between the start of CSF and repository operations when the CSF begins operation in 2020. This is because the highest arrival rate occurs prior to 2040 as can be seen in Figure A-55. Figure A-59 also shows that for $3000 \mathrm{MTHM} / \mathrm{yr}$ delaying the start of CSF operations until 2035 reduces the peak annual bare cask arrival rate because more UNF is placed into canisters at the reactor sites to maintain available used fuel pool capacity. 
Table A-28. Consolidated Storage Facility Logistics Summary Results

\begin{tabular}{|c|c|c|c|c|c|c|c|c|c|c|c|c|c|c|c|c|c|}
\hline \multirow[b]{3}{*}{ Scenario } & \multirow[b]{3}{*}{$\begin{array}{l}\text { Acceptance } \\
\text { Rate }\end{array}$} & \multirow[b]{3}{*}{$\begin{array}{l}\text { CSF } \\
\text { Start }\end{array}$} & \multirow[b]{3}{*}{$\begin{array}{l}\text { Repository } \\
\text { Start }\end{array}$} & \multicolumn{6}{|c|}{ Peak Annual Arrivals } & \multicolumn{8}{|c|}{ Peak Inventory in Storage } \\
\hline & & & & \multicolumn{3}{|c|}{ Bare Fuel Casks } & \multicolumn{3}{|c|}{ Canisters } & \multicolumn{2}{|c|}{$\begin{array}{c}\text { Bare Fuel } \\
\text { Assemblies }\end{array}$} & \multicolumn{2}{|c|}{ Vertical Canisters } & \multicolumn{2}{|c|}{ Horizontal Canisters } & \multicolumn{2}{|c|}{ Max MTHM } \\
\hline & & & & PWR & BWR & Total & $\begin{array}{c}\text { Horizontal } \\
\text { Total }\end{array}$ & $\begin{array}{c}\text { Vertical } \\
\text { Total }\end{array}$ & Total & PWR & BWR & 2-Year & Max & 2-Year & Max & Canisters & Bare \\
\hline \multirow{7}{*}{$\begin{array}{l}\text { Canisters Only - } \\
\text { Re-Package at } \\
\text { Repository }\end{array}$} & 1500 & 2020 & 2040 & & & & 27 & 142 & 147 & & & 259 & 2155 & 13 & 325 & 30377 & \\
\hline & 3000 & 2020 & 2040 & & & & 61 & 257 & 269 & & & 480 & 3981 & 45 & 825 & 59731 & \\
\hline & 6000 & 2020 & 2040 & & & & 156 & 476 & 520 & & & 900 & 5349 & 108 & 1581 & 86031 & \\
\hline & 1500 & 2020 & 2055 & & & & 50 & 142 & 147 & & & 259 & 3471 & 13 & 791 & 52824 & \\
\hline & 3000 & 2020 & 2055 & & & & 85 & 257 & 269 & & & 480 & 6506 & 45 & 1839 & 104054 & \\
\hline & 6000 & 2020 & 2055 & & & & 162 & 476 & 520 & & & 900 & 8241 & 108 & 2347 & 132300 & \\
\hline & 3000 & 2035 & 2055 & & & & 81 & 236 & 265 & & & 448 & 3696 & 71 & 1150 & 60081 & \\
\hline \multirow{7}{*}{$\begin{array}{l}\text { Canisters and } \\
\text { Bare Fuel - } \\
\text { Re-Package at } \\
\text { Repository }\end{array}$} & 1500 & 2020 & 2040 & 119 & 63 & 182 & 9 & 31 & 32 & 38746 & 60144 & 47 & 238 & 2 & 43 & 3010 & 27324 \\
\hline & 3000 & 2020 & 2040 & 202 & 133 & 325 & 11 & 47 & 49 & 79722 & 116490 & 84 & 346 & 7 & 91 & 4865 & 55216 \\
\hline & 6000 & 2020 & 2040 & 404 & 300 & 634 & 91 & 146 & 225 & 127651 & 174714 & 139 & 1790 & 14 & 953 & 32940 & 86605 \\
\hline & 1500 & 2020 & 2055 & 119 & 63 & 182 & 42 & 75 & 112 & 55862 & 77529 & 47 & 859 & 2 & 390 & 14834 & 37959 \\
\hline & 3000 & 2020 & 2055 & 202 & 133 & 325 & 80 & 143 & 215 & 112974 & 151895 & 84 & 1555 & 7 & 844 & 28925 & 76096 \\
\hline & 6000 & 2020 & 2055 & 404 & 300 & 634 & 91 & 146 & 225 & 156276 & 207289 & 139 & 1828 & 14 & 980 & 33280 & 104982 \\
\hline & 3000 & 2035 & 2055 & 146 & 112 & 258 & 79 & 139 & 218 & 49954 & 65464 & 84 & 1411 & 33 & 798 & 26671 & 33410 \\
\hline \multirow{7}{*}{$\begin{array}{l}\text { Canisters Only - } \\
\text { Re-Package at } \\
\text { CSF }\end{array}$} & 1500 & 2020 & 2040 & & & & 55 & 142 & 147 & & & 259 & 2155 & 13 & 835 & 30377 & \\
\hline & 3000 & 2020 & 2040 & & & & 87 & 262 & 348 & & & 480 & 3981 & 45 & 1430 & 59979 & \\
\hline & 6000 & 2020 & 2040 & & & & 162 & 476 & 520 & & & 900 & 5349 & 108 & 1713 & 86031 & \\
\hline & 1500 & 2020 & 2055 & & & & 55 & 142 & 147 & & & 259 & 3471 & 13 & 1459 & 52839 & \\
\hline & 3000 & 2020 & 2055 & & & & 87 & 262 & 348 & & & 480 & 6512 & 45 & 2213 & 105073 & \\
\hline & 6000 & 2020 & 2055 & & & & 162 & 476 & 520 & & & 900 & 8241 & 108 & 2407 & 132300 & \\
\hline & 3000 & 2035 & 2055 & & & & 98 & 236 & 265 & & & 448 & 3696 & 71 & 1652 & 60109 & \\
\hline \multirow{7}{*}{$\begin{array}{l}\text { Canisters and } \\
\text { Bare Fuel - Re- } \\
\text { Package at CSF }\end{array}$} & 1500 & 2020 & 2040 & 119 & 63 & 182 & 58 & 104 & 130 & 39280 & 60144 & 477 & 1721 & 2 & 839 & 30131 & 27324 \\
\hline & 3000 & 2020 & 2040 & 202 & 133 & 325 & 88 & 202 & 246 & 80044 & 116490 & 84 & 2800 & 7 & 1313 & 50451 & 55216 \\
\hline & 6000 & 2020 & 2040 & 404 & 300 & 634 & 91 & 146 & 225 & 127651 & 174714 & 139 & 1790 & 14 & 953 & 32940 & 86605 \\
\hline & 1500 & 2020 & 2055 & 119 & 63 & 182 & 58 & 104 & 130 & 55862 & 77529 & 47 & 2934 & 2 & 1459 & 52576 & 37959 \\
\hline & 3000 & 2020 & 2055 & 202 & 133 & 325 & 88 & 202 & 246 & 112974 & 151895 & 84 & 3203 & 7 & 1594 & 58845 & 76096 \\
\hline & 6000 & 2020 & 2055 & 404 & 300 & 634 & 91 & 146 & 225 & 156276 & 207289 & 139 & 1828 & 14 & 980 & 33280 & 104982 \\
\hline & 3000 & 2035 & 2055 & 146 & 112 & 258 & 94 & 219 & 252 & 49954 & 65464 & 84 & 3350 & 33 & 1655 & 59926 & 33410 \\
\hline
\end{tabular}

1. Peak BWR and PRW bare fuel cask arrivals do not necessarily sum to the total bare cask arrivals. The peaks do not necessarily occur on the same year.

2. Peak vertical and horizontal canister arrivals do not necessarily sum to the total canister arrivals. The peaks do not necessarily occur on the same year.

3. 2-Year UNF inventories are provided as these establish the storage capacity required for initial construction of the CSF (assumed part of the initial procurement and construction). 
Table A-29. Fuel Handling Logistics Summary Results

\begin{tabular}{|c|c|c|c|c|c|c|c|c|c|c|c|c|c|c|c|c|c|c|c|c|c|c|c|c|c|c|c|c|c|c|c|c|c|c|c|}
\hline \multirow[b]{3}{*}{ scenario } & \multirow[b]{3}{*}{$\begin{array}{c}\text { Acceptance } \\
\text { Rate }\end{array}$} & \multirow[b]{3}{*}{$\begin{array}{c}\text { ccs } \\
\text { start }\end{array}$} & \multirow[b]{3}{*}{$\begin{array}{l}\text { Repository } \\
\text { Start }\end{array}$} & \multirow[b]{3}{*}{$\begin{array}{l}\text { Disposal } \\
\text { canister } \\
\text { size }\end{array}$} & \multicolumn{6}{|c|}{ Peak Bare fuel Packaging } & \multicolumn{4}{|c|}{ Cumulative Bare Fuel Packaging } & \multicolumn{9}{|c|}{ Peak Canistered fuel Re-Packaging } & \multicolumn{7}{|c|}{ Cumulative Canistered fuel Re-Packaging } & \multicolumn{5}{|c|}{ Disposal Canister Generation } \\
\hline & & & & & \multicolumn{3}{|c|}{ Assemblies } & \multicolumn{3}{|c|}{ мтнм } & Assem & & $\mathrm{MT}$ & & & sters to $\mathrm{C}$ & & Assen & & & & МтнМ & & & isters toc & & Assem & & Mт & & PeakAr & & & cumulative & \\
\hline & & & & & PWR & BWR & Total & PWR & Bwi & Total & PWR & BWR & PWR & BWR & PWR & BWR & Total & PWR & BWR & Total & PWR & BWR & Total & PWR & BWR & Total & PWR & BWR & PWR & BWR & PWR & BWR & PWR & BWR & Total \\
\hline & 1500 & 2020 & 2040 & 4 & & & & & & & & & & & 100 & 64 & 132 & 2879 & 4071 & 6145 & 1262 & 720 & 1647 & 6998 & 4210 & 11208 & 205983 & 276420 & 89838 & 48897 & 719 & 453 & 51496 & 30714 & 82216 \\
\hline & 3000 & 2020 & 2040 & 4 & & & & & & & & & & & 236 & 123 & 348 & 5899 & 8060 & 13170 & 2737 & 1420 & 4022 & 6974 & 4190 & 11164 & 205983 & 276420 & 89838 & 48897 & 1474 & 896 & 51496 & 30714 & 82216 \\
\hline & 3000 & 2020 & 2040 & 12 & & & & & & & & & & & 236 & 123 & 348 & 5899 & 8060 & 13170 & 2737 & 1420 & 4022 & 6974 & 4190 & 11164 & 205983 & 276420 & 8938 & 48897 & 491 & 336 & 17166 & 11518 & 2868. \\
\hline & 3000 & 2020 & 2040 & 21 & & & & & & & & & & & 236 & 123 & 348 & 5899 & 8060 & 13170 & 2737 & 1420 & 4022 & 6974 & 4190 & 11164 & 205983 & 276420 & 89838 & 48897 & 281 & 183 & 9809 & 6283 & 1609: \\
\hline $\begin{array}{l}\text { Caninters Only- } \\
\text { Re-Package at Repositor }\end{array}$ & 6000 & 2020 & 2040 & 4 & & & & & & & & & & & 381 & 270 & 543 & 11300 & 17998 & 24904 & 4990 & 3141 & 6065 & 6964 & 4183 & 11147 & 205983 & 276420 & 89838 & 48897 & 2825 & 2000 & 51496 & 30714 & 82216 \\
\hline & 1500 & 2020 & 2055 & 4 & & & & & & & & & & & 100 & 64 & 132 & 2879 & 4071 & 6145 & 1262 & 720 & 1647 & 6998 & 4210 & 11208 & 205983 & 276420 & 89838 & 48897 & 720 & 452 & 51496 & 30714 & 82216 \\
\hline & 3000 & 2020 & 2055 & 4 & & & & & & & & & & & 236 & 123 & 348 & 5899 & 8060 & 13170 & 2737 & 1420 & 4022 & 6974 & 4190 & 11164 & 205983 & 276420 & 89838 & 48897 & 1474 & 896 & 51496 & 30714 & 82216 \\
\hline & 6000 & 2020 & 2055 & 4 & & & & & & & & & & & 410 & 274 & 546 & 12329 & 18022 & 24503 & 5394 & 3189 & 6063 & 6964 & 4183 & 11147 & 205983 & 276420 & 89838 & 48897 & 3082 & 2003 & 51496 & 30714 & 82216 \\
\hline & 3000 & 2035 & 2055 & 4 & & & & & & & & & & & 181 & 123 & 257 & 5261 & 8056 & 11756 & 2340 & 1421 & 3120 & 7001 & 4216 & 11217 & 205983 & 276420 & 89838 & 48897 & 1315 & 896 & 51996 & 30714 & 82216 \\
\hline & 1500 & 2020 & 2040 & 4 & 2306 & 3604 & 5236 & 1009 & 630 & 1471 & 56366 & 77529 & 24493 & 13686 & 98 & 62 & 130 & 2882 & 4029 & 6044 & 1264 & 715 & 1614 & 5145 & 3051 & 8196 & 199617 & 198891 & 65334 & 35212 & 720 & 455 & 51496 & 30714 & 82216 \\
\hline & 3000 & 2020 & 2040 & 4 & 4498 & 7208 & 10930 & 1970 & 1278 & 2935 & 114014 & 151895 & 49762 & 26782 & 179 & ${ }^{124}$ & 246 & 5194 & 8084 & 11658 & 2322 & 1425 & 3069 & 3190 & 1916 & 5106 & 91969 & 124525 & 40075 & 22116 & \begin{tabular}{|l|l|} 
& 1298 \\
\end{tabular} & 898 & 51496 & 30714 & 82216 \\
\hline & 3000 & 2020 & 2040 & 12 & 4498 & 7208 & 10930 & 1970 & 1278 & 2935 & 114014 & 151895 & 49762 & 26782 & 179 & 124 & 246 & 5194 & 8084 & 11658 & 2322 & 1425 & 3069 & 3190 & 1916 & 5106 & 91969 & 124525 & 40075 & 22116 & 432 & 337 & 17166 & 1518 & 2868. \\
\hline & 3000 & 2020 & 2040 & 21 & 4498 & 7208 & 10930 & 1970 & \begin{tabular}{|l|l|}
1278 \\
\end{tabular} & 2935 & \begin{tabular}{|l|l|}
140014 \\
\end{tabular} & 151895 & 49762 & 26782 & 179 & 124 & 246 & 5194 & 8084 & 11658 & 2322 & 1425 & 3069 & 3190 & 1916 & 5106 & 91969 & 124525 & 40075 & 22116 & 248 & 183 & 9809 & 6283 & 16092 \\
\hline 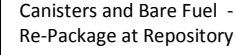 & 6000 & 2020 & 2040 & 4 & 9530 & 15592 & 29958 & 4162 & 2750 & 6011 & 157322 & 207289 & 68915 & 36522 & 139 & 118 & 216 & 4057 & 7642 & 9725 & 1752 & 1356 & 2670 & 1739 & 1073 & 2812 & 48661 & 69131 & 20923 & 12375 & 2768 & 1772 & 51496 & 30714 & 82216 \\
\hline & 1500 & 2020 & 2055 & 4 & 2306 & 3604 & 5236 & 1009 & 630 & 1471 & 56366 & 77529 & 24993 & 13686 & 98 & 62 & 130 & 2882 & 4029 & 6044 & 1264 & 715 & 1614 & 5145 & 3051 & 8196 & 149617 & 198891 & 65344 & 35212 & 721 & 456 & 51496 & 30714 & 82211 \\
\hline & 3000 & 2020 & 2055 & 4 & 4498 & 7208 & 10930 & 1970 & 1278 & 2935 & 114014 & 151895 & 49762 & 26782 & 179 & 124 & 246 & 5194 & 8084 & 11658 & 2322 & 1425 & 3069 & 3190 & 1916 & 5106 & 91969 & 124525 & 40075 & 22116 & 1298 & 898 & 51496 & 30714 & 82211 \\
\hline & 6000 & 2020 & 2055 & 4 & 9690 & 14144 & 21080 & 4270 & 2484 & 6023 & 157322 & 207289 & 68915 & 36522 & 153 & 121 & 225 & 4528 & 7801 & 10112 & 1953 & 1387 & 2814 & 1739 & 1073 & 2812 & 48661 & 69131 & 20923 & 12375 & 2439 & 1644 & 51496 & 30714 & 82210 \\
\hline & 3000 & 2035 & 2055 & 4 & 3520 & 6324 & 9076 & 1537 & \begin{tabular}{|l|}
1120 \\
\end{tabular} & 2472 & 50962 & 65464 & 22248 & \begin{tabular}{|l|l}
11597 \\
\end{tabular} & 179 & 126 & 252 & 5205 & 8223 & 11940 & 2315 & 1451 & 3085 & 5315 & 3232 & 8547 & 155021 & 210956 & \begin{tabular}{|l|l|}
67589 \\
\end{tabular} & 37301 & \begin{tabular}{|l|}
1302 \\
\end{tabular} & 914 & 51496 & 30714 & 82210 \\
\hline & 1500 & 2020 & 2040 & 4 & & & & & & & & & & & $\begin{array}{ll}95 \\
\end{array}$ & 73 & 142 & 2852 & 4743 & 6667 & 1232 & 785 & 1514 & 6998 & 4210 & 11208 & 205983 & 276420 & \begin{tabular}{|l|l|}
8938 \\
\end{tabular} & \begin{tabular}{|l|l|}
48897 \\
\end{tabular} & 713 & 527 & 51496 & 30714 & \begin{tabular}{|l|l|}
8221 \\
\end{tabular} \\
\hline & 3000 & 2020 & 2040 & 4 & & & & & & & & & & & 182 & 132 & 268 & 5081 & 8768 & 12670 & 2263 & 1458 & 3015 & 6974 & 4190 & 11164 & 205983 & 276420 & 89938 & 48897 & \begin{tabular}{|l}
1271 \\
\end{tabular} & 974 & 51496 & 30714 & 82216 \\
\hline & 3000 & 2020 & 2040 & 12 & & & & & & & & & & & 182 & 132 & 268 & 5081 & 8768 & 12660 & 2263 & 1458 & 3017 & 6974 & 4190 & 11164 & 205983 & 276420 & \begin{tabular}{|l}
8938 \\
\end{tabular} & 48897 & 424 & 365 & 17166 & 1518 & 2868. \\
\hline & 3000 & 2020 & 2040 & 21 & & & & & & & & & & & 182 & 132 & 268 & 5081 & 8768 & 12662 & 2263 & 1458 & 3019 & 6974 & 4190 & 11164 & 205983 & 276420 & 89838 & 48897 & 242 & 199 & 9809 & 6283 & 16092 \\
\hline 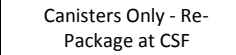 & 6000 & 2020 & 2040 & 4 & & & & & & & & & & & 426 & 274 & 530 & 12234 & 18022 & 24338 & 5297 & \begin{tabular}{|l|}
3189 \\
\end{tabular} & 6015 & 6964 & 4183 & 11147 & 205983 & 276420 & 89938 & 48897 & \begin{tabular}{|l|l}
3059 \\
\end{tabular} & 2002 & 51496 & 30714 & 82216 \\
\hline & 1500 & 2020 & 2055 & 4 & & & & & & & & & & & 95 & 73 & 142 & 2852 & 4743 & 6667 & 1232 & 785 & 1514 & 6998 & 4210 & 11208 & 205983 & 276420 & 89838 & 48897 & 713 & 527 & 514966 & 30714 & 82216 \\
\hline & 3000 & 2020 & 2055 & 4 & & & & & & & & & & & 182 & 132 & 268 & 5081 & 8768 & 12670 & 2263 & 1458 & 3015 & 6974 & 41900 & 11164 & 205983 & 276420 & 89838 & 48897 & 1271 & 974 & 51496 & 30714 & 82216 \\
\hline & 6000 & 2020 & 2055 & 4 & & & & & & & & & & & 426 & 274 & 530 & 12234 & 18022 & 24338 & 5297 & 3189 & 6015 & 6964 & 4183 & 11147 & 205983 & 276420 & 89838 & 48897 & 3059 & 2002 & 51496 & 30714 & 82216 \\
\hline & 3000 & 2035 & 2055 & 4 & & & & & & & & & & & 175 & 131 & 265 & 5077 & 8623 & 12486 & 2257 & 1454 & 3014 & 7001 & 4216 & 11217 & 205983 & 276420 & 89838 & 48897 & 1270 & 958 & 514966 & 30714 & 82216 \\
\hline & 1500 & 2020 & 2040 & 4 & 2296 & 4105 & 5587 & 1011 & \begin{tabular}{|l|l|}
726 \\
\end{tabular} & 1463 & \begin{tabular}{|l|l|}
56366 \\
\end{tabular} & 77529 & 24502 & 13697 & 94 & 68 & 127 & 2792 & 4351 & 6476 & 1212 & 765 & 1514 & \begin{tabular}{l|l|}
5145 \\
\end{tabular} & 3051 & 8196 & 149617 & \begin{tabular}{|l|l|}
198891 \\
\end{tabular} & \begin{tabular}{|l|l|}
653344 \\
\end{tabular} & 35212 & \begin{tabular}{|l|l|}
698 \\
\end{tabular} & 500 & 51496 & 30714 & 82211 \\
\hline & 3000 & 2020 & 2040 & 4 & 4498 & 7879 & 10881 & 1970 & \begin{tabular}{|l|l|}
1383 \\
\end{tabular} & 2929 & \begin{tabular}{|l|l|l|l|} 
\\
\end{tabular} & 151895 & 49773 & \begin{tabular}{|l|l|}
26794 \\
\end{tabular} & 174 & 124 & 248 & 5042 & 8084 & 12651 & 2253 & 1425 & 3012 & 3190 & 1916 & 5106 & 91969 & 124525 & 40075 & 22116 & 1260 & 975 & 51496 & 30714 & \begin{tabular}{|l|l|}
8221 \\
\end{tabular} \\
\hline & 3000 & 2020 & 2040 & 12 & 4498 & 7879 & 10881 & 1970 & \begin{tabular}{|l|l|}
1383 \\
\end{tabular} & 2929 & 114014 & 151895 & 49798 & 26812 & 174 & 124 & 248 & 5042 & 8084 & 12636 & 2253 & 1425 & 3013 & 3190 & 1916 & 5106 & 91969 & 124525 & 40075 & 22116 & 420 & 365 & 17166 & 11518 & 2868. \\
\hline & 3000 & 2020 & 2040 & 21 & 4498 & 7879 & 10881 & 1970 & \begin{tabular}{|l|l|}
1383 \\
\end{tabular} & 2929 & \begin{tabular}{|l|l|}
114014 \\
\end{tabular} & 151895 & 49850 & 26856 & 174 & 124 & 248 & 5050 & 8084 & 12620 & 2258 & \begin{tabular}{l|l|}
1425 \\
\end{tabular} & 3019 & 3190 & 1916 & 5106 & 91969 & 124525 & 40075 & 22116 & 241 & 199 & 9809 & 6283 & 16092 \\
\hline $\begin{array}{c}\text { Canisters and dare fuel. } \\
\text { Re-Package at CSF }\end{array}$ & 6000 & 2020 & 2040 & ${ }^{4}$ & 9859 & 14152 & 20601 & 4328 & 2486 & 5934 & 157322 & 207289 & 68929 & 36538 & 153 & 121 & 225 & 4528 & 7801 & 23828 & 1953 & 1387 & 6011 & 1739 & 1073 & 2812 & 48661 & 699131 & 20923 & 12375 & 2508 & 1736 & 51496 & 30714 & 8221 \\
\hline & 1500 & 2020 & 2055 & 4 & 2296 & 4105 & 5587 & 1011 & 726 & 1463 & 563666 & 77529 & 24995 & 13687 & 94 & 67 & 128 & 2810 & 4297 & 6476 & 1215 & 755 & 1515 & 5145 & 3051 & 8196 & 149617 & 198891 & 655344 & 35212 & 703 & 500 & 51496 & \begin{tabular}{|l|l|l|}
30714 \\
\end{tabular} & 82211 \\
\hline & 3000 & 2020 & 2055 & 4 & 4498 & 7879 & 10881 & 1970 & \begin{tabular}{|l|l|}
1383 \\
\end{tabular} & 2929 & \begin{tabular}{|l|l|}
114014 \\
\end{tabular} & 151895 & 49763 & 26783 & 174 & 124 & 248 & 5042 & 8084 & 12651 & 2253 & 1425 & 3011 & 3190 & 1916 & 5106 & 91969 & 124525 & 40075 & 22116 & 1260 & 975 & 51496 & 30714 & 8221 \\
\hline & 6000 & 2020 & 2055 & 4 & 9852 & 14152 & 20601 & 4325 & \begin{tabular}{|l|l|}
2486 \\
\end{tabular} & 5934 & 157322 & 207289 & 68920 & 36525 & 153 & 121 & 225 & 4528 & 7801 & 23828 & 1953 & 1387 & 6013 & 1739 & 1073 & 2812 & 48661 & 69131 & 20923 & 12375 & 2506 & 1736 & 51496 & 30714 & 8221 \\
\hline & 3000 & 2035 & 2055 & 4 & 3512 & 7102 & 9872 & 1533 & \begin{tabular}{|l|}
1254 \\
\end{tabular} & \begin{tabular}{|l|l|}
2500 \\
\end{tabular} & \begin{tabular}{|l|l|}
50962 \\
\end{tabular} & 65464 & \begin{tabular}{|l|l}
22249 \\
\end{tabular} & \begin{tabular}{|l|l|l}
1599 \\
\end{tabular} & 173 & 119 & 249 & 5021 & 7810 & 12440 & 2234 & 1375 & 3013 & \begin{tabular}{|l}
5315 \\
\end{tabular} & 3232 & 8547 & 155021 & 210956 & \begin{tabular}{|l}
67589 \\
\end{tabular} & 37301 & \begin{tabular}{|l}
1255 \\
\end{tabular} & 956 & 51496 & 30714 & \begin{tabular}{|l|l|}
8221 \\
\end{tabular} \\
\hline
\end{tabular}


Table A-30. CSF Logistic Results Figure Matrix

\begin{tabular}{|c|c|c|c|c|c|c|c|c|c|c|}
\hline & $\begin{array}{l}\text { Annual } \\
\text { Canister } \\
\text { Arrivals } \\
\text { at CSF }\end{array}$ & $\begin{array}{c}\text { Cumulative } \\
\text { Canister } \\
\text { Arrivals at } \\
\text { CSF }\end{array}$ & $\begin{array}{l}\text { Peak } \\
\text { Annual } \\
\text { Canister } \\
\text { Arrivals } \\
\text { at CSF }\end{array}$ & $\begin{array}{c}\text { CSF } \\
\text { Canister } \\
\text { Inventory }\end{array}$ & $\begin{array}{l}\text { Peak } \\
\text { CSF } \\
\text { Canister } \\
\text { Inventory }\end{array}$ & $\begin{array}{l}\text { Annual } \\
\text { Bare Fuel } \\
\text { Cask } \\
\text { Arrivals at } \\
\text { CSF }\end{array}$ & $\begin{array}{c}\text { Cumulative } \\
\text { Bare Fuel } \\
\text { Cask } \\
\text { Arrivals at } \\
\text { CSF }\end{array}$ & $\begin{array}{l}\text { Peak Annual } \\
\text { Bare Fuel } \\
\text { Cask } \\
\text { Arrivals at } \\
\text { CSF }\end{array}$ & $\begin{array}{c}\text { CSF } \\
\text { Bare } \\
\text { Fuel } \\
\text { Inventory }\end{array}$ & $\begin{array}{l}\text { Peak CSF } \\
\text { Bare Fuel } \\
\text { Inventory }\end{array}$ \\
\hline $\begin{array}{l}\text { Case } 1 \text { (all } \\
\text { canisters, re- } \\
\text { package at } \\
\text { repository) }\end{array}$ & $\begin{array}{c}\text { Figure } \\
\text { A-45 }\end{array}$ & $\begin{array}{c}\text { Figure } \\
\text { A-46 }\end{array}$ & $\begin{array}{c}\text { Figure } \\
\text { A-47 }\end{array}$ & $\begin{array}{c}\text { Figure } \\
\text { A-48 }\end{array}$ & $\begin{array}{c}\text { Figure } \\
\text { A-49 } \\
\\
\text { Figure } \\
\text { A-50 } \\
\end{array}$ & & & & & \\
\hline $\begin{array}{l}\text { Case } 2 \text { (canisters } \\
\text { and bare fuel, re- } \\
\text { package at } \\
\text { repository) }\end{array}$ & $\begin{array}{c}\text { Figure } \\
\text { A-51 }\end{array}$ & $\begin{array}{c}\text { Figure } \\
\text { A-52 }\end{array}$ & $\begin{array}{c}\text { Figure } \\
\text { A-53 }\end{array}$ & $\begin{array}{c}\text { Figure } \\
\text { A-54 }\end{array}$ & $\begin{array}{l}\text { Figure } \\
\text { A-55 } \\
\text { Figure } \\
\text { A-56 }\end{array}$ & $\begin{array}{c}\text { Figure } \\
\text { A-57 }\end{array}$ & $\begin{array}{c}\text { Figure } \\
\text { A-58 }\end{array}$ & $\begin{array}{c}\text { Figure } \\
\text { A-59 }\end{array}$ & $\begin{array}{c}\text { Figure } \\
\text { A-60 }\end{array}$ & $\begin{array}{c}\text { Figure } \\
\text { A-61 } \\
\text { Figure } \\
\text { A-62 }\end{array}$ \\
\hline $\begin{array}{l}\text { Case } 3 \text { (all } \\
\text { canisters, re- } \\
\text { package at CSF) }\end{array}$ & $\begin{array}{c}\text { Figure } \\
\text { A-63 }\end{array}$ & $\begin{array}{c}\text { Figure } \\
\text { A-64 }\end{array}$ & $\begin{array}{c}\text { Figure } \\
\text { A-65 }\end{array}$ & $\begin{array}{c}\text { Figure } \\
\text { B-66 }\end{array}$ & $\begin{array}{c}\text { Figure } \\
\text { A-67 } \\
\text { Figure } \\
\text { A-68 }\end{array}$ & & & & & \\
\hline $\begin{array}{l}\text { Case } 4 \text { (canisters } \\
\text { and bare fuel, re- } \\
\text { package at CSF) }\end{array}$ & $\begin{array}{c}\text { Figure } \\
\text { A-69 }\end{array}$ & $\begin{array}{c}\text { Figure } \\
\text { A-70 }\end{array}$ & $\begin{array}{c}\text { Figure } \\
\text { A-71 }\end{array}$ & $\begin{array}{c}\text { Figure } \\
\text { A-72 }\end{array}$ & $\begin{array}{c}\text { Figure } \\
\text { A-73 } \\
\text { Figure } \\
\text { A-74 } \\
\end{array}$ & $\begin{array}{c}\text { Figure } \\
\text { A-75 }\end{array}$ & $\begin{array}{c}\text { Figure } \\
\text { A-76 }\end{array}$ & $\begin{array}{c}\text { Figure } \\
\text { A-77 }\end{array}$ & $\begin{array}{c}\text { Figure } \\
\text { A-78 }\end{array}$ & $\begin{array}{c}\text { Figure } \\
\text { A-79 } \\
\text { Figure } \\
\text { A-80 } \\
\end{array}$ \\
\hline
\end{tabular}

The peak annual arrival rate of canisters, shown in Figure A-53, is a multi-variable function and depends primarily on the acceptance rate of fuel from the reactors and the time that acceptance begins. These variables influence how much UNF must be transferred to canisters to maintain used fuel pool capacity and therefore, how much UNF would be shipped to the CSF in re-useable transportation casks. Additional details can be found above in Section A-2.3.

The peak inventory of canisters in dry storage, shown in Figure A-55, and bare fuel assemblies in storage, shown in Figure A-61, increases for:

- Higher UNF acceptance rates,

- Longer period between the start of CSF and repository operations, and

- Later start of CSF operations.

Comparing Figure A-55 (Case 2) with Figure A-49 (Case 1) shows that the canister inventory in dry storage at the CSF is reduced when bare fuel is transported from the used fuel pools at reactor sites when the CSF begins operation. However, capacity to handle and store this fuel at the CSF is required with peak values shown in Figure A-61. This same trend can be seen for the total MTHM inventory in dry storage by comparing Figure A-50 (Case 1) with Figures A-56 and A-62 (Case 2).

\section{Case 3 - All Canisters, Re-Package at CSF}

The results for Case 3 are shown in Figures A-63 through A-68. Canisters continually arrive at the CSF until all fuel is removed from the reactor sites, as shown in Figures A-63 and A-64. This is because repackaging of all canisters occurs at the CSF. As stated in Section 3.3, it was assumed that UNF would follow a first-in-first-out shipping schedule from the CSF to the repository. After repository operations begin, UNF canisters are received from the reactors and placed into storage at the same time that UNF canisters are recovered from storage and sent to re-packaging. As shown in Figure A-66, the total CSF canister inventory increases until the repository begins operation, remains constant as canisters are added to and removed from dry storage, then decreases as UNF canisters are no longer being received from the reactors.

However, the canister inventory of vertical and horizontal canisters does not stay constant. This is because under the assumed YFF acceptance priority, the vertical canisters preferentially arrive first at the CSF and then are the first to be shipped from the CSF. The horizontal canisters arrive later and the inventory grows as they displace vertical canisters being removed from storage for re-packaging. This trend is shown in Figure A-66. 
The peak annual arrival rate of all canisters, shown in Figure A-65 (derived from the peaks shown in Figure A-63), increases for higher UNF acceptance rates, but does not depend on the period between CSF and repository operations. This is because the peak annual arrival rates occur before 2040. The total annual arrival rate of all canisters is dominated by the arrival of vertical canisters, which represents the majority of canisters that arrive at the CSF.

The peak inventory of canisters in dry storage, shown in Figure A-67 increases for:

- Higher UNF acceptance rates,

- Longer period between the start of CSF and repository operations, and

- Later start of CSF operations.

Comparing Figures A-67 (Case 3) and Figure A-49 (Case 1) shows that peak canister inventories at the CSF are slightly higher when re-packaging operations are done at the CSF as compared to the repository. This is difference is due to an increased inventory of horizontal canisters in storage. Figure A-63 shows that the rate of vertical canisters arrivals is highest when the CSF begins operation and reduces while the arrival of horizontal canisters increases after the CSF begins operation as a result of the assumed YFF acceptance preference from the reactors. The first-in-first-out CSF shipping assumption results in a preference for removal of vertical canisters from storage (and subsequent re-packaging) that is essentially replaced with newly arriving vertical canisters. However, since the rate of shipping horizontal canisters to the repository under the first-in-first-out assumption is less than the arrival rate, the inventory builds up until such time that the preference to ship horizontal casks increases. This can be seen in Figure A-66.

\section{Case 4 - Canisters and Bare Fuel, Re-Package at CSF}

The results for Case 4 are shown in Figures A-69 through A-80. Canisters and bare fuel casks continually arrive at the CSF until all fuel is removed from the reactor sites, as shown in Figures A-69, A-70, A-75 and A-76. This is because re-packaging of all canisters occurs at the CSF. After repository operations begin, UNF canisters and bare fuel are received from the reactors and placed into storage at the same time that UNF canisters and bare fuel are recovered from storage and sent to packaging/re-packaging.

As stated in Section 3.3, it was assumed that UNF would follow a YFF acceptance priority from the reactors and a first-in-first-out shipping schedule from the CSF to the repository. This results in a preference to transport bare fuel residing in the used fuel pools at the reactor sites to the CSF first, followed by canister shipments as shown in Figures A-70 (canisters) and A-76 (bare fuel).

As shown in Figures A-72 and A-78, the CSF canister and bare fuel inventory increases until the repository begins operation. The bare fuel inventory, as shown in Figure A-78, decreases first as a result of the assumed first-in-first-out shipment preference (bare fuel will arrive first under the YFF acceptance preference). Once bare fuel inventory is depleted, the canister inventory shown in Figure A-72 then decreases.

The peak annual arrival rate of bare fuel casks, shown in Figure A-71, increases for higher UNF acceptance rates, but does not depend on the period between the start of CSF and repository operations when the CSF begins operation in 2020. This is because the highest arrival rate occurs when the CSF begins operations as shown in Figure B-31. Figure A-71 also shows that delaying the start of CSF operations until 2035 reduces the peak annual bare cask arrival rate because more UNF is placed into canisters at the reactor sites to maintain available used fuel pool capacity.

As with Case 2, the peak annual arrival rate of canisters, shown in Figure A-73, is a multi-variable function and depends on the acceptance rate of fuel from the reactors and the time that acceptance begins. These variables influence how much UNF must be transferred to canisters to maintain used fuel pool capacity and therefore, how much UNF would be shipped to the CSF in re-useable transportation casks. Additional details can be found in Appendix A, Section A-2.3.

The peak inventory of bare fuel in storage, shown in Figure A-79, increases for: 
- Higher UNF acceptance rates,

- Longer period between the start of CSF and repository operations, and

- Later start of CSF operations.

The peak inventory of canisters in storage, shown in Figure A-73, depends on these same factors, but also depends on the amount of bare fuel received at the CSF. Higher acceptance rates from the reactors when the CSF begins operations results in more bare fuel being transported to the CSF and fewer canisters. However, higher acceptance rates increase the rate that these canisters arrive at the CSF. These two factors compete, leading to the results shown in Figure A-73 when the CSF starts operation in 2020. Delaying the start of the CSF from 2020 to 2030 results in more UNF being transferred to at-reactor dry storage leading to an increase in the rate that canisters are received and the canister inventory at the CSF (and a corresponding reduction in the rate that bare fuel is received, as shown in Figure A-33). 


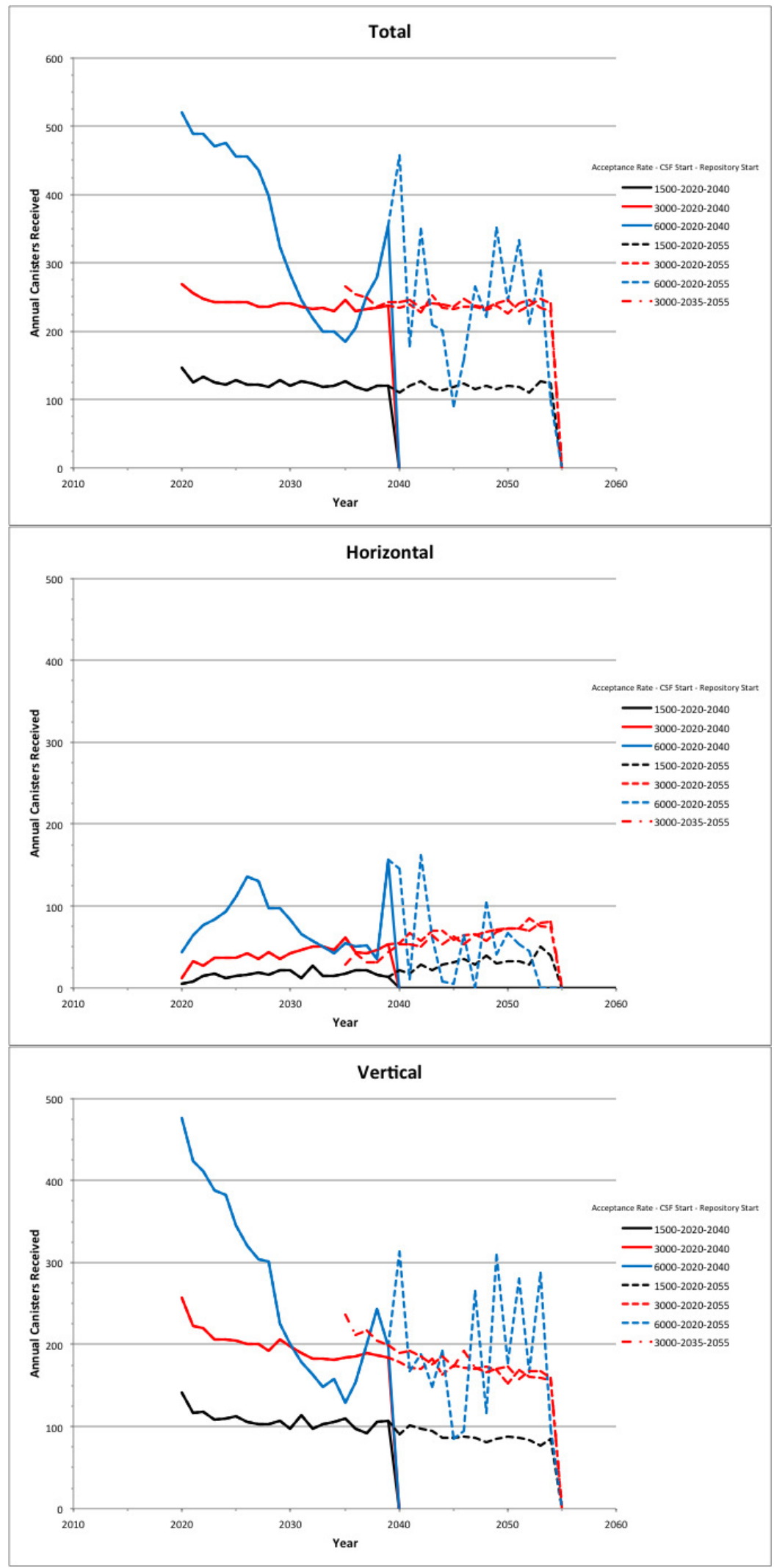

Figure A-45. Annual Canister Arrivals, Case 1 (All Canisters, Re-Package at Repository) 

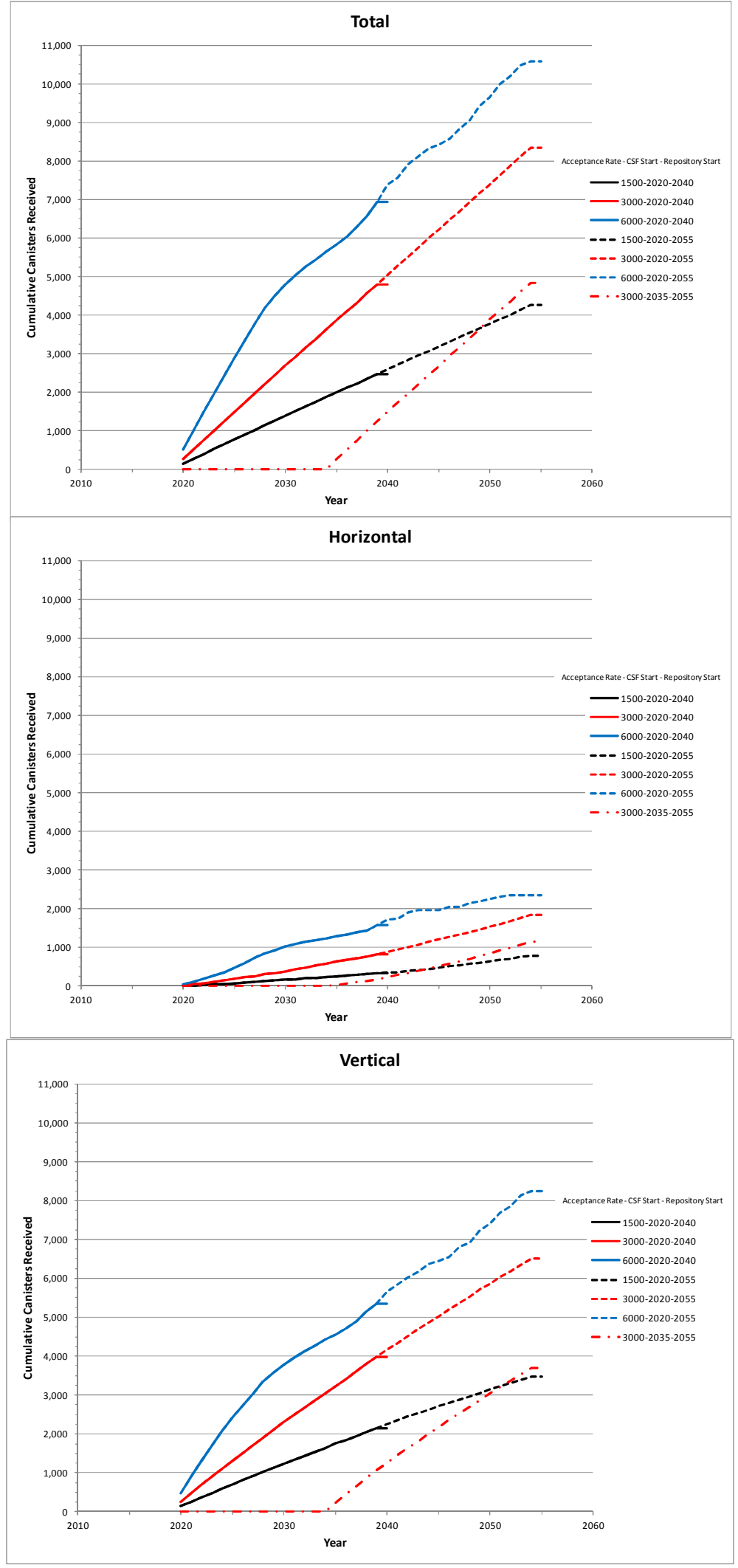

Figure A-46. Cumulative Canister Arrivals, Case 1 (All Canisters, Re-Package at Repository) 


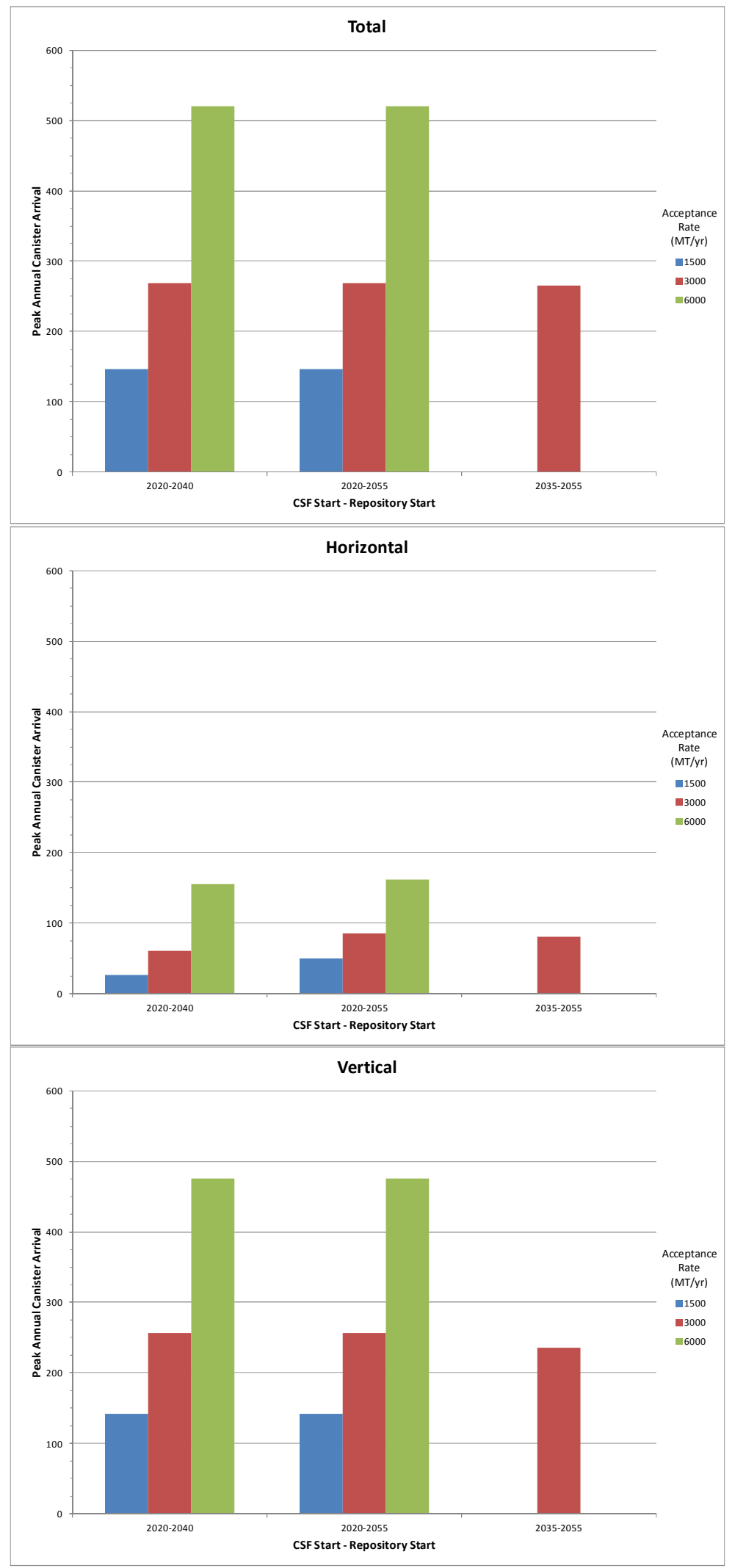

Figure A-47. Peak Canister Arrivals, Case 1 (All Canisters, Re-Package at Repository) 

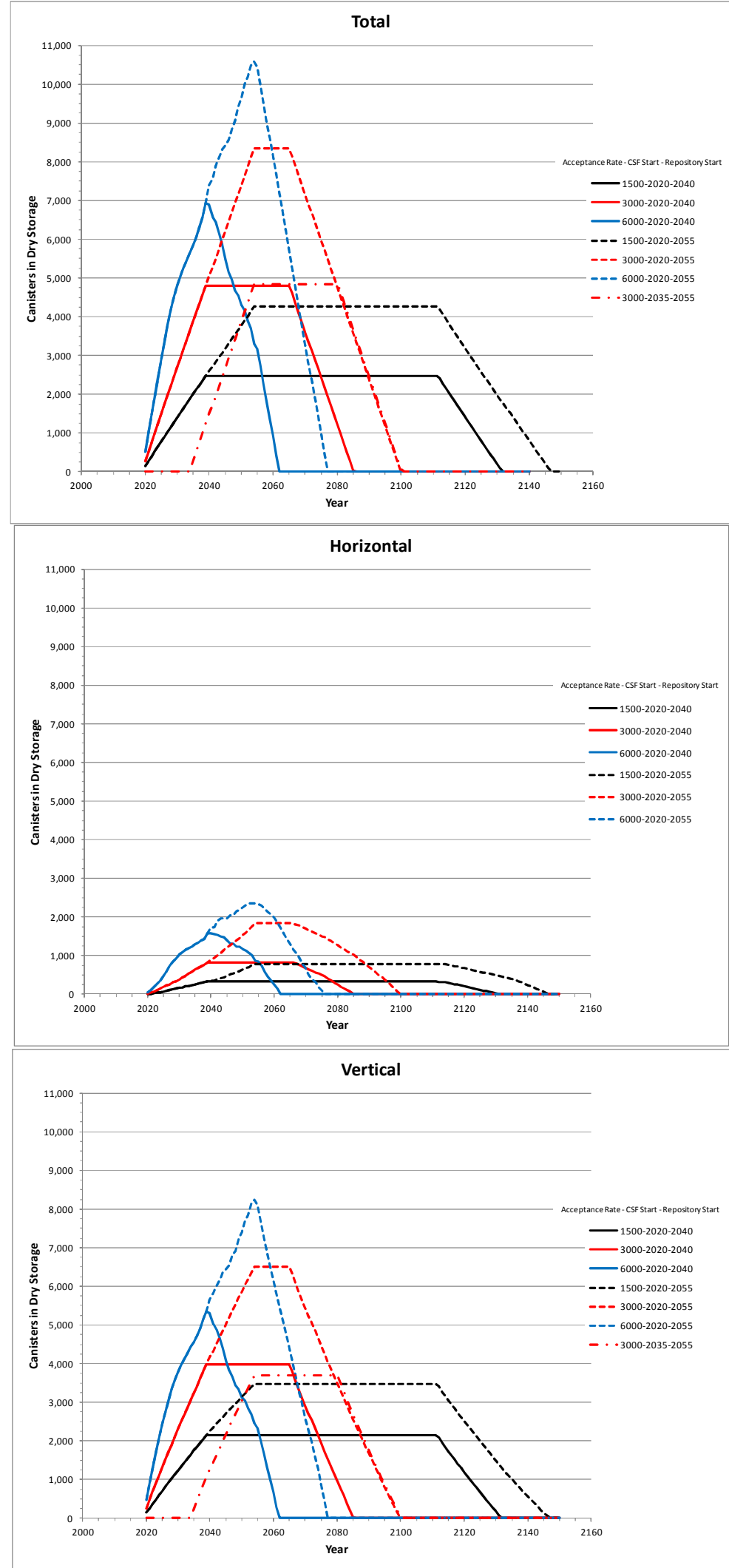

Figure A-48. CSF Dry Canister Inventory, Case 1 (All Canisters, Re-Package at Repository) 


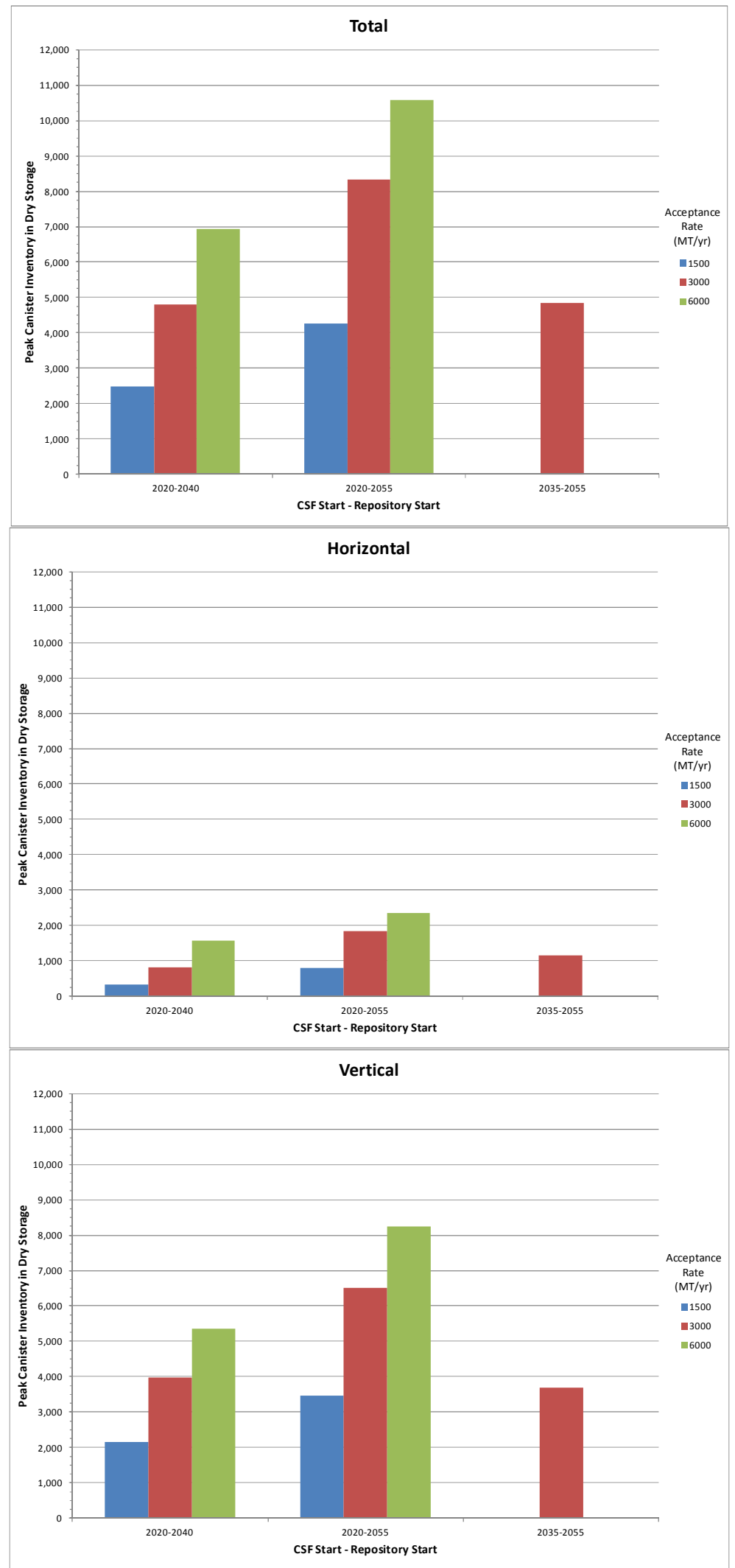

Figure A-49. Peak CSF Dry Canister Inventory, Case 1 (All Canisters, Re-Package at Repository) 


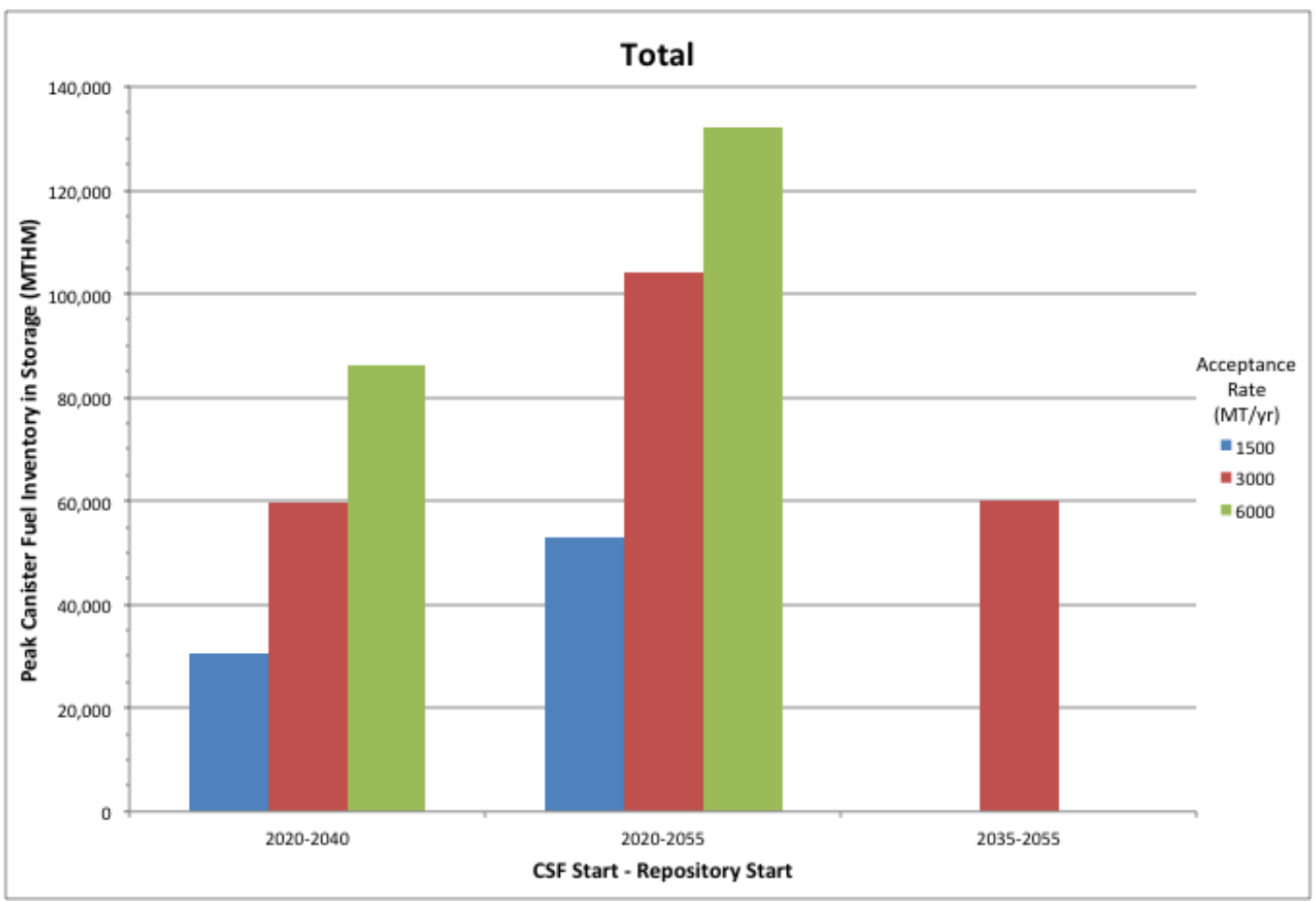

Figure A-50. Peak CSF Dry Inventory - MTHM, Case 1 (All Canisters, Re-Package at Repository) 


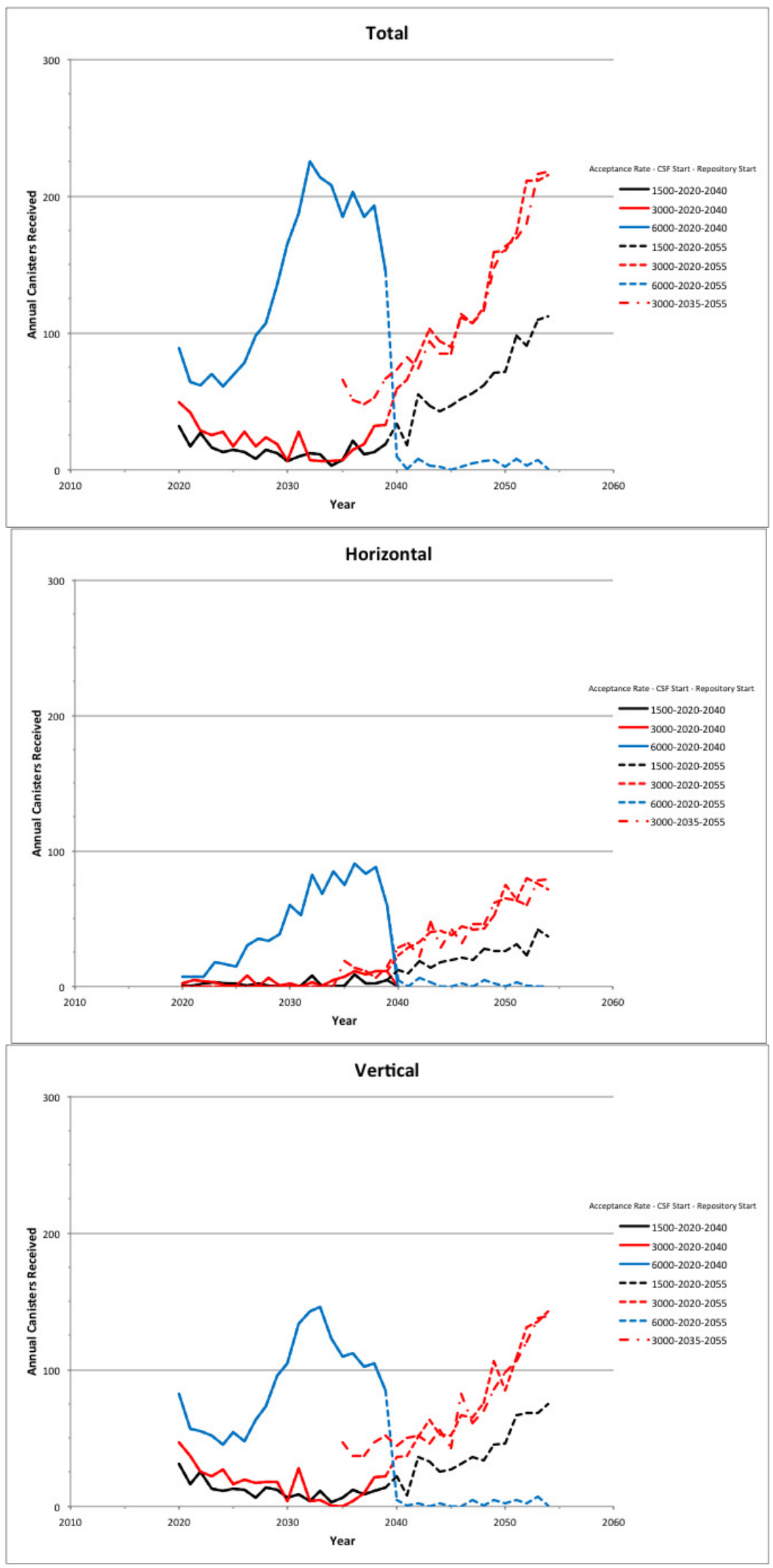

Figure A-51. Annual Canister Arrivals, Case 2 (Canisters and Bare Fuel, Re-Package at Repository) 


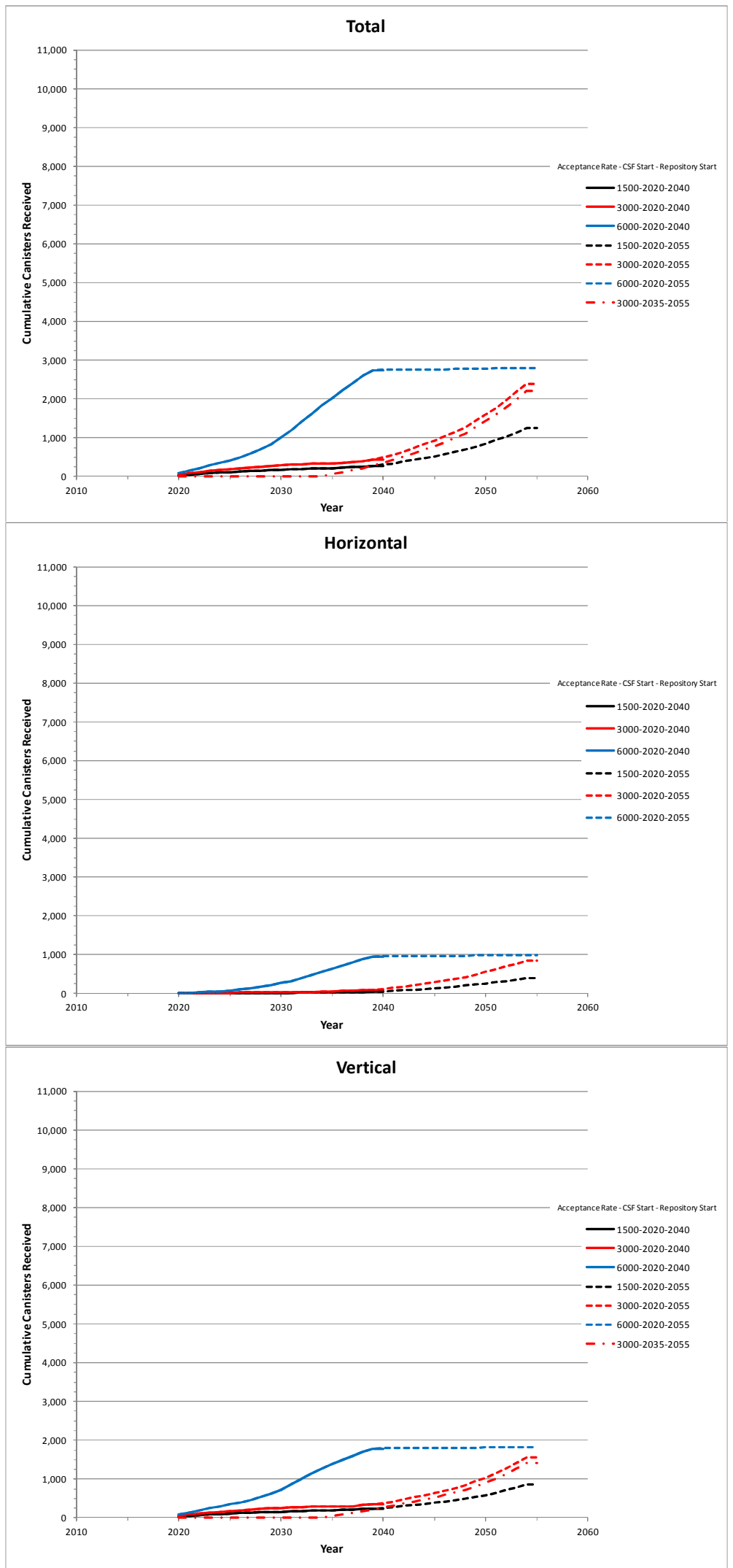

Figure A-52. Cumulative Canister Arrivals, Case 2 (Canisters and Bare Fuel, Re-Package at Repository) 


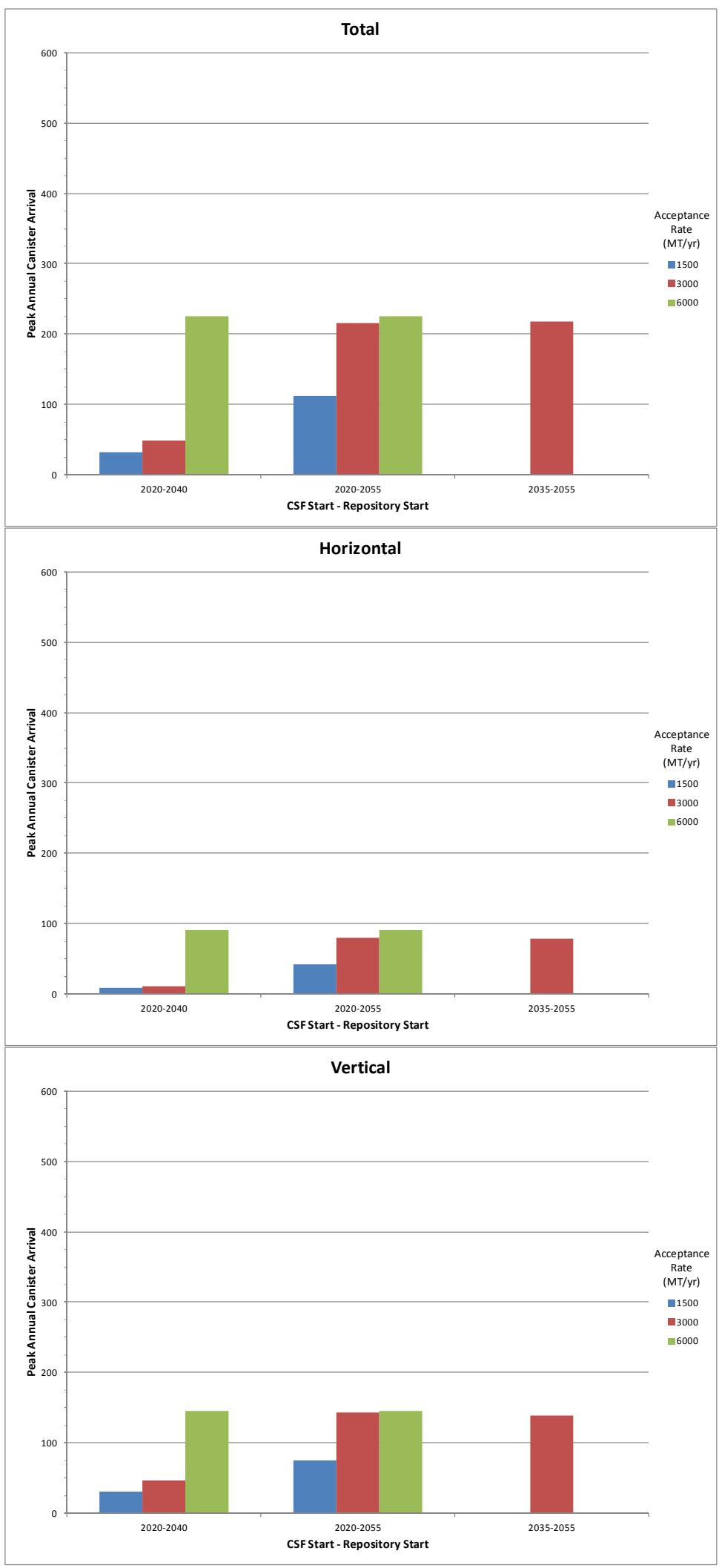

Figure A-53. Peak Canister Arrivals, Case 2 (Canisters and Bare Fuel, Re-Package at Repository) 


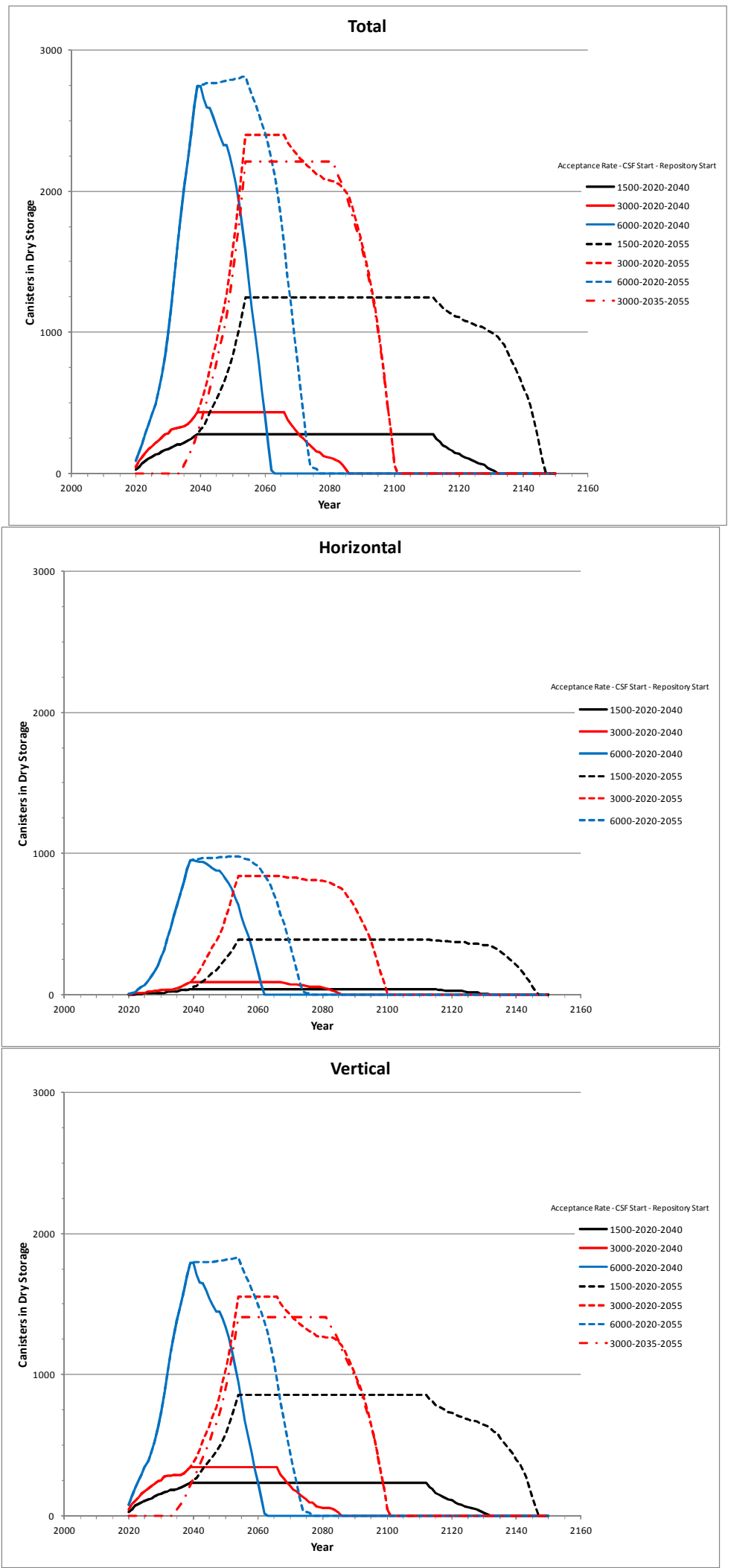

Figure A-54. CSF Dry Canister Inventory, Case 2 (Canisters and Bare Fuel, Re-Package at Repository) 


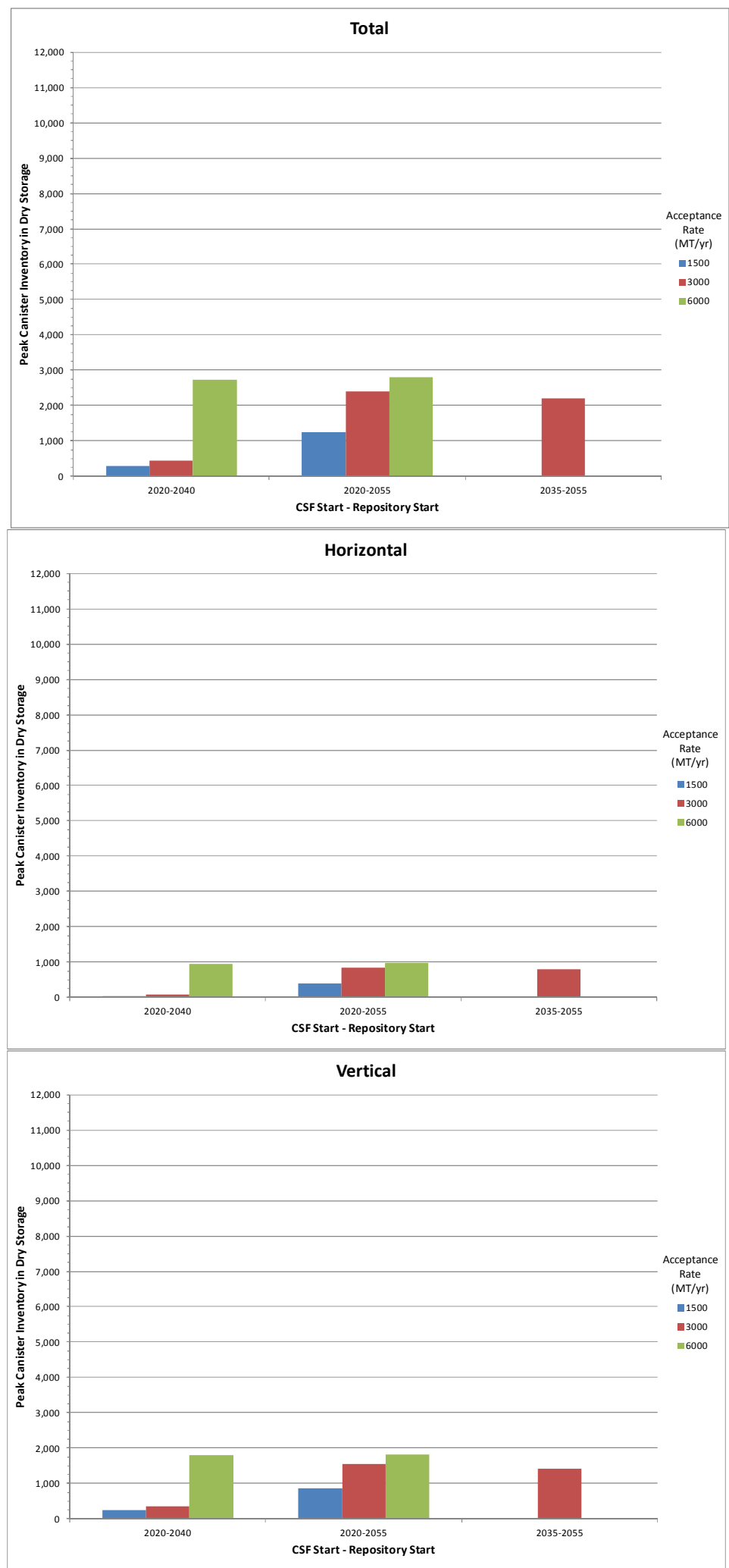

Figure A-55. Peak CSF Dry Canister Inventory, Case 2 (Canisters and Bare Fuel, Re-Package at Repository) 


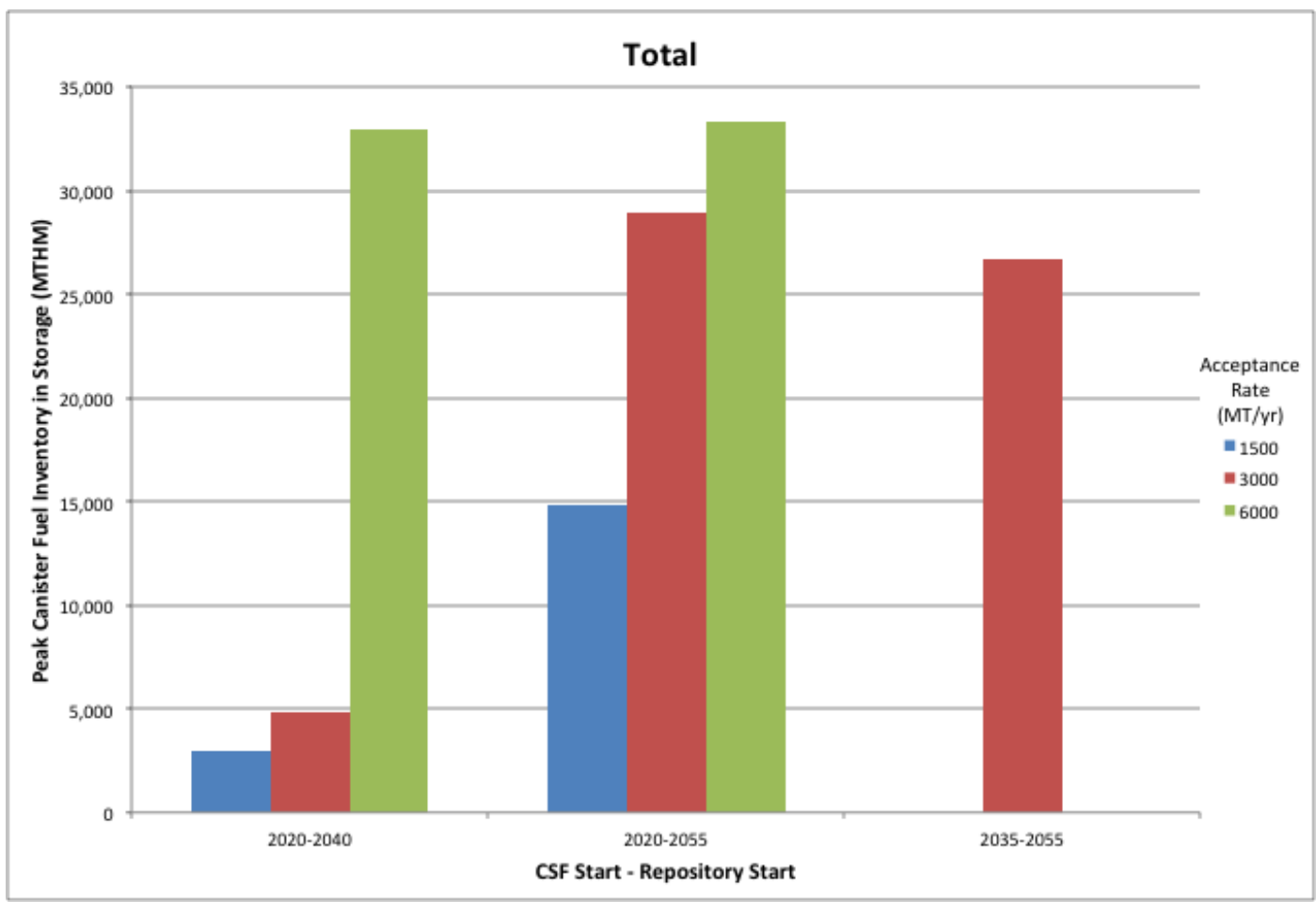

Figure A-56. Peak CSF Dry Inventory - MTHM, Case 2 (Canisters and Bare Fuel, Re-Package at Repository)

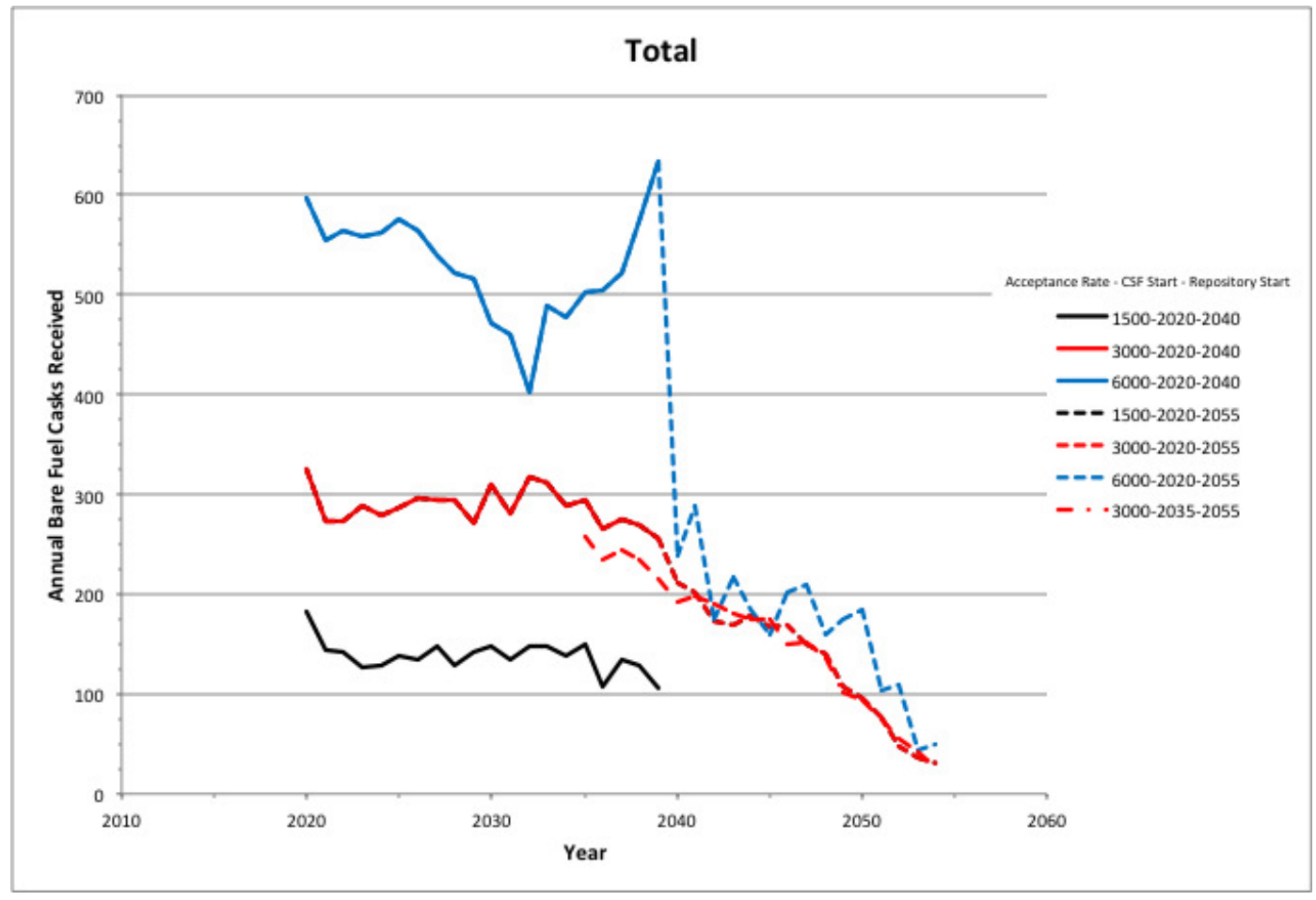

Figure A-57. Annual Bare Fuel Cask Arrivals, Case 2 (Canisters and Bare Fuel, Re-Package at Repository) 


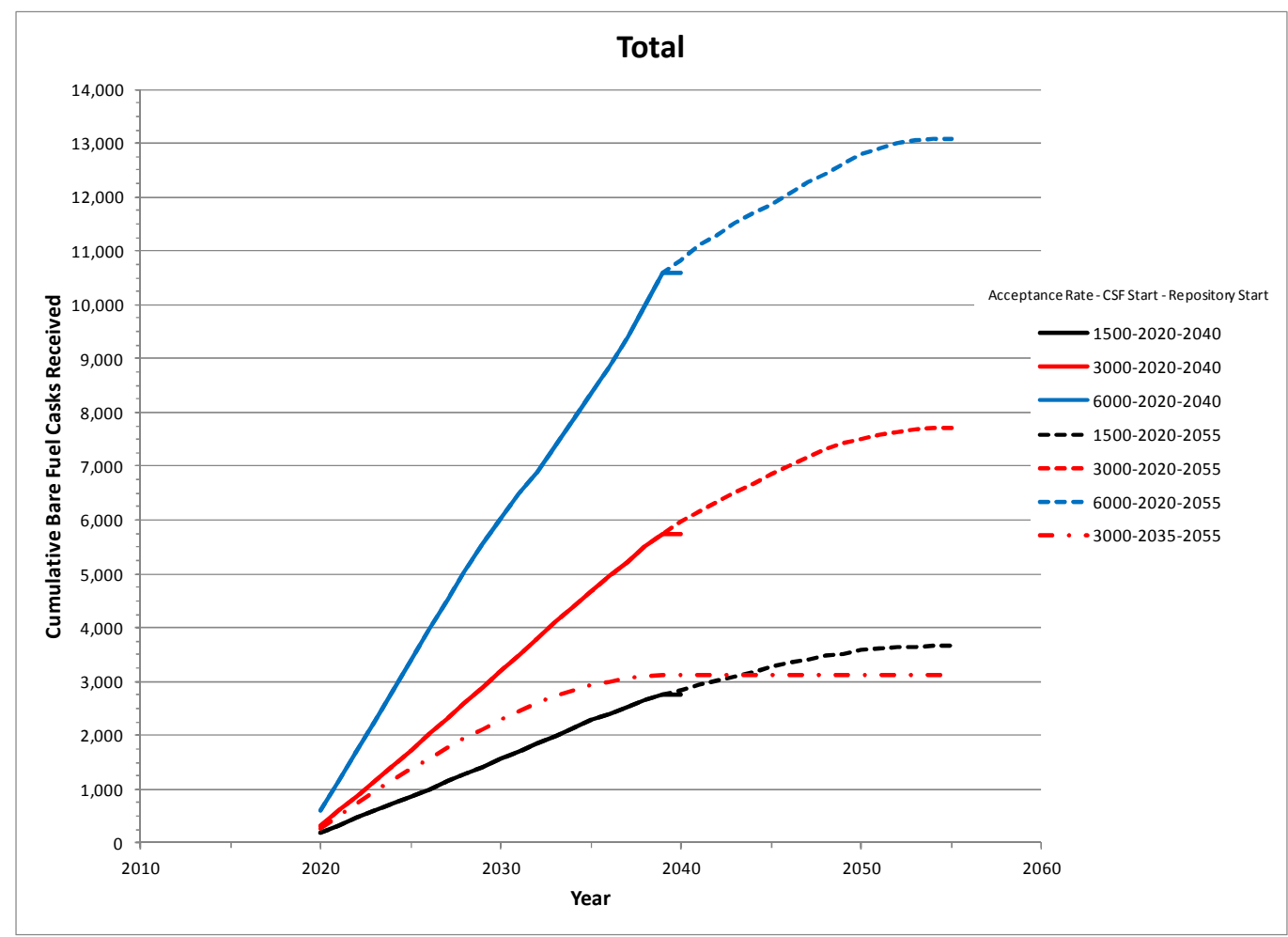

Figure A-58. Cumulative Bare Fuel Cask Arrivals, Case 2 (Canisters and Bare Fuel, Re-Package at Repository)

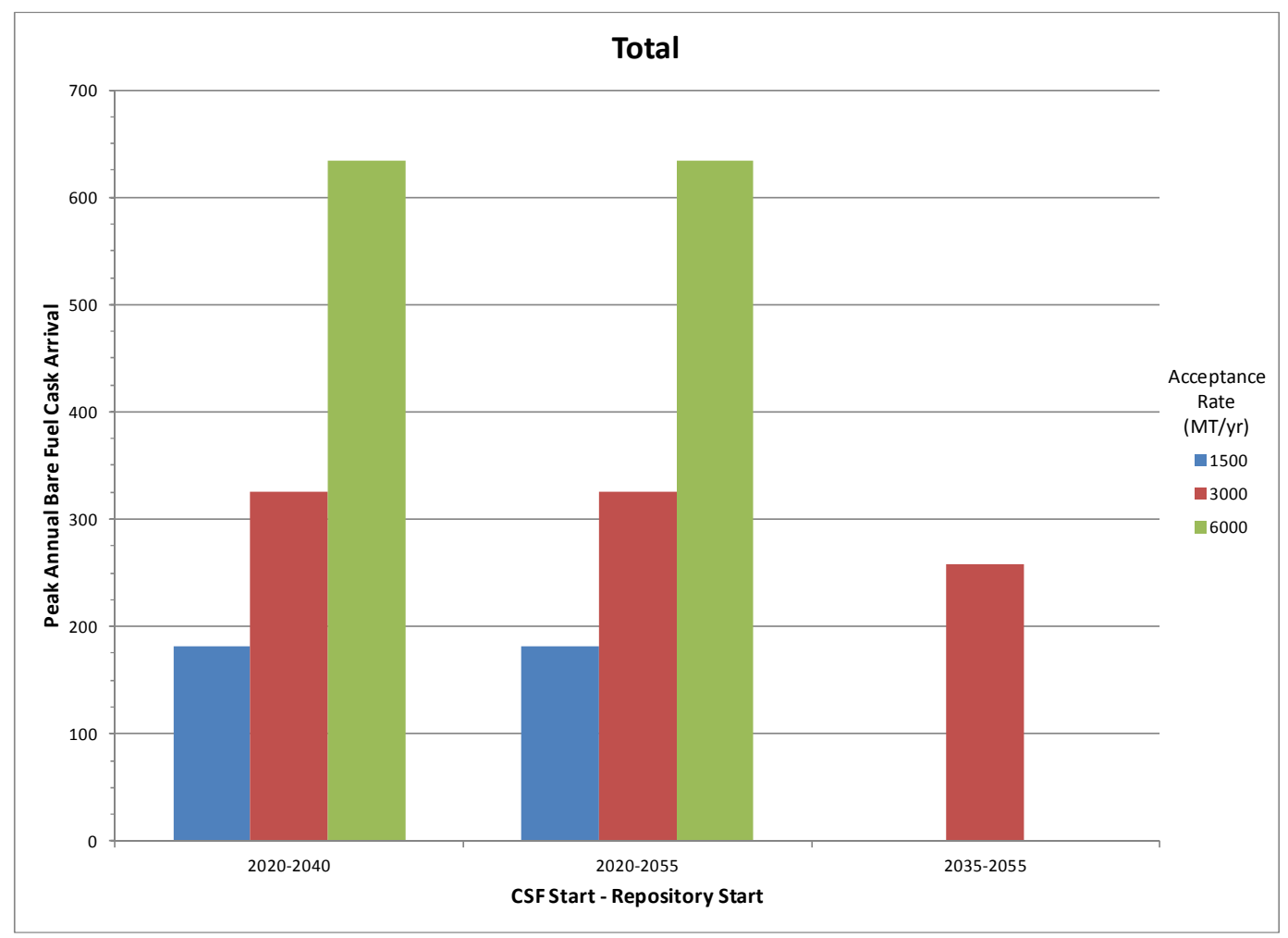

Figure A-59. Peak Bare Fuel Cask Arrivals, Case 2 (Canisters and Bare Fuel, Re-Package at Repository) 


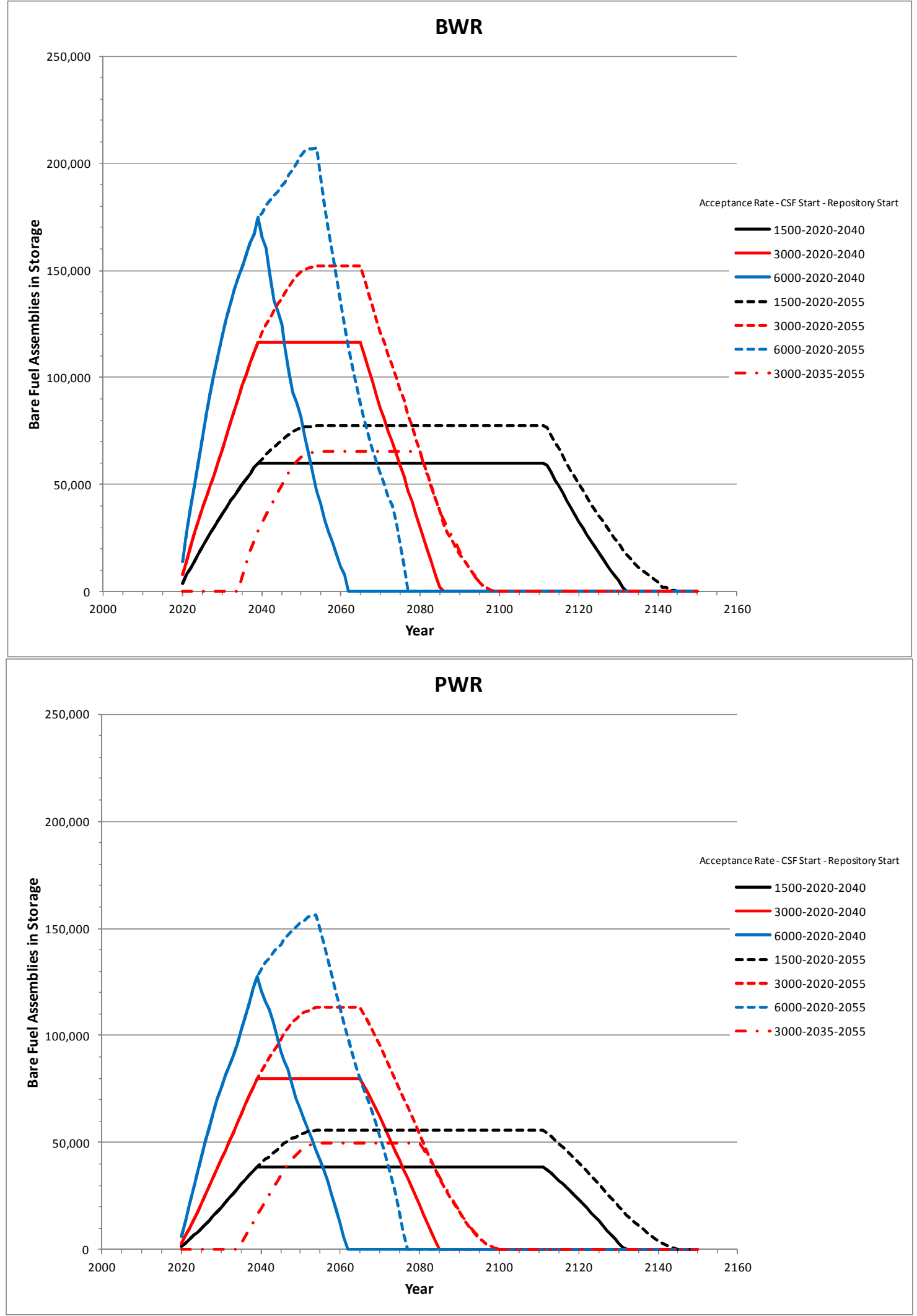

Figure A-60. Bare Fuel Inventory, Case 2 (Canisters and Bare Fuel, Re-Package at Repository) 


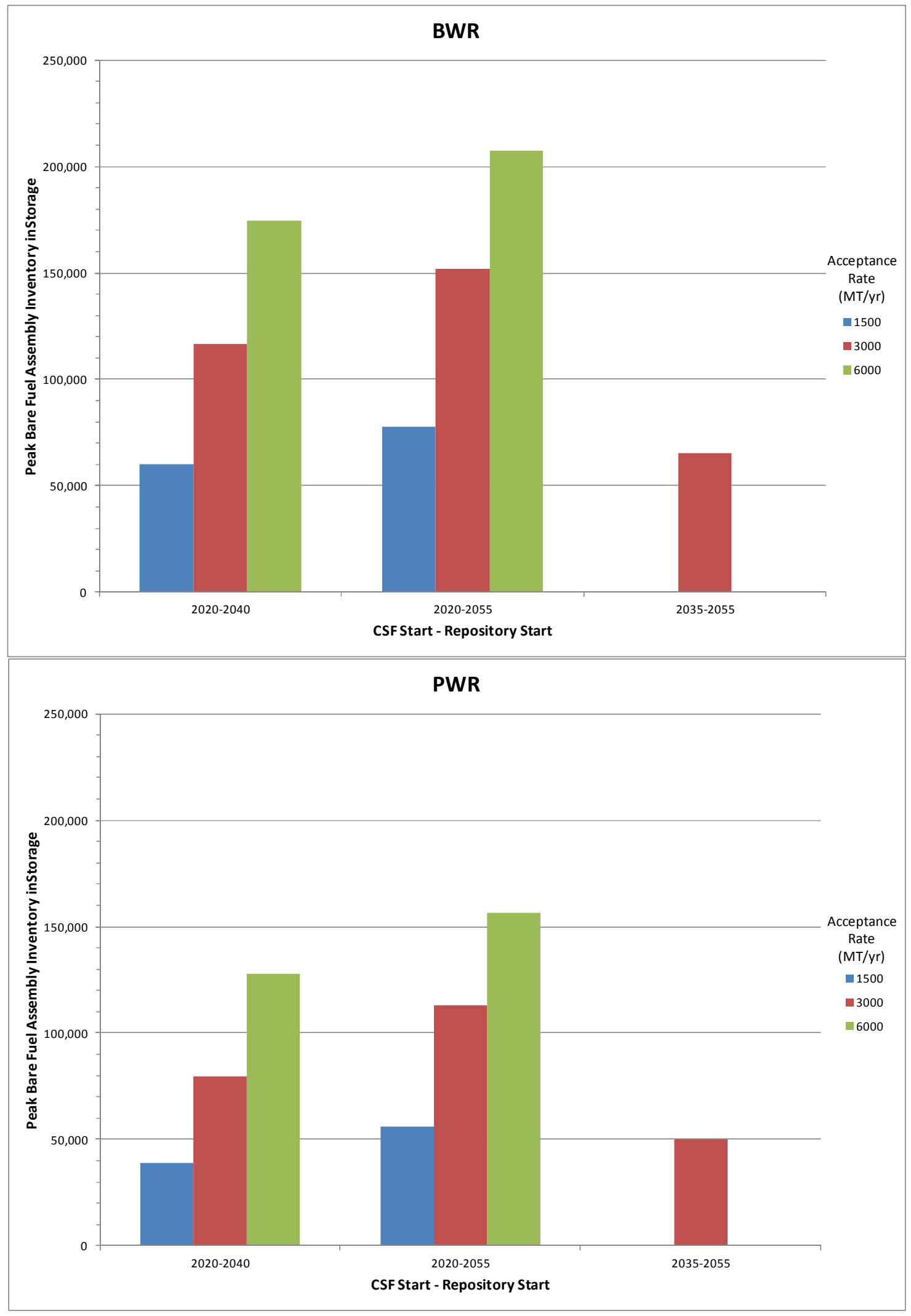

Figure A-61. Peak Bare Fuel Assembly Inventory, Case 2 (Canisters and Bare Fuel, Re-Package at Repository) 


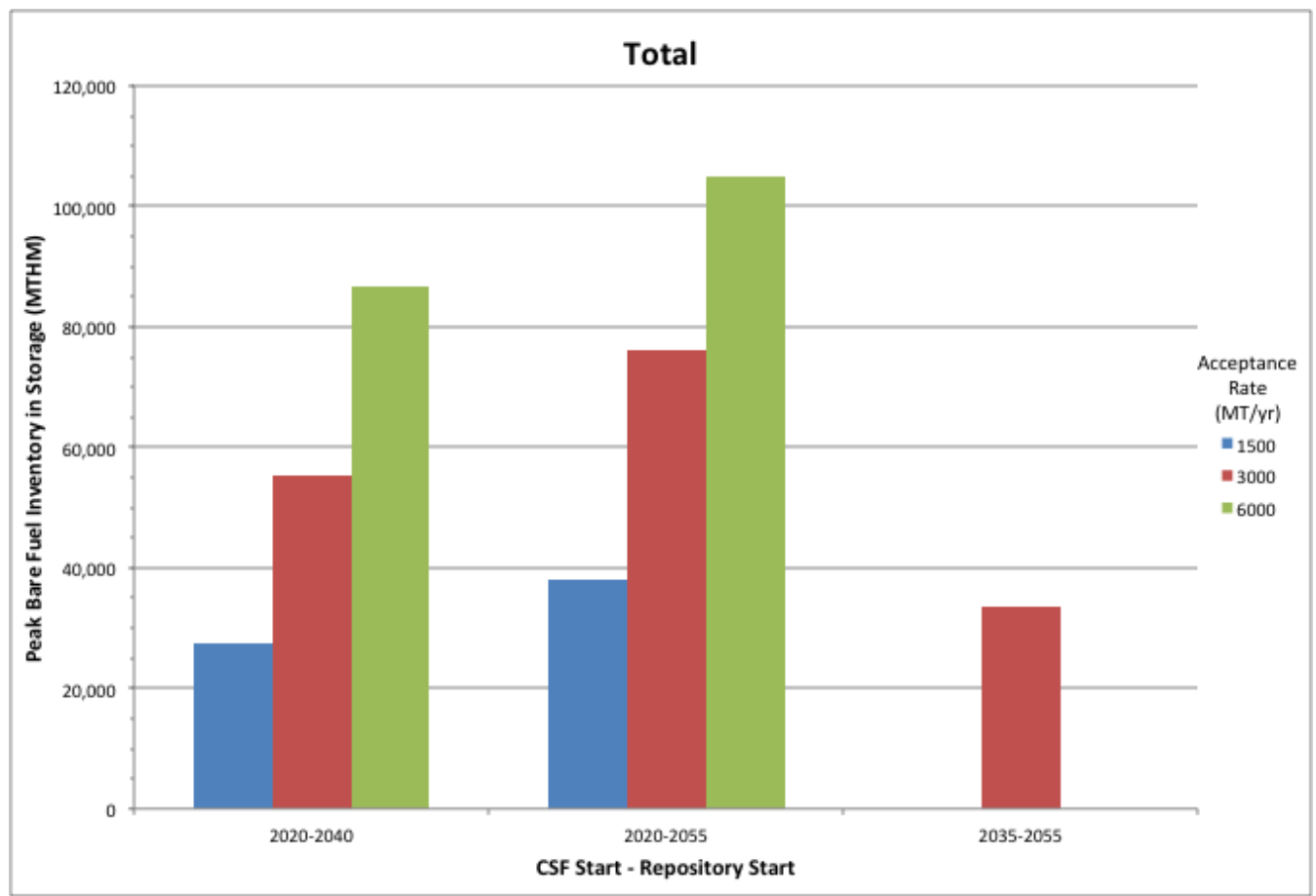

Figure A-62. Peak Bare Fuel MTHM Inventory, Case 2 (Canisters and Bare Fuel, Re-Package at Repository) 

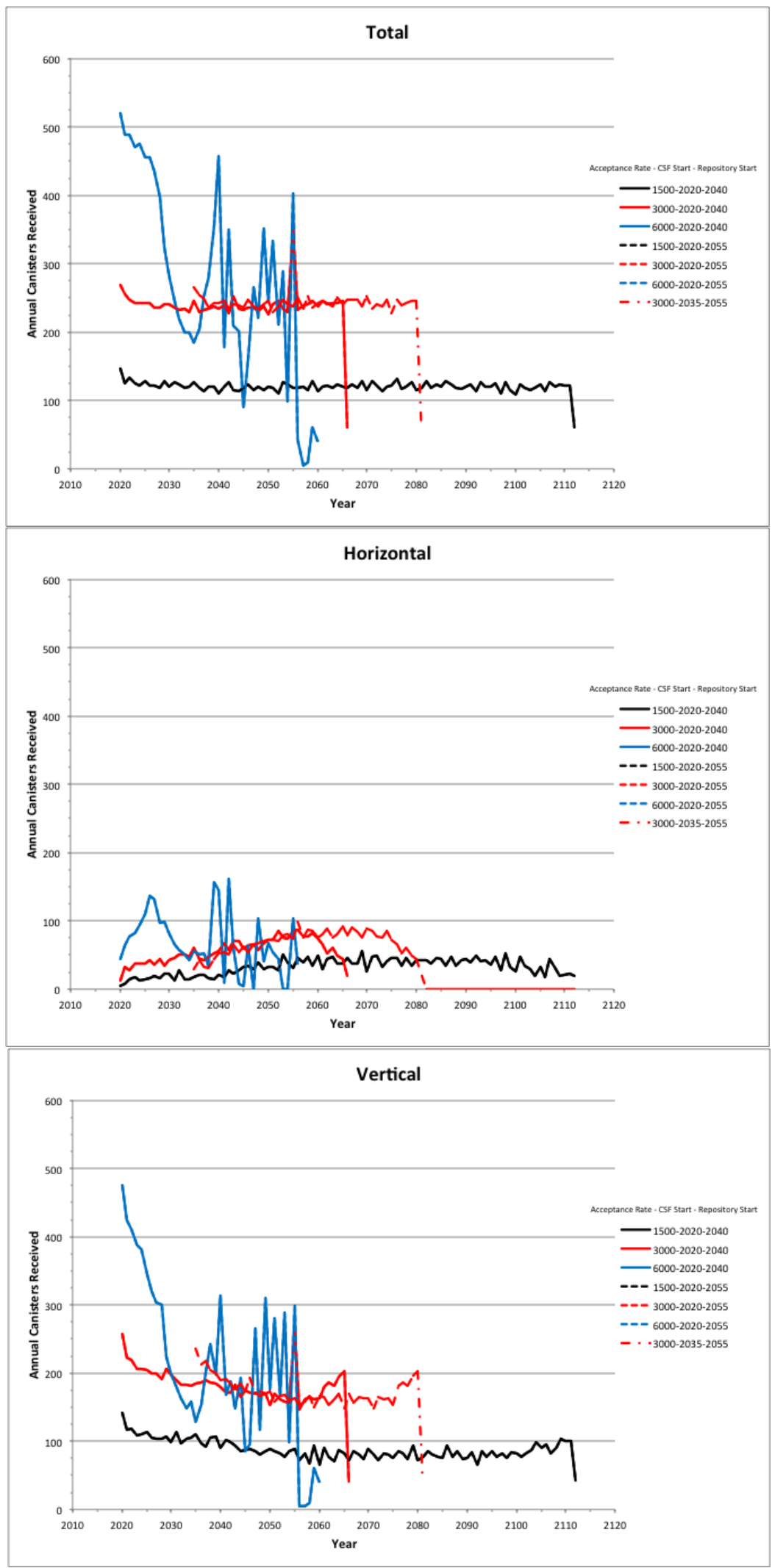

Figure A-63. Annual Canister Arrivals, Case 3 (All Canisters, Re-Package at CSF) 


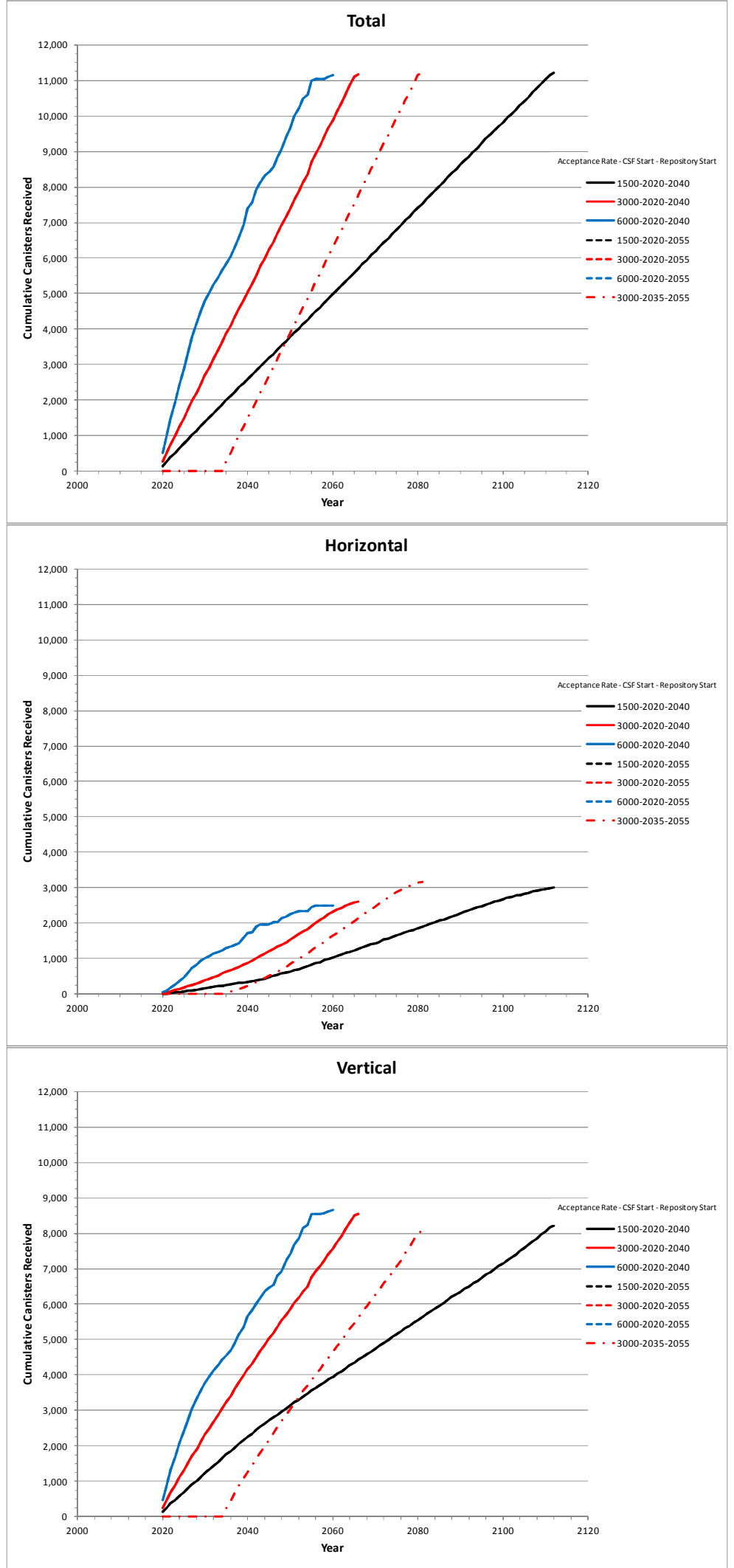

Note: $2020-2040$ and $2020-2055$ plots coincide as all bare fuel is processed through the CSF

Figure A-64. Cumulative Canister Arrivals, Case 3 (All Canisters, Re-Package at CSF) 


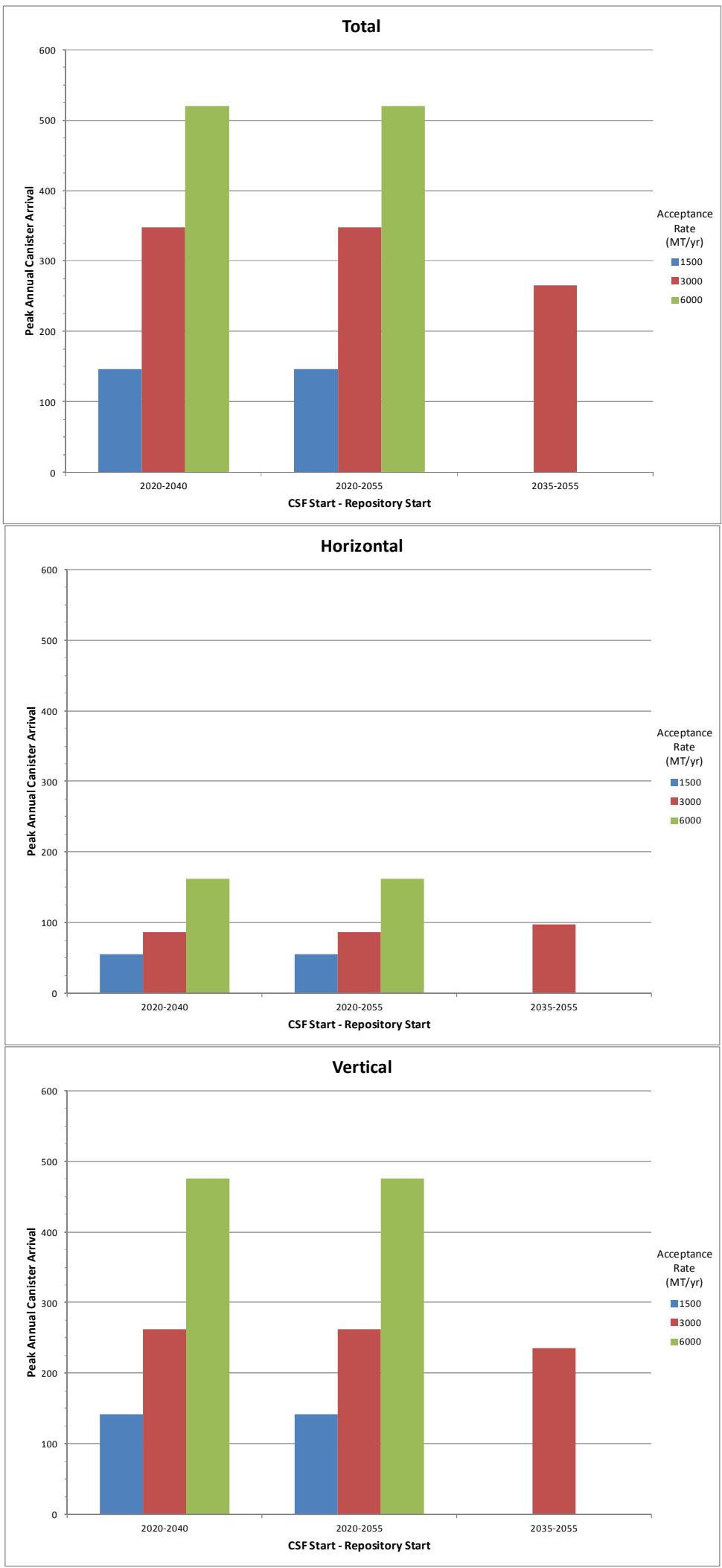

Figure A-65. Peak Canister Arrivals, Case 3 (All Canisters, Re-Package at CSF) 

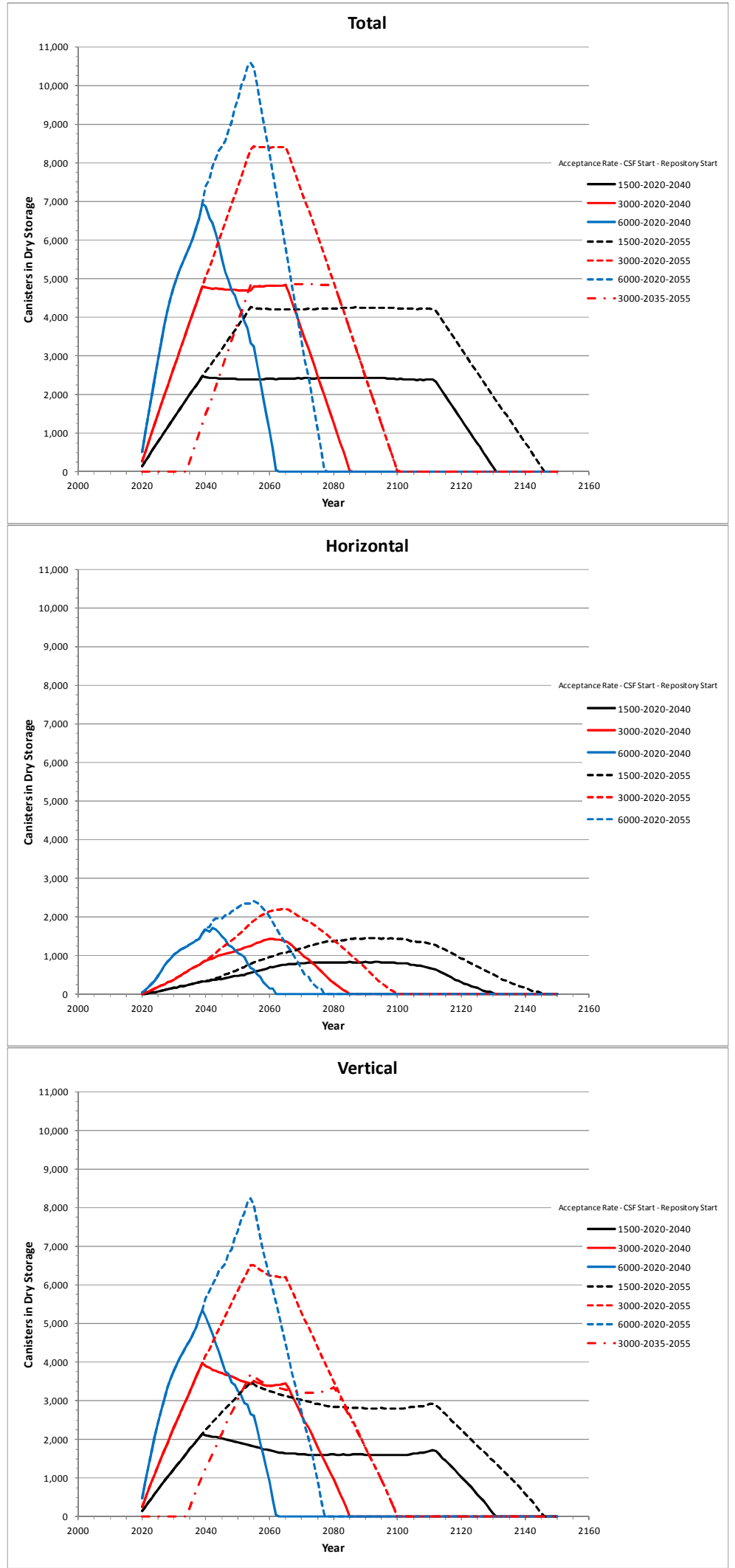

Figure A-66. CSF Dry Canister Inventory, Case 3 (All Canisters, Re-Package at CSF) 


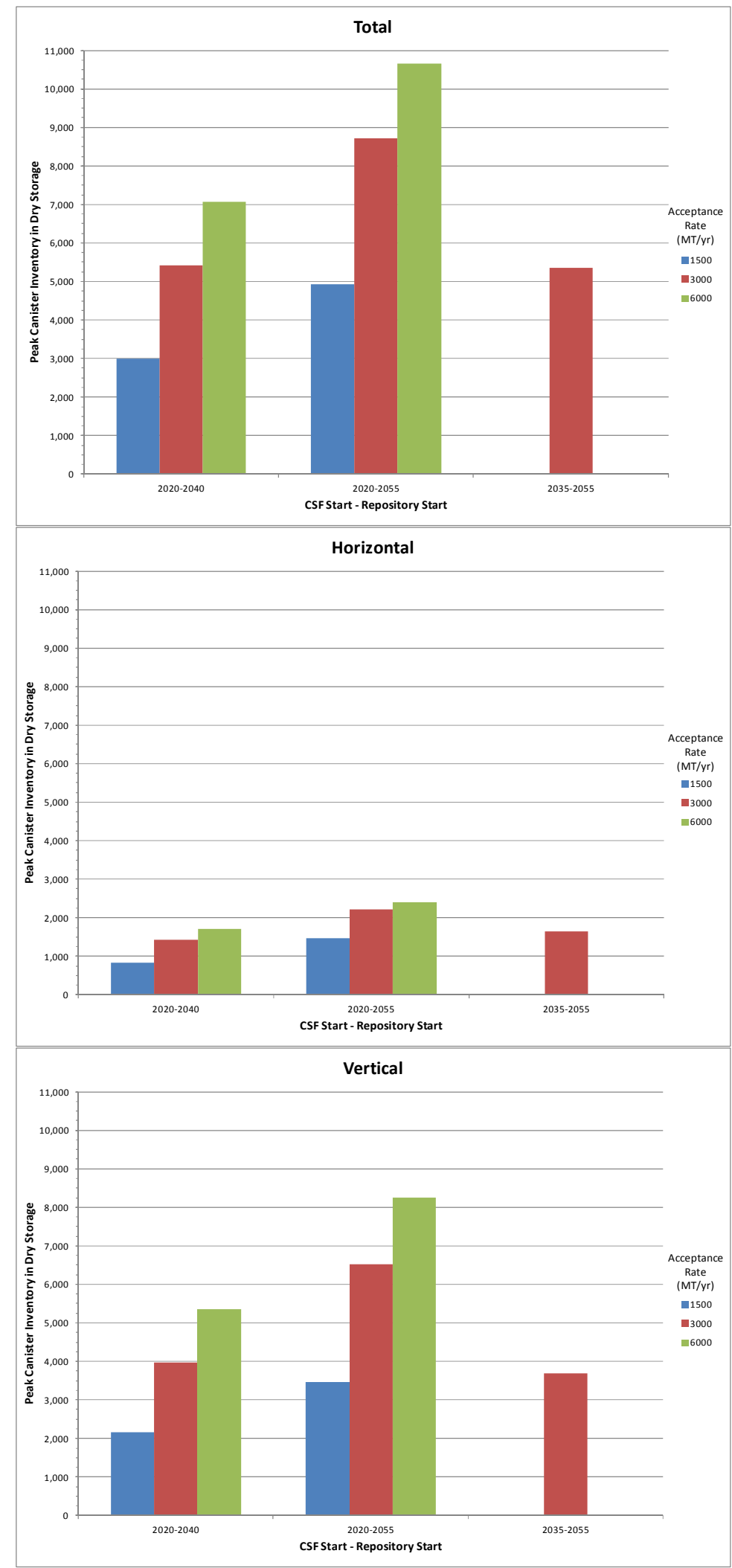

Figure A-67. Peak CSF Dry Canister Inventory, Case 3 (All Canisters, Re-Package at CSF) 


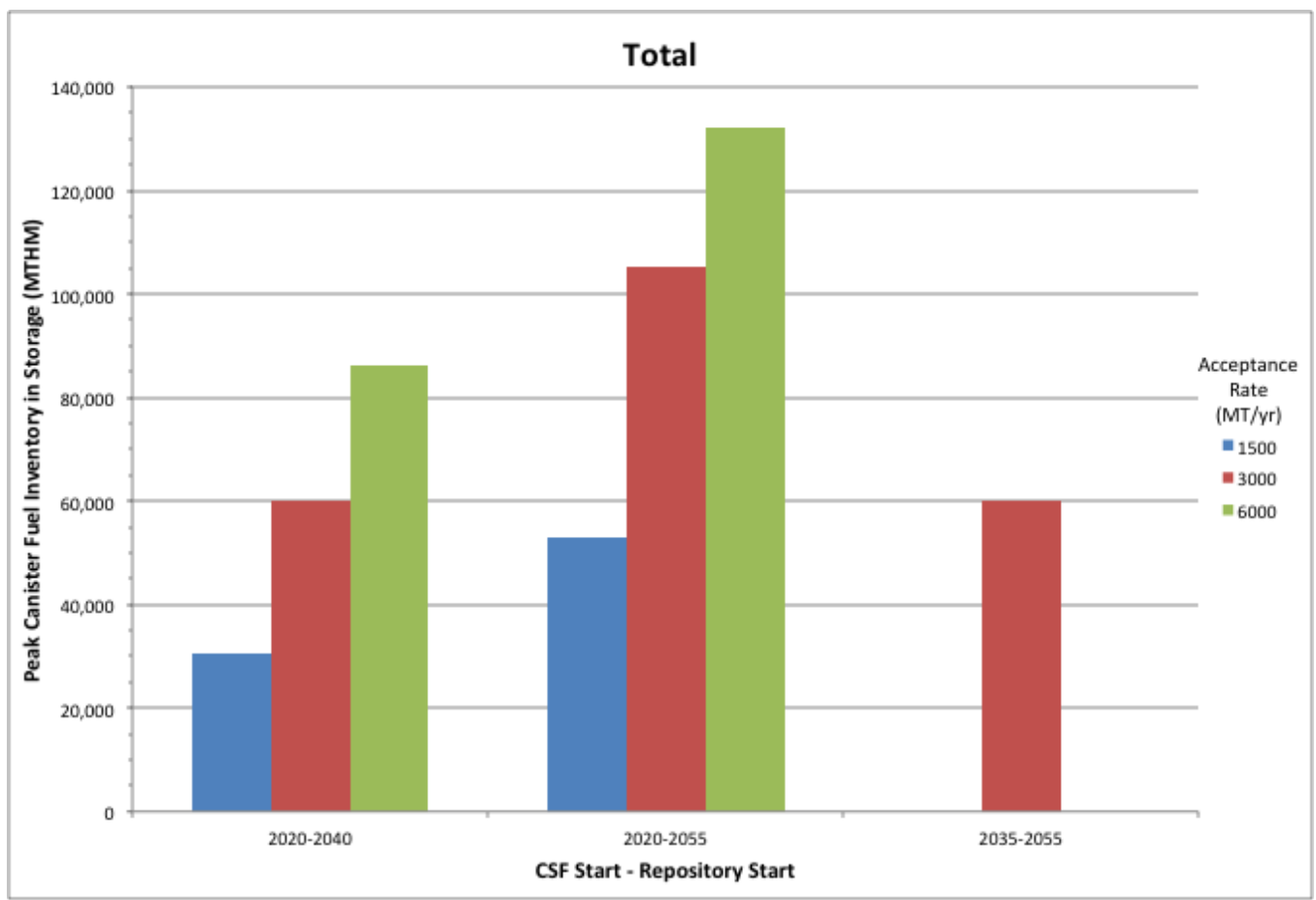

Figure A-68. Peak CSF Dry Inventory - MTHM, Case 3 (All Canisters, Re-Package at CSF) 

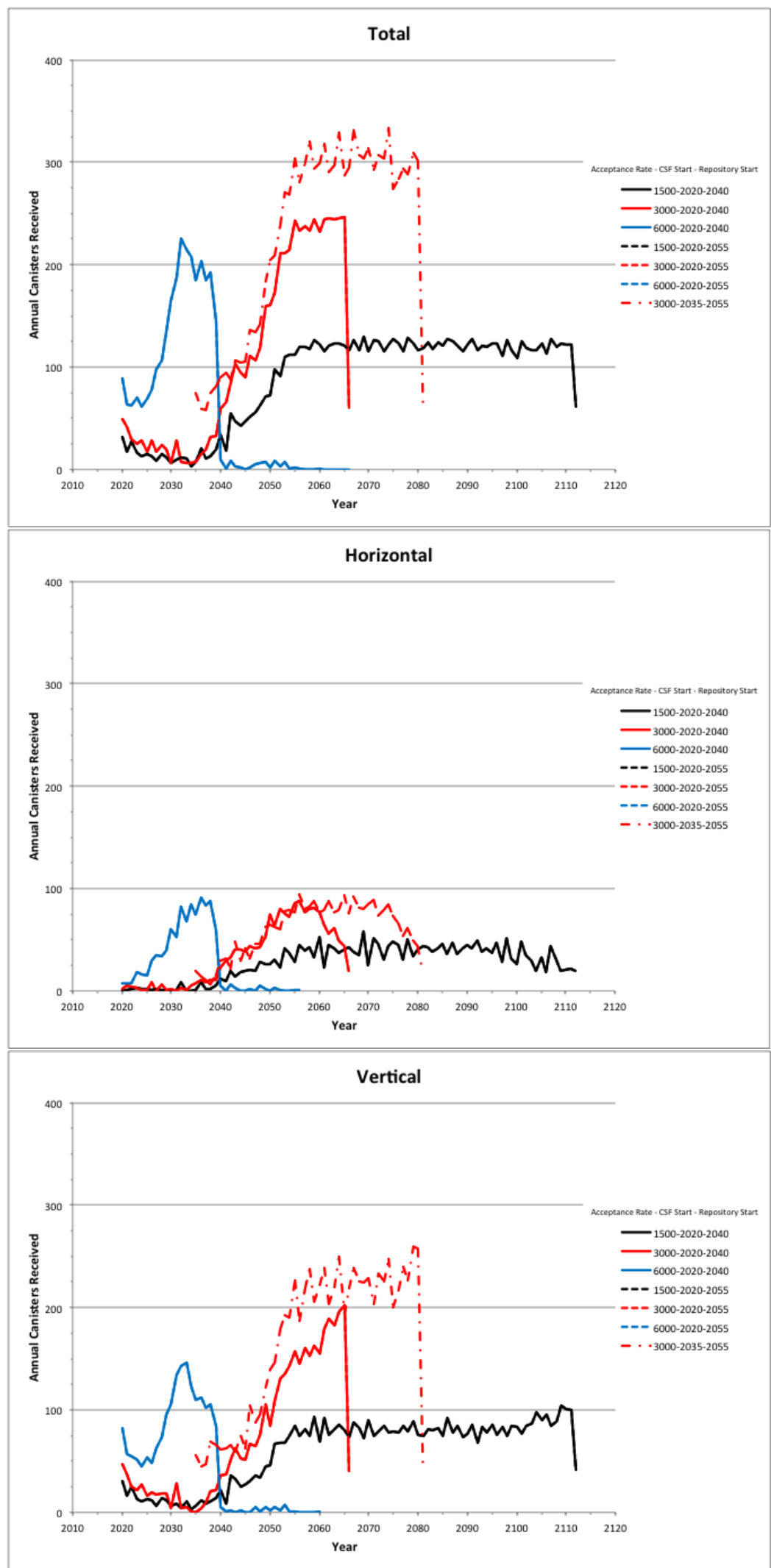

Figure A-69. Annual Canister Arrivals, Case 4 (Canisters and Bare Fuel, Re-Package at CSF) 

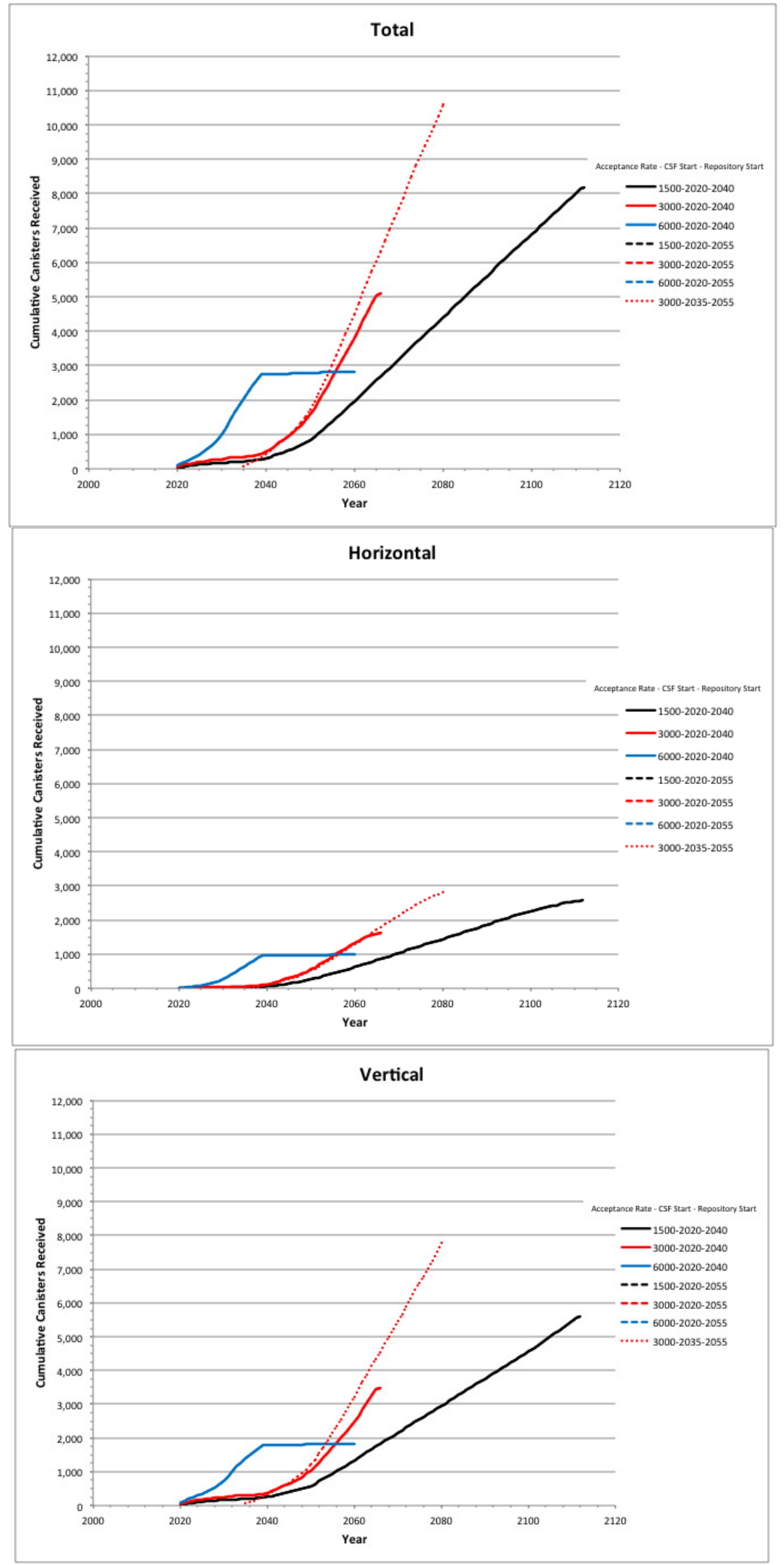

Note: $2020-2040$ and $2020-2055$ plots coincide as all canistered fuel is processed through the CSF

Figure A-70. Cumulative Canister Arrivals, Case 4 (Canisters and Bare Fuel, Re-Package at CSF) 


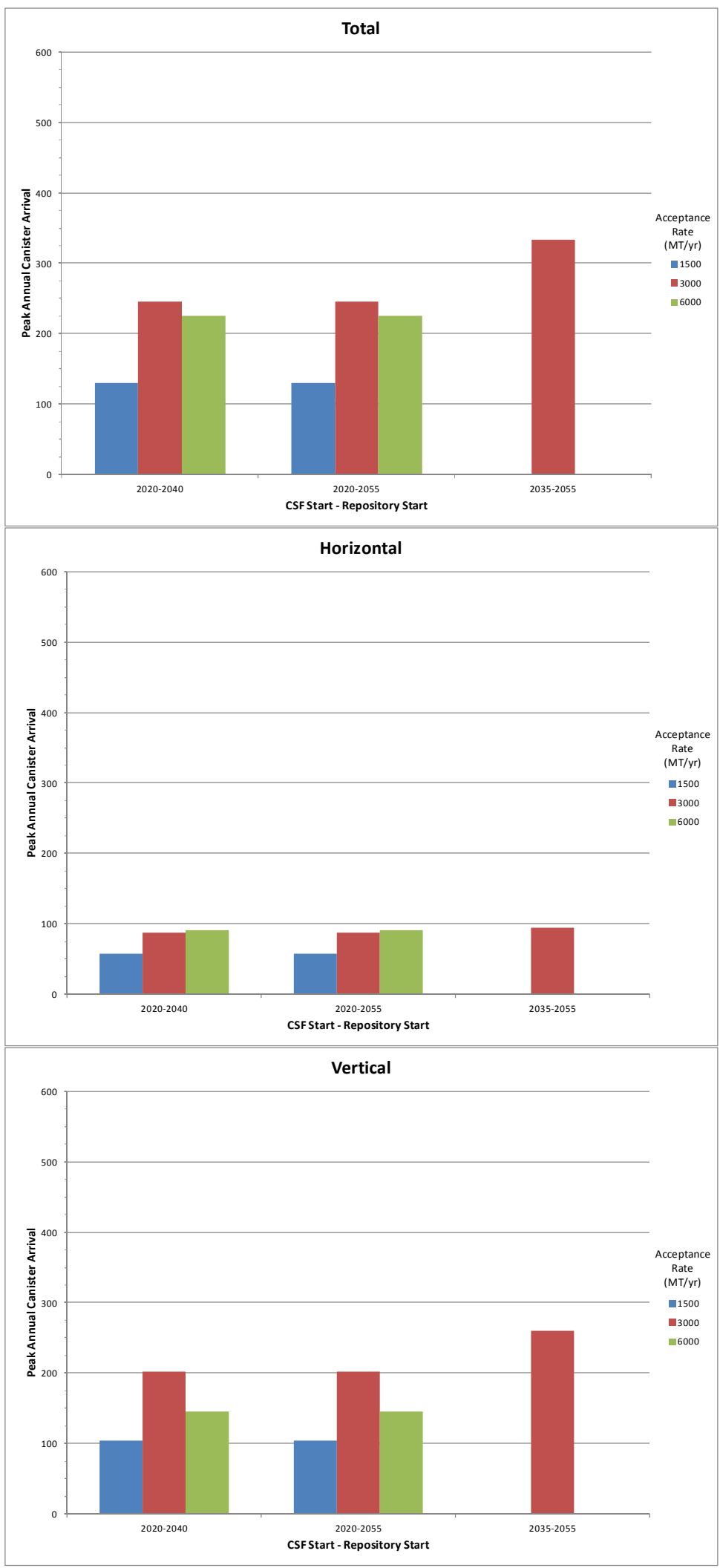

Figure A-71. Peak Canister Arrivals, Case 4 (Canisters and Bare Fuel, Re-Package at CSF) 

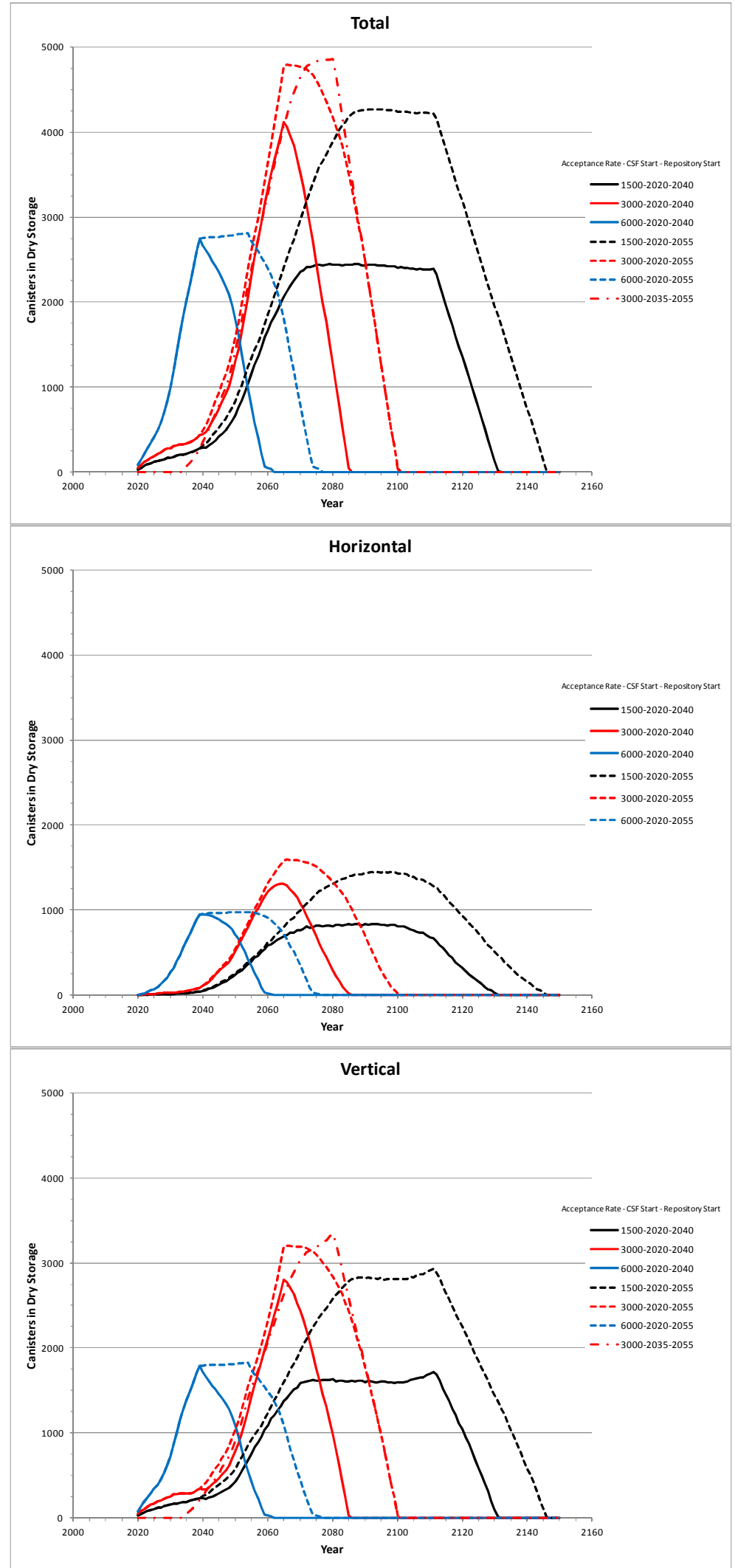

Figure A-72. CSF Dry Canister Inventory, Case 4 (Canisters and Bare Fuel, Re-Package at CSF) 


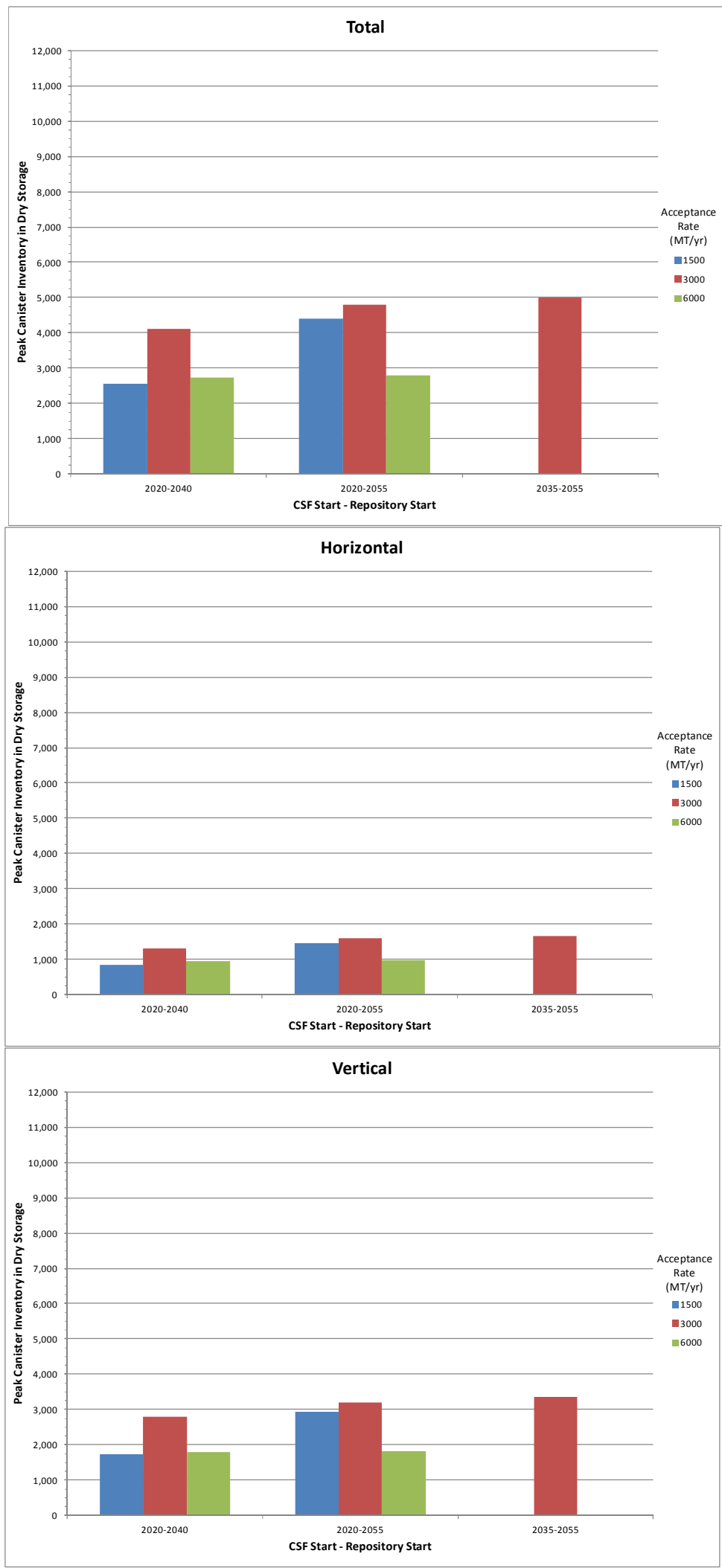

Figure A-73. Peak CSF Dry Canister Inventory, Case 4 (Canisters and Bare Fuel, Re-Package at CSF) 


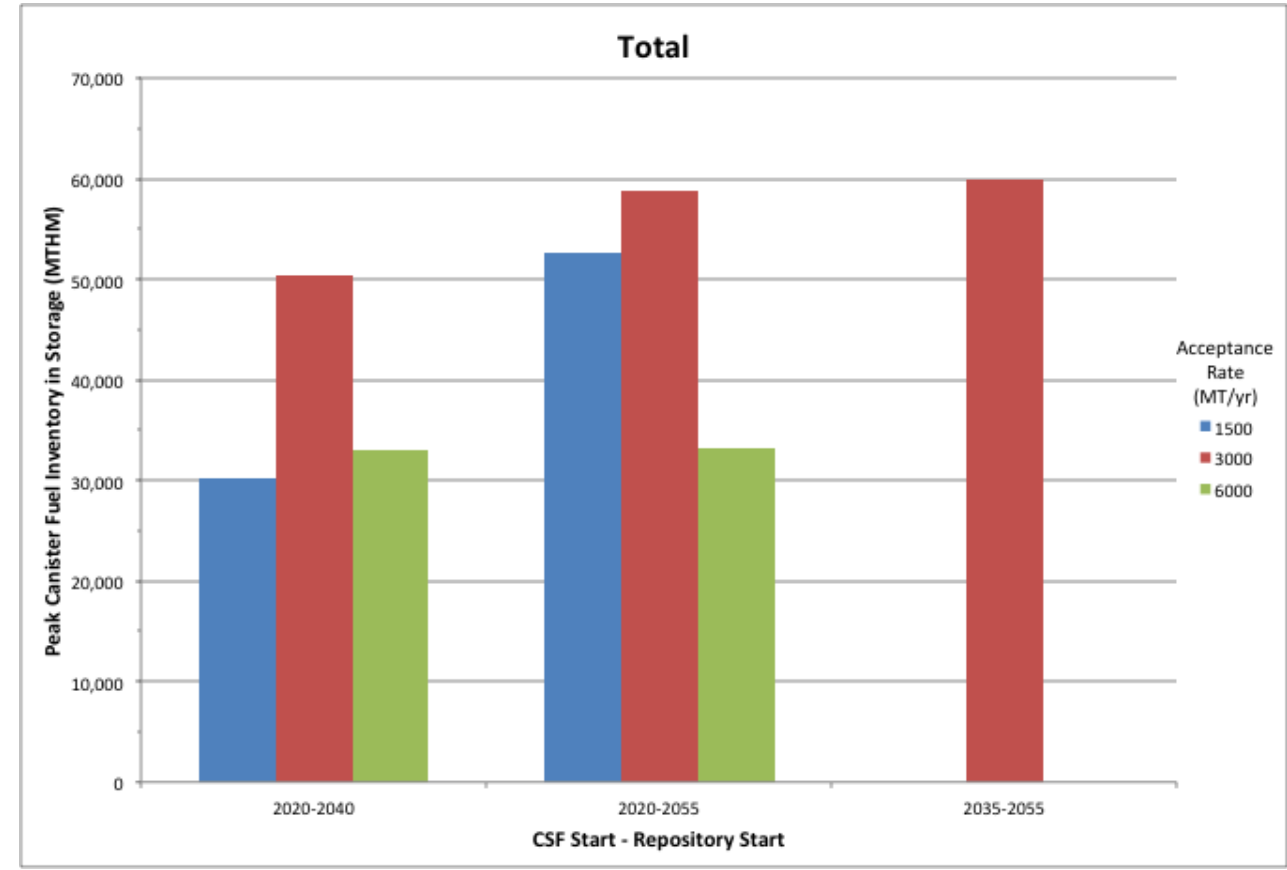

Figure A-74. Peak CSF Dry Inventory - MTHM, Case 4 (Canisters and Bare Fuel, Re-Package at CSF)

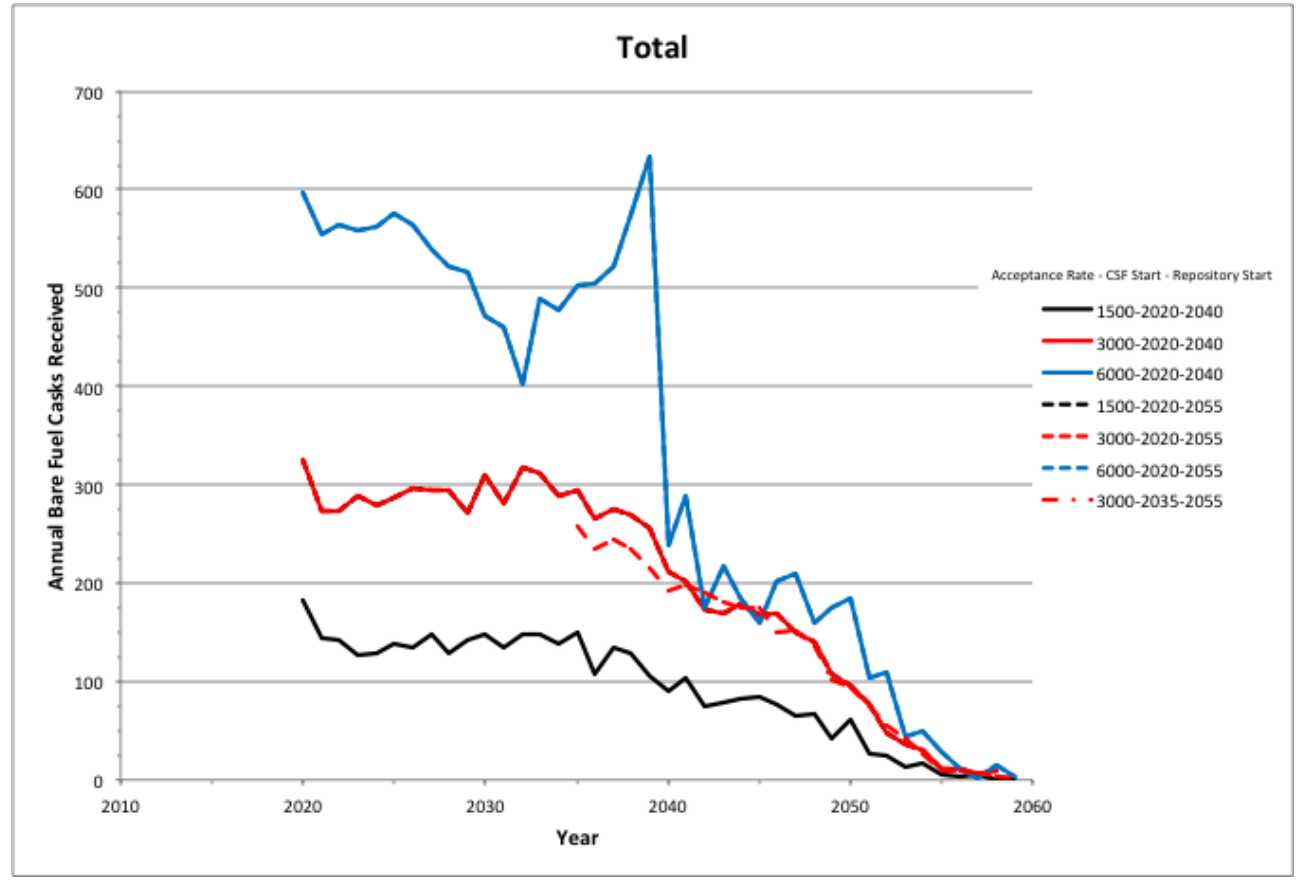

Note: $2020-2040$ and $2020-2055$ plots coincide as all bare fuel is processed through the CSF

Figure A-75. Annual Bare Fuel Cask Arrivals, Case 4 (Canisters and Bare Fuel, Re-Package at CSF) 


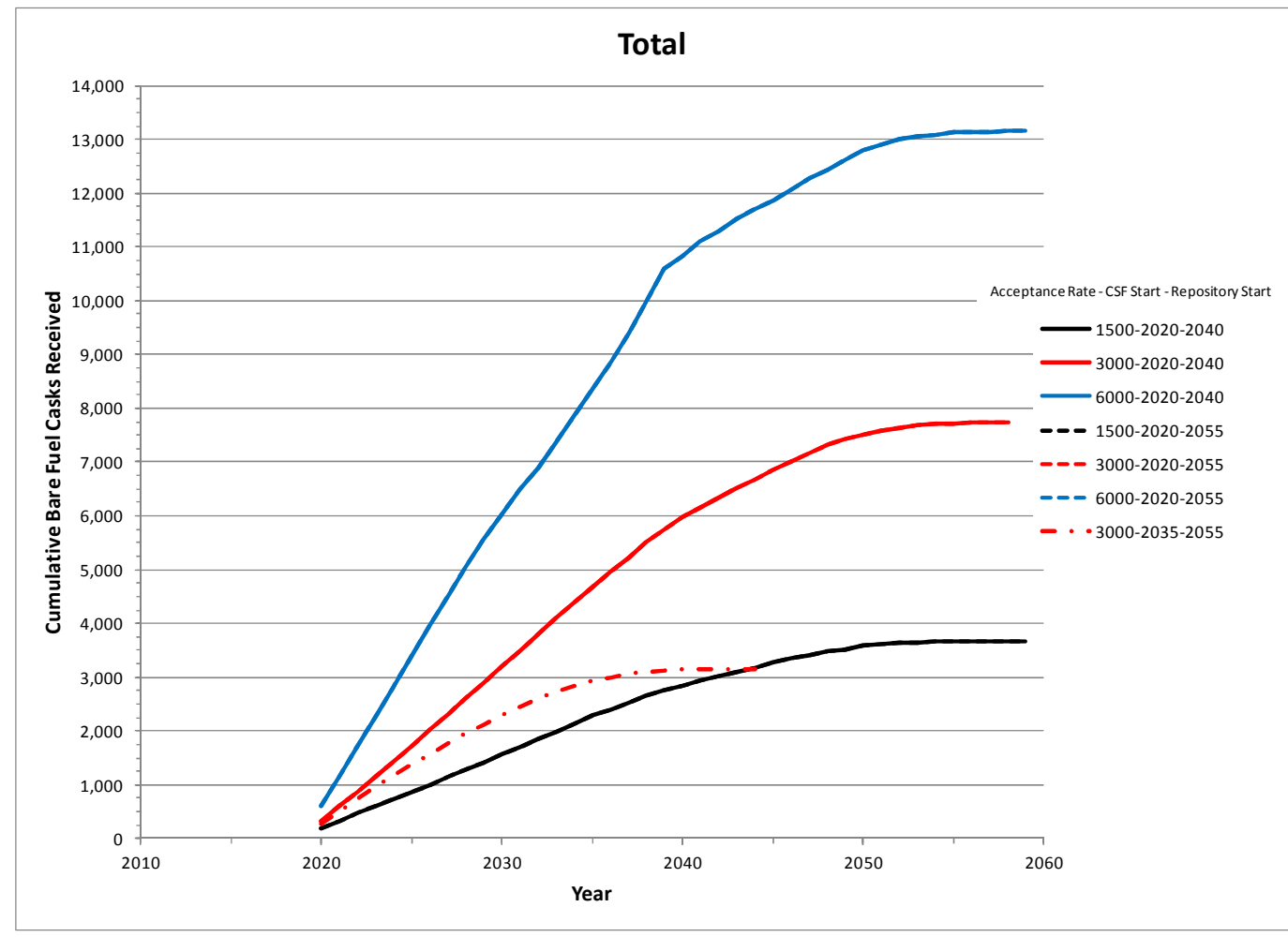

Figure A-76. Cumulative Bare Fuel Cask Arrivals, Case 4 (Canisters and Bare Fuel, Re-Package at CSF)

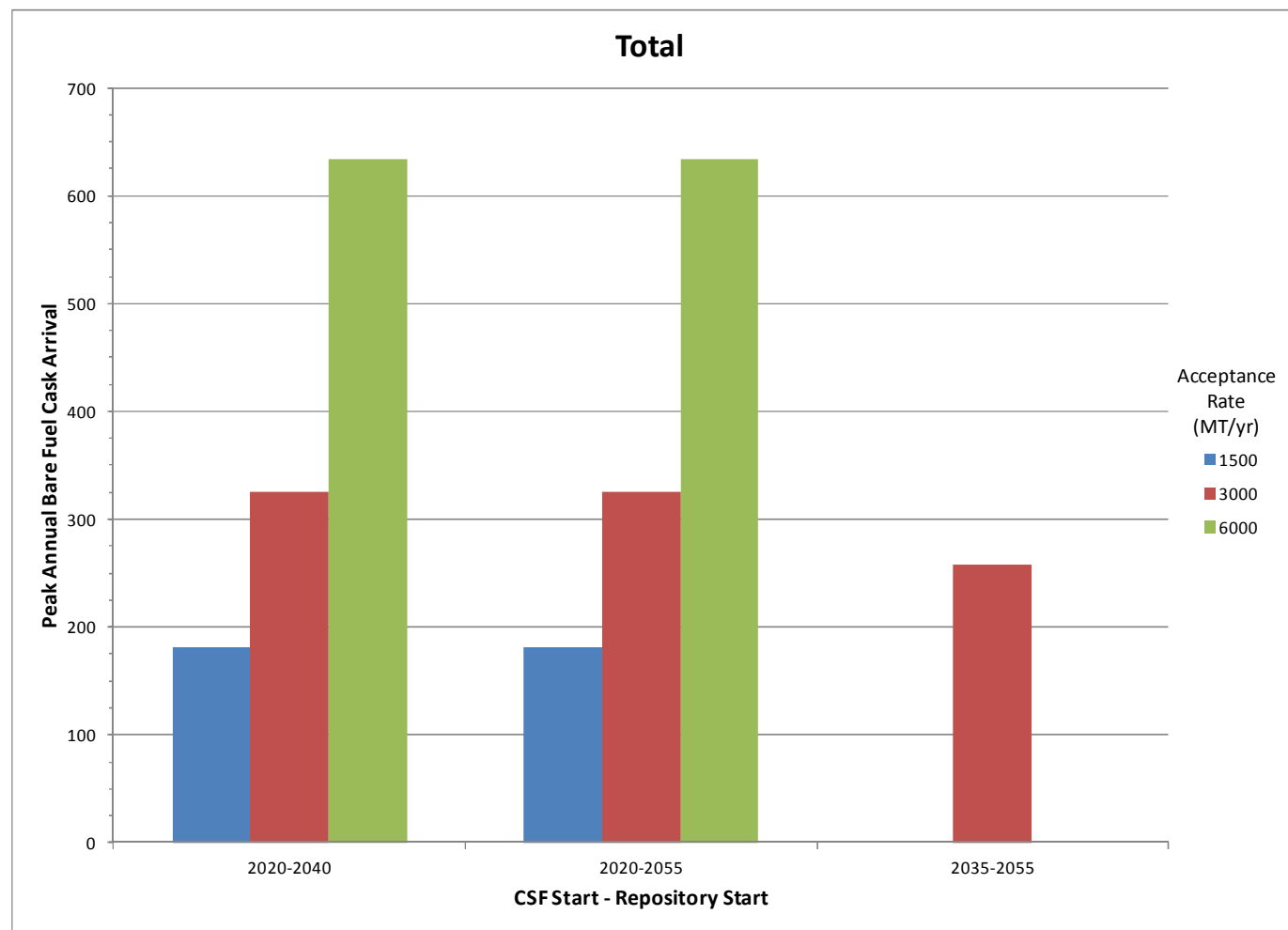

Figure A-77. Peak Bare Fuel Cask Arrivals, Case 2 (Canisters and Bare Fuel, Re-Package at CSF) 


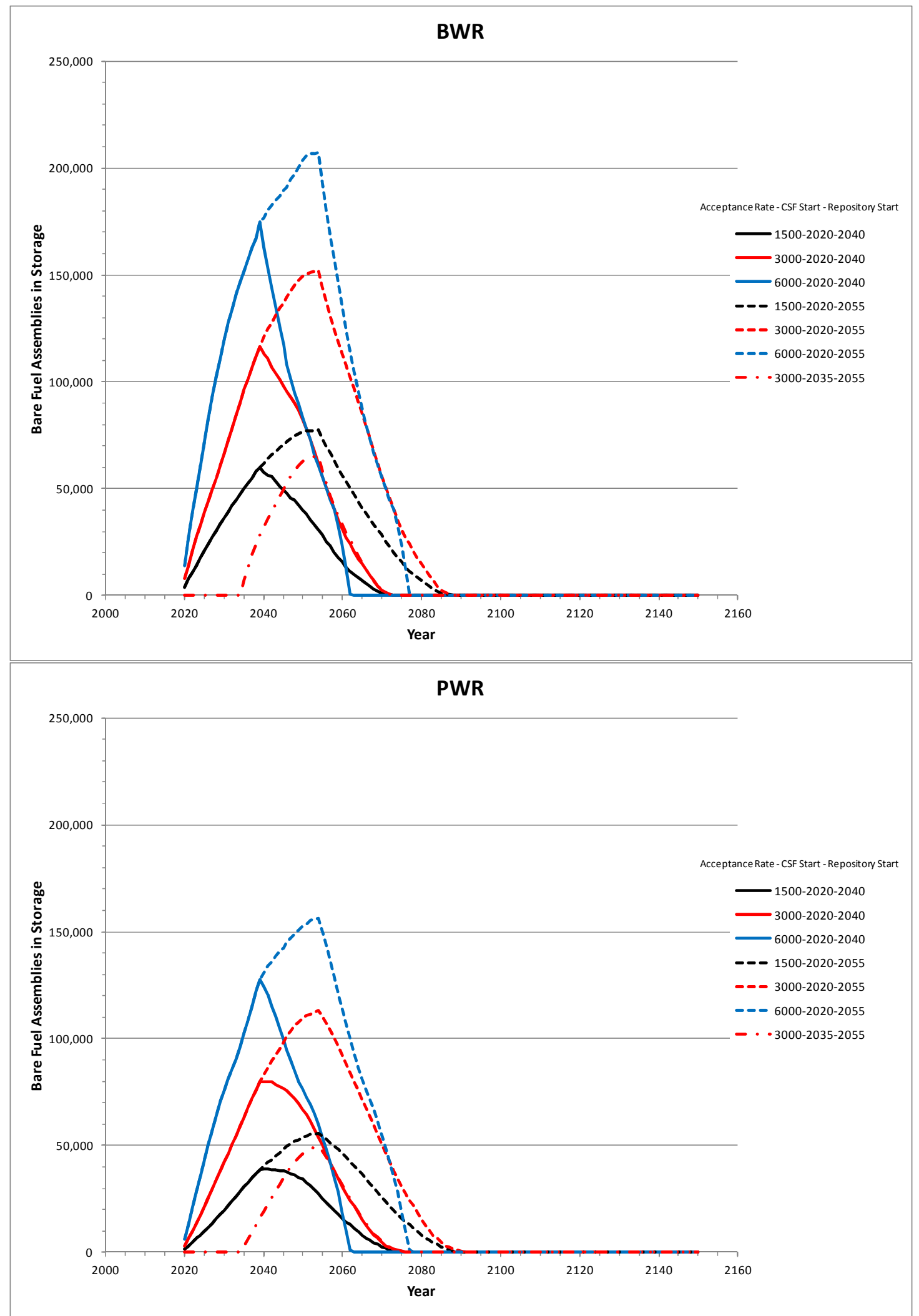

Figure A-78. Bare Fuel Inventory, Case 4 (Canisters and Bare Fuel, Re-Package at CSF) 


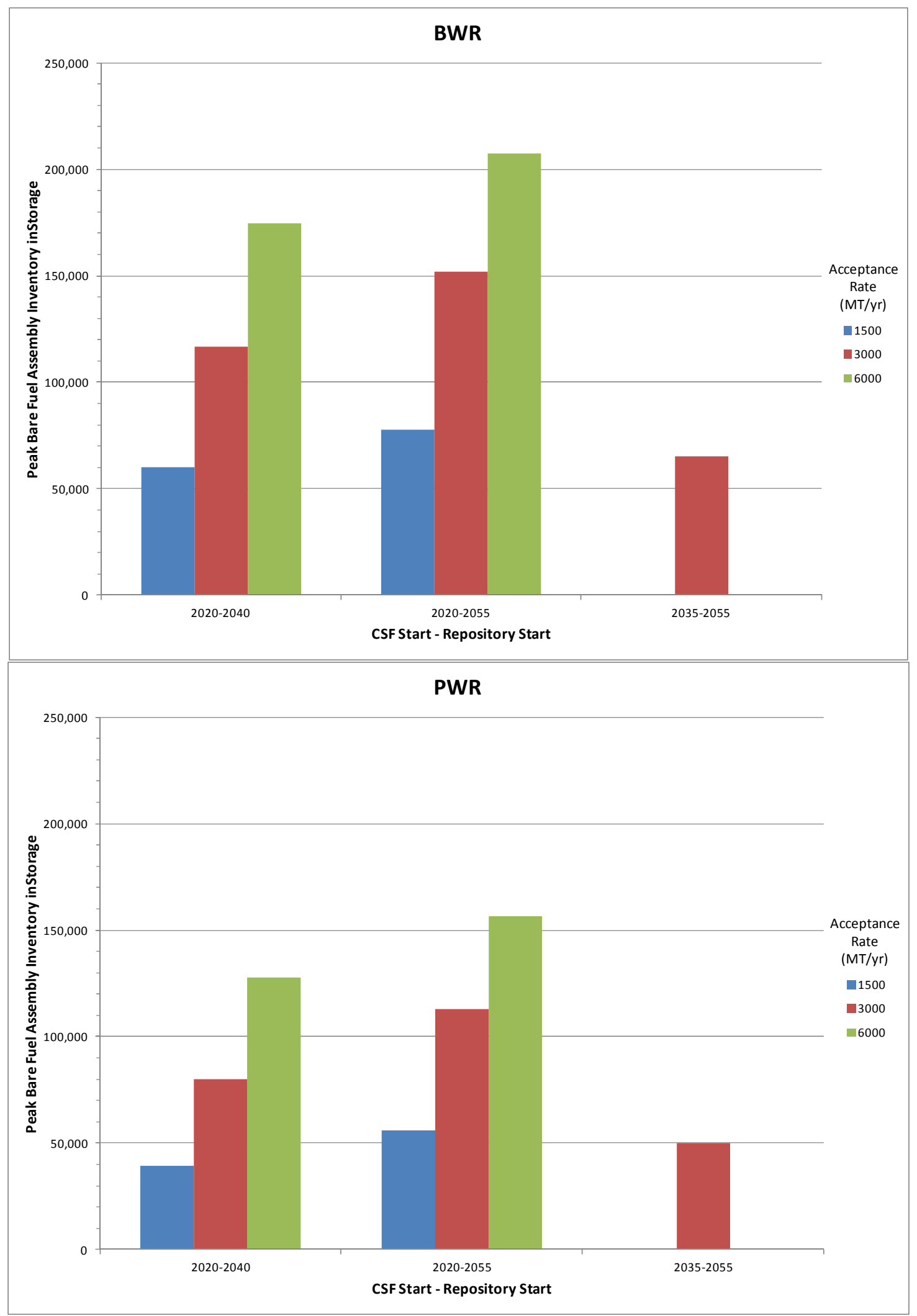

Figure A-79. Peak Bare Fuel Inventory, Case 4 (Canisters and Bare Fuel, Re-Package at CSF) 


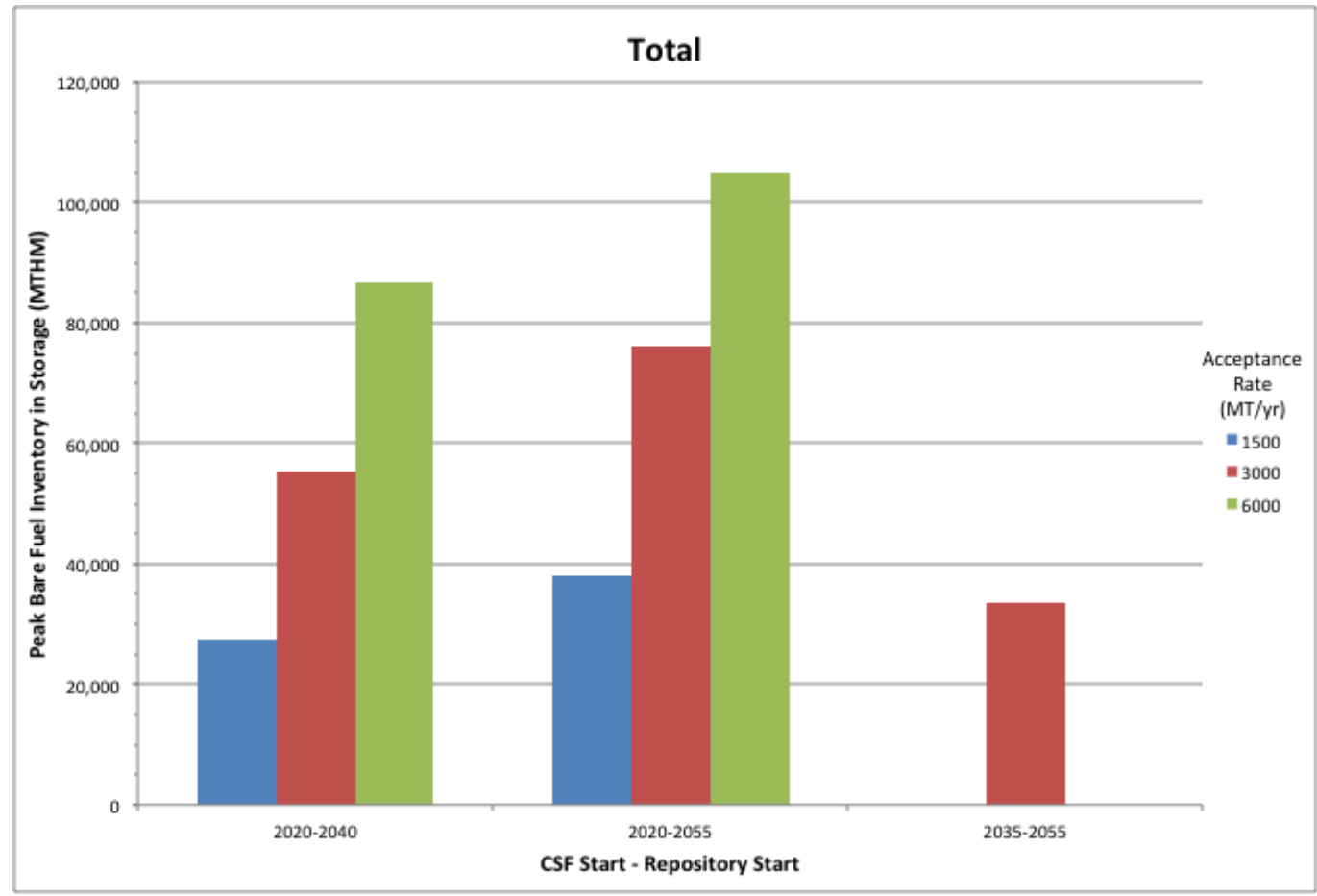

Figure A-80. Peak Bare Fuel MTHM Inventory, Case 4 (Canisters and Bare Fuel, Re-Package at CSF) 


\section{A-3.3 Detailed Results of Fuel Handling Logistics}

Detailed results for each of the cases are provided in a number of figures as shown in Table A-31. These figures show time-dependent and peak packaging/re-packaging rates for both canistered and bare fuel.

\section{Canister Re-Packaging}

When all UNF is transported from the reactors in existing size canisters (Cases 1 and 3) it must all be repackaged into disposable canisters. As shown in Table A-29, 11,150 - 11,200 canisters must ultimately be re-packaged. Because of the modeling assumption that once the repository is in operation it will accept UNF for disposal at the same rate it is accepted from the reactors, the annual rate that canisters are re-packaged is a simple function of the UNF acceptance rate, with higher acceptance rates leading to higher re-package rates. This trend is shown in Figures A-81, A-82, A-85, and A-88.

Canister re-packaging becomes more complex when bare fuel is transported from the at-reactor used fuel pools when the CSF becomes operational (Cases 2 and 4). The youngest-fuel-first (YFF) acceptance prioritization for shipments from the reactors and the first-in-first-out (FIFO) prioritization for shipments from the CSF affect when UNF in canisters is re-packaged.

When re-packaging occurs at the repository (Case 2, Figures A-84 and A-87) and repository operations begin in 2035, the YFF acceptance prioritization gives preference to shipping bare fuel from the reactor sites to the repository for packaging. Canisters are transported from the reactors to the repository for repackaging only when there is no bare fuel available for transport from the reactor sites. This trend is shown in Figure A-84.

When repository operations begin in 2035, all bare fuel has either been transported to the CSF and is being stored uncanisterized or has been transferred to canisters being stored either at reactor sites or at the CSF. No bare fuel is available for transport from the reactor sites when repository operations begin and canistered UNF is shipped from the reactors to the repository for re-packaging. Once the canistered UNF from the reactor sites is removed and re-packaged, the repository then begins drawing fuel from the CSF. The YFF-FIFO acceptance prioritization results in bare fuel first being shipped from the CSF to the repository, followed by the shipment of canisters. This trend is also shown in Figure A-84.

Table A-31. Packaging/Re-Packaging Logistic Results Figure Matrix

\begin{tabular}{|c|c|c|c|c|c|c|}
\hline & $\begin{array}{c}\text { Annual } \\
\text { Canister Re- } \\
\text { Packaging }\end{array}$ & $\begin{array}{c}\text { Peak } \\
\text { Annual } \\
\text { Canister } \\
\text { Re- } \\
\text { Packaging }\end{array}$ & $\begin{array}{c}\text { Annual } \\
\text { Bare Fuel } \\
\text { Packaging }\end{array}$ & $\begin{array}{c}\text { Peak } \\
\text { Annual } \\
\text { Bare Fuel } \\
\text { Packaging }\end{array}$ & $\begin{array}{l}\text { Cumulative } \\
\text { Bare Fuel } \\
\text { Assemblies } \\
\text { Packaged }\end{array}$ & $\begin{array}{c}\text { Cumulative } \\
\text { Canisters } \\
\text { Re- } \\
\text { Packaged }\end{array}$ \\
\hline $\begin{array}{l}\text { Case } 1 \text { (all } \\
\text { canisters, re- } \\
\text { package at } \\
\text { repository) }\end{array}$ & Figure A-81 & Figure A-85 & & & & \\
\hline $\begin{array}{l}\text { Case } 2 \\
\text { (canisters and } \\
\text { bare fuel, re- } \\
\text { package at } \\
\text { repository) }\end{array}$ & Figure A-83 & Figure A-87 & Figure A-89 & Figure A-91 & Figure A-93 & Figure A-94 \\
\hline $\begin{array}{l}\text { Case } 3 \text { (all } \\
\text { canisters, re- } \\
\text { package at CSF) }\end{array}$ & Figure A-82 & Figure A-86 & & & & \\
\hline $\begin{array}{l}\text { Case } 4 \\
\text { (canisters and } \\
\text { bare fuel, re- } \\
\text { package at CSF) }\end{array}$ & Figure A-84 & Figure A-88 & Figure A-90 & Figure A-92 & Figure A-93 & Figure A-94 \\
\hline
\end{tabular}


When repository operations begin in 2035, all bare fuel has either been transported to the CSF and is being stored uncanisterized or has been transferred to canisters being stored either at reactor sites or at the CSF. No bare fuel is available for transport from the reactor sites when repository operations begin and canistered UNF is shipped from the reactors to the repository for re-packaging. Once the canistered UNF from the reactor sites is removed and re-packaged, the repository then begins drawing fuel from the CSF. The YFF-FIFO acceptance prioritization results in bare fuel first being shipped from the CSF to the repository, followed by the shipment of canisters. This trend is also shown in Figure A-84. It should be noted that this would be the hottest fuel at the CSF and under actual conditions would not likely be the first fuel shipped to the repository.

The peak annual canister re-packaging rate when both bare fuel and canisters are packaged/re-packaged at the repository is not solely dependent on the acceptance rate, as shown in Figure A-87, as is the trend when all UNF is placed in canisters. The peak annual canister re-packaging rate is also influenced by the amount of fuel transferred to canisters at the reactor sites; with higher acceptance rates, more UNF is transported to the repository bare instead of being canisterized at the reactor sites first. This is evident in the decrease in the peak annual re-packaging rate for an acceptance rate of $6000 \mathrm{MT} / \mathrm{yr}$ as compared to $3000 \mathrm{MT} / \mathrm{yr}$.

Similar trends are seen when re-packaging occurs at the CSF (Case 4) and both bare fuel and canisters are transported to the CSF. The YFF-FIFI acceptance prioritization results in a preference for bare fuel packaging and subsequent transport to the repository when it becomes operational. This trend is shown in Figure A-84. The peak annual repackaging rate for re-packaging at the CSF, shown in Figure A-88, is similar in magnitude to and follows the same trend as when re-packaging occurs at the repository, shown in Figure A-87. These similarities result from roughly the same number of canisters having to be repackaged over the same period of time at either the CSF or at the repository.

\section{Bare Fuel Packaging}

The trends discussed above for canister re-packaging also influence bare fuel packaging trends as shown in Figures A-89 and A-90. In general, when bare fuel is being packaged it displaces the re-packaging of UNF in canisters.

Peak annual bare fuel packaging rates depend on the acceptance rate from the reactor sites as shown in Figures A-91 and A-92. Higher acceptance rates result in more UNF being shipped from the reactors to either the CSF or the repository. The peak annual bare fuel packaging rate for bare fuel packaging at the CSF, shown in Figure A-92, is similar in magnitude to and follows the same trend as when bare fuel packaging occurs at the repository, shown in Figure A-91. These similarities result from roughly the same number of bare fuel assemblies having to be packaged over the same period of time at either the CSF or at the repository.

\section{Cumulative Packaging and Re-Packaging}

As discussed above, when all UNF is transported from the reactors in canisters, 11,150 - 11,200 canisters must ultimately be re-packaged (Cases 1 and 3).

Transporting UNF as bare fuel to the CSF when it starts operations affects the total number of canisters that must be re-packaged and the amount of bare fuel that has to be packaged. Figure A-93 shows the cumulative number of bare fuel assemblies that are packaged and Figure A-94 shows the cumulative number of canisters that are re-packaged. Cumulative amounts are very similar for both Cases 2 (packaging/re-packaging at repository) and Case 4 (packaging/re-packaging at CSF) so single figures are provided. As the acceptance rate increases, less fuel is transferred to canisters and remains as bare fuel until it is packaged. 


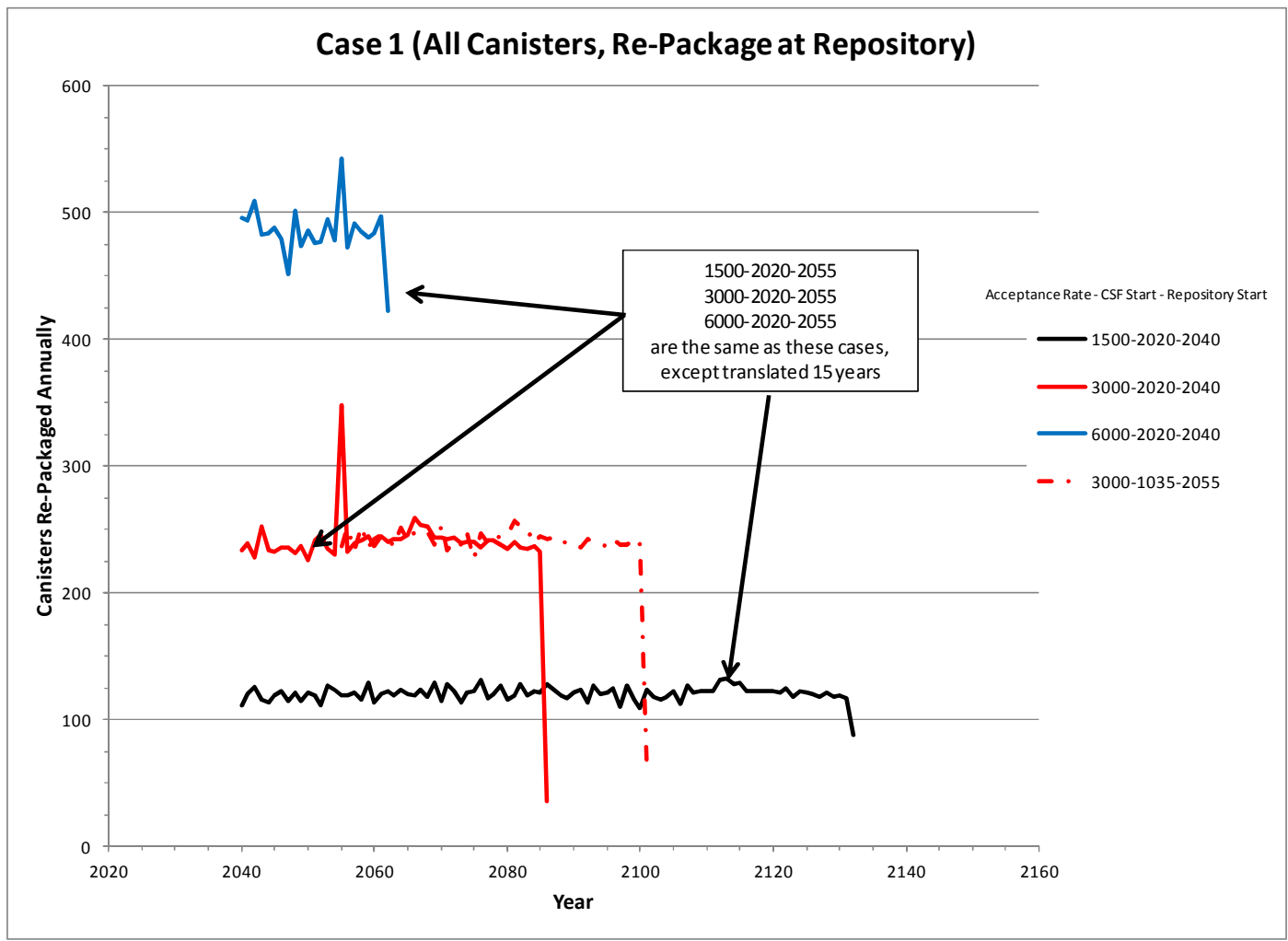

Figure A-81. Annual Canister Re-Packaging Rate, Case 1 (All Canisters, Re-Package at Repository)

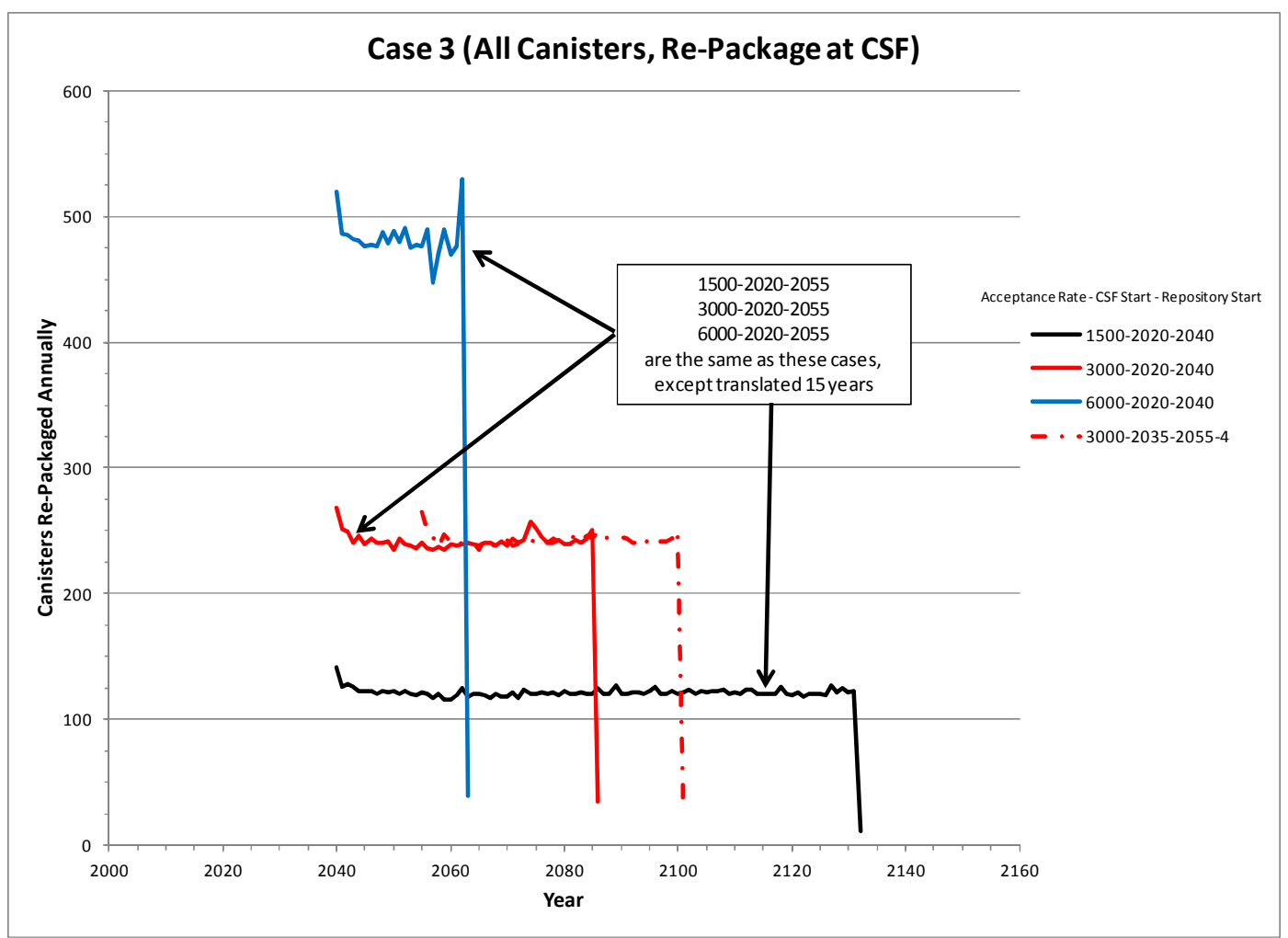

Figure A-82. Annual Canister Re-Packaging Rate, Case 3 (All Canisters, Re-Package at CSF) 

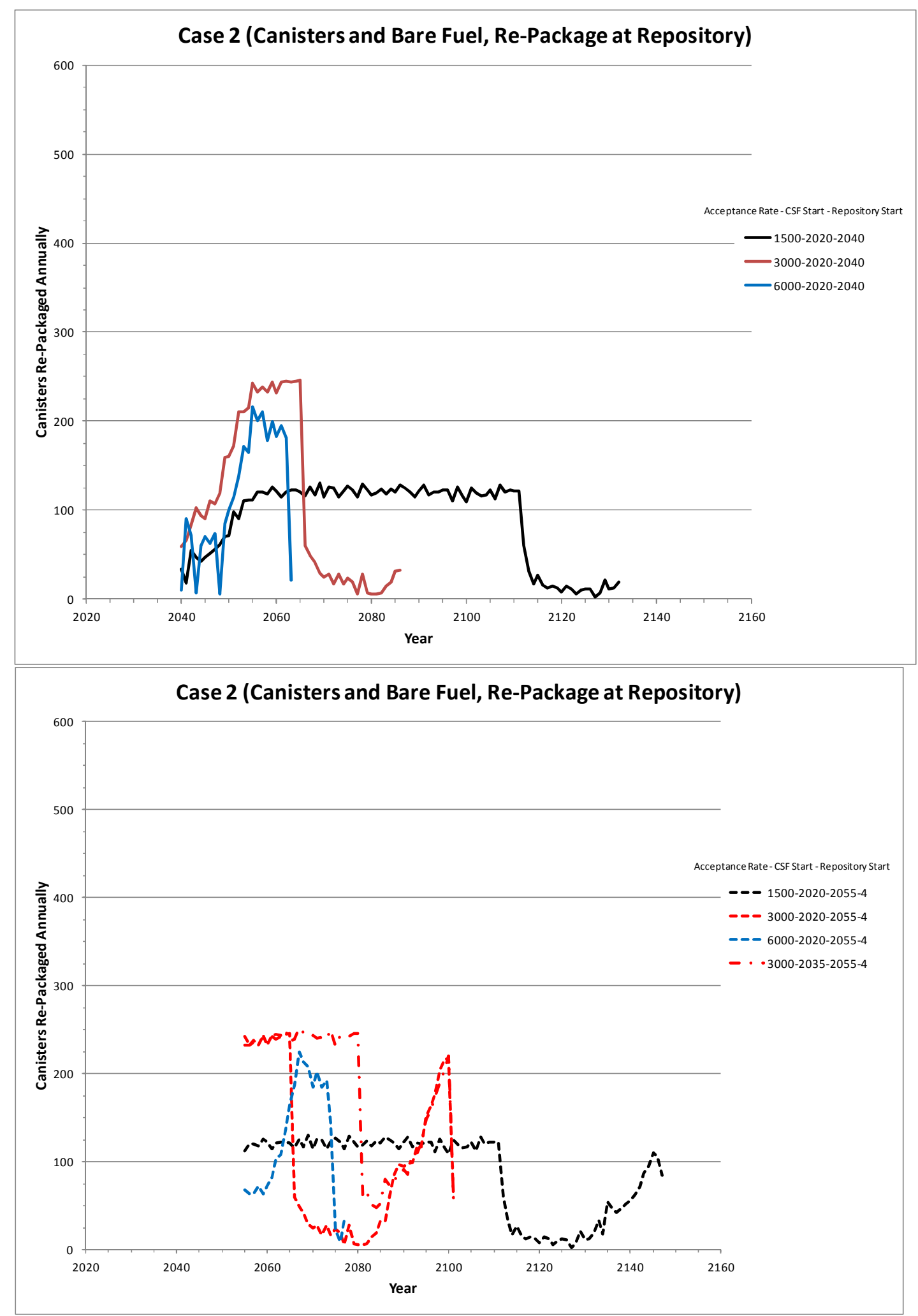

Figure A-83. Annual Canister Re-Packaging Rate, Case 2 (Canisters and Bare Fuel, Re-Package at Repository) 

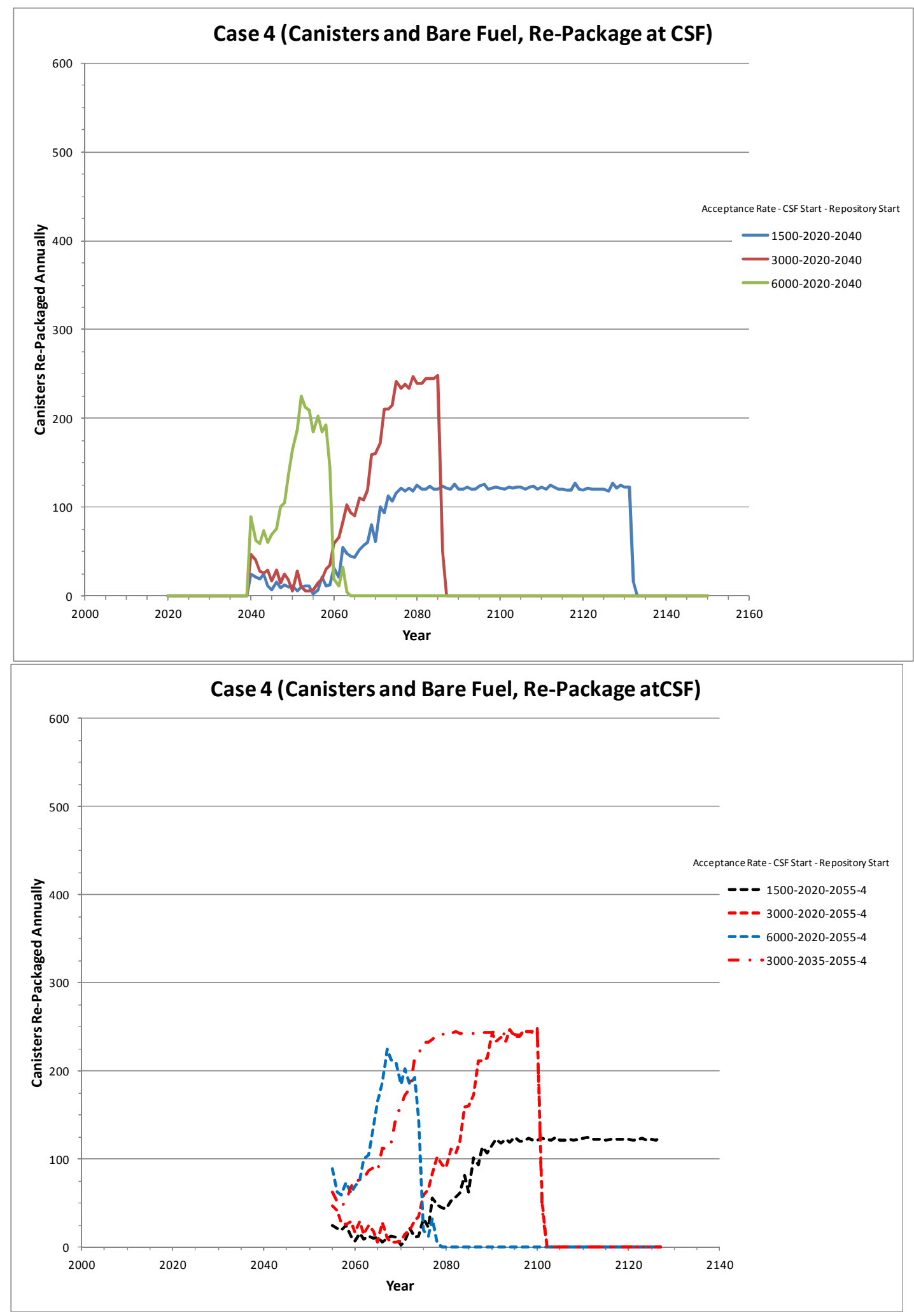

Figure A-84. Annual Canister Re-Packaging Rate, Case 4 (Canisters and Bare Fuel, Re-Package at CSF) 


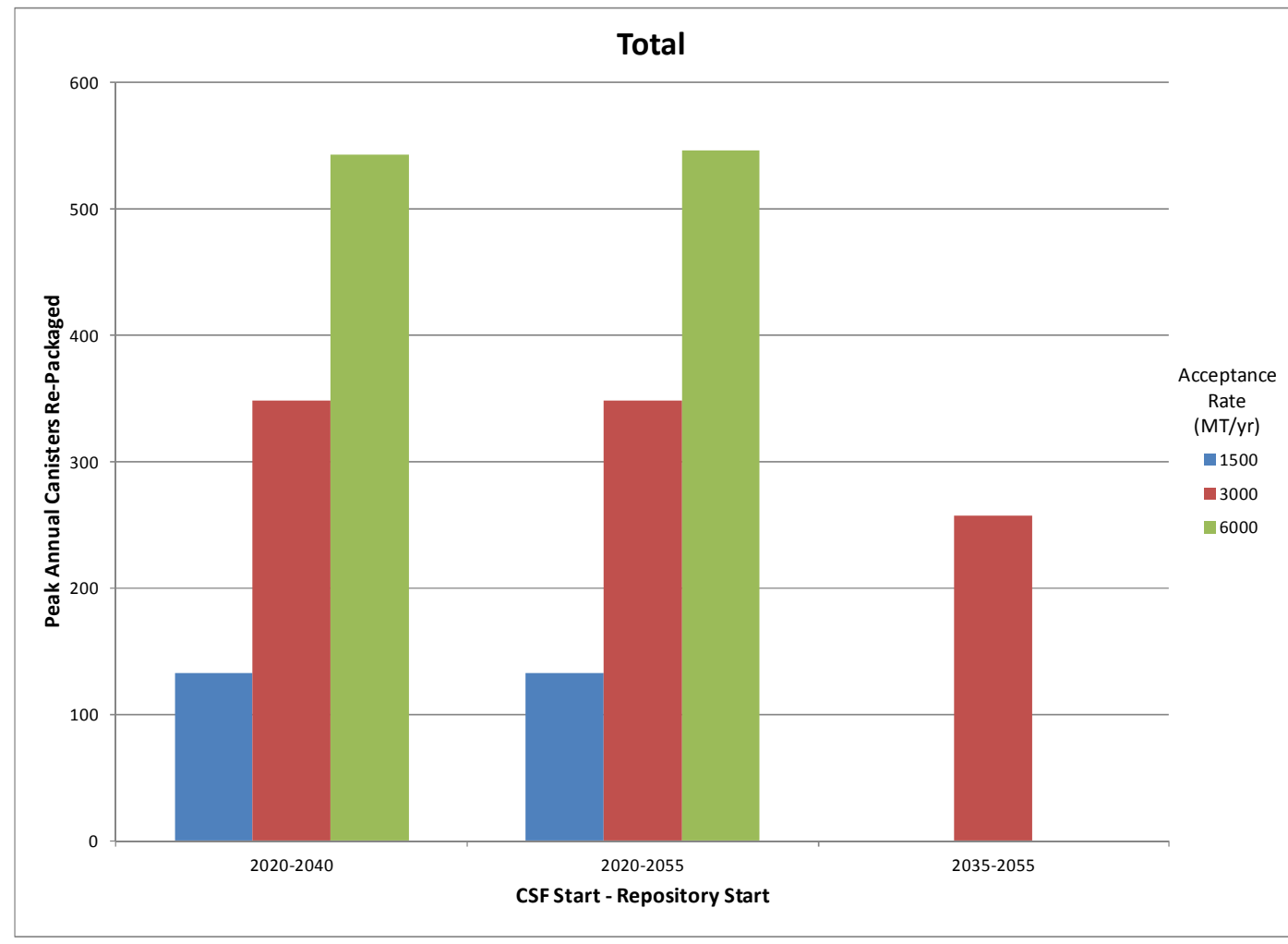

Figure A-85. Peak Annual Canister Re-Packaging Rate, Case 1 (All Canisters, Re-Package at Repository)

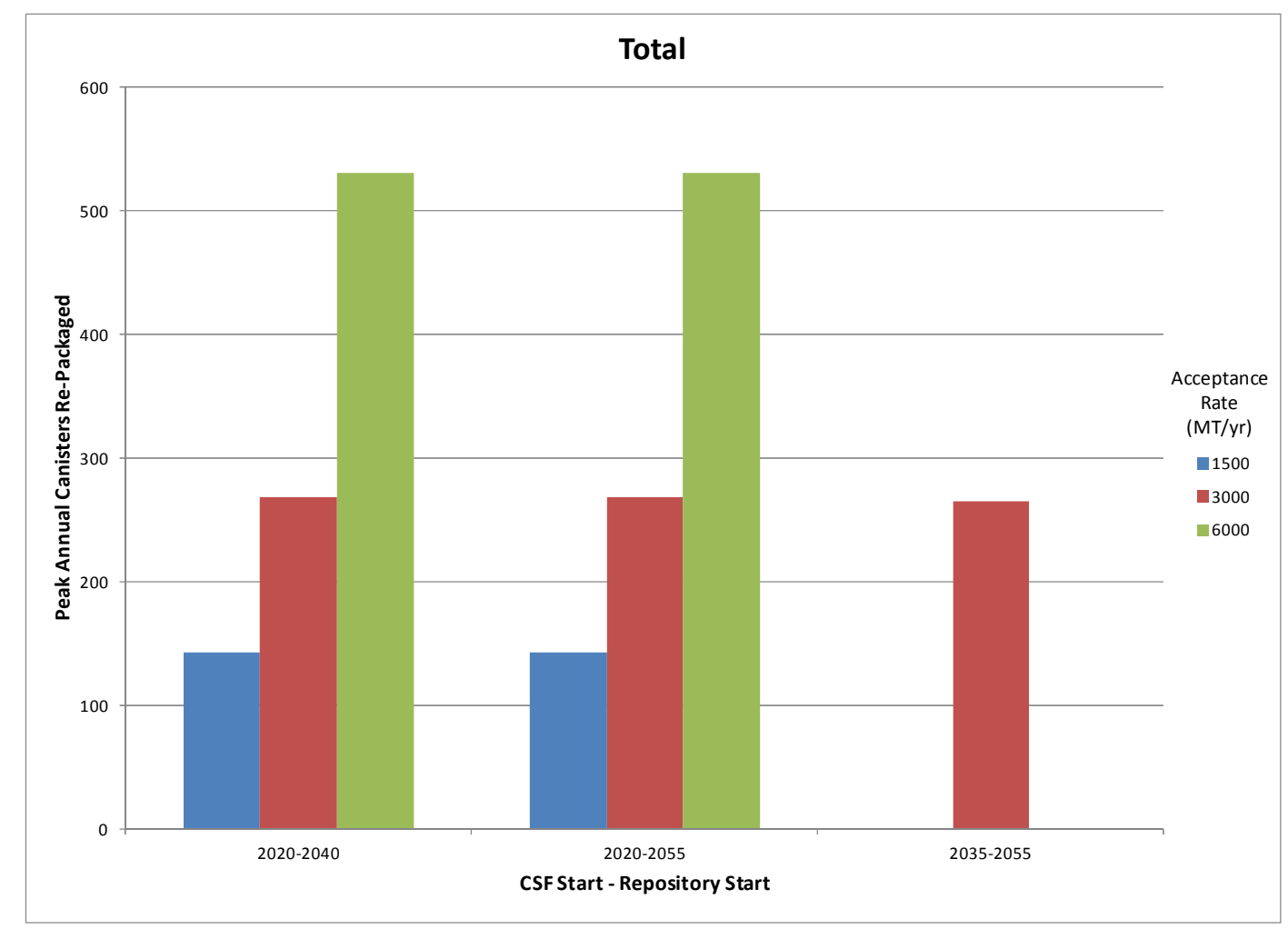

Figure A-86. Peak Annual Canister Re-Packaging Rate, Case 3 (All Canisters, Re-Package at CSF) 


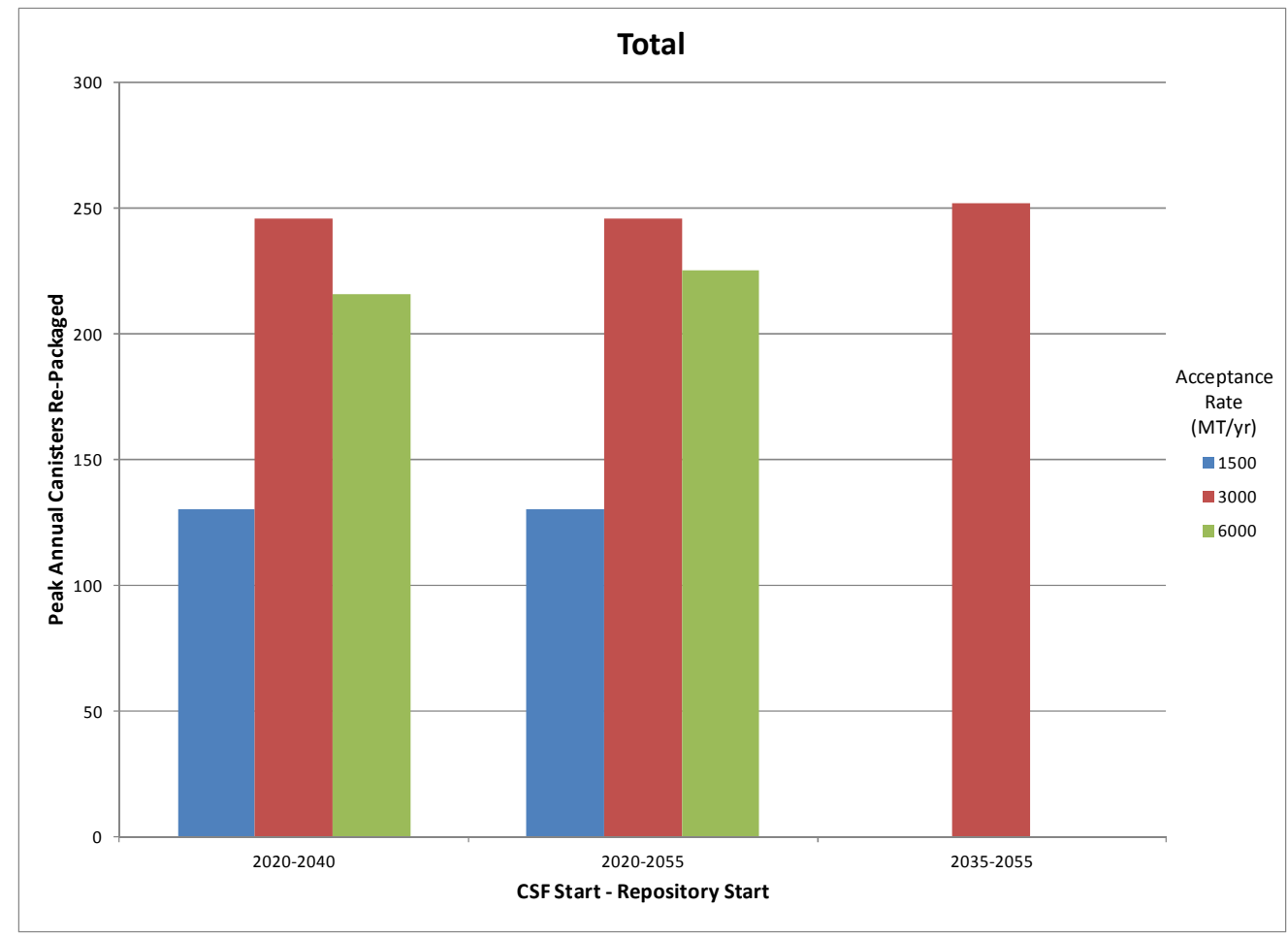

Figure A-87. Peak Annual Canister Re-Packaging Rate, Case 2 (Canisters and Bare Fuel, Re-Package at Repository)

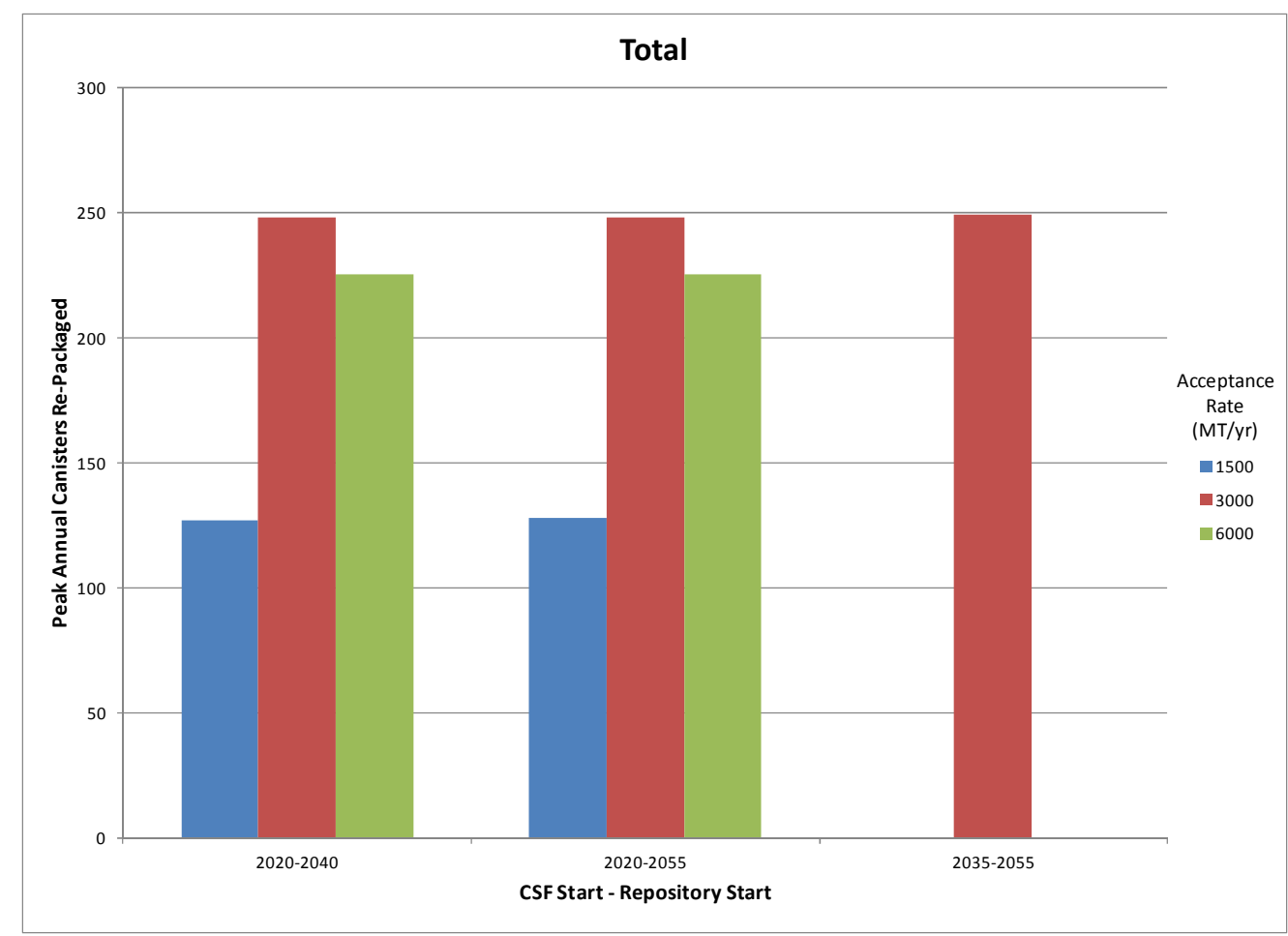

Figure A-88. Peak Annual Canister Re-Packaging Rate, Case 4 (Canisters and Bare Fuel, Re-Package at CSF) 

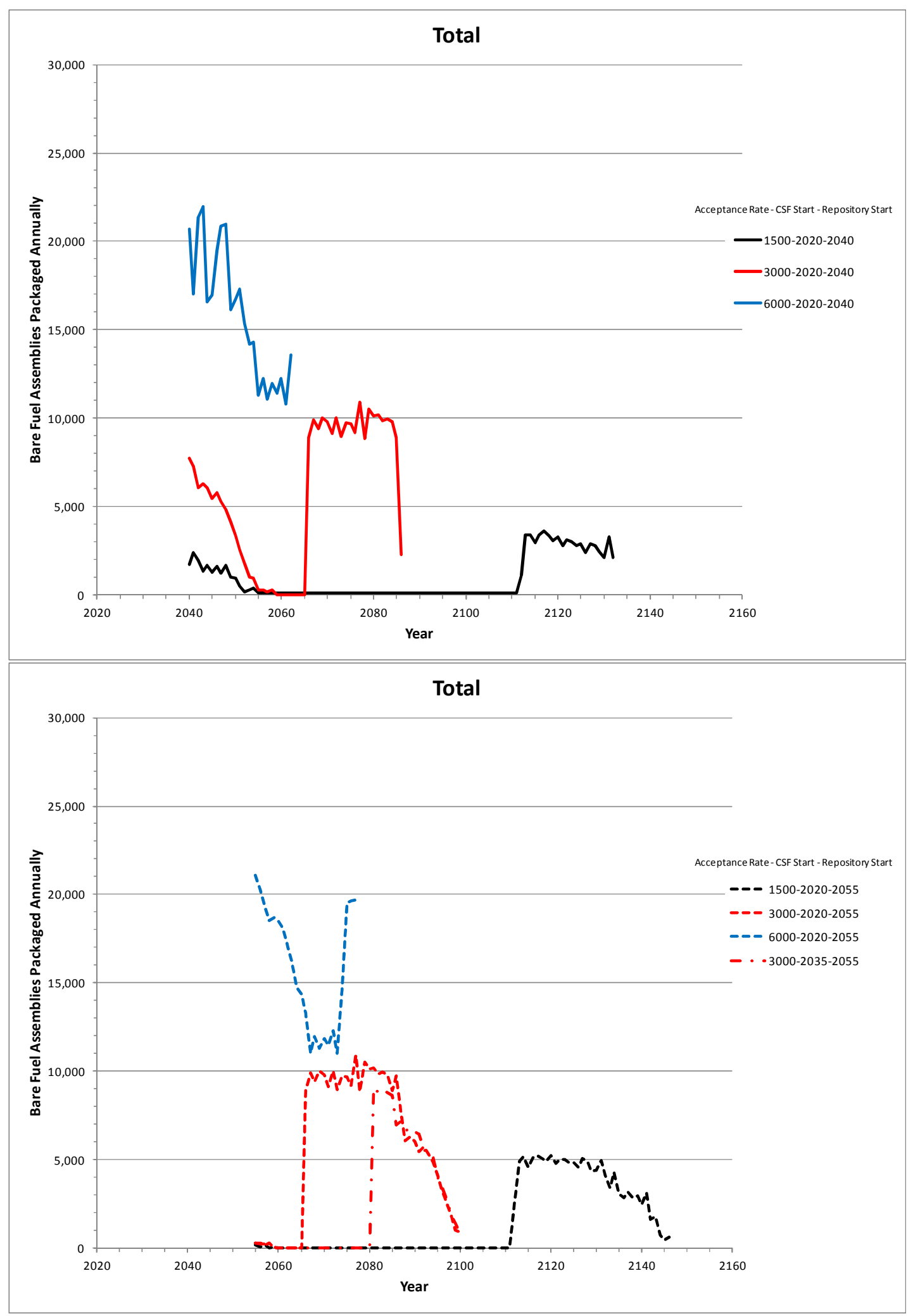

Figure A-89. Annual Bare Fuel Packaging Rate, Case 2 (Canisters and Bare Fuel, Re-Package at Repository) 


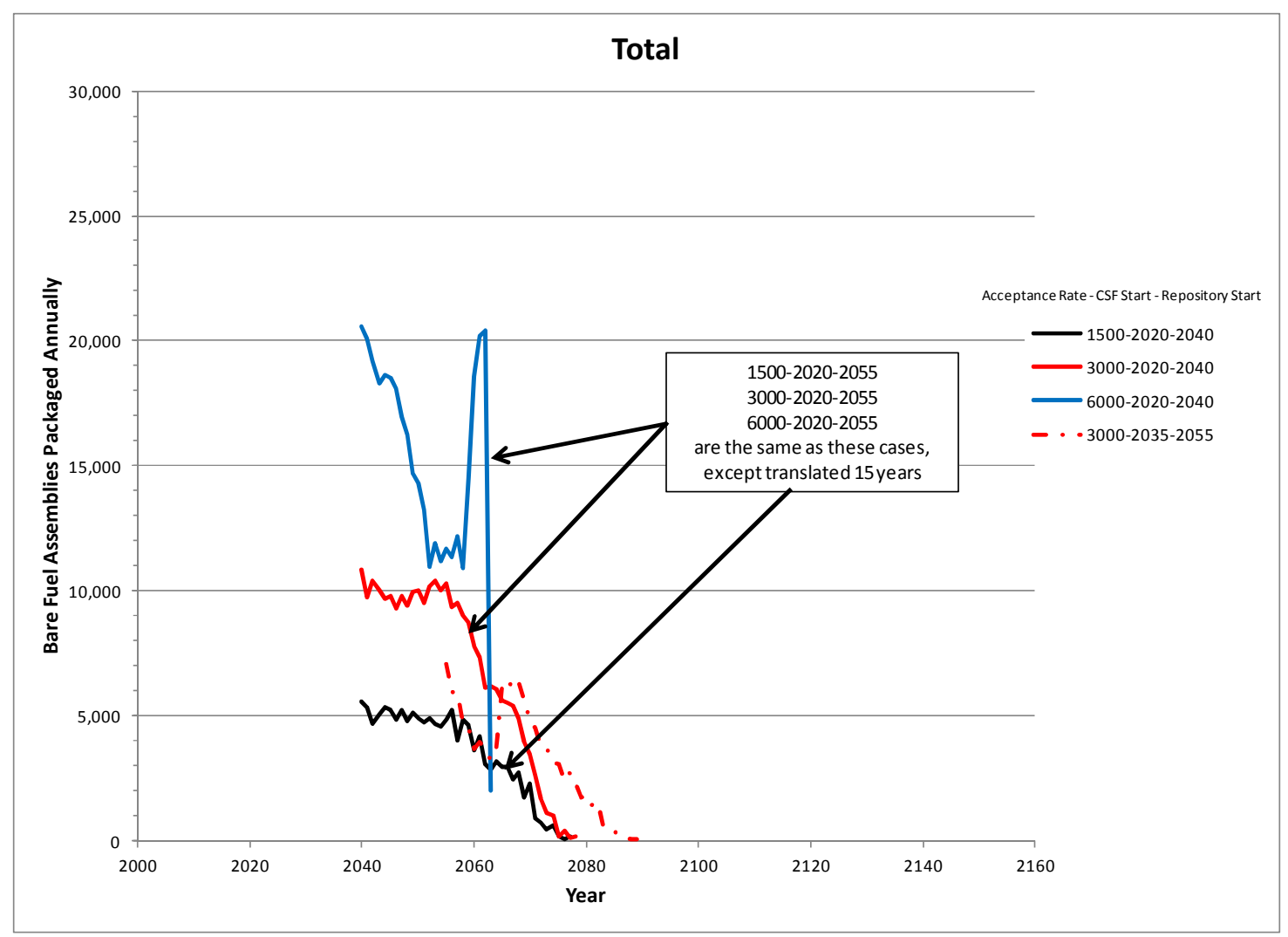

Figure A-90. Annual Bare Fuel Packaging Rate, Case 4 (Canisters and Bare Fuel, Re-Package at CSF) 


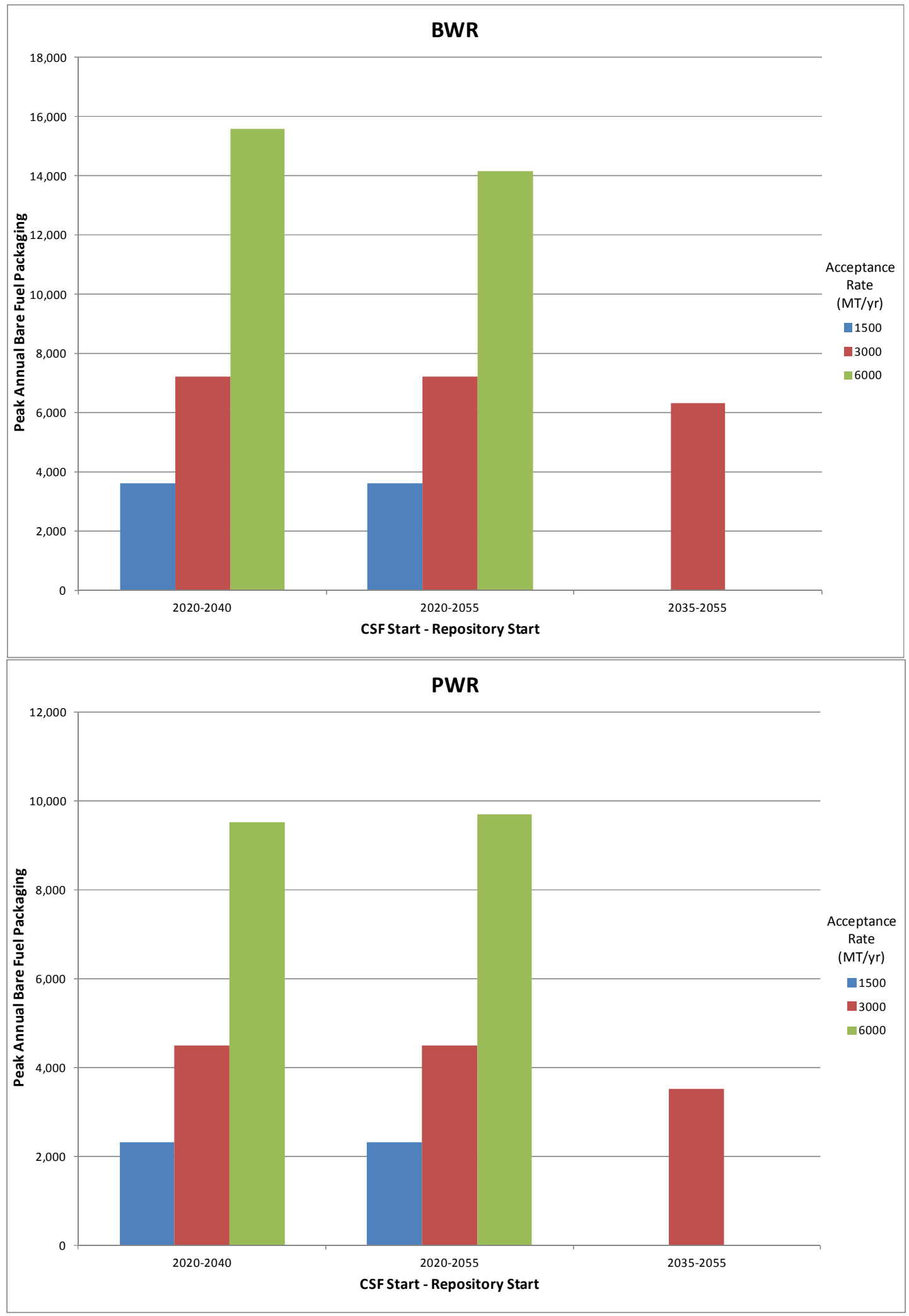

Figure A-91. Peak Annual Bare Fuel Packaging Rate, Case 2 (Canisters and Bare Fuel, Re-Package at Repository) 

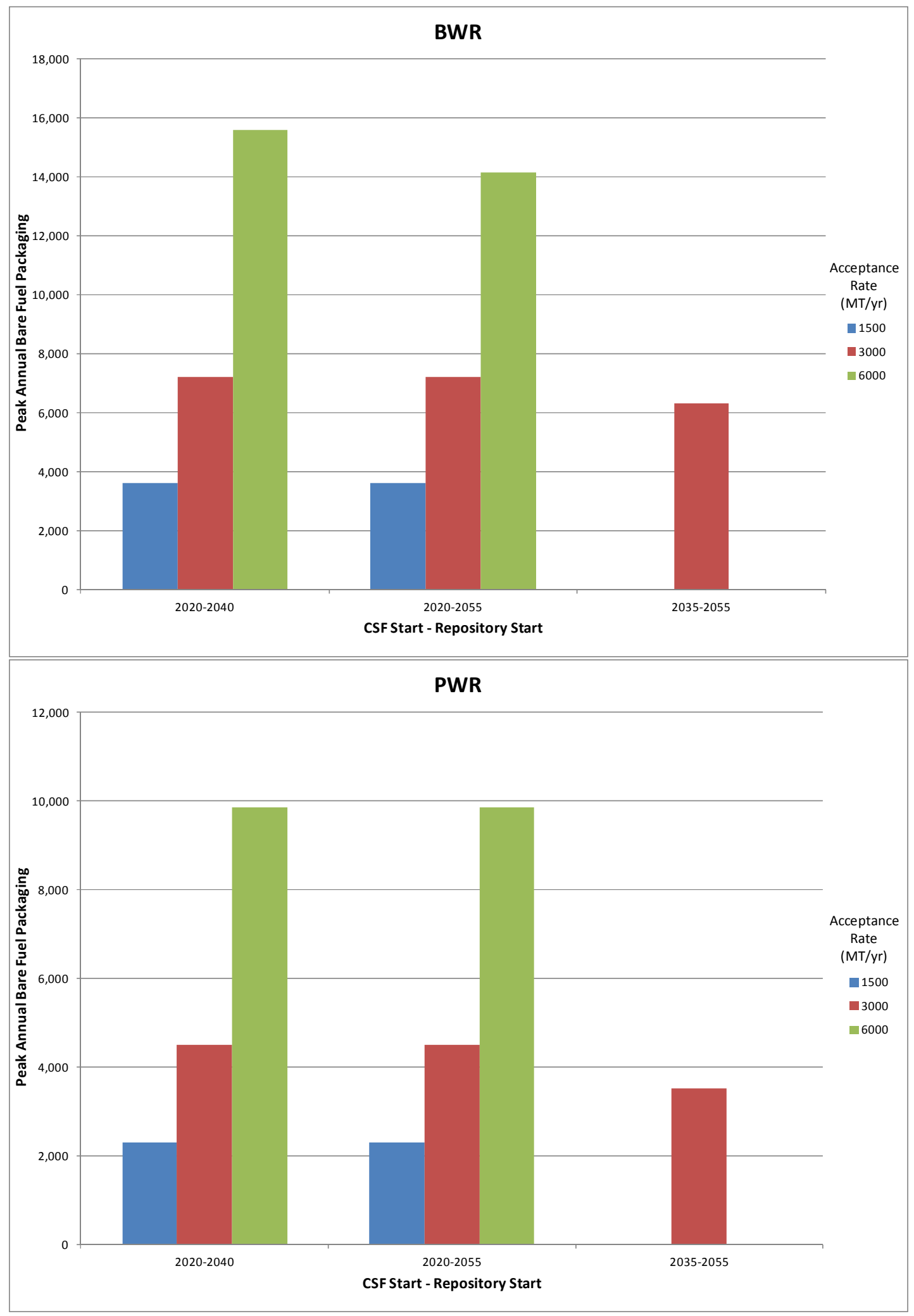

Figure A-92. Annual Bare Fuel Packaging Rate, Case 4 (Canisters and Bare Fuel, Re-Package at CSF) 

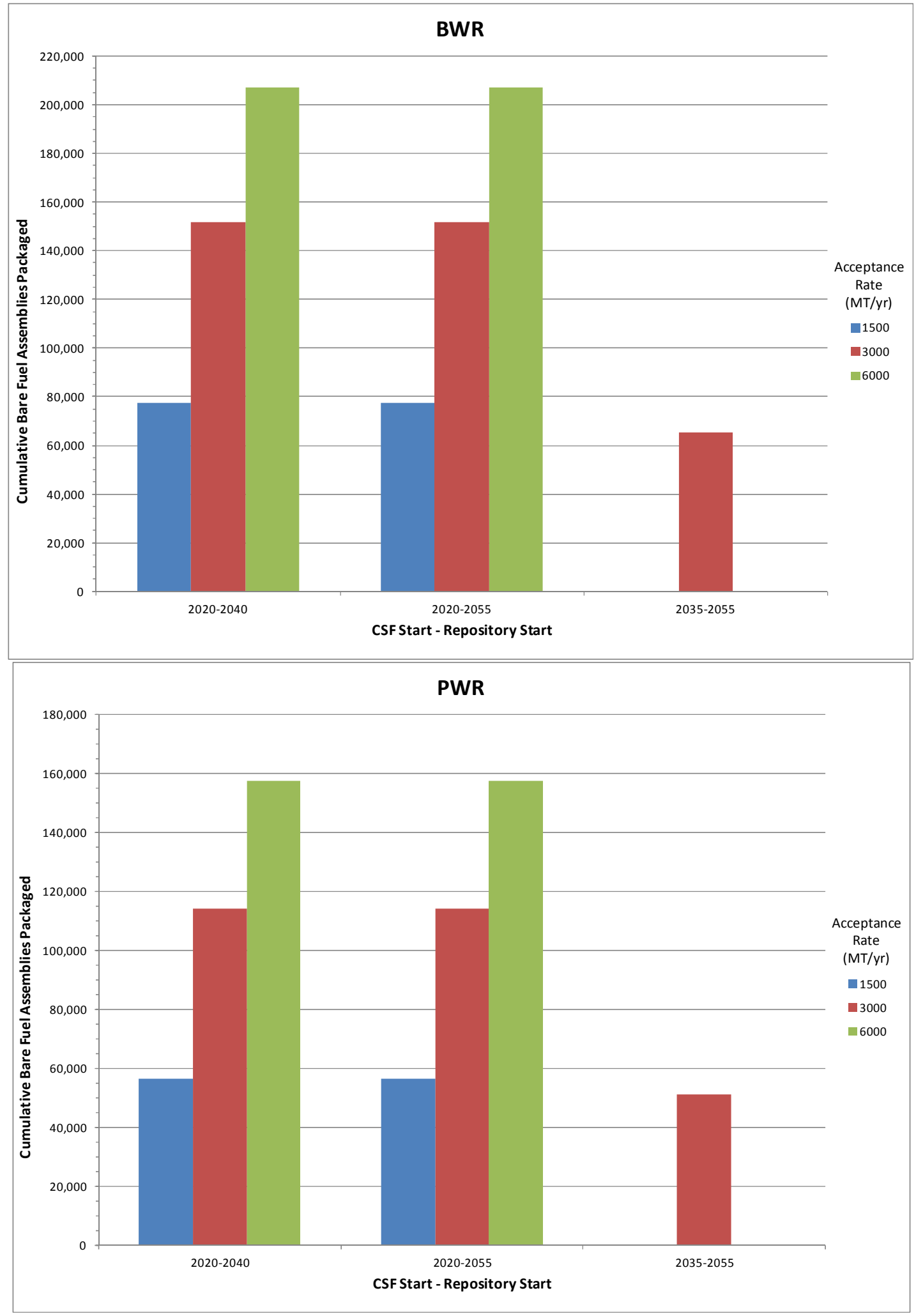

Figure A-93. Cumulative Bare Fuel Packaging, Cases 2 and 4 (Canisters and Bare Fuel, Re-Package at CSF / Repository) 


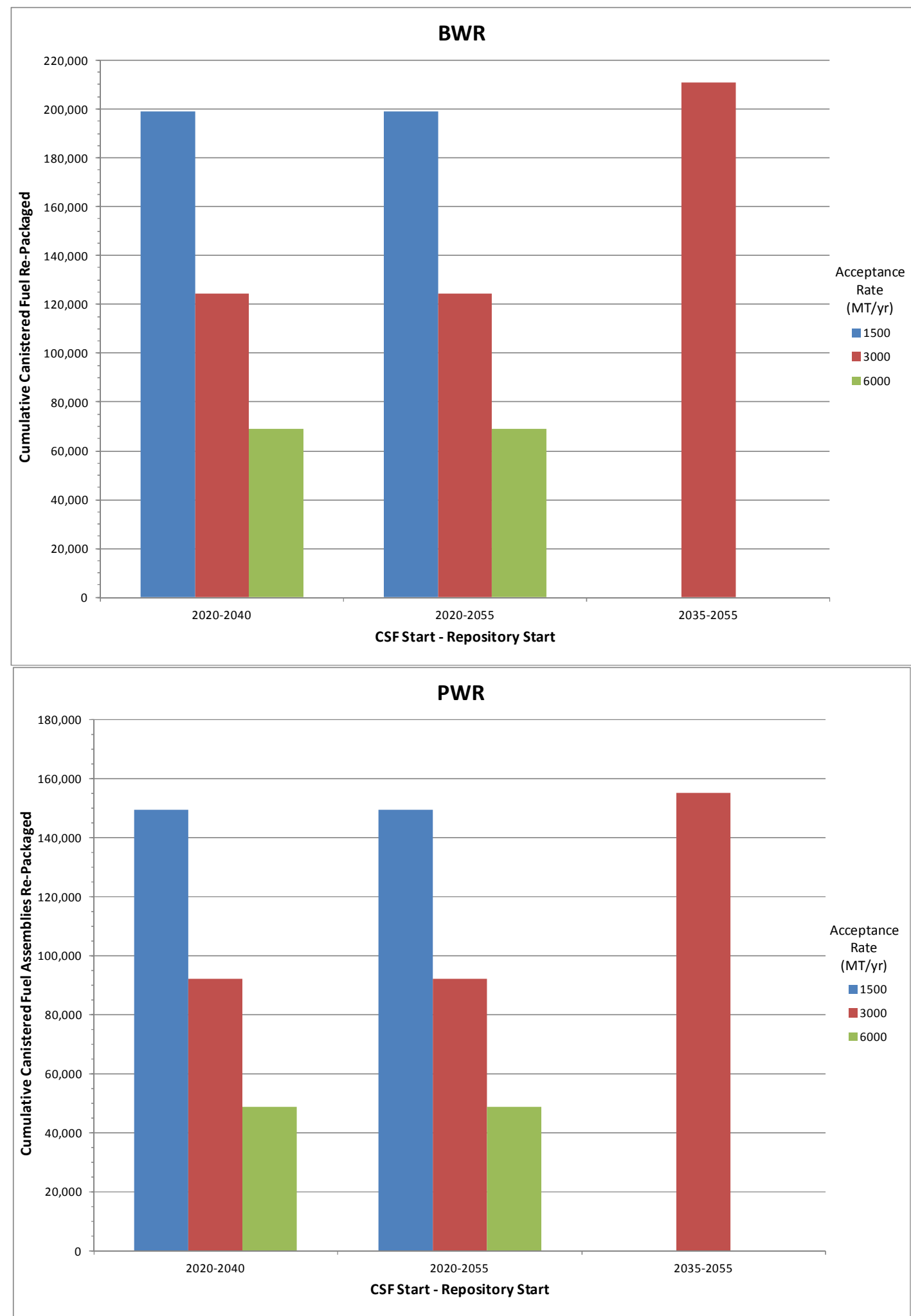

Figure A-94. Cumulative Canistered Fuel Packaging, Cases 2 and 4 (Canisters and Bare Fuel, Re-Package at CSF / Repository) 


\section{A-4. Transportation Logistics}

The cases and input parameters presented in Section 3 were simulated using the Transportation Operations Model (TOM) portion of the TSL simulation model (Busch et al. 2012) to evaluate transportation logistics implications for the cases considered (summarized in Table A-27 with details provided in Section 3.1). The assumptions and input/boundary conditions are provided in Section 3.3. The shipment of UNF from the reactors to the CSF or repository and from the CSF to the repository, depending on the specific case being evaluated, were calculated using the CALVIN portion of the TSL model and a shipping schedule was input into TOM for transportation logistics calculations.

A discussion of the transportation logistics (and ultimately estimated cost)s associated with moving UNF from its current (or projected) location to interim storage facilities and ultimately to a final destination repository requires an accounting of the assumptions and simplifications that must be made in such an analysis. These include assumption made in the transportation cost estimates, the transportation network, and the location of the storage facilities and repository.

While the present state of the transportation network is known, both rail and road (and to a much lesser extent, waterway) networks are changing entities, and an assumption implicit in the analysis is that the networks as they are now will be usable at such time as UNF is to be moved. Many sites have rails that go up to or into the utilities' control area, but in many cases these rail lines have not used since construction of the reactors. The lines are still in the network, but it may be that refurbishment would be required if those rail lines are to be utilized in the transportation of the UNF. Of particular concern are the rail crossings and the bridges. As the railroad infrastructure ages, it may no longer be capable of handling the weight of the cask cars. That said, the rail routes and truck routes for this analysis were developed using the transportation networks as they currently exist.

Not every reactor site currently has the capability for direct loading of canisters onto rail cars. However, when considering that all UNF is loaded into canisters at the reactor sites prior to transport either to the CSF or the repository, it was assumed that every site would be made capable of transporting these large canisters to the nearest railhead (i.e., by heavy-haul truck).

Another consideration is that while the cost of trailer transportation over the road network is, for the most part, proportional to the distance travelled, the cost of routing over the railroad network is not as straightforward. Each rail line is owned by a company, and that company will limit which other railroad companies can use the line. The routing of cargo by dedicated rail means that the shipment will be "handed off" from one carrier to another along its path, and contracting with each carrier may be required. Because the details of such contracting are impossible to model, the TOM software includes a proxy routing cost function based on distance.

It was assumed that there can only be one consist (i.e., cask car, escort car, security car) loading at the reactor at a time. There are economies of scale that can be realized by accomplishing all of the loading needed in a calendar year at once. In particular, when cranes have to be brought in to the reactor site, there is generally a deployment cost which covers the cost involved in bringing the crane to the site and setting it up. There is also a daily cost for having the crane on-site, as well as an hourly cost for the operation of the crane, which will cover the cost of the operating crew. However, these rates would likely again be contracted between whoever implements the transportation of UNF and the company providing the equipment and crews. Modeling the details of such contracting is complex, and the TOM model assumes that the crane deployment costs are charged for each rail consist.

It is assumed that the unloading capability at the interim storage facilities and repository are unlimited (unconstrained). While this would not likely be the actual case, it is assumed that the unloading capabilities will be constructed so as to not be the bottleneck in the transportation process. Once the details of the interim storage facilities and repository are established, a constraint could be added to TOM to ensure that the unloading infrastructure is not overtaxed. 
The maximum consist size was assumed at three cars for a rail transport and three trailers for a truck convoy. Train dynamics and weight restrictions on bridges limit the number of railcars that can be included in a consist. Security concerns limit the number of trucks that can be observed by an escort truck. Also, once casks are loaded on to transportation assets, they must be secured, and not all sites have the facilities for storage of railcars or trailers once they are loaded. Limiting the consist size to three cars mitigates these storage issues.

Train consists were assumed to travel with two buffer cars and an escort car containing security. Truck convoys were assumed to travel with an escort truck.

The transportation cycle modeled in TOM starts at the fleet maintenance facility, goes to the reactor site to pick up the SNF, transports it to the interim storage facility (or repository). After unloading, the consist (or convoy) goes to the cask maintenance facility and finally travels to the fleet maintenance facility.

It should be recognized that a computationally generated schedule is an idealized model of how shipments would be accomplished in a perfect world. Machinery does not fail, transportation assets are not delayed, and all needed equipment appears on schedule. In actuality, such a schedule is fragile. Introducing a "contingency factor" to purposefully introduce delays in the schedule would produce buffers that will help guard against such a problematic schedule, but would also tend to overestimate the resources required. It must be kept in mind that TOM is a planning model, and not an operations model.

Summary results of the transportation logistics modeling for each case is shown in Table A-32 and details are provided in Table A-33. Overall, the results show that higher UNF acceptance rates (either from the reactor sites or from the CSF) lead to larger infrastructure requirements, as expected.

When considering that all UNF is loaded into canisters at the reactor sites prior to transport either to the CSF or the repository (Cases 1 and 3), it can be seen that when the packaging/re-packaging facility is located at the CSF the required transportation fleet is larger. In a sense, essentially two transportation fleets are required to ship UNF both to the CSF and from the CSF to the repository. Recall, when the repository starts operations, the rate of UNF transfer to the repository is the same as the rate of UNF transfer from the reactors to the CSF.

In addition, a larger number of lower capacity canisters are shipped between the CSF and the repository to maintain the overall UNF transfer rate. This trend can be seen in Table A-32 where there is a difference in fleet requirements for 12 and 21 PWR assembly capacity canisters at a throughput of $3000 \mathrm{MTHM} / \mathrm{yr}$ when all UNF is transported canisters and re-packaging occurs at the CSF. No difference is seen when re-packaging occurs at the repository. The detailed acquisition schedule shown in Table A-33 shows that in cases where re-packaging occurs at the CSF there is a need to acquire a large number of casks, rail cars, etc. when the repository begins operation in either 2040 or 2055.

The results in Table A-32 show that the transportation fleet requirements are larger for the cases that transport UNF in the used fuel pools to the CSF when it begins operations (Cases 2 and 4). This is because of transportation thermal limits, the re-useable transportation casks are generally loaded to a lower capacity to allow immediate shipment offsite than are dual-purpose canisters that are stored onsite to allow cooling before shipment. In addition, those reactor sites that currently cannot directly load rail casks are assumed to utilize legal-weight truck shipping, although for purposes of this initial analysis the same sites are assumed to ship fuel in large canisters in the canister-only cases. This is evident by the need for a legal weight truck transportation fleet in addition to the rail fleet.

As before, the transportation fleet requirements are larger when bare fuel packaging and DPC repackaging occurs at the CSF for the same reasons as discussed above. 
Table A-32. Transportation Acquisition Summary

\begin{tabular}{|c|c|c|c|c|c|c|c|c|c|c|}
\hline Scenario & $\begin{array}{c}\text { Acceptance } \\
\text { Rate }\end{array}$ & $\begin{array}{l}\text { CSF } \\
\text { Start }\end{array}$ & $\begin{array}{l}\text { Repository } \\
\text { Start }\end{array}$ & $\begin{array}{c}\text { Disposal } \\
\text { Canister } \\
\text { Size }\end{array}$ & $\begin{array}{c}\text { Casks/ } \\
\text { Overpacks }\end{array}$ & $\begin{array}{l}\text { Buffer } \\
\text { Railcar }\end{array}$ & $\begin{array}{l}\text { Cask } \\
\text { Railcar }\end{array}$ & $\begin{array}{l}\text { Escort } \\
\text { Railcar }\end{array}$ & $\begin{array}{l}\text { Cask } \\
\text { Trailer }\end{array}$ & $\begin{array}{l}\text { Escort } \\
\text { Truck }\end{array}$ \\
\hline \multirow{9}{*}{$\begin{array}{c}\text { Canisters } \\
\text { Only - } \\
\text { Re-Package } \\
\text { at Repository }\end{array}$} & 1,500 & 2020 & 2040 & 4 & 174 & 18 & 18 & 9 & & \\
\hline & 3,000 & 2020 & 2040 & 4 & 176 & 38 & 43 & 19 & & \\
\hline & 3,000 & 2020 & 2040 & 12 & 176 & 38 & 43 & 19 & & \\
\hline & 3,000 & 2020 & 2040 & 21 & 176 & 38 & 43 & 19 & & \\
\hline & 6,000 & 2020 & 2040 & 4 & 257 & 48 & 61 & 24 & & \\
\hline & 1,500 & 2020 & 2055 & 4 & 185 & 18 & 18 & 9 & & \\
\hline & 3,000 & 2020 & 2055 & 4 & 197 & 34 & 40 & 17 & & \\
\hline & 6,000 & 2020 & 2055 & 4 & 316 & 42 & 61 & 21 & & \\
\hline & 3,000 & 2035 & 2055 & 4 & 166 & 28 & 33 & 14 & & \\
\hline \multirow{9}{*}{$\begin{array}{l}\text { Canisters and } \\
\text { Bare Fuel - } \\
\text { Re-Package } \\
\text { at Repository }\end{array}$} & 1,500 & 2020 & 2040 & 4 & 276 & 24 & 24 & 12 & 9 & 10 \\
\hline & 3,000 & 2020 & 2040 & 4 & 322 & 36 & 47 & 18 & 10 & 8 \\
\hline & 3,000 & 2020 & 2040 & 12 & 322 & 36 & 47 & 18 & 10 & 8 \\
\hline & 3,000 & 2020 & 2040 & 21 & 322 & 36 & 47 & 18 & 10 & 8 \\
\hline & 6,000 & 2020 & 2040 & 4 & 471 & 76 & 95 & 38 & 13 & 10 \\
\hline & 1,500 & 2020 & 2055 & 4 & 299 & 24 & 24 & 12 & 9 & 6 \\
\hline & 3,000 & 2020 & 2055 & 4 & 355 & 38 & 47 & 19 & 10 & 8 \\
\hline & 6,000 & 2020 & 2055 & 4 & 502 & 86 & 95 & 43 & 13 & 10 \\
\hline & 3,000 & 2035 & 2055 & 4 & 286 & 42 & 47 & 21 & 6 & 6 \\
\hline \multirow{9}{*}{$\begin{array}{l}\text { Canisters } \\
\text { Only - Re- } \\
\text { Package at } \\
\text { CSF }\end{array}$} & 1,500 & 2020 & 2040 & 4 & 535 & 74 & 101 & 37 & & \\
\hline & 3,000 & 2020 & 2040 & 4 & 516 & 138 & 200 & 69 & & \\
\hline & 3,000 & 2020 & 2040 & 12 & 260 & 66 & 90 & 33 & & \\
\hline & 3,000 & 2020 & 2040 & 21 & 245 & 56 & 75 & 28 & & \\
\hline & 6,000 & 2020 & 2040 & 4 & 664 & 266 & 395 & 133 & & \\
\hline & 1,500 & 2020 & 2055 & 4 & 537 & 76 & 102 & 38 & & \\
\hline & 3,000 & 2020 & 2055 & 4 & 515 & 146 & 213 & 73 & & \\
\hline & 6,000 & 2020 & 2055 & 4 & 664 & 252 & 377 & 126 & & \\
\hline & 3,000 & 2035 & 2055 & 4 & 506 & 138 & 198 & 69 & & \\
\hline \multirow{9}{*}{$\begin{array}{l}\text { Canisters and } \\
\text { Bare Fuel - } \\
\text { Re-Package } \\
\text { at CSF }\end{array}$} & 1,500 & 2020 & 2040 & 4 & 626 & 80 & 108 & 40 & 9 & 8 \\
\hline & 3,000 & 2020 & 2040 & 4 & 647 & 148 & 212 & 74 & 12 & 8 \\
\hline & 3,000 & 2020 & 2040 & 12 & 378 & 76 & 97 & 38 & 10 & 8 \\
\hline & 3,000 & 2020 & 2040 & 21 & 368 & 60 & 86 & 30 & 12 & 8 \\
\hline & 6,000 & 2020 & 2040 & 4 & 753 & 260 & 379 & 130 & 13 & 10 \\
\hline & 1,500 & 2020 & 2055 & 4 & 619 & 70 & 101 & 35 & 9 & 8 \\
\hline & 3,000 & 2020 & 2055 & 4 & 641 & 140 & 203 & 70 & 12 & 8 \\
\hline & 6,000 & 2020 & 2055 & 4 & 757 & 236 & 353 & 118 & 13 & 10 \\
\hline & 3,000 & 2035 & 2055 & 4 & 600 & 136 & 199 & 68 & 6 & 6 \\
\hline
\end{tabular}


Table A-32. Transportation Acquisition

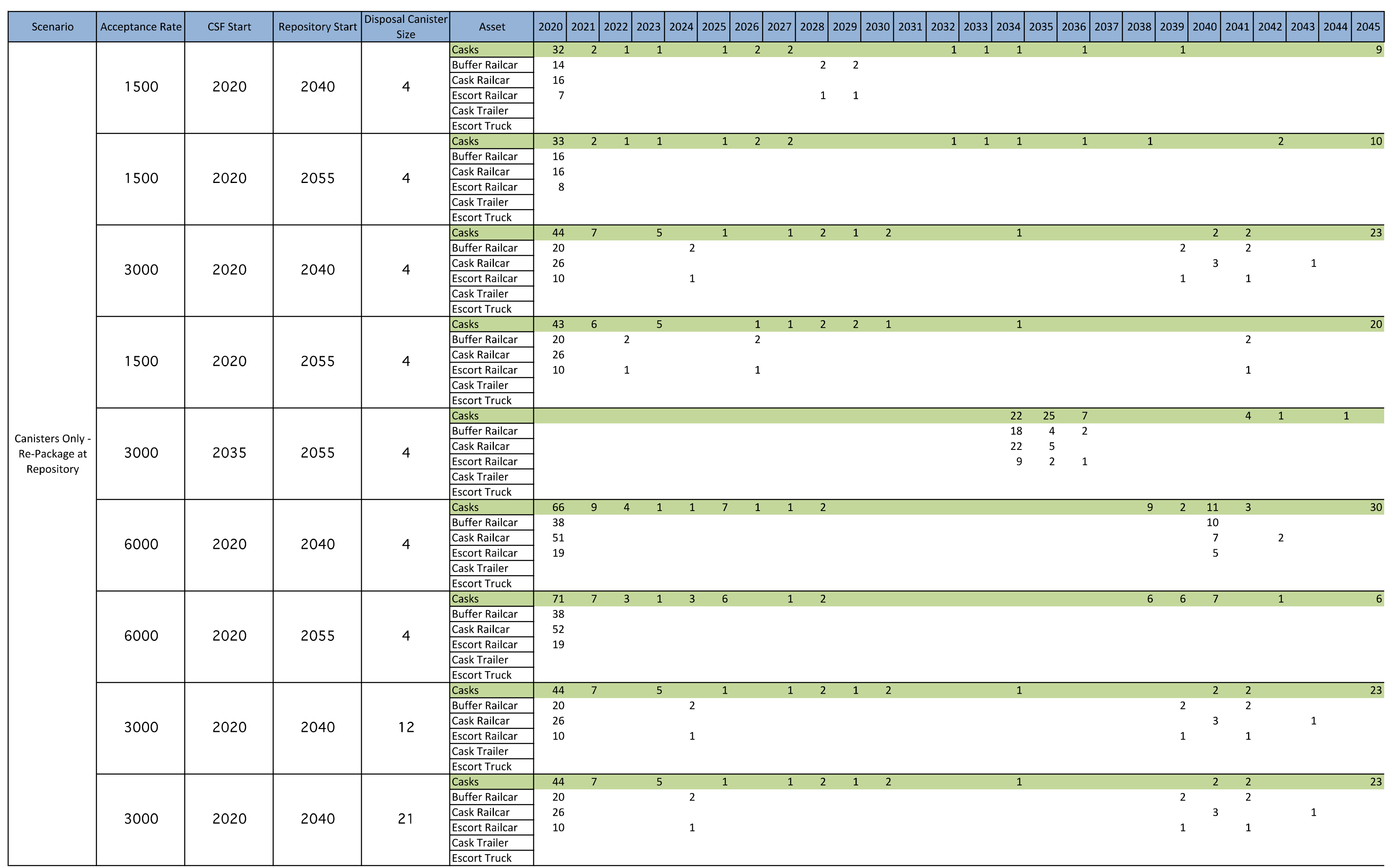


Table A-32. Transportation Acquisition (Continued)

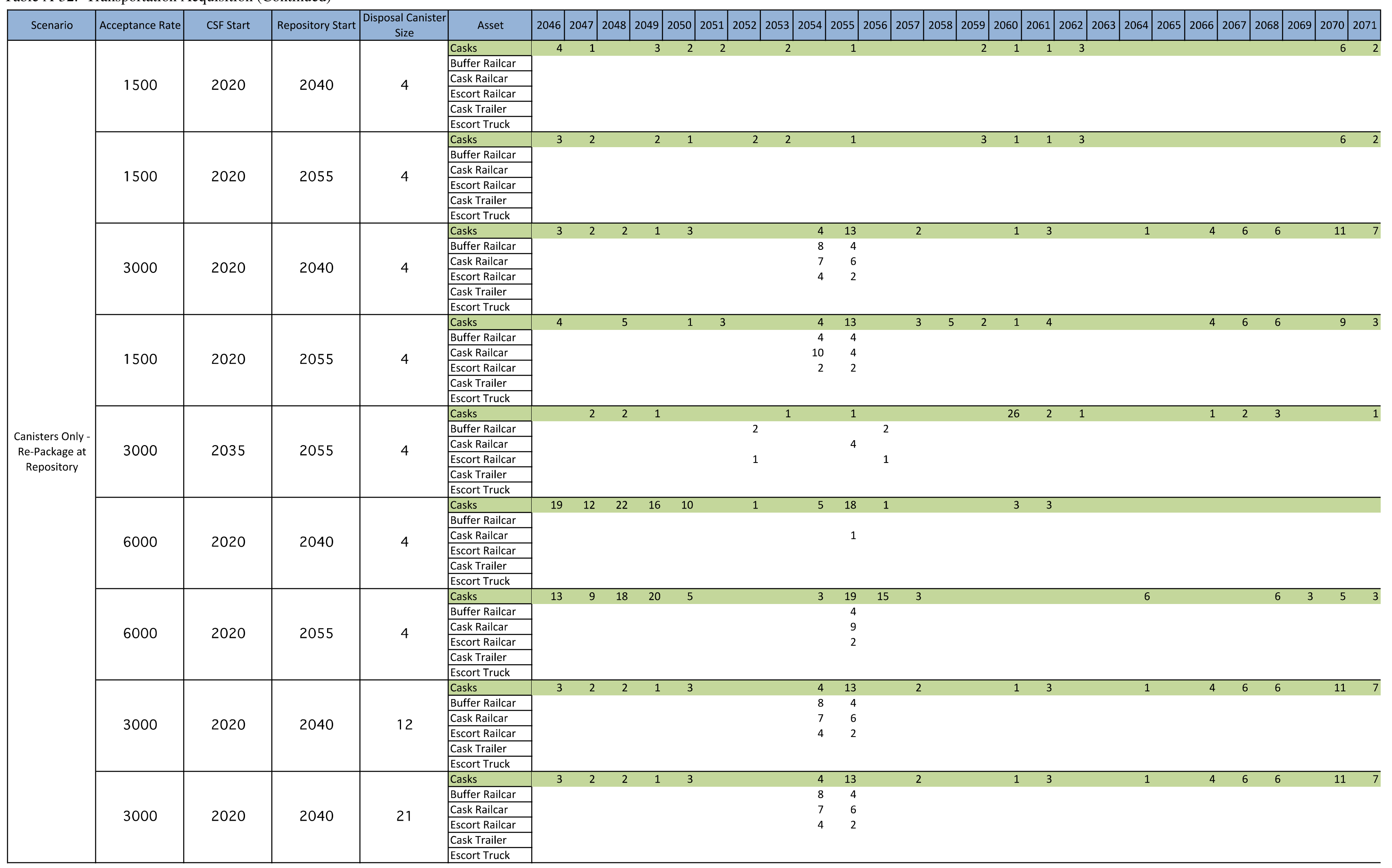




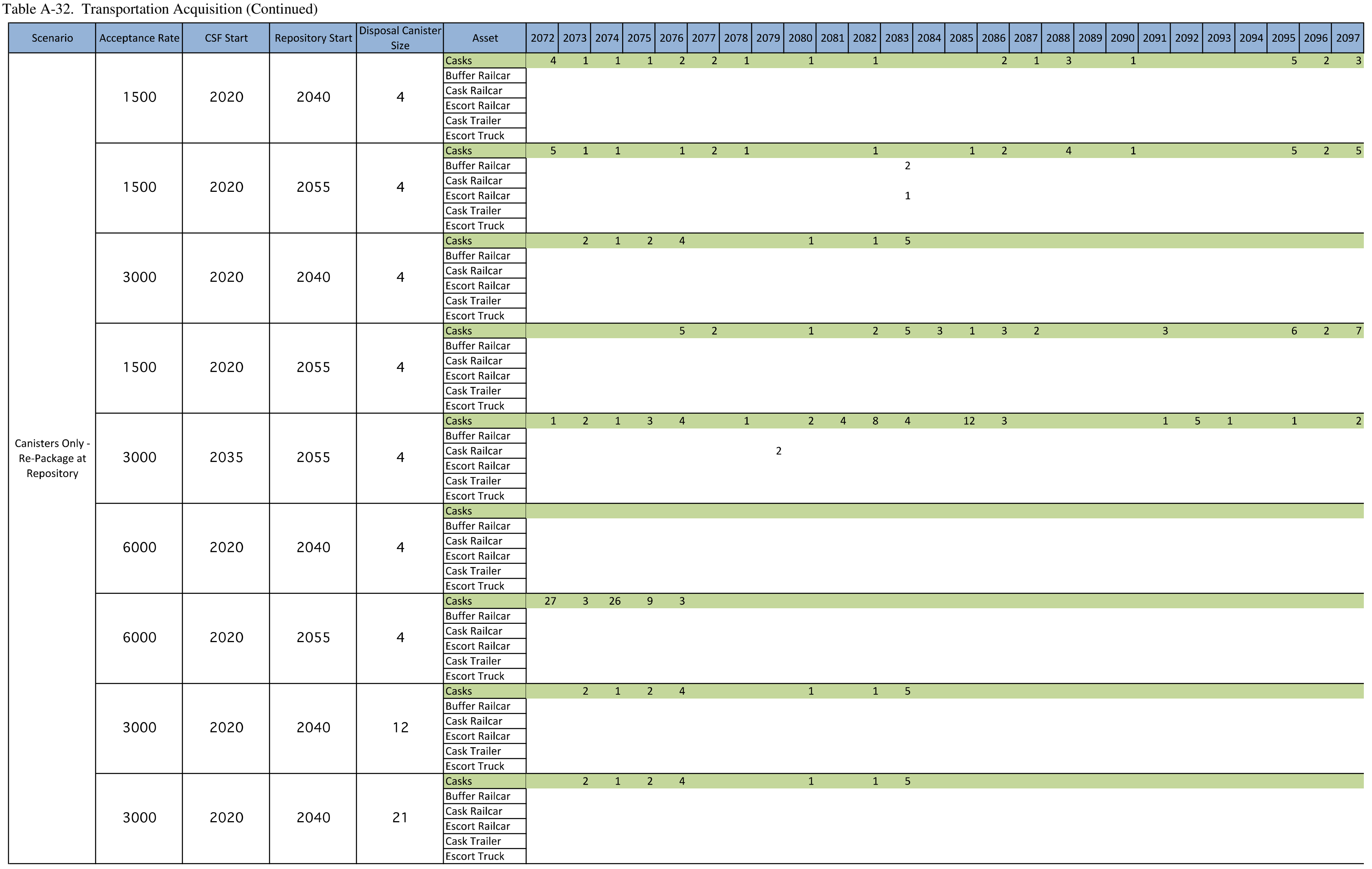




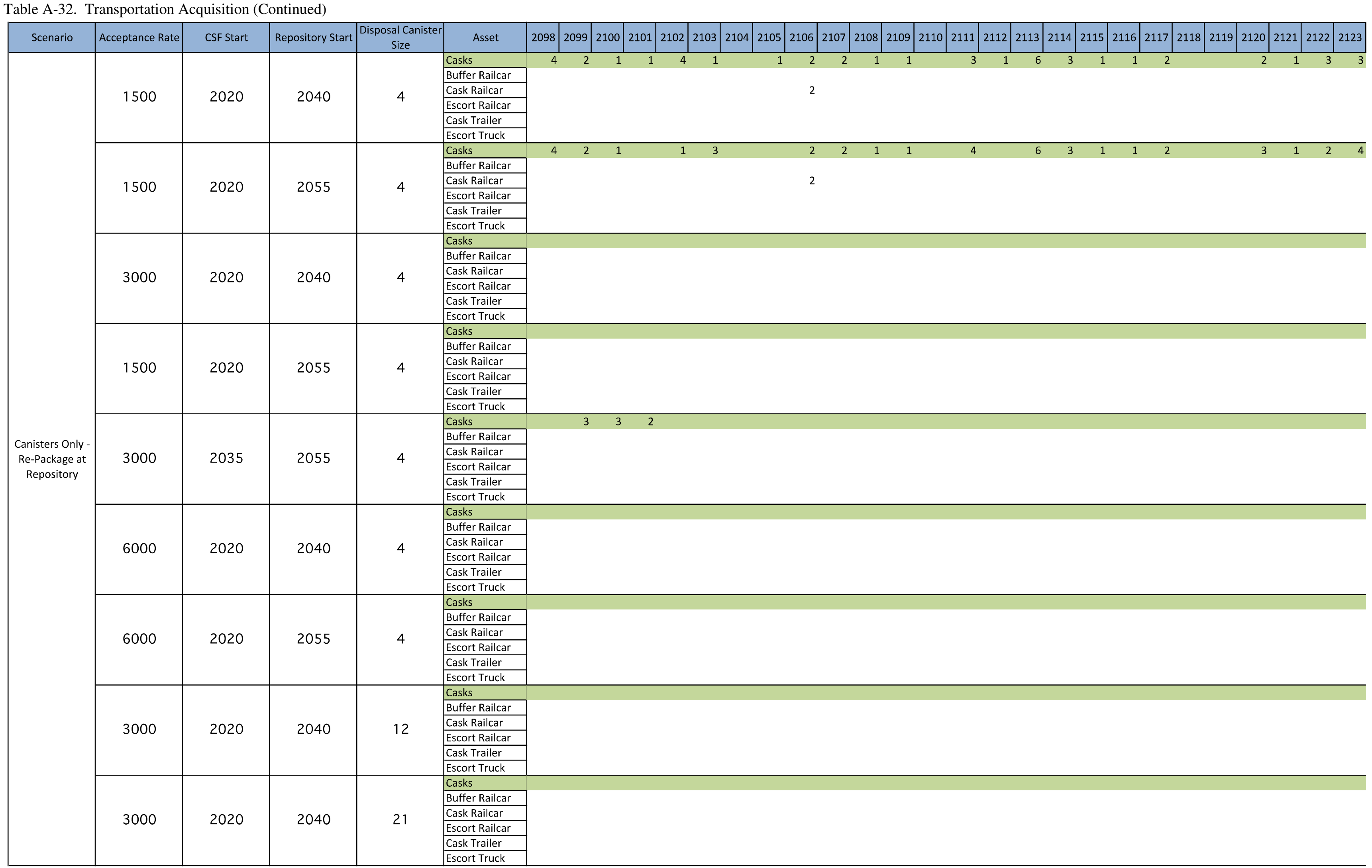


Table A-32. Transportation Acquisition (Continued)

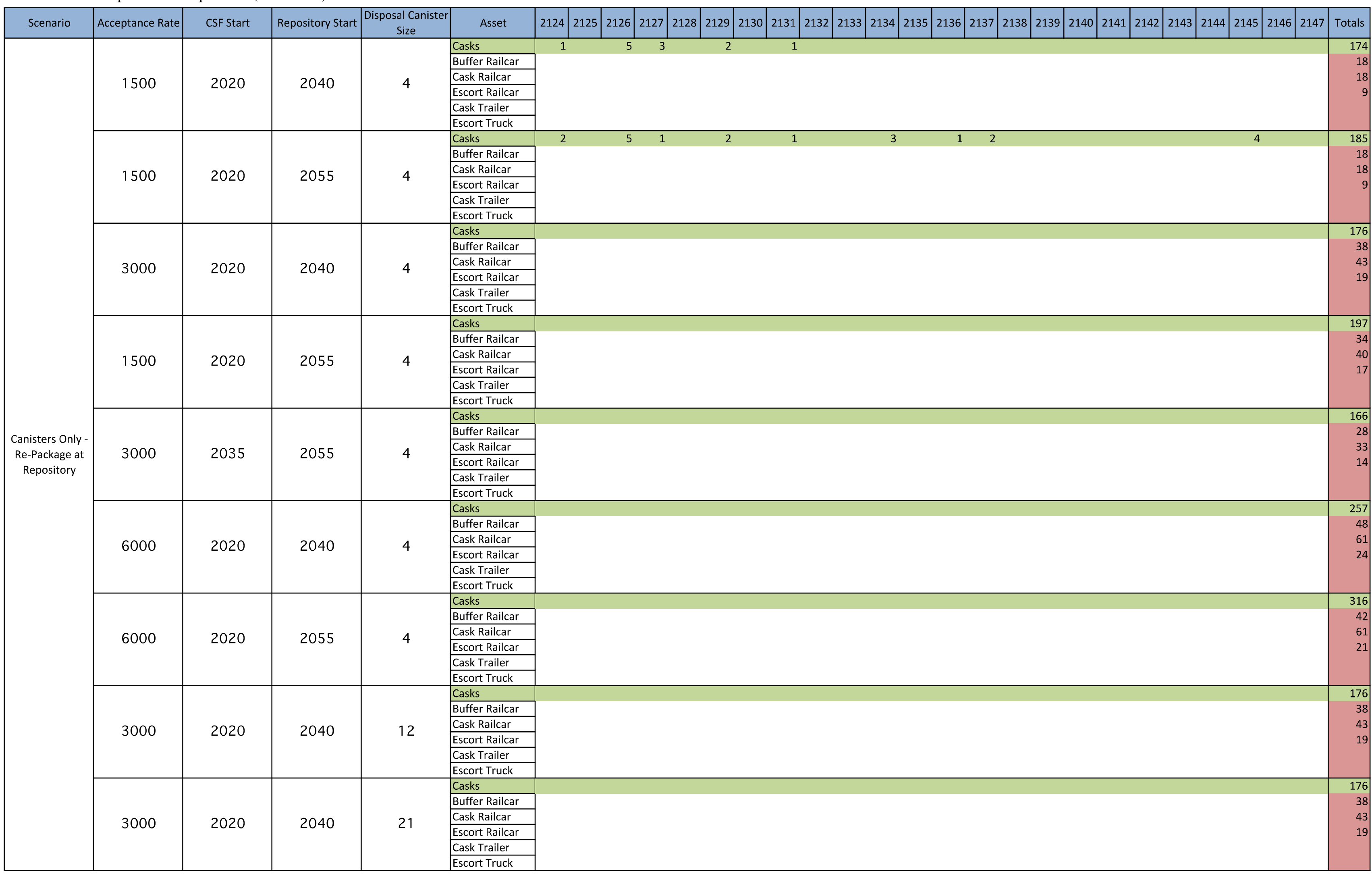


Table A-32. Transportation Acquisition (Continued)

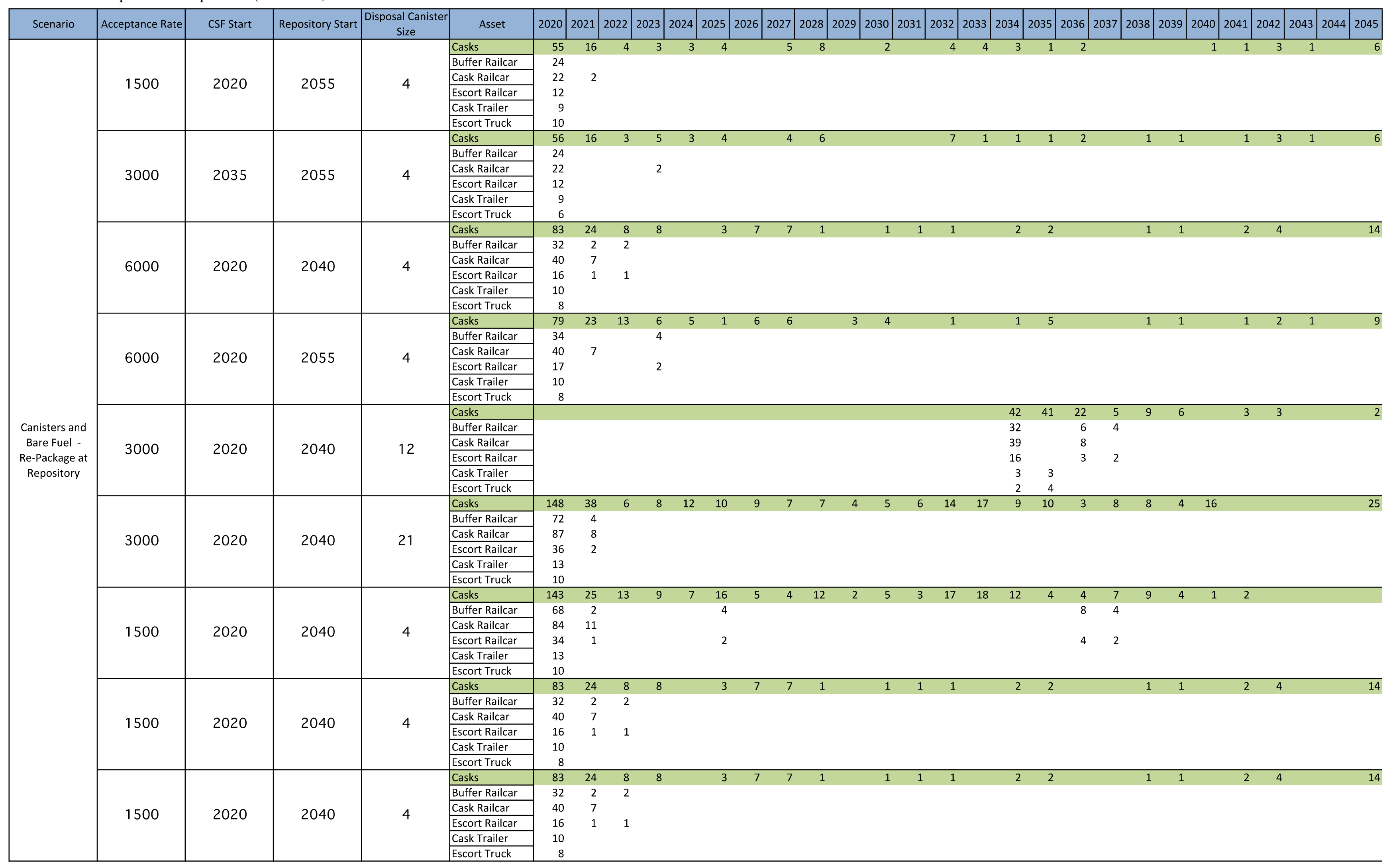




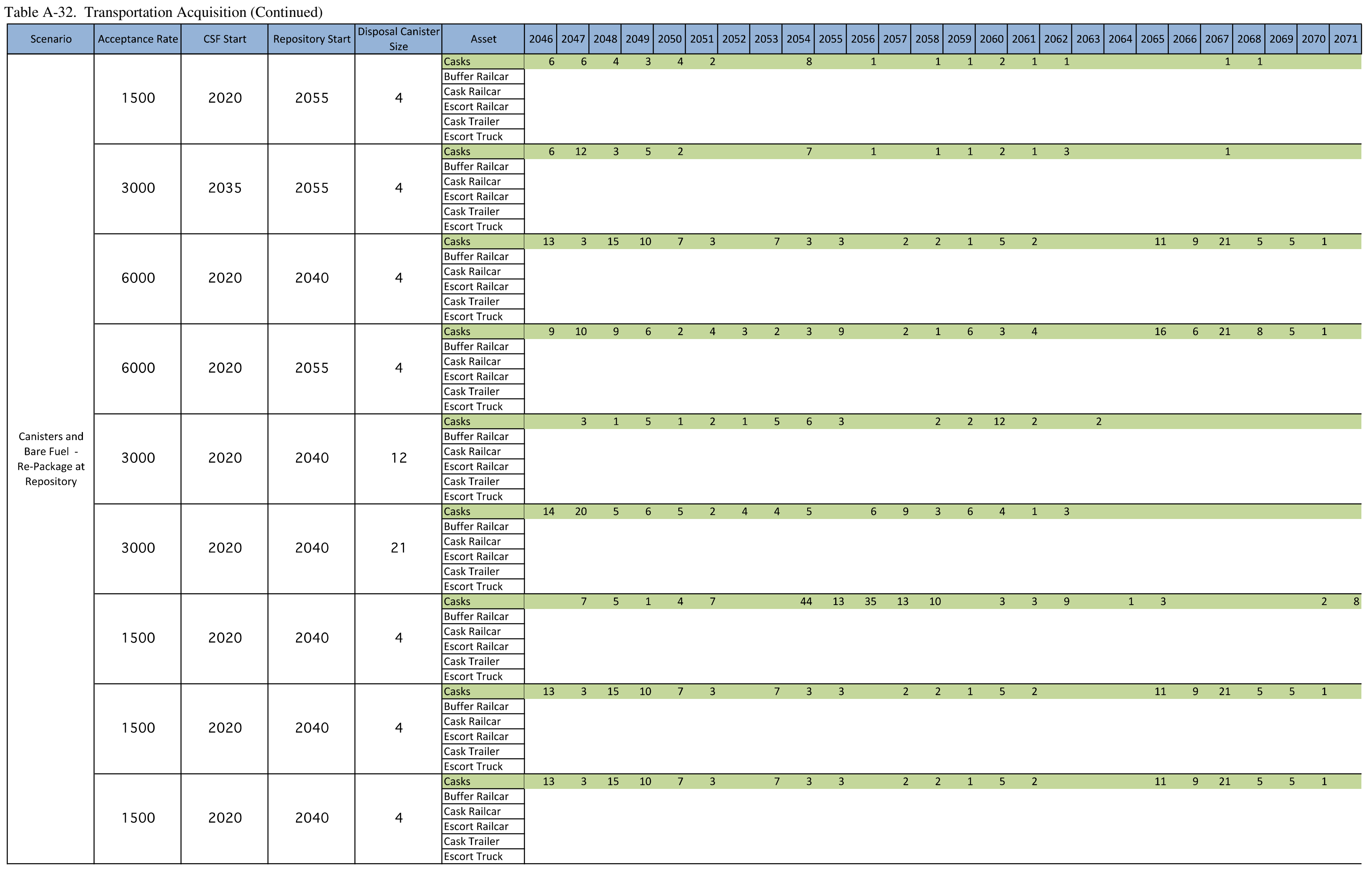


Table A-32. Transportation Acquisition (Continued)

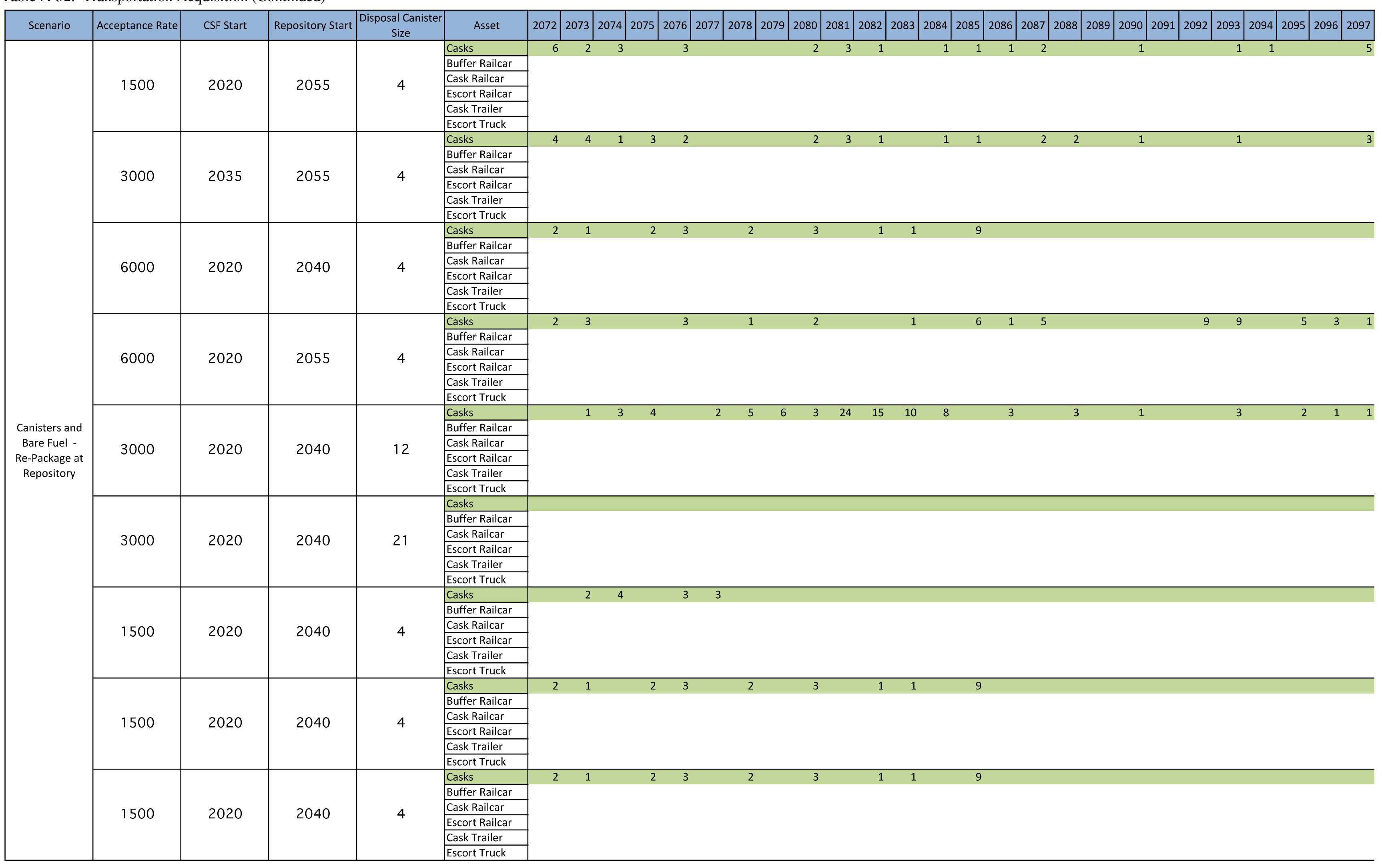




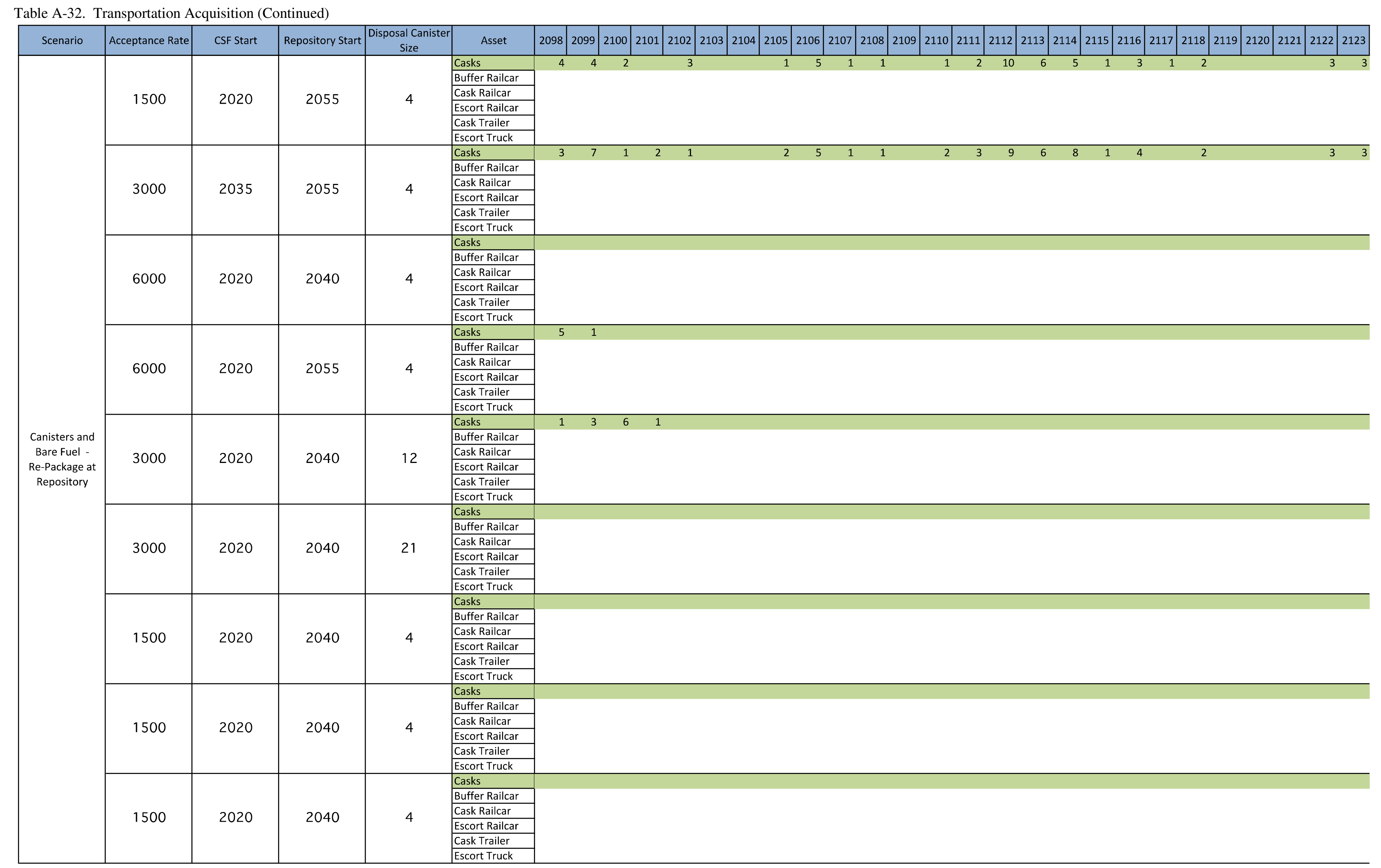


Table A-32. Transportation Acquisition (Continued)

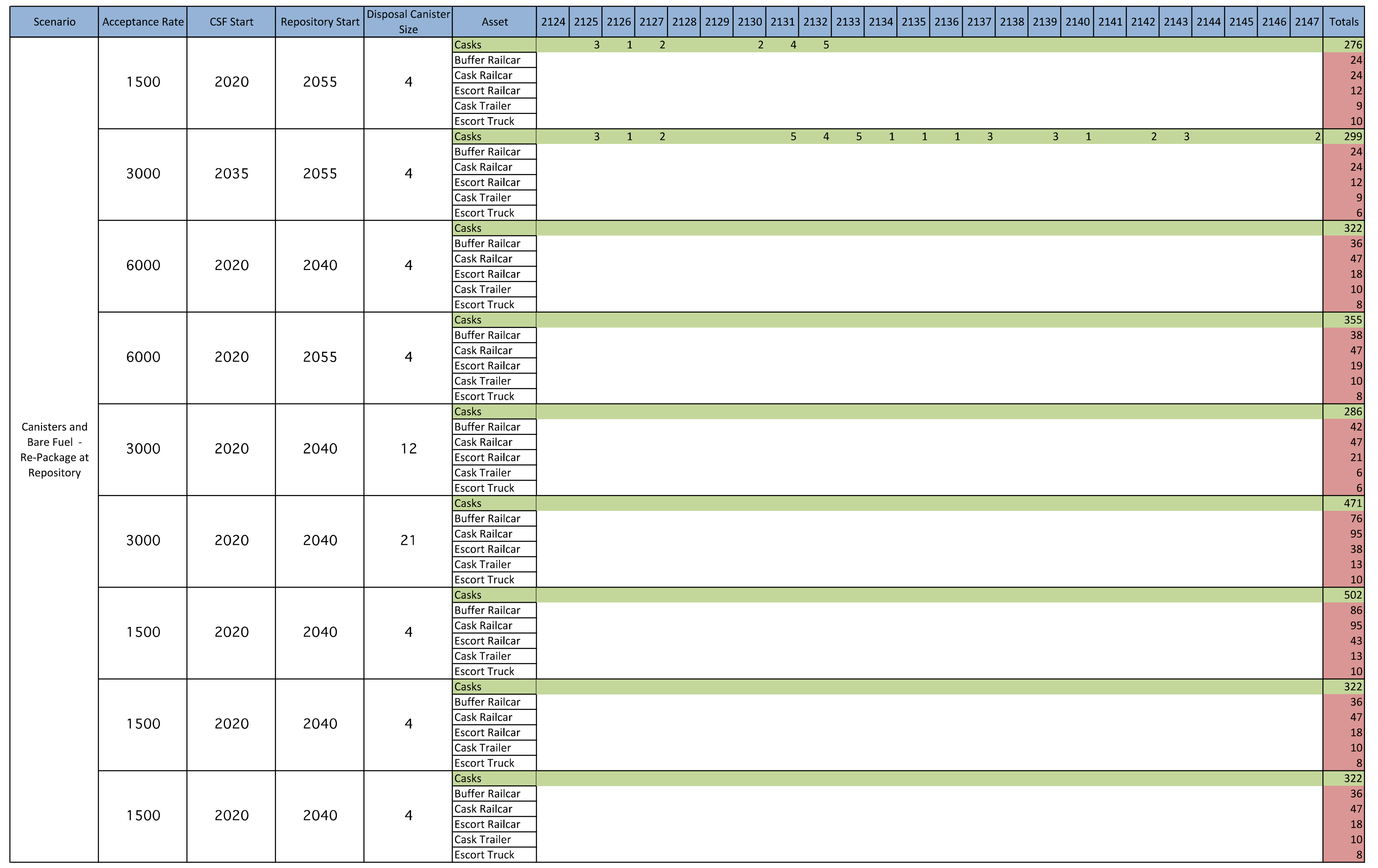


Used Fuel Management System Architecture Evaluation, Fiscal Year 2012

October 2012

Table A-32. Transportation Acquisition (Continued)

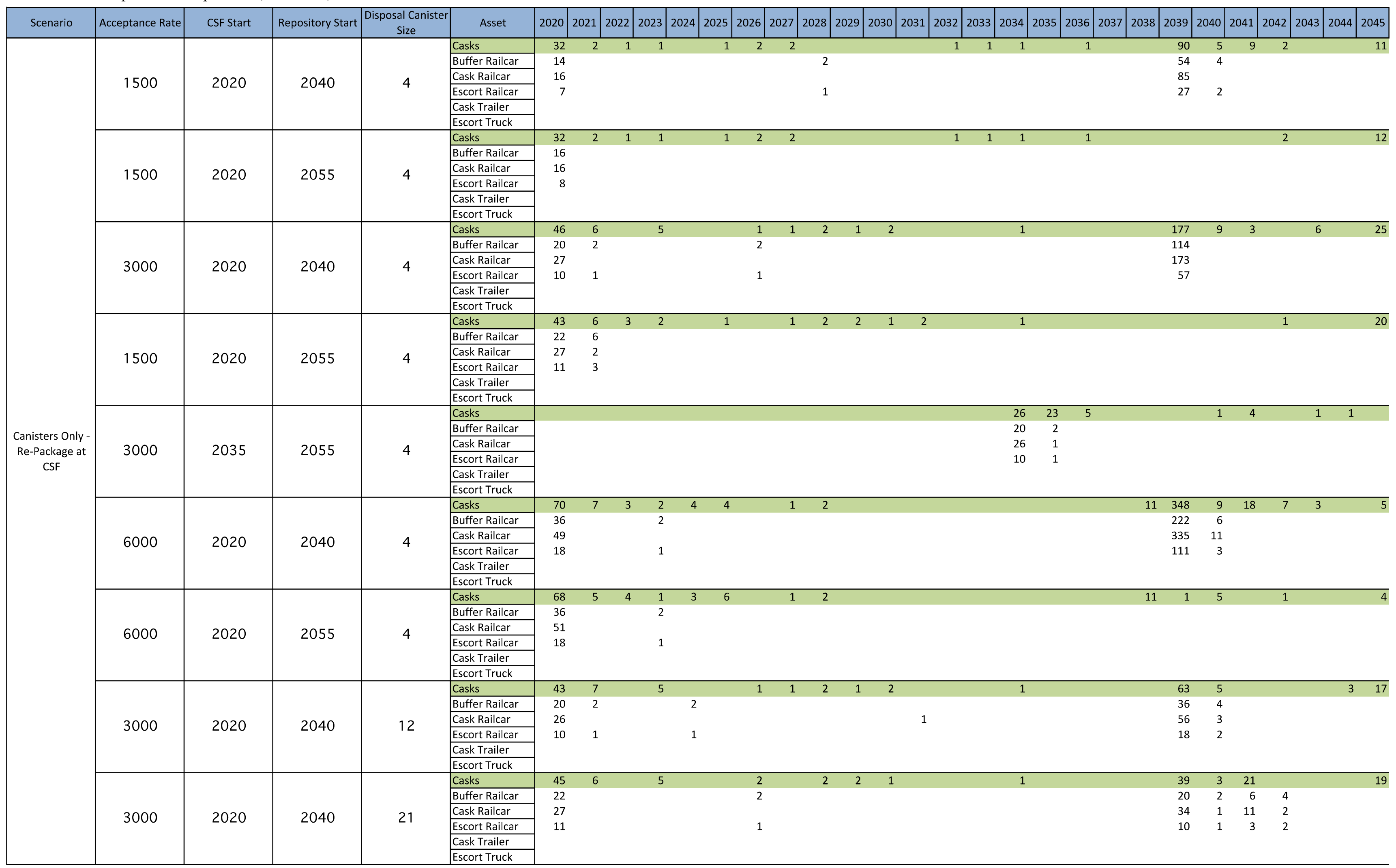


Table A-32. Transportation Acquisition (Continued)

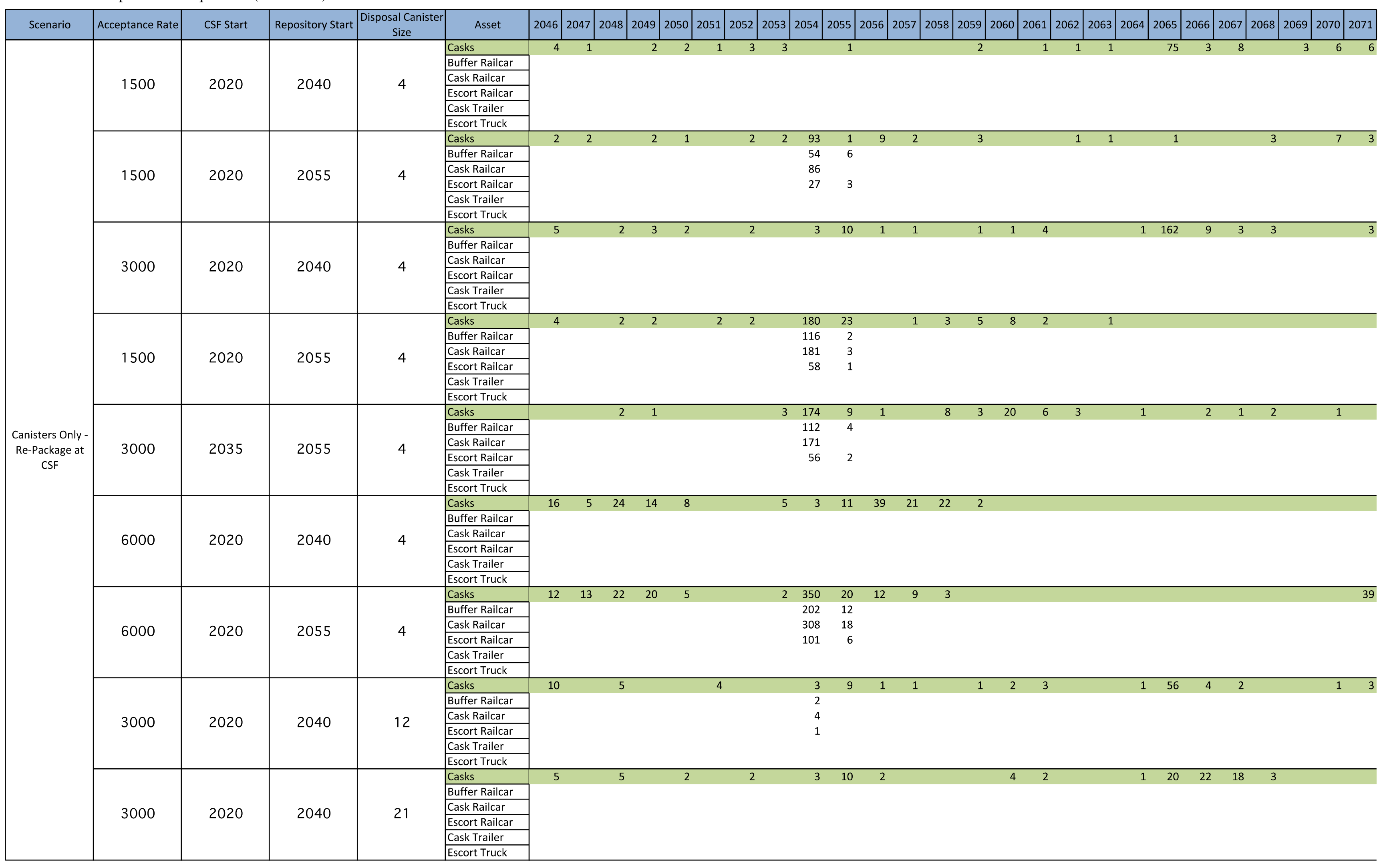


Table A-32. Transportation Acquisition (Continued)

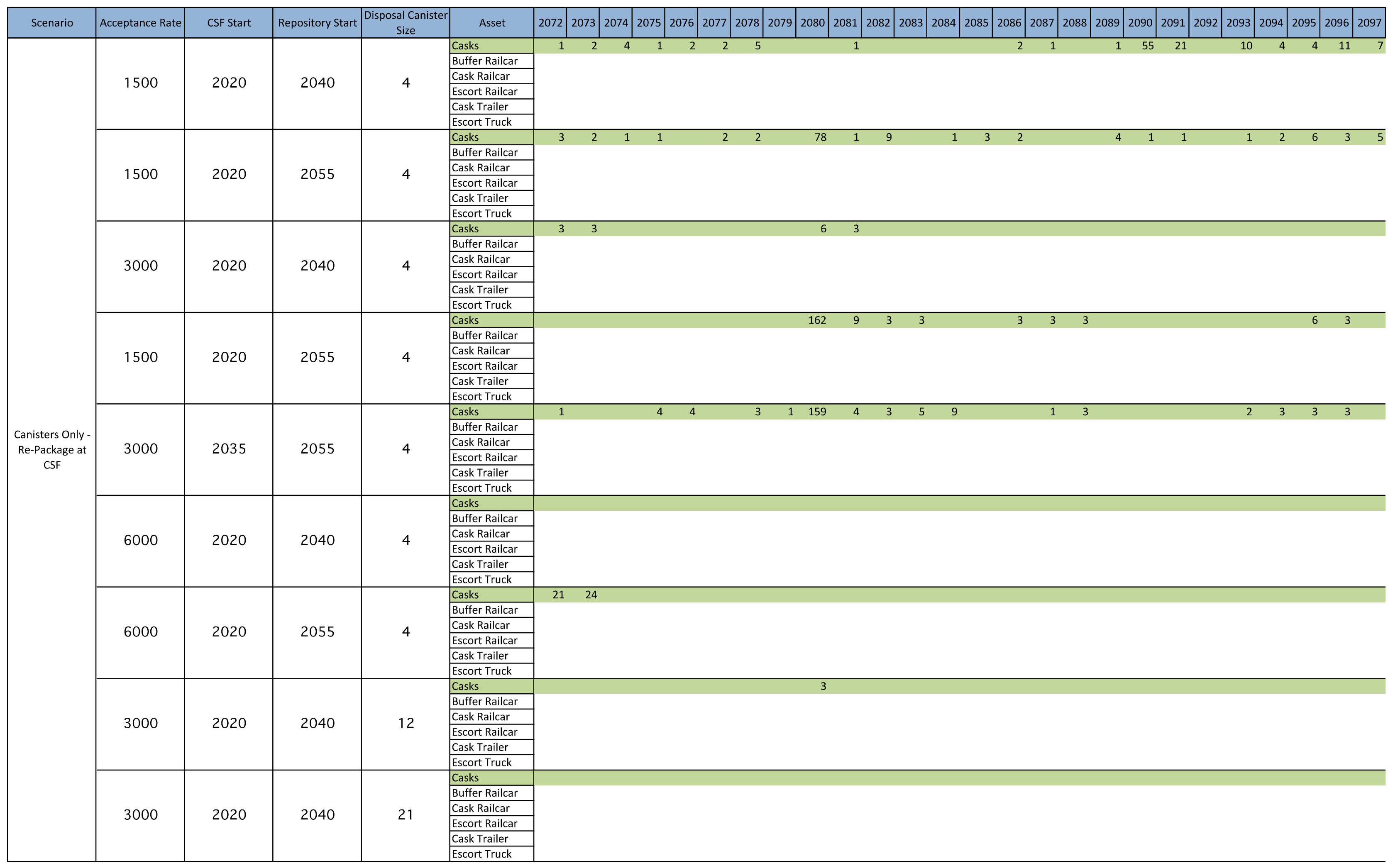


Table A-32. Transportation Acquisition (Continued)

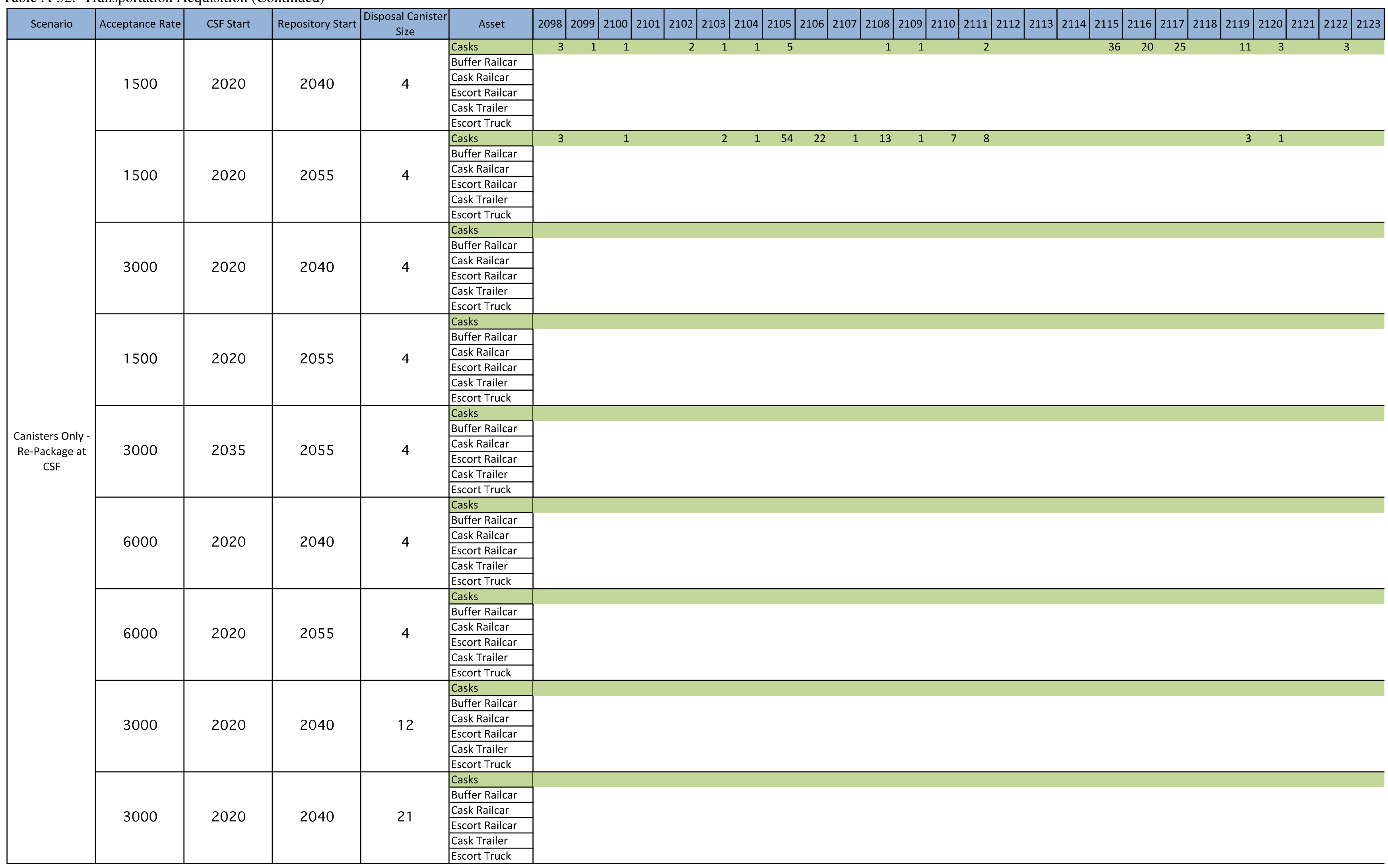


Table A-32. Transportation Acquisition (Continued)

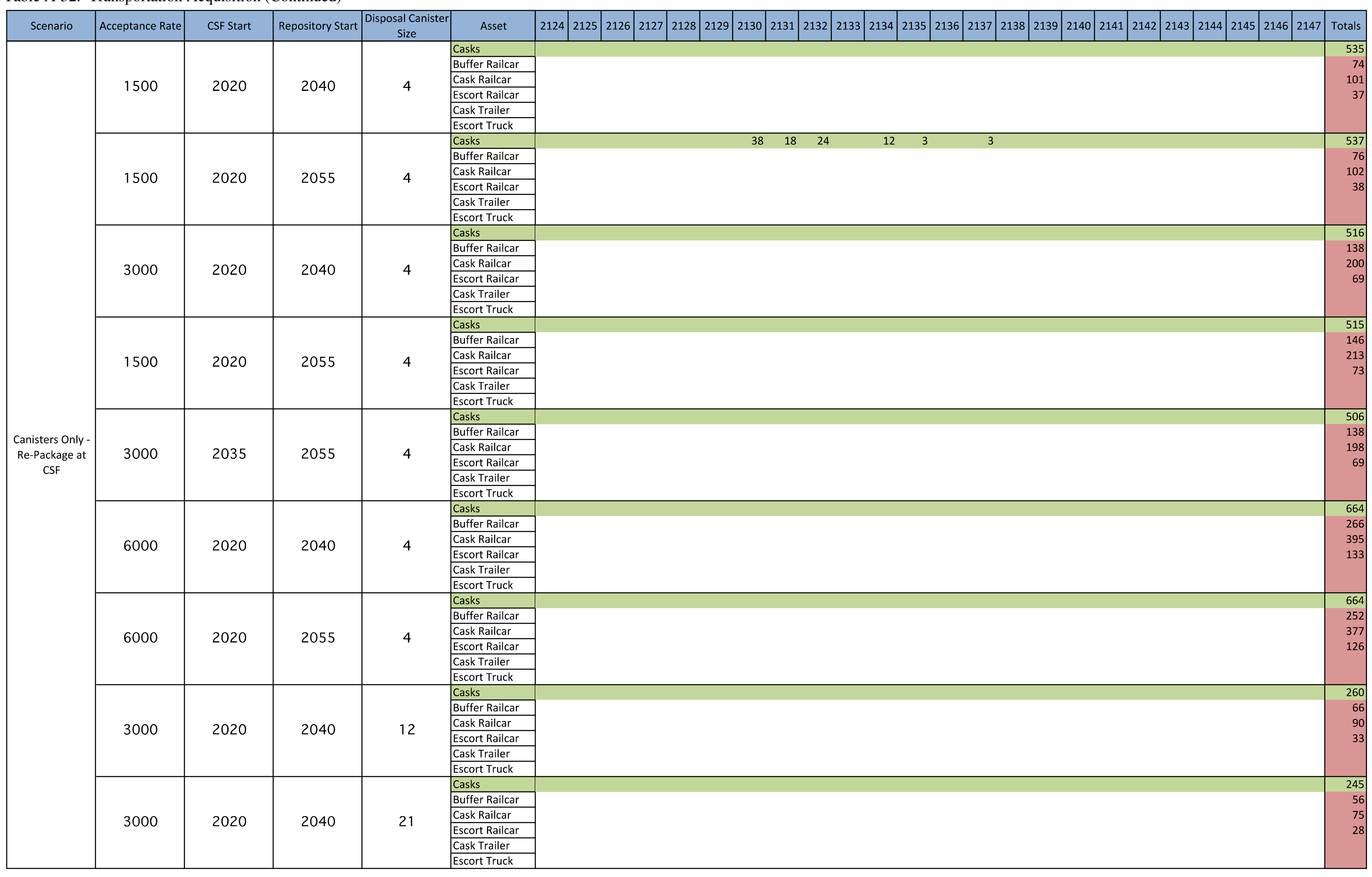


Table A-32. Transportation Acquisition (Continued)

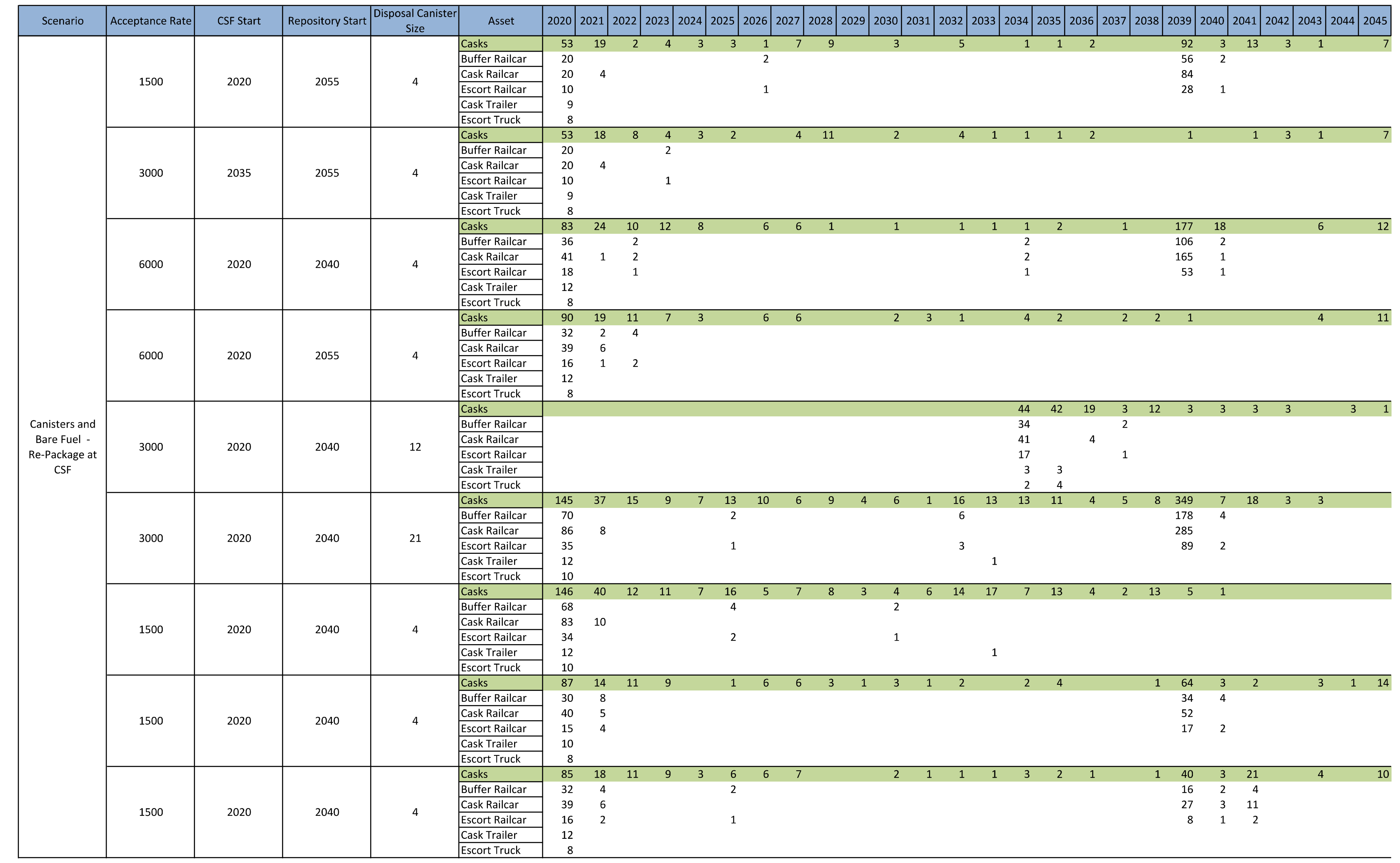


Table A-32. Transportation Acquisition (Continued)

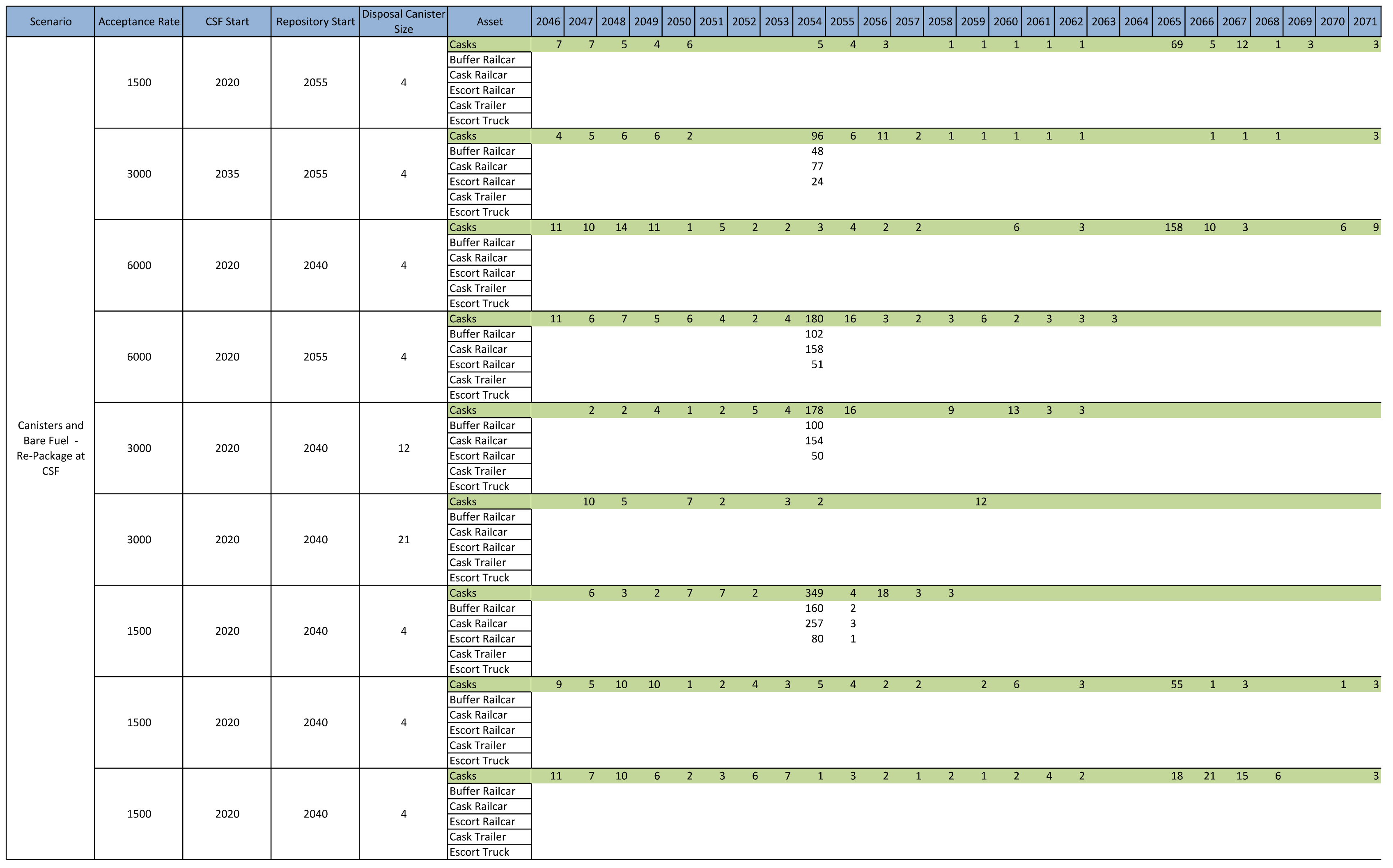


Table A-32. Transportation Acquisition (Continued)

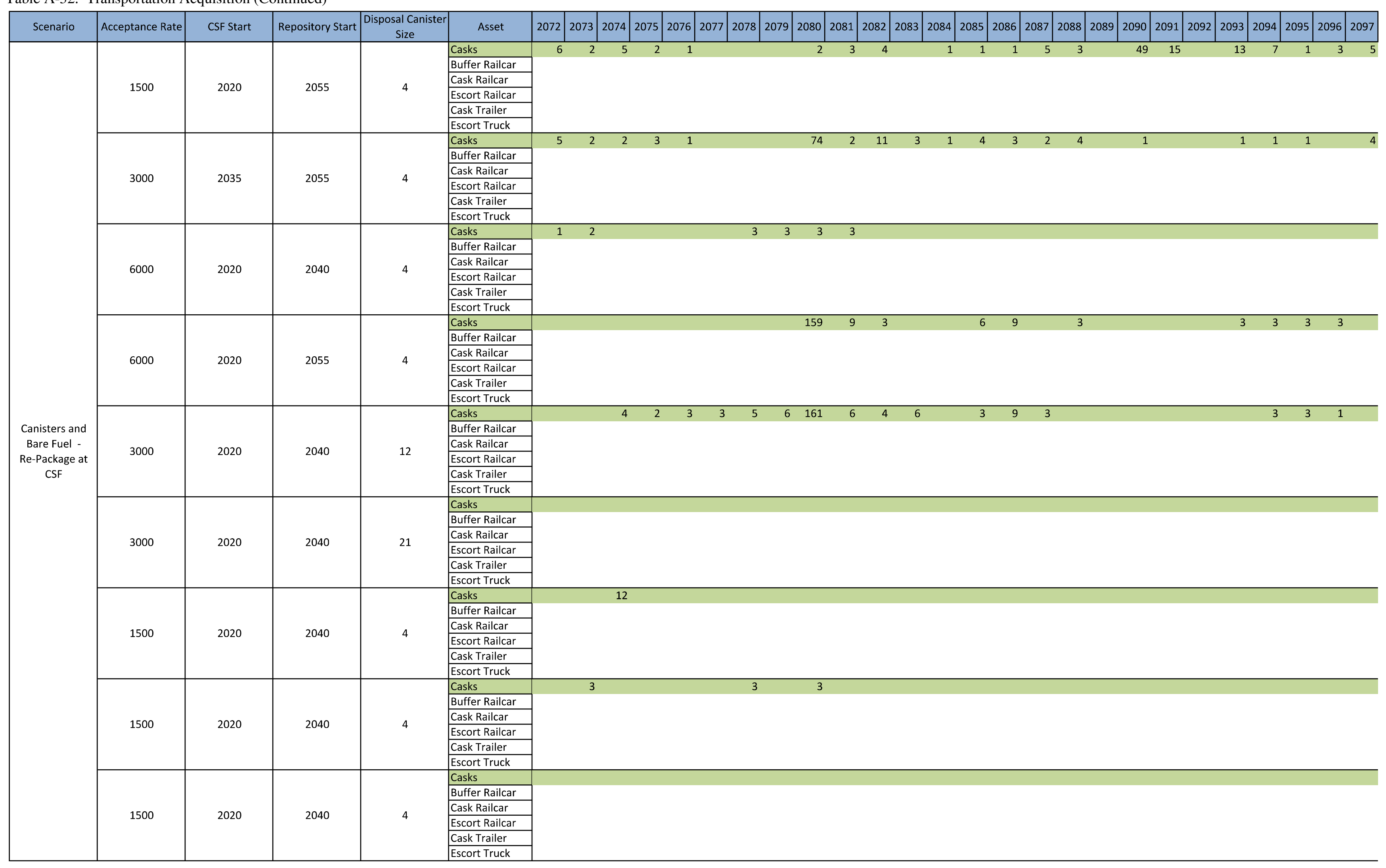


Table A-32. Transportation Acquisition (Continued)

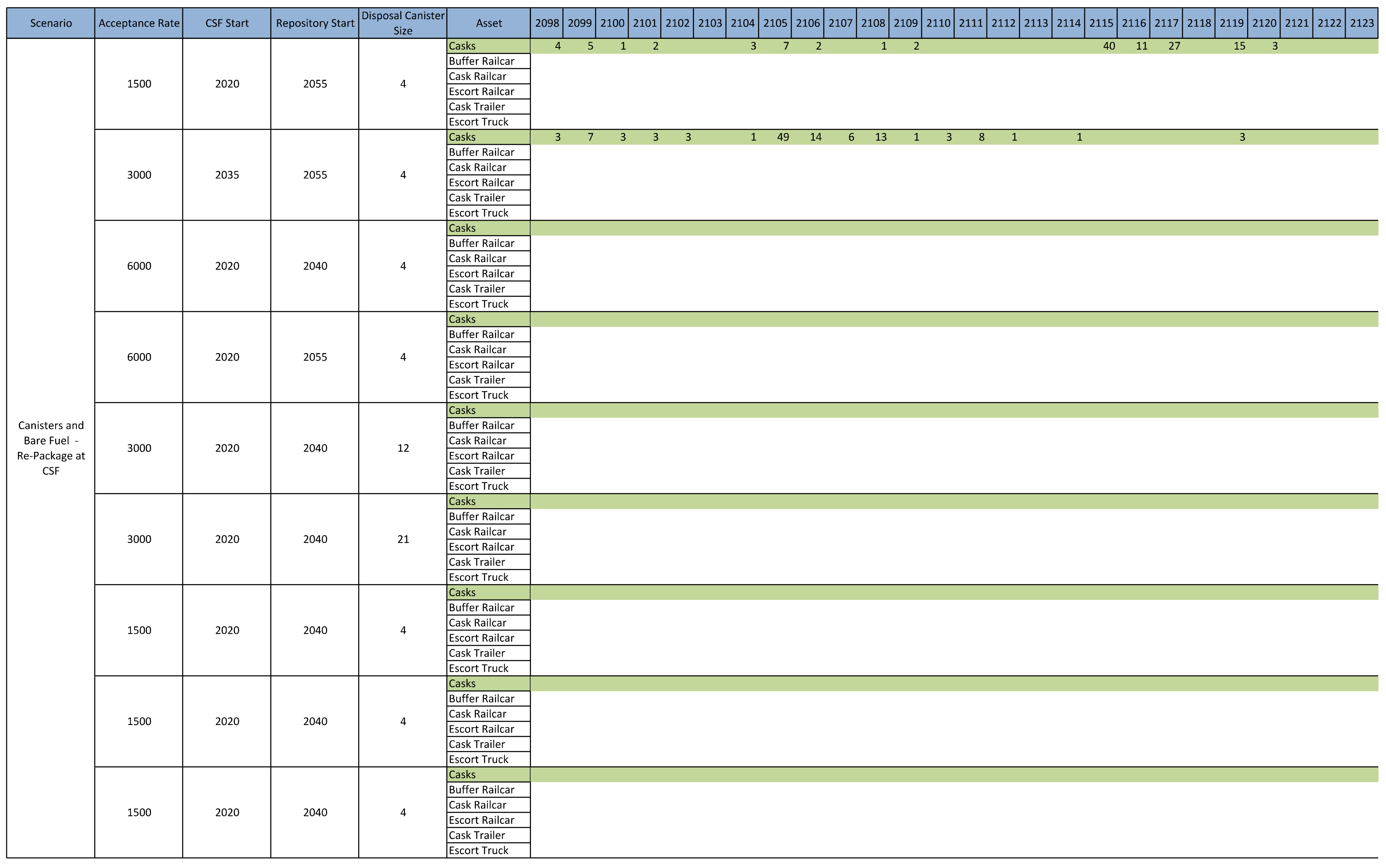


Table A-32. Transportation Acquisition (Continued)

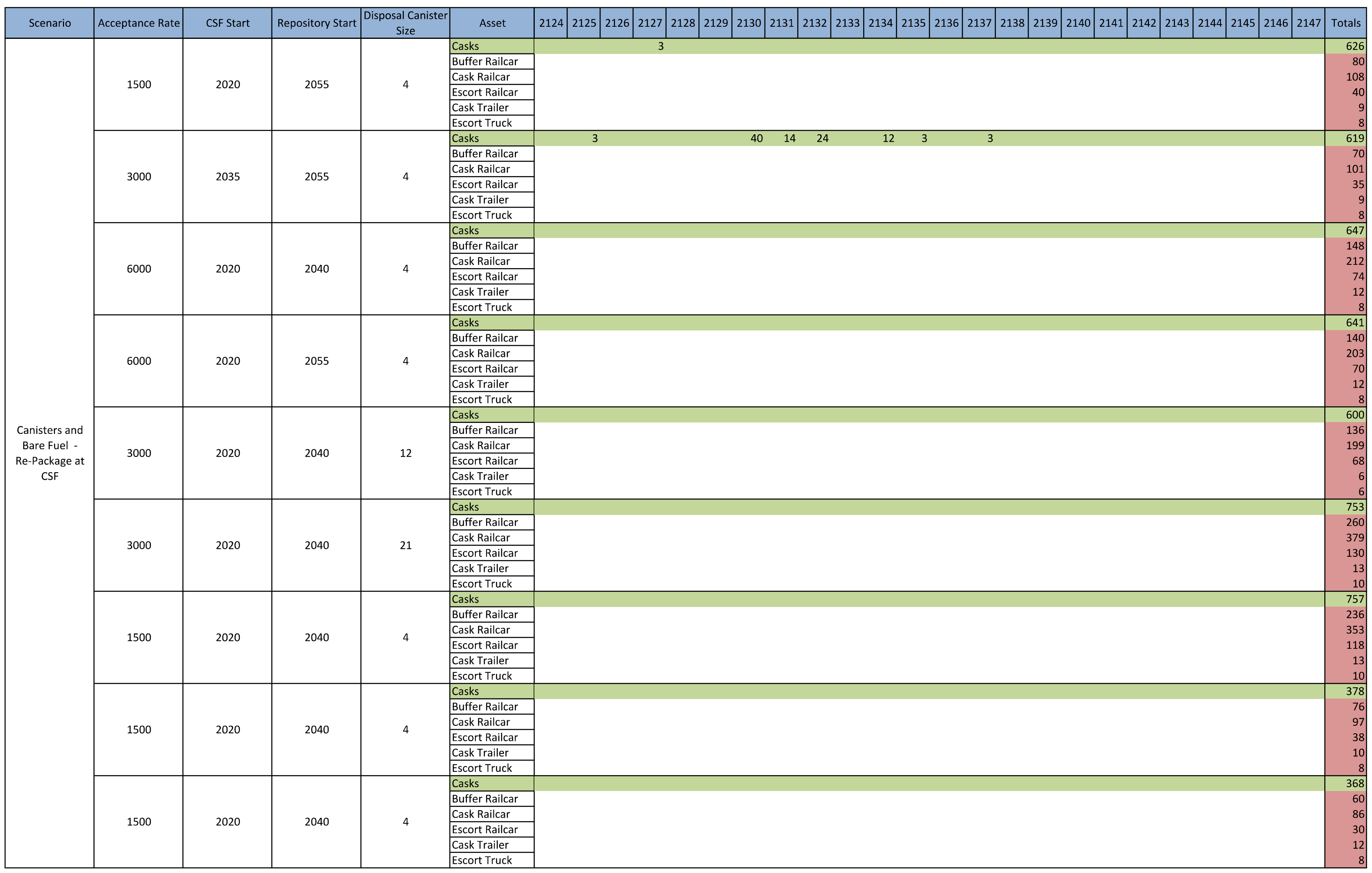




\section{REFERENCES FOR APPENDIX A}

Busch, I., Howard, R., Transportation Operations Model (TOM) Technical Manual, FCRD-NFST-2012000425, October 2012.

Carter, J.T., Luptak, A.J., Gastelum, J., Stockman, C., Miller, A., Fuel Cycle Potential Waste Inventory for Disposition, FCR\&D-USED-2010-000031 REV 5, July 2012.

Electric Power Research Institute (EPRI), Impacts Associated with Transfer of Spent Nuclear Fuel from Spent Fuel Storage Pools to Dry Storage After Five Years of Cooling. EPRI, Palo Alto, CA: 2010a. 1021049.

Electric Power Research Institute (EPRI), Industry Spent Fuel Storage Handbood. EPRI, Palo Alto, CA: 2010b. 102048.

Leblang, S., Entergy, Impact of Generic Issues on Individual Licenses, Presented at the U.S. Nuclear Regulatory Commission Spent Fuel Storage and Transportation Regulatory Conference, November 2-3, 2011. (Available at pbadupws.nrc.gov/docs/ML1131/ML113130539.pdf)

Leduc D.R., Carter J.T., Dry Storage of Used Fuel Transition to Transport, FCRD-UFD-2012-000253, August 2012.

Nutt, M., Morris, E., Puig, F., Kalinina, E., Gillespie, S., Transportation Storage Logistics Model CALVIN (TSL-CALVIN) , FCRD-NFST-2012-000424, October 2012.

Ux Consulting Company LLC, StoreFuel and Decommissioning Report, Vol. 13 No. 169, September 4, 2012

U.S. Department of Energy, Final Environmental Impact Statement for a Geologic Repository for the Disposal of Spent Nuclear Fuel and High-Level Radioactive Waste at Yucca Mountain, Nye County, Nevada, DOE/EIS-0250, February 2002

U.S. Nuclear Regulatory Commission (NRC), 2011-2012 Information Digest, NUREG-1350, Volume 23, August 2011, Appendix A

U.S. Government Accountability Office, Nuclear Waste Management - Key Attributes, Challenges, and Costs for the Yucca Mountain Repository and Two Potential Alternatives, GAO-10-48, November 2009.

J.A. Rice, Vice President - Nuclear Engineering, Virginia Electric and Power Company letter to the U.S. Nuclear Regulatory Commission, Virginia Electric and Power Company North Anna Power Station Units 2 and 2 Exemption Request for NUHOMS HD Dry Shielded Canisters Loaded to Incorrect Heat Load Limits Supplemental Information, September 28, 2011. (Available on the NRC ADAMS system, Accession No. ML11286A143). 


\section{Appendix B}

\section{UFD System Architecture Study Facilities Unit Operations and Facility Infrastructure Elements Sizing}




\section{B.1 FACILITY SIZING CONSIDERATIONS}

Facilities that are needed to support a given UNF system architecture scenario vary in size and configuration, relative to other scenarios, principally as a function of the type, total quantity (capacity) and annual quantity (throughput) of material that must be handled under that scenario.. To support its mission, a facility must be equipped with sufficient infrastructure to process the quantity of material, or through-put, assigned to each operational scenario as a function of the time needed to accomplish each material handling or processing sequence through each element of the facility. Thus, as a given throughput must be processed through all facility elements and different facility elements may require different time periods to process the same through-put quantities, each facility processing element must be sized accordingly.

As an example, a finite minimum time is needed to receive a UNF transportation cask carrier, unload its cask cargo and prepare the cask for further handling and processing steps. As a result, the number of each carriers each carrier bay can be considered to handle per shift, per day and per year is a function of the time that the carrier bay is occupied for one carrier and cask handling/processing cycle. As the throughput target is increased beyond the respective handling capacity for an element of a facility, additional facility elements must be included as needed to achieve the desired target through-put. As this concept is applied to each principal element of a facility for a given throughput scenario, it is then possible to "scale" or estimate the number of facility elements, of each type, needed to process the material through the facility for that scenario. The time needed to process material through the facility is typically broken down into various handling or "unit operations" steps and their estimated times. The total of the unit operations times associated with each key facility infrastructure element can then be used to determine the number of facility elements of each type needed to achieve the desired through-put target. While the unit operations times are not absolute and may vary as a function variations in material type and associated hardware, isolated handling variations etc., they can be viewed as average step times and operational total times considered over longer periods such an annual operating period.

This study assumed a set of standard operational considerations upon which to base the facility sizing for key infrastructure elements. They are summarized by the following:

- Facility operations are based on three eight hour shifts per day

- Two shifts per day are assigned for processing operations and one shift is assigned for maintenance, repairs, testing and calibrations etc.

- $\quad 75 \%$ utilization factor is assumed for each processing line

\section{B.1.1 Throughput Estimates}

Through-put is defined as the type and quantity of material that must be processed by facility modules and facility element ( module operation station) for each case and associated scenario. Below is the formula used to derive the throughput estimates. For the purpose of this study throughput simply means the number of component (casks, canisters, assemblies etc.) processed per facility element per year.

Desired Through-put Target $=\mathrm{D}_{\mathrm{TP}}$

Facility infrastructure element time (hours) associated with processing one unit $=$ Unit Operations Time $=(\mathrm{T})$

Operational Hours/shift $=(\mathrm{A})=8$ 
Shifts required $/$ Unit Processed $=(B)=(T) /(A)=T / 8$

Available Operating Shifts/day $=(D)=2$

Operating Calendar Days Required /Unit Processed $=(\mathrm{E})=(\mathrm{B}) /(\mathrm{D})=\mathrm{T} / 16$

Facility Element Assumed \% Utility or Availability $=75 \%$

Available calendar days/yr $=(\mathrm{F})=.75 \times 365=273$

Estimated Units that can be processed/year per Facility Element $=(\mathrm{U})=(\mathrm{F}) /(\mathrm{E})=273 /(\mathrm{T} / 16)=4368 / \mathrm{T}$

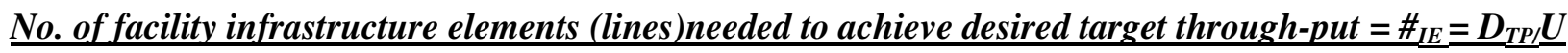
$\equiv\left(D_{T P} \underline{x T) / 4368}\right.$

Based on the above, key facility infrastructure elements were examined on the basis of their total unit operations processing time for a line of the respective facility infrastructure element. The total unit operations time is the sum of the times for individual unit operations steps that occupy the facility element during of one through-put item (one cask, one canister etc.). Typical facility infrastructure elements included carrier cask receipt bays, carrier cask release bays, cask and canister handling cranes, fuel pool fuel assembly handling cranes, canister welding stations etc.

\section{B.1.2 Capacity and Size Estimates}

Facility elements whose sizes are a function of capacity rather than throughput were sized based on the total capacity required for a given scenario. Typical examples of these include storage pad quantities and sizes, fuel pool fuel assembly total storage capacity etc.

\section{B.2 FACILITY DESCRIPTIONS AND PROCESSING ELEMENT UNIT OPERATIONS}

The following sections provide a summary description of the respective facilities addressed in this study, their typical unit operations times contributing to the sizing of the facility infrastructure elements associated with each scenario. The Used Fuel Disposition Storage, Transportation and Disposal Interface Cost Study (DOE 2012e), developed unit cost information for typical facilities which would be associated with the UNF receipt and disposition missions related to this study. Their associated facility descriptions from the referenced cost study are summarized below, for clarity and continuity in describing their functional elements, their unit operations activities and associated size estimates. Tables B-1- B-8 provide a summary of the unit operations facility element sizing data associated with the scenarios in this study.

\section{B.2.1 Consolidated Storage Facility (CSF) Scope}

The scope of the CSF includes the following major functions:

- Receiving fuel from reactors, currently in various wet and dry storage configurations, that will need to be considered by consolidated storage

- $\quad$ Storing dry fuel on pads

- Storing dry fuel in vaults (as an alternative to pads) 
- $\quad$ Storing bare fuel in pools

- Transferring fuel to a Repackaging Facility where it would be processed into waste package sized containers, with the size dependent upon specific repository geologic conditions

- Transferring fuel to a Repository for final disposition

\section{B.2.1.1 CSF Modules}

In order to provide the flexibility to respond to unknown situations and developments associated with accomplishing the back-end of the fuel cycle the CSF physical features were subdivide into the following potential stand-alone facility modules. These include:

- Cask Receipt and Canister Transfer (CRCT) Facility

- Dry Storage Pads (Vertical and Horizontal)

- Dry Storage Canister (DSC) Storage Vault Canister Transfer (SVCT) Facility

- $\quad$ Bare Fuel Receipt and Storage (FRS) Facility

Basic facility modules could then be combined into configurations necessary to the facility size and throughput requirements dictated by each given scenario. Sections 2.1.2 through 2.1.6 below summarize these facilities and associated operations. Section 2.1.7 summarizes the unit operations activities times associated with the facilities and tabulates the key facility element quantities (e.g. number of carrier receipt bays, number of carrier release bays, number of fuel pools and basins etc.) associated with the scenarios developed in this study.

\section{B.2.1.2 CSF Facility Modules Design Concepts}

The CSF will receive commercial UNF, currently in various wet and dry storage configurations, from reactor sites. If the fuel is packed in welded or bolted canisters, the canisters will be shipped in licensed transportation casks that measure approximately seven feet in diameter and 17 feet in length. Each cask may weigh as much as 175 tons, gross container weight, including fuel.

The CSF module dry storage design concepts are based on receiving and storing cask/canister systems whose designs are similar to the commercially available vertical and horizontal systems designs.

At no time, during the shipment or storage processes, beyond initial fuel canister loading at the reactor, would it be intended that the canisters be opened or the UNF fuel assemblies be directly handled until the fuel is subsequently transferred into the repackaging facility, in preparation for disposal.

In general, the cask and canister handling design concepts and storage design concepts are similar to concepts developed for DOE's past repository surface facility designs and the Private Fuel Storage L.L.C. (PFS) proposed for Toole County, Utah (. The receipt and the vertical storage design concepts are based closely on the facility that the US NRC licensed as PFS (US NRC-PFS). 


\section{B.2.1.3 Cask Receipt and Canister Transfer Facility}

The scope for the CRCT facility includes the following processes. The pre-conceptual layout of the CRCT facility is illustrated in Figures B-1-B-3:

- Receiving containerized fuel from the reactors in legacy existing storage systems

- Removing canisters from the transport over packs (or casks)

- Transferring canisters to facility over packs (or casks)

- Transferring fuel to CSF dry storage

The CRCT facility will be located at the CSF and is envisioned as a structural steel high-bay structure, consisting, of one or more receipt bays, each on the order of $168.5 \mathrm{ft}$. wide, $162 \mathrm{ft}$. long, and somewhere between 70 and $90 \mathrm{ft}$. high. This structure will have rail carrier access into each cask bay for cask handling and canister unloading/transfer operations. Each CRCT building transfer bay would contain a 250 ton, single trolley, overhead bridge crane. This crane would also be equipped with a 25 ton auxiliary hoist

Each bay would receive the transportation cask on its rail carrier, remove the transportation cask impact limiters and remove the transportation casks from the carriers, open the transportation casks, unload the UNF canisters from the cask, and place the canisters into the facility storage casks for transfer to the dry storage pads.

The loaded storage casks and empty transportation casks would be removed from the building by the same rail carrier "pusher engine" used to deliver the loaded rail cars from the Site security rail inspection siding, located at the site perimeter, as reflected in Figures B-4 and B-5. The CRCT facility will have the ability to provide localized (at the work site) HEPA filtered ventilation for cask venting and sampling operations.

Following removal of the loaded storage casks from the building, the transportation cask would be radiologically surveyed, decontaminated if necessary, and reassembled for shipment offsite. Each loading bay would provide for rail carrier pass through for fuel transportation cask receipt, and returns and storage cask transfer to dry storage.

Adjacent to the rail line, the structure would contain two below grade shielded wells (for placement of the loaded transportation cask and empty storage cask) approximately $20 \mathrm{ft}$. in depth, with one at approximately $8 \mathrm{ft}$. diameter and one at approximately $12 \mathrm{ft}$. diameter. Two additional, shallow inline wells would exist for temporary placement of the transportation cask lid and storage cask lid, with their tops resting at the level of the floor surface. Although other concepts and alternatives might be considered, the concept of use of the four casks and lids inline wells is suggested, vs. transfer cask handling and heavy component placement operations on and above the level of the CRCT building floor. The suggested concept would reduce overhead building height and allow a 50 ton floor running bell crane to travel over the shielded wells, transferring the UNF canisters from the transportation casks to the storage casks, on a standard handling cycle.

\section{B.2.1.4 Dry Storage Pads}

The loaded CSF storage cask would be mounted on a self-propelled modular transporter (SPMT) at the CRCT facility and transferred to a dry storage pad or vault. The storage casks are equipped with vents 
and channels that provide cooling by passive, natural convection processes. It is proposed that the storage casks and pads would be constructed, on an as needed basis, at the CSF to the respective licensed storage unit design. A concrete batch plant will be located within the boundary of the CSF to support on-site final fabrication of the storage units.

\section{B.2.1.4.1 Dry Storage - Pads (Horizontal)}

Horizontal storage casks would be transported from the CRCT building to a storage pad with a SPMT. At the storage pad the SPMT would position itself and the shielded cask for precise insertion of the canister into a horizontal storage module. This process of loading the storage casks is illustrated by pictures in Figures B-1-B-5.

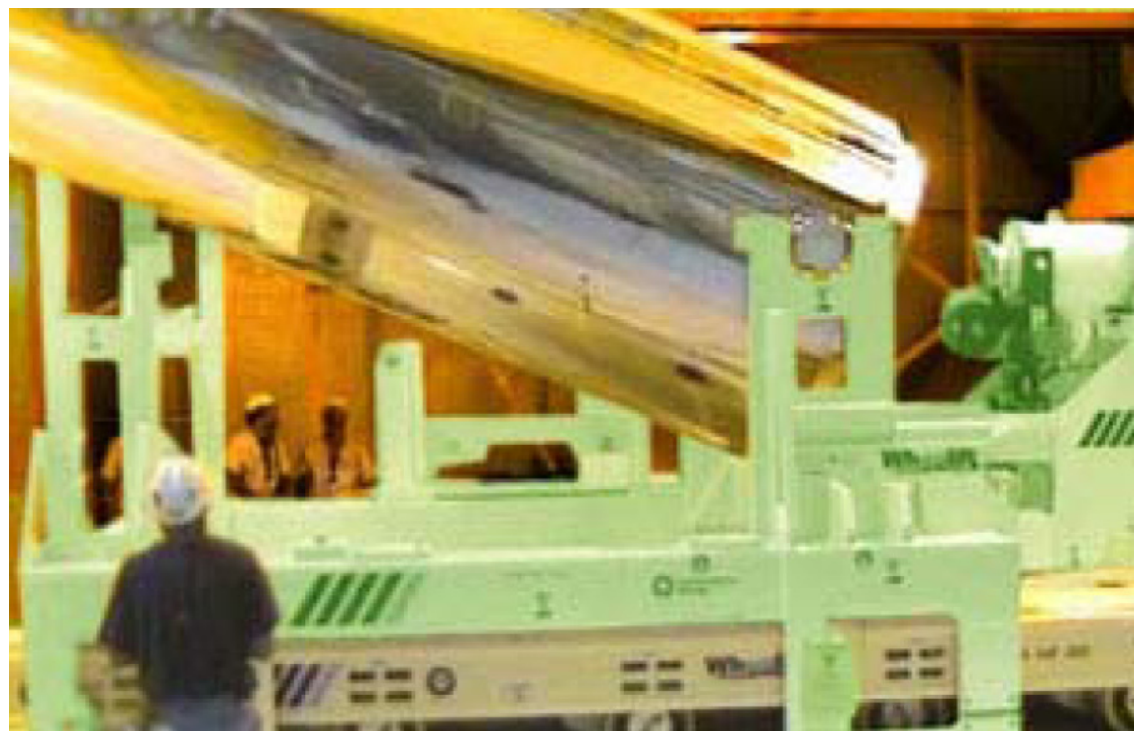

Figure B-1. Downending a horizontal shielded cask on an SPMT for transfer to the storage pad.

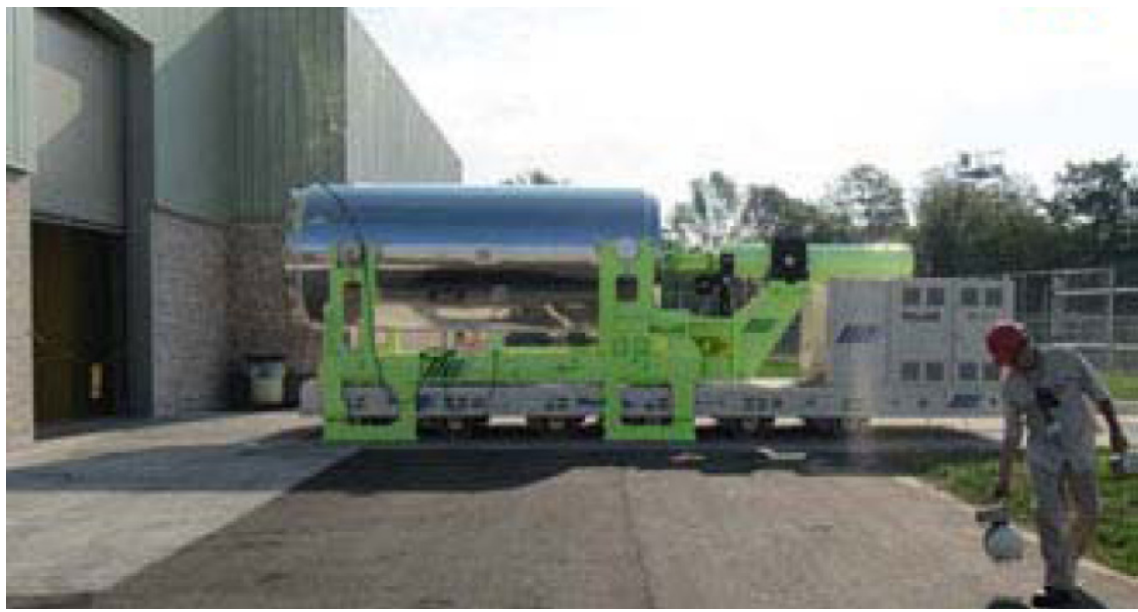

Figure B-2. SPMT transporting horizontal shielded cask and canister from the CRCT building. 


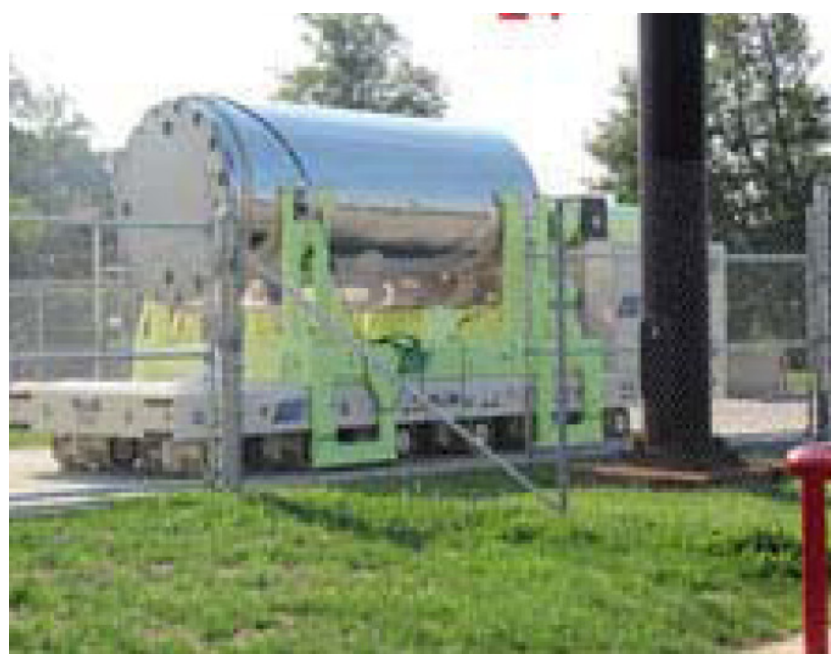

Figure B-3. SPMT in transit with horizontal shielded cask and canister.

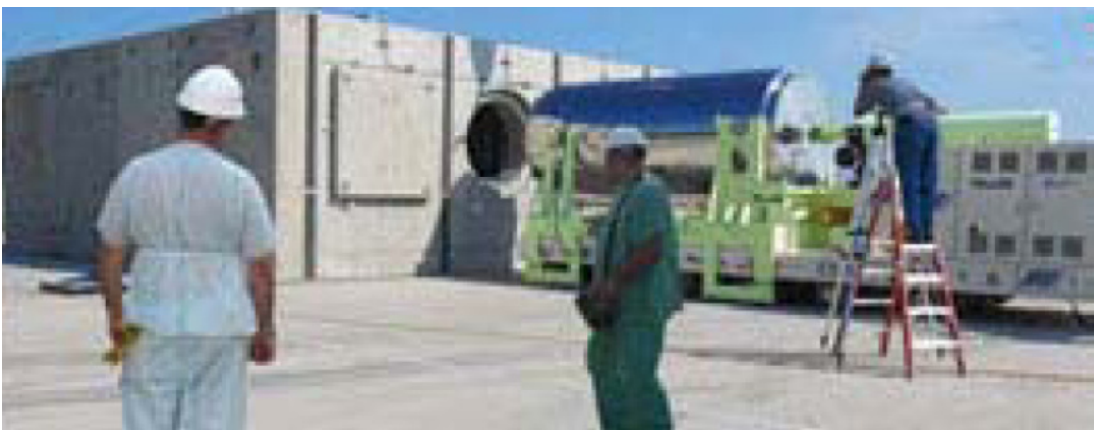

Figure B-4. Positioning SPMT for canister insertion into a horizontal storage module.

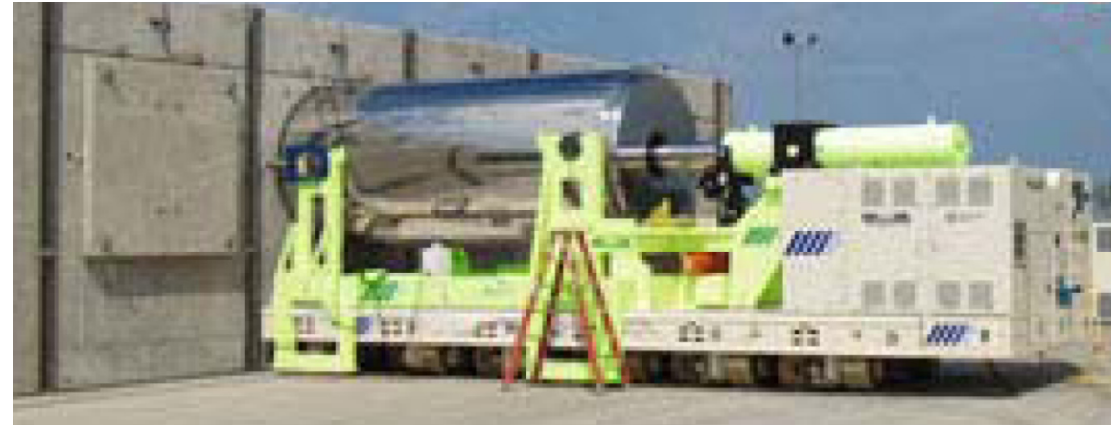

Figure B-5. SPMT canister insertion into a horizontal storage module.

Horizontal storage systems, such as shown in Figure B-6, can be aligned with multiple single-canister horizontal storage units in a module. Horizontal storage module arrays containing 12 canisters are assumed for layout purposes. Each module is approximately $52 \mathrm{ft}$. wide by $89 \mathrm{ft}$. long, containing 12 horizontal storage units. Rows of modules would be separated by approximately $50 \mathrm{ft}$. to allow access for the SPMT shielded transporter. Areas between the modules would have transporter access and be surfaced sufficiently to allow travel of heavy lift equipment. 


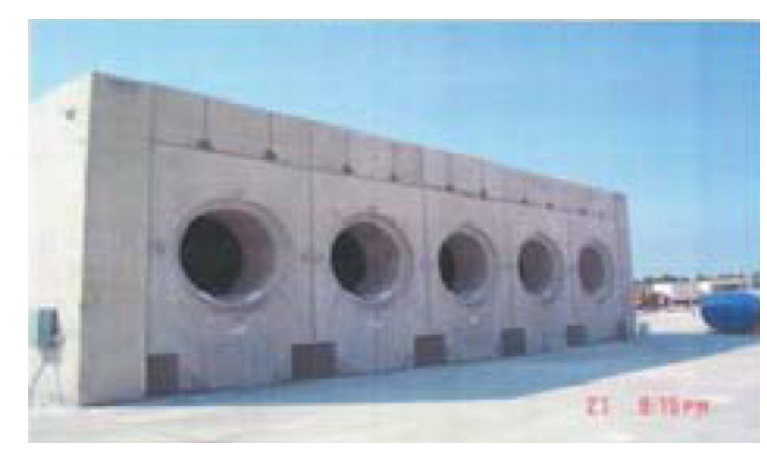

Figure B-6. Horizontal storage unit.

\section{B.2.1.4.2 Dry Storage - Pads (Vertical)}

Vertical storage casks would be transported from the CRCT facility to a storage pad with a SPMT as shown in Figure B-7. At the storage pad, the vertical cask would be lifted by a service crane or vertical cask crawler crane and placed on the storage pad.
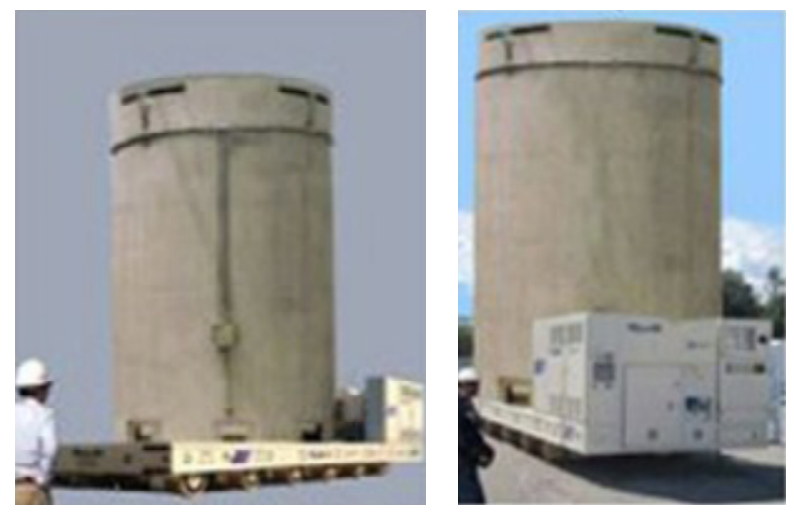

Figure B-7. SPMT carrying a UNF storage cask.

Figure B-8 illustrates a typical vertical cask storage pad. There are multiple licensed vertical dry storage systems and the vertical dry storage design concept would have to accommodate all vertical dry storage systems in use. Multiple vertical cask storage pads are needed. Each storage pad would be constructed flush with grade level and accept up to 8 storage casks in a $2 \times 4$ array. The typical vertical storage pad dimensions would be on the order of approximately 67 feet long by 30 feet wide by 3 feet thick. Each cask would contain $~ 10$ to 14 MTHM, assuming a basis of 68 BWR or 32 PWR assemblies in each cask, although canister loading densities will be a function of licensed canister design and actual loading densities achieved at reactor source sites. Although other pad configurations can be designed, each pad would be surrounded by a compacted gravel skirt, on the order of 30 feet wide. Areas between the pads would have transporter access and be surfaced sufficiently to allow travel of heavy lift and transfer equipment such as a SPMT. 


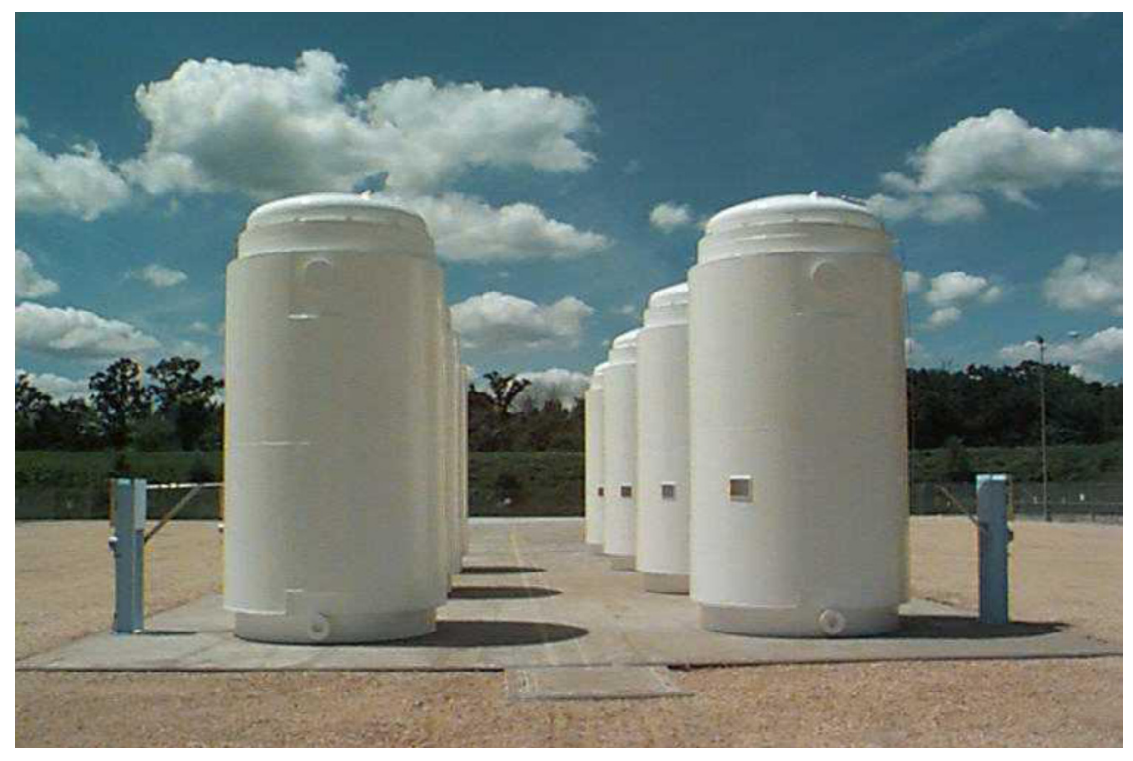

Figure B-8. Typical vertical cask dry storage pad.

\section{B.2.1.5 DSC Storage Vault and Canister Transfer Facility}

The scope for the Storage Vault Canister Transfer (SVCT) facility includes the following functions. The pre-conceptual layout of the SVCT has been illustrated in Figure B-21

- Receiving canisterized fuel from the reactors in either existing storage systems or newly designed disposal canisters that are compatible with future repository disposal criteria.

- Removing canisters from the transport over packs (or casks)

- Transferring canisterized fuel to CSF DSC vaults

A dry storage vault concept for the receipt, unloading, and storage of 3,000 MTHM of canisterized fuel was previously developed in the Engineering Alternative Study (EAS) for Separations -Summary Report (DOE 2007b). The basic receipt and storage concept has been adopted for the SVCT with an expansion of the module storage capacity to 7,500 MT.

The SVCT facility is envisioned to receive commercial UNF packaged in welded or bolted canisters in transportation overpacks. The UNF canisters will be transported to the facility by rail. The UNF canister will be transferred from the shipping over-pack to an underground vault for storage.

When received, the licensed transportation cask will be opened and unloaded by removal of its sealed fuel canister into a transfer cask. The canister will be moved to an underground transfer shuttle cart which moves the canister into the vault operations area. Overhead crane mounted shielded transfer casks are then used to locate the canister in the desired vault storage location. The underground vault ventilation is assumed to provide cooling by passive, natural convection; thermal analyses will be necessary to evaluate this assumption for canisters with higher burnup, newly discharged (5-10 years old) used fuel.. 
Following transfer of the fuel canisters to the storage vault, the transportation cask would be radiologically surveyed, decontaminated if necessary, and reassembled for shipment offsite. Each loading bay would provide for railroad pass through of fuel transportation cask carriers.

\section{B.2.1.6 Bare Fuel Receipt and Storage Facility}

The scope of the bare Fuel Receipt and Storage (FRS) Facility for the pool storage includes the following functions. The pre-conceptual layout is illustrated in Figure B-7, Figure B-8, and Figure B-9.

- Receiving bare fuel transportation cask on a transportation cask carrier from the reactor.

- Removing bare fuel transportation cask from the transportation cask carrier

- Transferring bare fuel from the transportation cask to CSF pool storage

A pool storage concept for the receipt, unloading, and storage of 3,000 MTHM was previously developed in the Engineering Alternative Study (EAS) for Separations -Summary Report (DOE 2007b). The basic receipt concept was adopted for this study with an expansion of the storage capacity to 7,500 MT.

The wet storage pool is separated from the cask receipt bays by an airlock. The transportation cask is moved from the cask receipt bays, through the airlock, lowered into the pool and flooded with water prior to removal of the inner container lid. Once the lid is removed, the individual fuel assemblies are transferred to the desired fuel assembly storage rack location.

The pool consists of 8 interconnected basins. Each basin is approximately $158 \mathrm{ft}$. long by $60 \mathrm{ft}$. wide and $55 \mathrm{ft}$. deep. Each basin contains 100 storage racks providing 35 assembly storage positions using a 15 by 15 inch array. Thus each fuel pool basin corresponds to 3500 bare fuel assembly storage positions.

If an 8-basin, 28,000 assembly pool (3500 assemblies per basin, separate PWR and BWR assembly basins) were fully loaded with high-burn-up UNF, on a ratio of $43 \%$ PWR fuel assemblies and 57\% BWR assemblies, the total assemblies of each type would be about 12,040 PWR and 15,960 BWR. On that basis, at approximately 0.436 MTU per PWR assembly and 0.179 MTU per BWR assembly, the total decay heat (60GWd/MT PWR fuel is $\sim 3,530$ watts/MT and $50 \mathrm{GWd} / \mathrm{MT}$ BWR fuel is $\sim 2,920$ watts/MT) would be about $26.9 \mathrm{MW}$. The decay heat is discharged to the atmosphere by cooling towers. Water treatment, ventilation, and support areas are adjacent to the pools.

Used nuclear fuel assemblies will arrive onsite via commercially licensed transport vehicle. The transport vehicle will consist of a special railcar or special truck with casks specifically designed for the safe and secure transport of UNF. All shipping casks will be NRC licensed, and contents will be within license constraints. For baseline planning purposes, it can be assumed that rail casks contain approximately 26 PWR fuel assemblies (or 61 BWR assemblies) and truck casks contain approximately 4 PWR fuel assemblies (or 9 BWR assemblies).

The fuel transportation casks will be received and initially staged in a receipt area where contamination surveys, other integrity checks and transportation \& shipment documentation verification can be performed, to assure that receipt documentation and package condition is in order and that decontamination or repairs are not required before unloading.

The transport,vehicle and transportation cask, upon completion of the radiological survey, will proceed to the Fuel Receipt and Storage (FRS) facility for removal, inspection, survey and storage of the bare spent fuel assemblies. Cameras, scanners, manipulators and similar equipment are likely to be required to 
perform this function. The used fuel assembly may require washing and de-scaling prior to release to storage.

\section{B.2.1.7 Facility Unit Operations Times and Facility Element Sizing Estimate Data}

This section summarizes facility unit operations and sizing data associated with the above facilities._Unit operations activities and estimated durations are summarized in Tables B-1 and B-2 and below. Tables B-3 and B-4 provide associated facility infrastructure element estimates.

Vertical storage cask pads and horizontal storage modules are sized on the basis of 8 vertical casks per pad and 12 horizontal storage units per horizontal storage module. Wet storage pools are sized on the basis of 3,500 UNF fuel assemblies per basin with 8 basins per fuel pool. The required CSF Facility infrastructure requirements are estimated on the basis of the following for the scenarios included in this study. 
Table B-1. Cask receipt and canister transfer bay unit operation

\begin{tabular}{|l|c|}
\hline \multicolumn{1}{|c|}{ Unit operations activities } & $\begin{array}{c}\text { Unit operations step } \\
\text { time } \\
\text { (hours) }\end{array}$ \\
\hline $\begin{array}{l}\text { Dock transport carrier, anchor, unload cask accompanied rigging/hardware, remove } \\
\text { shipping restraints, remove transport cask personnel barrier, remove cask impact limiters, } \\
\text { complete receipt documentation, perform rad surveys. }\end{array}$ & 8 \\
\hline $\begin{array}{l}\text { Install transport cask trunions and prepare cask for lifting, mount transport cask lifting } \\
\text { yoke }\end{array}$ & 2.85 \\
\hline $\begin{array}{l}\text { Upend transport cask, lift and transfer transport cask into transport cask receipt well, } \\
\text { position crane for next carrier. }\end{array}$ & 2.25 \\
\hline $\begin{array}{l}\text { Transfer empty storage casks into facility, anchor carrier, remove storage cask transfer } \\
\text { restraints, prepare storage cask for lift and prepare cask for canister receipt, remove } \\
\text { transport cask lifting yoke, install storage cask lifting yoke, lift and transfer storage cask } \\
\text { into storage cask receipt well. }\end{array}$ & \\
\hline $\begin{array}{l}\text { Prepare transport and storage casks outer lids for removal and canister transfer, perform } \\
\text { rad surveys. }\end{array}$ & 2 \\
\hline $\begin{array}{l}\text { Position shielded facility cask to transport cask for canister removal, remove cask lids, } \\
\text { transfer canister to storage cask, install cask lids, retire shielded facility cask and survey, } \\
\text { inspect casks and document. }\end{array}$ & 2.5 \\
\hline $\begin{array}{l}\text { Lift and transfer storage cask to facility shuttle carrier, anchor storage cask for shuttle } \\
\text { transfer to storage, survey for transfer. }\end{array}$ & \\
\hline $\begin{array}{l}\text { Mount transport cask lifting yoke, lift and transfer empty transport cask to carrier, } \\
\text { downend, remove and load tunions, install cask impact limiters, install cask personnel } \\
\text { barrier, install shipping restraints, load and release, finalize preparation for offside release, } \\
\text { remove carrier anchors. }\end{array}$ & \\
\hline Transfer transport cask carrier to rail yard and storage cask carrier to cask storage area. & \\
\hline Total Time in Cask Receipt Bay & \\
\hline Pan & \\
\hline
\end{tabular}

Notes:

- Durations shown are assumed to apply to all rail and truck cask/container packages received regardless of capacity.

- At approximately 32 hours estimated for canister transfer operations per bay, 4 shifts may be assumed considering shift changes and turnovers etc., per cask transfer cycle, per bay. Assuming approximately .75 utility per bay considering downtime for maintenance and repair this would relate to $365 \times .75$ days per year $=273$ days per year for operations $=273$ days $\times 2$ shifts per day $=546$ shifts $/ y$ for operations. At 4 shifts per container package processed, 546 shifts/4 shifts per container relates to processing of approximately 136 inbound cask and canister transfer per year per bay.

- Timeline activities do not account for bay down time, for for potential decon of inbound or outbound canisters, casks, or carriers. If required such activities can be assumed to occur in off shifts and at other locations as needed. Timeline does not account for bay occupancy/traffic time for return receipt and processing of casks/canister from downstream canister processing facilities or other traffic.

- Thus each cask receipt and canister transfer building bay supports cask receipt and canister transfers, on a two shift basis, on the basis of approximately 136 casks and canisters received per year per bay. An additional transfer bay would be allowed for each increment of 136 casks/canisters through-put per year beyond 136. 
Table B-2. Storage vault canister transfer (SVCT) bay unit operations

\begin{tabular}{|l|c|}
\hline \multicolumn{1}{|c|}{ Unit operations activities } & $\begin{array}{c}\text { Unit operations } \\
\text { step time } \\
\text { (hours) }\end{array}$ \\
\hline $\begin{array}{l}\text { Dock transport carrier, anchor, unload cask accompanied rigging/hardware, remove shipping } \\
\text { restraints, remove transport cask impact limiters, complete receipt documentation, perform rad } \\
\text { surveys }\end{array}$ & 8 \\
\hline Install transport cask trunions and prepare cask for lifting, mount transport cask lifting yoke. & 2.85 \\
\hline $\begin{array}{l}\text { Upend transport cask, lift and transfer transport cask into transport cask receipt well, position } \\
\text { crane for next carrier. }\end{array}$ & 2.25 \\
\hline $\begin{array}{l}\text { Position facility vault transfer cask in receipt bay, prepare vault transfer cask for canister } \\
\text { receipt. }\end{array}$ & 1 \\
\hline Prepare transport cask outer lid for removal and canister transfer, perform rad surveys. & 2.5 \\
\hline $\begin{array}{l}\text { Position facility vault transfer cask for canister removal, remove cask lid, transfer canister to } \\
\text { vault transfer cask, install cask lid, survey \& inspect casks and document. }\end{array}$ & 3.5 \\
\hline $\begin{array}{l}\text { Move transfer cask to vault, remove transfer cask lid, lift and transfer canister to vault storage } \\
\text { position, install vault shield plug and transfer cask lid. }\end{array}$ & 4 \\
\hline $\begin{array}{l}\text { Lift and transfer empty transport cask to carrier, downend, remove and load trunions, install } \\
\text { cask impact limiters, install cask personnel barrier, install shipping restraints, load and anchor } \\
\text { cask accompanied rigging/hardware, complete rad survey and transportation documentation } \\
\text { for release, finalize preparation for offsite release, remove carrier anchors. }\end{array}$ \\
\hline Transfer transport cask carrier to rail yard for release. & \\
\hline $\begin{array}{l}\text { Time In Storage Vault Canister Transfer Bay } \\
\text { Timal }\end{array}$ & \\
\hline
\end{tabular}

Notes:

- Durations shown are assumed to apply to all cask/container packages received regardless of capacity.

- At approximately 33 hours estimated for canister transfer operations per bay, 4 shifts may be assumed considering shift changes and turnovers etc., per cask transfer cycle, per bay. Assuming approximately .75 utility per bay considering downtime for maintenance and repair this would relate to $365 \times .75$ days per year $=$ 273 days per year for operations $=273$ days $\times 2$ shifts per day $=546$ shifts/y for operations. At 4 shifts per container package processed, 546 shifts/4 shifts per container relates to processing of approximately 136 inbound cask and canister transfers per year per SVCT bay.

- Thus each SVCT building receipt bay supports cask receipt and canister transfers, on a two shift basis, on the basis of increment of 136 casks/canister throughput per year beyond 136. 
Table B-3. CSF facility infrastructure receipt elements estimate

\begin{tabular}{|c|c|c|c|c|c|c|c|c|c|c|c|c|c|}
\hline & \multirow[b]{3}{*}{ Scenario } & \multirow[b]{3}{*}{$\begin{array}{c}\text { Acceptance } \\
\text { Rate }\end{array}$} & \multirow[b]{3}{*}{ CSF Start } & \multirow[b]{3}{*}{$\begin{array}{c}\text { Repository } \\
\text { Start }\end{array}$} & \multirow[b]{3}{*}{$\begin{array}{c}\text { Disposal } \\
\text { Canister } \\
\text { Size }\end{array}$} & \multicolumn{6}{|c|}{ Peak Annual Arrivals } & \multirow{2}{*}{\multicolumn{2}{|c|}{ Receipt Bay Requirements }} \\
\hline & & & & & & & Fuel Casks & & & Dry Canisters & & & \\
\hline & & & & & & PWR & BWR & Total & Horizontal Total & Vertical Total & Total & Canisters & Bare \\
\hline Case 1 Var Set 31 & \multirow{9}{*}{$\begin{array}{c}\text { Canisters } \\
\text { Only - } \\
\text { Re-Package } \\
\text { at Repository }\end{array}$} & 1,500 & 2020 & 2040 & 4 & & & & 27 & 142 & 147 & 2 & \\
\hline Case 1 Var Set 40 & & 3,000 & 2020 & 2040 & 4 & & & & 61 & 257 & 269 & 2 & \\
\hline Case 1 Var Set 67 & & 3,000 & 2020 & 2040 & 12 & & & & 61 & 257 & 269 & 2 & \\
\hline Case 1 Var Set 94 & & 3,000 & 2020 & 2040 & 21 & & & & 61 & 257 & 269 & 2 & \\
\hline Case 1 Var Set 49 & & 6,000 & 2020 & 2040 & 4 & & & & 156 & 476 & 520 & 4 & \\
\hline Case 1 Var Set 34 & & 1,500 & 2020 & 2055 & 4 & & & & 50 & 142 & 147 & 2 & \\
\hline Case 1 Var Set 43 & & 3,000 & 2020 & 2055 & 4 & & & & 85 & 257 & 269 & 2 & \\
\hline Case 1 Var Set 52 & & 6,000 & 2020 & 2055 & 4 & & & & 162 & 476 & 520 & 4 & \\
\hline Case 1 Var Set 44 & & 3,000 & 2035 & 2055 & 4 & & & & 81 & 236 & 265 & 2 & \\
\hline Case 2 Var Set 31 & \multirow{9}{*}{$\begin{array}{c}\text { Canisters and } \\
\text { Bare Fuel - } \\
\text { Re-Package } \\
\text { at Repository }\end{array}$} & 1,500 & 2020 & 2040 & 4 & 119 & 63 & 182 & 9 & 31 & 32 & 1 & 5 \\
\hline Case 2 Var Set 40 & & 3,000 & 2020 & 2040 & 4 & 202 & 133 & 325 & 11 & 47 & 49 & 1 & 8 \\
\hline Case 2 Var Set 67 & & 3,000 & 2020 & 2040 & 12 & 202 & 133 & 325 & 11 & 47 & 49 & 1 & 8 \\
\hline Case 2 Var Set 94 & & 3,000 & 2020 & 2040 & 21 & 202 & 133 & 325 & 11 & 47 & 49 & 1 & 8 \\
\hline Case 2 Var Set 49 & & 6,000 & 2020 & 2040 & 4 & 404 & 300 & 634 & 91 & 146 & 225 & 2 & 17 \\
\hline Case 2 Var Set 34 & & 1,500 & 2020 & 2055 & 4 & 119 & 63 & 182 & 42 & 75 & 112 & 1 & 5 \\
\hline Case 2 Var Set 43 & & 3,000 & 2020 & 2055 & 4 & 202 & 133 & 325 & 80 & 143 & 215 & 2 & 8 \\
\hline Case 2 Var Set 52 & & 6,000 & 2020 & 2055 & 4 & 404 & 300 & 634 & 91 & 146 & 225 & 2 & 17 \\
\hline Case 2 Var Set 44 & & 3,000 & 2035 & 2055 & 4 & 146 & 112 & 258 & 79 & 139 & 218 & 2 & 6 \\
\hline Case 3 Var Set 31 & \multirow{9}{*}{$\begin{array}{l}\text { Canisters } \\
\text { Only - Re- } \\
\text { Package at } \\
\text { CSF }\end{array}$} & 1,500 & 2020 & 2040 & 4 & & & & 55 & 142 & 147 & 2 & \\
\hline Case 3 Var Set 40 & & 3,000 & 2020 & 2040 & 4 & & & & 87 & 262 & 348 & 3 & \\
\hline Case 3 Var Set 67 & & 3,000 & 2020 & 2040 & 12 & & & & 87 & 262 & 348 & 3 & \\
\hline Case 3 Var Set 94 & & 3,000 & 2020 & 2040 & 21 & & & & 87 & 262 & 348 & 3 & \\
\hline Case 3 Var Set 49 & & 6,000 & 2020 & 2040 & 4 & & & & 162 & 476 & 520 & 4 & \\
\hline Case 3 Var Set 34 & & 1,500 & 2020 & 2055 & 4 & & & & 55 & 142 & 147 & 2 & \\
\hline Case 3 Var Set 43 & & 3,000 & 2020 & 2055 & 4 & & & & 87 & 262 & 348 & 3 & \\
\hline Case 3 Var Set 52 & & 6,000 & 2020 & 2055 & 4 & & & & 162 & 476 & 520 & 4 & \\
\hline Case 3 Var Set 44 & & 3,000 & 2035 & 2055 & 4 & & & & 98 & 236 & 265 & 2 & \\
\hline Case 4 Var Set 31 & \multirow{9}{*}{$\begin{array}{c}\text { Canisters and } \\
\text { Bare Fuel - } \\
\text { Re-Package } \\
\text { at CSF }\end{array}$} & 1,500 & 2020 & 2040 & 4 & 119 & 63 & 182 & 58 & 104 & 130 & 1 & 5 \\
\hline Case 4 Var Set 40 & & 3,000 & 2020 & 2040 & 4 & 202 & 133 & 325 & 88 & 202 & 246 & 2 & 8 \\
\hline Case 4 Var Set 67 & & 3,000 & 2020 & 2040 & 12 & 202 & 133 & 325 & 88 & 202 & 246 & 2 & 8 \\
\hline Case 4 Var Set 94 & & 3,000 & 2020 & 2040 & 21 & 202 & 133 & 325 & 88 & 202 & 246 & 2 & 8 \\
\hline Case 4 Var Set 49 & & 6,000 & 2020 & 2040 & 4 & 404 & 300 & 634 & 91 & 146 & 225 & 2 & 17 \\
\hline Case 4 Var Set 34 & & 1,500 & 2020 & 2055 & 4 & 119 & 63 & 182 & 58 & 104 & 130 & 1 & 5 \\
\hline Case 4 Var Set 43 & & 3,000 & 2020 & 2055 & 4 & 202 & 133 & 325 & 88 & 202 & 246 & 2 & 8 \\
\hline Case 4 Var Set 52 & & 6,000 & 2020 & 2055 & 4 & 404 & 300 & 634 & 91 & 146 & 225 & 2 & 17 \\
\hline Case 4 Var Set 44 & & 3,000 & 2035 & 2055 & 4 & 146 & 112 & 258 & 94 & 260 & 333 & 3 & 6 \\
\hline
\end{tabular}


Table B-4. CSF facility infrastructure storage elements estimate

\begin{tabular}{|c|c|c|c|c|c|c|c|c|c|c|c|c|c|c|c|c|c|c|}
\hline & \multirow[b]{2}{*}{ Scenario } & \multirow[b]{2}{*}{$\begin{array}{c}\text { Acceptance } \\
\text { Rate }\end{array}$} & \multirow[b]{2}{*}{ CSF Start } & \multirow[b]{2}{*}{$\begin{array}{l}\text { Repository } \\
\text { Start }\end{array}$} & \multirow[b]{2}{*}{$\begin{array}{l}\text { Disposal } \\
\text { Canister } \\
\text { Size }\end{array}$} & \multicolumn{2}{|c|}{ Vertical Canisters } & \multicolumn{2}{|c|}{ Horizontal Canisters } & \multicolumn{2}{|c|}{ Vertical Pads } & \multicolumn{2}{|c|}{ Horizontal Modules } & \multicolumn{2}{|r|}{ Pool Basins } & \multicolumn{3}{|c|}{ Maximum MTHM } \\
\hline & & & & & & 2-Year & Max & 2-Year & Max & 2-Year & Max & 2-Year & Max & \multicolumn{2}{|c|}{ Max } & Canisters & Bare & Total \\
\hline Case 1 Var Set 31 & \multirow{9}{*}{$\begin{array}{c}\text { Canisters } \\
\text { Only- } \\
\text { Re-Package } \\
\text { at Repository }\end{array}$} & 1,500 & 2020 & 2040 & 4 & 259 & 2,155 & 13 & 325 & 33 & 270 & 2 & 28 & 298 & & 30,377 & & 30,377 \\
\hline Case 1 Var Set 40 & & 3,000 & 2020 & 2040 & 4 & 480 & 3,981 & 45 & 825 & 60 & 498 & 4 & 69 & 567 & & \begin{tabular}{|l|l|}
59,731 \\
\end{tabular} & & 59,731 \\
\hline Case 1 Var Set 67 & & 3,000 & 2020 & 2040 & 12 & 480 & 3,981 & 45 & 825 & 60 & 498 & 4 & 69 & 567 & & 59,731 & & 59,731 \\
\hline Case 1 Var Set 94 & & 3,000 & 2020 & 2040 & 21 & 480 & 3,981 & 45 & \begin{tabular}{|l|}
825 \\
\end{tabular} & 60 & 498 & 4 & 69 & 567 & & \begin{tabular}{|l|l|}
59,731 \\
\end{tabular} & & 59,731 \\
\hline Case 1 Var Set 49 & & 6,000 & 2020 & 2040 & 4 & 900 & 5,349 & 108 & 1,581 & 113 & 669 & 9 & 132 & 801 & & 86,031 & & 86,031 \\
\hline Case 1 Var Set 34 & & 1,500 & 2020 & 2055 & 4 & 259 & 3,471 & 13 & 791 & 33 & 434 & 2 & 66 & 500 & & 52,824 & & 52,824 \\
\hline Case 1 Var Set 43 & & 3,000 & 2020 & 2055 & 4 & 480 & 6,506 & 45 & 1,839 & 60 & 814 & 4 & 154 & 968 & & \begin{tabular}{ll|}
104,054 \\
\end{tabular} & & 104,054 \\
\hline Case 1 Var Set 52 & & 6,000 & 2020 & 2055 & 4 & 900 & 8,241 & 108 & 2,347 & 113 & 1,031 & 9 & 196 & 1,227 & & 132,300 & & 132,300 \\
\hline Case 1 Var Set 44 & & 3,000 & 2035 & 2055 & 4 & 448 & 3,696 & 71 & 1,150 & 56 & 462 & 6 & 96 & 558 & & 60,081 & & 60,081 \\
\hline Case 2 Var Set 31 & & 1,500 & 2020 & 2040 & 4 & 47 & 238 & 2 & 43 & 6 & 30 & $\overline{11}$ & 4 & 34 & 29 & 3,010 & \begin{tabular}{|l|l|}
27,324 \\
\end{tabular} & 30,334 \\
\hline Case 2 Var Set 40 & & 3,000 & 2020 & 2040 & 4 & 84 & 346 & 7 & 91 & 11 & 44 & 1 & 8 & 52 & 57 & 4,865 & 55,216 & 60,081 \\
\hline Case 2 Var Set 67 & & 3,000 & 2020 & 2040 & 12 & 84 & 346 & 7 & 91 & 11 & 44 & 1 & 8 & 52 & 57 & 4,865 & 55,216 & 60,081 \\
\hline Case 2 Var Set 94 & Canisters and & 3,000 & 2020 & 2040 & 21 & 84 & 346 & 7 & 91 & 11 & 44 & 1 & 8 & 52 & 57 & 4,865 & 55,216 & 60,081 \\
\hline Case 2 Var Set 49 & Bare fuel - & 6,000 & 2020 & 2040 & 4 & 139 & 1,790 & 14 & 953 & 18 & 224 & 2 & 80 & 304 & 87 & 32,940 & 86,605 & 119,545 \\
\hline Case 2 Var Set 34 & re-package & 1,500 & 2020 & 2055 & 4 & 47 & 859 & 2 & 390 & 6 & 108 & 1 & 33 & 141 & 39 & 14,834 & 37,959 & 52,793 \\
\hline Case 2 Var Set 43 & & 3,000 & 2020 & 2055 & 4 & 84 & 1,555 & 7 & 844 & 11 & 195 & 1 & 71 & 266 & 76 & 28,925 & 76,096 & 105,021 \\
\hline Case 2 Var Set 52 & & 6,000 & 2020 & 2055 & 4 & 139 & 1,828 & 14 & 980 & 18 & 229 & 2 & 82 & 311 & 104 & 33,280 & \begin{tabular}{ll|}
104,982 \\
\end{tabular} & 138,262 \\
\hline Case 2 Var Set 44 & & 3,000 & 2035 & 2055 & 4 & 84 & 1,411 & 33 & 798 & 11 & 177 & 3 & 67 & 244 & 33 & 26,671 & 33,410 & 60,081 \\
\hline Case 3 Var Set 31 & & 1,500 & 2020 & 2040 & 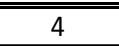 & 259 & 2,155 & \begin{tabular}{|c|}
13 \\
\end{tabular} & 835 & 33 & 270 & 2 & 70 & 340 & & 30,377 & & 30,377 \\
\hline Case 3 Var Set 40 & & 3,000 & 2020 & 2040 & 4 & 480 & 3,981 & 45 & 1,430 & 60 & 498 & 4 & 120 & 618 & & 59,979 & & 59,979 \\
\hline Case 3 Var Set 67 & & 3,000 & 2020 & 2040 & 12 & 480 & 3,981 & 45 & 1,430 & 60 & 498 & 4 & 120 & 618 & & 59,979 & & 59,979 \\
\hline Case 3 Var Set 94 & Canisters & 3,000 & 2020 & 2040 & 21 & 480 & 3,981 & 45 & 1,430 & 60 & 498 & 4 & 120 & 618 & & \begin{tabular}{|l|l|}
59,979 \\
\end{tabular} & & 59,979 \\
\hline Case 3 Var Set 49 & $\begin{array}{l}\text { Only-Re- } \\
\text { Pachage }\end{array}$ & 6,000 & 2020 & 2040 & 4 & 900 & 5,349 & 108 & 1,713 & 113 & 669 & 9 & 143 & 812 & & 86,031 & & 86,031 \\
\hline Case 3 Var Set 34 & & 1,500 & 2020 & 2055 & 4 & 259 & 3,471 & 13 & 1,459 & 33 & 434 & 2 & 122 & 556 & & 52,839 & & 52,839 \\
\hline Case 3 Var Set 43 & & 3,000 & 2020 & 2055 & 4 & 480 & 6,512 & 45 & 2,213 & 60 & 814 & 4 & 185 & 999 & & \begin{tabular}{|l|}
105,073 \\
\end{tabular} & & 105,073 \\
\hline Case 3 Var Set 52 & & 6,000 & 2020 & 2055 & 4 & 900 & 8,241 & 108 & 2,407 & 113 & 1,031 & 9 & 201 & 1,232 & & 132,300 & & 132,300 \\
\hline Case 3 Var Set 44 & & 3,000 & 2035 & 2055 & 4 & 448 & 3,696 & 71 & 1,652 & 56 & 462 & 6 & 138 & 600 & & \begin{tabular}{|l|}
60,109 \\
\end{tabular} & & 60,109 \\
\hline Case 4 Var Set 31 & & 1,500 & 2020 & 2040 & 4 & 47 & 1,721 & 2 & 839 & 6 & 216 & 1 & 70 & 286 & 29 & 30,131 & 27,324 & 57,455 \\
\hline Case 4 Var Set 40 & & 3,000 & 2020 & 2040 & 4 & 84 & 2,800 & 7 & 1,313 & 11 & 350 & 1 & 110 & 460 & 57 & 50,451 & 55,216 & 105,667 \\
\hline Case 4 Var Set 67 & & 3,000 & 2020 & 2040 & 12 & 84 & 2,800 & 7 & 1,313 & 11 & 350 & 1 & 110 & 460 & 57 & 50,451 & 55,216 & 105,667 \\
\hline Case 4 Var Set 94 & & 3,000 & 2020 & 2040 & 21 & 84 & 2,800 & 7 & 1,313 & 11 & 350 & 1 & 110 & 460 & 57 & 50,451 & $\begin{array}{ll}55,216 \\
\end{array}$ & 105,667 \\
\hline Case 4 Var Set 49 & Bare tuel - & 6,000 & 2020 & 2040 & 4 & 139 & 1,790 & 14 & 953 & 18 & 224 & 2 & 80 & 304 & 87 & 32,940 & 86,605 & 119,545 \\
\hline Case 4 Var Set 34 & & 1,500 & 2020 & 2055 & 4 & 47 & 2,934 & 2 & 1,459 & 6 & 367 & 1 & 122 & 489 & 39 & 52,576 & 37,959 & 90,535 \\
\hline Case 4 Var Set 43 & & 3,000 & 2020 & 2055 & 4 & 84 & 3,203 & 7 & 1,594 & 11 & 401 & 1 & 133 & 534 & 76 & 58,845 & $\begin{array}{l}76,096 \\
\end{array}$ & 134,940 \\
\hline Case 4 Var Set 52 & & 6,000 & 2020 & 2055 & 4 & 139 & 1,828 & 14 & 980 & 18 & 229 & 2 & 82 & 311 & 104 & 33,280 & 104,982 & 138,262 \\
\hline Case 4 Var Set 44 & & 3,000 & 2035 & 2055 & 4 & 84 & 3,350 & 33 & 1,655 & 11 & 419 & 3 & 138 & 557 & 33 & \begin{tabular}{ll|}
59,922 \\
\end{tabular} & 33,410 & 93,336 \\
\hline
\end{tabular}




\section{B.2.2 Repackaging Facility}

Current storage and transportation systems' design, operations, and licensing requirements do not consider disposal requirements because the disposal requirements are not defined and are not connected to storage and transportation functions. Nuclear utility decisions related to UNF storage are largely motivated by minimizing their cost and minimizing potential impacts to continued safe and cost-effective operation of their nuclear plants. This disconnect between storage and transportation and disposal, as well as insufficient pool storage capacity to enable continued reactor operation, has led to widespread use of large capacity dry storage casks. Direct disposal of the large canisters currently used by the commercial nuclear power industry is beyond the current experience base domestically and internationally, and represents significant engineering and scientific challenges. Repackaging of fuel from these larger canisters into smaller ones for disposal, may be required to avoid extensive surface decay storage, or to meet physical constraints on disposal systems, or because additional criticality controls are determined to be necessary.

For the purpose of this analysis, the repackaging of the canisters could be accomplished either at a standalone facility or a facility co-located with a CSF or Mined Geologic Repository. The scope for the Repackaging Facility (RF) includes the following processes. The pre-conceptual design of the RF is illustrated in Figure B-1, Figure B-2, and Figure B-3.

- Receiving bare fuel or canisterized fuel from either a utility site, a consolidated storage facility or both

- Unloading the transportation cask from the transportation carrier or unloading a transfer cask from a CSF SPMT

- Opening bare fuel transportation casks

- Cutting open welded dual purpose canisters

- Unloading used fuel assemblies from the casks or canisters and repackaging the assemblies into disposal canisters

- $\quad$ Transferring repackaged fuel to a CSF or a Repository

The RF module is sized for 1500 MTU/yr through-put. The main sub-structures within the module include a Carrier Receipt Bay, a Waste Handling Building (WHB), and a Carrier Release Bay. Two air locks are included - one between the Receipt Bay and the WHB and one between the WHB and the Release Bay (Figure B-9). The configurations of the Receipt Bay and the Release Bay may vary considerably depending on whether the RF is co-located with a CSF or a Mined Geologic Repository, or is a stand-alone facility. For example, if the repackaging facility is co-located with a Mined Geologic Repository, then the Release Bay would not necessarily be needed and could potentially be replaced with a transfer corridor to a facility for placing waste package over packs on the canisters as described in Disposal Concepts/Thermal Load Management (FY11/12 Summary Report) (DOE 2012c). 


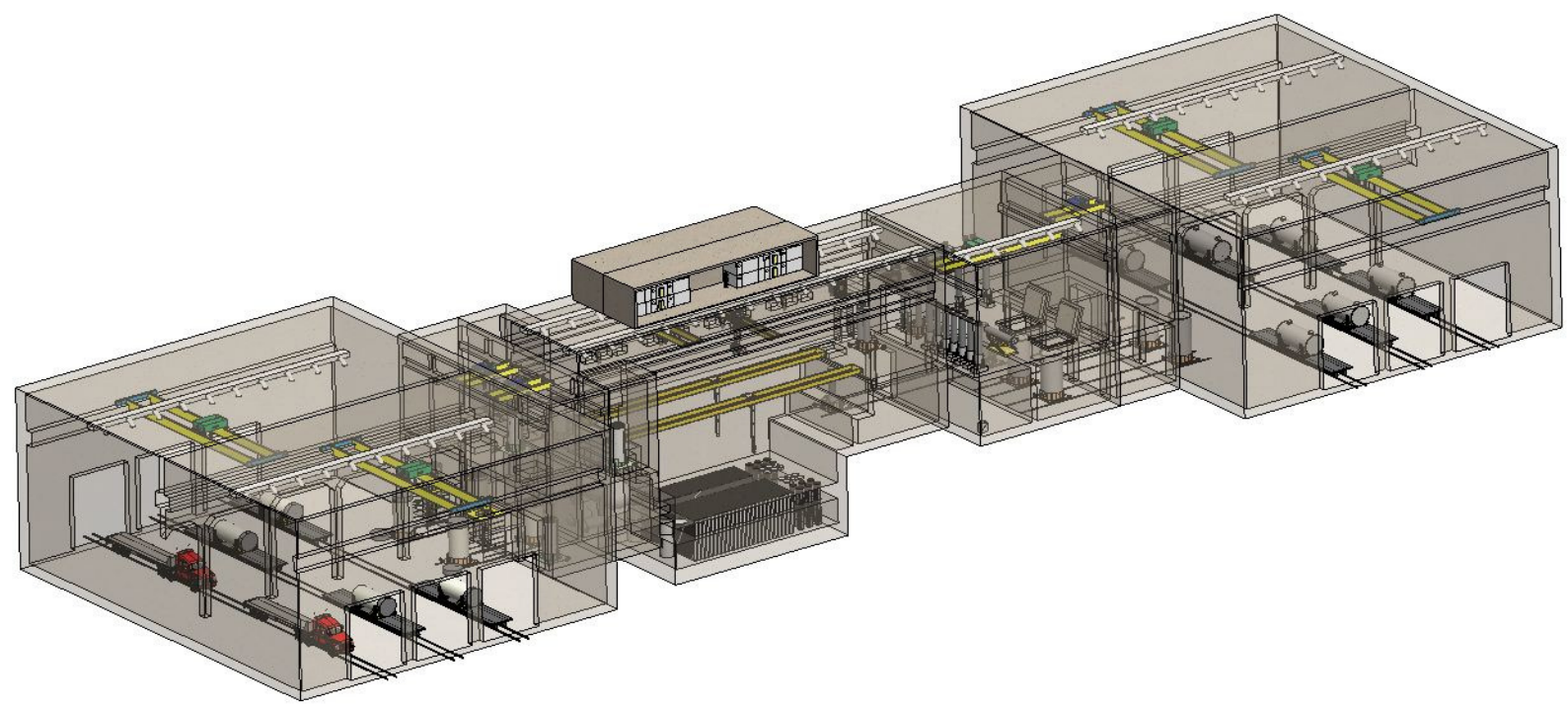

Figure B-9. Isometric view of the overall repackaging facility module concept, including carrier receipt bay, airlocks, waste handling building and carrier release bay.

Commercial UNF will be transported to RF in NRC-certified transportation casks. The waste will be transported by rail or road to the Facility Operations Area security station, where personnel will verify the shipping manifests, then inspect and survey the cask and its carrier. After the cask and its carrier enter the Radiologically Controlled Area, they will be staged in parking areas designated for either truck carriers or rail carriers. When the cask is scheduled for processing, a site prime mover will move the cask and carrier to the Carrier Receipt Bay. The facility operations security station is assumed to be a shared facility with either the CSF or the Repository, depending on where the RF is co-located. 


\section{B.2.2.1 Carrier Receipt Bay Features and Operations}

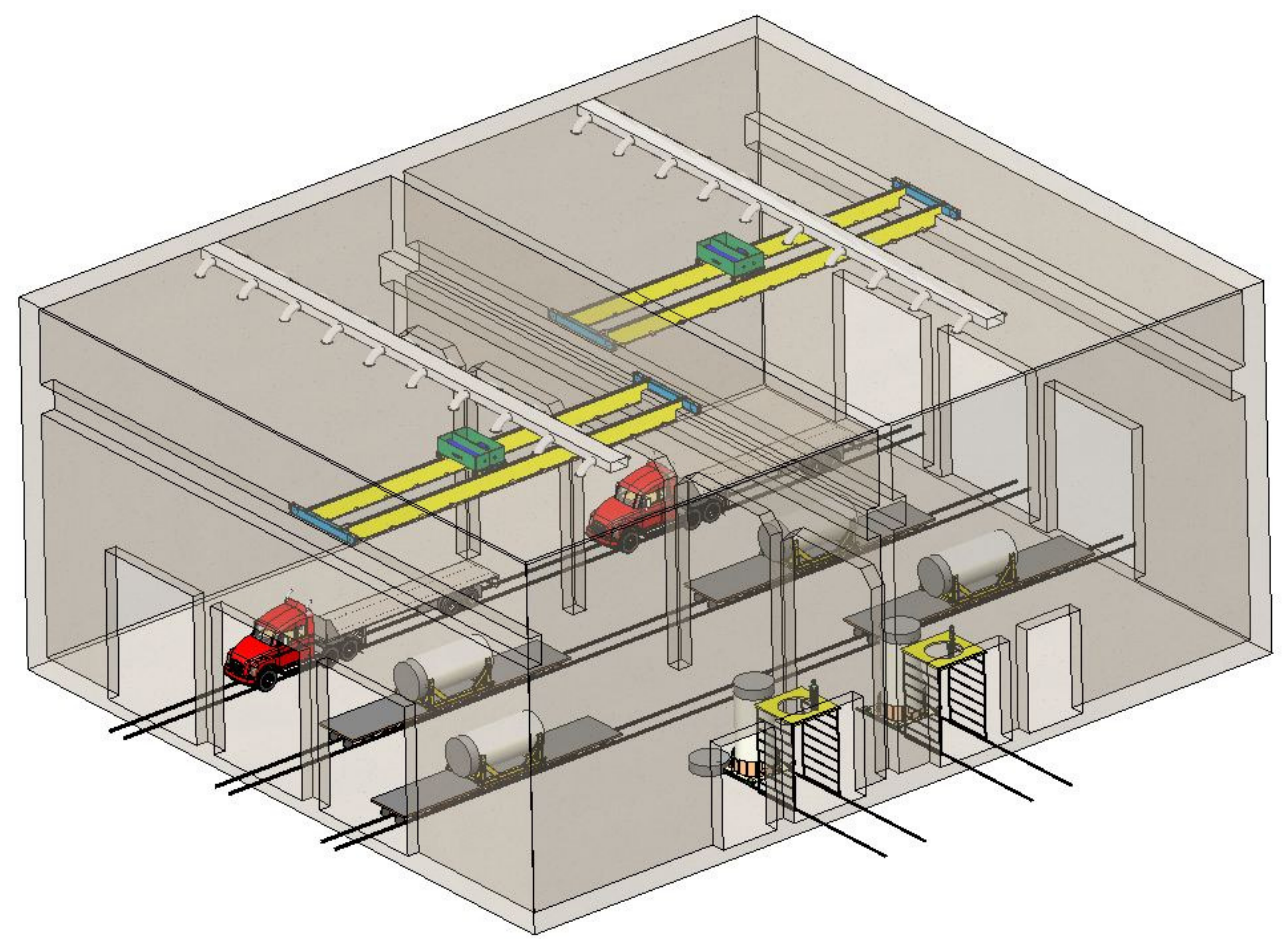

Figure B-10. Carrier receipt bay module.

The Carrier Receipt Bay Module is envisioned as a structural steel high-bay structure. The structure would be nominally $75 \mathrm{ft}$ high. For a $1500 \mathrm{MTU} / y e a r$ through-put, this structure would require at least two lines with two 250 ton overhead bridge cranes. A third line is shown in B-10 for additional flexibility. The actual number of lines and bays will be a function of projected through-put. The material handling system in the Carrier Receipt Bay will receive and inspect shipping casks from the carrier/cask transport system, prepare the casks for unloading, and unload transportation casks from the railcar or truck. The Carrier Receipt Bay will include sufficient space between lines for a Mobile Access Platform (MAP), Cask Stand for Tilt Frame Bare Fuel Transportation Casks and Horizontal Dual Purpose Canister (DPC) Transportation Casks, Tilt Frame, Transportation Cask Transfer Trolley, and lay down areas for impact limiters and other equipment. The MAP will allow personnel access to transportation casks brought in by rail or truck. The MAP bridges over the cask lying on the carrier. The MAP includes platforms to provide access by personnel to different features on the cask (e.g., personnel barriers and impact limiters). Railcars and trucks will enter and exit the Carrier Receipt Bay by $30 \mathrm{ft}$ high by $25 \mathrm{ft}$ wide roll up metal doors.

Receiving operations will include the following and were based on the operations documented in Civilian Radioactive Waste Management System (CRWMS) Management and Operations (M\&O) Attachment II, Section 1.3.2.1 (DOE 2000a)

- Performing a radiation survey of the carrier and the DPC transportation cask or bare fuel transportation cask

- Removing or retracting the personnel barrier(s)

- Sampling the cask exterior for contamination 
- Measuring the cask's temperature

- Removing or retracting the cask impact limiters

- Installing the cask's lifting attachments (if any).

Shipping operations for empty carriers/casks leaving the facility will include:

- Removing the cask's trunnions (if required)

- Checking the cask's tie-downs

- Installing the cask's impact limiters

- Performing another radiation survey of the cask

- Installing the personnel barriers.

\section{B.2.2.1.1 Transportation Casks Handling Operations}

The concept of operations assumes that two types of transportation casks will be received at the Repackaging Facility:

- Transportation casks that can be upended directly on the railcar to a vertical orientation for unloading (Direct Vertical Cask)

- Transportation casks that must be transferred from the railcar in a horizontal orientation to a tilting frame that is used to upend the cask to a vertical orientation for unloading (Tilt Frame Cask)

The handling operations differ based on the type of cask.

\section{B.2.2.1.2 Direct Vertical Casks Operations}

Associated operations include:

- Receive and move a Direct Vertical Transportation Cask on a railcar into preparation area for unloading

- Remove personnel barriers if present

- Remove impact limiters from transportation cask

- Attach lift yoke to transportation cask

- Upend cask

- Transfer transportation cask to cask transfer trolley/cart

- Move cask transfer trolley/cart into position in front of WHB Airlock

- Remove cask lid bolts, and attach cask lid lift fixture; remove and store lid

\section{B.2.2.1.3 Tilt Frame Cask Operations}

Associated operations include:

- Receive and move a Tilt Frame Transportation Cask on a railcar into preparation area for unloading

- Remove personnel barriers if present

- Attach slings to transportation casks for horizontal lift

- Make horizontal transfer of cask to cask stand

- Remove impact limiters from transportation cask

- Transfer cask stand to tilt frame

- Attach lift yoke to transportation cask

- Upend cask

- Transfer transportation cask to cask transfer trolley/cart 
- Move cask transfer trolley/cart into position in front of Waste Handling Building Airlock

- Remove cask lid bolts, and attach cask lid lift fixture; remove and store lid

\section{B.2.2.2 Waste Handling Building Features and Operations}

The WHB is a multi-level reinforced concrete structure made of noncombustible materials with interior and exterior shear walls, concrete floor, concrete roof slab diaphragms, concrete mat foundations, and a transfer pool. The nominal footprint of the WHB, including air locks, is about $282 \mathrm{ft}$ by $92 \mathrm{ft}$. The maximum height of the building is about $100 \mathrm{ft}$. above grade, with the majority of the building under a roof approximately $80 \mathrm{ft}$ above grade. The WHB pool substructure includes the rooms surrounding the pool that provide internal buttresses for the actual pool and space for make-up tanks, pumps and filters, ion exchangers, etc. The concrete base mat for the basement structure (pool and surrounding rooms) is 55 $\mathrm{ft}$ below the top of the at-grade concrete mat. The spent fuel pool is sized hold approximately $750 \mathrm{MTU}$ of used nuclear fuel (6 month worth of spent fuel assembly inventory for a 1500 MTU module.) This will allow some flexibility for fuel blending as a thermal management strategy as well as decouple waste receipt and unloading critical path operations from waste package canister loading and closure operations. The pool is split into separate basins and storage racks for BWR assemblies and PWR assemblies and includes separate spent fuel transfer machines (handling cranes) for BWR and PWR assemblies to avoid change out operations of lifting grapples.

The foundation for the WHB is a reinforced concrete mat at grade and another reinforced concrete mat below the pool having the necessary thickness to adequately support the structure. The foundation mat at grade for the Waste Handling structure is $3 \mathrm{ft}$ thick and the pool foundation is a 5 -ft-thick mat.

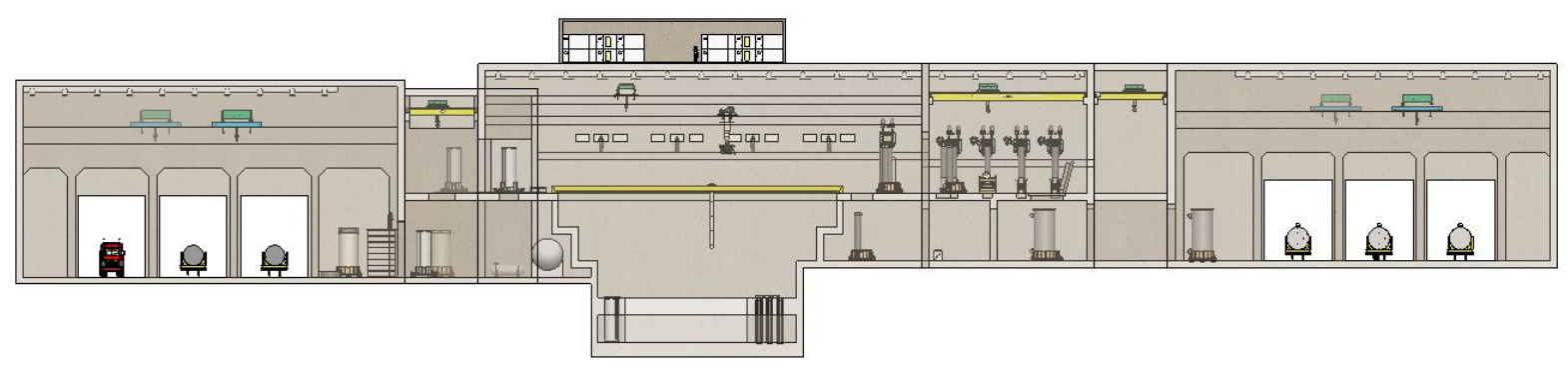

Figure B-11. Elevation of waste handling building.

An airlock structure connects the Receipt Bay with the WHB. The cask transfer cart will move the transportation cask into the air lock, which will have isolation doors to maintain a lower air pressure in the canister transfer work areas than in the carrier bay. The cart will take the cask through the air lock to the cask preparation area similar to the area in (Civilian Radioactive Waste Management System (CRWMS) Management and Operations (M\&O) Attachment II, Section 1.1.2.1 (2000b).

If the cask contains a DPC, an overhead bridge crane will lift the DPC out of the cask to the second level and the empty transportation cask will be decontaminated (if necessary) and returned to the Receipt Bay where it will be reconfigured for re-deployment to the transportation system. 


\section{B.2.2.2.1 Cask Cavity Gas Sampling Subsystem}

The cask cavity gas sampling system samples the gas inside a loaded transportation cask or DPC before it is opened to obtain an indication of the condition of the waste inside. The presence of gaseous fission products or gases other than helium is indicative of off-normal conditions inside the cask. The cask cavity gas sampling system also vents the cask or DPC to the HVAC system to equalize pressure with the room prior to opening the cask.

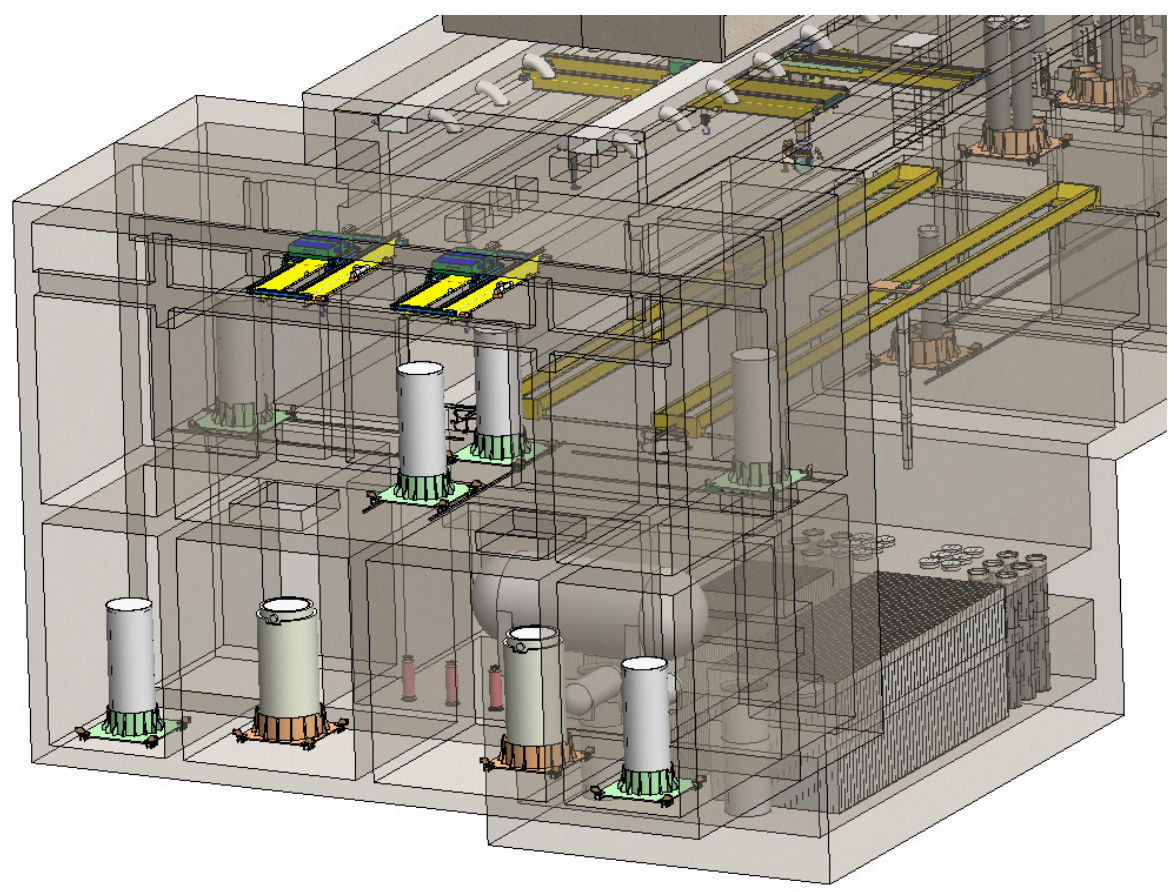

Figure B-12. View of receipt side airlock.

\section{B.2.2.2.2 Dual Purpose Canister Unloading Operations}

DPC cutting is done at the DPC cutting station in the cask preparation area. Although DPC lid cutting is done dry, within a hot cell area of the WHB, the reference concept assumes DPC UNF unloading is done wet.

- The DPC contained within a shielded site transfer cask is transferred to the DPC cutting station using the cask handling crane.

- A shield ring may be installed to limit personnel exposure.

- The DPC cutting jib crane is used to remove the shielded transfer cask lid.

- The DPC cutting machine is placed onto the DPC outer lid. The DPC outer lid weld is then cut, and the DPC cutting jib crane is used to remove the DPC cutting machine and the outer lid. (The DPC cutting machine is assumed to include an integral vacuum system to remove metal cuttings during the cutting process.)

- The DPC cutting machine is placed back onto the DPC to cut the siphon and vent port cover welds. The siphon and vent ports are used to sample and vent the DPC interior and fill the DPC with treated borated water in preparation for transfer to the pool. 
- The DPC cutting machine is then used to cut the final weld on either the inner lid or the shield plug, depending on the DPC type.

- If the DPC is a type that has an inner lid, the inner lid is removed from the DPC after the weld is cut. A lifting adapter is then attached to the shield plug. The lid to the shielded transfer cask is replaced using the DPC cutting jib crane. The shield ring is removed.

- Transfer casks with BWR DPC canisters are positioned next to BWR UNF basin and transfer casks with PWR DPC canisters are positioned next to the PWR UNF Basin.

- The shielded transfer cask containing the DPC is then transferred to the pool, where the shielded transfer cask lid is removed.

- If the DPC is a type that has a siphon tube attached to the shield plug, the shield plug is raised above the shielded transfer cask and the siphon tube is detached from the shield plug using the siphon tube shear tool. The detached siphon tube remains in the DPC.

- The shield plug is placed in a staging area in the pool.

- The used fuel transfer machine then accesses the interior of the DPC to remove the UNF assemblies. The UNF assemblies consist of PWR or BWR fuel; therefore, the used fuel transfer machine uses the PWR grapple or the BWR grapple to remove the UNF assemblies.

- The UNF assemblies are then transferred to a disposal canister or to the UNF staging rack. If the cask or canister contains damaged-fuel cans, these are transferred in the same manner. A limited number of special oversized cells are provided as part of the UNF staging racks to accommodate damaged-fuel cans or baskets shipped to the facility or encountered during UNF transfer within the WHB pool

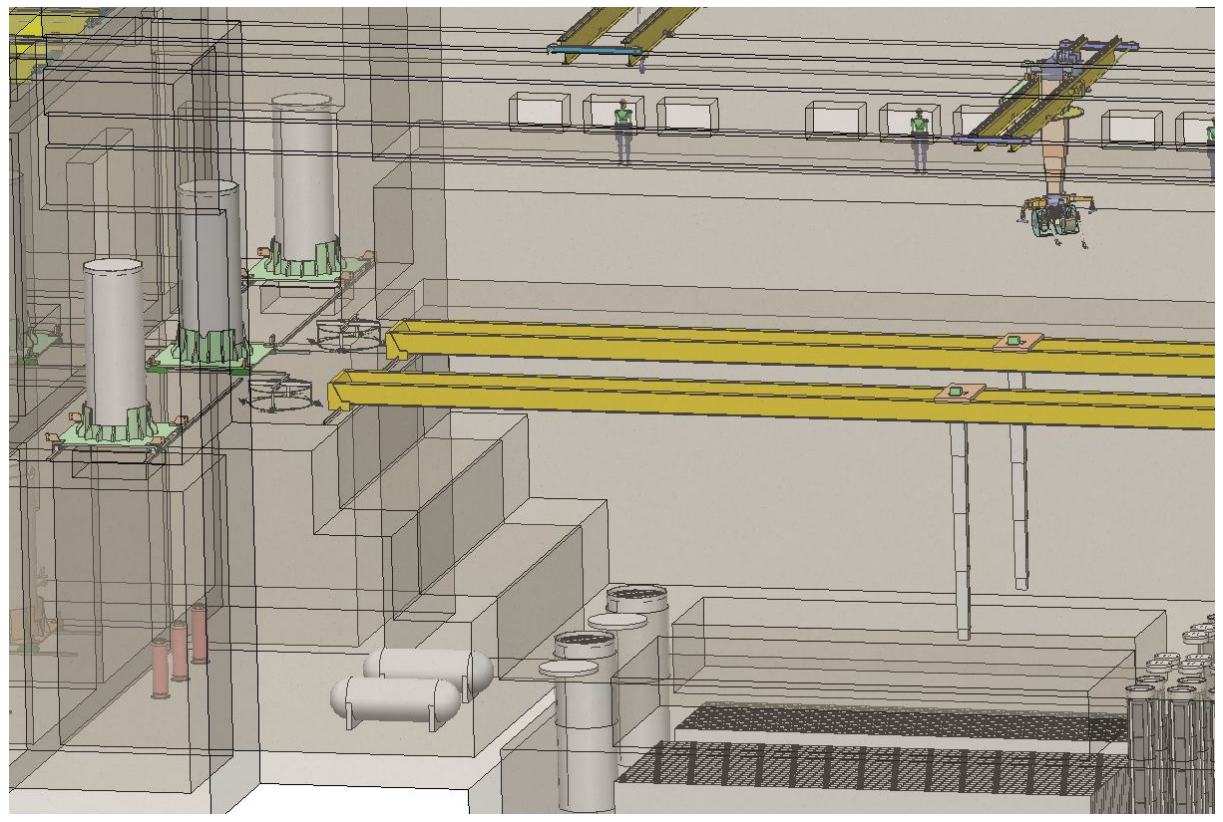

Figure B-13. View of DPC cutting stations adjacent to storage pool and canister unloading space. The observation corridor/control room is shown on the upper level.

\section{B.2.2.2.3 Disposal Canister Loading Operations}

- An empty disposal canister (and transfer cask if utilized) is placed on a disposal canister transfer trolley in the Carrier Release Bay 
- The empty disposal canister is moved through the Release Side Airlock and into the staging area below the canister welding and drying stations.

- A bridge crane lifts the empty disposal canister through the portal and places it in a disposal canister transfer trolley.

- The disposal canister transfer trolley positions the empty canister on the deck next to the canister loading area in the pool.

- The canister is filled with water and is lowered into the pool.

- The spent fuel transfer machine grapple is positioned over the UNF assembly to be moved. Once in position, the grapple is engaged and the assembly is lifted to the proper height under water for movement. The spent fuel transfer machine moves laterally to a position over the waste package canister and lowers the assembly into the canister, then disengages the grapple. This operation will repeat until the canister is full.

- The canister closure lid is seated on top of the canister using the overhead crane.

- The loaded canister (and transfer cask if utilized) is removed from the fuel pool, the canister is drained and placed in the canister transfer trolley.

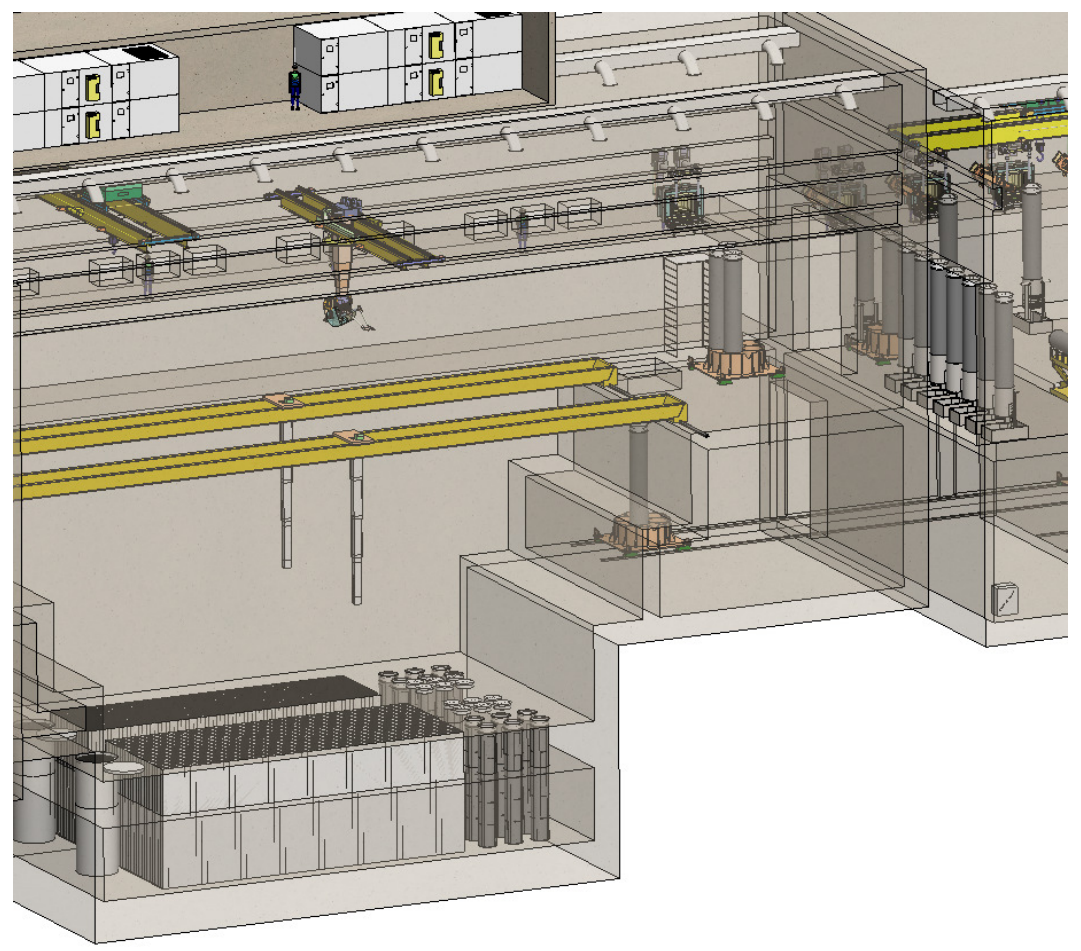

Figure B-14. Detail view of the disposal canister side of the packaging pool.

\section{B.2.2.2.4 Disposal Canister Closure}

- The Disposal Canister Transfer Trolley moves the loaded disposal canister to one of the welding stations

- The canister lid is welded onto the disposal canister

- The Disposal Canister Transfer Trolley moves the welded canister to one of the drying stations. 
- Residual water is removed from the canister cavity by either a vacuum or forced helium drying system

- The disposal canister is backfilled with helium to provide an inert atmosphere.

- An empty transportation cask is positioned in the room below the disposal canister closure and drying area using a transportation cask transfer cart/trolley.

- The overhead crane lowers the disposal canister into a transportation cask in the vertical position.

- The cask transfer cart moves the loaded transportation cask into the airlock between the WHB and the Carrier Release Bay.

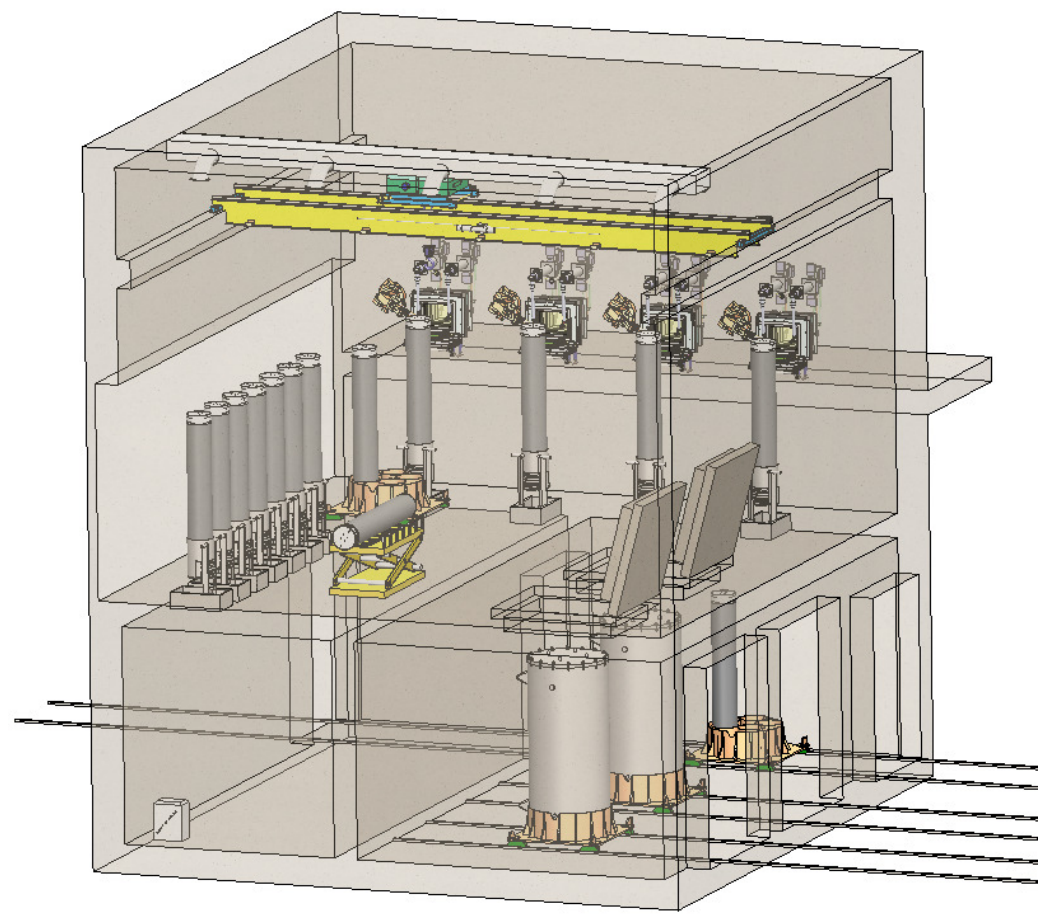

Figure B-15. View of disposal canister closure and drying/inerting area.

\section{B.2.2.2.5 Carrier Release Bay Features and Operations}

The Carrier Release Bay Module is envisioned as a structural steel high-bay structure similar in design and construction to the Carrier Receipt Bay Module described above. The main difference between the Receipt Bay and the Release Bay would likely be the number of lines required, with the Release Bay requiring more lines due to the greater number of canisters with smaller loads being shipped out for the same level of through-put. The material handling system in the Carrier Release Bay will receive and inspect empty transportation casks and empty disposal canisters from the carrier/cask transport system, prepare the casks for unloading, unload transportation casks from the railcar or truck, and reload the transportation cask and loaded disposal canister for release to the repository. Like the Receipt Bay, the Carrier Release Bay will include sufficient space between lines for a Mobile Access Platform (MAP), Cask Stand Tilt Frame, Transportation Cask Transfer Trolley, and lay down areas for impact limiters and other equipment. The MAP includes platforms to provide access by personnel to different features on the 
cask (e.g., personnel barriers and impact limiters). Railcars enter and exit the Carrier Release Bay by $30 \mathrm{ft}$ high by $25 \mathrm{ft}$ wide roll up metal doors.

Release operations will include:

- The cask transfer cart moves the loaded transportation cask from the airlock to the Carrier Release Bay.

- Placing the loaded transportation cask on a tilt frame and moving it to the horizontal position.

- Attaching impact limiters to the transportation cask

- Loading the transportation cask on the railcar

- Replacing the personnel barrier(s)

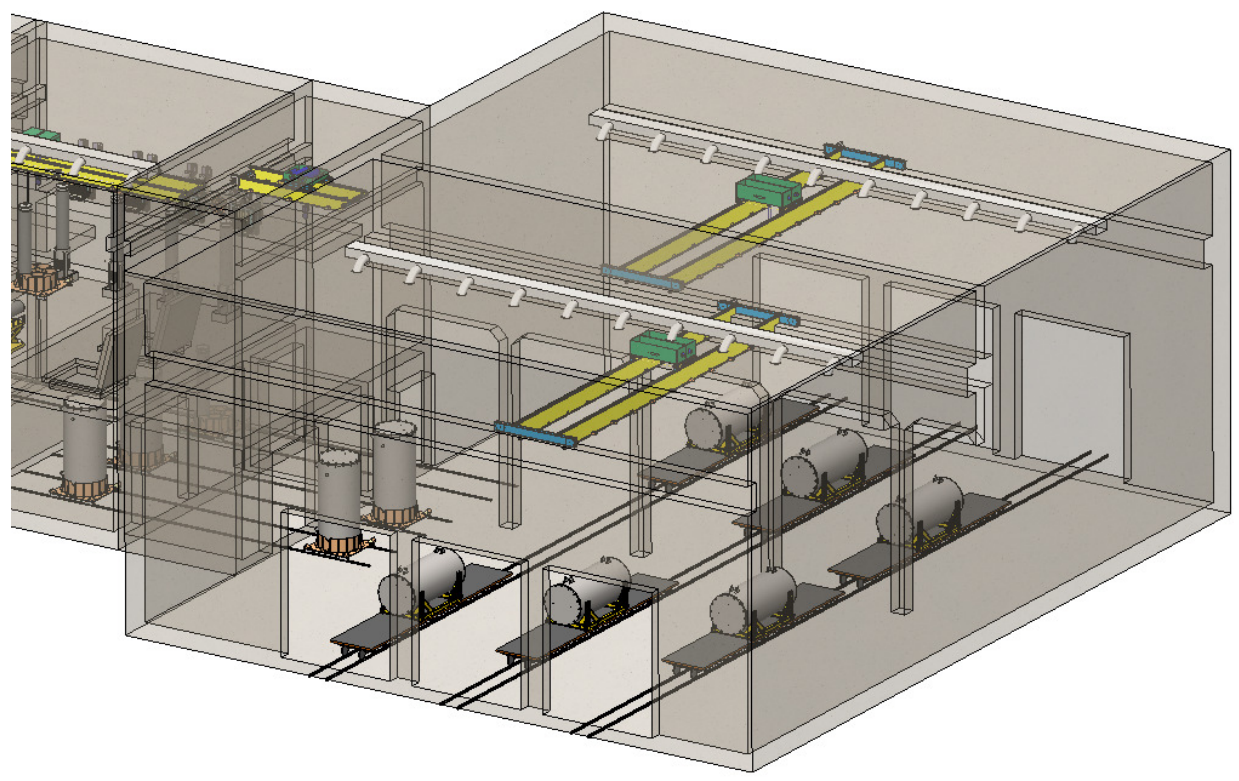

Figure B-16. Release side airlock and carrier release bay.

\section{B.2.2.3 Facility Unit Operations Times and Facility Element Sizing Estimate Data}

This section summarizes facility unit operations and sizing data associated with the above facility. Unit operations activities and estimated durations are summarized in Tables B-5 and B-6 below. Tables B-7 and B-8 provide associated facility infrastructure element estimates.

Repackaging facility carrier release bays are reduced by $50 \%$ for a repackaging facility located at the repository to account for reduced package handling for waste packages not requiring transportation overpack hardware preparation. Similarly repackaging facility carrier receipt bays are reduced by $50 \%$ for a repackaging facility located at the CSF to account for reduced package handling for storage casks not requiring transportation overpack hardware removal.

The required Repackaging Facility infrastructure requirements are estimated on the basis of the following for the scenarios included in this study. Repackaging facility lag storage pool capacity is estimated at half of the assigned annual through-put for each scenario. 
Table B-5. Repackaging facility inbound unit operations

\begin{tabular}{|c|c|c|c|c|c|c|c|c|c|c|}
\hline \multicolumn{11}{|c|}{ Repackaging facility inbound process unit operations (UOST hours) } \\
\hline Activity & \multicolumn{2}{|c|}{ Non-critical Path } & \multicolumn{2}{|c|}{$\begin{array}{l}\text { Package receipt bay (\# receipt bays } \\
\& \text { cranes }=[T p \text { (all annual casks) } x \\
\text { UOTT all casks }] / 4368\end{array}$} & \multicolumn{2}{|c|}{$\begin{array}{c}\text { Cask/Can Movement in Receipt } \\
\text { Side Airlock and Waste Handling } \\
\text { Building (\# transfer bays \& cranes } \\
=\text { Tp (all annual casks) x UOTT all } \\
\text { casks]/4368 } \\
\end{array}$} & \multicolumn{2}{|c|}{$\begin{array}{l}\text { Cask \& Can Opening (\# cutting stations } \\
=[\text { Tp (annual dry ONLY canisters) x U } \\
\text { OTT }] / 4368]\end{array}$} & \multicolumn{2}{|c|}{$\begin{array}{c}\text { Fuel Assembly Movement to Poo } \\
\text { (\# xfer bay fuel handling units }= \\
\text { [Tp (total all bare \& all dry } \\
\text { annual assemblies moved) } x \\
\text { 1]/4368) } \\
\end{array}$} \\
\hline In-Bound Packages & PWR & BWR & PWR & BWR & PWR & BWR & PWR & BWR & PWR & BWR \\
\hline $\begin{array}{l}\text { Receive in-bound transportation cask, inspect } \& \text { document } \\
\text { inbound packages }\end{array}$ & 40 & & & & & & & & & \\
\hline $\begin{array}{l}\text { Dock \& anchor carrier, remove shipping restraints, impact } \\
\text { limiters, personnel barriers, mount lifting hardware, upend, } \\
\text { cask inspection \& outer lid removal, move cask to transfer } \\
\text { bay UOST assumes receipt of off-site transportation } \\
\text { package steps and process is required (vs. receipt from } \\
\text { onsite transfer). }\end{array}$ & & & 23.85 & & & & & & & \\
\hline $\begin{array}{l}\text { Cut canister (if required) [UOST of } 20 \text { assumed for cutting } \\
\text { of all canistered fuel packages. UOST is } 0 \text { for all bare fuel } \\
\text { casks] }\end{array}$ & & & & & & & 20 & & & \\
\hline $\begin{array}{l}\text { Move loaded package into pool, water fill, remove empty } \\
\text { package from pool, dewater \& dry, move package out of } \\
\text { transfer bay to receipt bay. }\end{array}$ & & & & & 12 & & & & & \\
\hline $\begin{array}{l}\text { Move all fuel from canister into pool racks (@ } @ \text { hr. per } \\
\text { assembly). Computed separately for each case/scenario line } \\
\text { based on cask contents/size for bare and dry PWR \& BWR } \\
\text { fuel. UOST is total \# assemblies X 1hour }\end{array}$ & & & & & & & & & 1 & \\
\hline $\begin{array}{l}\text { Move package to receipt bay, install lid, inspect, move to } \\
\text { carrier, mount on carrier, install impact limiters, external } \\
\text { hardware, personnel protection, restraints and release }\end{array}$ & & & 23.25 & & & & & & & \\
\hline Finalize inspections, release paperwork and ship & 8 & & & & & & & & & \\
\hline Subtotal Inbound UOTT & 48 & 0 & 47.1 & 0 & 12 & 0 & 20 & 0 & 1 & 0 \\
\hline Subtotal Inbound all UOTT & 48 & & 47.1 & & 12 & & 20 & & 1 & \\
\hline
\end{tabular}

Legend: $\mathrm{Tp}$ = throughput; UST = unit operations step time (hours); UOTT - unit operations total time (hours) = sum of related UOST. 
Table B-6. Repackaging facility outbound unit operations

\begin{tabular}{|c|c|c|c|c|c|c|c|c|c|c|c|c|}
\hline \multicolumn{13}{|c|}{ Repackage facility inbound process unit operations (UOST hours); note: changes to UOST times revise throughput estimate number of lines needed } \\
\hline Activity & \multicolumn{2}{|c|}{ Non-critical Path } & \multicolumn{2}{|c|}{$\begin{array}{c}\text { Carrier \& package release bays } \\
\& \text { cranes }=[T p(\text { all annual casks } \\
\text { shipped) x UOTT all } \\
\text { casks }] / 4368\end{array}$} & \multicolumn{2}{|c|}{$\begin{array}{c}\text { Cask/recan canister } \\
\text { movement in transfer bay (\# } \\
\text { transfer bays \& OH cranes }= \\
\text { Tp (all annual re-can WPs) } x \\
\text { UOTT]/4368 }\end{array}$} & \multicolumn{2}{|c|}{$\begin{array}{c}\text { Re-canning closing/welding } \\
\text { (\#can welding stations }=[\text { Total } \\
\text { annual re-can canisters released) } \\
x \text { UOTT }] / 4368]\end{array}$} & \multicolumn{2}{|c|}{$\begin{array}{l}\text { Fuel assembly movement } \\
\text { from pool to re-cans (\# } \\
\text { transfer bay fuel handle units } \\
=[\text { Tp (total all repackaged } \\
\text { PWR \& BWR annual } \\
\text { assemblies moved) x 1]/4368) }\end{array}$} & \multicolumn{2}{|c|}{$\begin{array}{c}\text { Re-package canister fill time } \\
\text { (\#Re-Package Canning line } \\
\text { positions required = [Tp \# cans } \\
\text { loaded \{total cans repackaged)) } \\
\text { UOTT can loading time] plus } 1 \\
\text { hour can placement \& } \\
\text { positioning/4368] }\end{array}$} \\
\hline Outbounded packages & PWR & BWR & PWR & BWR & PWR & BWR & PWR & BWR & PWR & BWR & PWR & BWR \\
\hline $\begin{array}{l}\text { Finalize out-bound inspections and shipping } \\
\text { documentation, ship outbound WP/transportation } \\
\text { cask }\end{array}$ & 40 & & & & & & & & & & & \\
\hline $\begin{array}{l}\text { Dock \& anchor out-bound carrier, remove shipping } \\
\text { restraints, impact limiters personnel barriers, mount } \\
\text { lifting hardware, upend, cask inspection \& outer lid } \\
\text { removal. Water/dewater steps move cask from xfer } \\
\text { bay into carrier release bay. }\end{array}$ & & & 23.85 & & & & & & & & & \\
\hline $\begin{array}{l}\text { Weld re-can WP canister [UOST of } 25 \text { assumed for } \\
\text { welding of all WPs.] }\end{array}$ & & & & & & & 25 & & & & & \\
\hline $\begin{array}{l}\text { Move loaded package out of pool, drain, dewater\& } \\
\text { dry, inert, move welded WP package into shielded } \\
\text { transport package \& out of xfer bay. }\end{array}$ & & & & & 12 & & & & & & & \\
\hline $\begin{array}{l}\text { Move all fuel from pool rack into re-can WPs (@ } \\
1 \mathrm{hr} \text {. per assembly). Computed separately for each } \\
\text { case/scenario line based on cask contents/size for } \\
\text { bare and dry PWR \& BWR re-canned fuel. UOST } \\
\text { is total \# assemblies moved X } 1 \text { hour }\end{array}$ & & & & & & & & & 1 & & & \\
\hline $\begin{array}{l}\text { Move transport package to carrier release bay, } \\
\text { install lid, inspect move to carrier, mount on carrier, } \\
\text { install impact limiters, external hardware, personnel } \\
\text { protection, restraints and release. UOST assumes } \\
\text { offsite transportation package preparation is } \\
\text { required (vs. onsite transfer). }\end{array}$ & & & 23.25 & & & & & & & & & \\
\hline $\begin{array}{l}\text { \# Repackage canning lines positions required }=[\mathrm{Tp} \\
\text { \# cans loaded \{total cans repackaged\} x UOTT can } \\
\text { loading time] plus } 1 \text { hour per can for placement \& } \\
\text { positioning/4368, where loading UOTT is } 1 \text { hour } \\
\text { per assembly per can x \# assemblies per can. }\end{array}$ & & & & & & & & & & & 1 & \\
\hline Subtotal Outbound UOTT & 40 & 0 & 47.1 & 0 & 12 & 0 & 25 & 0 & 1 & 0 & 1 & 0 \\
\hline Subtotal Outbound all UOTT & 40 & & 47.1 & & 12 & & 25 & & 1 & & 1 & \\
\hline
\end{tabular}


Table B-7. Repackaging facility inbound infrastructure elements estimate

\begin{tabular}{|c|c|c|c|c|c|c|c|}
\hline \multirow[b]{2}{*}{ Scenario } & \multirow[b]{2}{*}{$\begin{array}{c}\text { Acceptance } \\
\text { Rate }\end{array}$} & \multirow[b]{2}{*}{ CSF Start } & \multirow[b]{2}{*}{$\begin{array}{l}\text { Repository } \\
\text { Start }\end{array}$} & \multirow[b]{2}{*}{$\begin{array}{c}\text { Disposal } \\
\text { Canister } \\
\text { Size }\end{array}$} & \multicolumn{2}{|c|}{ Peak Annual Receipts } & \multirow[b]{2}{*}{$\begin{array}{l}\text { Inbound Carrier } \\
\text { Receipt Bays }\end{array}$} \\
\hline & & & & & Dry Cans to Open & $\begin{array}{l}\text { Bare Cask } \\
\text { Received }\end{array}$ & \\
\hline \multirow{9}{*}{$\begin{array}{c}\text { Canisters } \\
\text { Only - } \\
\text { Re-Package } \\
\text { at Repository }\end{array}$} & 1,500 & 2020 & 2040 & 4 & 132 & & 2 \\
\hline & 3,000 & 2020 & 2040 & 4 & 348 & & 4 \\
\hline & 3,000 & 2020 & 2040 & 12 & 348 & & 4 \\
\hline & 3,000 & 2020 & 2040 & 21 & 348 & & 4 \\
\hline & 6,000 & 2020 & 2040 & 4 & 543 & & 6 \\
\hline & 1,500 & 2020 & 2055 & 4 & 132 & & 2 \\
\hline & 3,000 & 2020 & 2055 & 4 & 348 & & 4 \\
\hline & 6,000 & 2020 & 2055 & 4 & 546 & & 6 \\
\hline & 3,000 & 2035 & 2055 & 4 & 257 & & 3 \\
\hline \multirow{9}{*}{$\begin{array}{l}\text { Canisters and } \\
\text { Bare Fuel - } \\
\text { Re-Package } \\
\text { at Repository }\end{array}$} & 1,500 & 2020 & 2040 & 4 & 130 & 182 & 5 \\
\hline & 3,000 & 2020 & 2040 & 4 & 246 & 325 & 8 \\
\hline & 3,000 & 2020 & 2040 & 12 & 246 & 325 & 8 \\
\hline & 3,000 & 2020 & 2040 & 21 & 246 & 325 & 8 \\
\hline & 6,000 & 2020 & 2040 & 4 & 216 & 634 & 17 \\
\hline & 1,500 & 2020 & 2055 & 4 & 130 & 182 & 5 \\
\hline & 3,000 & 2020 & 2055 & 4 & 246 & 325 & 8 \\
\hline & 6,000 & 2020 & 2055 & 4 & 225 & 634 & 17 \\
\hline & 3,000 & 2035 & 2055 & 4 & 252 & 258 & 6 \\
\hline \multirow{9}{*}{$\begin{array}{l}\text { Canisters } \\
\text { Only - Re- } \\
\text { Package at } \\
\quad \text { CSF }\end{array}$} & $\begin{array}{l}1,500 \\
\end{array}$ & 2020 & 2040 & 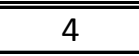 & 142 & & 2 \\
\hline & 3,000 & 2020 & 2040 & 4 & 268 & & 3 \\
\hline & 3,000 & 2020 & 2040 & 12 & 268 & & 3 \\
\hline & 3,000 & 2020 & 2040 & 21 & 268 & & 3 \\
\hline & 6,000 & 2020 & 2040 & 4 & 530 & & 6 \\
\hline & 1,500 & 2020 & 2055 & 4 & 142 & & 2 \\
\hline & 3,000 & 2020 & 2055 & 4 & 268 & & 3 \\
\hline & 6,000 & 2020 & 2055 & 4 & 530 & & 6 \\
\hline & 3,000 & 2035 & 2055 & 4 & 265 & & 3 \\
\hline \multirow{9}{*}{$\begin{array}{c}\text { Canisters and } \\
\text { Bare Fuel - } \\
\text { Re-Package } \\
\text { at CSF }\end{array}$} & 1,500 & 2020 & 2040 & 4 & 127 & & 2 \\
\hline & 3,000 & 2020 & 2040 & 4 & 248 & & 3 \\
\hline & 3,000 & 2020 & 2040 & 12 & 248 & & 3 \\
\hline & 3,000 & 2020 & 2040 & 21 & 248 & & 3 \\
\hline & 6,000 & 2020 & 2040 & 4 & 225 & & 3 \\
\hline & 1,500 & 2020 & 2055 & 4 & 128 & & 2 \\
\hline & 3,000 & 2020 & 2055 & 4 & 248 & & 3 \\
\hline & 6,000 & 2020 & 2055 & 4 & 225 & & 3 \\
\hline & 3,000 & 2035 & 2055 & 4 & 249 & & 3 \\
\hline
\end{tabular}


Table B-8. Repackaging facility outbound infrastructure elements estimate

\begin{tabular}{|c|c|c|c|c|c|c|c|c|c|c|c|}
\hline & Scenario & $\begin{array}{c}\text { Acceptance } \\
\text { Rate }\end{array}$ & CSF Start & $\begin{array}{l}\text { Repository } \\
\text { Start }\end{array}$ & $\begin{array}{l}\text { Disposal } \\
\text { Canister } \\
\text { Size }\end{array}$ & $\begin{array}{c}\text { \# of } 3500 \\
\text { Assembly Basins }\end{array}$ & $\begin{array}{l}\text { \# PWR WP Repac } \\
\text { Stations Req'd }\end{array}$ & $\begin{array}{l}\text { \# BWR WP Repac } \\
\text { Stations Req'd }\end{array}$ & $\begin{array}{c}\text { TOTAL\# } \\
\text { OUTBOUND } \\
\text { Repac. Stations } \\
\text { Req'd (PWR PLUS } \\
\text { BWR WPs) }\end{array}$ & \begin{tabular}{|c} 
\# Outbound Waste \\
Pkg. Welding \\
Station Lines \\
Required
\end{tabular} & $\begin{array}{c}\text { OutBound Carrier } \\
\text { Release Bays }\end{array}$ \\
\hline Case 1 Var Set 31 & \multirow{9}{*}{$\begin{array}{c}\text { Canisters } \\
\text { Only - } \\
\text { Re-Package } \\
\text { at Repository }\end{array}$} & 1,500 & 2020 & 2040 & 4 & 1.0 & 0.8 & 1.0 & 2 & 7 & 7 \\
\hline Case 1 Var Set 40 & & 3,000 & 2020 & 2040 & 4 & 2.0 & 1.7 & 2.1 & 4 & 14 & 13 \\
\hline Case 1 Var Set 67 & & 3,000 & 2020 & 2040 & 12 & 2.0 & 1.5 & 1.9 & 4 & 5 & 5 \\
\hline Case 1 Var Set 94 & & 3,000 & 2020 & 2040 & 21 & 2.0 & 1.4 & 1.9 & 4 & 3 & 3 \\
\hline Case 1 Var Set 49 & & 6,000 & 2020 & 2040 & 4 & 4.2 & 3.2 & 4.6 & 8 & 28 & 27 \\
\hline Case 1 Var Set 34 & & 1,500 & 2020 & 2055 & 4 & 1.0 & 0.8 & 1.0 & 2 & 7 & 7 \\
\hline Case 1 Var Set 43 & & 3,000 & 2020 & 2055 & 4 & 2.0 & 1.7 & 2.1 & 4 & 14 & 13 \\
\hline Case 1 Var Set 52 & & 6,000 & 2020 & 2055 & 4 & 4.3 & 3.5 & 4.6 & 9 & 30 & 28 \\
\hline Case 1 Var Set 44 & & 3,000 & 2035 & 2055 & 4 & 1.9 & 1.5 & 2.1 & 4 & 13 & 12 \\
\hline Case 2 Var Set 31 & \multirow{9}{*}{$\begin{array}{l}\text { Canisters and } \\
\text { Bare Fuel - } \\
\text { Re-Package } \\
\text { at Repository }\end{array}$} & 1,500 & 2020 & 2040 & 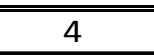 & 1.0 & 0.8 & \begin{tabular}{|c|}
1.0 \\
\end{tabular} & 2 & 7 & 7 \\
\hline Case 2 Var Set 40 & & 3,000 & 2020 & 2040 & 4 & 1.9 & 1.5 & 2.1 & 4 & 13 & 12 \\
\hline Case 2 Var Set 67 & & 3,000 & 2020 & 2040 & 12 & 1.9 & 1.3 & 1.9 & 4 & 5 & 5 \\
\hline Case 2 Var Set 94 & & 3,000 & 2020 & 2040 & 21 & 1.9 & 1.2 & 1.9 & 4 & 3 & 3 \\
\hline Case 2 Var Set 49 & & 6,000 & 2020 & 2040 & 4 & 3.9 & 3.2 & 4.1 & 8 & 26 & 25 \\
\hline Case 2 Var Set 34 & & 1,500 & 2020 & 2055 & 4 & 1.0 & 0.8 & 1.0 & 2 & 7 & 7 \\
\hline Case 2 Var Set 43 & & 3,000 & 2020 & 2055 & 4 & 1.9 & 1.5 & 2.1 & 4 & 13 & 12 \\
\hline Case 2 Var Set 52 & & 6,000 & 2020 & 2055 & 4 & 3.5 & 2.8 & 3.8 & 7 & 24 & 23 \\
\hline Case 2 Var Set 44 & & 3,000 & 2035 & 2055 & 4 & 1.9 & 1.5 & 2.1 & 4 & 13 & 12 \\
\hline Case 3 Var Set 31 & \multirow{9}{*}{$\begin{array}{l}\text { Canisters } \\
\text { Only - Re- } \\
\text { Package at } \\
\text { CSF }\end{array}$} & 1,500 & 2020 & 2040 & 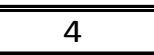 & \begin{tabular}{|c|}
1.1 \\
\end{tabular} & 0.8 & \begin{tabular}{c|}
1.2 \\
\end{tabular} & 3 & 8 & 14 \\
\hline Case 3 Var Set 40 & & 3,000 & 2020 & 2040 & 4 & 2.0 & 1.5 & 2.2 & 4 & 13 & 25 \\
\hline Case 3 Var Set 67 & & 3,000 & 2020 & 2040 & 12 & 2.0 & 1.3 & 2.1 & 4 & 5 & 9 \\
\hline Case 3 Var Set 94 & & 3,000 & 2020 & 2040 & 21 & 2.0 & 1.2 & 2.1 & 4 & 3 & 5 \\
\hline Case 3 Var Set 49 & & 6,000 & 2020 & 2040 & 4 & 4.3 & 3.5 & 4.6 & 9 & 29 & 55 \\
\hline Case 3 Var Set 34 & & 1,500 & 2020 & 2055 & 4 & 1.1 & 0.8 & 1.2 & 3 & 8 & 14 \\
\hline Case 3 Var Set 43 & & 3,000 & 2020 & 2055 & 4 & 2.0 & 1.5 & 2.2 & 4 & 13 & 25 \\
\hline Case 3 Var Set 52 & & 6,000 & 2020 & 2055 & 4 & 4.3 & 3.5 & 4.6 & 9 & 29 & 55 \\
\hline Case 3 Var Set 44 & & 3,000 & 2035 & 2055 & 4 & 2.0 & 1.5 & 2.2 & 4 & 13 & 25 \\
\hline Case 4 Var Set 31 & \multirow{9}{*}{$\begin{array}{c}\text { Canisters and } \\
\text { Bare Fuel - } \\
\text { Re-Package } \\
\text { at CSF }\end{array}$} & 1,500 & 2020 & 2040 & $\overline{44}$ & 1.0 & \begin{tabular}{|c|}
0.8 \\
\end{tabular} & 1.1 & 2 & \begin{tabular}{ll|}
7 \\
\end{tabular} & 13 \\
\hline Case 4 Var Set 40 & & 3,000 & 2020 & 2040 & 4 & 2.0 & 1.4 & 2.2 & 4 & 13 & 25 \\
\hline Case 4 Var Set 67 & & 3,000 & 2020 & 2040 & 12 & 2.0 & 1.3 & 2.1 & 4 & 5 & 9 \\
\hline Case 4 Var Set 94 & & 3,000 & 2020 & 2040 & 21 & 2.0 & 1.2 & 2.1 & 4 & 3 & 5 \\
\hline Case 4 Var Set 49 & & 6,000 & 2020 & 2040 & 4 & 3.7 & 2.9 & 4.0 & 7 & 25 & 46 \\
\hline Case 4 Var Set 34 & & 1,500 & 2020 & 2055 & 4 & 1.0 & 0.8 & 1.1 & 2 & 7 & 13 \\
\hline Case 4 Var Set 43 & & 3,000 & 2020 & 2055 & 4 & 2.0 & 1.4 & 2.2 & 4 & 13 & 25 \\
\hline Case 4 Var Set 52 & & 6,000 & 2020 & 2055 & 4 & 3.7 & 2.9 & 4.0 & 7 & 25 & 46 \\
\hline Case 4 Var Set 44 & & 3,000 & 2035 & 2055 & 4 & 1.9 & 1.4 & 2.2 & 4 & 13 & 24 \\
\hline
\end{tabular}




\section{B.2.3 Support Facilities}

To support the CSF and RF major facility modules described above, the team sub-divided the physical features into the following support facilities. A summary description of each support facility is provided below.

- Low Level Waste Building

- Operations/Maintenance/Radiological Protection and Health Physics Building

- $\quad$ Security Building

- Administrative Building (including training)

The LLW staging facility was derived from the FOEAS Early Fuel Receipt Sensitivity Analysis reflected in references 2 and 3. This facility was assumed to double in scale for the repackaging facility which is expected to generate more LLW from repackaging compared to fuel storage. The sizes of the other listed support facilities, derived from prior UFD studies, were not assumed to vary appreciably as a function of scenario through-put.

\section{B.2.3.1 Low Level Waste Building}

The Low Level WHB would be constructed adjacent to one of the facilities described in Section 2.1 and 2.2 would be of a single story steel frame construction. This building will provide space and utilities necessary to package low level solid waste, and solidify and package low level liquid waste for offsite disposal. This structure and accompanying facility area will also provide space for the interim staging of accumulated LLW packages pending offsite disposal. This building would also have the ability to provide localized (at the work site) HEPA filtered ventilation.

Long term storage of low level waste would not be provided at the facility. On this basis it would be interim-staged, for periodic disposal at a licensed disposal facility, offsite.

\section{B.2.3.2 Operations/Maintenance/Radiological Protection Building}

The Operations / Maintenance / Radiological Protection building would be of a single story steel frame construction. This building would provide space for light industrial maintenance shops, radiological laboratory facilities, and personnel office space.

\section{B.2.3.3 Security Building}

The Security building would be located at the entrance to the receipt facility. This building will provide office space for security staff and house security, communication, and normal service and back up emergency power electrical equipment needed for these personnel and services.

\section{B.2.3.4 Administrative Building and Other Services}

The Administration building would be of a single story steel frame construction. It would include office and record management space, an emergency response center, training facility, and meeting rooms. 
A fuel storage cask and horizontal storage module fabrication completion area would be provided, together with a batch plant, to allow fuel storage unit shells, provided by the licensed manufacturer, to be filled and cured, on site, according to the licensed design basis.

All buildings would be provided domestic water, sanitary waste, normal electric power, and controlled atmosphere ventilation services.

\section{B.2.4 Test and Validation Facility R\&D Module}

During FY 2012, a design concept for implementing DOE's Research Development \& Demonstration plan recommendations at a new "greenfield" CSF coupled with a test and validation facility (T\&VF) was developed and documented in the Used Fuel Research and Development Test and Validation Facility Cost Study (DOE 2012d)

Due to the limited through-put anticipated for this facility it was conceptualized on the basis of at least one radiological process line and one adjacent non-radiological mock up facility which provided the capability to simulate one process line. An alternative facility with two radiological process lines with a single line mock up facility was also conceptualized for scenarios that might involve higher test and validation through-puts.

The two line facility was assumed for this study. The scale of this facility was not adjusted as a function of scenario through-puts nor capacity in this study.

\section{B.3 REFERENCES FOR APPENDIX B}

1. (PFS)- Private Fuel Storage L.L.C. (PFS) facility proposed for Toole County, Utah (http://www.privatefuelstorage.com/project/facility.html)

2. (US NRC-PFS) - NRC News Release No. 06-028, February 22, 2006, NRC Issues License To Private Fuel Storage For Spent Nuclear Fuel Storage Facility In Utah

3. (DOE 2007a) - Follow-on Engineering Alternative Studies for Separations(FOEAS), Early Fuel Receipt \& Storage (EFR\&S) Considerations, FRS-G-ESR-G-00053, Rev. 0, September 2007, P. Rodwell, R. Geddes, S. McConnell

4. (DOE 2007b) -Engineering Alternative Studies(EAS) for Separations-Summary Report, EAS-GESR-G-00049, June 2007, S. McConnell, D. W. Ostby, et.al

5. (DOE 2008) - Follow-on Engineering Alternative Studies (FOEAS) Summary Report, Global Nuclear Energy Partnership (GNEP), GNEP-CFTC-PMO-MI-DV-2008-00087, March 2008, Consolidated Fuel Treatment Center

6. (DOE 2012c) - Disposal Concepts/Thermal Load Management FY11/12 Summary Report, 2012, Hardin et.al.

7. (DOE 2000a) - Civilian Radioactive Waste Management System (CRWMS) Management and Operations (M\&O) Attachment II, Section 1.3.2.1 
8. (DOE 2000b) - Civilian Radioactive Waste Management System (CRWMS) Management and Operations (M\&O) Attachment II, Section 1.1.2.1

9. (DOE 2012d) - Used Fuel Research and Development Test and Validation Facility Cost Study, FCRD-UFD-2012-000206, Rev. 0, August 2012, J. Carter, A. Delley, et. al

10. (DOE 2012e) Used Fuel Disposition Storage, Transportation and Disposal Interface Cost Study, FCRD-UFD-2012-000372, Revision 0, September 2012 
B.4 CONSOLIDATED STORAGE FACILITY LAY-OUT DRAWINGS

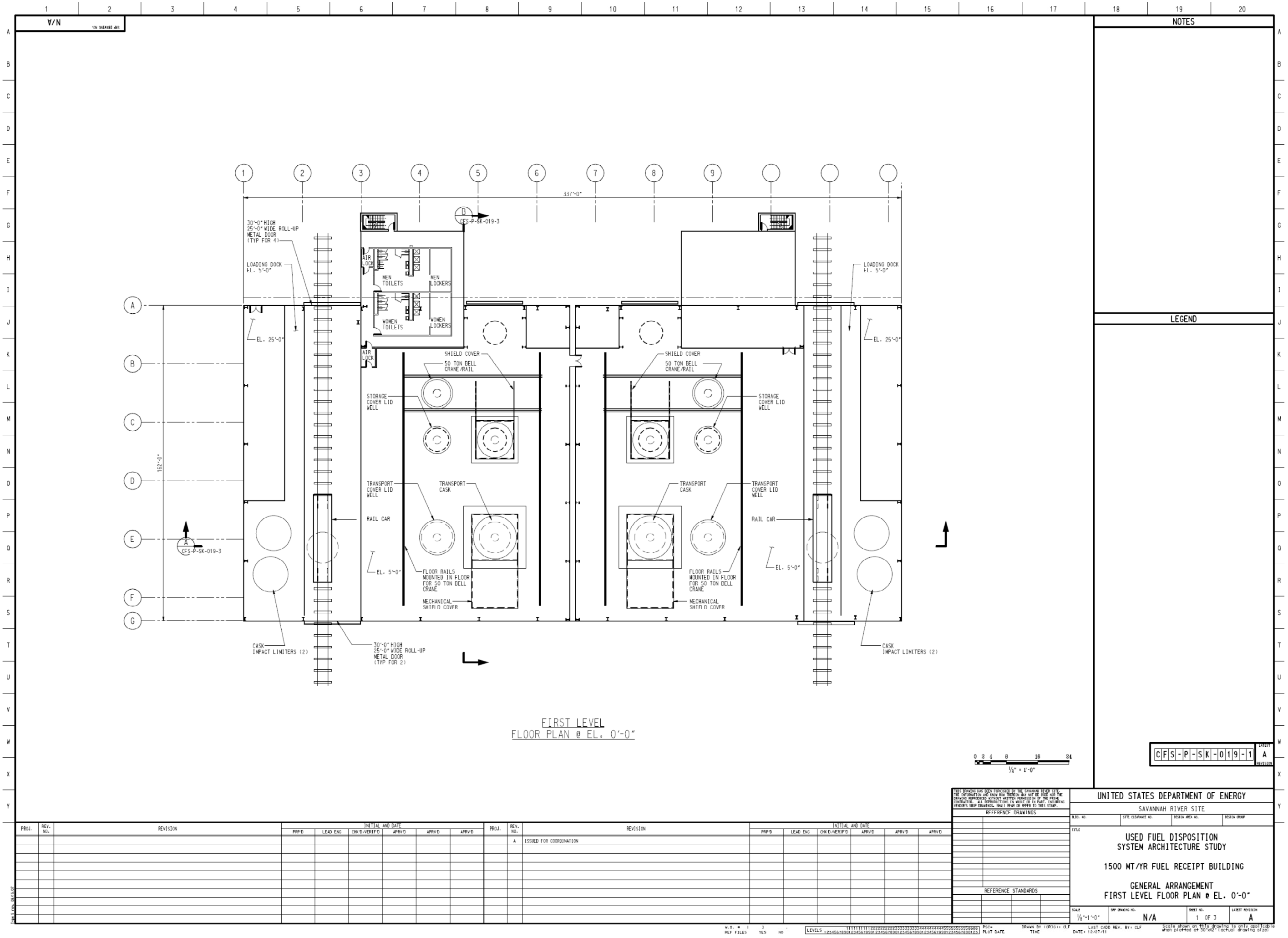




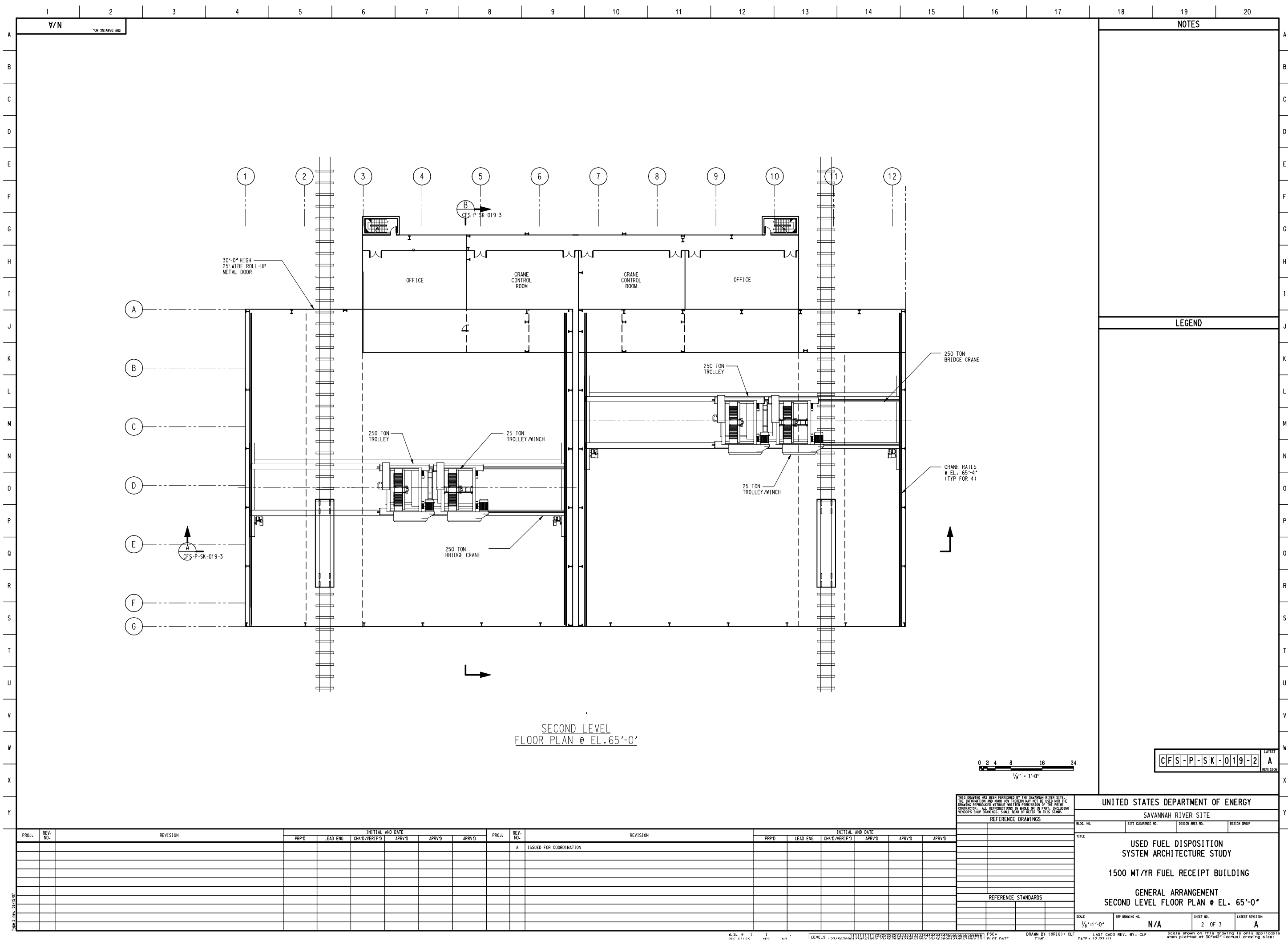

Figure B-17. 


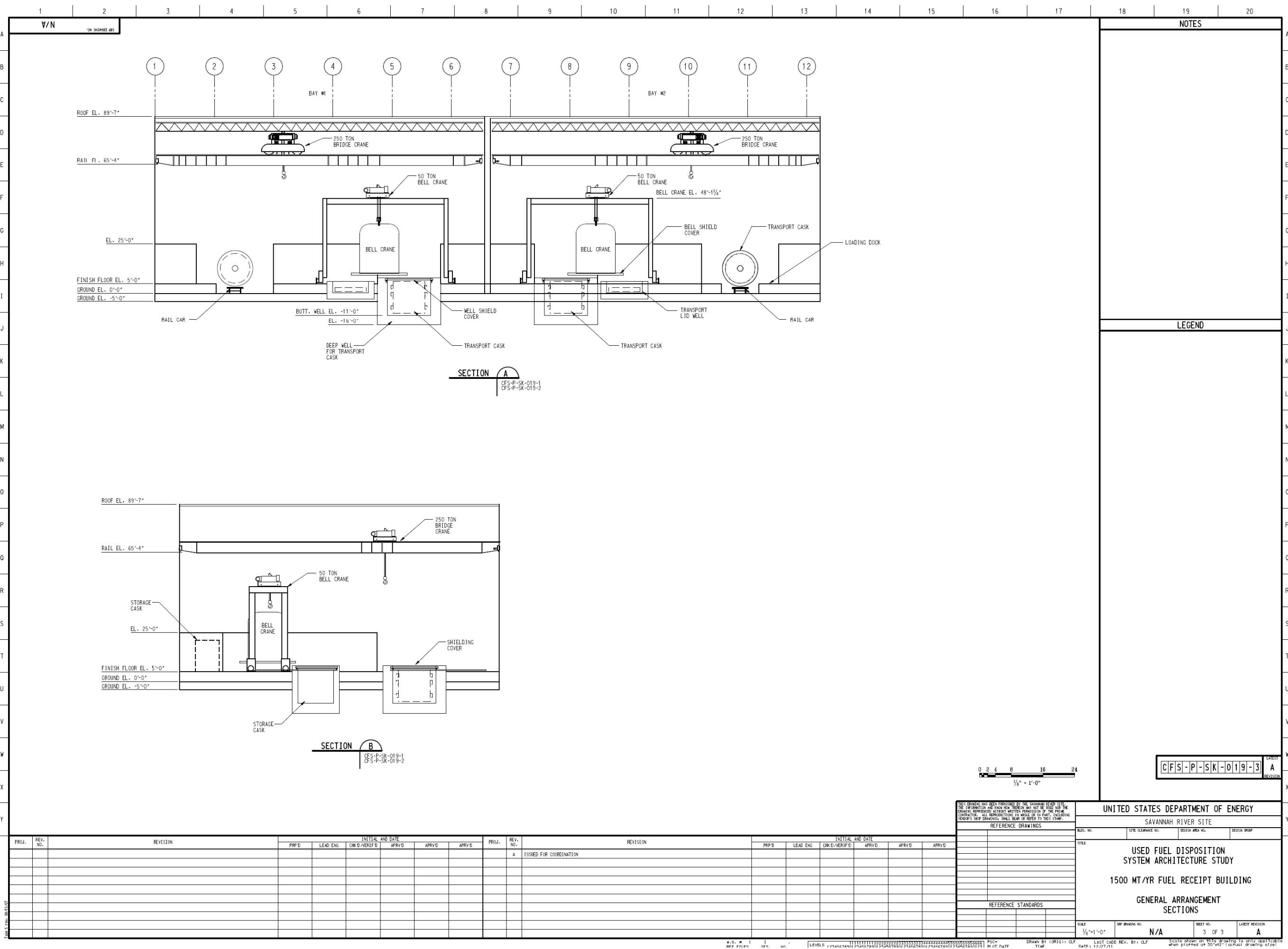

Figure B-18. 


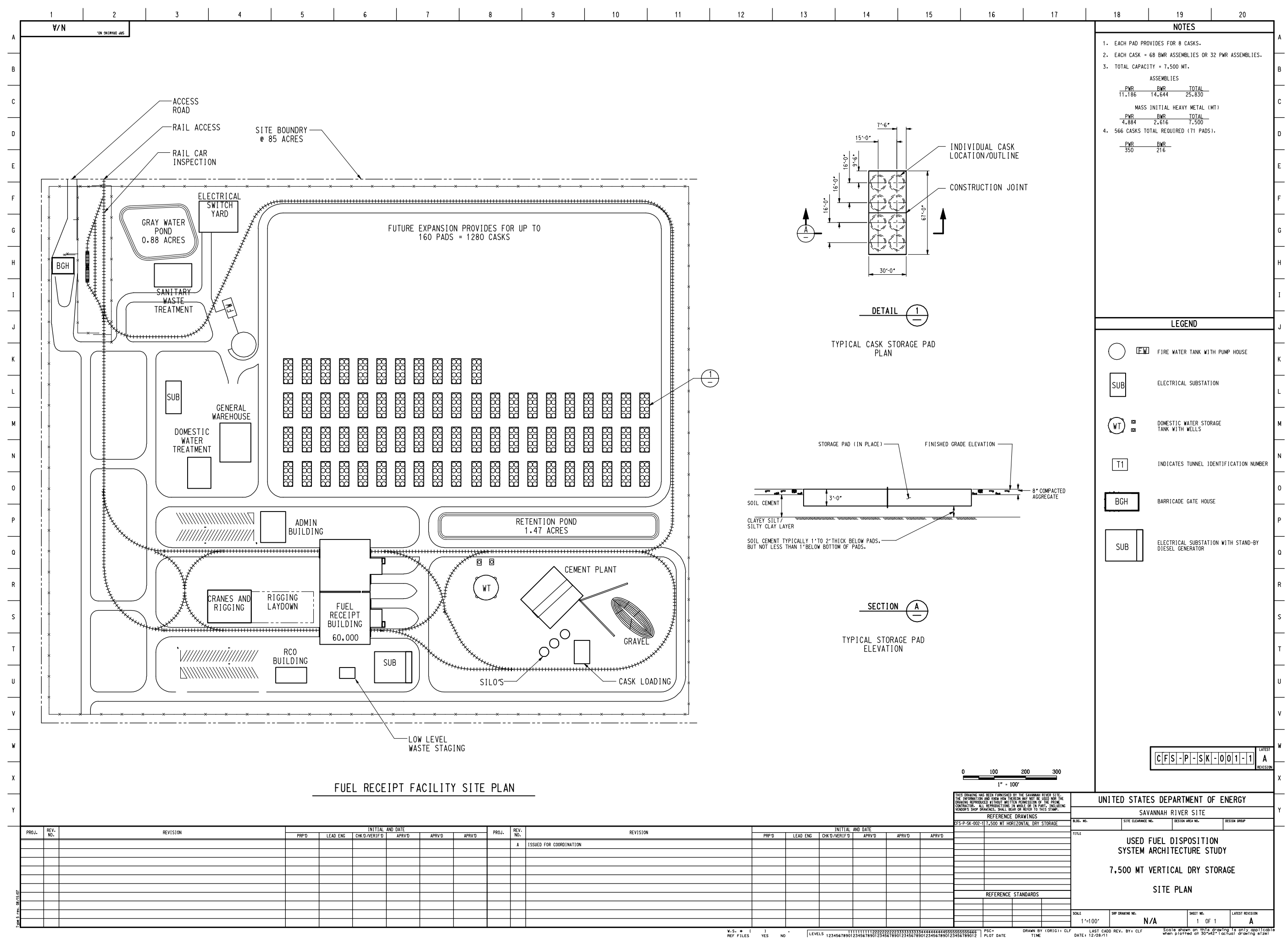




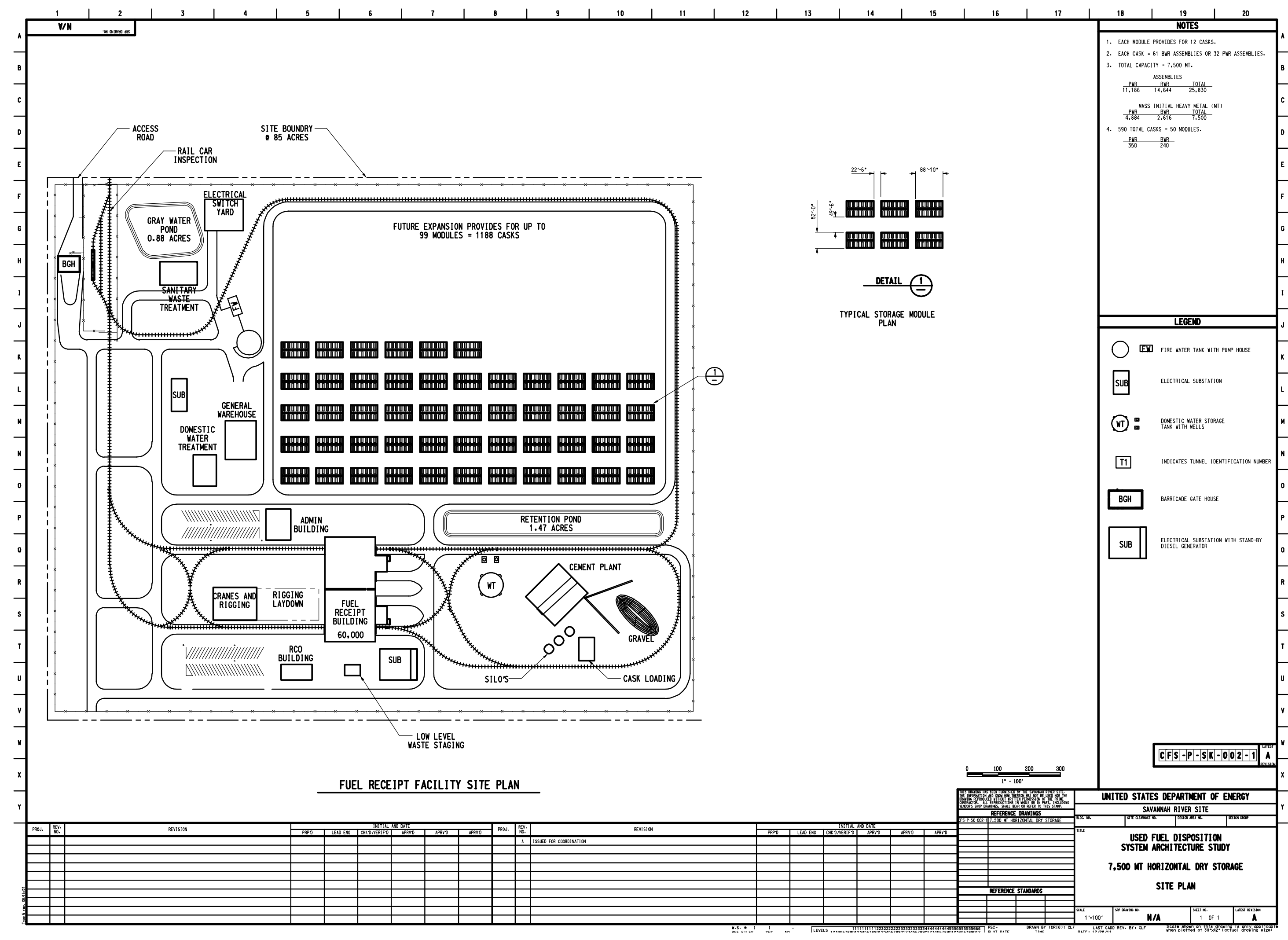

Figure B-20 


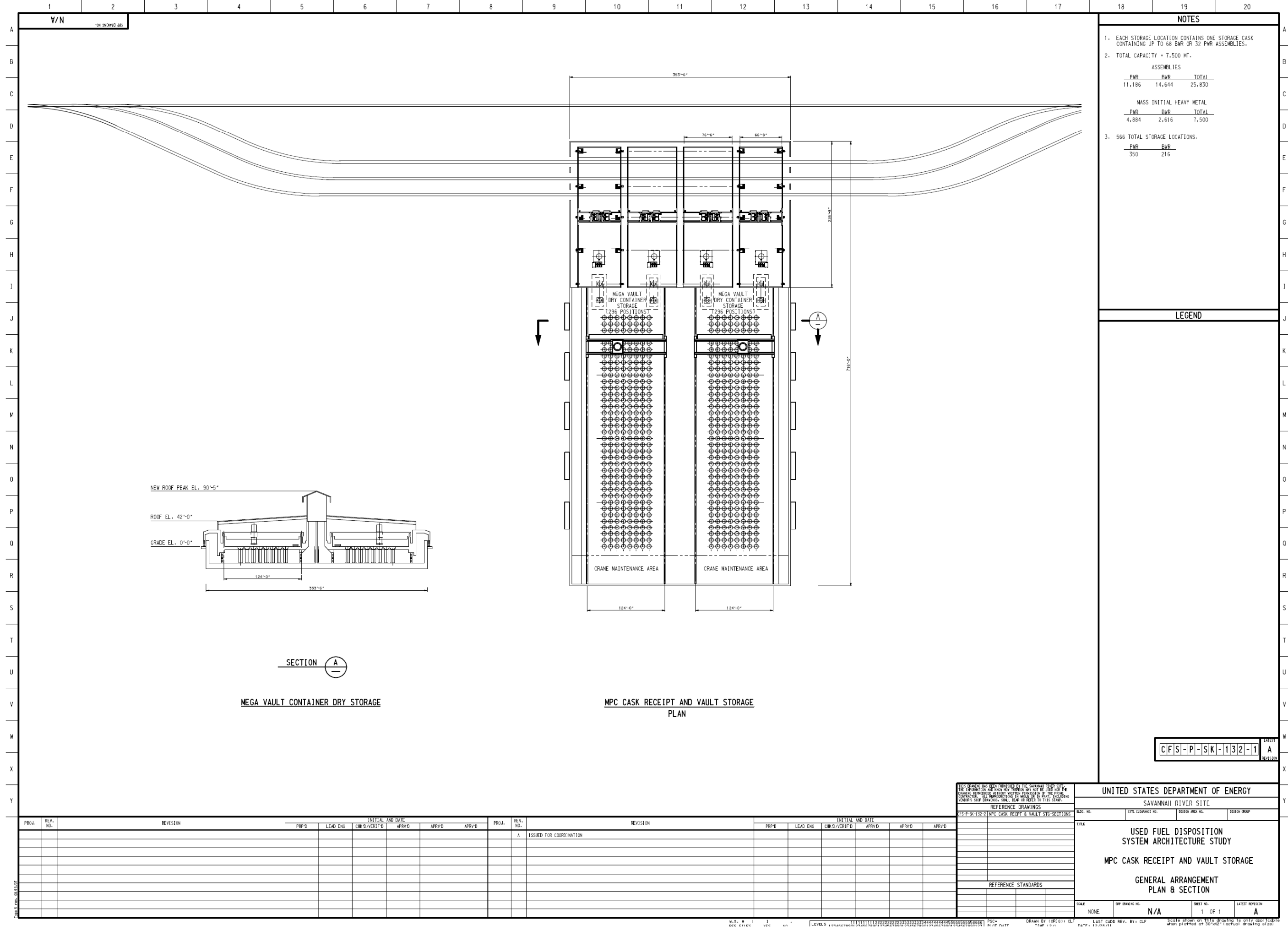

Figure B-21. 


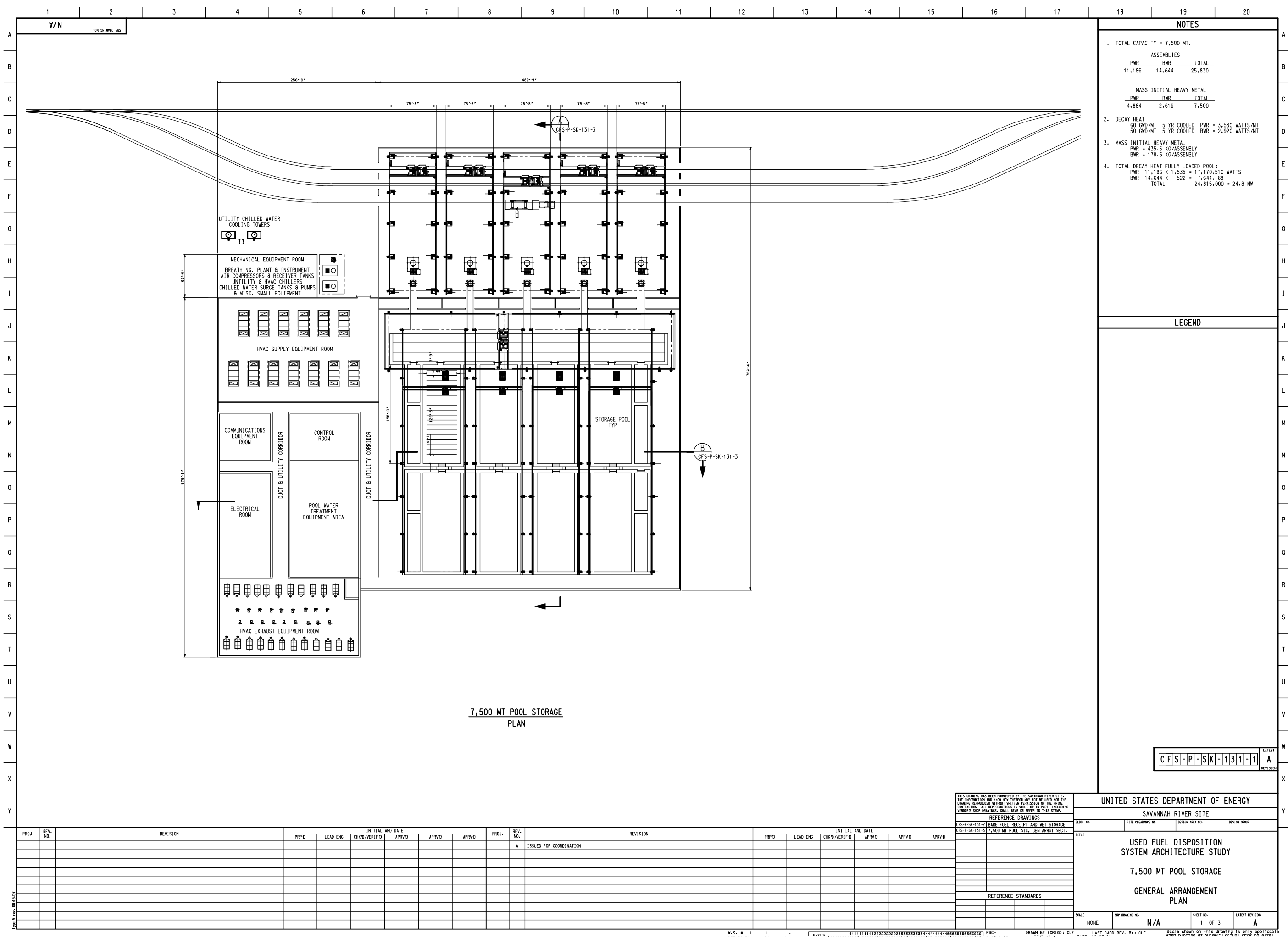

Figure B-22. 


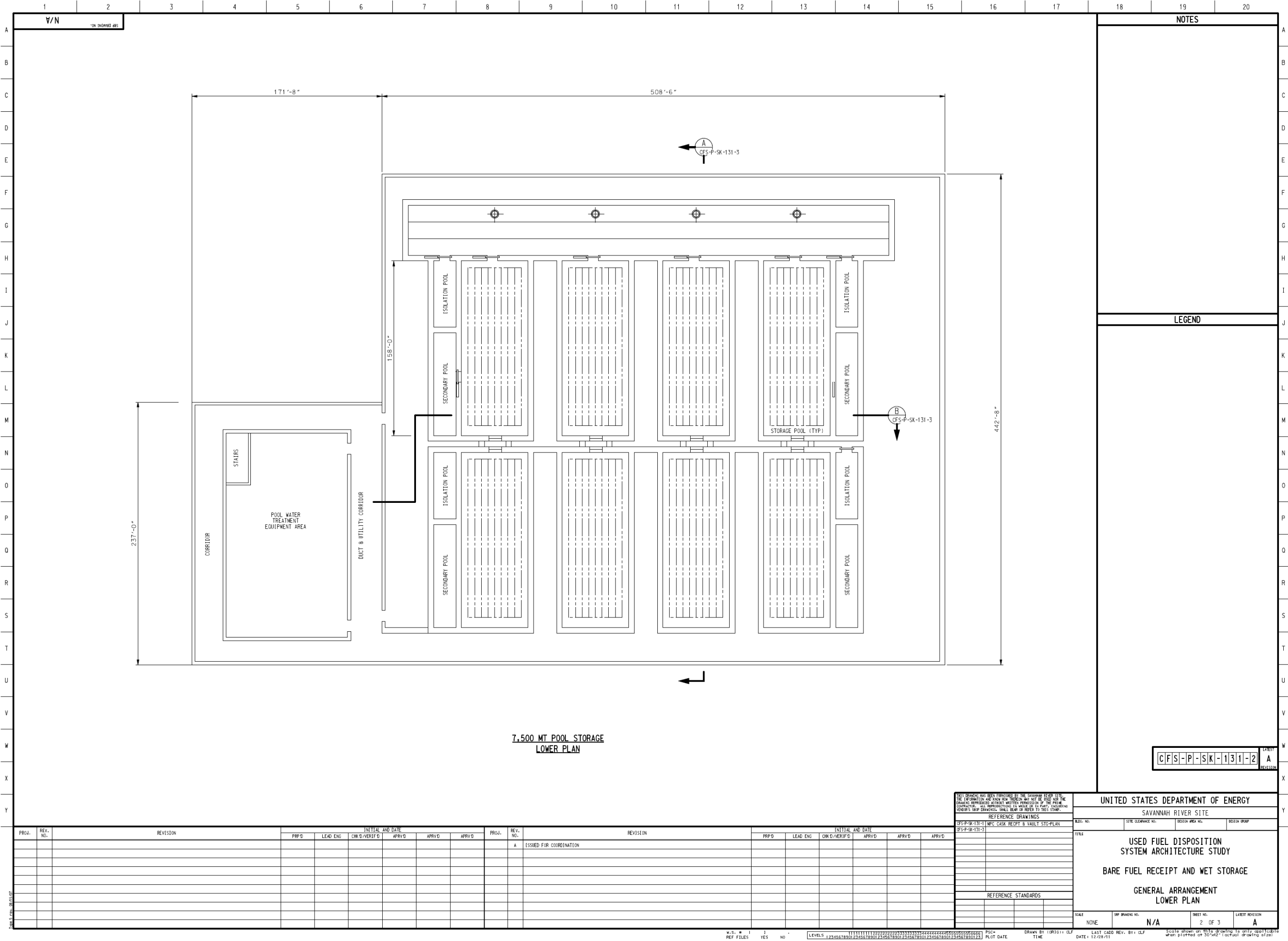

Figure B-23. 


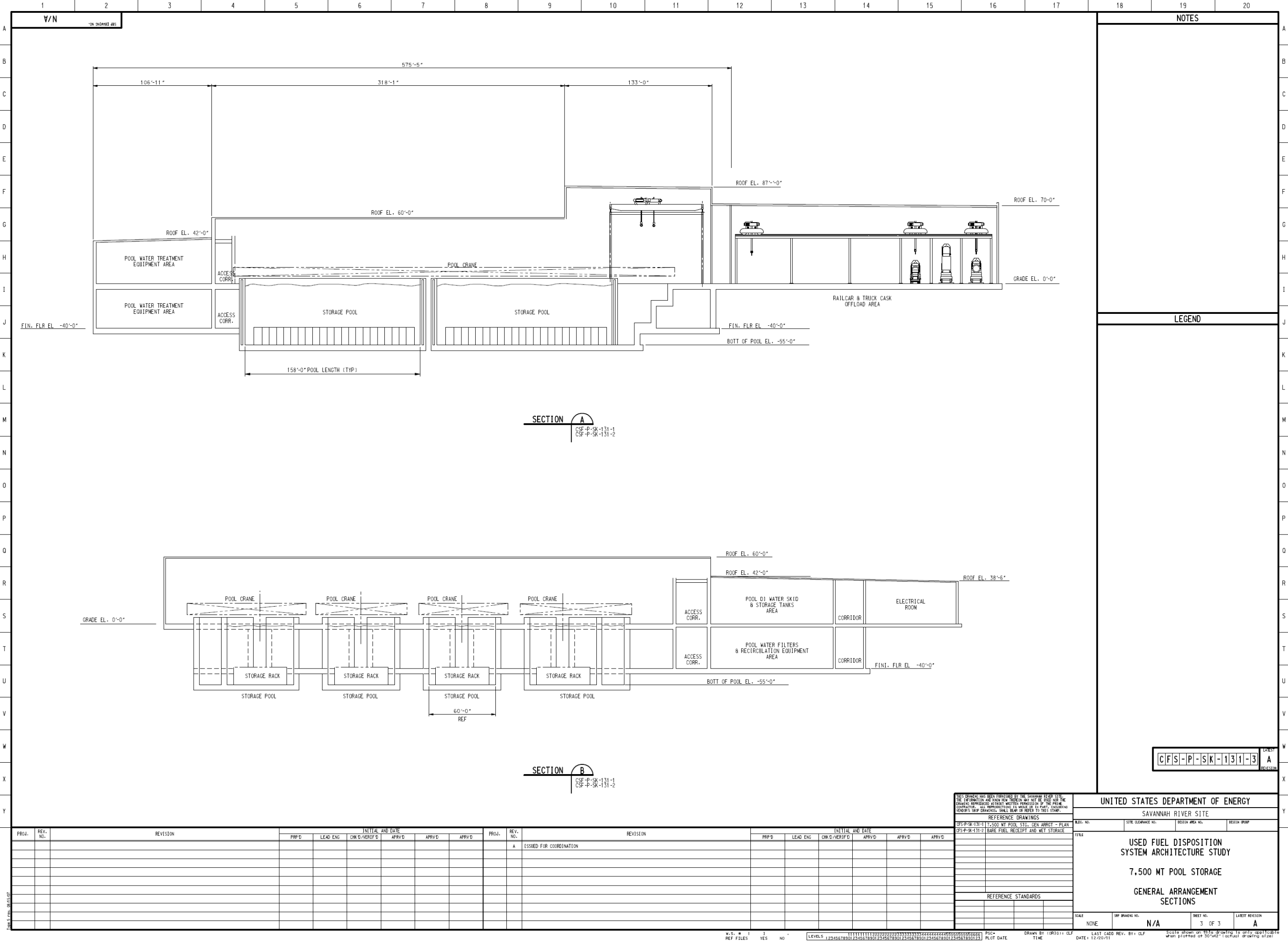

Figure B-24 
B.5 REPACKAGING FACILITY LAY-OUT DRAWINGS

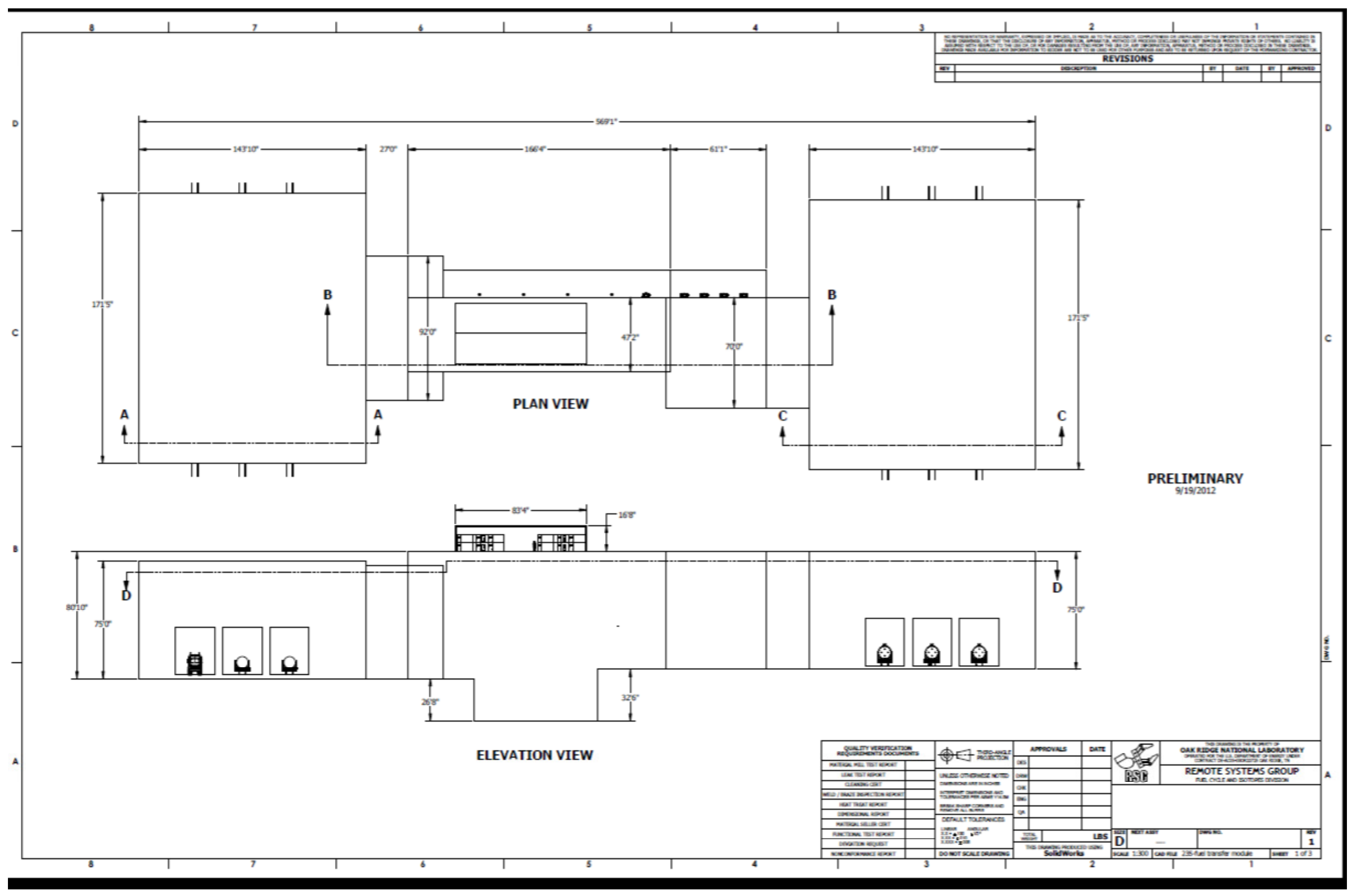

Figure B-25. 


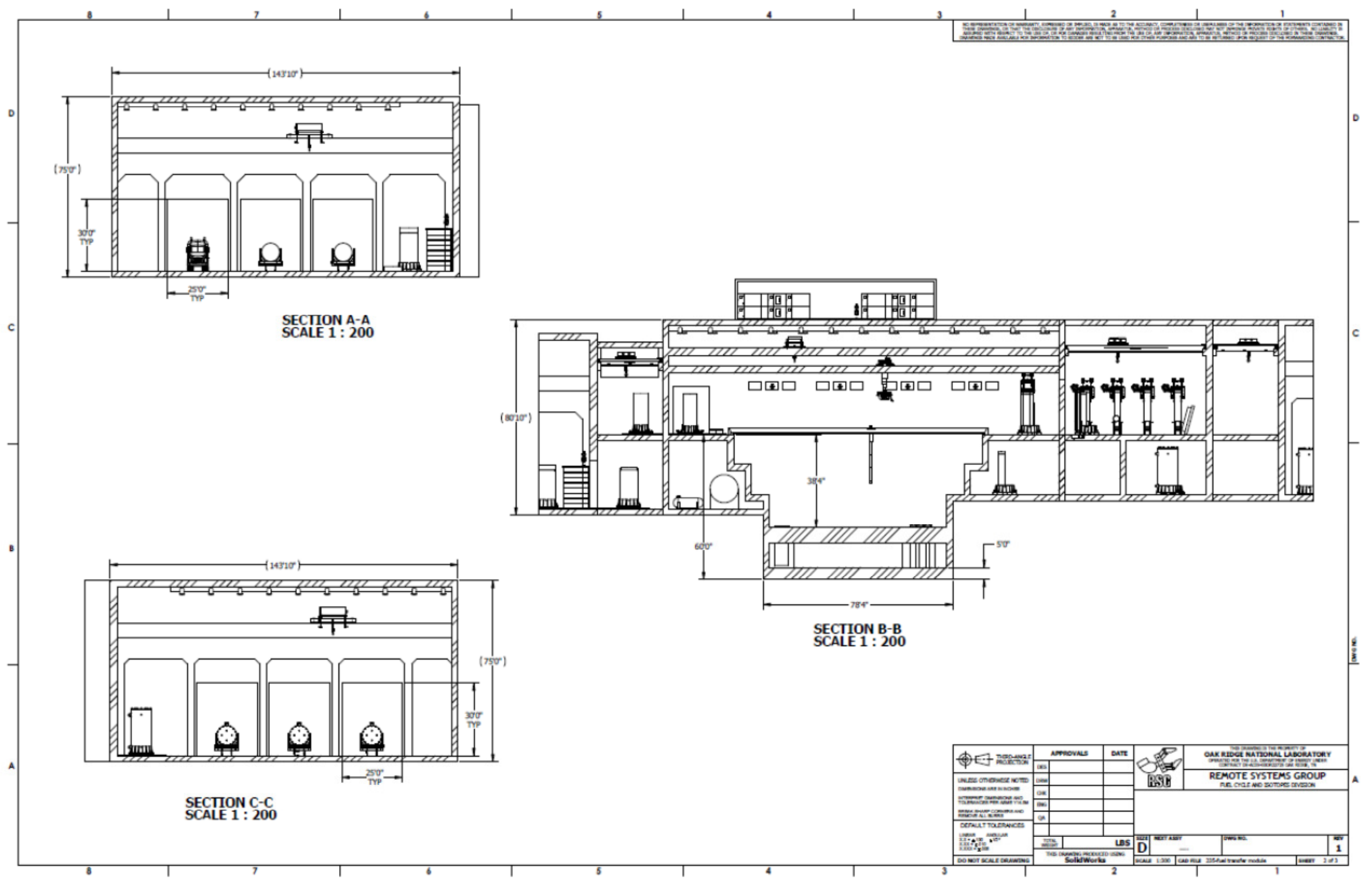




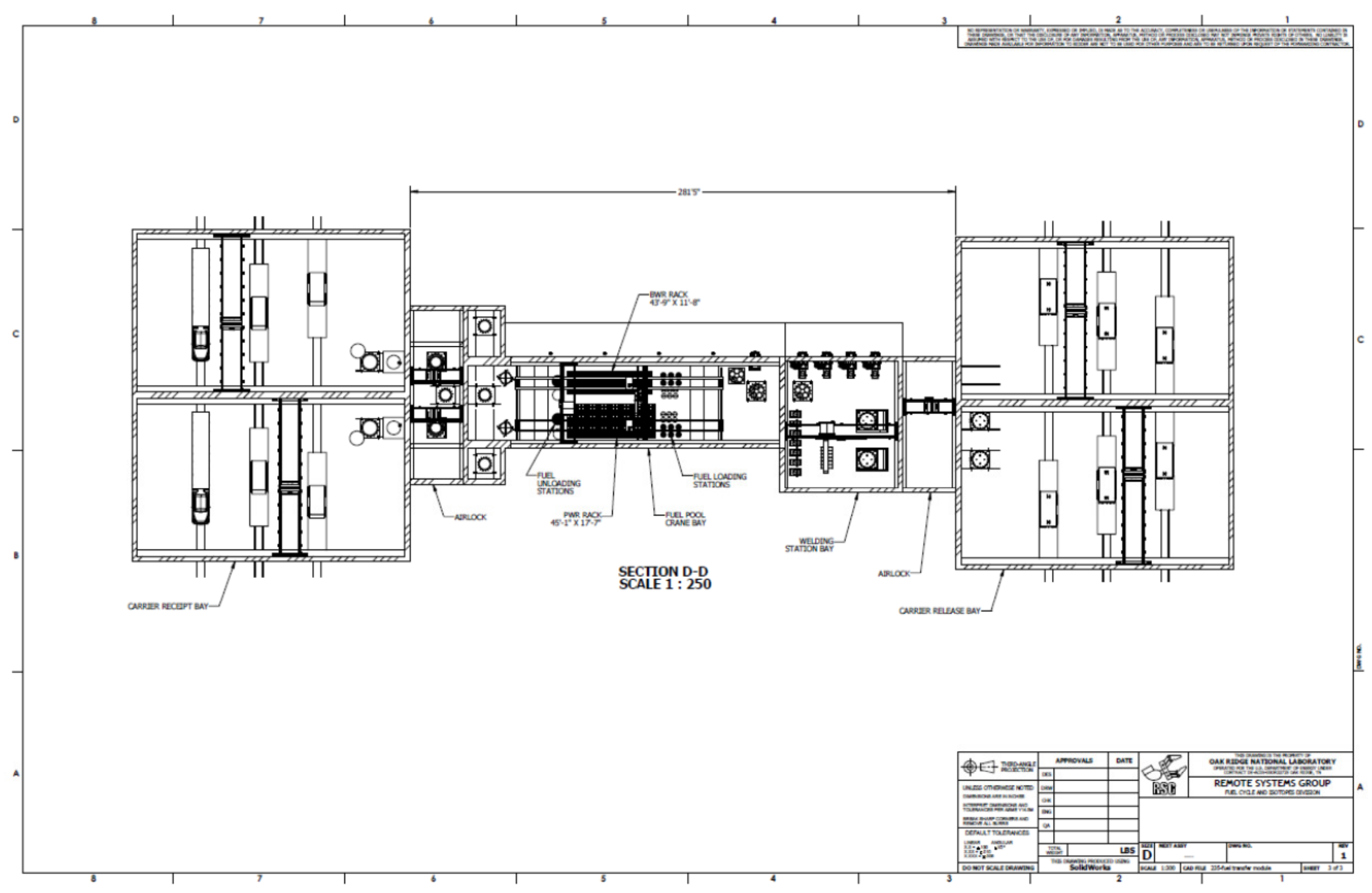


Used Fuel Management System Architecture Evaluation, Fiscal Year 2012

October 2012

INTENTIONALLY LEFT BLANK 


\section{APPENDIX C}

\section{ROUGH ORDER OF MAGNITUDE COST ESTIMATING METHODOLOGY}

Appendix $\mathrm{C}$ describes the methodology used to derive rough order of magnitude (ROM) unit cost estimates for the consolidated storage facility (CSF), test-validation facility (TVF) and packaging/repackaging facility (RF) modules described in Appendix B. This appendix provides a work breakdown structure (WBS) at a sub-module level. The individual WBS elements are used to derive costs for various system configurations, capabilities, throughput, and capacity. For the purpose of this evaluation, the unit cost estimates were allocated to the cost nomenclature defined below.

\section{Cost Nomenclature}

The unit costs estimates included the Total Project Costs (TPC) sometimes referred to as capital cost; the operations and maintenance cost (O\&M) during the operational period; and the total Life Cycle Cost (LCC) which combined the TPC and O\&M cost with additional capital project costs and facility decommissioning costs.

\section{Cost Basis}

Each of the costs defined above were determined parametrically based on prior cost estimates for "like" or extremely similar facilities and operational concepts. These parametric costs are discussed below in Sections C.1 and C.2.

The basis for these parametric costs were generally information contained in the working files supporting the Engineering Alternative Study (EAS) for Separations -Summary Report (DOE 2007b) and Follow-on Engineering Alternative Study (FOEAS) Summary Report, Global Nuclear Energy Partnership (DOE 2008) conducted for the U.S. Department of Energy, Office of Nuclear Energy (DOE- NE). These studies included three dry fuel storage methods; pool storage; receipt facilities for large dry storage canisters with transportation over packs; receipt facilities for bare fuel transportation casks; and many other site infrastructure and balance of plant (BOP) facilities.

Packaging/repackaging facilities were not directly included in these prior studies, but parametric costs for the similar hardened radioactive material processing buildings included in these studies were used to estimate applicable parts of this facility. A cost study for the TVF was recently completed and the results were documented in the Used Fuel Research and Development Test and Validation Facility Cost Study Report (DOE 2012d).

\section{C.1 Operation and Maintenance Costs}

Operations and Maintenance costs (O\&M) were estimated based on the full time equivalent (FTE) annual staffing for the following three categories: 1) management, 2) salaried, and 3) hourly. The staffing was converted to annual labor cost by the labor rates in Table A-1, and adding the labor overhead cost at $28.5 \%$ (21.5\% overheads and $7 \%$ fee). 
Table C-1 Staffing Labor Rates

\begin{tabular}{|l|r|}
\hline \multicolumn{1}{|c|}{$\begin{array}{c}\text { Labor } \\
\text { Category }\end{array}$} & $\begin{array}{c}\text { Annual } \\
\text { Labor Cost } \\
(\$ / \mathbf{y r})\end{array}$ \\
\hline Management & 150,000 \\
\hline Salaried & 150,000 \\
\hline Hourly & 83,000 \\
\hline
\end{tabular}

\section{C.1.1 Resource (FTE) Staffing Estimates}

The resource (FTE) staffing estimates for dry fuel receipt and storage were determined based on the FOEAS (DOE 2008) Early Fuel Receipt Sensitivity Analysis in which 8,000 MT of used fuel was received at a rate of 1,500 MT/year. The staffing was split into two groups; "direct" fuel handling support and BOP staffing. This staffing estimate is in Table C-2, which also describes the method used to vary staffing as a function of processing rate. This method will be applied to determine staffing for future dry storage receipt and storage scenarios.

The FTE staffing estimates for bare fuel receipts and wet storage were estimated by the team based on the EAS (DOE 2007b) in which 3,000MT/year of fuel was received and stored in a pool awaiting reprocessing. Table $\mathrm{C}-2$ provides this base staffing estimate along with the method used to vary staffing as a function of processing rate.

For a given system level analysis, the total direct CSF staffing is the maximum of the dry or bare fuel handling staff. The maximum is used as the bare and dry fuel arrive at the facility over two different time frames. CSF staffing is the total direct plus BOP staffing.

The TVF and RF staffing estimates determined by the team are also provided in Table C-2 along with the method used to vary the RF staffing as a function of capacity. The staffing for the TVF functions is assumed to be constant over the life of the facility.

\section{C.1.2 Annual Consumable Materials}

Consumable materials were estimated by algorithm based upon Savannah River Site (SRS) historical data. Consumable materials at SRS range from 7 to $15 \%$ of direct labor cost. This study assumes $10 \%$ of direct labor cost. Overheads at $28.5 \%$ are also applied. The RF materials cost also includes the cost of a thin wall (5/8") stainless steel dry storage canister. The unit cost for these canisters is provided in Table C-3. Previous more detailed studies of small disposable canisters (3 PWR) have used substantially thinner walls, from $3 / 16$ " to $3 / 8$ ", which could substantially reduce the relative cost of the small canisters (Morisette et al., 1989). This is one of many areas for possible future system optimization analysis. 
Table C-2 Staffing Estimates

\begin{tabular}{|c|c|c|}
\hline Base Staffing Description & $\begin{array}{l}\text { Full Time } \\
\text { Equivalent } \\
\text { (FTE) }\end{array}$ & Method of Scaling \\
\hline $\begin{array}{l}\text { Dry fuel receipt and inspection storage cask loading } \\
\text { and crane operations (30), } \\
\text { Cement Plant/Storage Cask Production (18), and } \\
\text { LLW Treatment and Packaging (7) }\end{array}$ & 55 & $\begin{array}{l}\text { Base staffing for } 1,500 \mathrm{MT} / \mathrm{yr} \text { staffing is } \\
\text { increased linear with receipt rate. The rate } \\
\text { adjusted value is compared to the rate adjusted } \\
\text { value for bare fuel handling (below) and the } \\
\text { maximum staffing is selected. }\end{array}$ \\
\hline $\begin{array}{l}\text { Bare Fuel Receipt and Inspection and Cask } \\
\text { Unloading }\end{array}$ & 116 & $\begin{array}{l}\text { Base staffing for 3,000 MT/yr staffing is } \\
\text { increased or decreased linear with receipt rate. } \\
\text { The rate adjusted value is compared to the rate } \\
\text { adjusted value for dry fuel handling (above) and } \\
\text { the maximum staffing is selected. }\end{array}$ \\
\hline $\begin{array}{l}\text { CSF BOP includes site management, engineering, } \\
\text { QA/QC, safety, radiation control, warehouse, entry } \\
\text { control, equipment maintenance, domestic and } \\
\text { sanitary water treatment, cranes and rigging support }\end{array}$ & 76 & Constant \\
\hline $\begin{array}{l}\text { TVF fuel handling includes fuel receipt/inspection, } \\
\text { cask and crane operations (10), } \\
\text { R\&D technicians and scientist (49), and } \\
\text { LLW staging (6) }\end{array}$ & 65 & Constant \\
\hline $\begin{array}{l}\text { TVF BOP includes site management, engineering, } \\
\text { QA/QC, safety, radiation control, equipment } \\
\text { maintenance, mock-up shop, and rigging support }\end{array}$ & 59 & Constant \\
\hline $\begin{array}{l}\text { RF fuel receipt/inspection, cask unloading and crane } \\
\text { operations }\end{array}$ & 145 & $\begin{array}{l}\text { Base facility operations for } 1500 \mathrm{MT} / \mathrm{yr} \text { capacity } \\
\text { for a } 4 / 9 \text { PWR/BWR size waste package. This } \\
\text { facility requires } 8 \text { final waste package welding } \\
\text { stations. The FTE staffing is a ratio to the } \\
\text { number of welding stations divided by } 8 \text {. }\end{array}$ \\
\hline $\begin{array}{l}\text { RF BOP includes site management, engineering, } \\
\text { QA/QC, safety, radiation control, warehouse, entry } \\
\text { control, equipment maintenance, domestic and } \\
\text { sanitary water treatment, cranes and rigging support }\end{array}$ & 76 & Constant \\
\hline
\end{tabular}

\section{C.1.3 Annual Utilities}

Utilities (electricity) estimates were based upon the power demand previously determined by the FOEAS (DOE 2008) Early Fuel Receipt Sensitivity Analysis for 1,500 MT/year receipt of dry fuel storage. Power consumption $(20,000 \mathrm{mw}-\mathrm{hr})$ is varied linearly with receipt rate and was doubled when pool storage is included to account for the additional mechanical equipment required. 
Table C-3 Repackaging Dry Storage Canister Unit Cost

\begin{tabular}{|c|c|c|c|}
\hline $\begin{array}{c}\text { Waste Package Compatible Size Canister } \\
\text { Description } \\
\text { (Assembly Capacity, Material, Thickness) }\end{array}$ & $\begin{array}{c}\text { Diameter } \\
(\mathbf{M})\end{array}$ & $\begin{array}{c}\text { Cost (\$) per } \\
\text { Package }\end{array}$ \\
\hline $4 \mathrm{PWR} / 9 \mathrm{BWR}$ & $5 / 8$ " Stainless & 0.82 & $\$ 60,000$ \\
\hline $12 \mathrm{PWR} / 24 \mathrm{BWR}$ & $5 / 8$ " Stainless & 1.29 & $\$ 90,000$ \\
\hline $21 \mathrm{PWR} / 44 \mathrm{BWR}$ & $5 / 8$ " Stainless & 1.60 & $\$ 117,000$ \\
\hline
\end{tabular}

Power consumption for the TVF was assumed to be 1/10 the FOEAS (DOE 2008) Early Fuel Receipt Sensitivity Analysis due to the low annual thru-put of the TVF. Power consumption for the RF was based upon the FOEAS (DOE 2008) for 1,500 MT/year. Power consumption was varied linearly with receipt rate. The cost of power was based on current Savannah River Site (SRS) cost of \$85/MW-hr.

\section{C.1.4 Contract Services and Spare Parts}

Contract services included janitorial services, equipment rentals (e.g. computers, non-routine heavy lift equipment), etc. These CSF costs were estimated based on the FOEAS (DOE 2008) Early Fuel Receipt Sensitivity Analysis at $\$ 250,000 / y r$. Contract services and spare parts were varied to account for the type of storage. The cost was assumed to double when both wet and dry storage is required.

Contract services and spare parts for TVF operations were assumed to be $\$ 250,000 /$ year. Contract services for RF was also estimated to have a base cost of $\$ 250,000 /$ year for the concepts described in Section 7.0. This cost was scaled to the number of package welding stations divided by the 4 (the number of welding stations in the based concept).

The cost of LLW disposal is also included as a contract service. One of the largest disposal costs is associated with discarding the DPC cut open at the RF. This is estimated by assuming the volume of a "typical" DPC, $2.5 \mathrm{~m}$ in diameter and $5 \mathrm{~m}$ in length and a disposal cost for Class A LLW of $\$ 1,650$ per cubic meter. The number of DPC required to be disposed annually is scenario specific.

\section{C.1.5 Annual O\&M to Life Cycle Operations Cost}

Table C-4 summarizes the operational years assumed for the facilities. The annual O\&M cost (labor, materials, utilities, contracts and spare parts) is converted to LCC operations cost by multiplying the annual O\&M cost by the total estimated number of operating years, plus an allowance for start-up (1 year prior to operations) staffing and initial decommissioning support (2 years).

For the CSF and TVF, the number of operating years is determined based on planned start of CSF (e.g., 2020 or 2035) to the end of the repository emplacement period. The end of the repository emplacement period is determined by the planned start of emplacement operations (e.g. 2040 or 2055) and the period required to emplace 140,000 MT at a specified rate of emplacement (e.g., 1,500, 3,000, or 6,000 MT/year). The resulting point value is then converted to a range by $80 \%$ low, $120 \%$ high algorithm

The RF is assumed to operate in support of repository emplacement. The operation time period is the waste emplacement time period plus an allowance of 1 year to build an initial inventory for emplacement and 3 years for start-up and decommissioning. The resulting point value is then converted to a range by $80 \%$ low, $120 \%$ high algorithm. 
Table C-4 Scenario Specific Operational Time Periods

\begin{tabular}{|c|c|c|c|}
\hline \multirow{2}{*}{\multicolumn{2}{|c|}{ Project Phase }} & \multicolumn{2}{|c|}{ Project Duration (yrs) } \\
\hline & & CSF and TVF & $\mathbf{R F}$ \\
\hline \multicolumn{2}{|c|}{ Operations Start-up } & 1 & $1+1$ \\
\hline \multicolumn{2}{|c|}{ CSF Operations } & & \\
\hline CSF Start & Repository Start & & \\
\hline 2020 & 2040 & 20 & \\
\hline 2020 & 2055 & 35 & \\
\hline 2035 & 2055 & 20 & \\
\hline \multicolumn{2}{|c|}{$\begin{array}{c}\text { Repository Operations Emplacement } \\
\text { Rate (MT/yr) }\end{array}$} & & \\
\hline \multicolumn{2}{|c|}{1,500} & 93 & 93 \\
\hline \multicolumn{2}{|c|}{3,000} & 47 & 47 \\
\hline \multicolumn{2}{|c|}{6,000} & 23 & 23 \\
\hline \multicolumn{2}{|c|}{ Decommissioning Support } & 2 & 2 \\
\hline
\end{tabular}

\section{C.1.6 Decommissioning and Dismantlement}

The life cycle operations cost also includes an allowance for D\&D. This cost is determined by algorithm where the D\&D costs is $10 \%$ of the TPC (Section C.2) plus 10\% of the additional capital used for building expansion (e.g. pool storage buildings) plus $2 \%$ of additional capital used for dry storage (e.g., storage over packs).

\section{C.2 Total Project Cost}

TPC is comprised of four major segments:

1) Conceptual Design

2) Site Improvements and Infrastructure

3) BOP facilities, and

4) Process Facilities

\section{C.2.1 Conceptual Design}

The study team estimated the conceptual design activities to range from $\$ 10,000 \mathrm{~K}$ to $\$ 15,000 \mathrm{~K}$ inclusive for all three of the major facilities (CSF, TVF and RF). A single conceptual design allowance was judged adequate given that all three facilities would be developed as an integrated facility. 


\section{C.2.2 Site Improvements and Infrastructure}

The site improvements and infrastructure estimate was based on the FOEAS (DOE 2008) Early Fuel Receipt Sensitivity Analysis in which a dry storage facility for 8,000 MT required approximately 50 acres. This area is representative of an initial capital investment needed to begin CSF operations site improvements and infrastructure. The cost is expected to range between $\$ 57,600 \mathrm{~K}$ and $\$ 74,900 \mathrm{~K}$ and is detailed below in Table C-5.

The same estimate is used for the RF site improvements and infrastructure cost since this facility may not be co-located with the CSF. The standalone RF will require these same WBS items. The TVF is to be co-located with the CSF. Therefore, no additional cost is assumed.

\section{C.2.3 Balance of Plant}

Similarly, BOP WBS items are also based upon FOEAS (DOE 2008) Early Fuel Receipt Sensitivity Analysis. These items are expected to range between $\$ 33,040 \mathrm{~K}$ and $\$ 42,170 \mathrm{~K}$ and are detailed below in Table C-6. The same estimate is used for the RF which may not be co-located with the CSF. The TVF is co-located with the CSF. Therefore, no additional cost is assumed.

\section{C.2.4 Process Facilities}

Process Facilities estimates are derived from the EAS (DOE 2007b), FOEAS (DOE 2008) Early Fuel Receipt Sensitivity Analysis and TVF Cost Study (DOE 2012d) estimates. These WBS items are summarized in Table C-7, which includes the data source study, the cost estimate range, a description of the sub-items included in the individual item and a resulting unit cost derived by this study.

For example, the 3,000 MT/yr (EAS (DOE 2007b)) reprocessing estimate included 6 horizontal storage vaults at a cost range of $\$ 125,796 \mathrm{~K}$ to $\$ 155,747 \mathrm{~K}$ for a unit cost range of $\$ 62,900 \mathrm{~K}$ to $\$ 77,900 \mathrm{~K}$ which will be used in system level scenarios requiring horizontal storage.

Cask receiving/shipping areas, pool storage areas, pool HVAC, and pool support areas were derived from the EAS (DOE 2007b) - Fuel Handling Building estimate using the foot print and equipment allocated to these areas. These sub-module WBS elements are used in system level analysis to derive system level costs for both bare fuel CSF functions and some RF functions.

The RF area for canister opening is $\sim 9,200$ sq.ft. and for canister closure is $\sim 15,200$ sq.ft. (Appendix B). These areas were converted to cost using the cost per sq.ft. parametric from the TVF $(\sim \$ 10,160 / \mathrm{sq}$. ft. to $\$ 14,730 /$ sq.ft.). Use of this parametric is judged acceptable since both processes will require a performance category 3 structure and both handle bare fuel (potentially damaged) assemblies, using remote handling techniques.

Table C-8 provides an example TPC roll-up for the RF facility with a through-put of 1,500 MT/year. Each of the facility segments in Table A-8 will be varied for specific system level analyses scenarios.

The LLW staging facility was derived from the FOEAS (DOE 2008) Early Fuel Receipt Sensitivity Analysis. This WBS item was assumed to double for the RF which is expected to generate more LLW from repackaging compared to fuel storage. The TVF was taken from the TVF Cost Study (DOE 2012d) which was specific to this WBS item.

\section{C.3 Additional Capital}

The TPC is scoped to provide 2 years of initial dry storage capacity or the first pool building which contains 8 basins and the associated support facilities (water treatment, HVAC, etc.) Storage requirements beyond the initial TPC require additional capital, which is scenario specific.

The same unit costs in Table C-7 are used to estimate the additional capital required for the CSF Life Cycle. Additional capital is not required for the TVF or RF. 
Table C-5 Site Improvements and Infrastructure Unit Costs

\begin{tabular}{|c|c|c|c|c|}
\hline WBS & Description & Source Data & $\begin{array}{c}\text { WBS } \\
\text { Low Range } \\
\text { Costs }(\$ 1000) \\
\end{array}$ & $\begin{array}{c}\text { WBS } \\
\text { High Range } \\
\text { Costs }(\$ 1000) \\
\end{array}$ \\
\hline 01.02.03 & Site Improvements \& Infrastructure & & $\$ 57,591$ & $\$ 74,870$ \\
\hline 01.02 .03 .01 & Clearing \& Grading & FOEAS SA2 Early Fuel Receipt ${ }^{1}$ & $\$ 5,749$ & $\$ 7,187$ \\
\hline 01.02.03.02 & $\begin{array}{l}\text { Construction Roads \& Laydown \& Central } \\
\text { Temporary Facilities (Including permanent Cement } \\
\text { Batch Plant and Silos) }\end{array}$ & FOEAS SA2 Early Fuel Receipt ${ }^{1}$ & $\$ 8,565$ & $\$ 10,706$ \\
\hline 01.02.03.03 & Retention Pond (1) \& Storm Drainage & FOEAS SA2 Early Fuel Receipt ${ }^{1}$ & $\$ 4,328$ & $\$ 5,410$ \\
\hline 01.02.03.05 & Paved Roads & FOEAS SA2 Early Fuel Receipt ${ }^{1}$ & $\$ 4,753$ & $\$ 5,941$ \\
\hline 01.02.03.06 & Parking Areas & FOEAS SA2 Early Fuel Receipt ${ }^{1}$ & $\$ 901$ & $\$ 1,126$ \\
\hline 01.02.03.07 & Landscaping & FOEAS SA2 Early Fuel Receipt ${ }^{1}$ & $\$ 1,643$ & $\$ 2,054$ \\
\hline 01.02.03.08 & Railroads & FOEAS SA2 Early Fuel Receipt ${ }^{1}$ & $\$ 10,942$ & $\$ 13,677$ \\
\hline 01.02.03.15 & Admin Building & FOEAS SA2 Early Fuel Receipt ${ }^{1}$ & $\$ 1,751$ & $\$ 2,501$ \\
\hline 01.02.03.19 & $\begin{array}{l}\text { Electrical Switch Yard (Including Site Elec. } \\
\text { Distribution) }\end{array}$ & FOEAS SA2 Early Fuel Receipt ${ }^{1}$ & $\$ 6,392$ & $\$ 9,132$ \\
\hline 01.02.03.24 & Cranes and Rigging Building & FOEAS SA2 Early Fuel Receipt ${ }^{1}$ & $\$ 5,465$ & $\$ 7,807$ \\
\hline 01.02 .03 .25 & Cranes and Rigging Laydown & FOEAS SA2 Early Fuel Receipt ${ }^{1}$ & $\$ 99$ & $\$ 123$ \\
\hline 01.02.03.26 & Rad Support Services & FOEAS SA2 Early Fuel Receipt ${ }^{1}$ & $\$ 4,197$ & $\$ 5,197$ \\
\hline 01.02.03.30 & General Warehouse & FOEAS SA2 Early Fuel Receipt ${ }^{1}$ & $\$ 2,806$ & $\$ 4,009$ \\
\hline
\end{tabular}

1. Information from the working files supporting the FOEAS sensitivity analysis \#2, Early Fuel Receipt (DOE 2008) 
Table C-6 Balance of Plant Facilities

\begin{tabular}{|c|c|c|c|c|}
\hline WBS & Description & Source Data & $\begin{array}{c}\text { WBS } \\
\text { Low Range } \\
\text { Costs }(\$ 1000) \\
\end{array}$ & $\begin{array}{c}\text { WBS } \\
\text { High Range } \\
\text { Costs }(\$ 1000) \\
\end{array}$ \\
\hline 01.02 .04 & Balance of Plant (BOP) Facilities & & $\$ 33,037$ & $\$ 42,171$ \\
\hline 01.02 .04 .11 & Site Boundary Entrance Control (1) & FOEAS SA2 Early Fuel Receipt $^{1}$ & $\$ 4,090$ & $\$ 4,980$ \\
\hline 01.02 .04 .21 & Domestic Water Treatment Plant & FOEAS SA2 Early Fuel Receipt ${ }^{1}$ & $\$ 9,117$ & $\$ 11,288$ \\
\hline 01.02.04.22 & $\begin{array}{l}\text { Domestic Water Storage Tank w Wells (1), Includes } \\
\text { supply and return system }\end{array}$ & FOEAS SA2 Early Fuel Receipt ${ }^{1}$ & $\$ 2,229$ & $\$ 2,694$ \\
\hline 01.02.04.23 & Sanitary Waste Treatment & FOEAS SA2 Early Fuel Receipt ${ }^{1}$ & $\$ 5,055$ & $\$ 6,258$ \\
\hline 01.02.04.25 & Fire Water Tank with Pump House (1) & FOEAS SA2 Early Fuel Receipt ${ }^{1}$ & $\$ 8,081$ & $\$ 11,544$ \\
\hline 01.02.04.37 & Gray Water Pond & FOEAS SA2 Early Fuel Receipt ${ }^{1}$ & $\$ 243$ & $\$ 303$ \\
\hline 01.02.04.38 & Electric Substation(1) & FOEAS SA2 Early Fuel Receipt ${ }^{1}$ & $\$ 4,222$ & $\$ 5,104$ \\
\hline
\end{tabular}

1. Information from the working files supporting the FOEAS sensitivity analysis \#2, Early Fuel Receipt (DOE 2008) 


\begin{tabular}{|c|c|c|c|c|c|c|}
\hline & & \multirow[b]{2}{*}{ Unit Cost Basis } & \\
\hline Description & Source Data & $\begin{array}{c}\text { WBS } \\
\text { Low Range } \\
\text { Costs }(\$ 1000) \\
\end{array}$ & $\begin{array}{c}\text { WBS } \\
\text { High Range } \\
\text { Costs }(\$ 1000) \\
\end{array}$ & & $\begin{array}{c}\text { WBS } \\
\text { Low Range } \\
\text { Costs }(\$ 1000) \\
\end{array}$ & $\begin{array}{c}\text { WBS } \\
\text { High Range } \\
\text { Costs }(\$ 1000) \\
\end{array}$ \\
\hline \multicolumn{7}{|l|}{ Dry Storage Concept WBS } \\
\hline Canister Transfer Building & FOEAS SA2 Early Fuel Receipt ${ }^{1}$ & $\$ 124,164$ & $\$ 170,876$ & 2 Transfer Stations & $\$ 124,164$ & $\$ 170,876$ \\
\hline Horizontal Dry Cask Storage & EAS $3000 \mathrm{MT} / \mathrm{yr}(\text { Benchmark 1) })^{2}$ & $\$ 125,796$ & $\$ 155,747$ & $\begin{array}{l}6 \text { Vaults for } 12 \text { DSC } \\
\text { Unit Cost per Vault }\end{array}$ & $\$ 62,898$ & $\$ 77,874$ \\
\hline $\begin{array}{l}\text { Pads \& Cask Storage } \\
\text { (Including Storage Casks) }\end{array}$ & FOEAS SA2 Early Fuel Receipt ${ }^{1}$ & $\$ 220,389$ & $\$ 272,863$ & $\begin{array}{l}30-8 \text { Cask Pads } \\
\text { Unit Cost per Pad }\end{array}$ & $\$ 7,346$ & $\$ 9,095$ \\
\hline DSC Dry Vault Storage & $\begin{array}{l}\text { EAS } 3000 \text { MT/yr (Benchmark 1) }{ }^{2}- \\
\text { Split Fuel Building }\end{array}$ & $\$ 459,989$ & $\$ 667,794$ & $\begin{array}{r}132 \text { positions } \\
\text { increased to } 269 \\
\text { positions }\end{array}$ & $\$ 1,031,490$ & $\$ 1,360,883$ \\
\hline \multicolumn{7}{|l|}{$\begin{array}{l}\text { Bare Fuel Handling Concept } \\
\underline{\text { WBS }}\end{array}$} \\
\hline Cask Receiving/Shipping & $\begin{array}{l}\text { EAS } 3000 \text { MT/yr (Benchmark 1) }{ }^{2}- \\
\text { Split Fuel Building }\end{array}$ & $\$ 633,088$ & $\$ 908,305$ & $\begin{array}{l}5 \text { Receipt Bays Unit } \\
\text { Cost per Bay }\end{array}$ & $\$ 126,618$ & $\$ 181,661$ \\
\hline Pool Area & $\begin{array}{l}\text { EAS } 3000 \text { MT/yr (Benchmark 1) }{ }^{2}- \\
\text { Split Fuel Building }\end{array}$ & $\$ 1,784,953$ & $\$ 2,508,755$ & $\begin{array}{r}4 \text { Basins Unit Cost } \\
\text { Per Basin }\end{array}$ & $\$ 446,238$ & $\$ 627,189$ \\
\hline Pool HVAC & $\begin{array}{l}\text { EAS } 3000 \text { MT/yr (Benchmark 1) }{ }^{2}- \\
\text { Split Fuel Building }\end{array}$ & $\$ 83,062$ & $\$ 144,076$ & $\begin{array}{r}\text { HVAC System for } \\
\text { every } 8 \text { Basins }\end{array}$ & $\$ 83,062$ & $\$ 144,076$ \\
\hline Pool Support Area & $\begin{array}{l}\text { EAS } 3000 \text { MT/yr (Benchmark 1) }{ }^{2}- \\
\text { Split Fuel Building }\end{array}$ & $\$ 134,289$ & $\$ 215,252$ & $\begin{array}{r}\text { Pool Support for } \\
\text { every } 8 \text { Basins }\end{array}$ & $\$ 134,289$ & $\$ 215,252$ \\
\hline \multicolumn{7}{|l|}{$\begin{array}{l}\text { Support Facility Concept } \\
\underline{\text { WBS }}\end{array}$} \\
\hline LLW Staging Area & FOEAS SA2 Early Fuel Receipt ${ }^{1}$ & $\$ 378$ & $\$ 468$ & Doubled for RF & $\$ 378$ & $\$ 468$ \\
\hline TVF Mock-Up Facility & T\&V Facility Report Table S-2 ${ }^{3}$ & $\$ 150,000$ & $\$ 220,000$ & Not Scaled & $\$ 150,000$ & $\$ 220,000$ \\
\hline Test and Validation Facility & T\&V Facility Report Table S- $2^{3}$ & $\$ 1,330,000$ & $\$ 1,940,000$ & $\begin{array}{l}\text { 78,570 Sq. Ft. Unit } \\
\text { Cost per Sq. Ft. }\end{array}$ & $\$ 10.16$ & $\$ 14.73$ \\
\hline
\end{tabular}

1. Information from the working files supporting the FOEAS sensitivity analysis \#2, Early Fuel Receipt (DOE 2008)

2. Information from the working files supporting the EAS for Separations - Summary Report (DOE 2007b)

3. Information from the Used Fuel research and Development Test and Validation Facility Cost Study (DOE 1012d) 
Table C-8 Example Roll-Up ROM Cost Estimate - 1,500 MT/yr Repackaging Facility TPC

\begin{tabular}{rcc}
\hline & \multicolumn{2}{c}{ Prior Study TPC } \\
\cline { 2 - 3 } Description & $\begin{array}{c}\text { WBS } \\
\text { Low Range } \\
\text { Costs } \mathbf{\$ 1 0 0 0})\end{array}$ & $\begin{array}{c}\text { WBS } \\
\text { High Range } \\
\text { Costs } \mathbf{\$ 1 0 0 0} \text { ) }\end{array}$ \\
\hline Pool Area & $\$ 253,235$ & $\$ 363,322$ \\
HVAC & $\$ 443,051$ & $\$ 622,709$ \\
Pool Support Area & $\$ 145,358$ & $\$ 252,132$ \\
Outbound shipping Bays & $\$ 16,666$ & $\$ 26,714$ \\
Canister Loading cells & $\$ 1,646,028$ & $\$ 2,361,592$ \\
TPC Total & $\$ 268,800$ & $\$ 389,760$ \\
\hline $\mathbf{2 , 8 1 9 , 8 6 9}$ & $\mathbf{\$ 4 0 8 3 , 9 8 8}$ \\
\hline
\end{tabular}

\section{C.4 REFERENCES FOR APPENDIX C}

1. (DOE 2007a) - Follow-on Engineering Alternative Studies for Separations(FOEAS), Early Fuel Receipt \& Storage (EFR\&S) Considerations, FRS-G-ESR-G-00053, Rev. 0, September 2007, P. Rodwell, R. Geddes, S. McConnell

2. (DOE 2007b) -Engineering Alternative Studies(EAS) for Separations-Summary Report, EAS-GESR-G-00049, June 2007, S. McConnell, D. W. Ostby, et.al

3. (DOE 2008) - Follow-on Engineering Alternative Studies (FOEAS) Summary Report, Global Nuclear Energy Partnership (GNEP), GNEP-CFTC-PMO-MI-DV-2008-00087, March 2008, Consolidated Fuel Treatment Center

4. (DOE 2012d) - Used Fuel Research and Development Test and Validation Facility Cost Study, FCRD-UFD-2012-000206, Rev. 0, August 2012, J. Carter, A. Delley, et. al

5. R. R Morissette et al., Commercial Radioactive Waste Management System Feasibility with the Universal Canister Concept, Volume I, General Atomics Report GA-A--1832o-Vol.L Prepared for OCRWM, January 1986; U.S. Department of Energy, MRS System Study Summary Report, DOE/RW-0235, June 1989. 